\title{
Improving Vortex Generators to Enhance the Performance of Air- Cooled Condensers in a Geothermal Power Plant
}

\author{
Manohar S. Sohal \\ September 2005
}

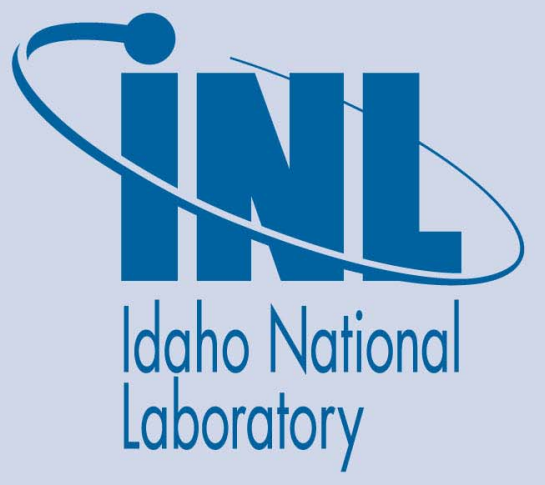

The INL is a U.S. Department of Energy National Laboratory operated by Battelle Energy Alliance 
INL/EXT-05-00627

\title{
Improving Vortex Generators to Enhance the Performance of Air-Cooled Condensers in a Geothermal Power Plant
}

\author{
Manohar S. Sohal
}

September 2005

Idaho National Laboratory
Idaho Falls, Idaho 83415

Prepared for the

U.S. Department of Energy

Assistant Secretary for Energy Efficiency and Renewable Energy Under DOE Idaho Operations Office

Contract DE-AC07-05ID14517 


\section{ABSTRACT}

This report summarizes work at the Idaho National Laboratory to develop strategies to enhance air-side heat transfer in geothermal air-cooled condensers such that it should not significantly increase pressure drop and parasitic fan pumping power.

The work was sponsored by the U.S. Department of Energy, NEDO (New Energy and Industrial Technology Development Organization) of Japan, Yokohama National University, and the Indian Institute of Technology, Kanpur, India.

A combined experimental and numerical investigation was performed to investigate heat transfer enhancement techniques that may be applicable to largescale air-cooled condensers such as those used in geothermal power applications. A transient heat transfer visualization and measurement technique was employed in order to obtain detailed distributions of local heat transfer coefficients on model fin surfaces. Pressure drop measurements were obtained for a variety of tube and winglet configurations using a single-channel flow apparatus that included four tube rows in a staggered array. Heat transfer and pressure drop measurements were also acquired in a separate multiple-tube row apparatus in the Single Blow Test Facility. In addition, a numerical modeling technique was developed to predict local and average heat transfer for these low-Reynoldsnumber flows, with and without winglets. Representative experimental and numerical results were obtained that reveal quantitative details of local finsurface heat transfer in the vicinity of a circular tube with a single delta winglet pair downstream of the cylinder. Heat transfer and pressure-drop results were obtained for flow Reynolds numbers based on channel height and mean flow velocity ranging from 700 to 6500 . The winglets were of triangular (delta) shape with a $1: 2$ or $1: 3$ height/length aspect ratio and a height equal to $90 \%$ of the channel height. Overall mean fin-surface heat transfer results indicate a significant level of heat transfer enhancement (in terms of Colburn j-factor) associated with deployment of the winglets with circular as well as oval tubes. In general, toe-in (common flow up) type winglets appear to have better performance than the toe-out (common flow down) type winglets. Comparisons of heat transfer and pressure drop results for the elliptical tube versus a circular tube with and without winglets are provided.

During the course of their independent research, all of the researchers have established that about 10 to $30 \%$ enhancement in Colburn j-factor is expected. However, actual increase in heat transfer rate from a heat exchanger employing finned tubes with winglets may be smaller, perhaps on the order of 2 to $5 \%$. It is also concluded that for any specific application, more full-size experimentation is needed to optimize the winglet design for a specific heat exchanger application. If in place of a circular tube, an oval tube can be economically used in a bundle, it is expected that the pressure drop across the tube bundle with the application of vortex generators (winglets) will be similar to that in a conventional circular tube bundle. It is hoped that the results of this research will demonstrate the benefits of applying vortex generators (winglets) on the fins to improve the heat transfer from the air-side of the tube bundle. 


\section{ACKNOWLEDGMENTS}

The author acknowledges the contribution of the following individuals who performed the research described in this report; their respective responsibilities are indicated:

J. E. O’Brien (Idaho National Laboratory), INL experimental work

T. D. Foust (former student at the University of Idaho and Idaho National Laboratory, now at the National Renewable Energy Laboratory); INL numerical work

P. S. Wallstedt (former student at the University of Idaho); INL experimental work

K. Torrii and students (Yokohama National University, Japan); NEDO-funded experimental work

K. Nishino (Yokohama National University, Yokohama, Japan); NEDO-funded experimental work

G. Biswas \& students (Indian Institute of Technology, Kanpur, India); NEDO-funded numerical work

G. L. Mines (Idaho National Laboratory); programmatic guidance and support.

This work was sponsored by the U.S. Department of Energy, Geothermal Program. The Idaho National Laboratory is operated by Battelle Energy Alliance. The author also acknowledges the support received from the Japanese New Energy and Industrial Technology Development Organization, under Research Grant 99EF1. 


\section{CONTENTS}

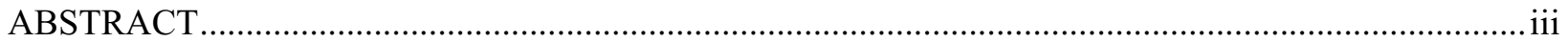

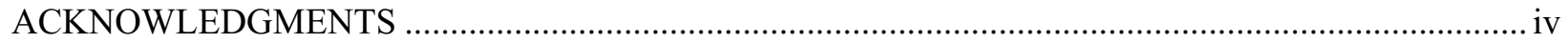

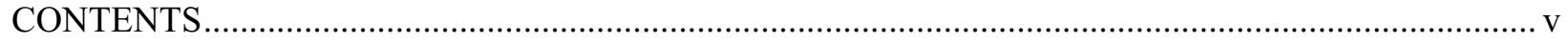

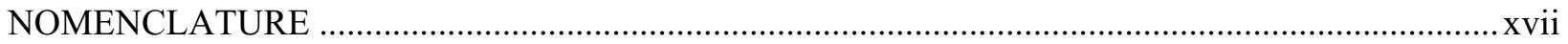

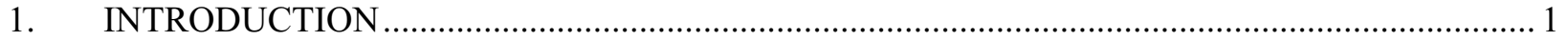

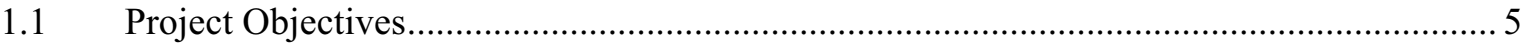

2. INL LABORATORY-SCALE EXPERIMENTS ON SINGLE TUBES .................................... 6

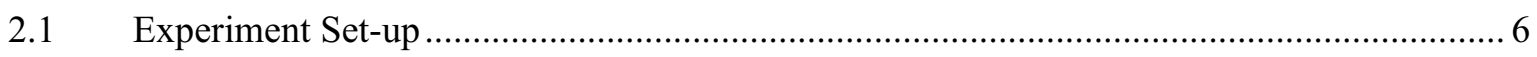

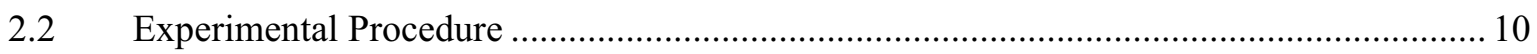

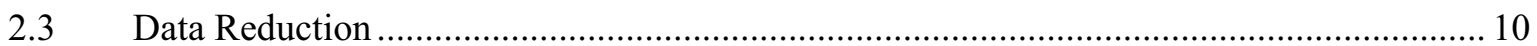

2.4 Heat Transfer with Single Cylindrical Tubes .............................................................. 11

2.5 Heat Transfer with Single Cylindrical Tubes and Winglets ............................................ 14

2.6 Heat Transfer with Single Oval Tubes and Winglets ...................................................... 19

2.7 Pressure Drop with Tube Bundle Model ........................................................................... 23

3. INL SINGLE BLOW TESTING OF A HEAT EXCHANGER TUBE BUNDLE ........................ 27

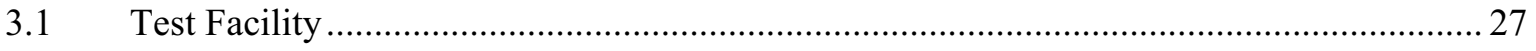

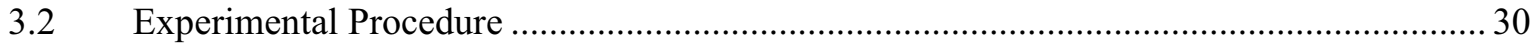

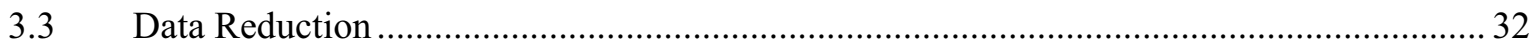

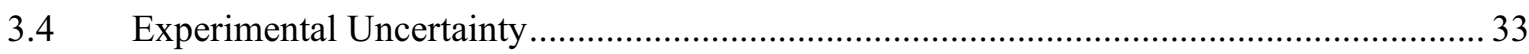

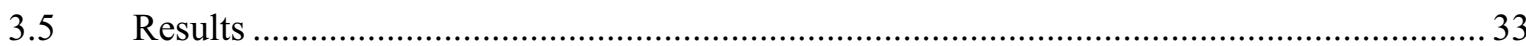

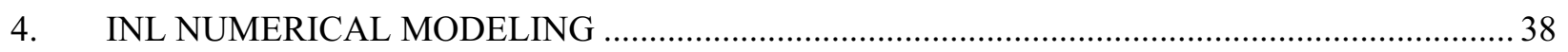

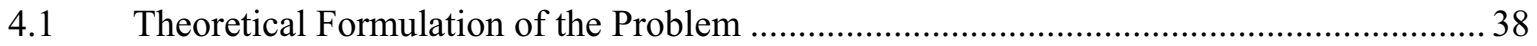

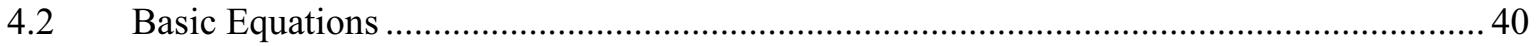

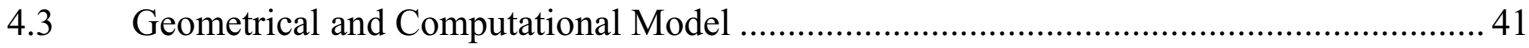

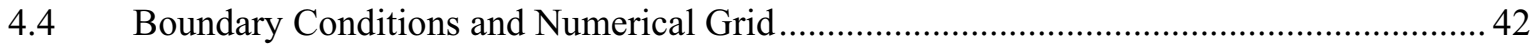

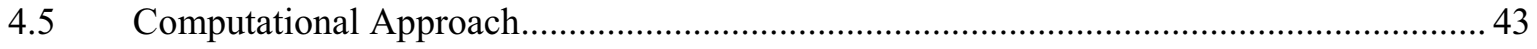

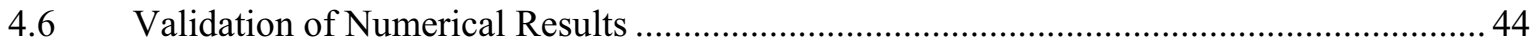

4.7 Single Oval and Circular Tube Comparison................................................................... 48

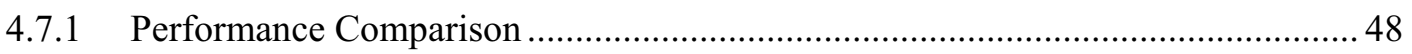

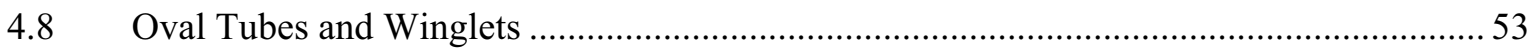

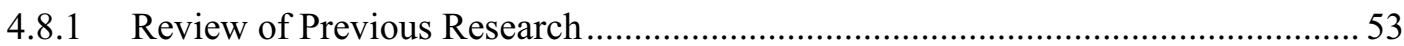

4.8.2 Winglet Configurations for a Single Oval Finned Tube .......................................55

4.8.3 Performance Ratio for Various Winglet Configurations on Finned Oval Tube ..... 57

4.9 Fluid Flow Pattern and Heat Transfer Contours........................................................... 58 


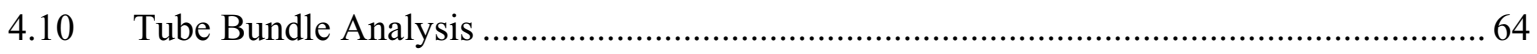

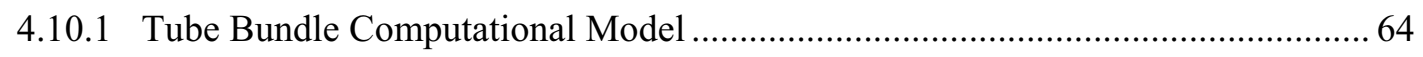

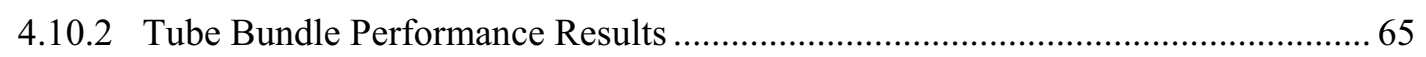

4.10.3 Comparison of In-line versus Staggered Tube Bundle Performance ....................... 68

4.11 Fluid Flow Contours and Heat Transfer Pattern for Tube Bundles..................................... 68

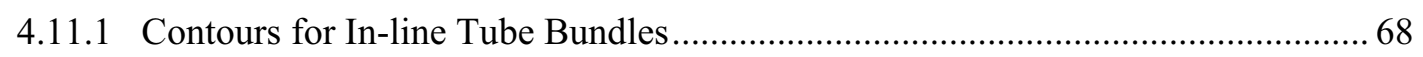

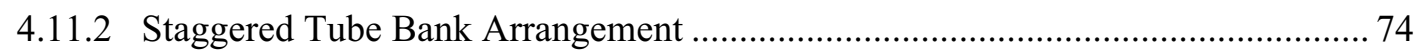

4.12 Conclusions from the Analysis of the Tube Bank Arrangement ...................................... 80

5. YOKOHAMA NATIONAL UNIVERSITY EXPERIMENTAL WORK .................................... 81

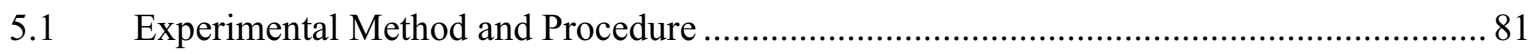

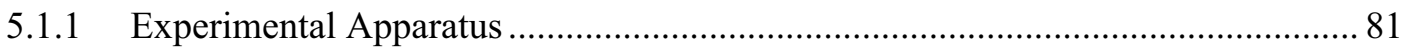

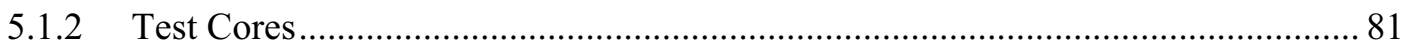

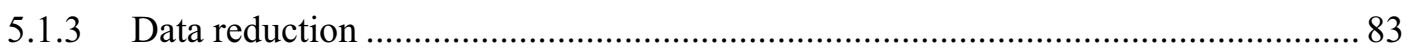

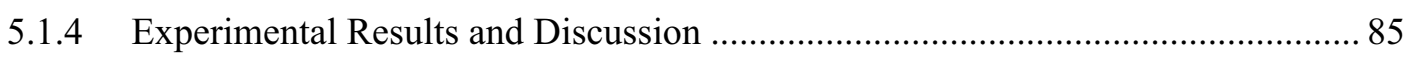

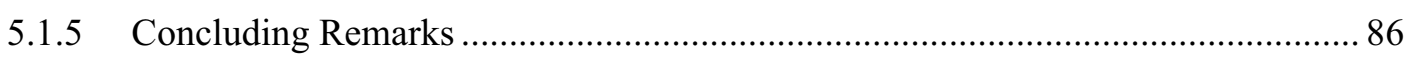

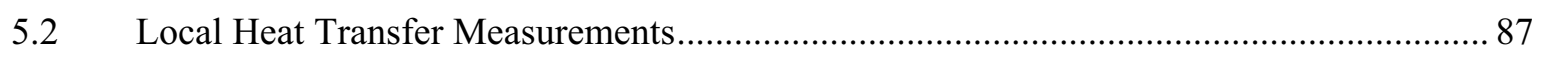

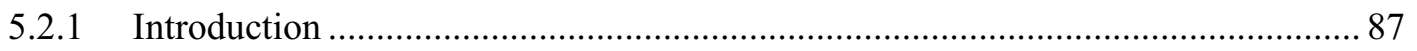

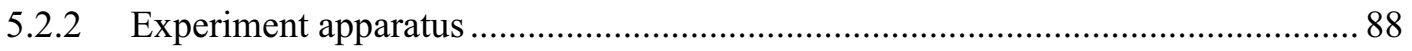

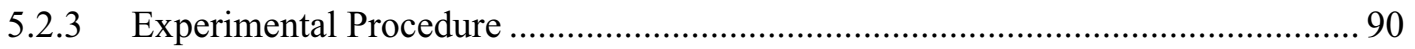

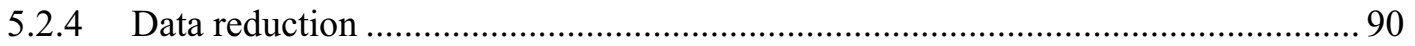

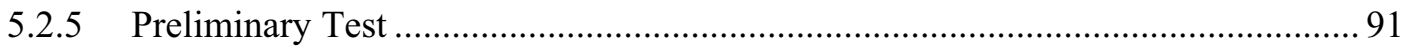

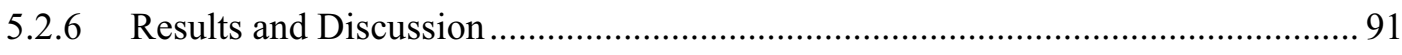

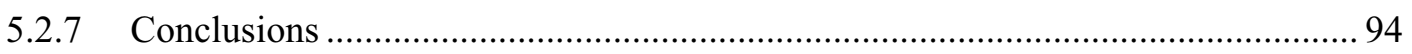

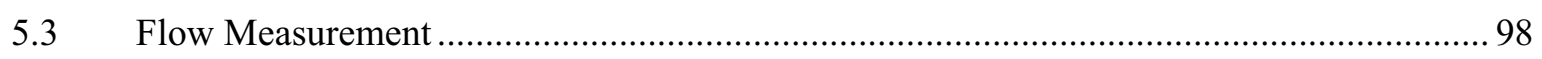

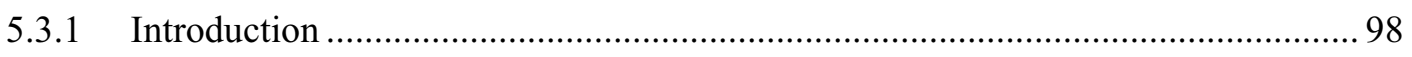

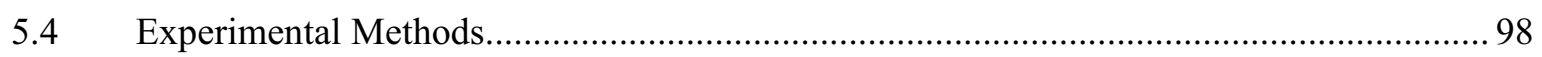

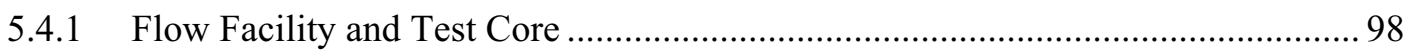

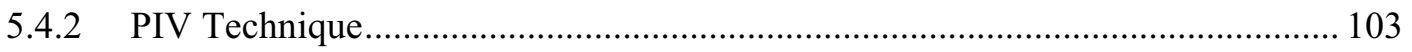

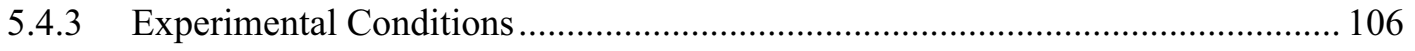

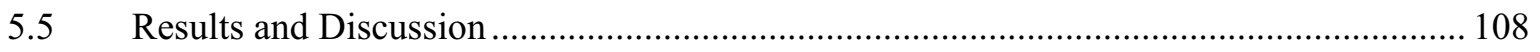

5.5.1 Flow Characteristics in Arrangement I (Single Row Tubes) ............................... 108

5.5.2 Advanced Analysis of Flow Fields in Arrangement I (Single Row Tubes) ......... 114

5.5.3 Flow Characteristics in Arrangement II (Three-Row In-Line Tube Bundle with Single Row Winglets) .................................................................... 121

5.5.4 Flow Characteristics in Arrangement III (Three-Row In-Line Tube Bundle with Three-Row Winglets). 
5.5.5 Flow Characteristics in Arrangement IV (Three-Row Staggered Tube Bundle) . 125

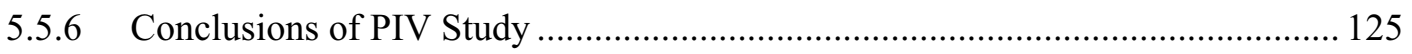

6. INDIAN INSTITUTE OF TECHNOLOGY NUMERICAL WORK (NEDO)............................ 131

6.1 Numerical Prediction of Flow and Heat Transfer in a Rectangular Channel with a

Built-in Circular Tube ......................................................................................................... 131

6.1.1 Formulation of the Problem............................................................................... 132

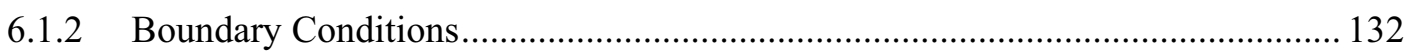

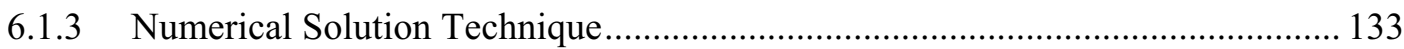

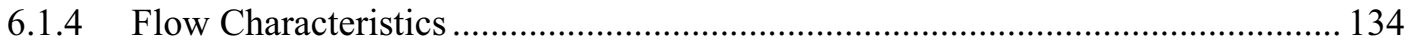

6.1.5 Flow Topology on the Horizontal and the Vertical Planes .................................. 134

6.1.6 Limiting Streamlines on the Tube Surface and the Bottom Plate ........................ 136

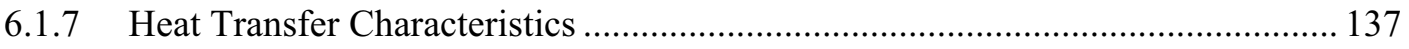

6.1.8 Validation of Results and Grid Independence ................................................ 142

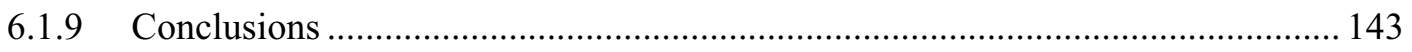

6.2 Winglet-Type Vortex Generators with Common-flow-up Configuration for Fin-Tube

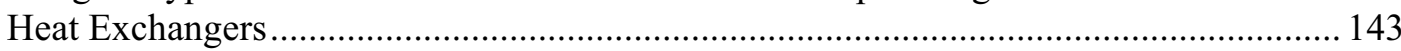

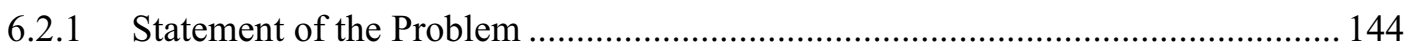

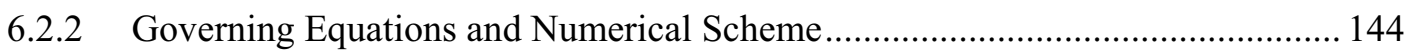

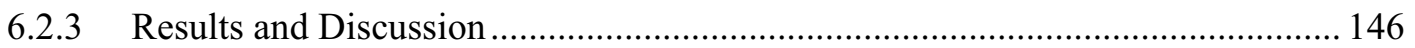

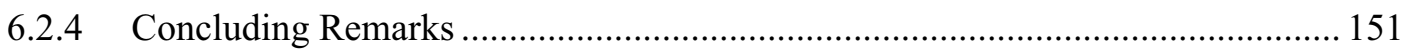

6.3 Numerical Prediction of Heat Transfer in a Channel with a Built-in Oval Tube ............. 151

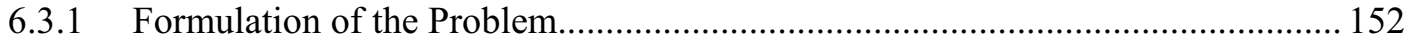

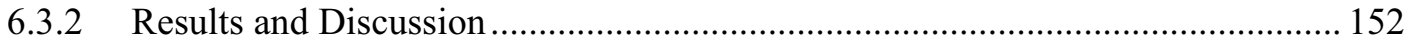

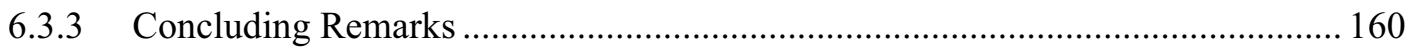

6.4 Heat Transfer Enhancement using Oval Tubes and Multiple Delta Winglets................. 160

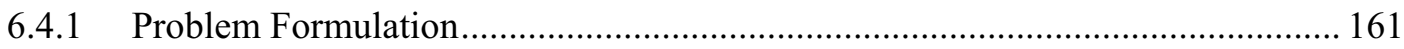

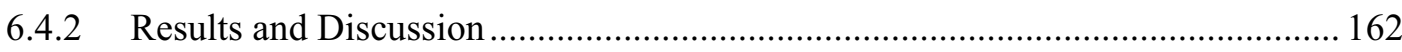

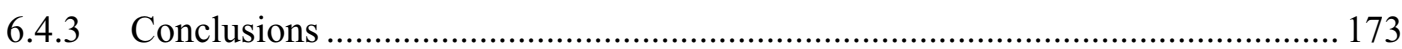

7. INDEPENDENT TESTING OF TUBE BUNDLES BY INTERTEK TESTING SERVICES .... 174

7.1 Test Tube Bundle Fabrication and Test Conditions ...................................................... 174

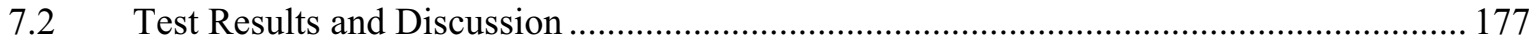

7.3 Review of the INL Single Blow Test Facility Data..................................................... 178

7.4 Possible Reasons for the Unexpected Performance of the Enhanced Tube Bundle ......... 182

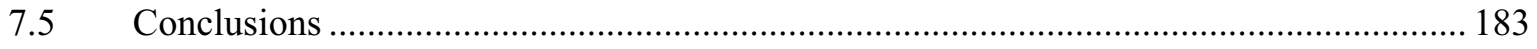

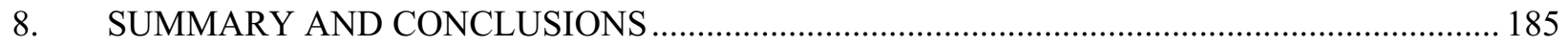

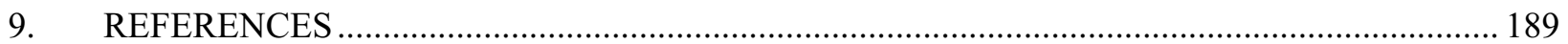




\section{FIGURES}

1-1. Basic phenomenon of generating horseshoe vortices with and without winglets. .......................... 2

1-2. Naturally generated vortices, and vortex generators for heat exchanger applications ..................... 4

2-1. Photograph of a bench-top experimental set-up with an oval tube under test................................ 6

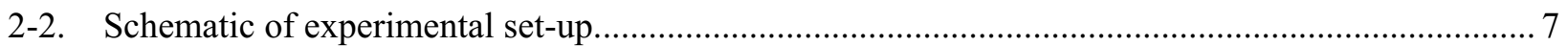

2-3. Test section top view and cross-sections with circular tube

2-4. Winglet locations and geometry: (a) winglet-only case, (b) circular cylinder plus winglets. ........... 9

2-5. Color contour map of local heat transfer coefficient, $\mathrm{h}\left(\mathrm{W} / \mathrm{m}^{2} \mathrm{~K}\right)$ for a circular cylinder, $\mathrm{Re}_{\mathrm{H}}=1000$.

2-6. Top centerline variation of heat transfer coefficient; bottom: transverse variation of heat transfer coefficient in wake region.

2-7. Cylinder stagnation region details, $\operatorname{Re}_{\mathrm{H}}=1080$ : (a) contour plot for heat transfer coefficient, $\mathrm{h},(\mathrm{b})$ variation in $\mathrm{h}$ along the centerline

2-8. Heat transfer distribution trends with the Reynolds number, circular tube.

2-9 Line plots of fin-surface heat transfer coefficient, circular tube: (a) streamwise variation along duct centerline, (b) wake region transverse variation, $\mathrm{y} / \mathrm{R}=1.20$.

2-10. Local fin-surface heat transfer coefficients for a delta-winglet pair.

2-11. Spanwise variation of local heat transfer coefficient for delta-winglet-pair configuration.

2-12 Local fin-surface heat transfer distributions for circular cylinder plus winglets.

2-13 Direct comparison of local heat transfer distributions for a circular cylinder, with and without winglets

2-14. Spanwise variation in local wake-region heat transfer coefficient, with and without winglets, $\operatorname{Re}_{\mathrm{H}}=1200$.

2-15 Mean Nusselt numbers for several cases 18

2-16. Winglet locations and geometry.

2-17. Local fin surface heat transfer coefficients for an oval tube plus a single winglet pair.

2-18. Local fin-surface heat transfer distributions for flow around an elliptical tube with two pairs of delta winglets in a staggered configuration.

2-19. Mean fin-surface Nusselt numbers for seven flow configurations, based on local heat transfer results. 23

2-20. Pressure-drop test section core 24

2-21 Friction factors measured for six flow configurations, steady-state and blow-down.................... 25

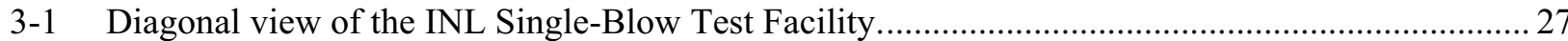

3-2. Schematic diagram of the Single-Blow Test Facility (all dimensions in inches)......................... 28

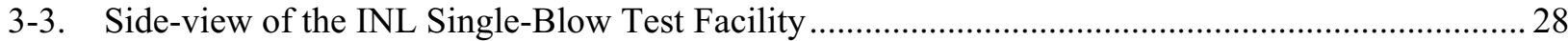

3-4. Low-heat-capacity heater for the Single Blow Test Facility ..................................................... 28

3-5 Test section (a) with only the edge half tubes, (b) with all tubes in place....................................2 29 
3-6. Geometric details of individual fins and winglets for the two cases studied: (a) 2:1 toe-out winglets, (b) 4:1 toe-in winglets

3-7. Individual fins and winglets for the two cases studied: (a) 2:1 toe-out (enhancement-1) winglets,

(b) 4:1 toe-in (enhancement-2) winglets.

3-8. Representative test section inlet and outlet temperatures after initiation of thermal transient,

$\operatorname{Re}_{\mathrm{Dh}}=550$

3-9. Nondimensional axial dispersion coefficient as a function of the Reynolds number. 33

3-10. Dependence of the friction factor and the Colburn $\mathrm{j}$-factor on the Reynolds number for three configurations.

3-11. Effect of winglet circumferential orientation and number of enhanced tube rows on friction factor and Colburn j-factor.

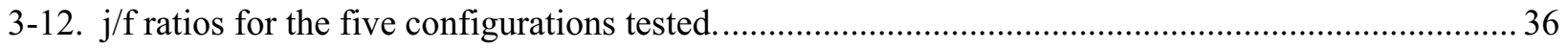

3-13. Performance criterion for two enhanced geometries and two variations........................................ 37

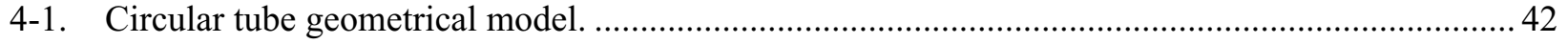

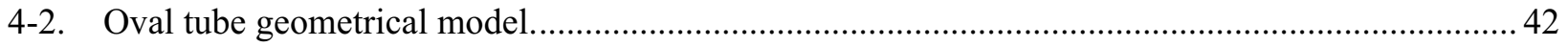

4-3. Contours of local heat transfer coefficients at $\mathrm{Re}_{\mathrm{H}}=500$ : (a) O'Brien and Sohal experimental data, (b) numerical results.

4-4. Contours of local heat transfer coefficients $\operatorname{Re}_{\mathrm{H}}=1240$ : (a) O'Brien and Sohal experimental data, (b) numerical results

4-5. Comparison of experimental and numerical values of fin-surface heat transfer coefficient $\left(\mathrm{W} / \mathrm{m}^{2} \mathrm{~K}\right)$ for an oval tube, along duct length centerline in the stagnation region.

4-6. Comparison of experimental and numerical values of fin-surface heat transfer coefficient $\left(\mathrm{W} / \mathrm{m}^{2} \mathrm{~K}\right)$ for an oval tube, along duct width in the wake region at $\mathrm{y} / \mathrm{a}=1.10$.

4-7. Horseshoe vortices in the stagnation region upstream of the oval tube wall (in yellow) at $\mathrm{Re}_{\mathrm{H}}=500$.

4-8. Horseshoe vortices in the stagnation region upstream of the oval tube wall (in yellow) at $\mathrm{Re}_{\mathrm{H}}=1240$

4-9. $\quad\left(\left(\mathrm{j} / \mathrm{j}_{\mathrm{o}}\right) /\left(\mathrm{f} / \mathrm{f}_{\mathrm{o}}\right)\right)$ ratio for oval and circular tubes versus the Reynolds number.

4-10. Fin surface heat transfer coefficient $(\mathrm{W} / \mathrm{m} 2 \cdot \mathrm{K})$ contour plots for $\mathrm{ReH}=500$ : (a) oval tube, (b) circular tube

4-11. Fin surface heat transfer coefficient $\left(\mathrm{W} / \mathrm{m}^{2} \cdot \mathrm{K}\right)$ contour plots for $\mathrm{Re}_{\mathrm{H}}=900$ : (a) oval tube,

(b) circular tube.

4-12. Fin surface heat transfer coefficient $(\mathrm{W} / \mathrm{m} 2 \cdot \mathrm{K})$ contour plots, for $\mathrm{ReH}=1240$ : (a) oval tube, (b) circular tube

4-13. Line plots of fin-surface heat transfer coefficient $\left(\mathrm{W} / \mathrm{m}^{2} \cdot \mathrm{K}\right)$ : streamwise variation along duct centerline from tube front stagnation point (in $\mathrm{m}$ ).

4-14. Line plots of fin-surface heat transfer coefficient $(\mathrm{W} / \mathrm{m} 2 \cdot \mathrm{K})$ : transverse variation in wake region from tube centerline (in $\mathrm{m}$ ), $\mathrm{y} / \mathrm{r}=1.10$ for circular tube and $\mathrm{y} / \mathrm{a}=1.10$ for oval tube ....................51

4-15. Velocity vectors for horseshoe vortices for a circular tube (in yellow) at $\operatorname{Re}_{\mathrm{H}}=1240 \ldots \ldots \ldots \ldots \ldots \ldots . .52$

4-16. Pressure (in $\mathrm{Pa}$ ) contours for $\mathrm{Re}_{\mathrm{H}}=1240$, (a) Oval tube, (b) Circular tube. ............................... 53 
4-17. Vortex generator configurations generating co-rotating and counter-rotating vortices. .54

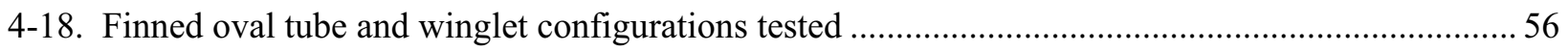

4-19. Performance comparison for four winglet pairs on four finned oval tubes. ................................... 57

4-20. A baseline case without winglets: (a) velocity ( $\mathrm{m} / \mathrm{s}$ ) pattern, (b) surface heat transfer coefficient $\left(\mathrm{W} / \mathrm{m}^{2} \cdot \mathrm{K}\right)$ distribution.

4-21. Configuration 1: (a) velocity $(\mathrm{m} / \mathrm{s})$ contours, (b) surface heat transfer coefficient $\left(\mathrm{W} / \mathrm{m}^{2} \cdot \mathrm{K}\right)$ distribution.

4-22. Configuration $2:$ (a) velocity $(\mathrm{m} / \mathrm{s})$ contours, (b) surface heat transfer coefficient $\left(\mathrm{W} / \mathrm{m}^{2} \cdot \mathrm{K}\right)$ distribution.

4-23. Configuration 3: (a) velocity $(\mathrm{m} / \mathrm{s})$ contours, (b) surface heat transfer coefficient $\left(\mathrm{W} / \mathrm{m}^{2} \cdot \mathrm{K}\right)$ distribution.

4-24. Configuration 4: (a) velocity $(\mathrm{m} / \mathrm{s})$ contours, (b) surface heat transfer coefficient $\left(\mathrm{W} / \mathrm{m}^{2} \cdot \mathrm{K}\right)$ distribution.

4-25. Velocity vectors for winglet Configuration 1.

4-26. Velocity vectors for winglet Configuration 2.

4-27. Velocity vectors for winglet Configuration 3.

4-28. Velocity vectors for winglet Configuration 4

4-29. Tube bundle configurations: (a) in-line configuration, (b) staggered configuration.

4-30. Performance ratio, $\left(\mathrm{j} / \mathrm{j}_{0}\right) /\left(\mathrm{f} / \mathrm{f}_{0}\right)$, results for in-line oval tube bundles with winglets in $1,1-2$, and $1-2-3$ rows.

4-31. Performance ratio, $\left(\mathrm{j} / \mathrm{j}_{0}\right) /\left(\mathrm{f} / \mathrm{f}_{0}\right)$, results for staggered oval tube banks with winglets in $1,1-2$, and $1-2-3$ rows.

4-32. Comparison (ratio) of performances ratios, $\left(\mathrm{j} / \mathrm{j}_{0}\right) /\left(\mathrm{f} / \mathrm{f}_{0}\right)$, for staggered versus in-line tube bundles.. 68

4-33 a and b. Base case with no winglets: (a) velocity ( $\mathrm{m} / \mathrm{s}$ ) contours, (b) surface heat transfer coefficient $\left(\mathrm{W} / \mathrm{m}^{2} \cdot \mathrm{K}\right)$ distribution.

4-33 c and d. First row WVGs: (c) surface heat transfer coefficient $(\mathrm{W} / \mathrm{m} 2 \cdot \mathrm{K})$ distribution, (d) contours of velocity magnitude $(\mathrm{m} / \mathrm{s})$.

4-33 e and f. First and second row WVGs: (e) surface heat transfer coefficient $\left(\mathrm{W} / \mathrm{m}^{2} \cdot \mathrm{K}\right)$ distribution, (f) contours of velocity magnitude $(\mathrm{m} / \mathrm{s})$.

4-33 g. First, second, and third row WVGs: $(\mathrm{g})$ surface heat transfer coefficient $\left(\mathrm{W} / \mathrm{m}^{2} \cdot \mathrm{K}\right)$ distribution, (h) contours of velocity magnitude $(\mathrm{m} / \mathrm{s})$.

4-34. Dynamic-pressure gradients (pascals) for in-line tube bank arrangement .................................. 71

4-35 a. Velocity vectors for in-line tube bank baseline case (no WVGs) ............................................. 72

4-35 b. Velocity vectors for in-line tube bank, 1 row of WVGs........................................................... 72

4-35 c. Velocity vectors for in-line tube bank, 2 rows of WVGs........................................................ 73

4-35 d. Velocity vectors for in-line tube bank, 3 rows of WVGs....................................................... 73

4-36 a and b. Base case, no WVGs: (a) surface heat transfer coefficient $\left(\mathrm{W} / \mathrm{m}^{2} \mathrm{~K}\right)$ distribution, (b) contours of velocity magnitude $(\mathrm{m} / \mathrm{s})$. 
4-36 c and d. First row WVGs: (c) surface heat transfer coefficient $\left(\mathrm{W} / \mathrm{m}^{2} \mathrm{~K}\right)$ distribution,

(d) contours of velocity magnitude $(\mathrm{m} / \mathrm{s})$

4-36. e and f. First and second row WVGs: (e) surface heat transfer coefficient (W/m $\mathrm{K}$ )

distribution, (f) contours of velocity magnitude $(\mathrm{m} / \mathrm{s})$.

4-36 g. First, second, and third row WVGs: (g) surface heat transfer coefficient $\left(\mathrm{W} / \mathrm{m}^{2} \mathrm{~K}\right)$

distribution, (h) contours of velocity magnitude $(\mathrm{m} / \mathrm{s})$. .76

4-37. Dynamic-pressure gradients (pascals) for staggered tube bank arrangement................................ 76

4-38a. Velocity vectors for staggered tube bank baseline case (no WVGs) …....................................... 78

4-38b. Velocity vectors for in-line tube bank, one row of WVGs ....................................................... 78

4-38c. Velocity vectors for in-line tube bank, two rows of WVGs ....................................................... 79

4-38d. Velocity vectors for in-line tube bank, three rows of WVGs .................................................... 79

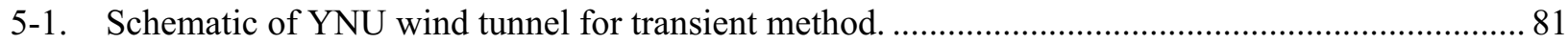

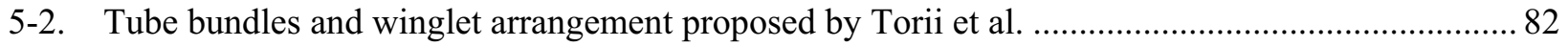

5-3. Tube bundle with winglet arrangement proposed by Fiebig et al............................................... 83

5-4. The comparison of $\mathrm{j} / \mathrm{j}_{\mathrm{Go}}$ and $\mathrm{f} / \mathrm{f}_{\mathrm{Go}}$ with respect to Reynolds number for the winglet configuration suggested by Feibig et al.

5-5. Comparison of $\mathrm{j} / \mathrm{j}$ Go and $\mathrm{f} / \mathrm{fGo}$ with respect to the Reynolds number for the in-line tube bundle and a winglet configuration suggested by Torii et al..

5-6. Comparison of $\mathrm{j} / \mathrm{j}_{\mathrm{Go}}$ and $\mathrm{f} / \mathrm{f}_{\mathrm{Go}}$ with respect to the Reynolds number for the staggered tube bundle and a winglet configuration suggested by Torii et al...................................................... 87

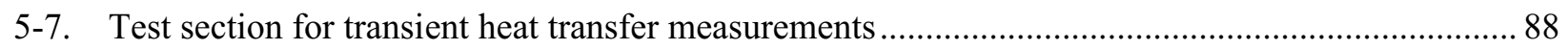

5-8. Schematic of the experimental apparatus for measuring local heat transfer .................................. 89

5-9. Calibration curve for calculating the temperature from an IR camera measurement ...................... 91

5-10. Geometrical details of test section with finned tubes and winglets ............................................ 92

5-11. The ratio of span-averaged Nusselt number distributions with and without vortex generators

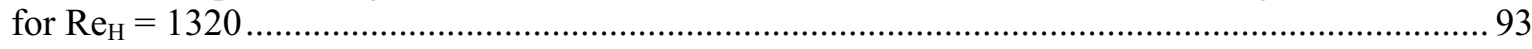

5-12. Geometrical details of a test section with finned tubes and proposed winglets............................... 94

5-13. The ratio of local Nusselt numbers with and without vortex generators on the bottom surface

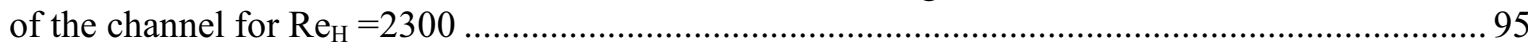

5-14. Longitudinal variation of the heat transfer enhancement in terms of the Nusselt number ratio for $\mathrm{Re}_{\mathrm{H}}=2300$

5-15. Spanwise variation of the heat transfer enhancement in terms of the Nusselt number ratio at $\mathrm{x} / \mathrm{D}=0.7$ for $\mathrm{ReH}=2300$

5-16. Spanwise variation of the heat transfer enhancement in terms of the Nusselt number ratio at $\mathrm{x} / \mathrm{D}=3.2$ for $\mathrm{ReH}=2300$

5-17. Spanwise variation of the heat transfer enhancement in terms of the Nusselt number ratio at $\mathrm{x} / \mathrm{D}=5.7$ for $\mathrm{ReH}=2300$.

5-18. Re-circulating water channel facility at Yokohama National University. 
5-19. Test core tube bundle arrangement, (a) in-line tubes, (b) staggered tubes ................................... 100

$5-20$. Various arrangements of tube bundles and winglets studied..................................................... 101

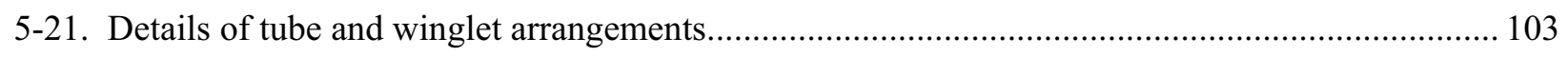

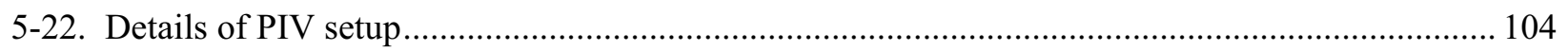

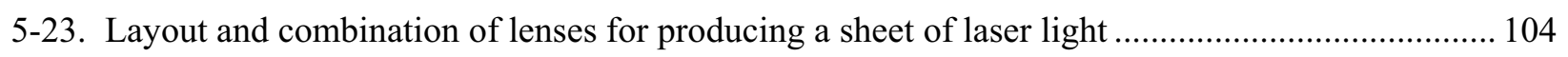

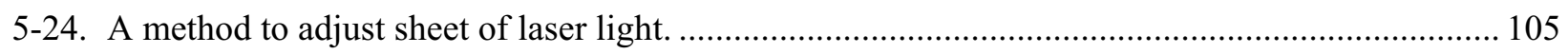

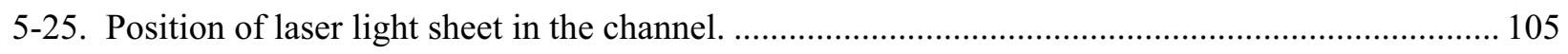

5-26. Example of a pair of PIV images and a result of velocity distribution......................................... 106

5-27. Velocity profiles for Types A-D of tube bundle Arrangement I. ................................................ 107

5-28. Flow patterns for Type A of Arrangement I (single row tubes). .................................................. 109

5-29. Flow patterns for Type B of Arrangement I (single row tubes). ................................................. 110

5-30. Flow patterns for Type $\mathrm{C}$ of Arrangement I (single row tubes). ................................................ 111

5-31. Flow patterns for Type D of Arrangement I (single row tubes). ............................................... 112

5-32. Mean velocity profile along a horizontal line for Arrangement I............................................. 113

5-33. RMS of velocity variation along a horizontal line for Arrangement I....................................... 115

5-34. Distribution of RMS values of streamwise and spanwise velocity fluctuations in a horizontal plane $0.5 \mathrm{~mm}$ above the bottom channel wall: Type C of Arrangement I................................. 116

5-35. Mean velocity vectors in Y-Z planes for Type C of Arrangement I........................................... 117

5-36. Mean velocity vectors in X-Y planes for Type C of Arrangement I. .......................................... 118

5-37. Mean velocity vectors in Y-Z planes for Type D of Arrangement I. ........................................... 119

5-38. Mean velocity vectors in X-Y planes for Type D of Arrangement I. .......................................... 120

5-39. Total wall shear stress distribution for Types A-D of Arrangement I. ....................................... 121

5-40. Flow patterns of Type A of Arrangement II (three row in-line tube bundle)............................... 122

5-41. Flow patterns of Type B of Arrangement II (three row in-line tube bundle). .............................. 123

5-42. Flow patterns of Type C of Arrangement II (three row in-line tube bundle). ............................... 124

5-43. Flow patterns for Type B of Arrangement III (three-row in-line tube bundle with three-row

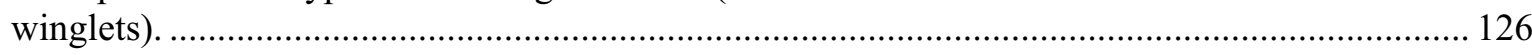

5-44. Flow patterns for Type D of Arrangement III (three-row in-line tube bundle with three-row

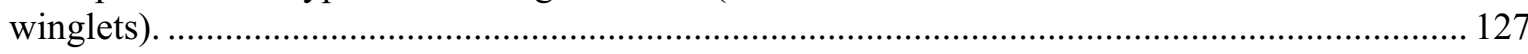

5-45. Flow patterns for Type A of Arrangement IV (three-row staggered tube bundle with no

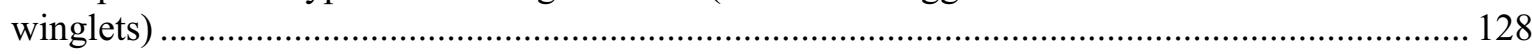

5-46. Flow patterns for Type $\mathrm{C}$ of Arrangement IV (three-row staggered tube bundle with one row of winglets).

5-47. Flow patterns for Type D of Arrangement IV (three-row staggered tube bundle with one row

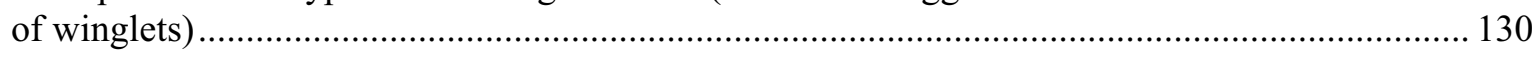

6-1 Schematic of the core region of a fin-tube heat exchanger ..................................................... 131 
6-2. Module of the heat exchanger.

6-3. Schematic of the three-dimensional grid.

6-4. Schematic representation of the singular points.

6-5. Streamline representation of the flow past a circular tube placed in a channel on the horizontal mid-plane for (a) instantaneous flow field and (b) time-averaged flow field.....

6-6. Structure of three-dimensional flow (particle path)........................................................................ 135

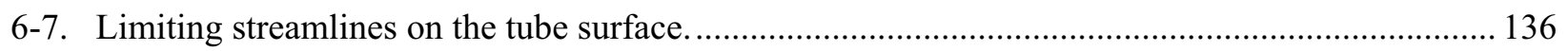

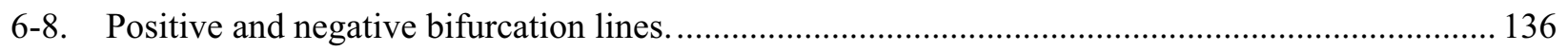

6-9. Limiting streamlines of the flow past a built-in circular tube in a channel at the bottom plate corresponding to (a) instantaneous flow field and (b) time-averaged flow field.................. 137

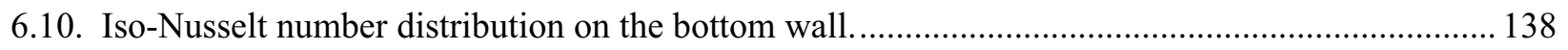

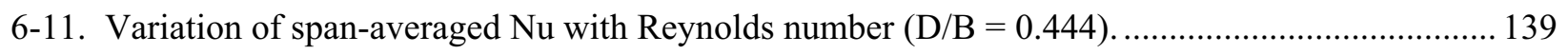

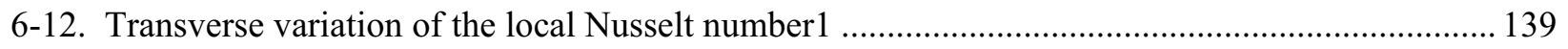

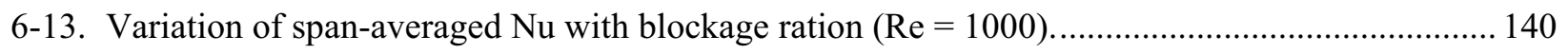

6-14. Comparison of the span-averaged Nusselt number at three different times with the time-averaged span-averaged Nusselt number.

6-15. Pressure variation along the length of the channel for different values of the Reynolds number. . 141

6-16. Comparison of the transverse heat transfer coefficient in the wake region ................................... 142

6-17. Comparison of the span- and time-averaged Nusselt number for two different grids................... 143

6-18. Heat exchanger module considered in the present investigation................................................ 144

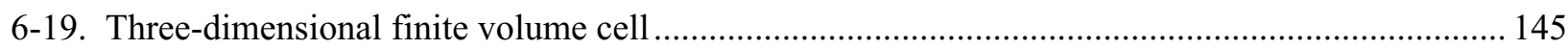

6-20. Limiting streamlines on a plane close to the bottom plate of a channel with a built-in circular tube and a pair of delta winglets in a toe-in (common flow up) configuration. ............................. 146

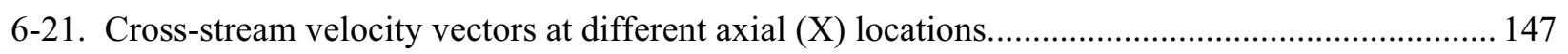

6-22. Streamlines on the cross-stream planes at different axial (X) locations...................................... 148

6-23. Nusselt number distribution on the bottom plate of a channel with built-in circular tube and delta winglet pair in toe-in (common-flow-up) configuration............................................... 148

6-24. Span-averaged Nusselt number distribution on the bottom plate of the channel for $\mathrm{Re}=1000 \ldots 149$

6-25. Span-averaged Nusselt number distribution for two configurations and two Reynolds numbers.. 150

6-26. Comparison between the numerical results and experimental data of Torii et al. ....................... 150

6-27. Heat exchanger module with oval tube and vortex generators ................................................... 151

6-28. The schematic of the grid system on a two-dimensional plane .................................................... 152

6-29. Steamlines on the horizontal mid-plane of the channel............................................................ 153

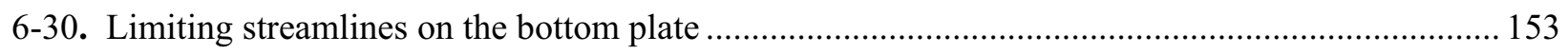

6-31. Cross stream velocity vectors at axial location $X=6: 125$ : (a) oval tube, $(b)$ oval tube and winglet pair. 
6-32. Nusselt number distribution on the bottom plate for $\operatorname{Re}=1300$.

6-33. Comparison of experimental and numerical span-averaged Nusselt number for $\mathrm{Re}=1090$.

6-34 Nusselt number distribution on the bottom plate with an oval tube and a winglet pair for $\operatorname{Re}=1300$.

6-35. Span-averaged Nusselt number distribution for various Re in a channel with an oval tube and a winglet pair.

6-36. Transverse variation of local Nusselt number for $\mathrm{Re}=1300$.

6-37. Span-averaged Nusselt number distribution for various angles of attack for $R e=1300$.

6-38. Local Nusselt number distribution on the bottom plate of a channel for (a) a delta winglet pair and (b) a rectangular winglet pair.

6-39. Span-averaged Nusselt number distribution on the bottom plate.

6-40. Two-dimensional representation of the computational domain.

6-41. Various configurations of the winglet pairs: (a) one winglet pair (W1) toe-out,

(b) two winglet pairs (W1 toe-out; W2 toe-in, (c) two winglet pairs (W1 and W2 both toe-out),

(d) three winglet pairs (W1, W2 and W3 toe-out), and (e) four winglet pairs (W1 and W3 toe-out, and W2 and W4 toe-in).

6-42. (a) Streamline plot of the instantaneous flow field at the mid-plane, showing vortex shedding (circular tube without winglets). (b) Streamline plot of the instantaneous field at the mid-plane (oval tube without winglets and having the same perimeter as the circular tube). (c) Streamline plot of the time-averaged field at the mid-plane (oval tube without winglets).

6-43. (a) Streamline plot of the time-averaged field on the bottom plate (in the case of oval tube without winglets), (b) iso-Nusselt number distribution on the bottom plate (in the case of oval tube without winglets).

6-44. (a) Limiting streamlines on the bottom plate for flow past a built-in oval tube with one pair of winglets, (b) Iso-Nusselt number distribution on the bottom plate for flow past a built-in oval tube with one winglet pair.

6-45. Iso-Nusselt number distribution on the bottom plate for flow past a built-in oval tube with two winglet pairs (configuration shown in Figure 6-44b)

6-46. Iso-Nusselt number distribution on the bottom plate for flow past a built-in oval tube with three winglet pairs

6-47. Iso-Nusselt number distribution on the bottom plate for flow past a built-in oval tube with three winglet pairs (configuration shown in Figure 6-41d)

6-48. Iso-Nusselt number distribution on the bottom plate for flow past a built-in oval tube with four winglet pairs.

6-49. Comparison of span-averaged Nusselt number for a plane channel, channel with oval tube, channel with oval tube, and one and two winglet pairs

6-50. Comparison of the span-averaged Nusselt number for different axial locations of one winglet pair.

6-51. Comparison of span-averaged Nusselt number for different angles of attack for flow past a built-in oval tube with two winglet pairs. 
6-52. Comparison of the span-averaged Nusselt number for flow past a built-in oval tube with one, two, and three winglet pairs.

6-53. Comparison of span-averaged Nusselt number for flow past a built-in oval tube with two and four winglet pairs.

6-54. Comparison of span-averaged Nusselt number for different Reynolds numbers for flow past a built-in oval tube with one winglet pair 172

6-55. Comparison of the span-averaged Nusselt number for three different grid sizes 173

7-1. Schematic of Concept 2, toe-in (common flow up) winglet design 175

7-2. A (6 rows $\times 3$ column $)$ staggered tube bundle. $)$ 175

7-3. Photograph of the test apparatus with enhanced finned tubes and inlet and outlet plena. 176

7-4. Close-up of the finned tubes with toe-in (common flow up) winglets. ......................................... 176

7-5. Raw pressure drop data versus Reynolds number from the Single Blow Facility....................... 180

7-6. Friction factors for the baseline and toe-in (Concept 2) cases.................................................... 180

7.7. Colburn j-factors for the baseline and toe-in cases from the Single Blow Test Facility.

\section{TABLES}

1-1. Plant cost summary

3-1. Unit-cell dimensions and hydraulic diameter for the three test configurations ............................. 32

4-1. Summary of geometrical arrangements of test-cores and winglets............................................. 82

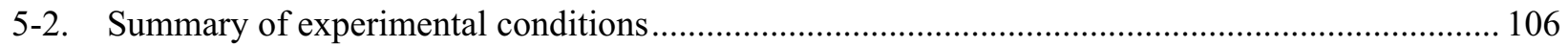

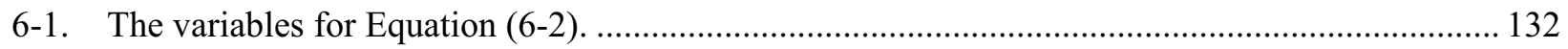

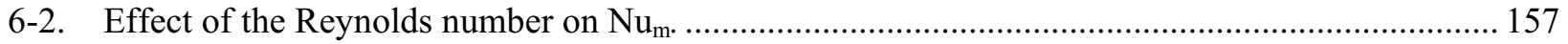

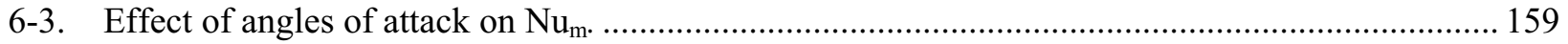

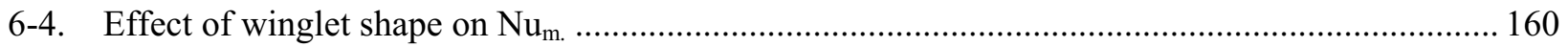

6-5. Comparison of mean Nusselt number for different configurations .............................................. 169

6-6. Comparison of mean Nusselt number for different angles of attack. .......................................... 170

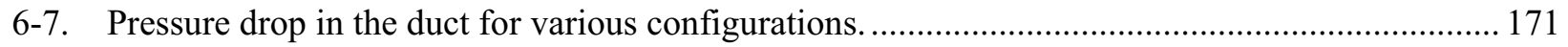

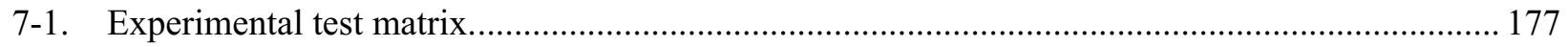

7-2. Test data for a baseline and the INL enhanced finned tube bundles. ......................................... 177

7-3. Unit-cell dimensions and hydraulic diameter for the three INL test bundles in SBTF................ 178 


\section{NOMENCLATURE}

a

A

$\mathrm{A}_{\text {min }}$

b

c

Dh

f

G

$\mathrm{h}_{\mathrm{W}}$

$\mathrm{H}$

$\mathrm{k}$

L

$\dot{m}$

$\mathrm{Nu}_{\mathrm{H}}=\mathrm{hH} / \mathrm{k}$

$\Delta \mathrm{P}$

$\mathrm{Re}_{\mathrm{H}}=\rho \mathrm{UH} / \mu$

$\mathrm{Re}_{\mathrm{Dh}}=\mathrm{GDh} / \mu$

$\mathrm{t}$

$\mathrm{T}$

$\mathrm{T}_{\mathrm{i}}$

$T_{\infty}$

U

W

$\alpha$

$\rho$

$\gamma=\frac{h \sqrt{t}}{\sqrt{\rho c k}}$

$\theta=\frac{T(0, t)-T_{i}}{T_{\infty}-T_{i}}$

$\mu$ elliptical tube major axis half length, $\mathrm{cm}$

tube bundle heat transfer surface area, $\mathrm{m}^{2}$

minimum flow cross-sectional area, $\mathrm{m}^{2}$

elliptical tube minor axis half-length, cm

specific heat, $\mathrm{J} / \mathrm{kg} \mathrm{K}$

duct hydraulic diameter, cm

friction factor

air flow mass flux, $\mathrm{kg} / \mathrm{m}^{2} \mathrm{~s}$

heat transfer coefficient, $\mathrm{W} / \mathrm{m}^{2} \mathrm{~K}$

winglet height, $\mathrm{cm}$

channel height, $\mathrm{cm}$

thermal conductivity, $\mathrm{W} / \mathrm{m} \mathrm{K}$

test section length, tube bundle length, $\mathrm{cm}$

air mass flow rate, $\mathrm{kg} / \mathrm{s}$

Nusselt number based on channel height

pressure drop across tube bundle, $\mathrm{Pa}$

Reynolds number based on channel height

Reynolds number based on hydraulic diameter

time, $s$

temperature, $\mathrm{K}$

initial temperature, $\mathrm{K}$

flow mean temperature, $\mathrm{K}$

mean flow velocity, $\mathrm{m} / \mathrm{s}$

channel width, cm

lexan thermal diffusivity, $\mathrm{m} 2 / \mathrm{s}$

density, $\mathrm{kg} / \mathrm{m} 3$

nondimensional heat transfer coefficient

nondimensional temperature difference

air absolute viscosity, $\mathrm{N} \mathrm{s} / \mathrm{m} 2$ 
xviii 


\section{Improving Vortex Generators to Enhance the Performance of Air-Cooled Condensers in a Geothermal Power Plant}

\section{INTRODUCTION}

Binary geothermal plants, lacking a supply of water for an evaporative cooling system, reject heat directly to the ambient air. In an air-cooled binary geothermal power plant, hot geothermal fluid (brine) is used to heat and vaporize a working fluid (hydrocarbon/isobutene, iC4) in a series of heat exchangers. The hot working fluid vapor is then expanded through a turbine to generate electricity. The exhaust from the turbine is condensed in an air-cooled condenser. The condensed working fluid is pumped back to the evaporator to be heated by the hot brine. The air-cooled condenser is a major component in a geothermal power plant. An EPRI report ${ }^{1}$ indicates that the cost of an air-cooled condenser can be up to about 22 to $30 \%$ of the total plant cost (including well field). Table 1-1 compares costs of the air-cooled condensers in five 50-MW binary geothermal power plants. ${ }^{1}$ The total plant cost of air-cooled binary cycles primarily comprises the costs of the geothermal well-field, turbine, air-cooled condenser, brine-toworking-fluid heat exchanger, (fluid) gathering system, and several pumps. The air-cooled condenser was sized to give a $34^{\circ} \mathrm{F}$ approach on the cold end, with a $14.8^{\circ} \mathrm{F}$ pinch temperature. The pinch temperature is the minimum temperature difference between the hot and cold streams at any given point on the condenser's heat curve. In the first column, the name of the site and the resource (brine) temperature $\left(265-510^{\circ} \mathrm{F}\right)$ are given. In the next line of the same column is the turbine inlet pressure and the condensing temperature $\left(74-87^{\circ} \mathrm{F}\right)$.

Table 1-1. Plant cost summary.

\begin{tabular}{|c|c|c|c|c|c|c|}
\hline Plant Specifications & $\begin{array}{l}\text { Condenser } \\
\text { Cost }(\mathrm{M} \$)\end{array}$ & $\begin{array}{l}\text { Well-field } \\
\text { Cost (M\$) }\end{array}$ & $\begin{array}{l}\text { Total Plant } \\
\text { Cost }(\mathrm{M} \$)\end{array}$ & $\begin{array}{l}\text { Specific Cost } \\
(\$ / \mathrm{MW})\end{array}$ & $\begin{array}{l}\text { Brine Rate } \\
(\mathrm{Mlb} / \mathrm{h})\end{array}$ & $\begin{array}{l}\text { Specific } \\
\text { Output, } \\
\text { (kWh/klb) }\end{array}$ \\
\hline \multicolumn{7}{|l|}{ Vale, OR } \\
\hline $500 \mathrm{psia}, 85^{\circ} \mathrm{F}$ & 31.726 & 33.441 & 122.000 & 2.440 & 8.678 & 5.76 \\
\hline $610 \mathrm{psia}, 85^{\circ} \mathrm{F}$ & 31.500 & 31.500 & 117.800 & 2.356 & 8.348 & 5.99 \\
\hline \multicolumn{7}{|l|}{$\begin{array}{l}\text { Surprise Valley, CA } \\
\quad @ 375^{\circ} \mathrm{F}\end{array}$} \\
\hline $500 \mathrm{psia}, 84^{\circ} \mathrm{F}$ & 30.616 & 31.000 & 108.300 & 2.166 & 6.800 & 7.35 \\
\hline 850 psia, $84^{\circ} \mathrm{F}$ & 23.600 & 25.500 & 105.750 & 2.115 & 5.889 & 8.49 \\
\hline \multicolumn{7}{|l|}{$\begin{array}{l}\text { Thermo Hot Springs, UT } \\
\text { (a) } 265^{\circ} \mathrm{F}\end{array}$} \\
\hline 205 psia, $74^{\circ} \mathrm{F}$ & 67.910 & 82.125 & 226.900 & 4.538 & 22.402 & 2.23 \\
\hline 235 psia, $74^{\circ} \mathrm{F}$ & 60.499 & 76.875 & 209.400 & 4.188 & 20.482 & 2.23 \\
\hline \multicolumn{7}{|l|}{$\begin{array}{l}\text { Raft River, ID } \\
\qquad 300^{\circ} \mathrm{F}\end{array}$} \\
\hline $325 \mathrm{psia}, 85^{\circ} \mathrm{F}$ & 41.186 & 47.250 & 147.250 & 2.945 & 12.384 & 4.04 \\
\hline \multicolumn{7}{|l|}{$\begin{array}{l}\text { Glass Mountain, CA } \\
\text { @ } 510^{\circ} \mathrm{F}\end{array}$} \\
\hline $500 \mathrm{psia}, 87^{\circ} \mathrm{F}$ & 29.395 & 38.500 & 107.100 & 2.142 & 4.257 & 11.75 \\
\hline 800 psia, $87^{\circ} \mathrm{F}$ & 25.474 & 36.500 & 103.600 & 2.072 & 3.830 & 13.05 \\
\hline
\end{tabular}


Most thermal resistance in an air-cooled condenser is on the air-side of the tubes. An air-cooled condenser rejects the entire heat load sensibly to the air. Air is a less-than-ideal heat transfer fluid because its density, heat capacity, and related convective heat transfer coefficient are low. Consequently, high air-flow rates and extensive heat exchanger areas are required, and the power required to force the air over the heat exchange area is significant. The thermal resistance can be decreased by increasing the air flow rate, which increases the convective heat transfer coefficient. But this also increases the pressure drop across the tube bundle and the fan pumping power. A cost effective method to provide the large surface area is to use externally finned tubes, which increase the outer surface area by a factor of about 20. Air-cooled condensers, even with finned tubes, however, are large and structurally dominate plant installation. Thus, another requirement of an enhancement device(s) is not to significantly increase the pressure drop and the parasitic fan pumping power. The enhancement is to be accomplished without increasing the capital or operating cost of the total heat rejection system. This leads to the decision to improve the heat transfer coefficient on the air-side of an air-cooled condenser.

One area of research to enhance heat transfer is the application of vortex generators (also called winglets) on the tube fins. Whenever there is a cylindrical object perpendicular to a fluid flowing along a flat surface (for example, flow around a bridge support pillar in a river-bed), vortices, called horseshoe vortices, are generated in the flow stream near the base of the cylinder (see Figure 1-1). The same phenomenon occurs in a tube-fin arrangement. An excellent review of heat transfer enhancement through use of longitudinal vortices is given by Feibig ${ }^{2}$ and Jacobi and Shah. ${ }^{3}$ Vortices are generated when the boundary layer flow gets separated from a curved cylinder-like wall surface. The boundary layer on the fin surface upstream of the cylinder is in a region of adverse pressure gradient. At some distance upstream of the cylinder, the boundary layer undergoes a three-dimensional separation. The separated boundary layer rolls-up downstream of the separation line to form a system of vortices, moves around the cylinder base, and finally trails off. The vortices in the wake region behind a cylinder are caused by the presence of rotation in the boundary layer. In some situations, these vortices may remain embedded in the boundary layers. In the case of a finned tube, the vortices form in front of the tube in the bounded space between two fin surfaces, move around the tube, and may even reach the rear of the tube. The spiraling motion of the vortices enables intermixing of the fluid near the tube surface and that in the main flow, leading to good heat transfer. The wake behind the cylinder is a stagnant region, where the fluid gets trapped and does not mix with the main-stream flow. This is the cause of poor heat transfer in the wake region. These observations by various researchers elucidate the flow behavior around a cylindrical

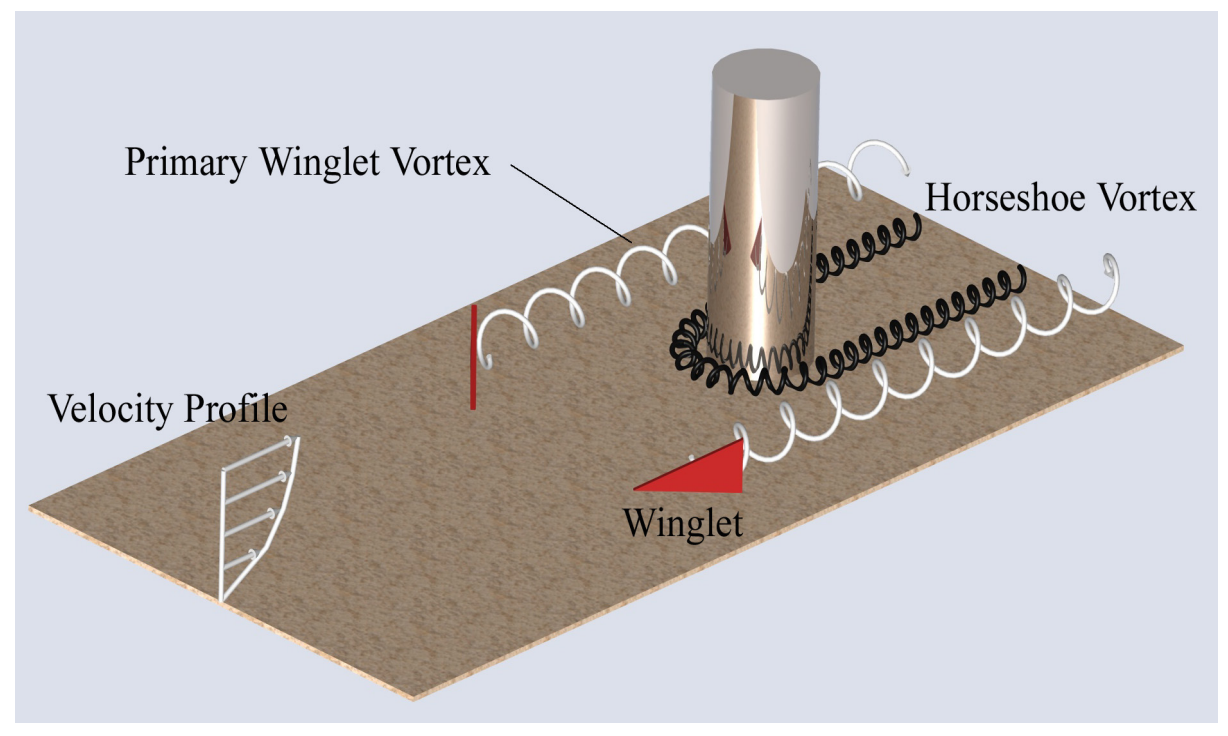

Figure 1-1. Basic phenomenon of generating horseshoe vortices with and without winglets. 
object on a plane surface, which represents a tube-fin heat exchanger. Several numerical and experimental results lead to a theoretical, but ideal postulation for improving heat transfer from a tube-fin arrangement. To enhance heat transfer from finned tubes, the fluid near the tube surface, especially in the wake region of the cylinder, should mix with the mainstream flow. Heat transfer in the wake region can be improved with the help of vortex generators, or winglets. Vortex generators (on the fins) induce vortices (swirling flow), which disrupt the formation of the boundary layer and mixes the flow between the fins. It also reduces the stagnant wake region behind the tube.

If the axis of rotation of the vortices is mainly parallel to the main flow direction, the vortices are called longitudinal (or streamwise) vortices. If the axis of rotation is mainly perpendicular to the main flow direction, the vortices are called transverse vortices. The wake region of a cylinder in cross-flow generally has transverse vortices. When the span of a vortex generator is attached to the wall, it is called a wing; when its chord is attached to the wall, it is called a winglet. Figure 1-2 (from Jacobi and Shah) ${ }^{3}$ shows the formation of horseshoe vortices in a finned tube arrangement and application of various forms of delta and rectangular wings and winglets to generate longitudinal vortices. It has been observed that in steady flow, transverse vortices can enhance heat transfer locally but not globally. It has been shown that if both heat transfer enhancement and pressure loss increase are taken into account, longitudinal vortices appear to be more efficient than the transverse vortices. The longitudinal vortices cause an exchange of fluid in the core and wall region, leading to heat transfer enhancement. The enhancement mechanism in transverse flow consists of unsteady flow and flow reversals at some locations, because swirling takes place around an axis perpendicular to the main flow direction. It is logical to expect that for the same rate of core and wall fluid exchange, less energy is required to swirl the flow around an axis parallel to the main flow than that required for swirling around an axis perpendicular to the flow. Therefore, longitudinal vortices are preferred over transverse vortices.

There are mainly two common winglet shapes, rectangular and delta (triangular). By optimizing the shape, size, and placement of the winglets, the resulting vortices can minimize the wake (stagnant flow) region behind a cylinder and improve heat transfer. As shown by Fiebig, ${ }^{2}$ the winglets enhance heat transfer by about $10-15 \%$, but are also likely to increase pressure drop. Vortex generators enhance heat transfer by an exchange of heat and fluid flow between the wall layer and the core. This enhances the heat transfer mainly in the downstream region of the vortex generators. If the vortex generators are placed appropriately, the flow behavior in the wake region of a cylindrical tube can also be changed. The main flow gets partly diverted toward the tube. The longitudinal vortices cause increased mixing of the main stream fluid and the fluid in the tube wake. The dead wake zone with low heat transfer gets reduced. This leads to a significant increase in heat transfer in the wake region. The application of the vortex generator beyond the separation point on the tube does not affect heat transfer in the stagnation region in front of the tube nor at the leading edge of the fin.

A second heat transfer enhancement strategy is to use oval tubes instead of circular tubes in heat exchanger design. Oval tubes are being used in low-pressure applications to enhance heat transfer in other industries, especially the automotive industry. This enhancement strategy is not practical in all cases, however, because of the higher cost of manufacturing the oval tubes and the fact that circular tubes can withstand higher pressures than do oval tubes of the same wall thickness. In any case, obvious advantages exist for oval tubes, including reduced form drag and increased tube-surface area for the same cross-sectional internal flow area. Webb and Iyengar ${ }^{4}$ have shown about $32 \%$ better heat transfer and $17 \%$ lower pressure drop by using oval tubes compared to using cylindrical tubes. It is anticipated that by combining both concepts, the air-side heat transfer coefficients in binary plant air-cooled condensers can be increased without imposing additional pressure drop and parasitic fan power.

From the available heat transfer enhancement techniques, the two enhancement techniques discussed above were selected. This report documents the investigation relating to these techniques, specifically to the case of air-cooled condensers used in geothermal power plants. The research was undertaken with the 
aim of devising viable heat transfer enhancement to improve air-cooled condenser heat transfer performance (overall heat transfer coefficient) by at least $\sim 15 \%$, resulting in lowering condenser cost without increasing (or minimizing the increase in) the air-side pressure drop and fan parasitic power consumption. It would mean generating more power and reducing cost $/ \mathrm{kWh}$.

There are two common winglet shapes, rectangular and delta (triangular). The delta winglet (a triangle on its longer side) is the preferred shape for the enhancement. As shown in Figure 1-2, the angle of attack of a wing form can change the type of vortices. If the angle of attack is small, mainly longitudinal vortices are produced, whereas if the angle of attack is 90 degrees, mainly transverse vortices are generated. A horseshoe or corner vortex develops in the stagnation region of the winglet. It wraps around the winglet along the corner between the winglet and the flat surface of the fin. A Karman vortex street may develop at the trailing edge of the winglet. At the leading edge of a winglet, flow division may also lead to development of a transverse vortex. The details of the vortex system are strongly influenced by the geometry of the winglet and the Reynolds number. The vortices and fluctuations, in turn, influence the heat transfer between the winglet and the fin surface. Highly nonlinear interactions exist between various parameters, all of which influence heat transfer and flow losses. These vortices lead to heat
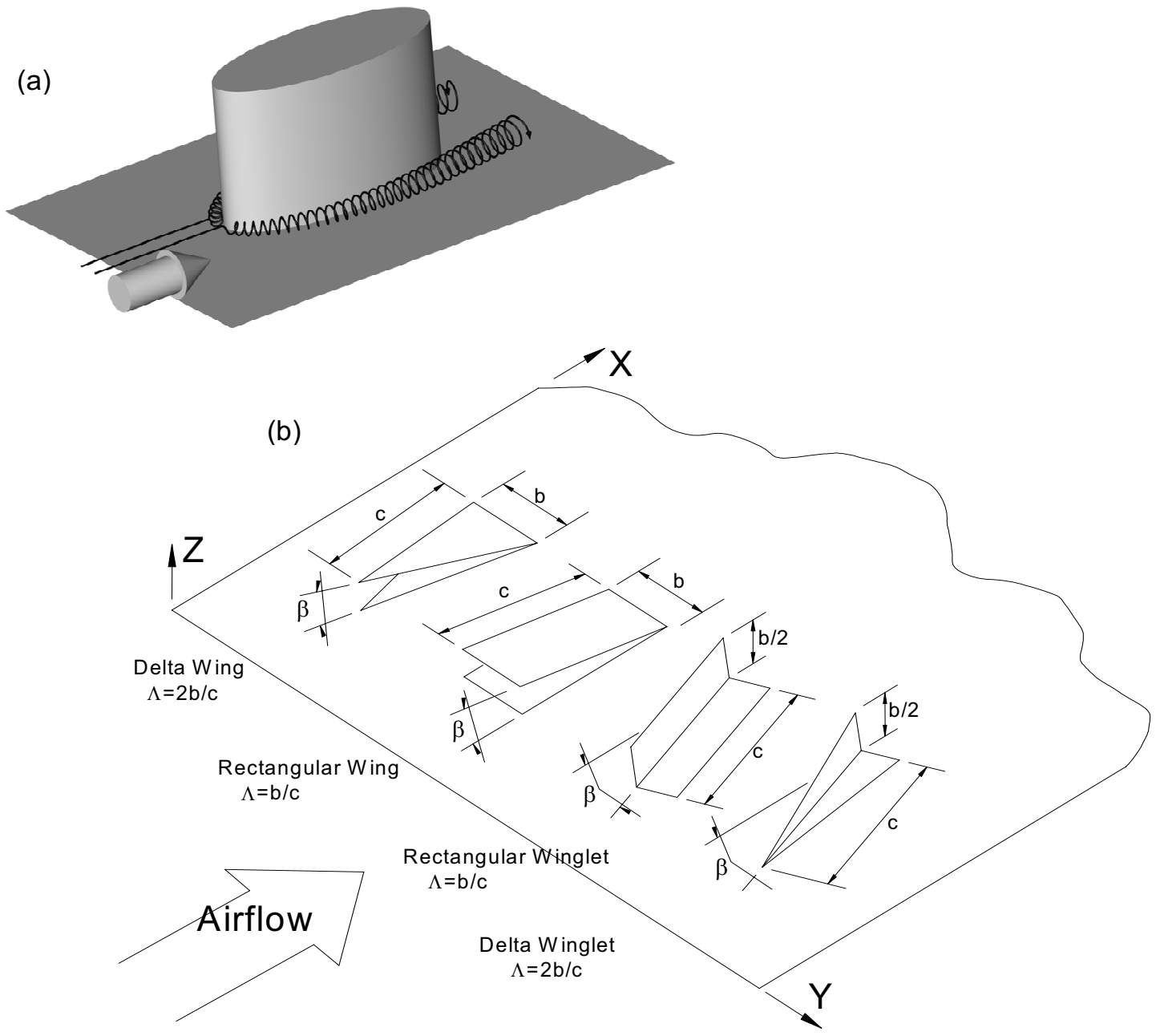

Figure 1-2. Naturally generated vortices, and vortex generators for heat exchanger applications: ${ }^{3}$ (a) the natural formation of a laminar horseshoe vortex at a fin-tube junction; (b) common vortex generators and associated geometrical definitions. 
transfer enhancement (to or from the wall) caused by increased temperature gradient near the wall. In longitudinal vortices, flow is nearly three-dimensional. There is increased form drag resulting from the presence of vortex generators, and the vortices increase wall shear, which leads to increased pressure drop compared to a finned tube without a vortex generator. However, the heat transfer and pressure drop are not directly related. The pressure drop is related to the wall friction, which is related to the derivative of streamwise velocity. However, convective heat transfer is related to spanwise and transverse (normal) velocities. Heat transfer enhancement with double rows of longitudinal vortex-generators (delta-winglet pairs) in a channel flow without tubes has been evaluated experimentally by Tiggelbeck et al. ${ }^{5}$ An investigation of the mechanisms of heat transfer enhancement associated with delta-wing vortex generators was performed by Torii et al. ${ }^{6}$ The specific configuration of vortex generators and circular tubes used in the present study was based on recommendations by Fiebig et al. ${ }^{19}$ We also investigated the use of oval tubes instead of circular tubes, with and without winglets, ${ }^{8-9}$ for the geothermal application. Baseline local heat transfer measurements for both circular and oval tubes without winglets obtained by the present author(s) are presented in Reference 10.

\subsection{Project Objectives}

The objectives of this project directly relate to a DOE programmatic goal: "reducing the levelized cost of generating geothermal power to $3-5$ cents/kWh by 2010." The technical objectives of this project are the following:

- Improve the air-side convective heat transfer coefficient of air-cooled condenser tubes in a geothermal power plant compared to the current practice of using finned tubes

- $\quad$ Minimize the pressure drop increase across the tube bundle as a result of employing the proposed heat transfer enhancement techniques in a tube bundle

- $\quad$ Collaborate with a condenser manufacturer to cost-effectively implement the proposed enhancement device in a practical tube bundle case.

This project was undertaken with the expectation that the research results would demonstrate the benefits of applying vortex generators (winglets) on the fins to improve the heat transfer from the air side of the tube bundle. Eventually, about 10 to $15 \%$ enhancement in heat transfer is expected. If oval tubes can be economically used in a bundle, it is expected that the pressure drop across the tube bundle with the application of vortex generators (winglets) will be similar to that in a conventional circular tube bundle. If circular tubes must be used, the increase in pressure drop from the winglet will be small enough to maintain the benefits of using vortex generators to enhance the heat transfer.

A combined experimental/computational effort was pursued with the objective of designing and demonstrating significant improvements in the performance of air-cooled condensers. A computational fluid dynamics code, FLUENT, was used to model heat transfer and pressure drop in various tube-fin configurations of an air-cooled condenser. The purpose of the modeling was to (a) verify and develop confidence in the experimental and modeling results, (b) optimize the size and location of the vortex generators/winglets without resorting to numerous experiments, and (c) predict trends and performance in cases of interest. Collaboration with a heat exchanger manufacturer was pursued to commercially fabricate a prototype heat exchanger based on the concept and commercialize the technology. 


\section{INL LABORATORY-SCALE EXPERIMENTS ON SINGLE TUBES}

\subsection{Experiment Set-up}

The experiments were performed in a narrow rectangular duct designed to simulate a single passage of a fin-tube heat exchanger. A photograph of the test section is shown in Figure 2-1. The dimensions of the flow channel were $\mathrm{W}=11.4 \mathrm{~cm}, \mathrm{H}=1.0 \mathrm{~cm}(4.5 \times 0.4 \mathrm{in})$. It is scaled up to about double-size that of a typical fin-tube condenser heat exchanger. The duct was fabricated primarily out of lexan polycarbonate. For the first series of tests, a 5.08 -cm (2.0-in)-diameter circular disk, representing the circular tube of a fin-tube heat exchanger, also fabricated from lexan, was positioned in the center of the duct, as shown. The test section length was $27.94 \mathrm{~cm}(11.0 \mathrm{in}$.), yielding $\mathrm{L} / \mathrm{H}=27.5$. A flow development section with $\mathrm{L} / \mathrm{H}=30$ was located upstream of the test section. Consequently, depending on the Reynolds number, the flow is hydrodynamically about fully developed as it enters the test section.

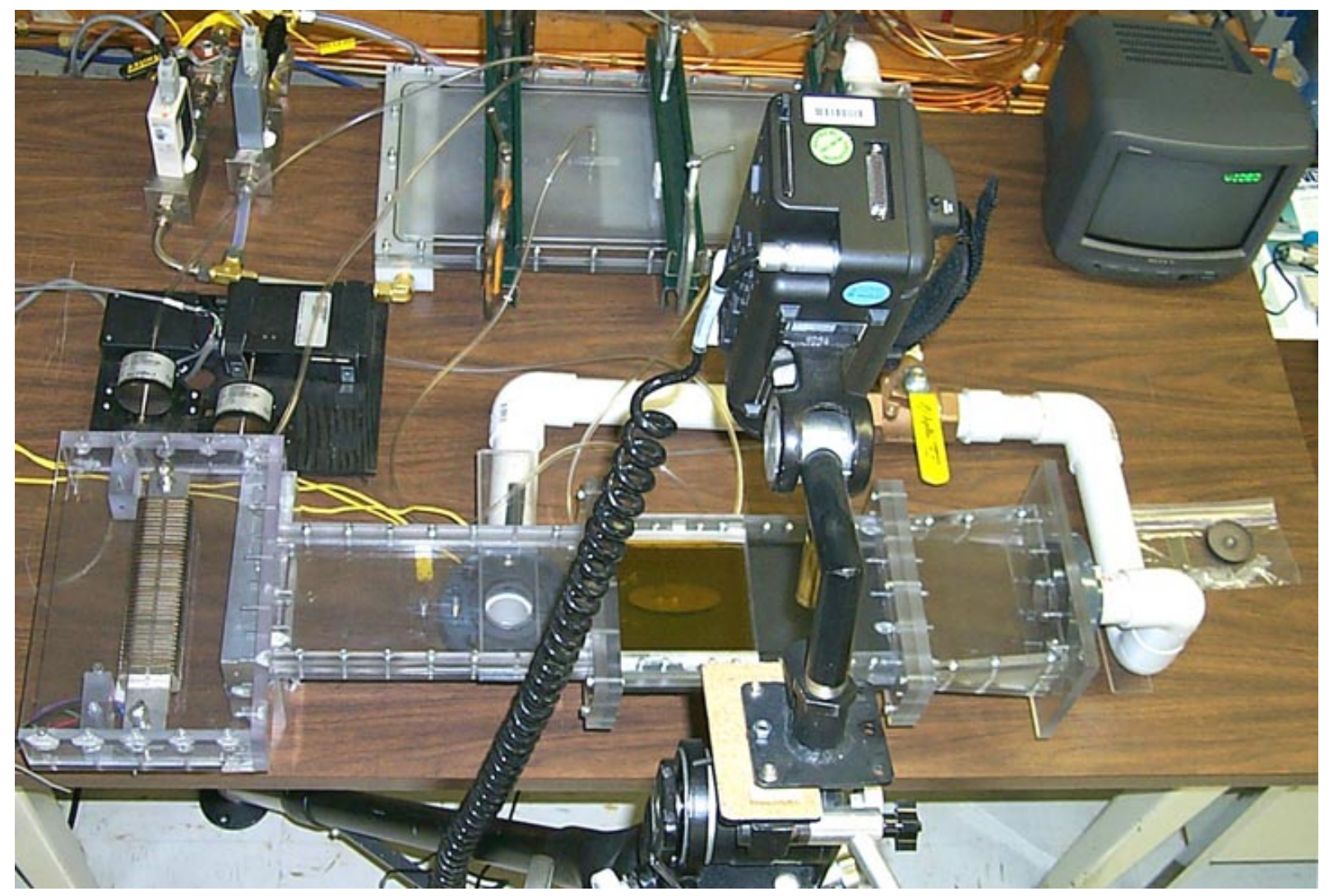

Figure 2-1. Photograph of a bench-top experimental set-up with an oval tube under test.

In order to enable thermal visualization of the test section bottom surface (representing the fin surface), the top wall of the flow duct in the vicinity of the circular tube was formed by a calcium fluoride $\left(\mathrm{CaF}_{2}\right)$ window. Initial testing (single circular tube, delta-winglet pair) was performed using two $\mathrm{CaF}_{2}$ windows, each $12.7 \mathrm{~cm} \times 6.35 \mathrm{~cm} \times 6 \mathrm{~mm}(5 \times 2.5 \times 0.24$ in. $)$. Subsequent testing with the circular tube and winglets was performed using a single larger window: $12.7 \mathrm{~cm} \times 12.7 \mathrm{~cm} \times 6 \mathrm{~mm}(5 \mathrm{in} . \times 5$ in. $\times 0.24$ in.). The $\mathrm{CaF}_{2}$ windows enabled viewing of the test section bottom surface with an imaging infrared camera. Lexan is opaque in the sensitive wavelength range of the camera (3.6 to $5 \mu \mathrm{m})$. The transmissivity of the $\mathrm{CaF}_{2}$ windows is very high (>95\%) in this wavelength range. The test section bottom surface (polycarbonate) was painted black using ultra-flat black paint in order to achieve a surface emissivity very close to 1.0. This emissivity value was verified over a wide temperature range in separate 
camera-calibration tests by comparing camera-indicated temperatures with surface temperatures measured using a precision thin-foil flush-mounted thermocouple bonded to a black-painted polycarbonate test surface. Therefore, no emissivity corrections were required for the infrared temperature measurements. A transient heat transfer measurement technique was employed for obtaining detailed local heat transfer measurements on the model fin surface. A schematic of the flow loop is shown in Figure 2-2. Inlet air was heated to a desired setpoint temperature using an in-line feedback-controlled finned-element air heater $(350 \mathrm{~W})$. The heated air initially flowed through a bypass line until the desired air temperature and flow rate were established. The air was then suddenly diverted through the test section by changing the position of a 3-way valve. Using this technique, the room-temperature fin/tube model was suddenly exposed to a uniformly heated airflow, thereby inducing a heat conduction transient in the lexan substrate. Local surface temperatures on the substrate increase at a rate that depends on the value of the local heat transfer coefficient. This transient local heating was quantitatively recorded using an imaging infrared camera. Values of local heat transfer coefficients were then determined from an inverse heat conduction analysis.

\section{DAS/ System Controller}

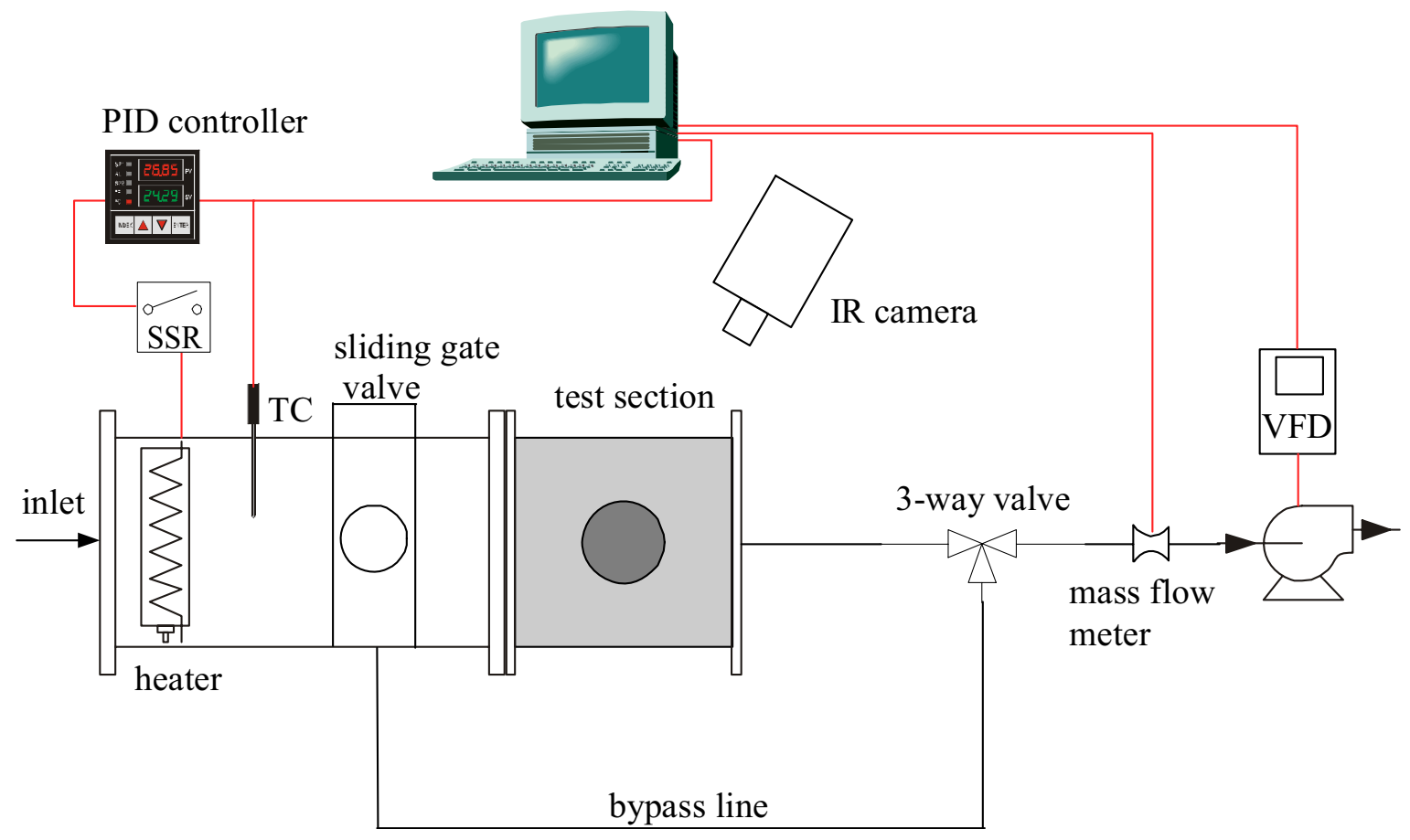

Figure 2-2. Schematic of experimental set-up.

The bypass flow was diverted from the main flow duct through a circular hole ( $4.85 \mathrm{~cm}$ diameter) cut into the bottom of the flow-development section. The center of the hole is located $8.25 \mathrm{~cm}(3.25 \mathrm{in}$.) upstream of the test section entrance. The duct walls downstream of this location were not preheated during the flow establishment period. Therefore, this distance represented a thermal entry length. The flow was suddenly initiated through the test section by changing the position of the 3-way valve, which resulted in covering the flow bypass hole in the bottom of the flow development section by sliding a flat lexan cover (sliding gate valve) over the hole. This procedure provided a continuous flat smooth flow surface, eliminating any concern about flow disturbance associated with a cavity (hole). 
Heater control was accomplished using a PID controller (Yokogawa Model 514) coupled to a solidstate relay with a pulsed relay output. A thermocouple mounted inside the duct measured the process variable. Air temperature uniformity across the duct was verified via infrared imaging of the test surface during preliminary tests with no test cylinder in place. Airflow rate was monitored through the use of an in-line precision mass-flow meter (Kurz model 504FT) plumbed into the exhaust line. Test-section massaverage velocities and Reynolds numbers were calculated based on the standard $\mathrm{m} 3 / \mathrm{hr}$ (SCMH) values obtained from the mass-flow meter. Air was drawn through the test apparatus by a centrifugal blower (1/3 HP, $240 \mathrm{~V}, 3$-phase) located at the apparatus exit. Blower speed was controlled by a sub-micro inverter variable-frequency drive (AC Tech model SF215), which in turn was controlled by a computergenerated 4-20-mA control signal. System flow rate varied linearly with blower speed over the range used in this study from about $1.51 \times 10-3$ to $14.0 \times 10-3 \mathrm{~kg} / \mathrm{s}$. These flow rates correspond to a ductheight Reynolds number $(\mathrm{ReH}=\rho \mathrm{UH} / \mu=\dot{m} / \mu \mathrm{W})$ range of $670-6300$, with a duct of $1.106 \mathrm{~cm}$ and a duct width-to-height ratio, $\mathrm{W} / \mathrm{H}$, of 11.25 .

Two flush-mounted thin-foil thermocouples were bonded to the bottom surface of the test section near the test section inlet. These thermocouples provided a continuous indication of surface temperature at two locations and were used to help determine the exact start time of each test, which occurs when the heated airflow is diverted through the test section.

Quantitative thermal visualization images are obtained using a precision imaging infrared camera (FLIR PRISM DS). This camera uses a fully calibrated $320 \times 244$ platinum-silicide IR CCD focal plane array detector, which operates at a temperature of $77 \mathrm{~K}$. The detector temperature is maintained by a mechanical split-stirling-cycle helium cryo-cooler. In its base mode of operation, the camera can be used to measure infra-red intensities corresponding to temperatures in the -10 to $250^{\circ} \mathrm{C}$ range, with extended ranges available through the use of filters up to $1500^{\circ} \mathrm{C}$. The camera detector has a 12-bit digital dynamic range and a minimum discernible temperature difference of $0.1{ }^{\circ} \mathrm{C}$ at $30^{\circ} \mathrm{C}$. It is equipped with a $25-\mathrm{mm}$ standard lens, which provides a $17 \times 13$ degree field of view. All radiometric information is stored in binary digital files on flash memory cards for subsequent analysis. Infra-red thermography has several advantages over thermochromic liquid crystals for surface temperature mapping, including a wide available temperature range, high spatial resolution, excellent thermal resolution, and full-field direct digital data acquisition and processing.

The thermal image binary data files created by the camera on-board processor are stored in a special 16-bit TIFF gray-scale format (file extension .ana). These files include not only the image pixel values, but also a large amount of camera and test-specific information, such as camera and firmware identifiers, date and time of image acquisition, camera settings at image acquisition, and temperature/pixel calibration data points. This information is included in the TIFF file in the form of "private tags" (see Reference 12). Specific file-format information provided by FLIR was used in conjunction with general information about the TIFF standard found in Reference 12 to fully decode the binary data files for subsequent thermal analysis using a Labview program created for this purpose.

Signals from loop instrumentation were fed into a modular multiplexing data-acquisition system (Hewlett Packard 3852A), which in turn was interfaced to a system-controller computer via an IEEE-488 bus. For this experiment, the data acquisition unit was configured with a 20-channel FET multiplexer with thermocouple compensation, a 5 1/2-digit integrating voltmeter, and a 4-channel voltage/current DAC. The DAC module was used to provide control signals (4-20 mA) to the variable-frequency blower drive. The mass-flow meter was configured to communicate directly with the computer using an RS-232 interface. Data-acquisition and instrument-control system programming was accomplished using Labview (National Instruments Version 5.1) software. The data files included time histories of the thermocouple and mass-flow meter signals with updates at 0.7 -s intervals. 
Heat transfer results were determined for three experimental configurations: circular tube, deltawinglet pair, and circular tube plus delta-winglet pair. The test section geometry for the circular tube is presented in Figure 2-3. The specific geometries for the delta-winglet pair and the circular tube plus delta-winglet pair are presented in Figure 2-4. The winglets had a 1:2 height/length aspect ratio and were
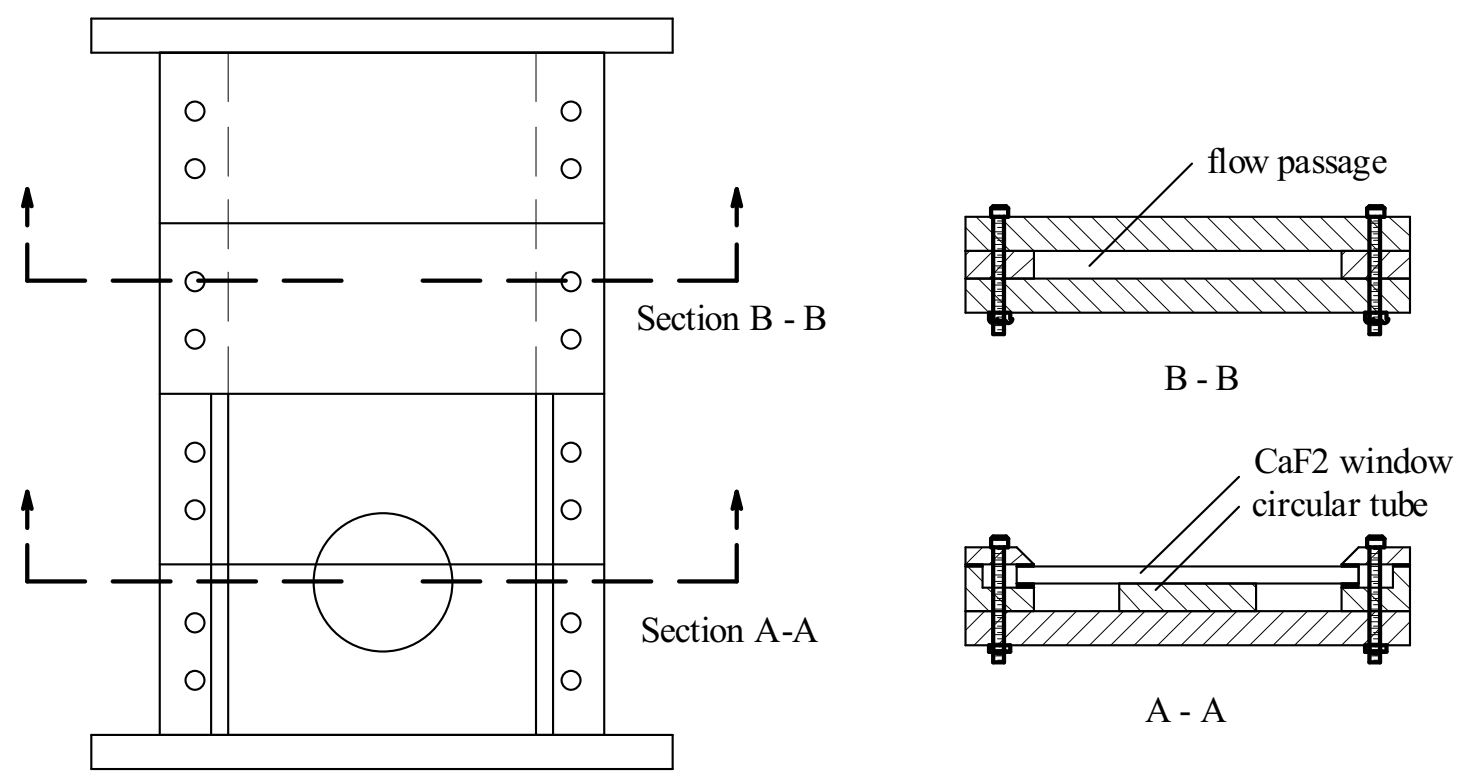

Figure 2-3. Test section top view and cross-sections with circular tube.

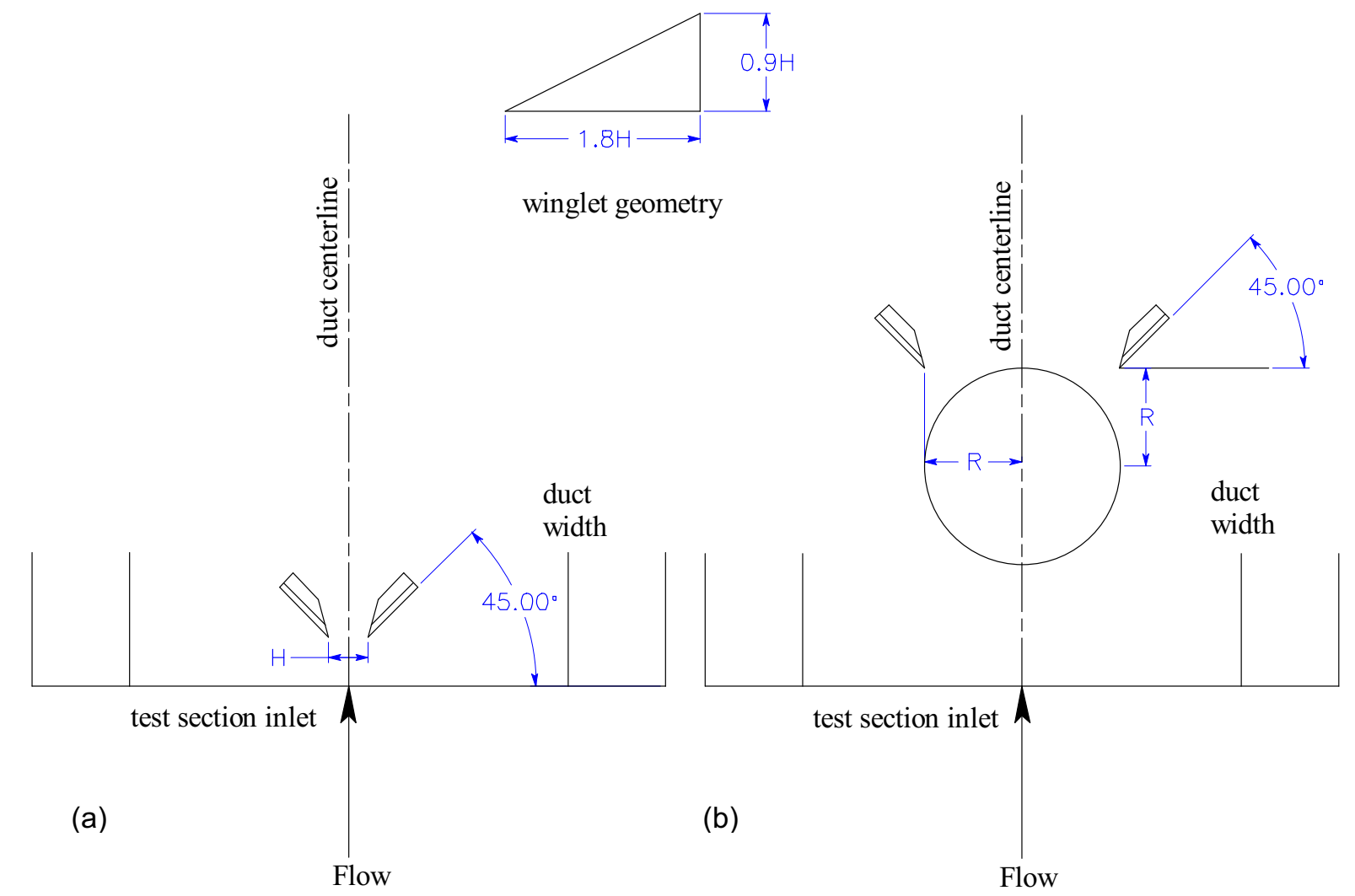

Figure 2-4. Winglet locations and geometry: (a) winglet-only case, (b) circular cylinder plus winglets. 
oriented at a 45 degree angle to the flow. The height of the winglets was $90 \%$ of the channel height. This height was chosen rather than the full channel height in order to avoid damage to the CaF2 windows. The winglets were machined from lexan polycarbonate and were bonded to the test surface. The test configuration for the winglet-only tests is shown in Figure 2-4a. For these tests, the winglets were located near the test section inlet and were spaced one channel height apart. The test configuration for the circular tube plus delta-winglet pair tests is shown in Figure 2-4b. The location of the winglets for these tests was based on the geometry recommended in Reference 6 . The winglet tips are located downstream of the test cylinder at the intersection of the horizontal and vertical cylindrical-tube tangent lines. The angular orientation with respect to the flow was, again, 45 degrees.

\subsection{Experimental Procedure}

As mentioned, a transient heat transfer technique was employed in order to obtain measurements of local heat transfer coefficients on model fin surfaces. Details of the experimental procedure are given here. After the IR camera was powered up and the detector array reached its operating temperature, the camera was positioned above the test section at an appropriate height for observing either the entire portion of the test section or close-up. In order to avoid IR reflections of the warm camera body off the $\mathrm{CaF}_{2}$ windows, the camera was positioned at a small angle off vertical. The camera gain and level adjustments were set such that the minimum observable temperature corresponds to the initial temperature, with a temperature range of $10-15^{\circ} \mathrm{C}$. The software clock on the camera was synchronized with the clock on the data acquisition computer to within $\pm 0.5 \mathrm{~s}$. The 3-way valve was set to the bypass position, and the sliding gate valve was opened. Flow was initiated through the bypass line by adjusting the blower RPM until the desired flow rate was achieved. The air temperature was established by adjusting the PID controller set point value to the desired level, typically $45^{\circ} \mathrm{C}$. Before diverting heated air through the test section, a pretest thermal image of the test section was acquired. At this time, the data acquisition system was set to begin writing data to disk. The 3-way valve position was then changed, and the sliding gate valve was closed to divert the heated airflow through the test section, initiating the convective thermal transient. A number of thermal images of the test section (typically 5) were acquired during the first 5-60 s of the transient. These images were stored on (PCMCIA) flash memory cards and were transferred to the system controller computer after each test.

\subsection{Data Reduction}

The objective of the tests was to obtain detailed maps of local heat transfer coefficient. The IR images provide local surface temperatures at specified times after initiation of the transient. In order to obtain heat transfer coefficients from the measured surface temperatures, the bottom surface of the test section is assumed to behave locally as a one-dimensional semi-infinite solid undergoing a step change in surface heat transfer coefficient. For the $1.27-\mathrm{cm}$ thickness of the lexan test surface, the semi-infinite assumption is valid for at least $88 \mathrm{~s}$ after initiation of the transient. The time-dependent, one-dimensional temperature field within a semi-infinite solid subjected to this boundary condition is given by Incropera and Dewitt: $:^{13}$

$$
\frac{T(0, t)-T_{i}}{T_{\infty}-T_{i}}=\operatorname{erfc}\left(\frac{x}{2 \sqrt{\alpha t}}\right)-\left[\exp \left(\frac{h x}{k}+\frac{h^{2} \alpha t}{k^{2}}\right)\right]\left[\operatorname{erfc}\left(\frac{x}{2 \sqrt{\alpha t}}+\frac{h \sqrt{\alpha t}}{k}\right)\right] .
$$

The time-dependent surface $(\mathrm{x}=0)$ temperature is therefore given by

$$
\frac{T(0, t)-T_{i}}{T_{\infty}-T_{i}}=1-\exp \left(\frac{h^{2} \alpha t}{k^{2}}\right) \operatorname{erfc}\left(\frac{h \sqrt{\alpha t}}{k}\right) .
$$


And if we let

$$
\theta=\frac{T(0, t)-T_{i}}{T_{\infty}-T_{i}} ; \gamma=\frac{h \sqrt{\alpha t}}{k}=\frac{h \sqrt{t}}{\sqrt{\rho c k}}
$$

the equation reduces to

$$
\theta=1-\exp \left(\gamma^{2}\right) \operatorname{erfc}(\gamma)
$$

This equation represents the relationship between the heat transfer coefficient and surface temperature measured at a specific time after the start of the test. It must be solved iteratively for $\gamma$. However, since the camera pixel array includes over 78,000 pixels, it is not practical to directly solve the equation at every pixel. Instead, a look-up table approach was used in the data reduction scheme. The measured temperature range for each thermal image was divided into 100 increments and a value of heat transfer coefficient was obtained for each of these 100 temperatures by iteratively solving Equation (2-4). Each actual pixel temperature was then converted to a heat transfer coefficient by linear interpolation among the 100 increments.

Estimates of the experimental uncertainties of the Reynolds numbers and heat transfer coefficients presented in this report have been obtained based on constant-odds, 95\% confidence level. ${ }^{14}$ For the Reynolds number, individual uncertainties in pitot probe pressure differential, barometric pressure, air temperature, and air viscosity were considered. Results indicate a relative uncertainty of $17 \%$ at $\mathrm{ReH} \sim 1000$ and only $1.7 \%$ at $\mathrm{ReH} \sim 6300$. For the heat transfer coefficients, individual uncertainties in image capture time, surface temperature, air temperature, and substrate thermal product were considered. Results indicate that the relative uncertainty in heat transfer coefficient ranges from $20 \%$ at $\mathrm{h} \sim 10 \mathrm{~W} / \mathrm{m}^{2} \cdot \mathrm{K}$ to $10 \%$ at $\mathrm{h} \sim 120 \mathrm{~W} / \mathrm{m}^{2} \cdot \mathrm{K}$.

\subsection{Heat Transfer with Single Cylindrical Tubes}

The first baseline configuration studied was a single circular tube. Local fin-surface heat transfer results for this configuration are presented in Figures 2-5 through 2-9. Heat transfer results are presented as a function of Reynolds number based on channel height, $\mathrm{H}$. Heat transfer coefficients are based on the test section inlet temperature. A color contour map of local heat transfer coefficient for $\mathrm{ReH}=1000$ is presented in Figure 2-5.

The figure reveals several interesting features, including the heat transfer signature of the horseshoe vortex system that forms in the forward stagnation region around the base of the cylinder and is swept downstream as longitudinal vortices. Heat transfer coefficients on the fin surface in the forward stagnation region are about a factor of 10 higher than corresponding fully developed duct values. A double peak in local heat transfer is evident in the

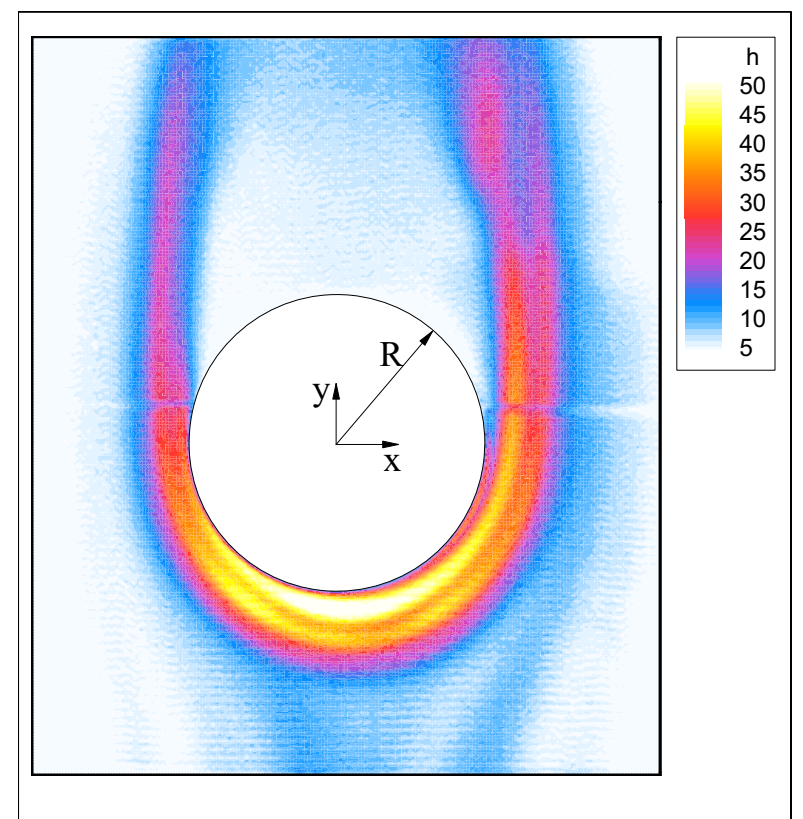

Figure 2-5. Color contour map of local heat transfer coefficient, $\mathrm{h}\left(\mathrm{W} / \mathrm{m}^{2} \mathrm{~K}\right)$ for a circular cylinder, $\operatorname{Re}_{\mathrm{H}}=1000$.

forward stagnation region. This double peak has been observed in several previous studies ${ }^{15-17}$ using both 
mass transfer analogy techniques and liquid crystal thermography. The double peak is associated with the complex multiple-vortex structure that becomes established in the forward stagnation region at the base of a bluff object such as a circular cylinder when a laminar boundary layer flow encounters the object. A detailed study of the fluid mechanics of laminar horseshoe vortices is presented in Reference 18.

Figure 2-5 indicates that flow separation occurs at approximately 90 degrees from the forward stagnation line, which is characteristic of laminar flow separation. The wake region is quite large at this Reynolds number and heat transfer coefficients in the wake region are very low. The coordinate system used in subsequent figures is indicated in Figure 2-5 with the origin located at the center of the cylinder.

Two line plots of local heat transfer coefficient obtained from Figure 2-5 are presented in Figure 2-6. The top plot represents the variation in local heat transfer along the duct centerline at

$\mathrm{x} / \mathrm{R}=0$. The double peak in heat transfer coefficient in the forward stagnation region can also be seen in this figure. Heat transfer coefficients are very high in the forward stagnation region, peaking at around $58 \mathrm{~W} / \mathrm{m} 2 \mathrm{~K}$. Upstream of the stagnation region, $\mathrm{h}$ values are near $10 \mathrm{~W} / \mathrm{m} 2 \mathrm{~K}$. Wake region values are closer to $5 \mathrm{~W} / \mathrm{m} 2 \mathrm{~K}$.

A cross-plot of the transverse variation of local heat transfer coefficient obtained from Figure 2-5 is presented in the lower plot of Figure 2-6. This figure reveals the variation in local heat transfer coefficient across the duct span at a fixed value of $y / R=1.38$. Two major peaks associated with the horseshoe vortex system are evident. The rightmost of these peaks has two subpeaks, remnants of the multiple-vortex structure of the horseshoe vortex.

A close-up of the cylinder stagnation region at $\mathrm{Re}_{\mathrm{H}}=1080$ is presented in Figure 2-7, along with a plot of the centerline variation of heat transfer coefficient. In order to avoid reflection from the $\mathrm{CaF}_{2}$ windows, the thermal images are acquired with the camera positioned not directly above the test cylinder. Rather, the camera views the test section from a small angle. Therefore, the edge of the test cylinder can be seen in the image, as indicated in Figure 2-7. The close-up
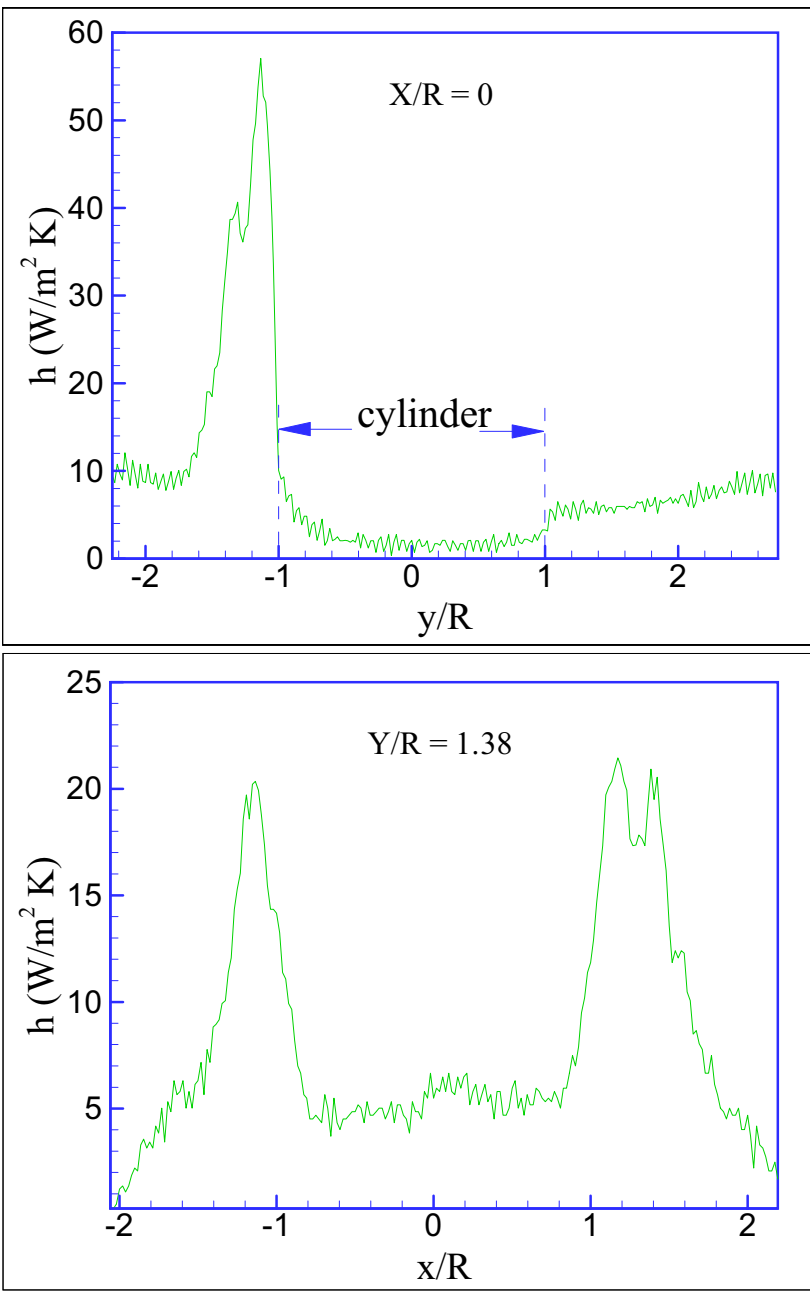

Figure 2-6. Top centerline variation of heat transfer coefficient; bottom: transverse variation of heat transfer coefficient in wake region. X-Y coordinates correspond to those in Figure 2-5. heat transfer contour plot again clearly shows the double peak in heat transfer in the forward stagnation region. A higher resolution line plot of the centerline variation in heat transfer coefficient in the forward stagnation region is shown in the right portion of the Figure. 


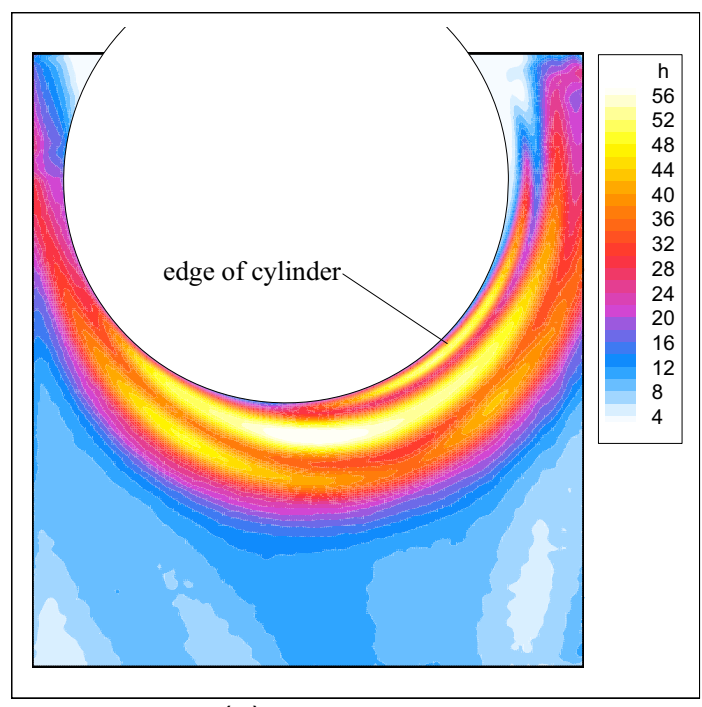

(a)

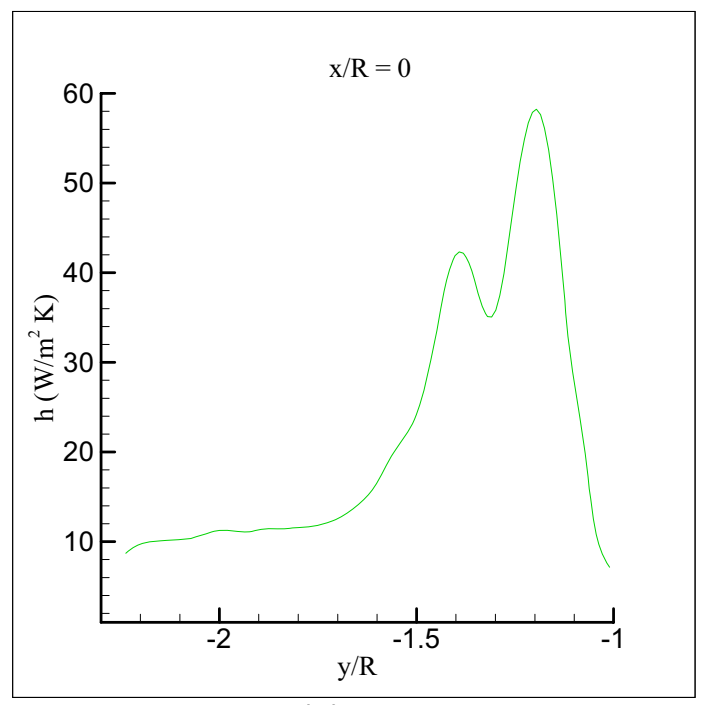

(b)

Figure 2-7. Cylinder stagnation region details, $\operatorname{Re}_{\mathrm{H}}=1080$ : (a) contour plot for heat transfer coefficient, $\mathrm{h},(\mathrm{b})$ variation in $\mathrm{h}$ along the centerline.

Heat transfer distributions at three additional Reynolds numbers are presented in Figure 2-8. At $\mathrm{ReH}=1375$, the duct flow is laminar, and flow separation occurs near 90 degrees. The approach flow at this Reynolds number is characterized by four distinct longitudinal streaks, deflected around the cylinder. A hint of similar streaks is also visible at $\mathrm{ReH}=1000$ (Figure 2-7) and in Figure 2-8 at $\mathrm{ReH}=2650$, but they are by far the most evident at $\mathrm{ReH}=1375$. These streaks may be caused by secondary flows that become established in the rectangular-duct flow development section. At $\mathrm{ReH}=1375$, the horseshoe vortices downstream of the cylinder begin to bend inward toward the wake region. At $\mathrm{ReH}=2650$, flow separation is delayed to about 110 degrees from the stagnation line, and the wake region begins to "fill in" with relatively high heat transfer coefficients due to increased turbulent mixing. This trend continues at $\mathrm{ReH}=5200$ with a much larger region of high heat transfer coefficients. Note that many commercial heat exchangers, including geothermal air-cooled condensers operate at $\mathrm{ReH}$ values below 1000 . At low Reynolds numbers, the regions of increased heat transfer are constrained to relatively small regions just upstream and to the sides of the tube and in the immediate vicinity of the horseshoe vortices downstream of the tube. Therefore, at low Reynolds numbers, there is great potential for significant heat transfer enhancement through the usage of strategically placed vortex generators on fin surfaces.

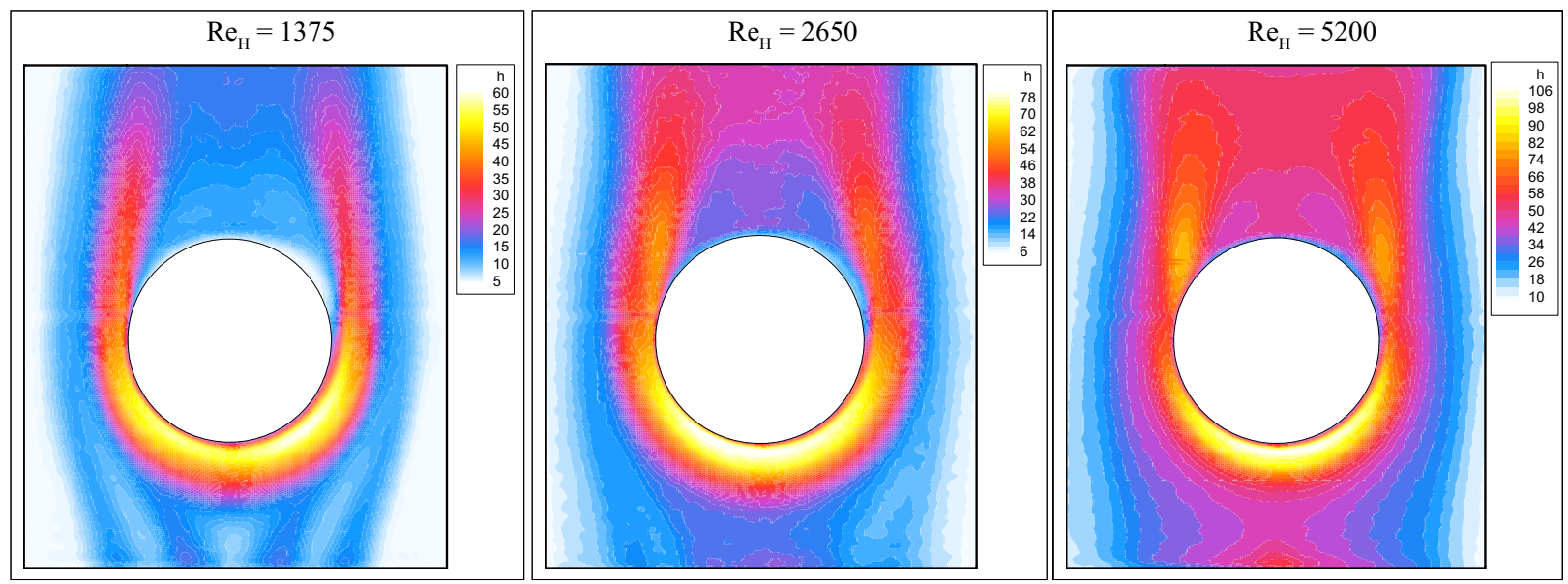

Figure 2-8. Heat transfer distribution trends with the Reynolds number, circular tube. 
Line plots of local fin-surface heat transfer coefficient trends with the Reynolds number are presented in Figure 2-9. Figure 2-9a represents duct-centerline heat transfer coefficients along $x / R=0$ and Figure $2-9 \mathrm{~b}$ represents the transverse variation in heat transfer coefficient in the wake region at $y / R=1.2$. The large peak in heat transfer coefficient in Figure $2-9 a$, near $y / R=-1.3$ is associated with the forward stagnation region horseshoe vortex. Heat transfer coefficients in the wake region $(y / R>1)$ are very close to zero for the lowest Reynolds number shown and increase dramatically with increasing Reynolds number. The two peaks seen in Figure 2-9b at each Reynolds number result from the downstream longitudinal portion of the horseshoe vortex. Examination of these figures reinforces the fact that heat transfer coefficients increase with Reynolds number and are more uniform at higher Reynolds numbers due to turbulent mixing. At low Reynolds numbers, relatively high heat transfer coefficients are confined to very small areas in the stagnation and horseshoe vortex regions.

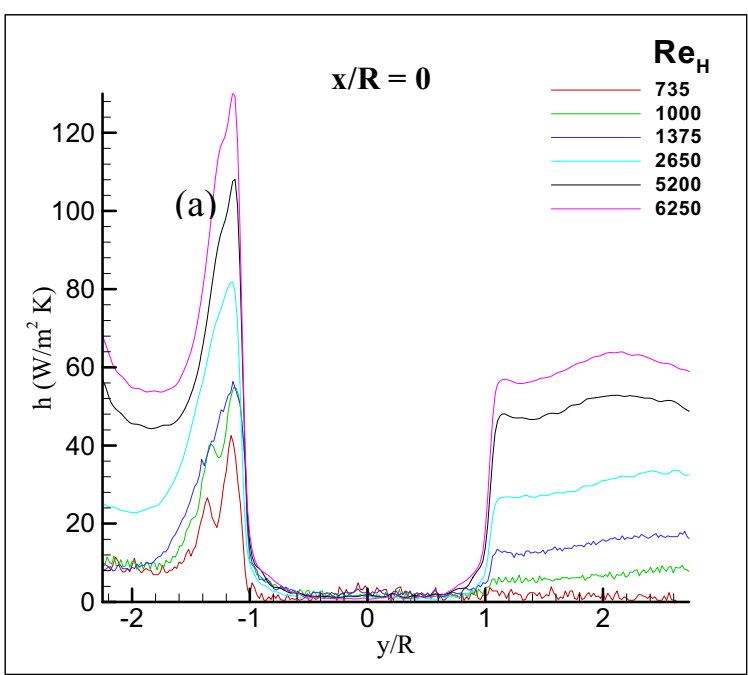

(a)

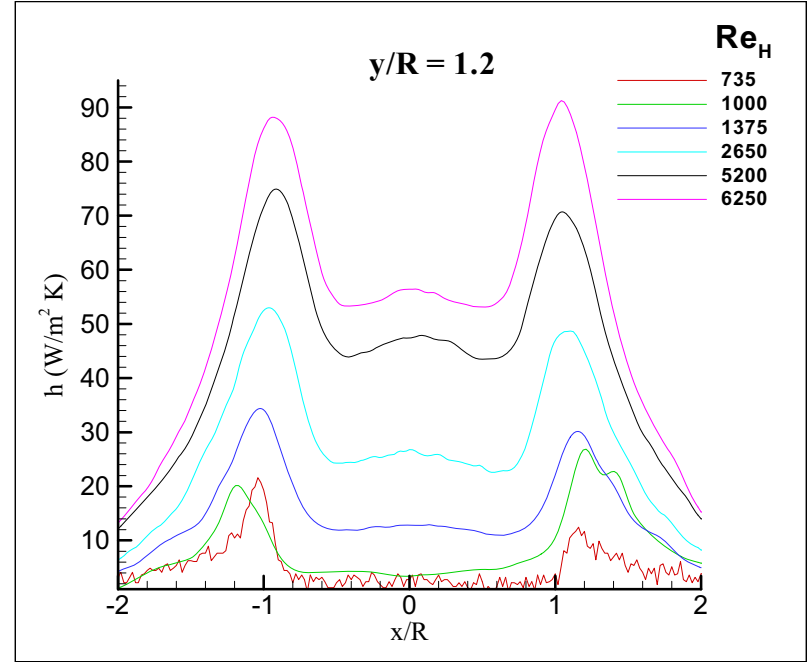

(b)

Figure 2-9. Line plots of fin-surface heat transfer coefficient, circular tube: (a) streamwise variation along duct centerline, (b) wake region transverse variation, $\mathrm{y} / \mathrm{R}=1.20$.

\subsection{Heat Transfer with Single Cylindrical Tubes and Winglets}

Local surface heat transfer contour plots for the delta-winglet-pair configuration (design per Figure 2-4) are presented in Figure 2-10. Heat transfer results are presented as a function of the Reynolds number based on channel height, $\mathrm{H}$. Heat transfer coefficients are based on the test section inlet temperature. The results presented in Figure 2-10 reveal the heat transfer effects of a double-vortex system associated with each winglet. The vortices are swept downstream as longitudinal vortices. The main vortex, located directly downstream of the vortex generators, is formed by flow separation along the leading edge of the winglets. The corner vortex, located outside of the main vortex, develops like a horseshoe vortex on the upstream-facing pressure side of the winglets. The heat transfer effects of the vortices persist for many channel heights (at least 15) downstream of the winglet location. The horizontal line near the center of each heat transfer distribution is an artifact caused by the boundary between two of the $\mathrm{CaF} 2$ windows. The small circular white region in the center of each image just below the horizontal window boundary is a bolt hole used to attach a circular disk for the fin/tube studies, discussed later in this report. This hole was covered with tape on the outside substrate surface to avoid any flow disturbance. Apparent heat transfer coefficient values on the winglets themselves are not valid, since the one-dimensional semi-infinite assumption does not apply at this location.

At low Reynolds numbers, maximum fin-surface heat transfer coefficients are observed in the main vortex downstream of the winglets. At higher Reynolds numbers, maximum values are associated with the corner vortex, very close to the outer corner of the winglets. The magnitudes of these peak values are 
similar to the peak values observed in the same duct in the stagnation region of a circular cylinder, as shown in Figures 2-9. The peak values are about four to five times larger than corresponding fully developed duct values. However, mean surface heat transfer coefficient values for the configuration displayed in Figure 2-10 are just about equal to the corresponding fully developed duct values, as predicted using the Dittus-Boelter correlation, with the Reynolds number based on the hydraulic diameter of the rectangular duct. The individual vortices appear to be most well-defined at the lowest Reynolds number shown. Lowest heat transfer coefficients occur in the corner regions of the rectangular duct, which are at the sides of the images shown in Figure 2-10.
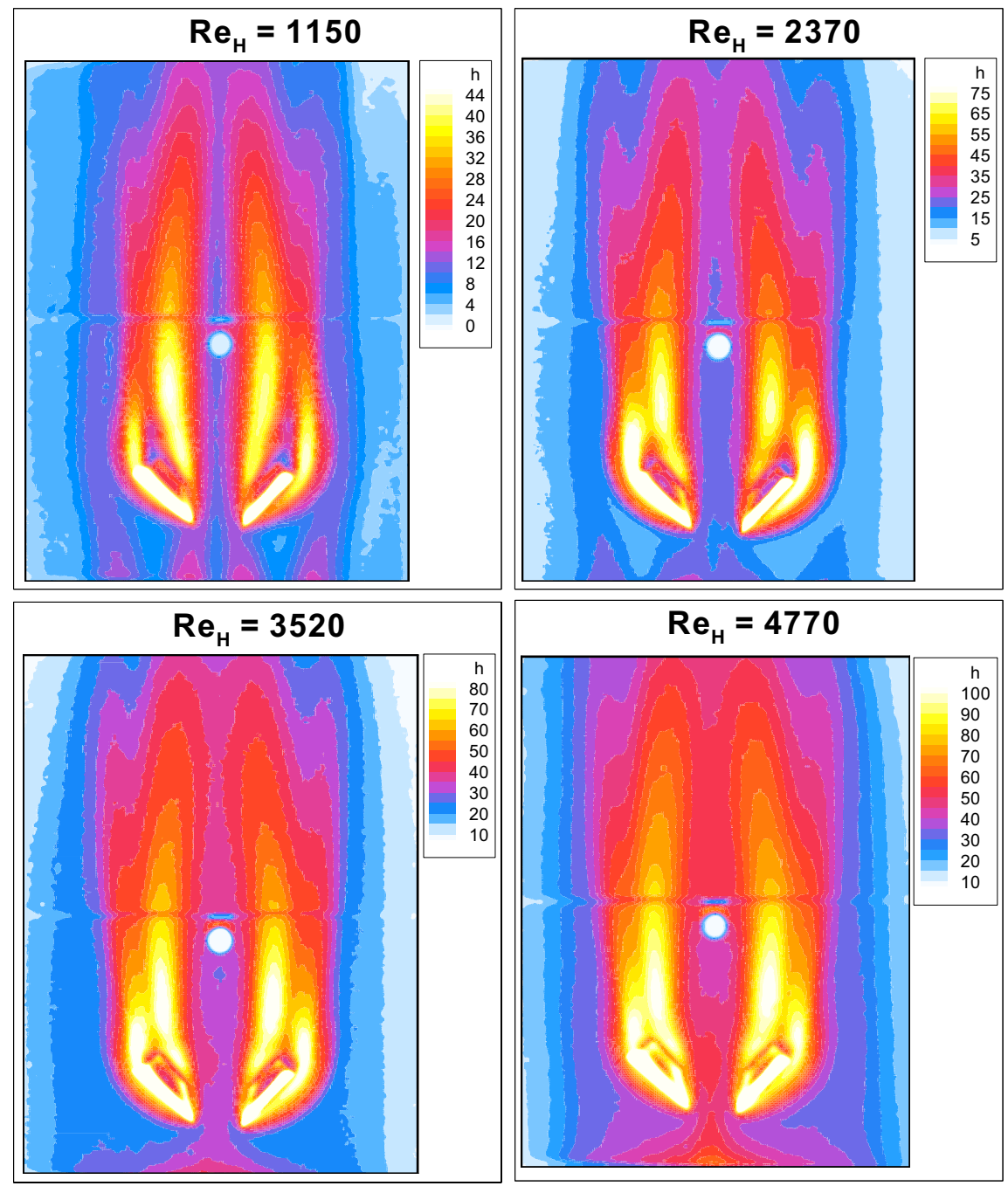

Figure 2-10. Local fin-surface heat transfer coefficients for a delta-winglet pair.

The spanwise variation in local fin-surface heat transfer coefficient for the delta-winglet-pair configuration is presented in Figure 2-11 at two different axial measurement locations. The coordinate system used is defined in the figure. These plots clearly show the heat transfer effects of the wingletinduced double-vortex system. The local spanwise variation in heat transfer coefficient at $\mathrm{x} / \mathrm{H}=3.75$, which is just downstream of the winglets, shows very sharp double peaks in local heat transfer associated with each vortex generator. For all of the Reynolds numbers shown, the highest heat transfer coefficients at each axial location are associated with the primary vortex. The magnitude of the local heat transfer coefficient decreases sharply from the peak values to a minimum near the sides of the rectangular duct. 
At $\mathrm{y} / \mathrm{H}=7.50$, the spanwise heat transfer variations are broader, and the peak associated with the corner vortex is visible, but indistinct.

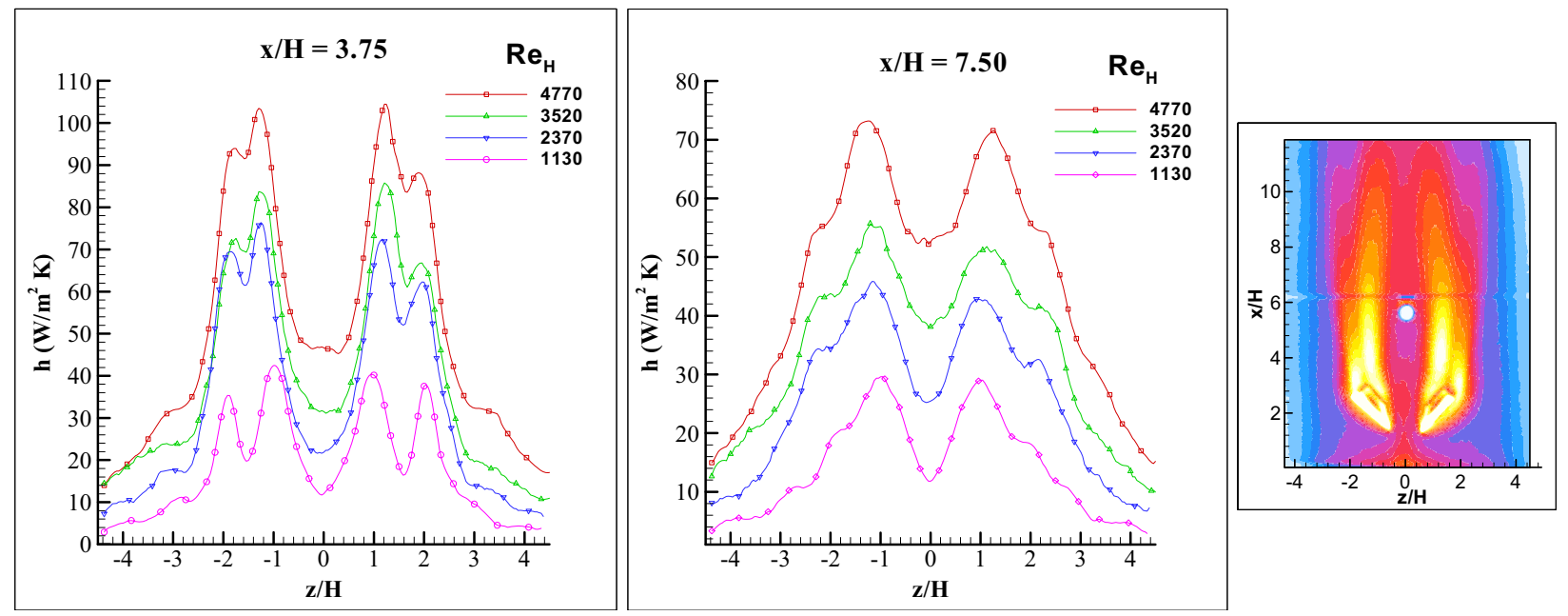

Figure 2-11. Spanwise variation of local heat transfer coefficient for delta-winglet-pair configuration.

The second configuration considered for this study was a circular cylinder and winglets (see Figure 2-4 for the design of the winglets). Local fin-surface heat transfer results for this configuration are presented in Figure 2-12 for four Reynolds numbers. The addition of winglets yields a reduction in the size of the low-heat-transfer wake region and also enhances local heat transfer in the vicinity of the winglets similar in magnitude to the local enhancement observed for the delta-winglet-pair cases shown in Figure 2-12. With the exception of the lowest Reynolds number case, peak local heat transfer coefficients in the vicinity of the winglets are similar to the peak values observed in the cylinder stagnation region. A distinct double peak in local heat transfer coefficient can be seen in the cylinder stagnation region at $\mathrm{ReH}$ $=670$. O'Brien and Sohal ${ }^{11}$ have discussed this double peak for a cylinder without winglets. The spanwise streaks evident near the test section inlet for $\mathrm{ReH}=1224$ are associated with secondary flows that become established in the flow development section of the rectangular duct.

The horseshoe vortex that forms in the tube stagnation region is swept downstream along the side of the tube as a longitudinal vortex, producing a streak of high fin-surface heat transfer that persists well downstream of the tube. Each winglet produces two vortices: a primary vortex and a corner, horseshoetype vortex. The primary vortex, located directly downstream of the winglet, is formed by flow separation along the top edge of the winglet. The corner vortex, located outside of the main vortex, develops like a horseshoe vortex on the upstream-facing pressure side of the winglets. A direct comparison of local heat transfer distributions for a circular cylinder without and with winglets at $\mathrm{Re}_{\mathrm{H}}=\sim 1200$ is presented in Figure 2-13. The comparison reveals that, for this winglet location, the horseshoe vortex produced by the interaction of the flow with the circular cylinder is disrupted by the winglets. There is a reduction in the width of the low-heat-transfer wake region, but the heat transfer coefficients directly downstream of the cylinder are actually slightly reduced for the winglet case compared to the no-winglet case. Stagnationregion heat transfer coefficients are slightly higher for the winglet case compared to the no-winglet case. A plot of the spanwise variation in local wake-region heat transfer coefficient at an axial location just downstream of the winglets is presented in Figure 2-14 for the same two data sets presented in Figure 2-13. 
Figure 2-12 (Right). Local fin-surface heat transfer distributions for circular cylinder plus winglets.

Figure 2-13 (Below). Direct comparison of local heat transfer distributions for a circular cylinder, with and without winglets.

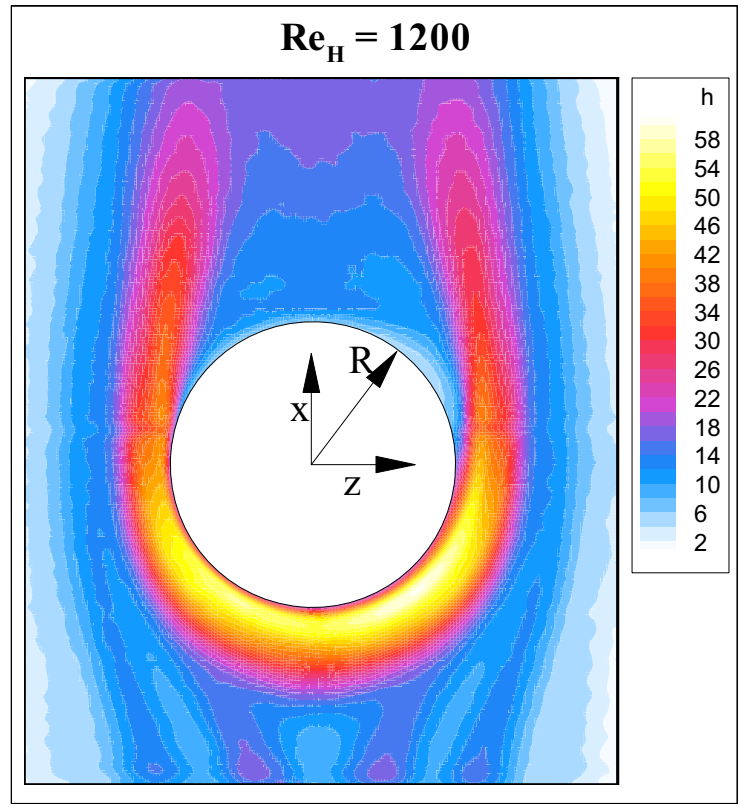

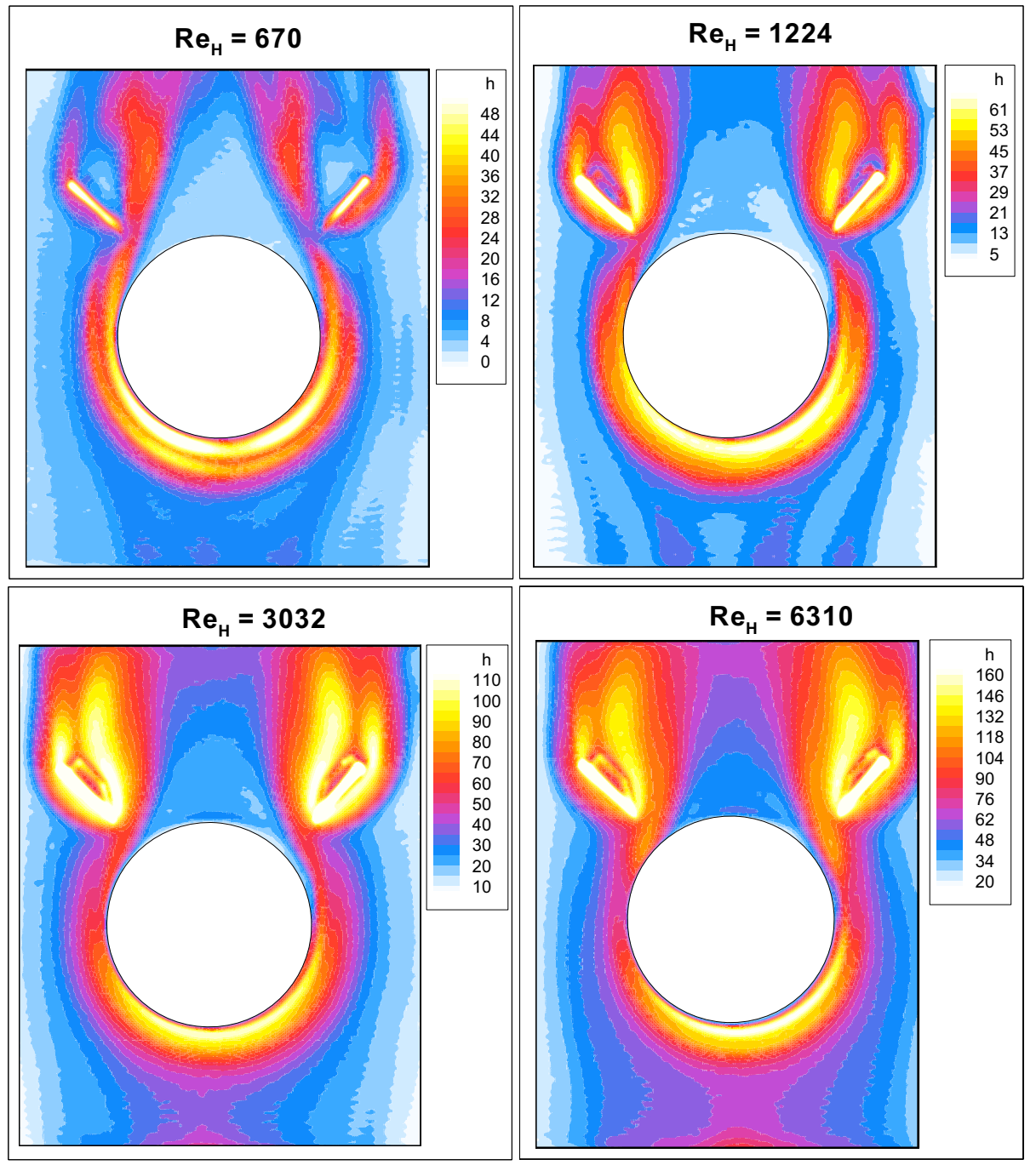

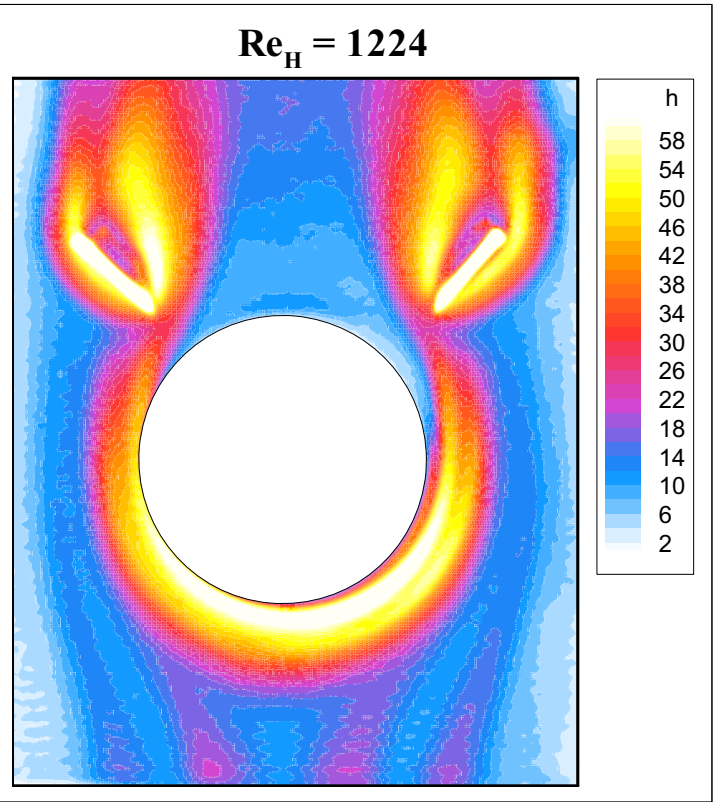


The spanwise variation for the winglet case clearly shows a double peak associated with each winglet. A single peak associated with each horseshoe vortex is evident in the no-winglet curve.

Overall mean fin-surface Nusselt numbers calculated from the heat-transfer-coefficient data files are presented in Figure 2-15. For calculation of mean Nusselt numbers, the surface areas occupied by the winglets and the cylinder (if applicable) were not included. The characteristic dimension used here for both the Reynolds number and the Nusselt number is the channel height, $\mathrm{H}$ and chosen for consistency with related literature3-9. Results from three configurations are included in the figure: delta-winglet pair (no cylinder), circular cylinder without winglets, and circular cylinder with winglets. In addition, a curve representing the Dittus-Boelter correlation for turbulent duct flow is also included.

Per accepted Practice 13, the Dittus-Boelter results were calculated using the duct hydraulic diameter as the characteristic dimension. The Reynolds and Nusselt numbers were then converted to the form based on the duct height, $\mathrm{H}$. Note that since the hydraulic diameter for this duct is almost twice the duct height, transition to turbulence would be expected to occur at an $\mathrm{ReH}$ value of about 1250.

Results presented in Figure 2-15 indicate a significant level of heat transfer enhancement associated with the deployment of the winglets with the circular cylinder. At the lowest Reynolds numbers (which correspond to the laminar operating conditions of existing geothermal air-cooled

condensers), the enhancement level is nearly a factor of two. At higher Reynolds numbers, the enhancement level is close to 50\%. Mean Nusselt numbers for the cylinder-only case and the wingletonly case were very similar to each other and very close to the Dittus-Boelter correlation. The agreement

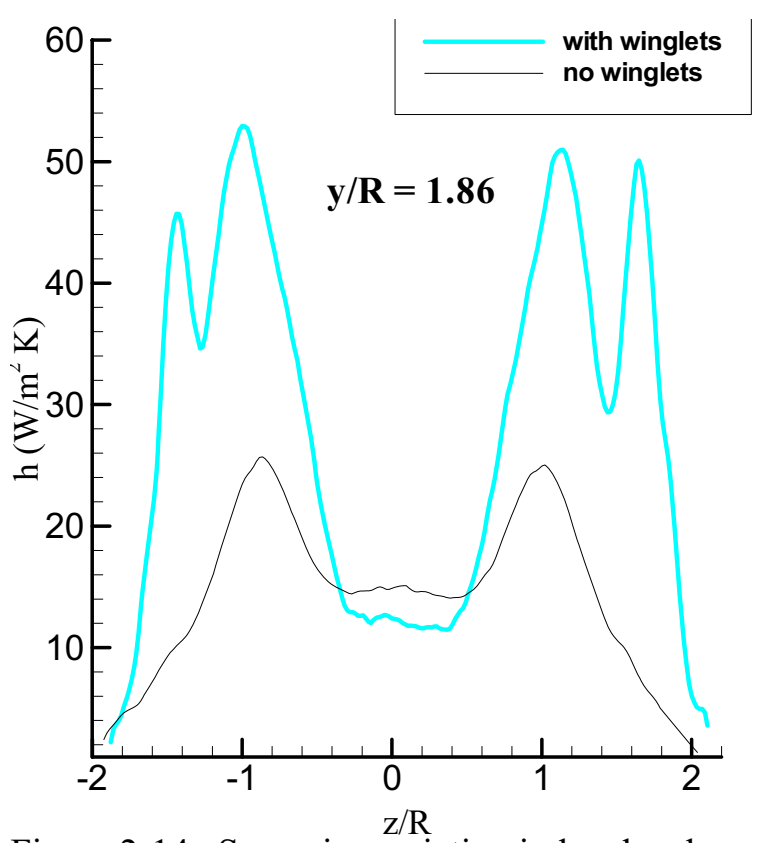

Figure 2-14. Spanwise variation in loeal wakeregion heat transfer coefficient, with and without winglets, $\mathrm{Re}_{\mathrm{H}}=1200$.

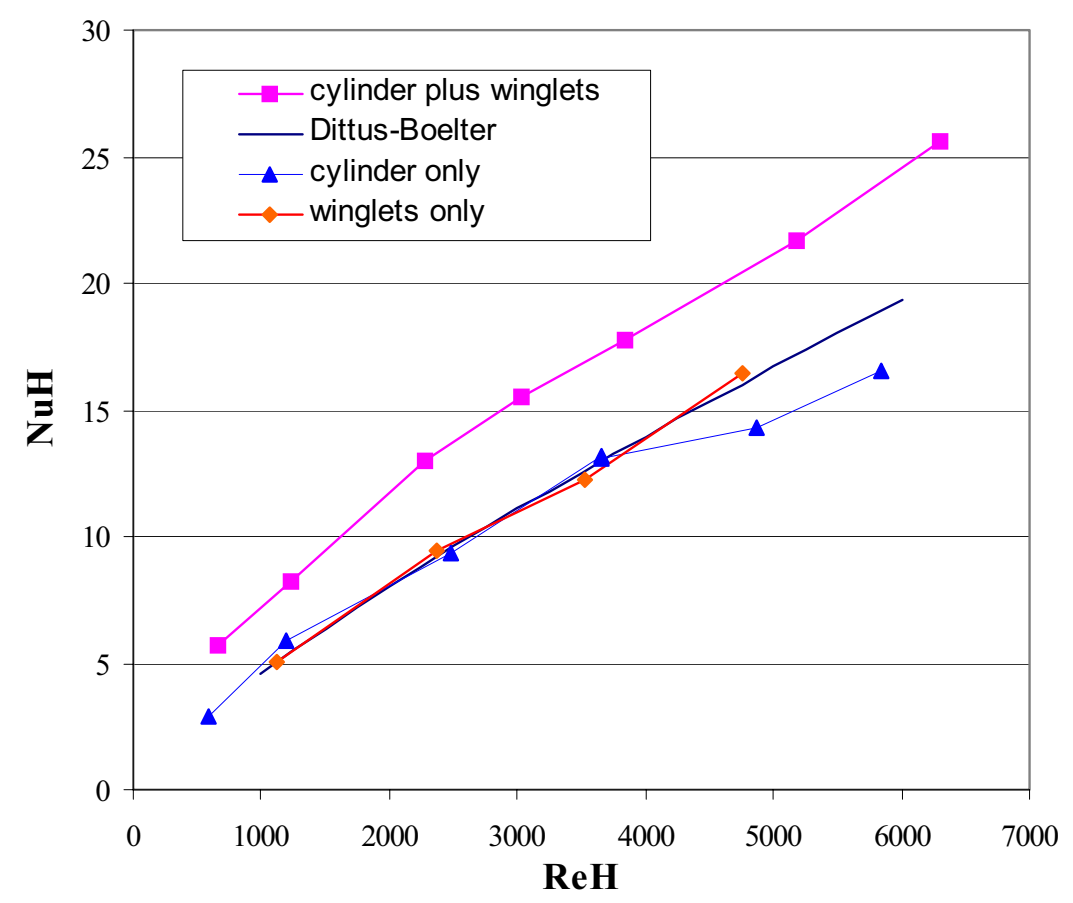

Figure 2-15. Mean Nusselt numbers for several cases. 
with Dittus-Boelter is somewhat surprising, because this correlation applies to fully developed turbulent duct flow without any protuberances in the flow. Low heat transfer coefficient values in the corner regions of the duct were included in the calculation of the mean values. Also, when the cylinder is in place, high stagnation-region local heat transfer is offset by the low wake-region values.

\subsection{Heat Transfer with Single Oval Tubes and Winglets}

Heat transfer results are presented for three experimental configurations: oval (elliptical) tube, oval tube plus a single delta-winglet pair, and oval tube plus two delta winglet pairs in a staggered array. The geometric details of the oval tube, the winglets, and the winglet deployment locations are presented in Figure 2-16. The elliptical tube was fabricated with the same cross-sectional area as the circular tube used in earlier tests. It has a 3:1 aspect ratio between the major and minor axis lengths. The resulting minor and major axis lengths were $2 \mathrm{~b}=2.93 \mathrm{~cm}(1.15 \mathrm{in}$.) and $2 \mathrm{a}=8.80 \mathrm{~cm}$ (3.464 in.), respectively. The winglets had a 1:2 height/length aspect ratio and were oriented at a 30-degree angle to the flow. The height of the winglets was $90 \%$ of the channel height. This height was chosen rather than the full channel height in order to avoid damage to the $\mathrm{CaF} 2$ windows. The winglets were machined from lexan polycarbonate and were bonded to the test surface. The winglet deployment geometry for these tests was based on the geometry recommended in Reference 10 .
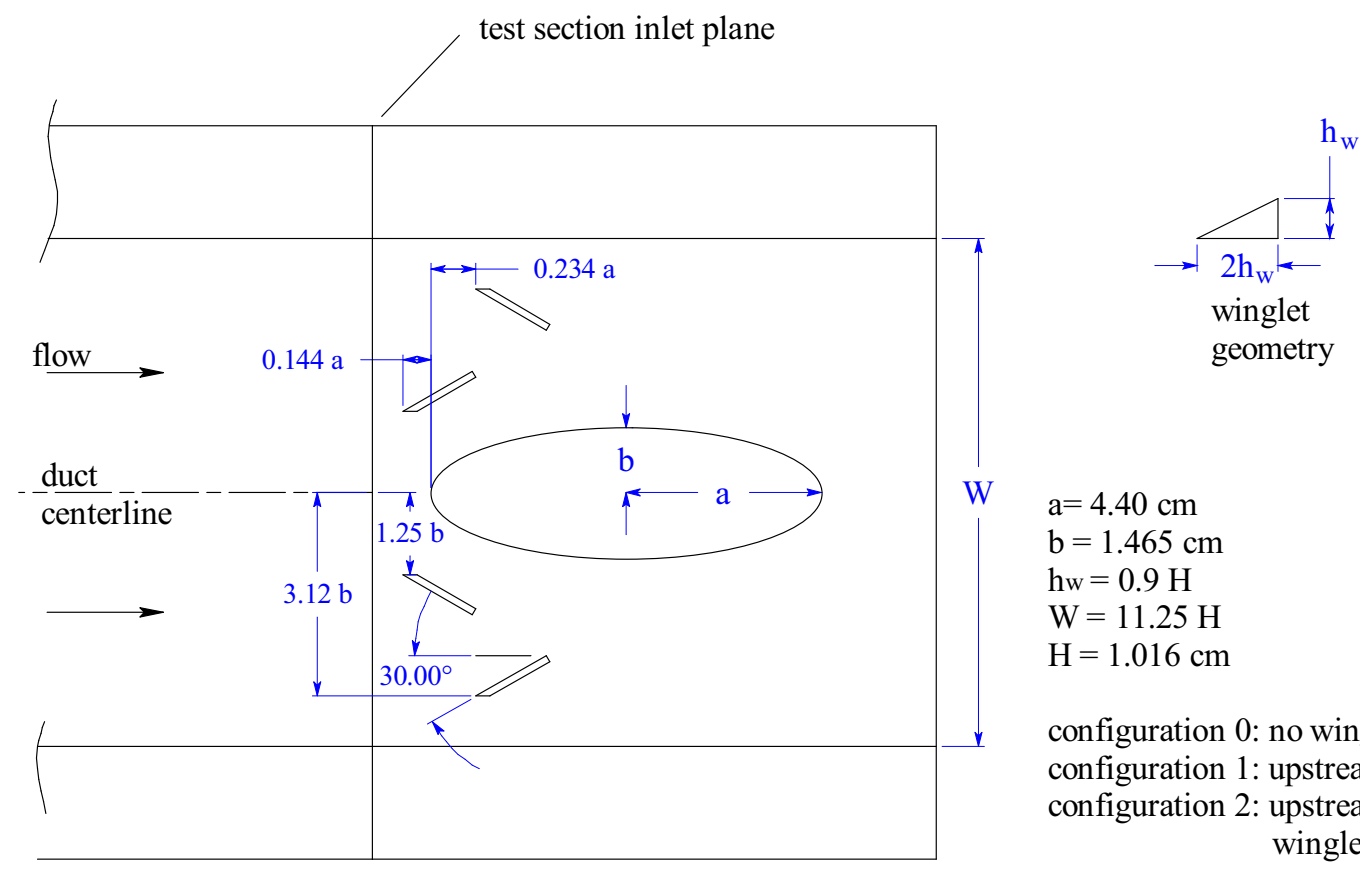

$$
\begin{aligned}
& \mathrm{a}=4.40 \mathrm{~cm} \\
& \mathrm{~b}=1.465 \mathrm{~cm} \\
& \mathrm{~h}_{\mathrm{w}}=0.9 \mathrm{H} \\
& \mathrm{W}=11.25 \mathrm{H} \\
& \mathrm{H}=1.016 \mathrm{~cm}
\end{aligned}
$$

configuration 0: no winglets

configuration 1: upstream winglet pair only configuration 2: upstream plus downstream winglet pairs

Figure 2-16. Winglet locations and geometry.

Four contour plots of local heat transfer coefficient for an oval tube with a single winglet pair for four Reynolds numbers are presented in Figure 2-17. The distributions appear to be slightly asymmetric. The slight asymmetry visible in the heat transfer images is due to the effect of the slightly off-vertical viewing angle of the IR camera, as indicated previously in the discussion of Figure 2-7. These contour plots exhibit trends that are similar to the circular-tube heat transfer distributions presented in Figure 2-8. In these figures, flow is from bottom to top. The winglets are visible in the figures near the upstream leading edge of the oval tubes. Apparent heat transfer coefficients on the winglets themselves are not valid since the winglets protrude into the flow and therefore do not behave as a semi-infinite solid. At the lowest Reynolds number shown, $\mathrm{ReH}=1410$, longitudinal streaks are evident, similar to the streaks seen 
in Figure 10 at $\mathrm{ReH}=1375$. These streaks do not appear to be directly related to the horseshoe vortex system, but rather may be formed as secondary flows in the rectangular flow-development duct well upstream of the elliptical tube. The streaks are again most obvious only at one of the Reynolds numbers shown, $\mathrm{ReH}=1410$. They can also be seen at $\mathrm{ReH}=2800$, but not as distinctly. As in the circular-tube cases, a horseshoe vortex system is also established for the elliptical tube cases. It results in the high stagnation-region heat transfer coefficients seen in Figure 2-6. In addition, longitudinal vortices are produced and swept downstream of the stagnation region, resulting in enhanced heat transfer along the sides and downstream of the elliptical tube. The heat transfer results indicate that the flow separation point moves downstream, and that the size of the low-heat-transfer wake region shrinks significantly with increasing Reynolds number.
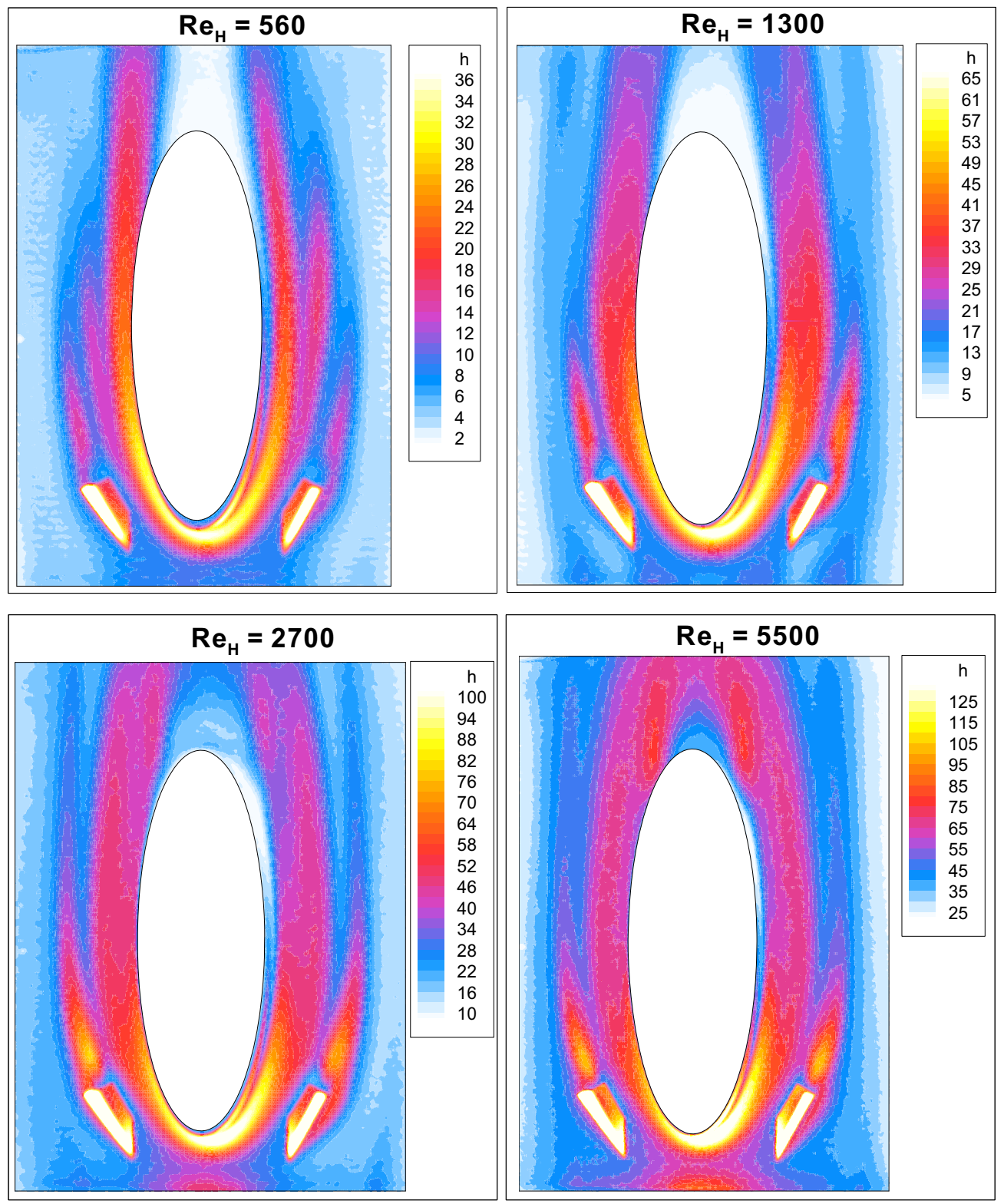

Figure 2-17. Local fin surface heat transfer coefficients for an oval tube plus a single winglet pair. 
Results presented in Figure 2-17 clearly indicate local areas of high fin-surface heat transfer in the stagnation region of the oval tube, along the side of the oval tube, and downstream of the winglets. As discussed in case of a cylindrical tube, highest heat transfer coefficients are in the tube stagnation region. Because of momentum and thermal entry lengths preceding the test section, very high heat transfer coefficients that would normally be expected to occur at the fin leading edge in an actual finned tube are absent. The horseshoe vortex formed in the tube stagnation region is swept downstream along the side of the tube as a longitudinal vortex, producing a streak of high fin-surface heat transfer that persists well downstream of the tube. Each winglet produces two vortices: a primary vortex and a corner, horseshoetype vortex. The primary vortex, located directly downstream of the winglets, is formed by flow separation along the top edge of the winglets. The corner vortex, located outside of the main vortex, develops like a horseshoe vortex on the upstream-facing pressure side of the winglets. The fin-surface heat transfer enhancement associated with the primary vortex is visible in the images of Figure 2-17 directly downstream of each winglet, starting about a half-winglet-length directly axially downstream of each winglet. The heat transfer enhancement associated with the corner vortex of each winglet is visible in the images as a streak that begins on the upstream side of each winglet and is swept to the outside and downstream. The heat transfer effects of all three of these vortices are most distinctly visible in the lowest Reynolds number case presented in Figure 2-17. In this figure, moving outward spanwise from the side of the tube, the tube horseshoe vortex, the primary winglet vortex, and the corner winglet vortex are all visible. At low Reynolds number, the wake region downstream of the oval tube represents a very low heat transfer region. However, at higher Reynolds numbers, this region "fills in" due to the transition to turbulent flow in the duct and the boundary layer that forms on the tube wall. The increase in overall heat transfer associated with the deployment of the winglets will be discussed below. Results for the case of two delta winglet pairs in a staggered array are presented in Figure 2-18.

Local heat transfer results for an oval tube with two winglet pairs deployed near the forward stagnation region in a staggered configuration are presented in Figure 2-18. These results are similar to those shown in Figure 2-17. Local regions of high heat transfer associated with the tube stagnation region, the tube horseshoe vortex, and the primary and corner vortices produced by each winglet are all visible in the images. Trends with increasing Reynolds number are also similar to those in the singlewinglet-pair case.

Mean fin-surface heat transfer coefficients have been calculated based on the local heat transfer results for the seven flow configurations studied so far. These configurations, listed in Figure 2-19 are (1) open channel, (2) circular cylinder with a pair of toe-out winglets in the tube wake, (3) circular cylinder without any winglets, (4) a pair of toe-out winglets in a rectangular duct, (5) an oval tube, (6) and an oval tube with a pair of toe-out winglets on the upstream (front) side of the tube, and (7) an oval tube with two (toe-out and toe-in) pairs of winglets on the upstream side of the tube. For these calculations, only the active fin area is considered. The areas covered by the tubes (circular or oval) or the winglets are not included. Results of these calculations are presented in Figure 2-19 in the form of the Nusselt number based on channel height, $\mathrm{NuH}$, versus the Reynolds number based on channel height, ReH. A small schematic of each flow configuration is shown in the top of the figure. Highest heat transfer coefficients were observed for the case of a circular tube plus winglets with the winglets located on the downstream side of the cylinder, oriented at a 45-degree angle to the flow (configuration a). The cases of oval tube plus one pair of winglets and oval tube plus two pairs of winglets yielded similar mean heat transfer results, with the single-winglet-pair configuration actually producing higher heat transfer at the highest Reynolds numbers. The addition of the single winglet pair to the oval-tube geometry yielded significant heat transfer enhancement, averaging 38\% higher than the oval-tube, no-winglet case. Mean Nusselt numbers for the cases of a circular tube without winglets and a single delta-winglet pair with no tube yielded similar results. Heat transfer results for the oval tube without winglets were quite low. Lowest heat transfer coefficients, as expected, were produced by the open-channel configuration, because in the absence of a tube and winglets, no vortices are generated in the fluid flowing in the duct. Even in the case 
of an oval tube (configuration d), the Nusselt number is lower when compared with a circular tube because its streamline shape gives rise to lower vortices that are less effective in enhancing the heat transfer compared with a case of circular tube.
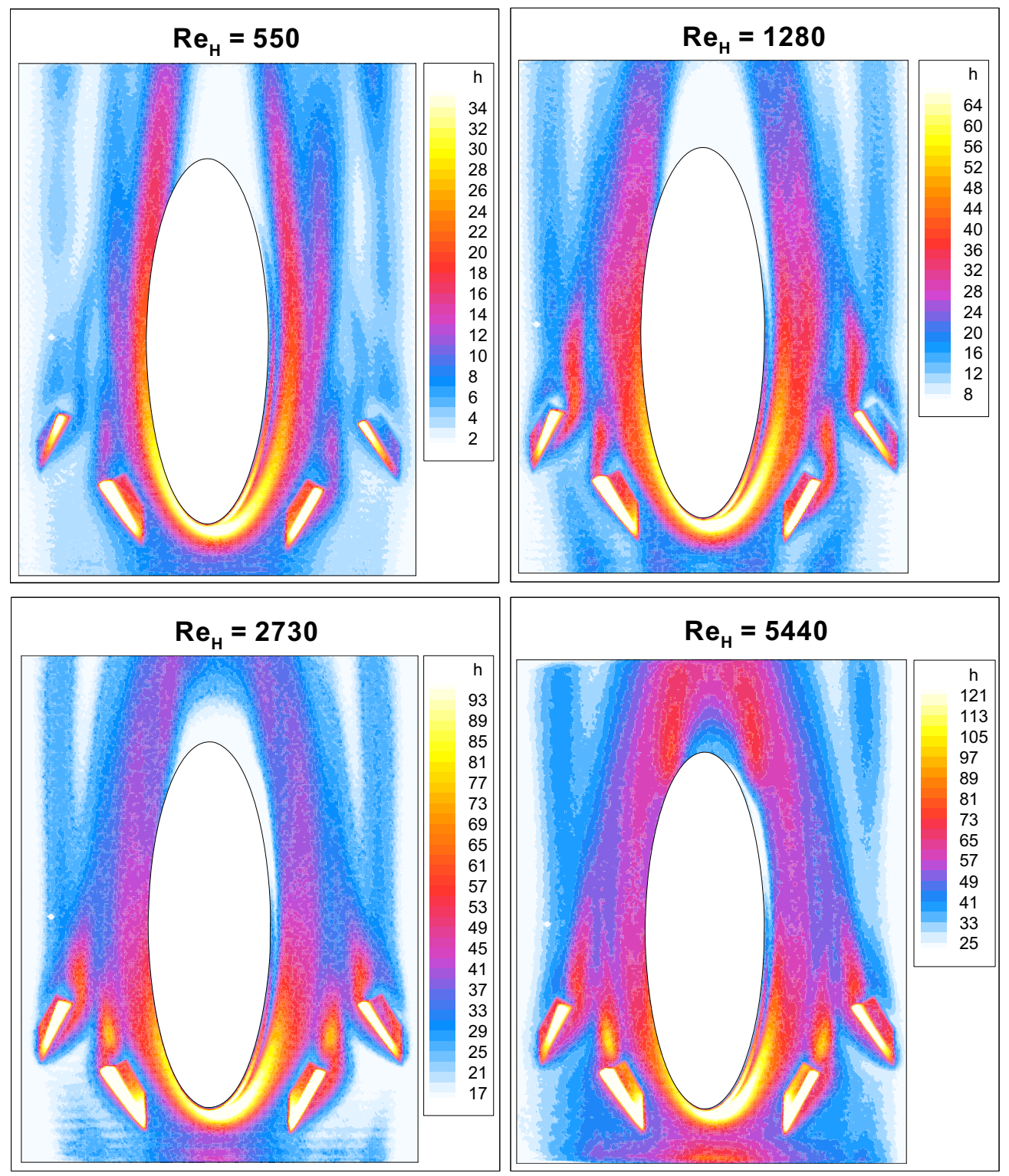

Figure 2-18. Local fin-surface heat transfer distributions for flow around an elliptical tube with two pairs of delta winglets in a staggered configuration. 


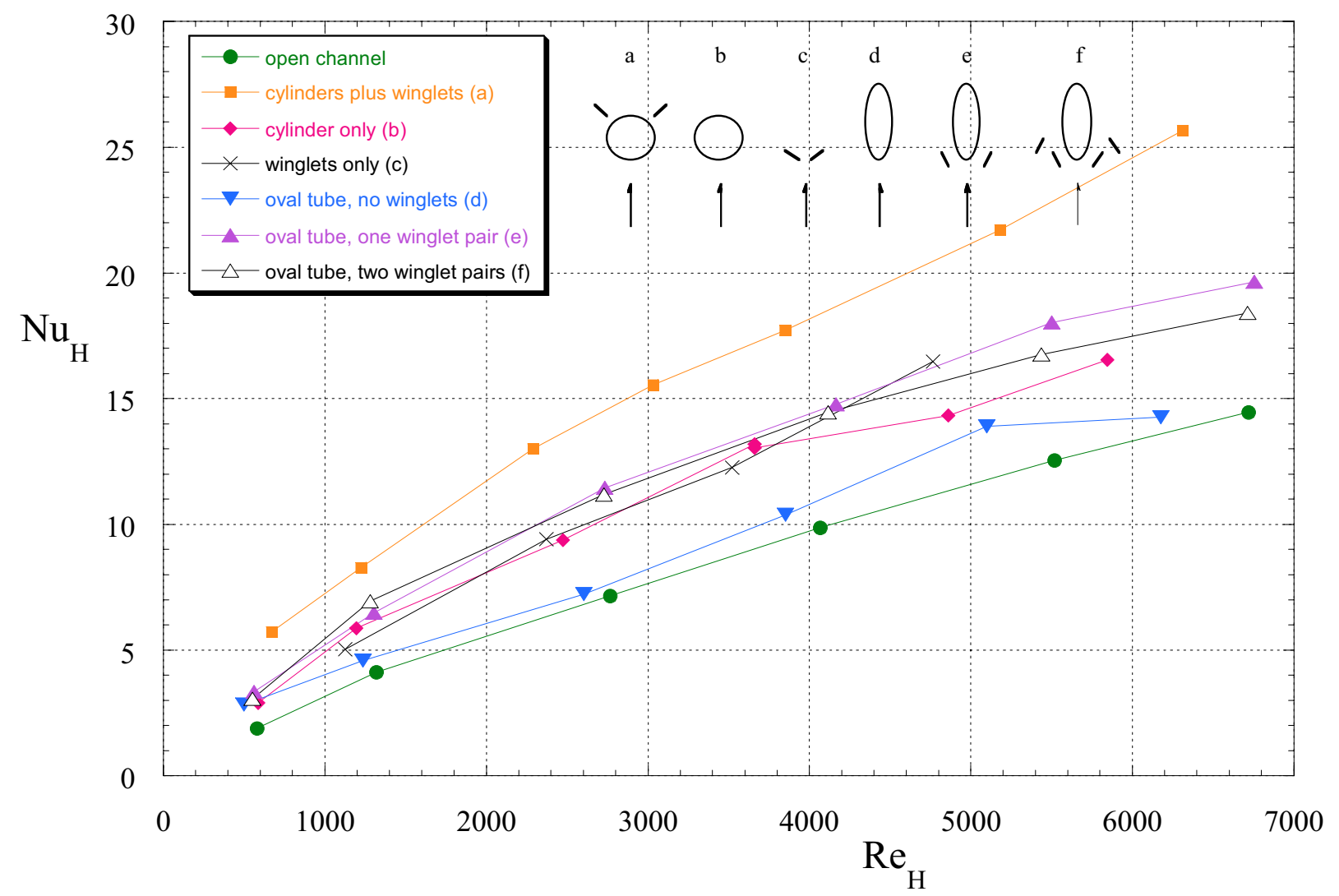

Figure 2-19. Mean fin-surface Nusselt numbers for seven flow configurations, based on local heat transfer results.

\subsection{Pressure Drop with Tube Bundle Model}

A separate single-channel multiple-tube-row apparatus was fabricated for evaluating the pressuredrop measurements corresponding to various tube and winglet configurations studied in the heat transfer experiments. A drawing of the pressure-drop test section core is shown on the left side of Figure 2-20 for the case of oval tubes with two pairs of winglets. The axial and spanwise tube spacing is shown in the figure. The circular tube arrays had the same spanwise and axial tube spacing, with a tube diameter of $2.54 \mathrm{~cm}$. The oval and circular tubes were designed to have the same cross-sectional flow area. Dried and filtered shop air enters the flow channel from a slot located $24 \mathrm{~cm}$ upstream of the tube bundle. The flow channel dimensions are $0.254 \mathrm{~cm}$ high $\times 22.86 \mathrm{~cm}$ wide. A row of six pressure taps was drilled along the channel centerline upstream and downstream of a four-tube row staggered array of either circular or elliptical tubes, with or without winglets. The tube array simulates a single passage in a platefin heat exchanger. The tubes and winglets were machined from a solid sheet of lexan using a CNC mill. A close-up photograph of the oval tubes and winglets is shown on the right side of Figure 2-2. Pressure drop was measured using a precision differential pressure transducer (MKS Model 223BD, 1 and 10-Torr ranges). Air flow rates were obtained from a precision mass-flow meter (Hasting Model HFM, 500 and 300 slpm ranges). 


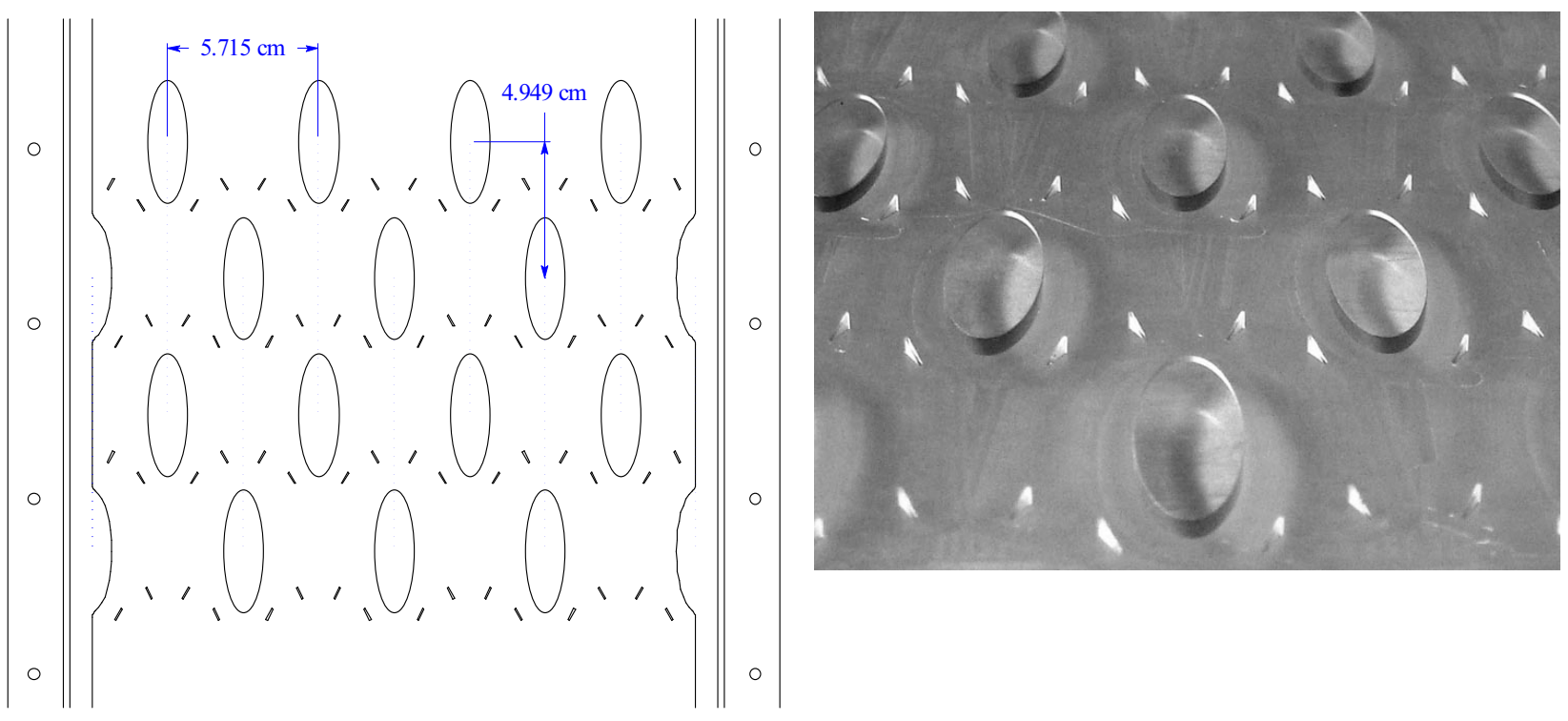

Figure 2-20. Pressure-drop test section core.

Instrumentation signals from both the heat transfer and pressure-drop flow loops were fed into a modular multiplexing data-acquisition system (Hewlett Packard 3852A), which in turn was interfaced to a system-controller computer via an IEEE-488 bus. For this experiment, the data acquisition unit was configured with a 20-channel FET multiplexer with thermocouple compensation, a $51 / 2$ digit integrating voltmeter, and a 4-channel voltage/current DAC. The DAC module was used to provide control signals $(4-20 \mathrm{~mA})$ to the variable-frequency drive for the blower. The mass-flow meter was configured to communicate directly with the computer using an RS-232 interface. Data-acquisition and instrumentcontrol system programming was accomplished using Labview software. The data files included time histories of the thermocouple mass-flow meter, and pressure-transducer signals with updates at $0.7-\mathrm{s}$ intervals.

Two techniques were used for the measuring pressure-drop: steady-flow and blow-down. Steadyflow tests were performed by establishing a steady air flow rate through the apparatus using a flowcontrol valve, then acquiring a large number of averages of both flow rate and pressure-drop at a specific flow rate. Blow-down tests were performed by pressurizing the compressor storage tank with air to a predetermined value, then discharging the air through the test apparatus while continuously monitoring instantaneous flow rate and pressure drop. The air compressor was disabled during the blow-down test. Blow-down testing allowed pressure-drop measurements to be obtained over a much broader flow range (both higher and lower flows) than steady-flow tests. It also provided a complete pressure-drop versus flow rate data set over the entire flow rate range of interest in a single test. Results obtained with the steady-state technique and the blow-down technique were in excellent agreement.

Pressure-drop results are presented in terms of friction factor $\mathrm{f}$, defined as

$f=\frac{\Delta P \rho}{G^{2} / 2\left(4 L / D_{h}\right)}$

where $\Delta \mathrm{P}$ is the pressure drop across the tube bundle, $\rho$ is the density of air, $\mathrm{G}$ is the mass flux at the minimum flow area, and $\mathrm{L}$ is the axial length of the tube bundle. The hydraulic diameter, $\mathrm{D}_{\mathrm{h}}$, is given by 


$$
D_{h}=\frac{4 L A_{\min }}{A}
$$

where $\mathrm{A}_{\min }$ is the minimum flow cross-sectional area, and $\mathrm{A}$ is the total heat transfer surface area. Friction factors are parameterized using the Reynolds number based on hydraulic diameter, $\mathrm{Re}_{\mathrm{Dh}}=$ $\mathrm{GD}_{\mathrm{h}} / \mu$. Note that this friction factor definition does not include any entrance or exit effects. Only core friction is considered, since the measured pressure drop only corresponds to core friction.

Results of the pressure-drop measurements obtained with the single-channel, multiple-tube-row pressure drop apparatus are presented in Figure 2-21 in terms of friction factor versus Reynolds number based on hydraulic diameter, as defined in Equations (2-5) and (2-6). Based on this definition, in the laminar regime, the highest friction factors were observed for the open channel, followed by the oval-tube cases and finally by the circular-tube cases. Actual pressure drop magnitudes are in the opposite order.

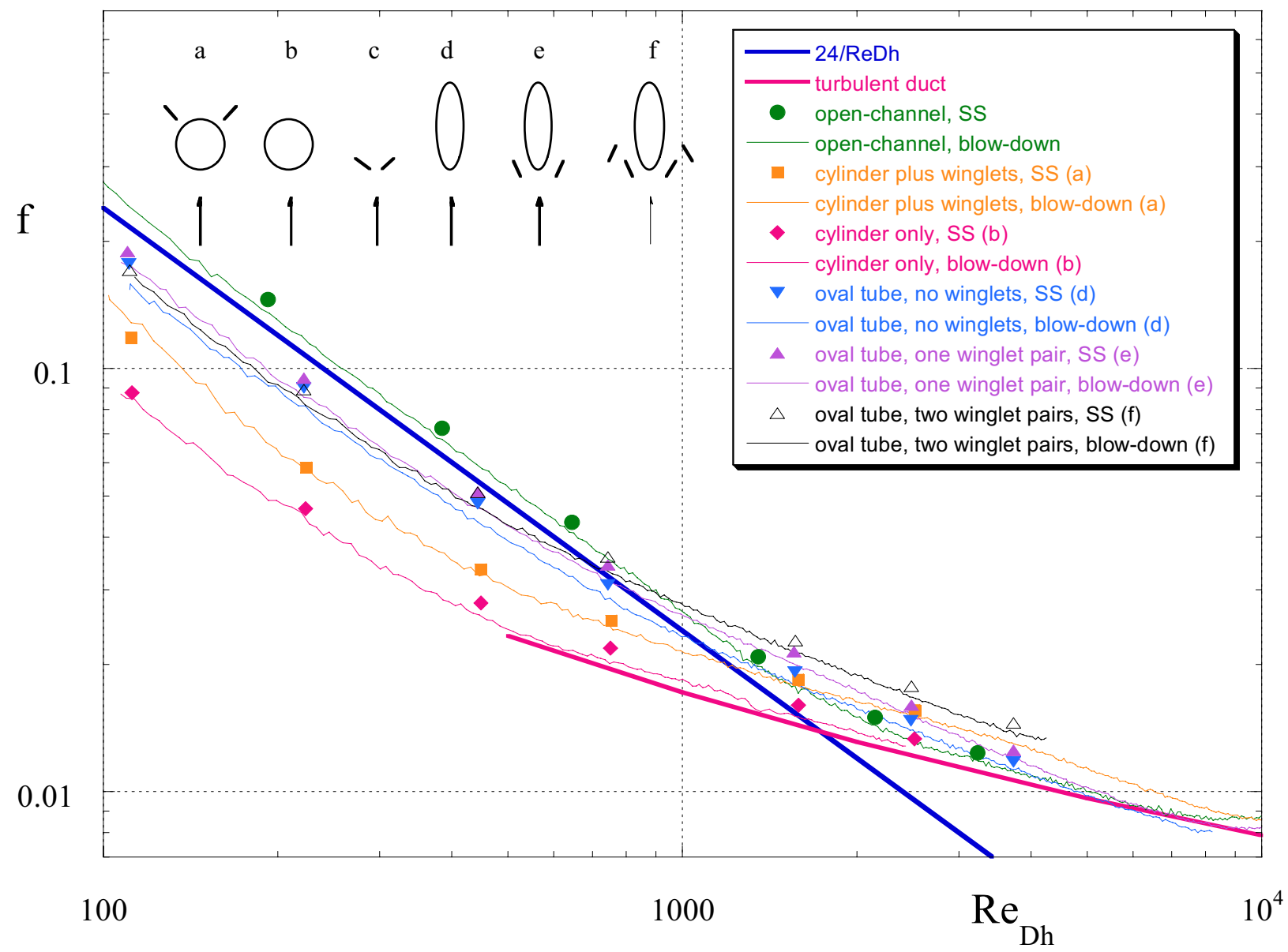

Figure 2-21. Friction factors measured for six flow configurations, steady-state and blow-down.

The friction factor results reflect the fact that the minimum-area mass flux, $\mathrm{G}$, is successively higher for the oval-tube cases and the circular-tube cases for a specified total mass flow rate (note that G2 appears in the denominator of the friction factor definition). Open-channel data agree well with the theoretical value of $24 / \mathrm{ReDh}$ for laminar flow in a parallel-plate channel. The friction factor data for all cases tend to converge at higher (turbulent) Reynolds numbers, approaching the Petukhov ${ }^{20}$ correlation for turbulentflow friction factor. Friction factor results for cases with winglets are higher than their respective baseline cases since the only quantity that is different in calculating those friction factors is the magnitude 
of the pressure drop. No correction is made to the minimum-flow area for the winglet cases. Note that because the mean heat transfer results were obtained from the single-tube heat transfer tests, and the friction factor results were obtained from a multiple-tube-row configuration, the heat transfer and friction factor results do not correspond to identical flow situations. This is why the mean heat transfer results have not been converted to $\mathrm{j}$-factors for presentation on the same graph as the friction factors, as is standard practice for presentation of heat exchanger results. Local heat transfer results clearly indicate areas of high fin-surface heat transfer in the stagnation region of the oval tube, along the side of the oval tube, and downstream of the winglets. Highest heat transfer coefficients are in the tube stagnation region. The local heat transfer enhancement associated with both the primary vortex and the corner, horseshoetype vortices produced by each winglet are visible in the heat transfer images. Evaluation of mean finsurface heat transfer coefficients indicate that the addition of the single winglet pair to the oval-tube geometry yielded significant heat transfer enhancement, averaging 38\% higher than the oval-tube, nowinglet case. The corresponding increase in friction factor was very modest, less than $10 \%$ at $\mathrm{ReDh}=$ 500 and less than 5\% at $\mathrm{ReDh}=5000$. The highest mean heat transfer coefficients were observed for the case of a circular tube plus winglets with the winglets located on the downstream side of the cylinder, oriented at a 45-degree angle to the flow. However, this case also yielded very high friction factors. 


\section{INL SINGLE BLOW TESTING OF A HEAT EXCHANGER TUBE BUNDLE}

INL developed an open-circuit air-flow loop, designated a single-blow test facility (Figures 3-1, -2, and -3), for pressure-drop and heat transfer performance testing of heat exchanger finned-tube bundles. The testing has evaluated several enhanced heat transfer geometries to be used with individually finned tubes in which winglet vortex generators have been punched into the fin surfaces at specific locations.

\subsection{Test Facility}

The test flow loop includes a 4:1 inlet contraction nozzle, designed to provide a uniform inlet velocity profile. The nozzle is followed by a square-cross-section flow channel, $25.4 \mathrm{~cm}$ on a side, $82 \mathrm{~cm}$ in length. A special low-heat-capacity, high-surface-area stainless-steel foil heater, developed for this facility based on a design recommended by Mochizuki, ${ }^{21}$ is flanged to the downstream end of the square flow channel. The heater surface is formed from stainless-steel ribbon, $0.178 \mathrm{~mm}$ thick, $19.0 \mathrm{~mm}$ wide, wound back and forth across the flow channel at a 3.17-mm spacing, making a total of 80 passes across the channel (Figure 3-4). The heater, therefore, also serves as an effective flow staightener. Total electrical resistance of the stainless-steel ribbon is about $4.5 \mathrm{ohm}$. The heater is followed by a section of square flow channel, $30.5 \mathrm{~cm}$ in length, which is followed by the test section. The test section can accommodate a prototypic tube bundle, with $25.4-\mathrm{cm}$ tube lengths in a $4 \times 4$ fin-tube bundle (Figures 3-5a and -b). The tube bundle tested in this study was a staggered equilateral array, with a tube spacing of 6.35 $\mathrm{cm}$. In order to maintain symmetry and simulate a larger staggered array, there are two tube rows that include three full tubes and three half-tubes. Test section inlet and outlet mean temperatures are measured with fast response (time constant about $0.1 \mathrm{~s}$ ) using a $2.5-\mathrm{m}$ length of $0.102-\mathrm{mm}$-diameter nickel wire used as the sensing element in a constant-current anemometry circuit. These wires are stretched diagonally across the test section inlet and outlet planes with multiple (16) passes, in order to provide accurate cross-sectional mean air temperatures. A thermocouple (1.59-mm SS, sheathed, type K) is also located between the heater and the test section and another downstream of the test section. The pressure drop across the test section is monitored continuously using a precision pressure transducer (MKS 223 BD, 1 Torr range). Four flush-mounted pressure taps (one on each side of the square duct) are located five tube diameters upstream and downstream of the test section, respectively.

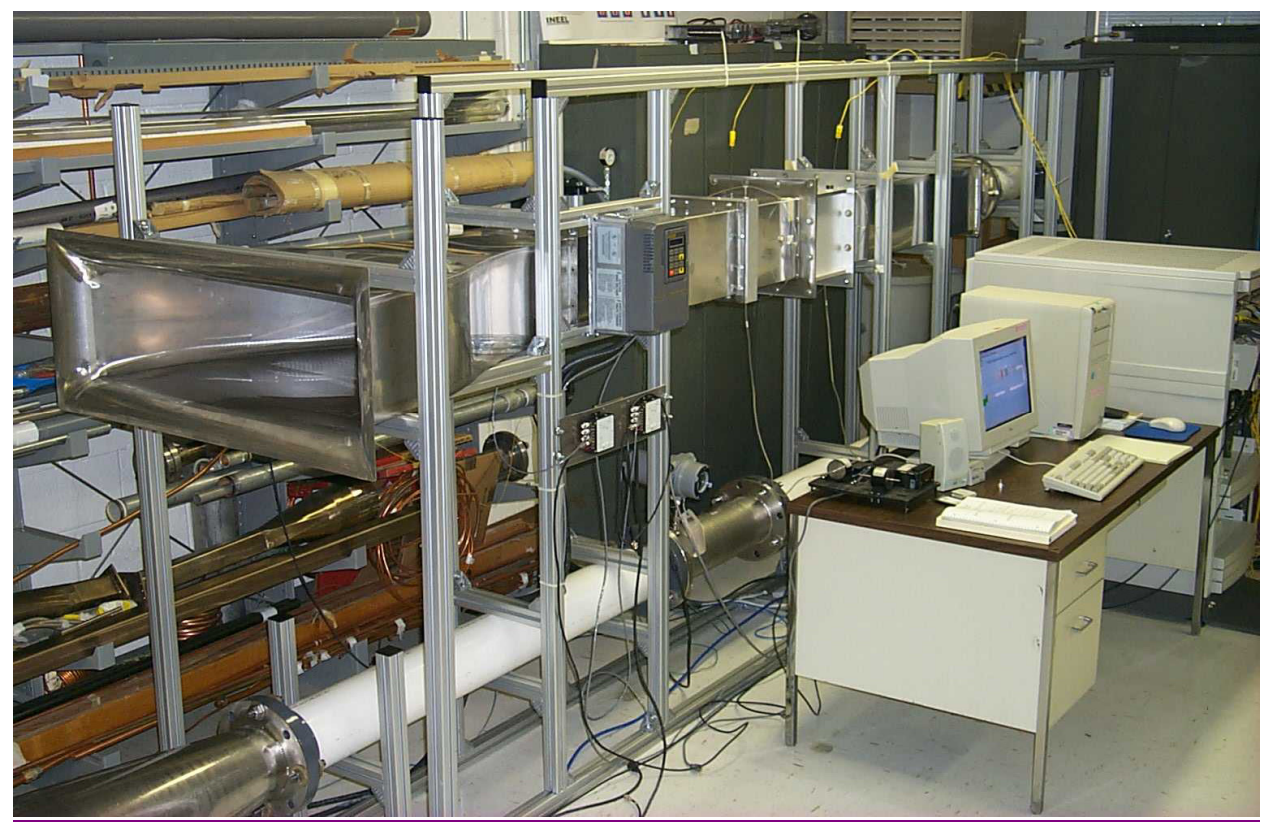

Figure 3-1. Diagonal view of the INL Single-Blow Test Facility. 


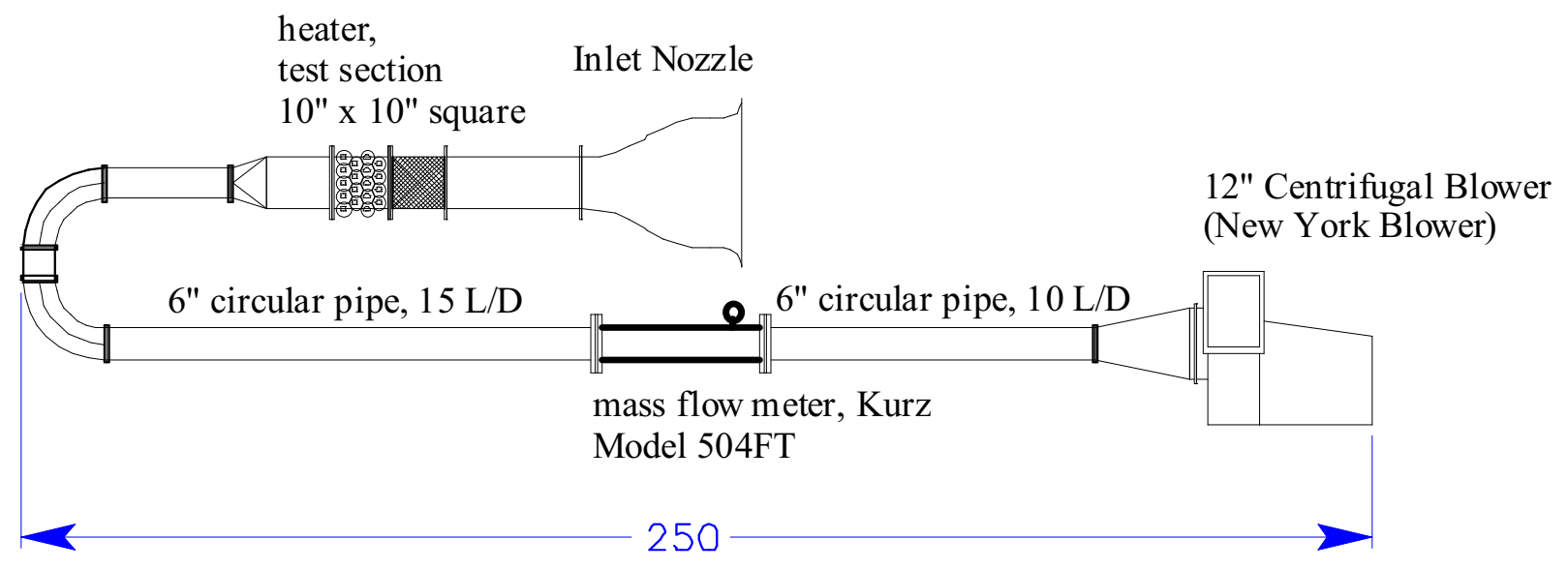

Figure 3-2. Schematic diagram of the Single-Blow Test Facility (all dimensions in inches).
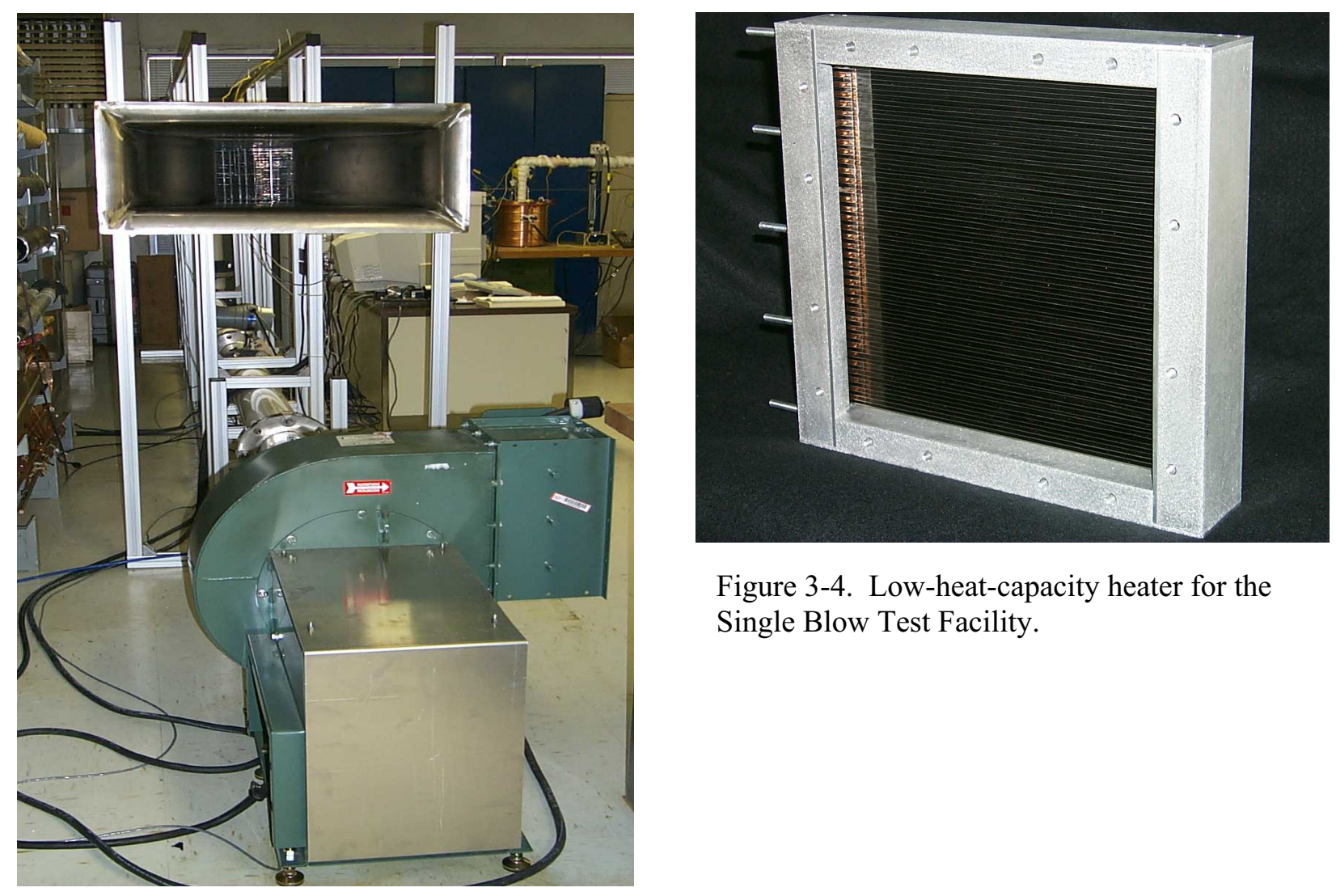

Figure 3-4. Low-heat-capacity heater for the Single Blow Test Facility.

Figure 3-3. Side-view of the INL SingleBlow Test Facility. 


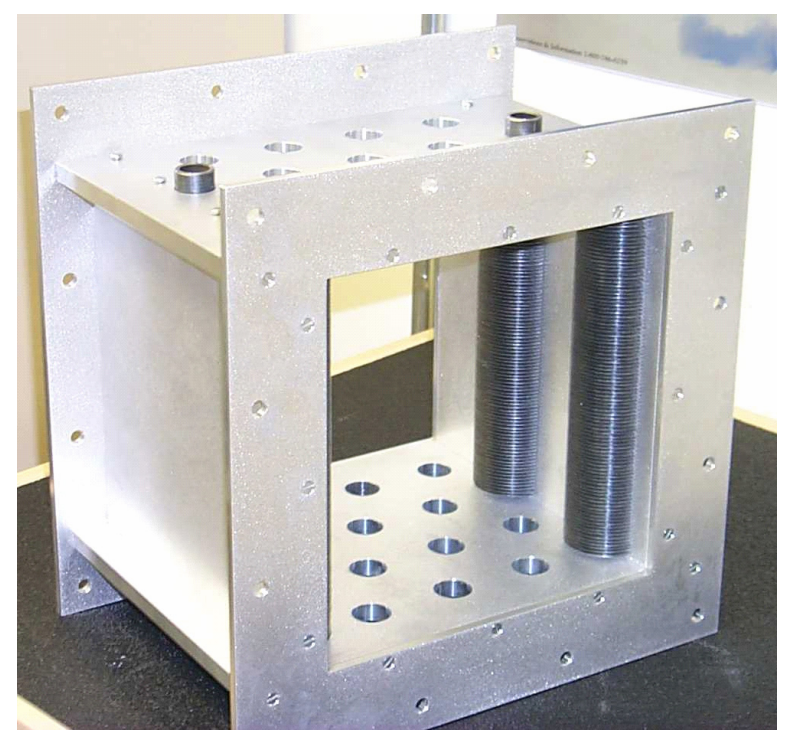

(a)

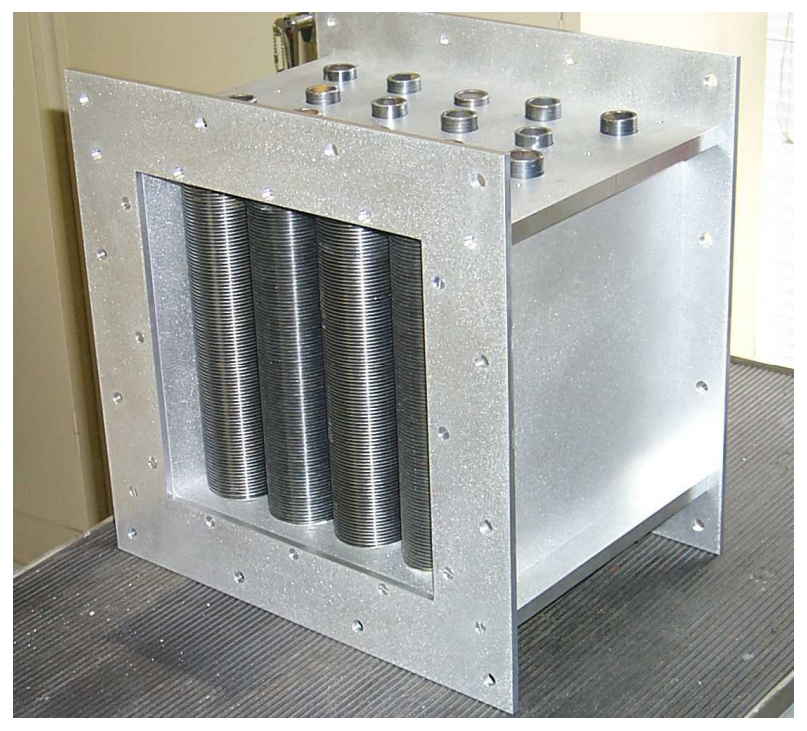

(b)

Figure 3-5a and $\mathrm{b}$. Test section, (a) with only the edge half tubes, (b) with all tubes in place.

The four pressure taps are tied together to provide an average reading at the two axial stations. Downstream of the test section, the flow channel transitions from square to circular cross-section. Air flow rate is monitored continuously using an in-line mass-flow meter (Kurz Model 504Ft-96). With the heater and test section in place, flow rate through the loop can range from 50 to $1500 \mathrm{SCMH}$, yielding a Reynolds number range based on hydraulic diameter from 100 to 3000 . The geothermal air-cooled condensers typically operate with a hydraulic-diameter Reynolds number, $\mathrm{Re}_{\mathrm{Dh}}$, of about 900 . Air is drawn through the flow loop using a $30.5-\mathrm{cm}$ centrifugal blower. Blower speed is controlled from the data acquisition/instrument control computer via a 4-20-mA process-control signal fed to a variablefrequency drive.

The objective of these experiments was to evaluate the heat transfer performance of several heat exchanger tube bundles, consisting of individually finned tubes with circular fins, with and without vortex-generating winglets. In order to meet this objective, three different fin geometries were created, a baseline geometry with no winglets, and two different enhanced geometries with winglets. Geometric details of the enhanced fin geometries are provided in Figure 3-6. Photographs of completed individual enhanced fins are presented in Figure 3-7. In both cases, the winglets are located adjacent to the downstream side of the tube. This location was selected in order to increase local fin-surface heat transfer in a region of relatively low heat transfer and to attempt to decrease the size of the low-heat-transfer wake region behind the tubes. The first enhanced geometry, designated enhanced-1 or toe-out (also called common flow down) is shown in Figures 3-6a and 3-7a. It includes a pair of 2-to-1 aspect ratio deltashaped winglets located on the downstream side of the tube in a 45-degree "common flow down" orientation. This geometry produced significant enhancement in local heat transfer. ${ }^{22}$ The second enhanced geometry, designated enhanced- 2 or toe-in (also called common flow up) is shown in Figures $3-6 \mathrm{~b}$ and $3-7 \mathrm{~b}$. This geometry makes use of a pair of 4-to-1 aspect ratio delta-shaped winglets, also located near the downstream side of the tube in a 45-degree "common-flow-up" orientation. The fins were fabricated individually from aluminum stock $(0.203 \mathrm{~mm}$ thick) by a manual punching operation in two or three steps. Baseline fins (with no winglets) were formed in a two-step process in which the initial donut shape is sheared from the aluminum stock, followed by a forming operation to develop the central collar seen in the Figure 3-7. The collar serves as a spacer between adjacent fins, establishing the fin pitch, when the fins are mounted on a supporting tube. For the purpose of this experiment, using the 
single-blow technique, it would be desirable to have all the materials in the test core (fins and tubes) fabricated from the same material (and the same gauge of that material). Since this would require that the tube material be too thin to support the fins, polycarbonate tubes were used to support the aluminum fins. This low-conductivity material was chosen in order to minimize the heat transfer participation of the support tubes. Thermal participation of the polycarbonate is minimal during the test times considered. The relevant heat capacity of a tube wall is provided by the aluminum collars. Therefore, the heat transfer results obtained from this testing represent mean external heat transfer coefficients for the fin and tube surfaces. Fin efficiency and contact resistance effects are not accounted for in this experiment. Fabrication of the enhanced fins with winglets required an additional step to form the winglets via shearing along the hypotenuse and short leg of the delta triangle, and bending along the long leg of the triangle (designated the fold edge in Figure 3-6). Each finned tube used in the test (several are visible in Figure 3-5) is created by mounting about 100 individual fins on the polycarbonate tube, with a nominal fin pitch of $2.54 \mathrm{~mm}$, over a finned length of $24.9 \mathrm{~cm}$. The actual average fin pitch values of the completed tubes were $23.1 \mathrm{~mm}$ for the baseline and enhanced- 1 cases, and $28.2 \mathrm{~mm}$ for the enhanced-2 case.

(a)
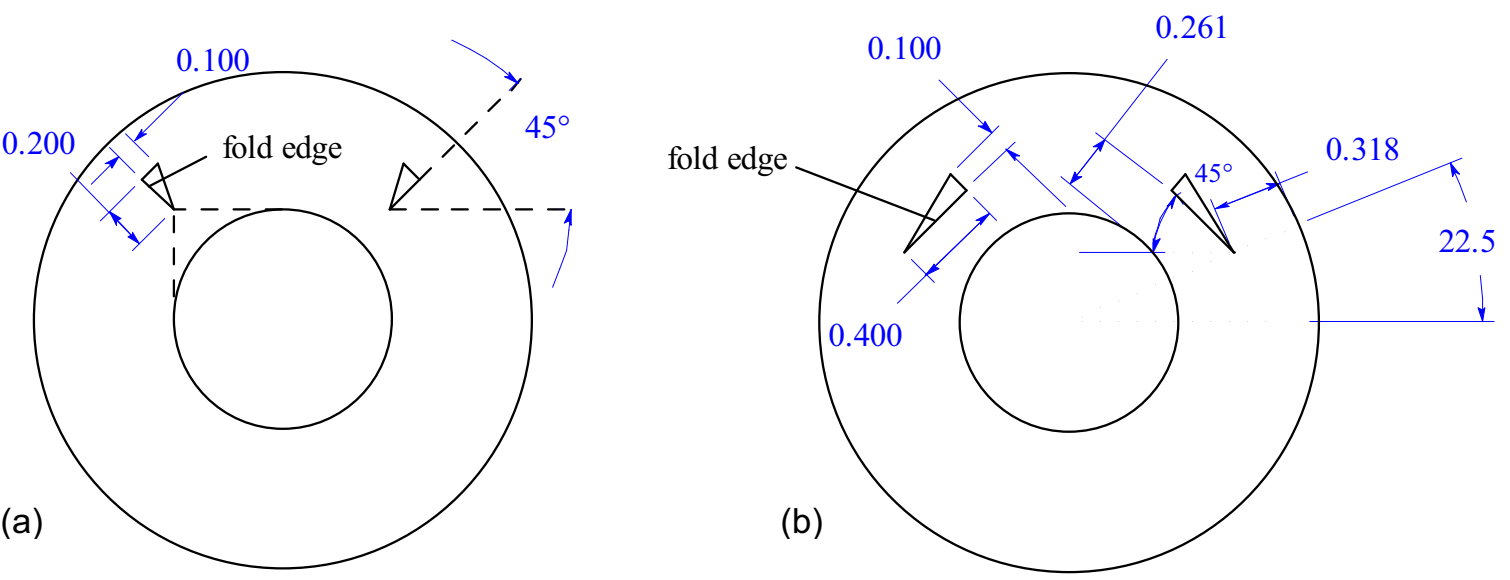

Figure 3-6. Geometric details of individual fins and winglets for the two cases studied: (a) 2:1 toe-out (enhancement-1) winglets, (b) 4:1 toe-in (enhancement-2) winglets (flow is from bottom to top).

\subsection{Experimental Procedure}

For each test section configuration, both pressure-drop and heat transfer tests are performed.

Pressure-drop data are acquired at steady-state conditions. For each blower speed, after steady state is attained, output signals from the pressure transducer and mass-flow meter are recorded continuously for about $30 \mathrm{~s}$ at a rate of about one reading per second. These data are averaged, yielding the mean pressuredrop value for each flow rate. Testing is completed for numerous flow rates over the range of interest.

The single-blow heat transfer tests are performed in a thermal transient fashion. Each test begins with establishing a steady desired flow rate. Power is then suddenly applied to the heater, resulting in a transient heat-up of the air exiting the heater and entering the test section. Heat transfer occurs from the heated air to the fins representing thermal capacitance of the tube bundle, yielding a test-section-outlet temperature response that lags behind the inlet-temperature curve according to the magnitude of the mean tube-bundle heat capacity and heat transfer coefficient. A representative temperature history showing this behavior is presented in Figure 3-8 at a flow Reynolds number, $\mathrm{Re}_{\mathrm{Dh}}$, of 550. Four thermal response curves are shown in the figure: inlet thermocouple, inlet wire, outlet thermocouple, and outlet wire. The inlet thermocouple is located downstream of the heater and upstream of the test section. It provides a single-point, slow-response temperature measurement. Note that its response lags significantly behind the 
inlet wire response. The inlet wire response, representing the transient heating of the air, is generally very well fitted by an exponential first-order response curve, as shown in Figure 3-8. The time constant of this first-order system response (i.e., heater outlet air temperature) varies from about $20 \mathrm{~s}$ at the lowest air flow rate to about $4 \mathrm{~s}$ at the highest flow rate tested. The response of the test-section outlet wire represents the mean test-section outlet temperature variation with time. Its shape is determined by the heat capacity and heat transfer characteristics of the heat exchanger core. The final response curve shown in Figure 30 is obtained from the outlet thermocouple. It is also only a single-point measurement, with a relatively slow response that lags behind the outlet wire response.

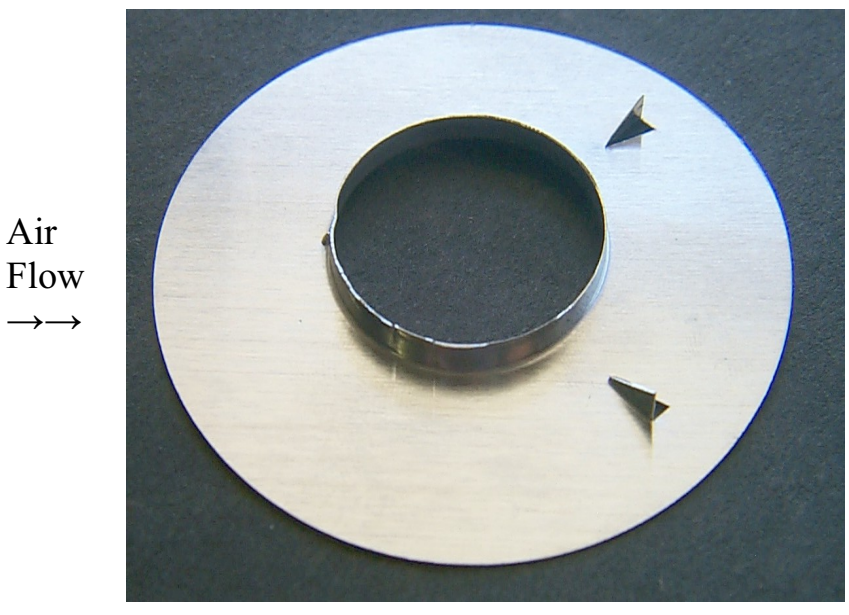

(a)

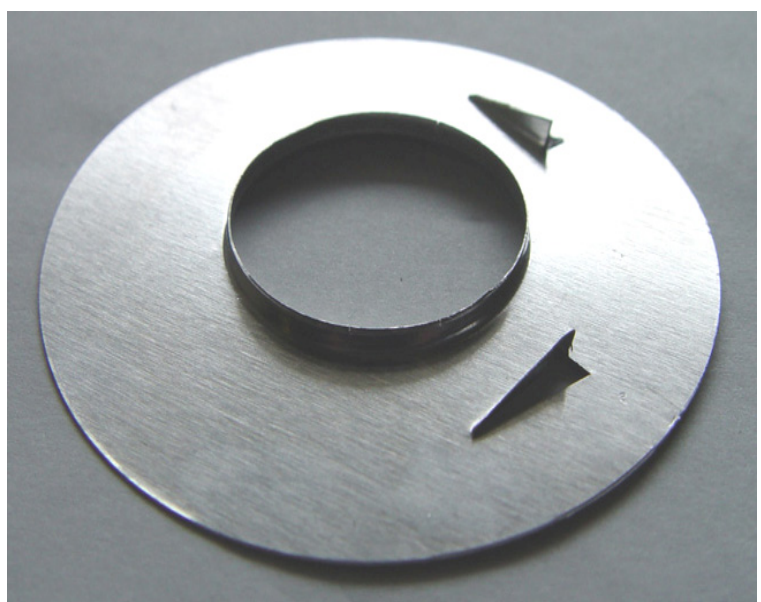

(b)

Figure 3-7. Individual fins and winglets for the two cases studied: (a) 2:1 toe-out (enhancement-1) winglets, (b) 4:1 toe-in (enhancement-2) winglets, (flow is from left to right).

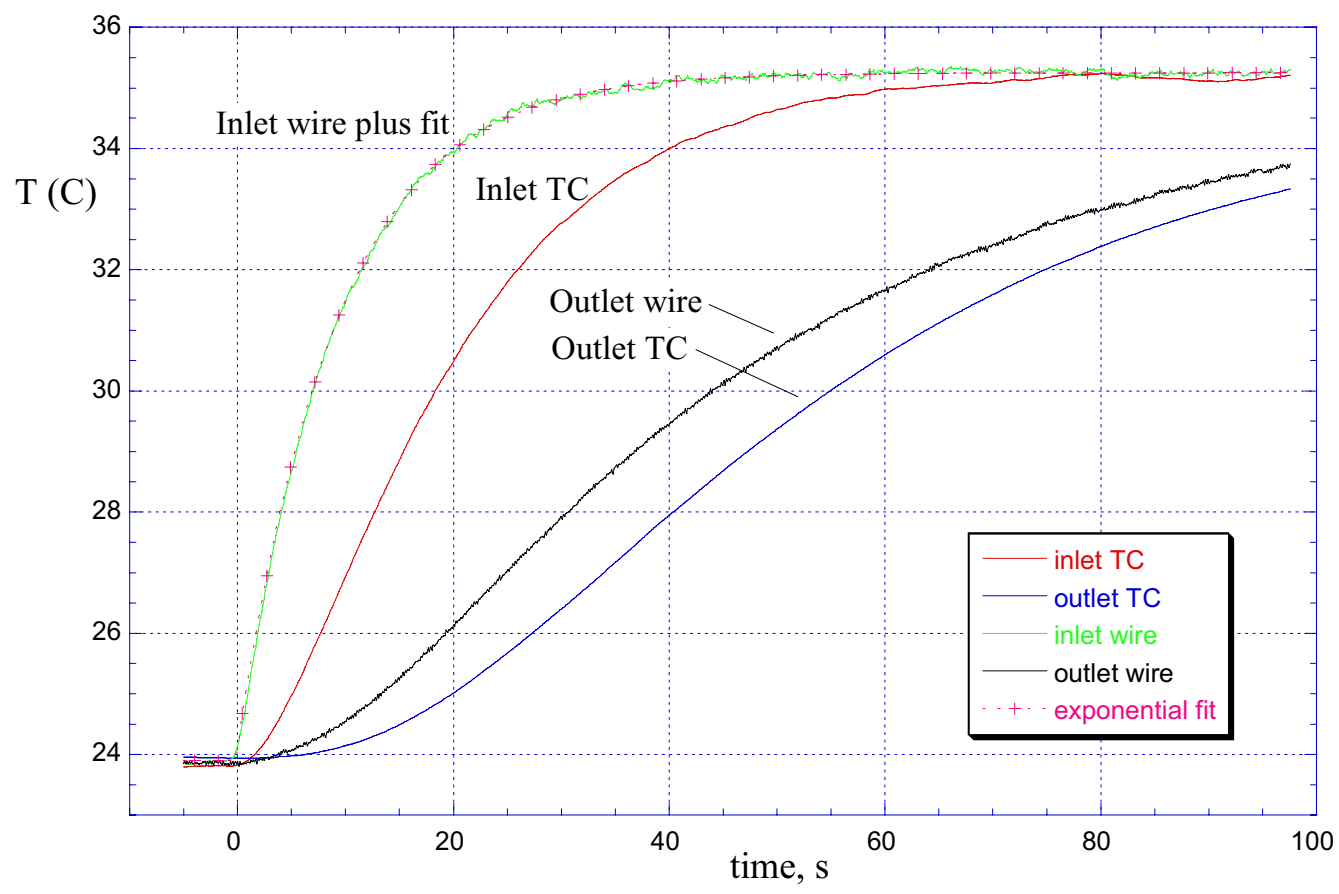

Figure 3-8. Representative test section inlet and outlet temperatures after initiation of thermal transient, $\mathrm{Re}_{\mathrm{Dh}}=550$. 


\subsection{Data Reduction}

Values for $\mathrm{L}, \mathrm{A}_{\min }, \mathrm{A}$, and $\mathrm{D}_{\mathrm{h}}$, defined on a unit-cell basis, are listed in Table 3-1 for the three fintube configurations considered in this study. The friction factor definition given in Equation (2-5) is commonly employed in the analysis of compact heat exchangers. Friction factors are presented as a function of the Reynolds number based on hydraulic diameter. Heat transfer results will be presented in the form of the Colburn $\mathrm{j}$-factor, defined as:

$j=\frac{h}{G c_{p}} \operatorname{Pr}^{2 / 3}=N T U \frac{A_{\min }}{A} \operatorname{Pr}^{2 / 3}$

where $h$ is the heat transfer coefficient, $c_{p}$ is the air specific heat, Pr is the Prandtl number, and NTU is the number of transfer units,

$$
N T U=\frac{h A}{\dot{m} c_{p}} .
$$

Table 3-1. Unit-cell dimensions and hydraulic diameter for the three test configurations

\begin{tabular}{lcccc}
\hline & $\begin{array}{c}\mathrm{L} \\
(\mathrm{cm})\end{array}$ & $\begin{array}{c}\mathrm{A}_{\min } \\
\left(\mathrm{cm}^{2}\right)\end{array}$ & $\begin{array}{c}\mathrm{A} \\
\left(\mathrm{cm}^{2}\right)\end{array}$ & $\begin{array}{c}\mathrm{D}_{\mathrm{h}} \\
(\mathrm{cm})\end{array}$ \\
\hline $\begin{array}{l}\text { Baseline (with no winglets) } \\
\begin{array}{l}\text { Enhanced-1 (toe-out, common flow } \\
\text { down) }\end{array}\end{array}$ & 5.5 & 0.8089 & 43.20 & 0.4119 \\
$\begin{array}{l}\text { Enhanced-2 (toe-in, common flow up) } \\
\text { Enhy }\end{array}$ & 5.5 & 0.8089 & 43.20 & 0.4119 \\
\hline
\end{tabular}

Values for NTU and $\mathrm{j}$ are obtained by an advanced curve-fitting technique in which the measured test-section outlet temperature response is compared to the corresponding response obtained from a complex analytical model of the test section heat capacitance, using the software package

TAIHE/SBTT. ${ }^{23}$ The specific model used for reduction of the present thermal-response data assumes an exponential change in the inlet fluid temperature and incorporates an axial heat dispersion term to account for flow maldistribution associated with flow through the fin-tube bundle. ${ }^{24}$ An important parameter that appears in the governing energy equation for the case in which axial heat dispersion is considered is the axial-dispersion Peclet number,

$$
P e=\frac{\dot{m} c_{p} L}{D A_{\min }}
$$

where $\mathrm{D}$ is the axial dispersion coefficient $(\mathrm{W} / \mathrm{m} \mathrm{K})$, and $\mathrm{L}$ is the length of the test core.

The TAIHE/SBTT software allows for simultaneous solution of both NTU and the nondimensional axial dispersion coefficient, $1 / \mathrm{Pe}$. The values of $1 / \mathrm{Pe}$ obtained from these simultaneous solutions exhibited a strong Reynolds number dependency with a fair amount of data scatter. Therefore, in order to maintain consistency, for the final data reduction (for all cases), a correlation for 1/Pe was used, based on a curve fit of the values obtained from the initial simultaneous solutions. This correlation is shown in Figure 3-9. The magnitudes of the 1/Pe values shown in the figure indicate that axial dispersion is indeed an important consideration for the tube-bundle geometry, as might be expected due to the presence of recirculating flows in the tube wake regions. The final NTU (and Colburn-j factor) values 
were obtained from the TAIHE/SBTT solver, solving for NTU only, using values of $1 / \mathrm{Pe}$ obtained from the correlation at each Reynolds number.

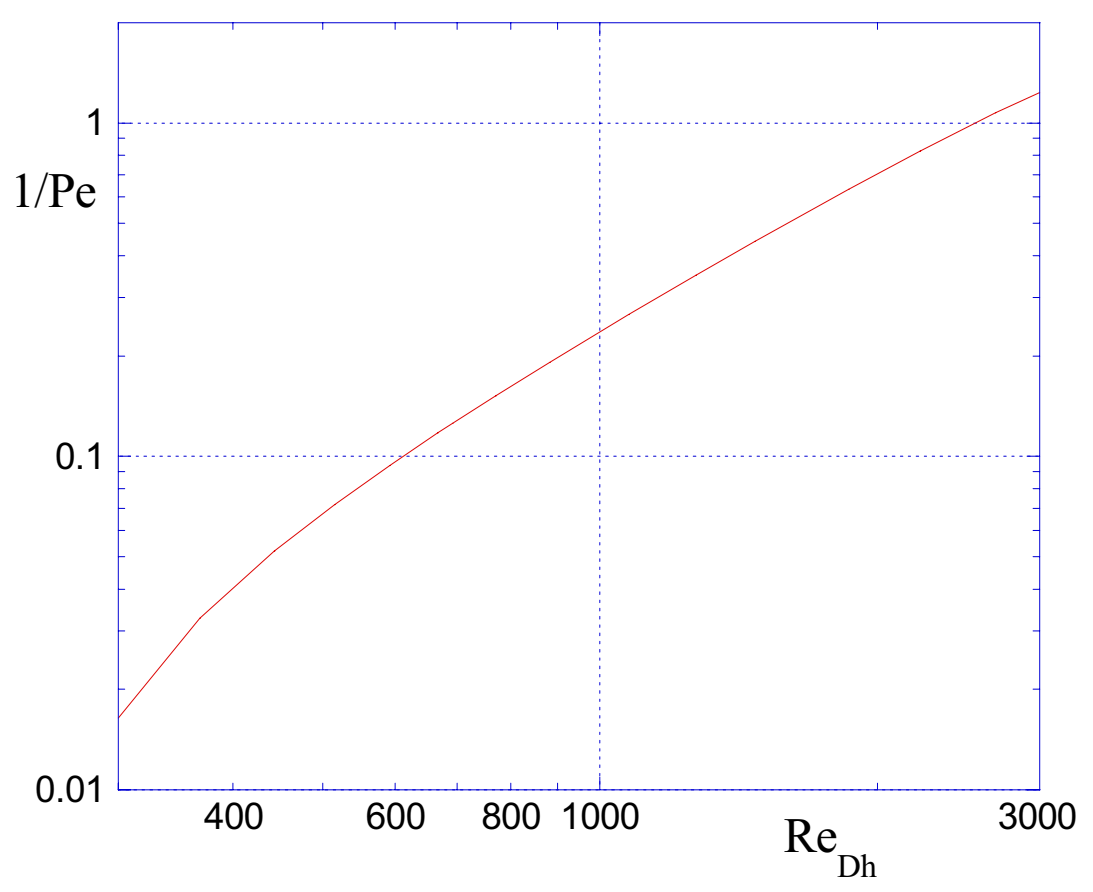

Figure 3-9. Nondimensional axial dispersion coefficient as a function of the Reynolds number.

\subsection{Experimental Uncertainty}

Estimates of the experimental uncertainties of the Reynolds numbers, friction factors, and $\mathrm{j}$-factors presented in this report have been obtained based on constant-odds, $95 \%$ confidence level. ${ }^{25}$ For Reynolds numbers, the uncertainty associated with the mass-flow meter was the primary contributor. Based on the manufacturer's specifications for the mass-flow meter $(2 \%$ of reading plus $0.5 \%$ of fullscale), the relative uncertainty for the Reynolds number ranged from $13 \%$ at the $\operatorname{Re}_{\mathrm{D}}=300$ to $3 \%$ at $\operatorname{Re}_{\mathrm{Dh}}$ $=3000$. These uncertainty estimates are much lower if actual calibration data are used for the mass-flow meter. Uncertainties in friction factor include contributions from both the mass-flow and pressure-drop measurements. The relative uncertainty for friction factor ranges from $25 \%$ at $\operatorname{Re}_{\mathrm{Dh}}=300$ to $6 \%$ at $\mathrm{Re}_{\mathrm{Dh}}$ $=3000$. For the $\mathrm{j}$-factors, uncertainties in inlet and outlet mean temperature, system heat capacity, mass flow rate, and axial dispersion coefficient were considered. Relative uncertainty estimates for $\mathrm{j}$-factor ranged from $14 \%$ at $\mathrm{Re}_{\mathrm{Dh}}=300$ to $20 \%$ at $\mathrm{Re}_{\mathrm{Dh}} \sim 1000$ to $6 \%$ at $\mathrm{Re}_{\mathrm{Dh}}=3000$. The peak in uncertainty in the mid-range of Reynolds numbers is due to a peak in the relative uncertainty of the axial dispersion coefficient in the mid-range.

\subsection{Results}

Pressure-drop and heat transfer results of this study are summarized in Figures 3-10 and -11 in terms of friction factor and Colburn j-factor. Figure 3-10 presents measured friction factors and j-factors for the baseline tubes and the two different enhanced tubes as a function of the Reynolds number. The friction factor curves are slightly nonlinear on this log-log plot, with a steeper slope at a low Reynolds number. Lowest friction factors were measured for the baseline case. Friction factors for the enhanced-1 (toe-out) case averaged $8.9 \%$ higher than the baseline values. For the enhanced-2 (toe-in) case, friction factors averaged $24 \%$ higher than the baseline values. The friction factor data fits shown in the figure (and in 
Figure 3-11) were obtained from a linear combination of four power-law functions, using exponents of $-0.3,-0.4,-0.5$, and -0.6 on the Reynolds number. Colburn j-factor results were well represented by a single power-law fit for the cases shown in Figure 3-10. The slopes (exponents) of the j-factor power-law fits were $-0.426,-0.415$, and -0.403 for the baseline, enhanced- 1 , and enhanced- 2 cases, respectively. Colburn j-factor values for the enhanced- 1 and enhanced- 2 cases averaged 28 and $40 \%$ higher than the baseline $\mathrm{j}$-values, respectively. Because the fin pitch and resultant unit-cell dimensions for the enhanced- 2 case were different than those for the baseline case, the values of friction factor and Colburn- $j$ factor do not directly reflect increased pressure-drop and heat transfer coefficient values. In fact, pressure drop values at a specified Reynolds number were significantly lower for the enhanced- 2 case than for the baseline case, due to the larger fin spacing.

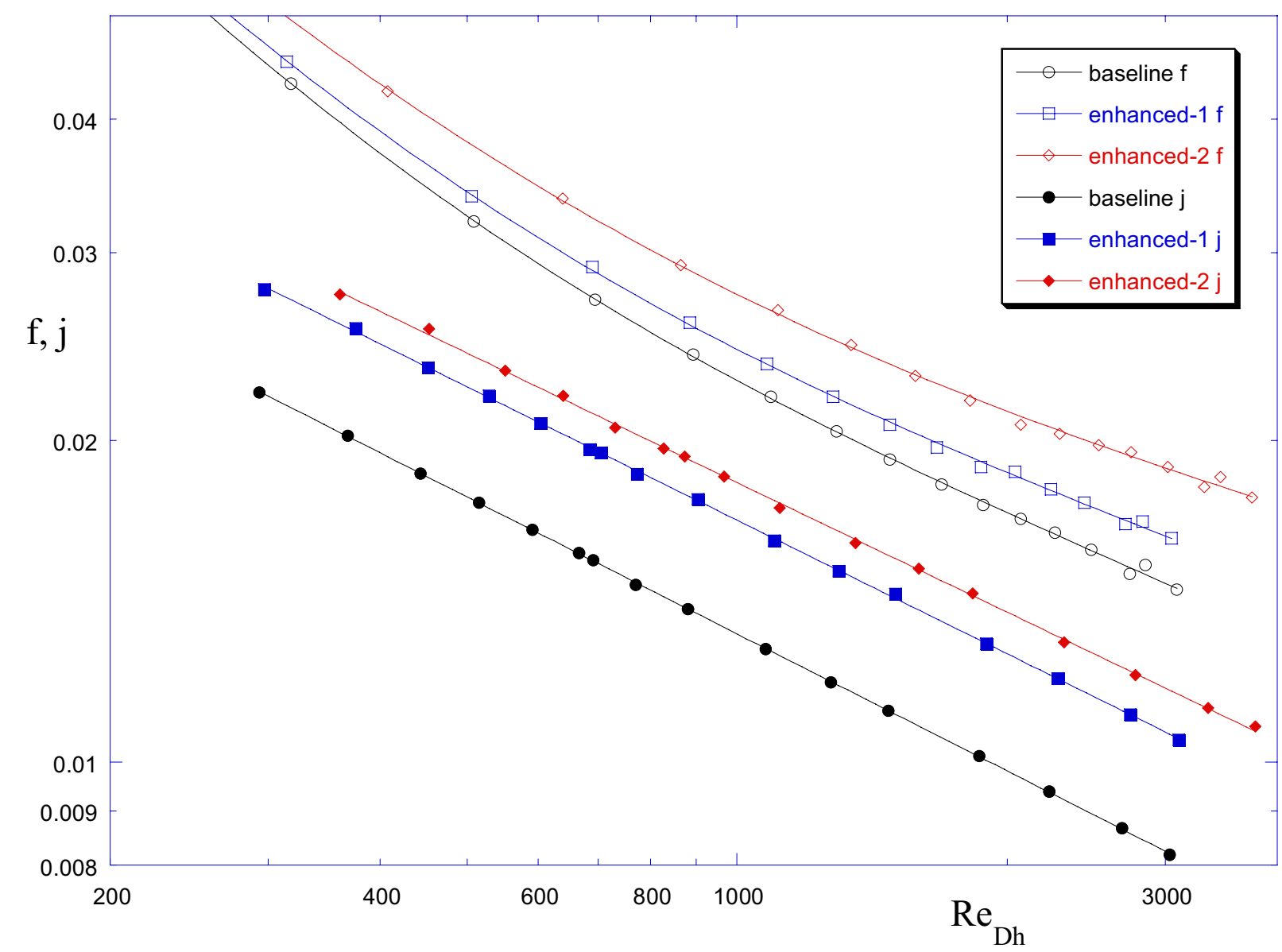

Figure 3-10. Dependence of the friction factor and the Colburn j-factor on the Reynolds number for three configurations.

Two variations of the enhanced-1 geometry were also tested. In order to assess the importance of the circumferential alignment of the winglets, a series of tests was performed in which all of the enhanced-1 tubes were rotated 180 degrees about their axes, such that the winglets were located on the upstream side of the tubes, facing backward. This test series was designated E180. An additional test series was run to determine the effect of replacing only the most upstream row of baseline tubes with enhanced- 1 tubes, in the correct circumferential orientation. The remaining three tube rows consisted of baseline tubes for this case. This test series is designated 1-row. Friction factor and j-factor results of the E180 tests and the 1-row tests are presented in Figure 3-11. Friction factors for the 1-row case are virtually identical to the 
baseline values at low Reynolds numbers and are only 3\% increased at high Reynolds numbers compared to those for the baseline case. Friction factors for the E180 case are slightly higher than the values obtained for the enhanced-1 case, averaging 10\% higher than the baseline case. Colburn j-actor data for the E180 and 1-row-enhanced cases could not fit well with a single power law; therefore, a linear combination of power laws was again used. Colburn $\mathrm{j}$-factors for the 1-row case exhibit very little improvement above the baseline case at low-Reynolds-numbers. At high Reynolds numbers, however, a distinct improvement of up to $20 \%$ is observed. Colburn j-factor values for the E180 case lie about $10 \%$ above the baseline for low Reynolds numbers and about 30\% above the baseline at the highest Reynolds number.

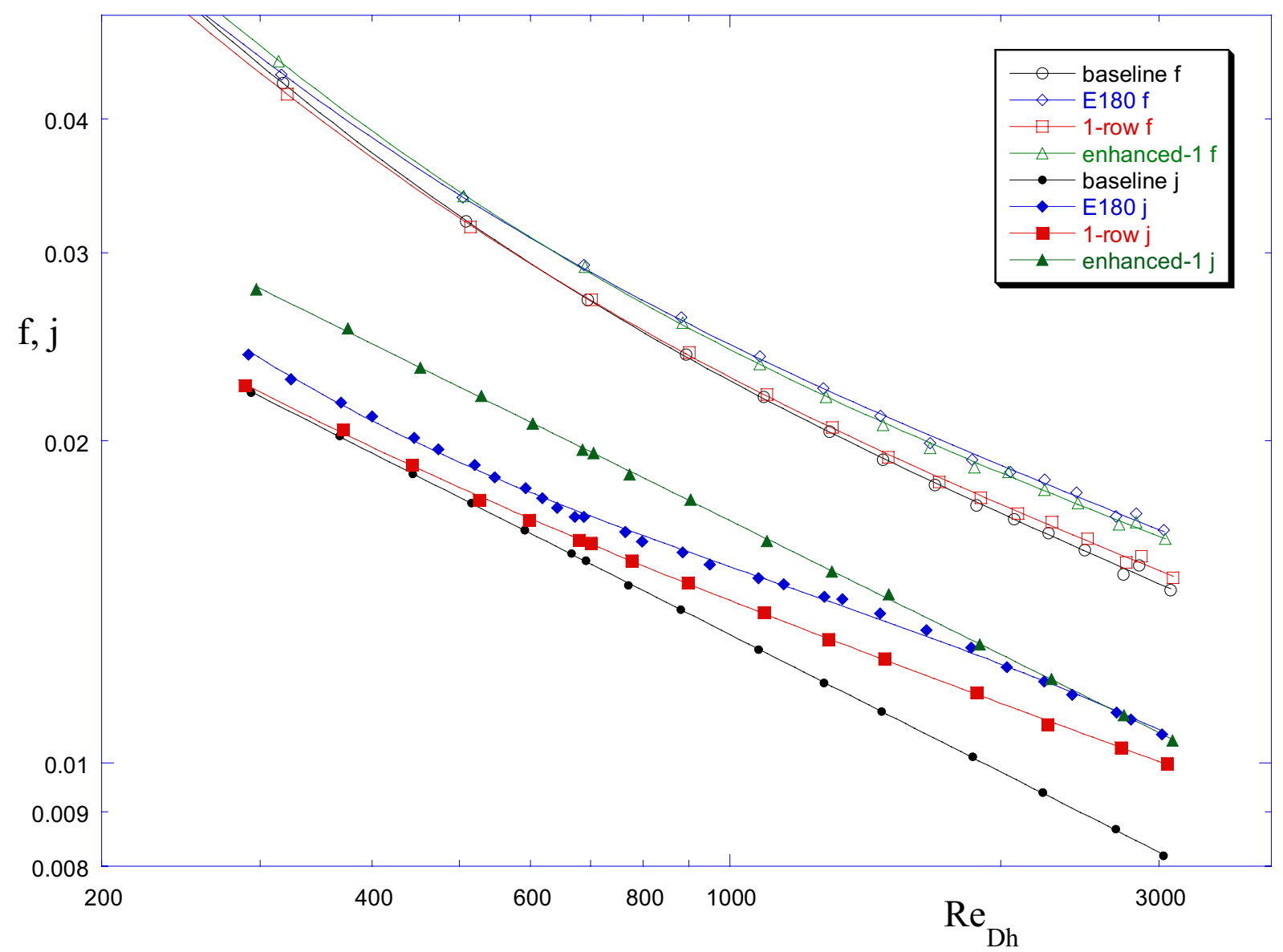

Figure 3-11. Effect of winglet circumferential orientation and number of enhanced tube rows on friction factor and Colburn j-factor.

One indication of the effectiveness of any heat transfer enhancement strategy is the ratio of Colburn $\mathrm{j}$-factor to friction factor for the same configuration. Values of this ratio are presented in Figure 3-12 as a function of the Reynolds number for the five configurations tested. These ratios are based on the curve fits of the friction factor and $\mathrm{j}$-factors shown in Figures 3-10 and -11. The lowest values of $\mathrm{j} / \mathrm{f}$ were obtained for the baseline case, ranging from 0.49 at a Reynolds number of 300 to 0.58 at a Reynolds number of 1000, and dropping off slightly at higher Reynolds numbers. Values of $\mathrm{j} / \mathrm{f}$ for the two enhanced geometries followed similar trends, ranging from $\sim 0.59$ at a Reynolds number of 300 to about 0.67 at a Reynolds number of 1000 , again dropping off slightly at higher Reynolds numbers. Values of $\mathrm{j} / \mathrm{f}$ for the E180 case and the 1-row case increased monotonically over the entire Reynolds number range, 
with values close to the baseline case at the lowest Reynolds numbers and with values close to the enhanced cases at the highest Reynolds numbers.

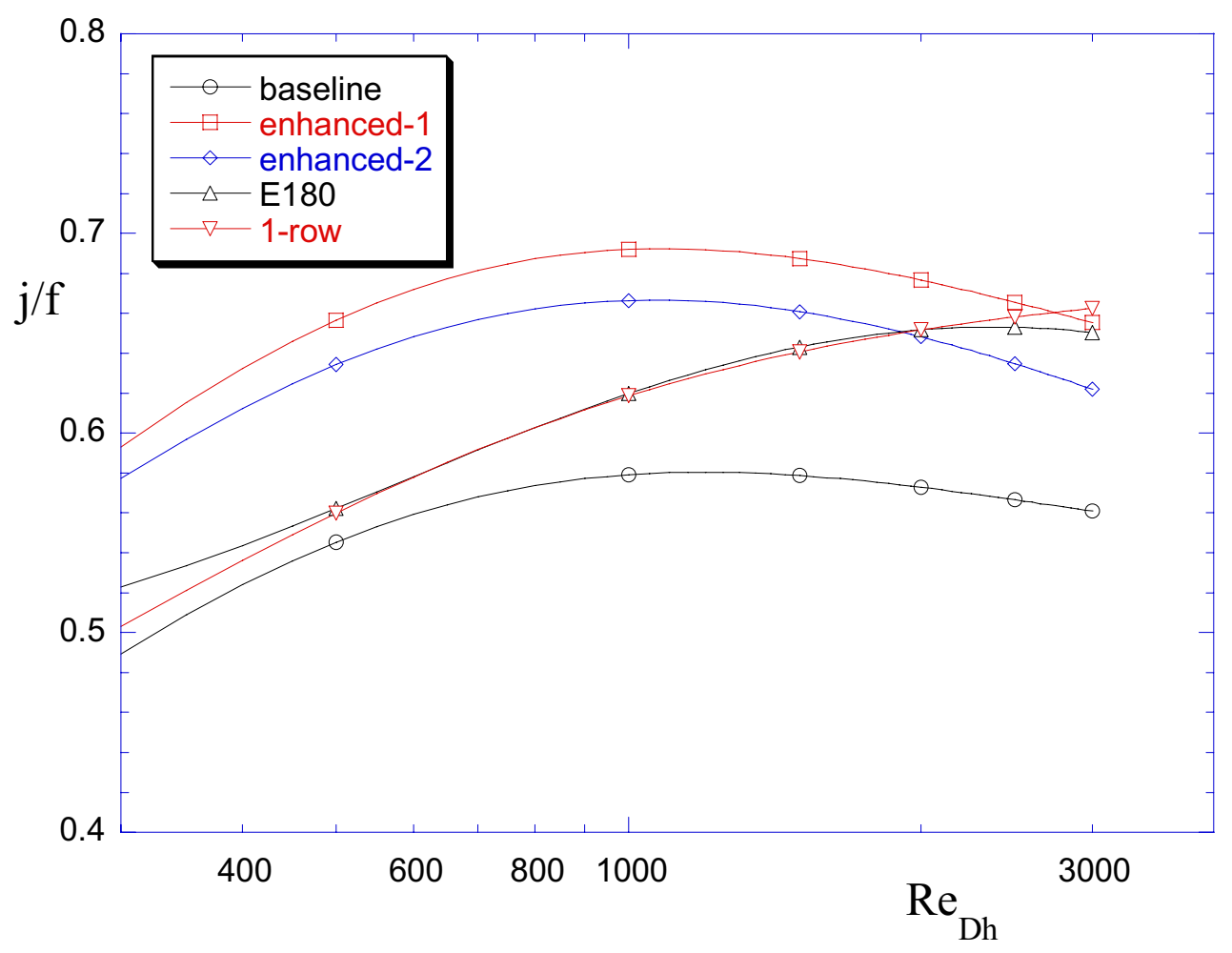

Figure $3-12 . \mathrm{j} / \mathrm{f}$ ratios for the five configurations tested.

Under the constraint of constant pumping power, an appropriate performance evaluation criterion (PEC) for a proposed enhanced heat transfer surface that considers both heat transfer and pressure drop is given by $\mathrm{Wbb}:^{26}$

PEC (at constant Pumping power) $=\frac{\left(j / j_{o}\right)}{\left(f / f_{o}\right)^{1 / 3}}$

where $\mathrm{f}_{\mathrm{o}}$ and $\mathrm{j}_{\mathrm{o}}$ represent the friction factor and Colburn $\mathrm{j}$-factor for the baseline case. Values for this parameter are presented in Figure 3-13 as a function of the Reynolds number for the two enhanced geometries and the two variations considered previously. Based on this criterion, the enhanced-2 geometry clearly yields the best performance at the target Reynolds number of 900 . At the highest Reynolds numbers, in the turbulent regime, the various geometries show similar performance.

Results of the experimental studies show that the heat transfer enhancement levels of $20-30 \%$ were obtained over the tested Reynolds number range of 100-3000, with an increase in pressure drop of only $4-12 \%$, over the same range. Enhanced-" concept appears to be the preferred choice considering performance. 


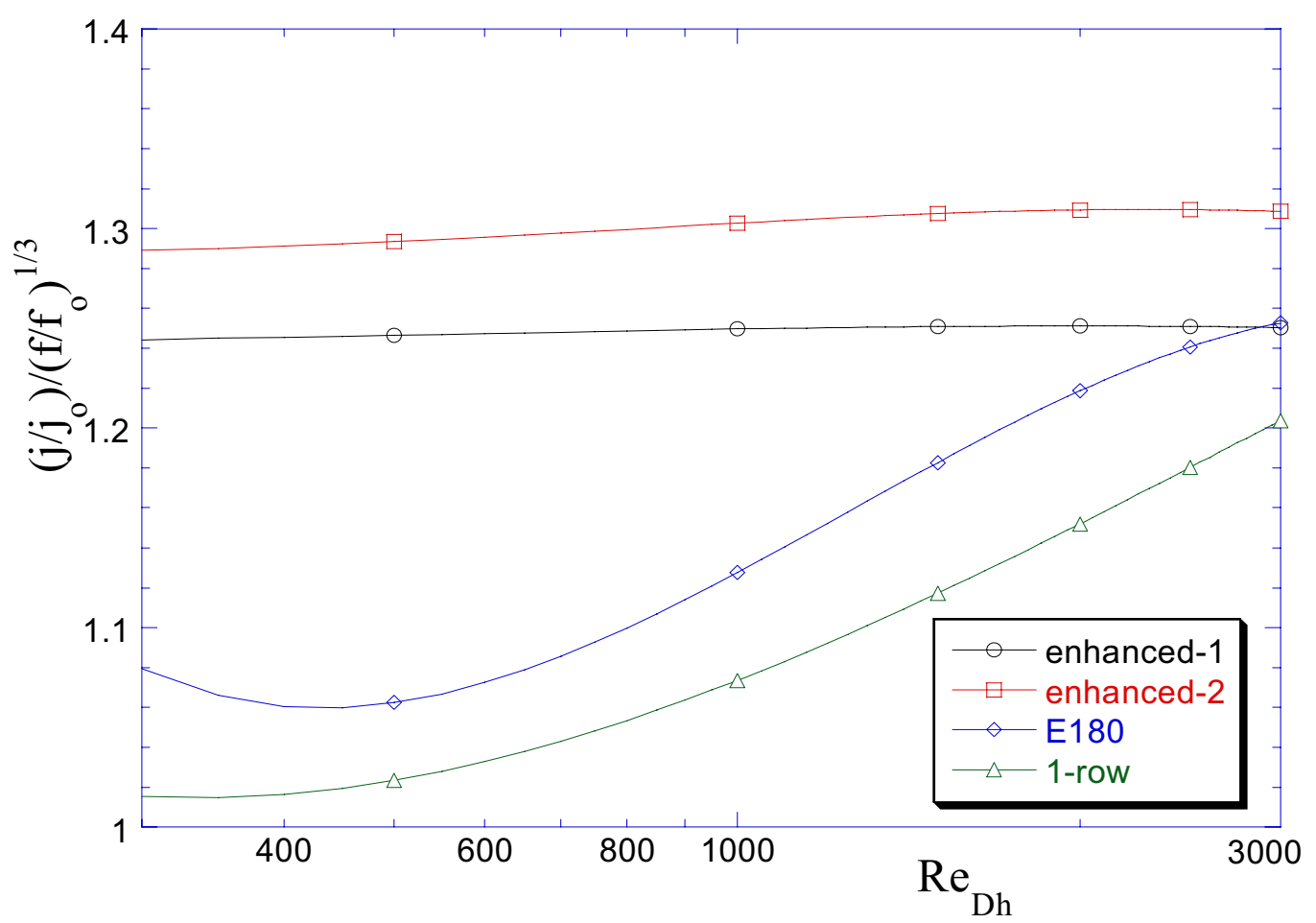

Figure 3-13. Performance criterion for two enhanced geometries and two variations. 


\section{INL NUMERICAL MODELING}

Foust ${ }^{27}$ performed a numerical study of the flow and heat transfer characteristics of finned oval and circular tubes with and without winglet vortex generators, and most of this section is taken from his $\mathrm{PhD}$ thesis. ${ }^{27}$ The numerical solution was based on a solution of the Navier-Stokes and energy equations using a finite-volume formulation. Four winglet vortex generator configurations in combination with a 3:1 axis ratio oval tube were analyzed over the range of $\mathrm{Re}_{\mathrm{H}}$ from 450 to 1700 , with a goal of finding the configuration that produced the best (heat transfer ratio/friction factor ratio) defined as $\left(\left(j / j_{0}\right) /\left(f / f_{o}\right)\right)$. A detailed analysis of the interrelated flow and heat transfer fields was performed.

\subsection{Theoretical Formulation of the Problem}

Heat transfer between the fin and the flowing air depends on the flow characteristics and the temperature distribution on the fin. The flow pattern is three-dimensional (3-D) in nature in both the stagnation region due to the horseshoe vortex formation and in the wake region due to the 3-D vortex shedding. Therefore, 3-D models would be expected to provide better results than those provided by the 2-D models. Atkinson et al. ${ }^{28}$ performed a detailed comparison of the performance of 2-D versus 3-D models for overall heat transfer in louvered-fin compact heat exchangers. Their results indicate that although both approaches gave accurate predictions of pressure losses, the 3-D models gave heat transfer predictions that were in better agreement with experimental observations. Therefore, we decided to develop 3-D models for our purpose.

Chen et al. ${ }^{9,}{ }^{29}$ presented numerical results for laminar, steady-state flow at a low Reynolds number, $\operatorname{Re}_{\mathrm{DH}}<1000$. For automotive and other low air-side Reynolds number (200-1200) applications, laminar, steady-state flow assumption appears reasonable. Zhang et al. ${ }^{30}$ compared heat transfer results at $\operatorname{Re}_{\mathrm{H}}>1000$ for the steady-state versus unsteady-state flow assumptions. He demonstrated that for some parameters of interest, such as the Colburn $\mathrm{j}$ factor and the friction factor $f$, an unsteady-state solution technique was utilized for the higher Reynolds numbers. For the present analysis, unsteady-state assumption was used.

Heat transfer between a solid wall (the fin) and a fluid flow is usually calculated according to the following correlation for convective heat transfer.

$q=h A_{s}\left(T_{w}-T_{\infty}\right)$

The local fin temperature is not an independent variable, as it is a function of the fluid flow conditions and the thermophysical properties of the fin. In addition, the local heat transfer coefficient, $h$, generally varies along the fin surface coordinates, and for unsteady heat transfer it is time-dependent. If $x_{i}$ denotes a coordinate direction of fluid flow over the fin, then Equation (4-1) may be written as:

$$
q=N u_{i j} \frac{k_{f}}{x_{i}}\left(T_{w}-T_{\infty}\right)
$$

where $N u_{x i}$ is the local Nusselt number.

In general, the local surface temperature of the fin depends not only on the $x_{i}$-coordinates but also on the thermal conductivity of the fin. However, a low Biot Number $(B i<<1$, convection being much smaller than conduction) will yield a near-uniform fin temperature distribution. If this is the case, a viable solution technique can be to initially specify the fin surface temperature as a constant temperature surface boundary condition. An internal-flow laminar forced cooling formulation could be solved numerically according to the procedure outlined by Cebeci and Bradshaw. ${ }^{31}$ The converged results would 
yield the local heat flux interrelated to the flow field. Local heat transfer coefficients could then be determined from the numerical results using Newton's law of cooling. Conversely, if the Biot number is large ( $B i>>1$, convection being much larger than conduction), the uniform fin temperature distribution approximation is not realistic, and the flow and energy equations need to be solved simultaneously. This simultaneous solution technique is commonly referred to as the conjugate heat transfer problem. Luikov ${ }^{32}$ has addressed the boundary conditions of the conjugate heat transfer problem and given an analytical solution. In his formulation, boundary conditions of the third kind corresponding to convective heat transfer (definition of the local heat transfer coefficient),

$k_{f}\left(\frac{\partial T}{\partial n}\right)_{w}+h_{i j}\left(T_{w}-T_{\infty}\right)=0$

are substituted by the following boundary condition:

$$
-k_{f}\left(\frac{\partial T}{\partial n}\right)_{w}=-k_{s}\left(\frac{\partial \Theta}{\partial n}\right)_{w}
$$

where $T$ is fluid temperature, and $T_{w}=\Theta_{\mathrm{w}}$.

Luikov formulated a clever analytical technique for calculating the local Nusselt number on the fin surface by substituting this boundary condition into the differential heat transfer equation. Sparrow and Acharya ${ }^{33}$ provided a numerical solution to the conjugate heat transfer problem by simultaneously solving the conduction equation in the fin and the natural convective heat transfer for the cooling fluid, which Sparrow and $\mathrm{Chyu}^{34}$ extended to laminar forced cooling for a one-dimensional fin.

Using their approach and extending it to a two-dimensional fin, the fin energy balance for forced convection is as follows:

$$
\partial^{2} T_{f} / \partial x_{i}^{2}=\left(h_{i j} / k_{w}\right)\left(\left(T_{w}\right)_{i j}-T_{\infty}\right)
$$

where the local heat transfer coefficient $h$ may vary with $\mathrm{x}$ and $\mathrm{y}$.

Recasting in dimensionless form by the substitutions

$$
X_{i}=x_{i} / L, \quad\left(\theta_{s}\right)_{i j}=\left(\left(T_{s}\right)_{i j}-T_{\infty}\right) /\left(T_{f i}-T_{\infty}\right)
$$

leads to

$$
\partial^{2}\left(\theta_{s}\right)_{i j} / \partial X_{i}^{2}=\hat{h} N_{c c}\left(\theta_{s}\right)_{i j}
$$

where $\hat{h}$ is a dimensionless form of the local heat transfer coefficient, and $N_{c c}$ is the conductionconvection number, defined as follows:

$$
\hat{h}=h L / k_{f} \operatorname{Re}_{L^{1 / 2}}, \quad N_{c c}=\left(k_{w} L / k_{f}\right) \operatorname{Re}_{L^{1 / 2}} .
$$


For the present numerical analysis, it was decided to implement the solution to the conjugate heat transfer problem in a manner similar to the technique outlined above. This choice was made over solving the simpler case of initial uniform fin temperature distribution for the following reasons. No a priori decision is necessary for determining the importance of fin conduction contribution. The proposed solution is for a more general case, where fin conjugate effects may or may not have a significant effect. If computational resources are sufficient for this more general case, this approach is more suitable in modeling actual fin-tube heat exchangers.

\subsection{Basic Equations}

The velocity and temperature fields are calculated by a time-marching solution of the unsteady threedimensional Navier-Stokes and energy equations for an incompressible flow with constant properties neglecting viscous dissipation, pressure work, and kinetic energy terms. For laminar unsteady forced convection flow, the governing 3-D differential equations representing mass, momentum, and energy conservation in rectangular Cartesian coordinates using index notation are as follows:

Continuity equation

$$
\frac{\partial u_{i}}{\partial x_{i}}=0
$$

Momentum equation

$$
\rho \frac{\partial}{\partial t}\left(u_{i}\right)+\rho \frac{\partial}{\partial x_{j}}\left(u_{i} u_{j}\right)=-\frac{\partial p}{\partial x_{i}}+\frac{\partial \tau_{i j}}{\partial x_{j}}+\rho g_{i}
$$

where

$$
\tau_{i j}=\mu\left(\frac{\partial u_{i}}{\partial x_{j}}+\frac{\partial u_{j}}{\partial x_{i}}\right)-\frac{2}{3} \mu \frac{\partial u_{k}}{\partial x_{k}} \delta_{i j}
$$

Energy equation for the fluid

$$
\rho\left[\frac{\partial}{\partial t}\left(h_{e n t}\right)+\frac{\partial}{\partial x_{i}}\left(u_{i} h_{e n t}\right)\right]=\frac{\partial}{\partial x_{i}}\left[k \frac{\partial T}{\partial x_{i}}\right]
$$

Energy equation for the fin

$$
\rho \frac{\partial}{\partial t} h_{e n t}=\frac{\partial}{\partial x_{i}}\left(k \frac{\partial T}{\partial x_{i}}\right)+\dot{q}
$$

To form a closed set of equations, additional relations are required to link the thermodynamic and transport properties of the air (pressure, density, temperature, enthalpy, specific heat, viscosity, and thermal conductivity). Air properties are assumed to be constant over the narrow temperature range of the experiment. Air is assumed to behave according to the ideal gas law, and, therefore, enthalpy is only a function of temperature. The relationship is:

$$
d h_{\text {ent }}=c_{p}(T) d T \text {. }
$$


For the test section, the change in air temperature is small, so $c_{p}$ can be assumed to be constant and evaluated at the mean air temperature. The viscosity of the air is also a function only of the temperature, and is obtained from Sutherland's law:

$$
\mu=1.45 \times 10^{-6} \frac{T^{3 / 2}}{(T+110)} \frac{\mathrm{kg}}{\mathrm{m} \cdot \mathrm{s}}
$$

The commercial code Fluent was used to solve the governing equation set. Fluent utilizes a finite volume formulation. The control volume technique converts the governing equations to algebraic equations that can be solved numerically. This control volume technique consists of integrating the governing equations about each control volume, yielding discrete equations that conserve each quantity on a control-volume basis.

The solution technique employed in Fluent for the present analysis follows the following steps.

For the first iteration, the fluid properties are taken from the initialized solution and, thereafter, are updated based on the current solution.

The $\mathrm{x}, \mathrm{y}$, and $\mathrm{z}$ momentum equations are each solved in turn, using current values for pressure and facial mass fluxes in order to update the velocity field.

Since the fluid velocity obtained in Step 1 may not satisfy the continuity equation locally, a poissontype equation for the pressure correction is derived from the continuity equation and the linearized momentum equations. The pressure correction equation is then solved to obtain the necessary corrections to the pressure, velocity and facial mass fluxes such that the continuity equation is satisfied.

The fluid energy equation is solved with the known velocity field and the updated temperature values for the fluid and the fin surface.

The local heat flux to the fin is calculated.

The solid (fin) energy equation is solved with the local heat fluxes spread as a uniform flux in the first surface node on the fin surface.

This process is repeated until a converged solution is obtained with a convergence criterion of $10^{-4}$ for continuity and momentum equations, and $10^{-8}$ for energy equations.

\subsection{Geometrical and Computational Model}

This numerical analysis work was conducted in tandem with related experimental work conducted by O'Brien and Sohal. ${ }^{11,22}$ Their experimental test section was exactly modeled in order to provide a one-toone comparison of numerical and experimental results. Their transient technique provides fin surface heat transfer contours and flow behavior, which are very revealing. The details of the experiments are given in Sections 2.1-2.7 for circular and oval tubes. This step can validate the numerical approach of calculating fin surface heat transfer coefficients.

The geometry of the computational domain was based on the experimental test section. Figures 4-1 and 4-2 show the computational domains for the circular and oval tube, respectively. The computational model matches the test section exactly, with the exception that symmetry conditions were used about the test section centerline, both in the vertical and horizontal directions (W/2 and H/2 in Figures 2-1, -2, -3). This effectively reduces the computational domain to one forth of the full test section and saves considerable computational resources. An entry length of $8.25 \mathrm{~cm}$ was included in the computational models as a thermal entry length before the test section to duplicate the experimental setup. A fully developed velocity profile was specified as an inlet condition to the entry section. This accurately 
simulates the experimental procedure conducted by O'Brien and Sohal, ${ }^{11,22}$ while reducing computational resources required, since the full entry length does not need to be included in the computational models.

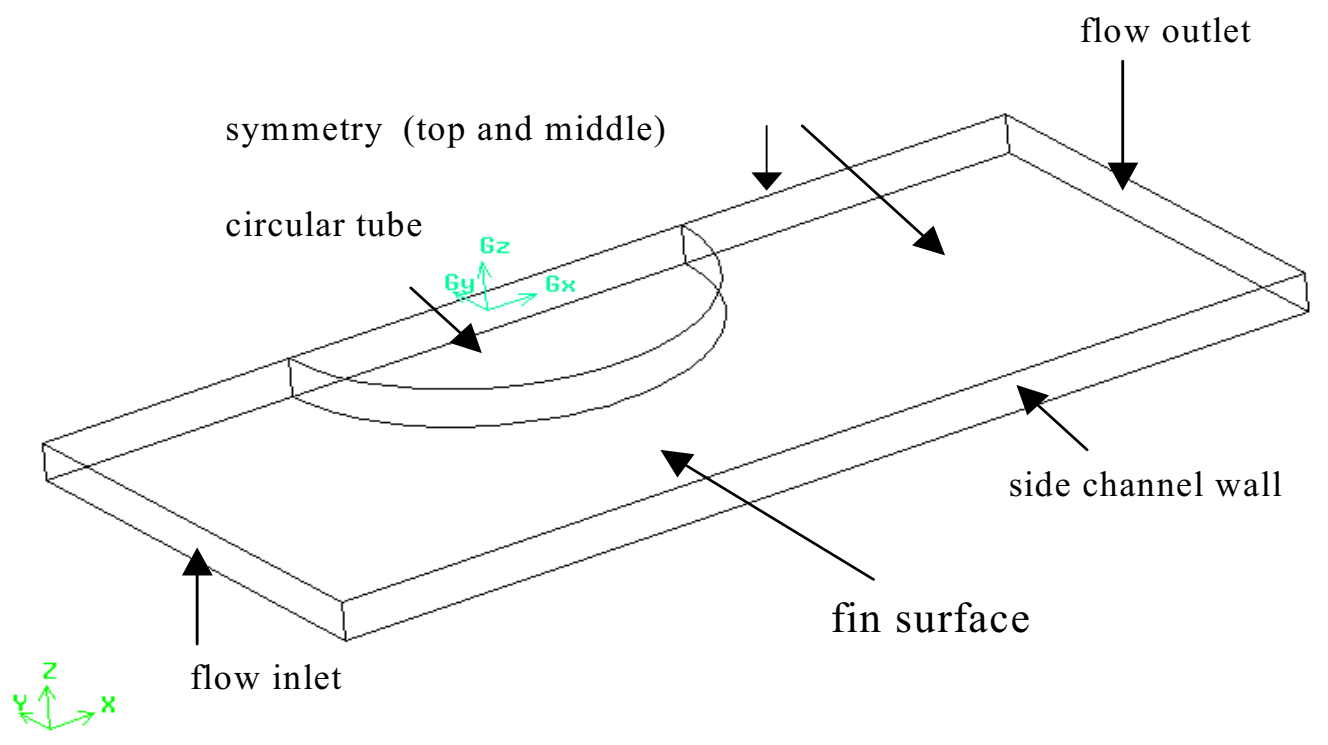

Figure 4-1. Circular tube geometrical model.
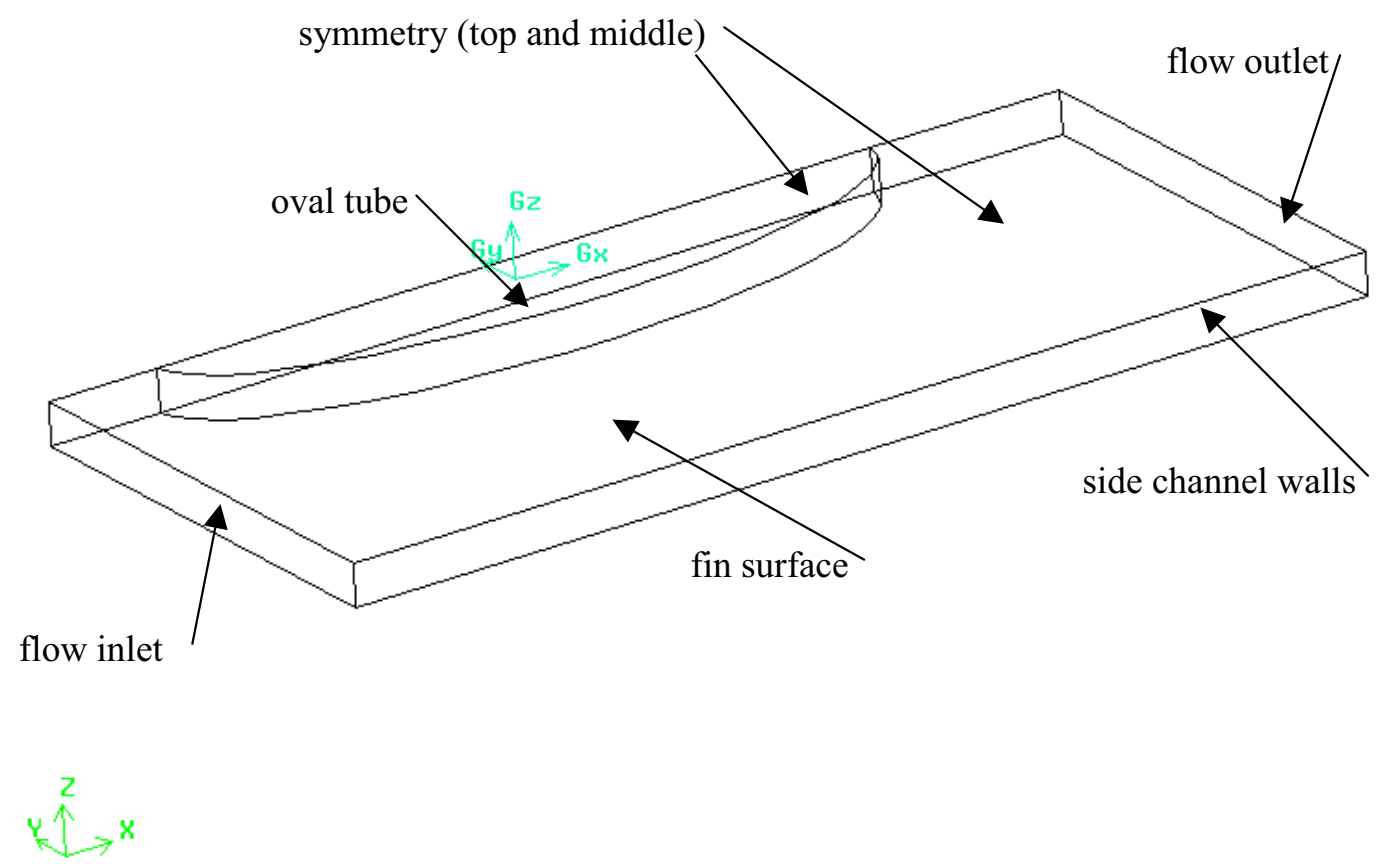

Figure 4-2. Oval tube geometrical model.

\subsection{Boundary Conditions and Numerical Grid}

Boundary conditions were set to accurately reflect conditions in the test section. The inlet condition was set as a mass-flow inlet at atmospheric pressure, with a fully developed velocity profile specified. 
The mass flow rate is set to the specified flow rate for the specific Reynolds number being analyzed. The section and tube mid-plane $\mathrm{W} / 2$ were set as symmetry planes. The outlet of the test section was set as a pressure outlet specified at atmospheric pressure. All test section walls were specified as nonslip for momentum boundary conditions. Test section sidewalls were specified as zero heat transfer walls for the thermal boundary condition, which is a reasonable assumption for the short time period of the transient simulations. A transient simulation was necessary to account for the unsteadiness of the flow due to vortex shedding and also to simulate the transient experiment performed by O'Brien and Sohal. ${ }^{11,22}$

The numerical setup of the conjugate heat transfer problem outlined above requires that the test section bottom surface be set as a two-sided wall. To accomplish this, the bottom lexan surface was included in the computational domain and meshed. This allows specifying the bottom surface as a twosided wall, with the coupled convective-conductive boundary condition modeled implicitly. This approach is consistent with the numerical solution technique for the conjugate heat transfer problem outlined above. The incoming air temperature was set at $45^{\circ} \mathrm{C}$, and the initial temperature of the test section was set at room temperature, $23^{\circ} \mathrm{C}$. At these mild temperature differences, the properties of air will be essentially constant, and variable property effects can be ignored. In order to obtain accurate determinations of surface heat transfer coefficients, a very fine grid spacing is required for the first couple of cells normal to the fin surface. Since grid criteria require aspect ratios (cell length/height) $<5$ to minimize numerical diffusion, horizontal cell length can only be five times cell height. Therefore, the size of the computational domain is a function of vertical spacing squared (i.e., for every doubling of vertical refinement, the computational domain increases by a factor of four). Since a transient solution technique was utilized, these calculations are computationally intensive, and, therefore, a good balance between desired accuracy and computational time was found. Therefore, three grids were developed, with vertical spacing of 10, 20, and 30 vertical cells. For this analysis, the 10 -vertical-cell grid could be considered a relatively coarse grid scheme, and the 30 -vertical-cell grid a fine grid scheme. This corresponds to cell vertical heights of $0.5,0.25$, and $0.167 \mathrm{~mm}$, with corresponding cell length set to keep aspect ratios on all cells in the test section $\leq 5$. This approach led to $200,503,356,448$, and 802,010 computational control volumes for the 10, 20, and 30 vertical cell grids, respectively.

\subsection{Computational Approach}

To reduce the computational domain, the outer quarter of the test section channel was removed from computational domain, effectively halving the computation size. Although this approach was deemed to be sufficient for determining fin surface heat transfer coefficients in the vicinity of the tube, there was concern that this approach would skew the pressure-drop calculations. The circular tube has a diameter of $2.54 \mathrm{~cm}$, and this modeling approach reduces the computational domain width to $2.85 \mathrm{~cm}$. This reduces the flow channel between the circular tube edge and the flow channel side to $0.31 \mathrm{~cm}$. The oval tube has a minor axis of $2.93 \mathrm{~cm}$; so, in the oval tube case, the flow channel width would be $1.43 \mathrm{~cm}$. Therefore, pressure drop calculations might be artificially skewed against the circular tube. Further compounding the problem was the fact that results for the 10 and 20 vertical cell grids appeared to depend on mesh size.

Clearly, this approach for meshing the computational domain was not acceptable, and alternative methods had to be developed. In an attempt to overcome this dilemma, an alternative boundary layer meshing approach ${ }^{35}$ was utilized. This approach uses a boundary layer type meshing approach on the fin surface. The fin surface has very fine cells, and the bulk flow region has coarse cells. For the boundary layer mesh, the first grid point was specified at $0.015 \mathrm{~mm}$ above the fin surface, with a growth factor of 1.20. This grid configuration reduced the number of vertical cells to 13 , while exceeding the refinement of the 30-vertical-cell grid approach near the fin. The approach resulted in a $\sim 30 \%$ reduction in computational control volumes compared to the 30-uniform-cell vertical grid. Although this meshing technique reduced transient run times to about 4 to 5 days of elapsed time, run times of this length were still onerous at best. 
After about 8 months into this work, the Sun Enterprise workstation was replaced with a SGI Origin with 64-400 MHz MIPS R12000 (IP35) processors and 64 GB of main memory. A parallel license and server were obtained, which allowed computations to be performed in an 8-parallel-processor mode. Improved computational capabilities reduced transient simulation run times on 100,000 cell grids from 4 or 5 days to a matter of hours. In addition, the enhanced computational resources allowed computational domains to be increased to 500,000 cells or more, while still maintaining very reasonable run times, thus allowing finer meshes and better results. All calculations were performed again with this enhanced computational capability, and the computational domain was expanded to the full half-width to avoid any skewing of the pressure-drop calculations.

Fluent is a computational fluid dynamics software that allows computations to be performed on unstructured meshes. Unstructured meshes significantly reduce the time required to mesh the tube portion of the test section. The mesh used for this analysis was a hybrid, ${ }^{36}$ being an unstructured hexagonal mesh in the tube section region and a structured quadratic mesh in the inlet and outlet regions. This meshing approach was used since it was deemed the best approach toward achieving the accuracy desired with the minimum number of mesh volumes.

\subsection{Validation of Numerical Results}

The baseline configuration studied for this validation was a single oval tube in the test section. Experimental and numerical results are compared for $\mathrm{Re}_{\mathrm{H}}$ values of 500 and 1240 , which correspond to $\mathrm{Re}_{\mathrm{DH}}$ of 950 and 2250, respectively. The coarse girds of 10 and 20 vertical cells failed to resolve the horseshoe vortices and other important physical aspects of the flow, and exhibited grid dependence. Hence, they were dropped. The 30 -vertical-cell grid and the boundary layer mesh grid produced nearly identical results, indicating grid independence. Since the boundary layer meshing approach significantly reduced required computational resources compared to the 30 vertical cell mesh, this approach was used exclusively for this analysis.

The first step was to compare the numerical predictions with experimental results obtained by O'Brien and Sohal, ${ }^{11,22}$ Contour plots of fin-surface heat transfer coefficients in the vicinity of the oval tube are presented in Figures 4-3a and - $b$ for $\operatorname{Re}_{\mathrm{H}}=500$ and in Figures 4-4a and $-\mathrm{b}$ for $\operatorname{Re}_{\mathrm{H}}=1240$. Figures 4-3a and 4-4a present the experimental results, and Figures 4-3a and -b present the numerical results. The slight asymmetrical nature in Figures 4-3a and 4-4a is due to the slightly off-normal viewing angle of the infrared camera, as explained by O'Brien and Sohal. Figure 4-5 presents the local streamwise fin-surface heat transfer coefficients for $\mathrm{Re}_{\mathrm{H}}=1240$ along duct length centerline. Figure 4-6 presents the transverse fin-surface heat transfer coefficients for $\mathrm{Re}_{\mathrm{H}}=1240$ in the wake region. Note that in Figures 4-5 and 4-6, the $\mathrm{x}$-axis is along the transverse direction, and the $y$-axis is along the streamwise direction. Comparison of the experimental and numerical contour plots shows very good agreement for the fin surface heat transfer coefficients and the shape of the contour profiles. Inspection of the line plots for numerical versus experimental results also show good agreement for the $\mathrm{Re}_{\mathrm{H}}=500$ and 1240 cases in both the stagnation and wake regions.

Both the experimental and numerical results depict longitudinal streaks located outside of the primary horseshoe vortex. The streaks are most evident at $\mathrm{Re}_{\mathrm{H}}=500$, but are also evident at $\mathrm{Re}_{\mathrm{H}}=1240$. O'Brien and Sohal ${ }^{11,22}$ stated that for the experimental results these streaks do not appear to be directly related to the horseshoe vortex system, but rather may be formed as secondary flow in the rectangular duct upstream of the oval tube. The numerical results predict that the secondary flow develops in closer proximity to the tube than that indicated by the experimental results. Hence, the numerical results show a smaller secondary peak in the stagnation region and a smaller transverse distance between the peaks in the wake region. However, these streaks may also be evidence of induced vortices related to the primary horseshoe vortices. Exploring this possibility further, Figures 4-7 and 4-8 are vector plots in a vertical plane along the tube major axis and normal to the tube wall in the stagnation region for $\mathrm{Re}_{\mathrm{H}}=500$ and 
1240, respectively. The yellow area represents the tube wall surface in both Figures. The flow is from left (upstream) to right (downstream).

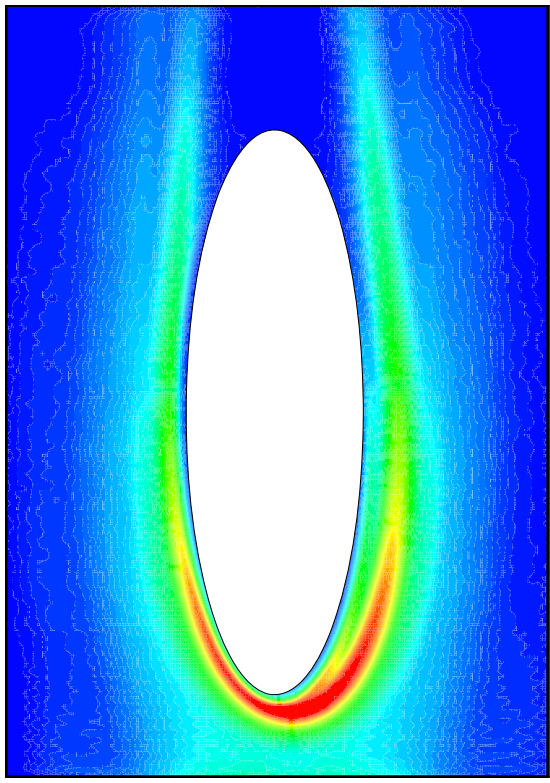

(a) Experimental data

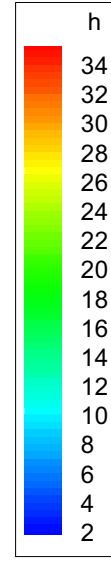

(b) Numerical results
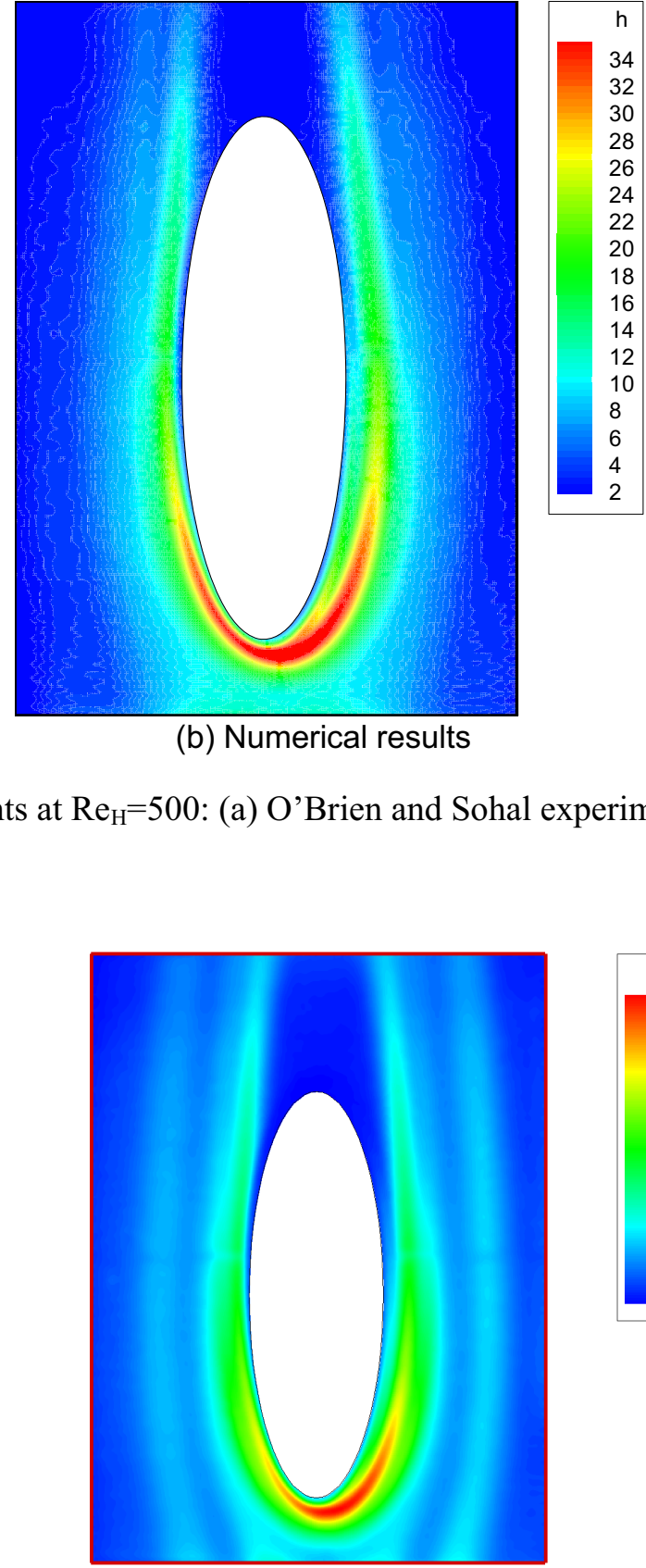

(b) Numerical results

(a) Experimental data

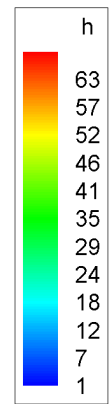

Figure 4-4. Contours of local heat transfer coefficients $\mathrm{Re}_{\mathrm{H}}=1240$ : (a) O'Brien and Sohal experimental data, (b) numerical results. 


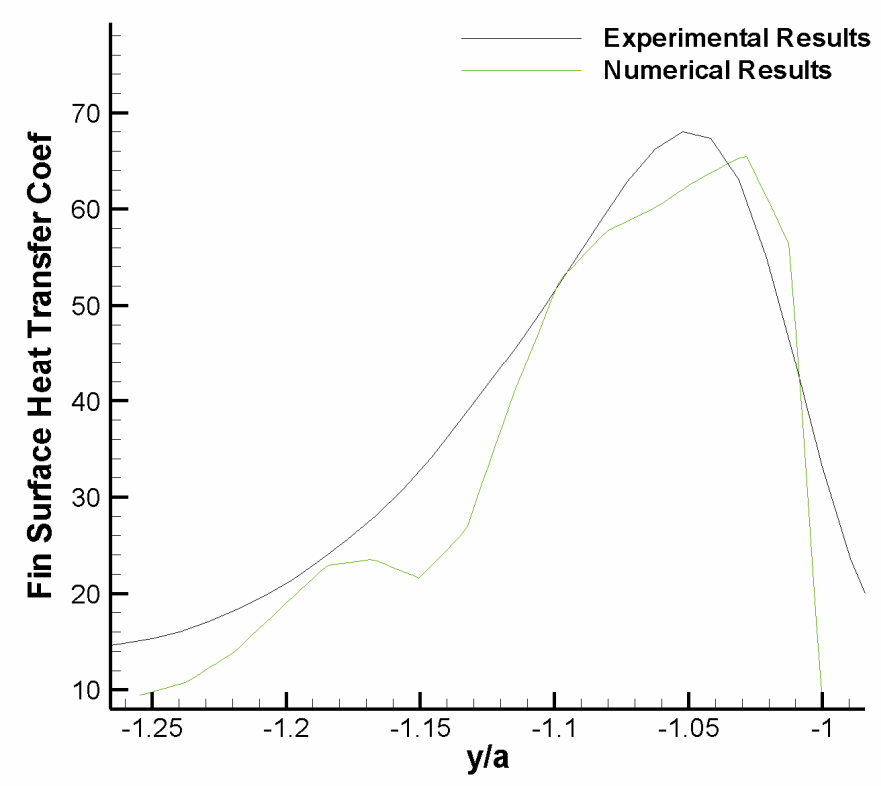

Figure 4-5. Comparison of experimental and numerical values of fin-surface heat transfer coefficient $\left(\mathrm{W} / \mathrm{m}^{2} \mathrm{~K}\right)$ for an oval tube, along duct length centerline in the stagnation region.

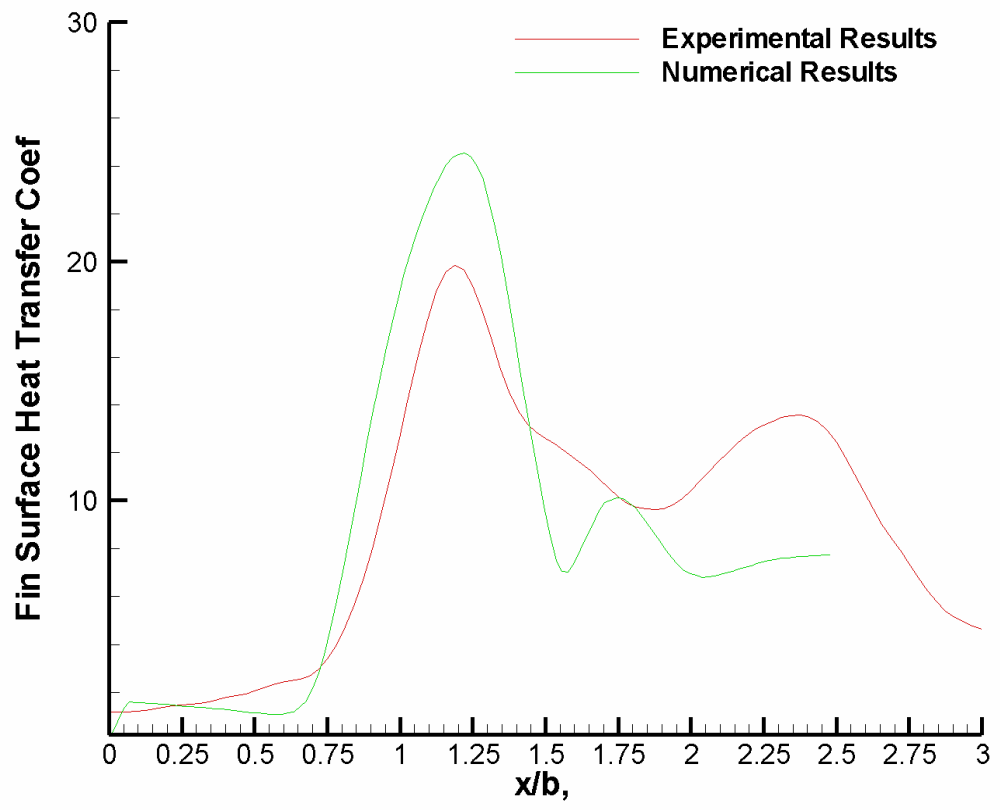

Figure 4-6. Comparison of experimental and numerical values of fin-surface heat transfer coefficient $\left(\mathrm{W} / \mathrm{m}^{2} \mathrm{~K}\right)$ for an oval tube, along duct width in the wake region at $\mathrm{y} / \mathrm{a}=1.10$. 


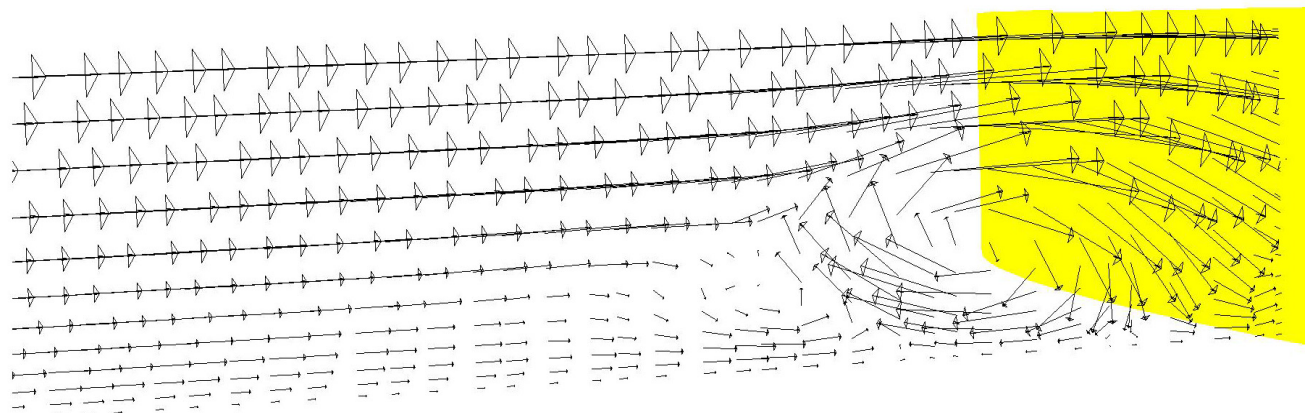

Figure 4-7. Horseshoe vortices in the stagnation region upstream of the oval tube wall (in yellow) at $\mathrm{Re}_{\mathrm{H}}=500$.

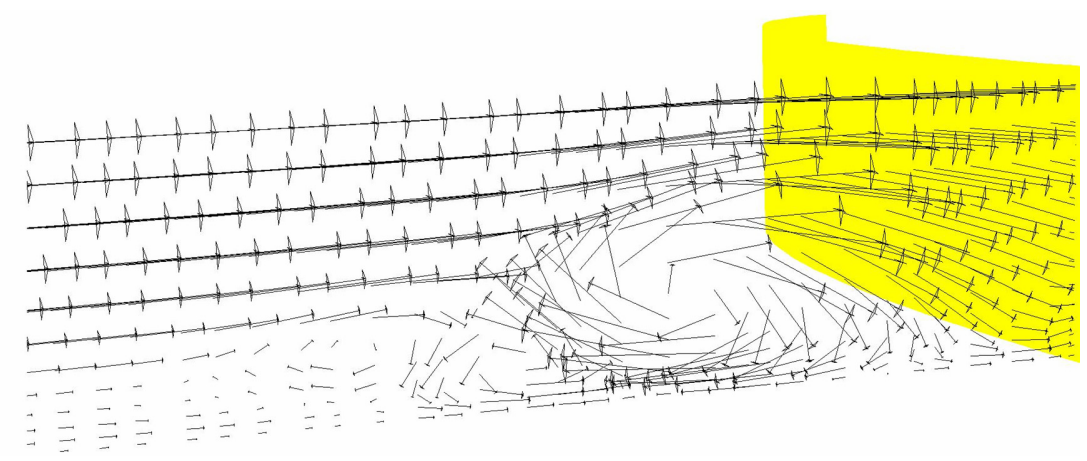

Figure 4-8. Horseshoe vortices in the stagnation region upstream of the oval tube wall (in yellow) at $\operatorname{Re}_{\mathrm{H}}=1240$.

Both $\mathrm{Re}_{\mathrm{H}}$ cases show an induced co-rotating vortex outside the primary horseshoe vortex. In the case of $\operatorname{Re}_{\mathrm{H}}=500$, the induced vortex is very weak and does not appear to penetrate the boundary layer; hence, the surface heat transfer coefficient plot (Figure 4-3b) does not show heat transfer enhancement associated with this vortex. In the case of $\mathrm{Re}_{\mathrm{H}}=1240$, the induced co-rotating vortex is much stronger and does break up the boundary layer, resulting in a double peak in surface heat transfer coefficients, as depicted in Figures 4-4(b) and 4-5. O'Brien and Sohal ${ }^{11,22}$ also observed this double peak in their experimental results for circular tubes and show evidence of their existence for oval tubes. This induced vortex accounts for the second peak in the wake region, evident in the numerical results presented in Figure 4-6.

Both numerical and experimental results show that longitudinal horseshoe vortices are produced and swept downstream of the stagnation region, resulting in enhanced heat transfer along the sides and downstream of the oval tube.

Both experimental and numerical results also indicate that the flow separation point moves downstream and that the size of the low-heat-transfer wake region shrinks significantly with increasing an Reynolds number. The wake region transverse numerical results predict slightly higher fin surface heat transfer coefficients than the experimental results show. The secondary peak in the numerical results is due to the induced vortex as it is swept downstream, whereas the experimental secondary peak is due to the secondary flows. Both experimental and numerical results indicate that the peak stagnation region fin-surface heat transfer coefficient is about an order of magnitude higher than the corresponding fully developed duct values. The numerical results show very good agreement with the experimental results 
and are well within the uncertainties associated with experimental and numerical research. This validates the numerical approach used to obtain the fin surface heat transfer coefficients.

The convective heat flux from the fin surface is determined directly from the numerical solution of the energy equation. The local value of the surface heat transfer coefficient is determined by dividing the heat flux by the temperature difference between the wall and incoming free stream air temperature. O'Brien and Sohal ${ }^{11,22}$ report relative experimental uncertainty in heat transfer coefficient ranges from $20 \%$ at $\mathrm{h}=\sim 10 \mathrm{~W} / \mathrm{m}^{2} \cdot \mathrm{K}$ to $10 \%$ at $\mathrm{h}=\sim 120 \mathrm{~W} / \mathrm{m}^{2} \cdot \mathrm{K}$. In addition, there is uncertainty in the value of $\sqrt{\rho c k}$ for the lexan (duct wall material). Auxiliary open-channel experiments have indicated that the value used in the oval-tube study $\left(543 \mathrm{~W} / \mathrm{m}^{2} \cdot \mathrm{K} \cdot \mathrm{s}^{1 / 2}\right)$ may be about $10 \%$ low. Therefore, given the experimental uncertainty and two different methods used for calculating fin surface heat transfer coefficients, the experimental and numerical results show exceptionally good agreement, both as to magnitude and shape of contours. Atkinson et al. ${ }^{28}$ had a $70 \%$ over-prediction of the average computed Stanton number for the heat transfer surface when using a 2-D model over the measured value and successfully reduced this to about $10 \%$ by using a 3-D model. Although the experimental setup for the present research did not allow measurements of the overall heat flux, good agreement between experimental and numerical measurements of the overall or average heat flux would be expected given the good agreement between the local distribution of the experimental and numerical heat flux values.

\subsection{Single Oval and Circular Tube Comparison}

\subsubsection{Performance Comparison}

When the oval tubes are oriented such that the major axis is parallel to the flow direction, they present a smaller frontal area than the circular tubes of the same cross sectional area. This leads to delayed flow separation and a smaller wake region, which reduces pressure drop compared to that in athe case of circular tubes. However, recent studies ${ }^{37,38}$ show that oval tubes have lower heat transfer from the fin surface than from fins on circular tubes. To compare the performance of oval and circular tubes with the same cross sectional area, a performance criterion ratio (Colburn heat transfer degradation ratio/friction factor reduction ratio) was calculated over a range of Reynolds numbers $\left(\mathrm{Re}_{\mathrm{H}}\right)$. Figure 4-9 presents the results of this comparison for $\mathrm{Re}_{\mathrm{H}}$ values of 500, 900, and 1240. In the figure, subscript $\mathrm{o}$ (zero) refers to the baseline cylindrical case, being the reference value. Heat transfer from the oval tube fin surface is $14.3,9.5$, and $13.6 \%$ lower than that for the circular tube fin surface $\left(\mathrm{j} / \mathrm{j}_{\mathrm{o}}\right)$ case for $\mathrm{Re}_{\mathrm{H}}=$ 500,900 , and 1240, respectively. The pressure drop for the oval tube is 58.3, 61.3, and $62.2 \%$ lower than that for the circular tube case $\left(\mathrm{f} / \mathrm{f}_{\mathrm{o}}\right)$ for $\mathrm{Re}_{\mathrm{H}}=500,900$, and 1240 , respectively.

Across the range of $\mathrm{Re}_{\mathrm{H}}$ numbers analyzed, the oval tubes averaged a $12.5 \%$ lower heat transfer rate and a $60.6 \%$ lower pressure drop compared to corresponding values for circular tubes. Therefore, as can be seen in Figure 44, the oval tubes produce a favorable $\left(j / j_{o}\right) /\left(f / f_{o}\right)$ for Reynolds numbers of 500-1240 and are preferable when internal operating pressures are low enough to allow their usage. This result is consistent with previous experimental results presented in the literature ${ }^{38,39}$.

Figures 4-10 through 4-12 depict the numerically computed contour plots of fin surface heat transfer coefficients for the oval and circular tubes for $\mathrm{Re}_{\mathrm{H}}$ of 500, 900, and 1240, respectively, and Figures 48a and $48 \mathrm{~b}$ show computed line plots of fin-surface heat transfer coefficients for the stagnation and the wake regions, respectively. Examination of Figures 4-10 through 4-12 shows that for the circular-tubes, increased area on the fin surface is influenced by the horseshoe vortices, which leads to enhanced heat transfer. This observation can also be confirmed from Figures 4-13 and 4-14, which show the line plots of heat transfer coefficients at three Reynolds numbers for both circular and oval tubes. For all Reynolds numbers, it can be seen that for circular tubes, increased heat transfer is prevalent for a longer distance upstream from the tube stagnation point. Peak heat transfer coefficients in the stagnation region are also 
slightly higher for the circular tubes than for the corresponding values for oval tubes and are about a factor of ten higher than correspondingly fully developed duct flow. For all cases, with the exception of the oval tube case at $\mathrm{Re}_{\mathrm{H}}=500$, a double peak in fin surface heat transfer coefficients is evident in the stagnation region.

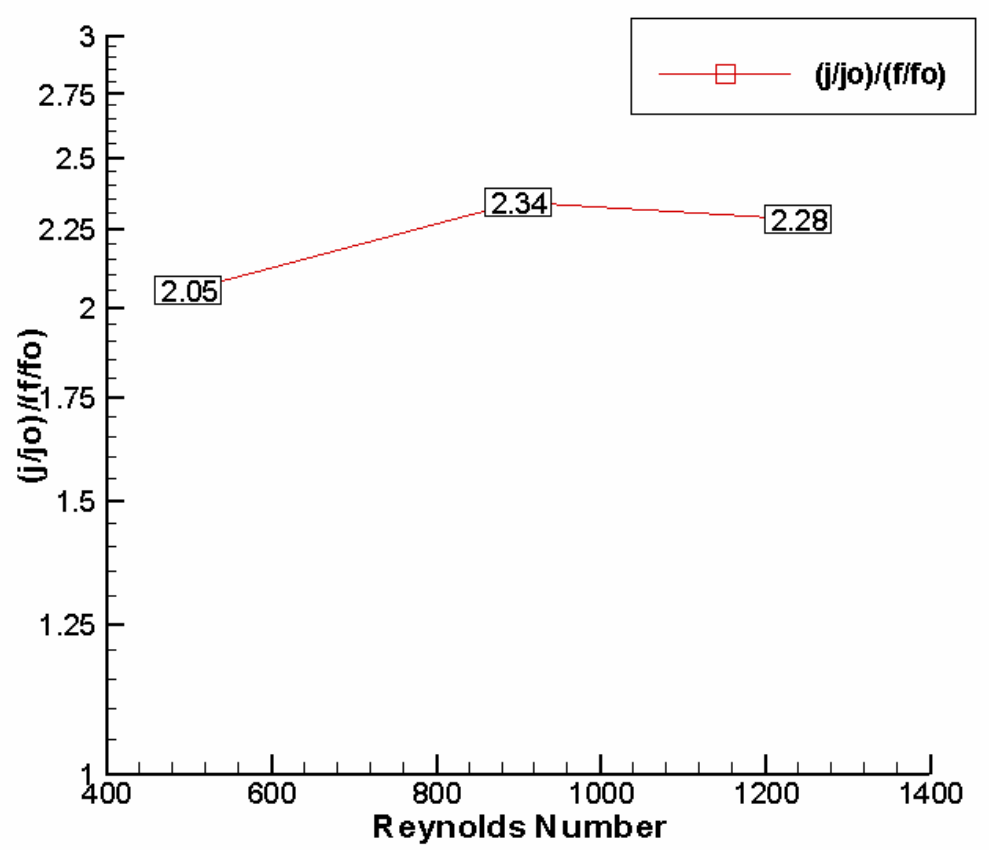

Figure 4-9. $\left(\left(\mathrm{j} / \mathrm{j}_{\mathrm{o}}\right) /\left(\mathrm{f} / \mathrm{f}_{\mathrm{o}}\right)\right)$ ratio for oval and circular tubes versus the Reynolds number.

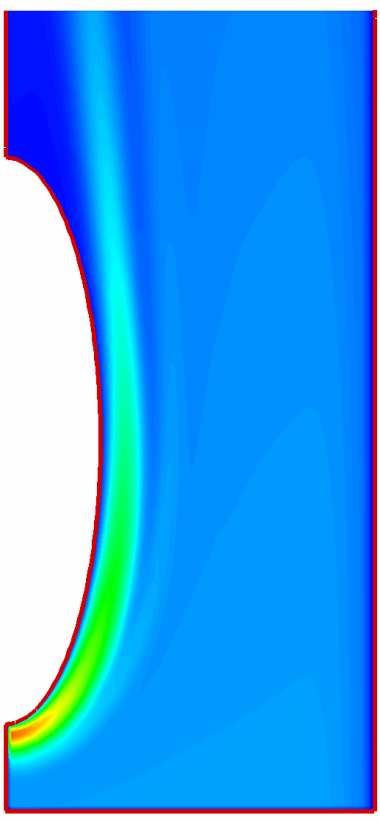

(a)

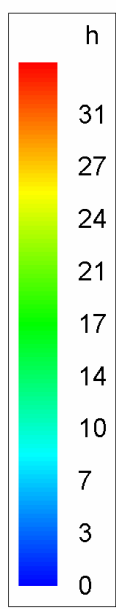

(b)

Figure 4-10. Fin surface heat transfer coefficient $(\mathrm{W} / \mathrm{m} 2 \cdot \mathrm{K})$ contour plots for $\mathrm{ReH}$ $=500$ : (a) oval tube, (b) circular tube. 


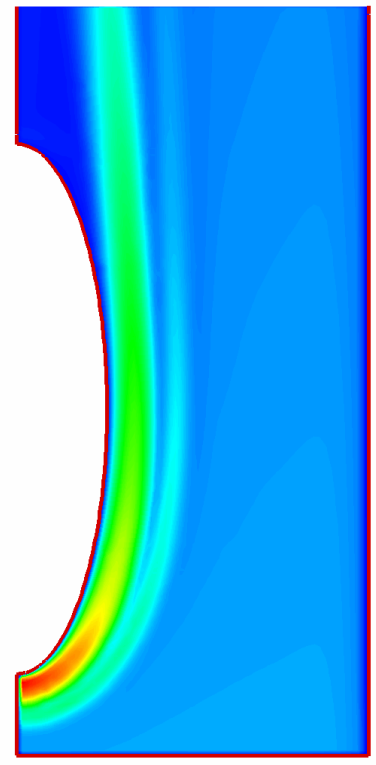

(a)

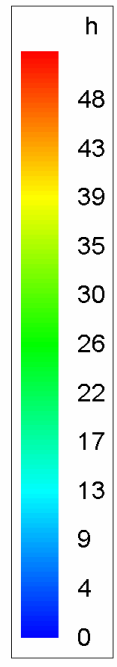

Figure 4-11. Fin surface heat transfer coefficient $\left(\mathrm{W} / \mathrm{m}^{2} \cdot \mathrm{K}\right)$ contour plots for $\mathrm{Re}_{\mathrm{H}}=$ 900: (a) oval tube, (b) circular tube.

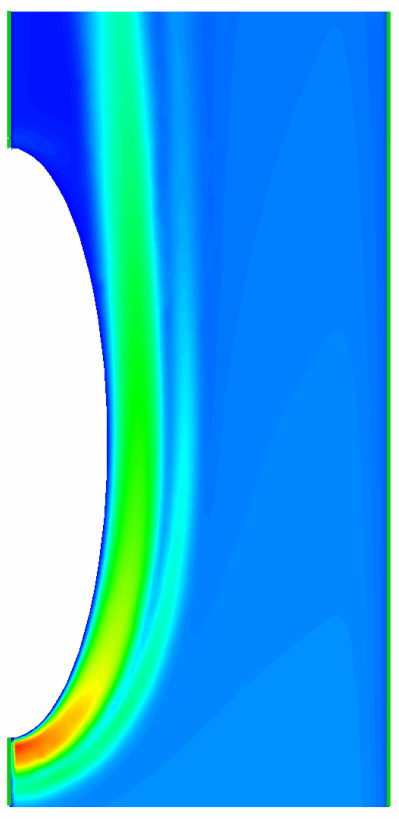

(a)

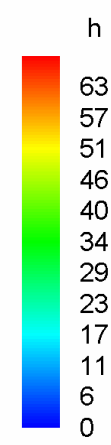

Figure 4-12. Fin surface heat transfer coefficient $(\mathrm{W} / \mathrm{m} 2 \cdot \mathrm{K})$ contour plots, for $\mathrm{ReH}=1240$ : (a) oval tube, (b) circular tube.

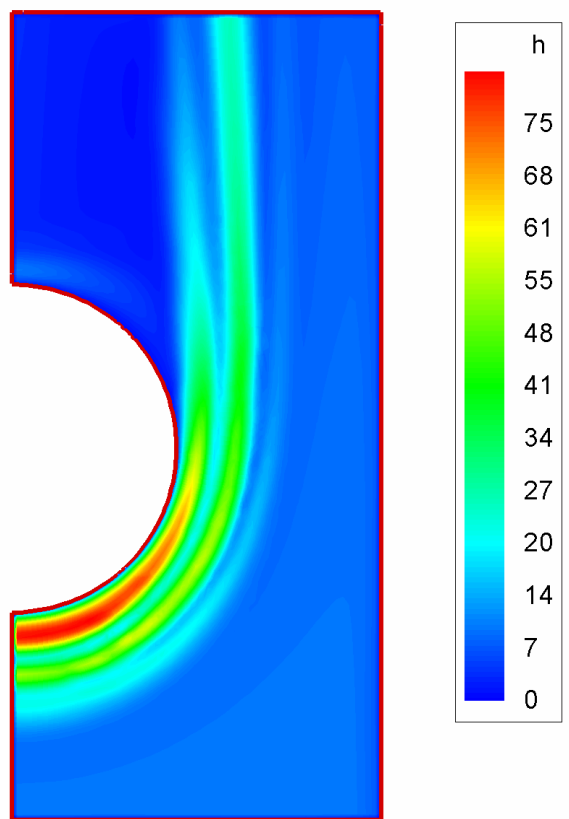

(b) 


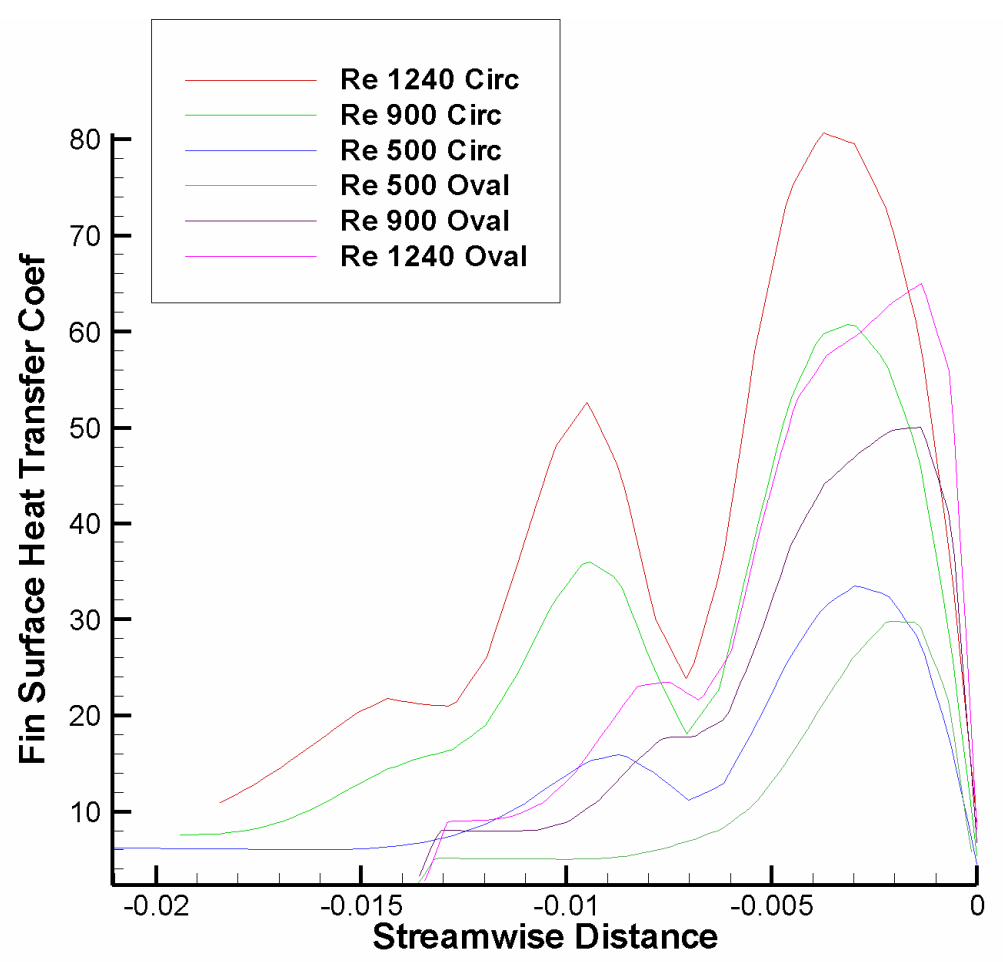

Figure 4-13. Line plots of fin-surface heat transfer coefficient $\left(\mathrm{W} / \mathrm{m}^{2} \cdot \mathrm{K}\right)$ : streamwise variationalong duct centerline from tube front stagnation point (in $\mathrm{m}$ ).

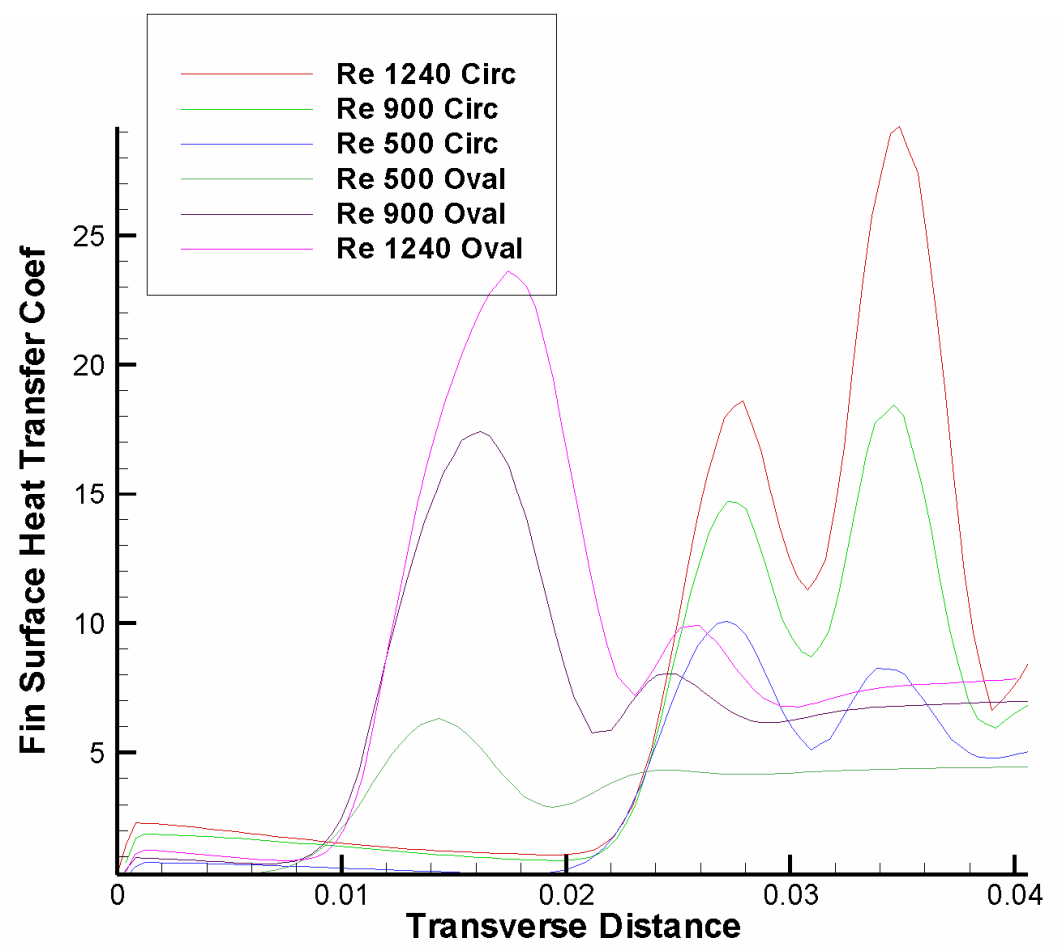

Figure 4-14. Line plots of fin-surface heat transfer coefficient $(\mathrm{W} / \mathrm{m} 2 \cdot \mathrm{K})$ : transverse variation in wake region from tube centerline (in $\mathrm{m}$ ), $\mathrm{y} / \mathrm{r}=1.10$ for circular tube and $\mathrm{y} / \mathrm{a}=1.10$ for oval tube. 
This double-peak observation is consistent with results presented in the literature ${ }^{16,17}$ that used mass transfer analogies and liquid crystal thermography techniques, respectively, to calculate heat transfer coefficients. The double peak is characteristic of the complex horseshoe vortex system that establishes in the forward stagnation region near the base of a bluff body when a boundary layer flow encounters the bluff body. The double peak in fin-surface heat transfer is more prevalent for the circular tube case than for the oval tube case. Figure 4-15 is a vector plot in a vertical plane normal to the tube wall in the stagnation region for a circular tube at $\mathrm{Re}_{\mathrm{H}}=1240$. Again, the yellow area on the right side of Figure 4-15 represents the tube wall surface. Figure 4-15 clearly shows the co-rotating double vortex system that establishes in the stagnation region. Close inspection of Figure 54-150 also reveals a much weaker third counter-rotating vortex induced outside the secondary vortex. The presence of this third vortex is also evident in Figure 4-12b. Comparison of Figure 4-15 for a circular tube at $\operatorname{Re}_{\mathrm{H}}=1240$ and Figure 43 for an oval tube at $\mathrm{Re}_{\mathrm{H}}=1240$ shows that the secondary induced co-rotating vortex is much stronger in the case of the circular tube. This explains why the secondary peaks in surface heat transfer coefficients for the circular-tubes are much higher in both the stagnation and wake regions.

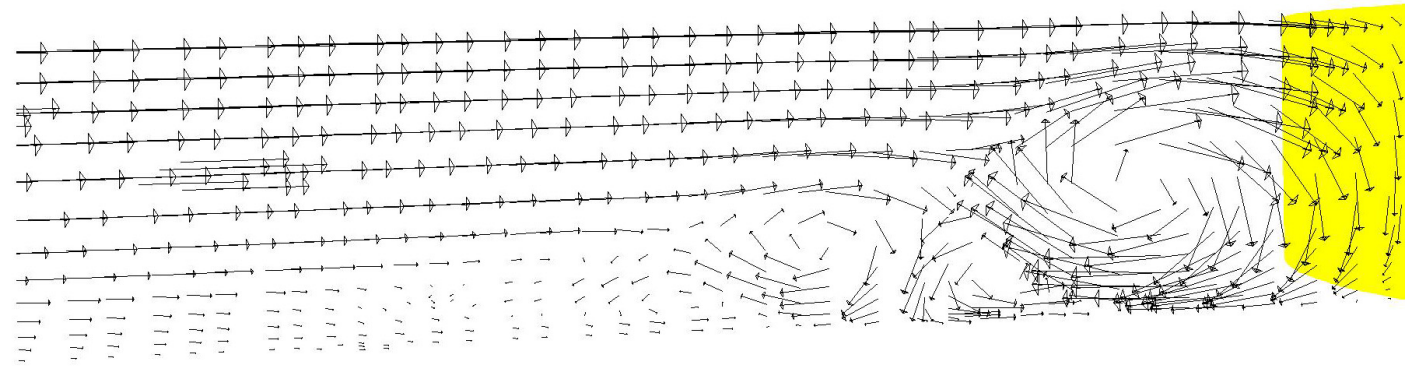

Figure 4-15. Velocity vectors for horseshoe vortices for a circular tube (in yellow) at $\operatorname{Re}_{\mathrm{H}}=1240$.

For all three Reynolds numbers for the circular tube, flow separation occurs at about $90-100^{\circ}$ from the forward stagnation point, which is characteristic of laminar flow separation. Examination of the wake region reveals that flow separation is delayed for the oval tube for all three cases analyzed and that separation (as indicated by the surface heat transfer contours) is not as distinct as for the circular tube. Examination of Figure 4-14 reveals that for both the oval and circular tube cases, for all three Reynolds numbers, the transverse distance from the tube centerline to the two peaks remains constant, and the magnitude of the peak increases with increase in Re number, as would be expected. Because geothermal air-cooled condensers typically operate at $\mathrm{Re}_{\mathrm{H}}<1750$, the optimal location of the vortex generators should be the same for the range of $\mathrm{Re}_{\mathrm{H}}$ for normal condenser operation.

Figure 4-16 shows pressure contours for (a) the oval case, and for (b) the circular-tube case at $\mathrm{Re}=1240$. As shown in the Figure, the circular tube presents a much larger resistance to flow than the oval tube. The peak pressure in the stagnation region for the circular tube is higher than for the oval tube, and the total area of this higher pressure is considerable larger for the circular-tube case. This provides insight into the considerable advantage in the frictional pressure drop of oval tubes compared to circular tubes.

The results of this analysis show the performance criterion ratio (Colburn heat transfer degradation ratio/friction factor reduction ratio) of oval tubes (axis ratio 3:1) compared to that for circular tubes of the same cross sectional area. Over the range of Reynolds numbers analyzed, oval tubes yielded slightly lower average heat transfer $(12.5 \%)$ from the fin surface, but with a considerably lower average pressure drop $(60.6 \%)$, thus producing a very favorable performance criterion ratio. 


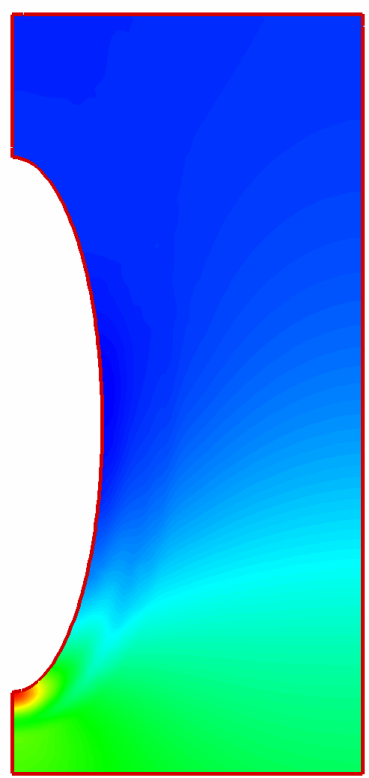

(a)

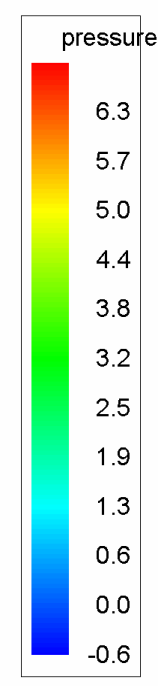

(b)

Figure 4-16. Pressure (in Pa) contours for $\mathrm{Re}_{\mathrm{H}}=1240$, (a) Oval tube, (b) Circular tube.

\subsection{Oval Tubes and Winglets}

\subsubsection{Review of Previous Research}

Russell et al. ${ }^{40}$ probably were the first to examine special finned oval (flat) tube geometry with triangular and rectangular vortex generators (winglets). They showed that winglets with an angle of attack $\beta=20-30$ degrees and winglet height $h=H / 2$ (half of gap between two successive fins) are the most promising They reported that the ratio of the Colburn factor, $j$, to the apparent friction factor, $f$, is over 0.5 in the Reynolds number range of 500-2000 for a staggered tube bundle arrangement. Further experimental investigations, Torii et al., ${ }^{41,42}$ investigated the effect of one winglet pair at different angles of attack $(\beta=5,15,25,35,45$, and 60 degrees $)$ and winglet heights $(2,10,15$, and $25 \mathrm{~mm})$ in both toe-in (common flow up) and toe-out (common flow down) configurations. They observed that near the winglet the main heat transfer enhancement mechanism appeared to be the vortical motion of the main longitudinal vortices. At some location away from the winglet, transition to turbulence also contributes to the enhanced heat transfer. In the area far downstream, the longitudinal vortices increase the heat transfer rate by up to $140 \%$. The onset of the transition to turbulence mainly depends on the strength of the vortex, which is a function of (a) the ratio, winglet height/angle of attack, $\beta$, and (b) $\beta$.

Yanagihara and Torii ${ }^{43-46}$ also studied different winglet configurations (Figure 4-17) with different spacings, heights, and angles of attack that generate vortices rotating either in the same direction or in a direction counter to the neighboring vortices. They found that counter-rotating winglet pairs are better suited for heat transfer enhancement than are co-rotating winglet pairs for the same angles of attack. Their results also show higher heat transfer enhancement for larger angles of attack up to $\beta=45$ degrees, with heat transfer enhancement subsequently decreasing asis further increased from 45 to 90 degrees. They reported better heat transfer enhancement results with rectangular winglets than with delta winglets. They attributed this result to winglets with larger frontal areas producing stronger main and corner vortices, since they chose to base the comparison on winglet height remaining constant, versus constant 
frontal area. However, they did not measure flow losses associated with the delta and rectangular winglets of different frontal areas.

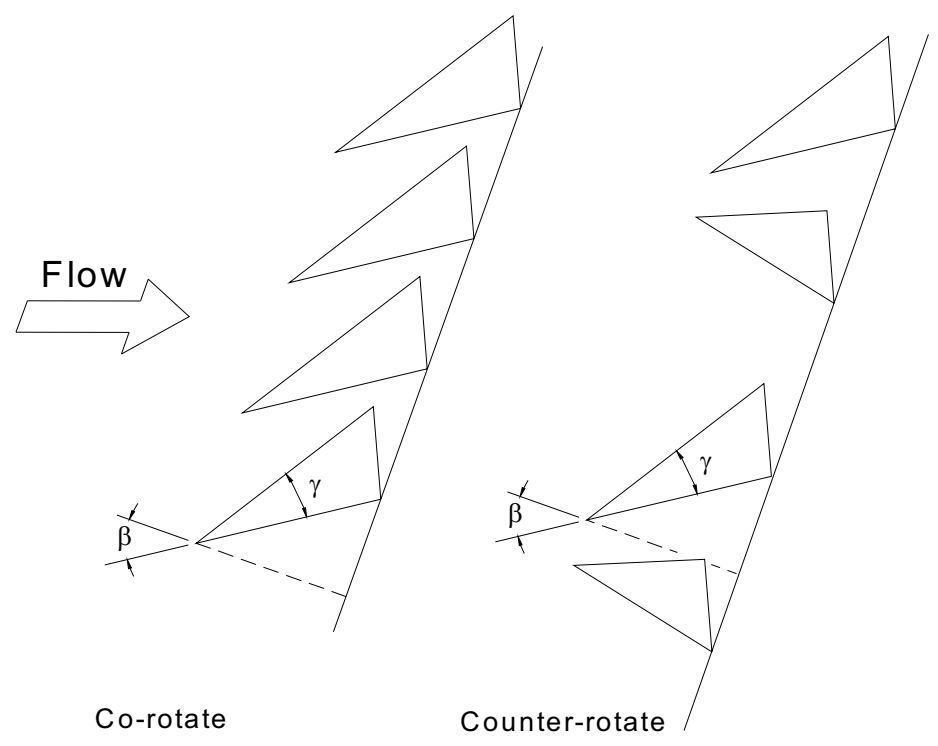

Figure 4-17. Vortex generator configurations generating co-rotating and counter-rotating vortices.

Fiebig et al. ${ }^{47}$ performed an experimental parametric study of different vortex generator shapes and angles of attacks at various Reynolds numbers for a single pair of vortex generators. Their results indicate local heat transfer enhancement of several hundred percent and mean heat transfer enhancement of more than $50 \%$ over a surface area of more than 50 times the winglet surface area. Tiggelbeck et al. ${ }^{48}$ examined two rows of delta winglet pairs in both aligned and staggered orientation. Their results indicate a slightly higher heat transfer enhancement for the aligned configuration than for the staggered configuration. They reported heat transfer enhancement of $\sim 80 \%$ and increased drag of $\sim 160 \%$. Tiggelbeck ${ }^{49}$ and Güntermann ${ }^{50}$ investigated experimentally and numerically the influence of aspect ratio $(\Lambda)$ of the winglets and the angle of attack on the performance of the plate heat exchangers. They found that the best ratio of heat transfer enhancement and frictional pressure increase for a delta winglet pair $(\Lambda=2, h=H / 2)$, was obtained for $\beta=45$ degrees for a developing flow in a duct with $\operatorname{Re}_{H}=2300$, while $\beta=15$ degrees produced the best result for a very compact arrangement of rectangular winglet pairs $(\Lambda=$ $0.5, \mathrm{~h}=\mathrm{H} / 2$ ) in a periodically fully developed flow with $\mathrm{Re}_{\mathrm{H}}=1000$. They concluded that a winglet with $\Lambda=1.5$ generates maximum heat transfer enhancement, while a winglet with $\Lambda=2.0$ produces the best heat transfer enhancement and frictional pressure increase ratio. Valencia et al. ${ }^{51}$ showed that heat transfer enhancement by triangular winglet $(\beta=45$ degrees, $\Lambda=2.0, \mathrm{~h}=\mathrm{H})$ in finned flat (oval) tubes is more pronounced than in circular tubes, and that triangular winglets should be located on the upstream side of the tube, with a distance of two tube widths between the tips of the winglets. Chen et al. ${ }^{10}$ performed a numerical investigation for a finned flat (oval) tube with triangular winglet pairs and studied the influences of $\Lambda, \beta$, and winglet location at $\mathrm{Re}_{\mathrm{H}}=300$ in a toe-in (common flow up) configuration. They investigated three angles of attack $(\beta=20,30$, and 45 degrees) and two aspect ratios $(\Lambda=1.5$ and 2$)$ and found the combination of $\beta=30$ degrees and $\Lambda=2$ to provide the best performance ratio with $\left(\mathrm{j} / \mathrm{j}_{\mathrm{O}}\right)$ / $\left(\mathrm{f} / \mathrm{f}_{\mathrm{O}}\right)=1.04$. Their results also indicate that by increasing the winglets from 1 to 2 to 3 , reduces the performance ratio, indicating that multiple winglets for a single finned tube is not justified.

Biswas and Chattopadhyay ${ }^{52}$ performed a numerical parametric analysis of a triangular winglet pair, including the effects of the hole on the fin surface. They found that for a long channel at $\operatorname{Re}_{\mathrm{H}}=500$ with a winglet at an angle of attack of 26 degrees and no hole adjacent to it, the average Nusselt number increased by $30 \%$, while the friction factor increased by $79 \%$ over the corresponding values for plain 
channel geometry. For other identical conditions but with a hole adjacent to the winglet, the average Nusselt number increased by $10 \%$, and the friction factor increased by $48 \%$ over the plain channel geometry. They attributed this to a downward normal flow being induced near the wing, which reduces the strength of the longitudinal vortices generated by the delta winglet when the presence of the hole is included. Prabhakar et al. ${ }^{53}$ also performed a numerical investigation of winglets on a finned oval tube. They started with the configuration recommended by Chen et al. ${ }^{10}$ of $\beta=30^{\circ}$ and $\Lambda=2$ in a commonflow-up configuration and extended the analysis to a range of $\mathrm{Re}_{\mathrm{H}}$ from 560 to 3500, looking at HTE only for $\beta=30,35$, and 45 degrees. They reported maximum heat transfer enhancement at $\beta=45$ degrees. They also looked at rectangular winglets in a common-flow-up configuration with $\beta=30$ degrees and reported better heat transfer enhancement with a triangular winglet configuration.

Recently, Torii et al.$^{54}$ proposed that the winglets could be oriented such that they can enhance heat transfer and at the same time decrease pressure loss compared to a plain tube for both staggered and inline circular tube banks. They theorized that this new configuration creates constricted passages in the aft region of the tube, which accelerates the air in the constricted passages, and as a consequence the point of separation shifts downstream. Narrowing of the wake region and suppression of vortex shedding are perceived outcomes of such a configuration, which reduce form drag. Since the air is accelerated in the passage, the zone of poor heat transfer on the fin surface is also removed from the near-wake of the tube. They further theorized that in a low-Reynolds-number flow in the absence of any winglets, the poor heat transfer zone is created widely on the fin surface in the near-wake of the tube and may extend far downstream, even to the next row of the tube bank. Hence, they expected and experimentally verified that their proposed configuration would be most effective for low-Reynolds-number flows. Another advantage they cited for their proposed configuration was that only one winglet pair for the first row of tubes was required for their configuration, versus three rows of winglets for the configuration proposed by Fiebig et al. ${ }^{19}$ Jain et al. ${ }^{55}$ performed a numerical investigation of the same winglet configuration suggested by Torii et al. ${ }^{54}$ for a single circular tube. They reported significant heat transfer enhancement for this configuration, which persists for long distances downstream, consistent with the experimental results obtained by Torii et al. for circular tubes. However, if this orientation of winglets could also be used effectively on oval tubes, significant performance advantages could be obtained.

\subsubsection{Winglet Configurations for a Single Oval Finned Tube}

Four selected winglet configurations were examined for application in a single-finned oval tube with an aspect ratio of 3:1. The selected configurations tested are as shown in Figure 4-18 with dimensions given in Table 4-1. These configurations were chosen based on a review of the available literature as the most promising configurations to produce the optimal performance. Since the bulk of the literature work is for circular tubes, or in the case of Configuration 4, a flat tube, this work modifies the chosen configurations for the low ratio oval tube used in this analysis. The goal of this analysis is to determine the best winglet configuration for a single oval tube.

The first configuration is shown on the left (a) of Figure 53 and is based on a configuration suggested by Fiebig et al. ${ }^{19}$ for a circular tube. A toe-out (common flow down) orientation of the winglet pair placed in the wake region was used. The second and third configurations are those suggested by Torii et al., ${ }^{54}$ adapted for a 3:1 aspect ratio oval tube. In these configurations, the winglets are oriented in a Toe-in (common flow-up) configuration, as shown in Figure 53b and c. Two angles of attack were investigated, $\beta=150$ degrees (or 30 degrees) for Configuration 2 and $\beta=165$ degrees (or 15 degrees) for Configuration 3. Torii et al. ${ }^{54}$ positioned the trailing edge of the winglets at $\beta=110$ degrees (or 70 degrees), such that the location of accelerating flow coincides with the point where boundary layer separation begins. This delays flow separation, reduces the size of the wake region, and suppresses vortex shedding, which reduces the form drag. Since location of flow separation for an oval tube is at a point downstream than that for a circular tube, the trailing edge of the winglets were located at $\beta=120$ degrees close to the point of flow separation. 

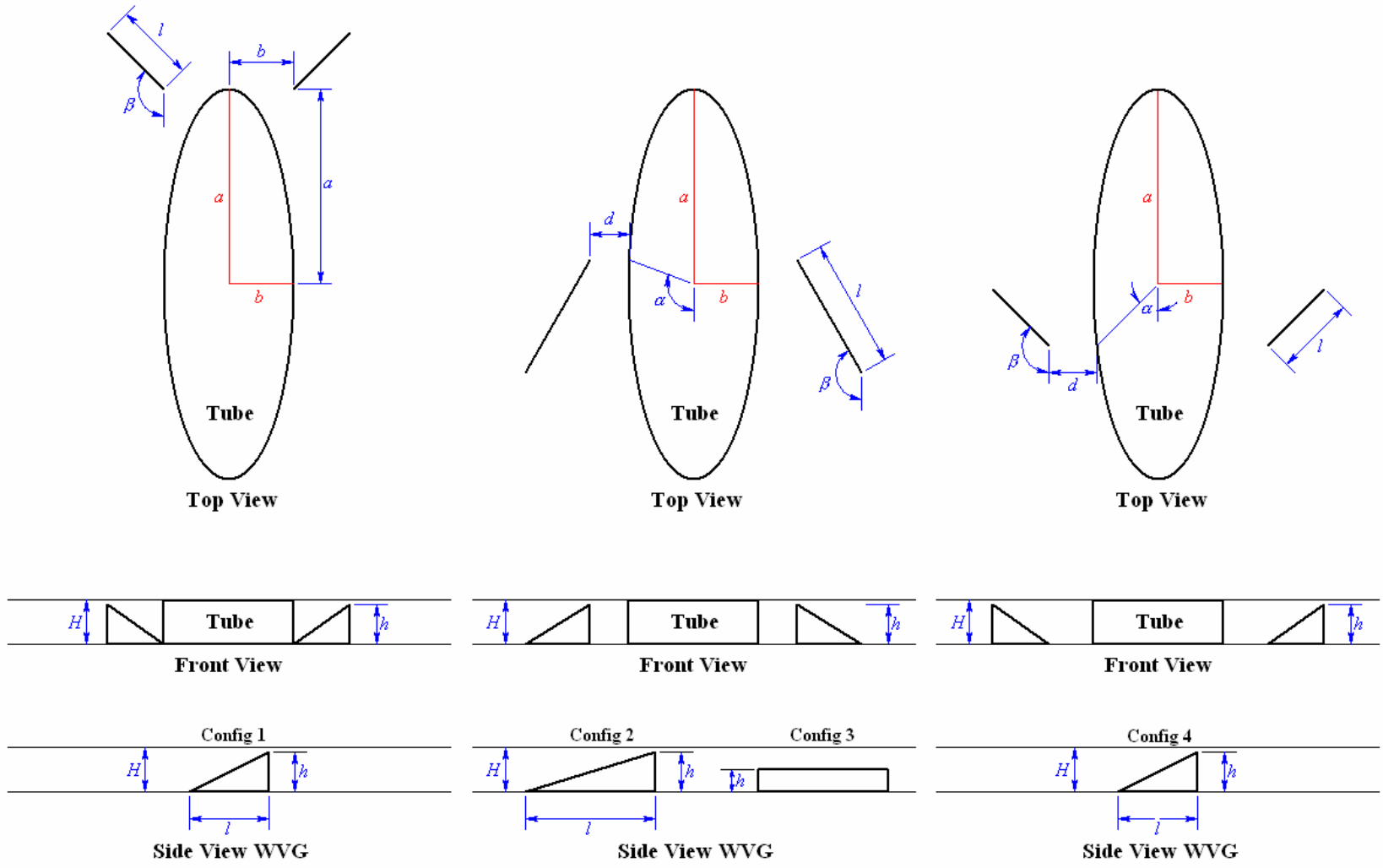

(a)

(b)

(c)

(d)

Figure 4-18. Finned oval tube and winglet configurations tested.

Table 4-1. Summary of finned oval tube and winglet configurations tested.

\begin{tabular}{cllll}
\hline Variables & Configuration 1 & Configuration 2 & Configuration 3 & Configuration 4 \\
\hline $\begin{array}{c}\text { Tube aspect ratio } \\
\text { (a:b) }\end{array}$ & $3: 1$ & $3: 1$ & $3: 1$ & $3: 1$ \\
$\beta$ & 135 degrees & 150 degrees & 165 degrees & 135 degrees \\
$\alpha$ & N/A & 120 degrees & 120 degrees & 45 degrees \\
1 & $2 \mathrm{~h}$ & $2 \mathrm{~b}$ & $2 \mathrm{~b}$ & $2 \mathrm{~h}$ \\
$\mathrm{~d}$ & $\mathrm{~B}$ & $0.3(2 \mathrm{~b})$ & $0.3(2 \mathrm{~b})$ & $0.37(2 \mathrm{~b})$ \\
$\mathrm{h}$ & $0.9 \mathrm{H}$ & $0.9 \mathrm{H}$ & $0.5 \mathrm{H}(\mathrm{rect})$ & $0.9 \mathrm{H}$ \\
\hline
\end{tabular}

For the case of $\beta=165$ degrees, the triangular winglet was changed to a rectangular winglet with half of the fin gap height to keep the frontal surface area and the aspect ratio of the winglet the same for the rectangular and triangular winglets, consistent with the approach suggested by Fiebig et al. ${ }^{19}$ However, since the frontal surface area and aspect ratio were maintained constant, it is expected that changes in heat transfer enhancement and frictional pressure drop increase associated with the change form triangular to rectangular winglet will be minor, based on the comparison of results for triangular/rectangular winglets given by Fiebig et al. ${ }^{19}$ In Configuration 4, the winglets are placed in the forward region of the tube in a toe-out (common flow down) orientation, shown in Figure 4-18d. This arrangement was suggested by Chen et al., ${ }^{10}$ modified for the lower aspect ratio oval tube. 


\subsubsection{Performance Ratio for Various Winglet Configurations on Finned Oval Tube}

The four configurations were analyzed over a Reynolds number range of 500-1240. The heat transfer and friction factor ratios representing performance are presented in Figure 4-19. Configurations 1 and 3 produce favorable $(\mathrm{j} / \mathrm{j} 0) /(\mathrm{f} / \mathrm{f} 0)$, whereas Configurations 2 and 4 produce unfavorable results. Configuration 3 yielded the best performance of the four configurations tested. Over the range of $\mathrm{ReH}$ values tested, Configuration 3 exhibited $\sim 17 \%$ heat transfer enhancement, which was about average for all the configurations. However, the frictional pressure loss increase for this configuration was very low, only $3.3 \%$ over the range of $\mathrm{ReH}$ tested, with a decease in pressure drop for the case, $\mathrm{ReH}=1240$.

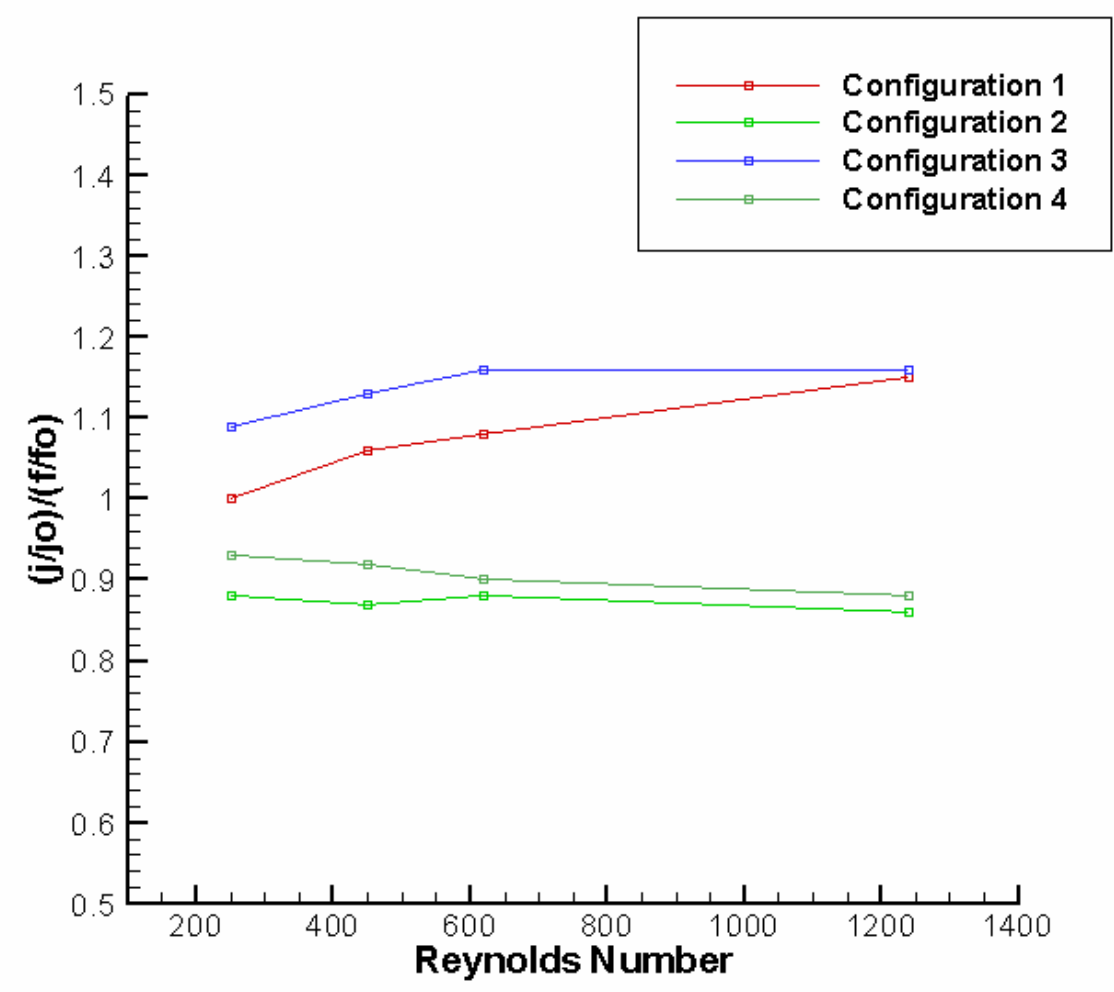

Figure 4-19. Performance comparison for four winglet pairs on four finned oval tubes.

This resulted in favorable $(\mathrm{j} / \mathrm{j} 0) /(\mathrm{f} / \mathrm{f} 0)$ ratios of $1.09,1.13,1.16$, and 1.16 for $\mathrm{ReH}=250,450,620$, and 1240, respectively. This is the only configuration that produced a favorable $(\mathrm{j} / \mathrm{j} 0) /(\mathrm{f} / \mathrm{f} 0)$ for all $\mathrm{ReH}$ values tested. Configuration 3 is based on the suggestions by Torii et al. ${ }^{54}$ for in-line and staggered circular-tube banks, as adapted for oval tubes. Torii et al. ${ }^{54}$ positioned the trailing edge of the winglets 110 degrees from the leading point of the circular tube, which was modified for this study to 120 degrees to account for the delayed flow separation from an oval tube. The second best configuration, based on the numerical results, was Configuration 1. This configuration averaged an $8.5 \%$ heat transfer enhancement and a $1.6 \%$ frictional pressure loss over the range of $\mathrm{ReH}$ values evaluated. This corresponds to a $(\mathrm{j} / \mathrm{j} 0) /(\mathrm{f} / \mathrm{f} 0)$ ratio of $1.0,1.06,1.08$, and 1.15 for $\mathrm{ReH}$ values of $250,450,620$, and 1240 , respectively. These results indicate that locating the winglets in the wake region has the lowest overall impact on the flow field. Configuration 4 produced good results for heat transfer enhancement, averaging $19.8 \%$ over the range of $\mathrm{ReH}$ tested. However, pressure loss for this configuration was high, averaging $31.0 \%$ over the same $\mathrm{ReH}$ range. These results correspond to a $(\mathrm{j} / \mathrm{j} 0) /(\mathrm{f} / \mathrm{f} 0)$ ratio in the range of $0.88-0.93$ for $\mathrm{ReH}$ in the 250-1240 range. These data suggest that this configuration significantly disrupts the flow field. But high heat transfer enhancement also leads to high pressure loss and results in unfavorable $(\mathrm{j} / \mathrm{j} 0) /(\mathrm{f} / \mathrm{f} 0)$ ratio. Configuration 2 produced the best results for heat transfer enhancement, averaging $27.3 \%$ over the 
range of ReH tested. However, pressure loss for this configuration was very high, averaging $45.7 \%$ over the range of $\mathrm{ReH}$ tested. This corresponds to an unfavorable $(\mathrm{j} / \mathrm{j} 0) /(\mathrm{f} / \mathrm{f} 0)$ ratio of $\sim 0.88$ for $\mathrm{ReH}$ tested. Configuration 2 was designed to test how critical $\beta$ is in the configuration suggested by Torii et al. The results indicate that this configuration is very $\beta$ sensitive, and that the best performance $\mathrm{ratio},(\mathrm{j} / \mathrm{j} 0) /(\mathrm{f} / \mathrm{f0})$, is obtained with a $\beta$ of 165 degrees (or 15 degrees).

\subsection{Fluid Flow Pattern and Heat Transfer Contours}

Although performance ratio, $(\mathrm{j} / \mathrm{j} 0) /(\mathrm{f} / \mathrm{f} 0)$, comparison results are very useful in determining the optimal single oval tube winglet configuration, these results do not provide much insight into why a configuration performed as it did. Detailed analysis of the coupled behavior of fluid flow and heat transfer pattern may provide significant insight into the performance results and be useful in determining optimal design.

Figure 4-20 presents surface heat transfer and flow velocity magnitude plots for the four tested configurations at $\mathrm{ReH}=450$. The solid blue lines evident in the surface heat transfer coefficient plots are an artifact of the meshing and the postprocessing software used and should be ignored. Figure 4-20 clearly indicates that the placement and orientation of the winglets can have significant impacts on the primary and secondary flow. The winglet placement in Configurations 1 and 3 (Figures 4-21 and 4-23) appear to have only secondary effects on the flow field and heat transfer when compared to the corresponding parameters for an oval tube without winglets. These figures also show that these configurations do not impact the primary flow, but only the secondary flow. However, for both of these configurations, especially for Configuration 1 (Figure 4-21), the flow separation from the tube appears to be delayed, resulting in lower pressure loss. In contrast to Configurations 1 and 3, Configurations 2 and 4 (Figures 4-22 and 4-24) appear to have both primary and secondary affects on flow pattern and heat transfer. Both Configurations 2 and 4 accelerate the flow in the area immediately downstream of the winglets, resulting in correspondingly higher fin surface heat transfer coefficients in this region. Also, in Configurations 2 and 4, the lower flow velocity near the tube wall results in lower fin surface heat transfer coefficients in this area. 


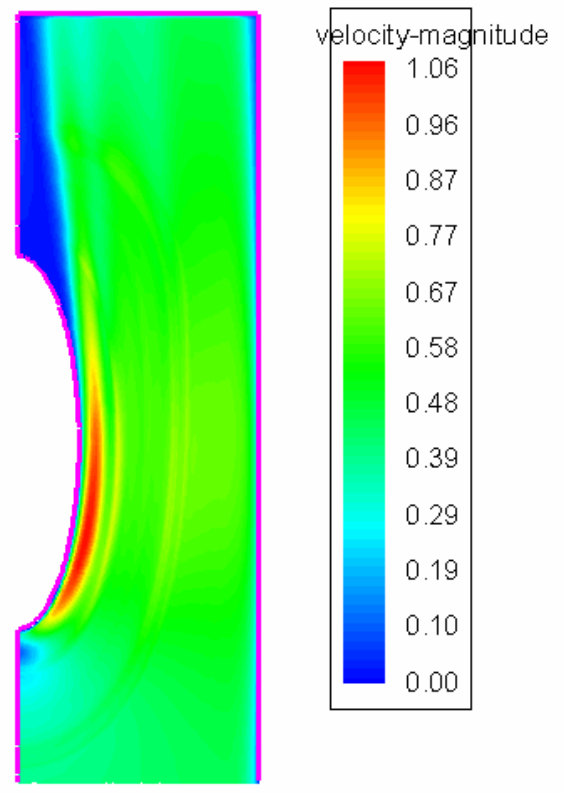

(a)

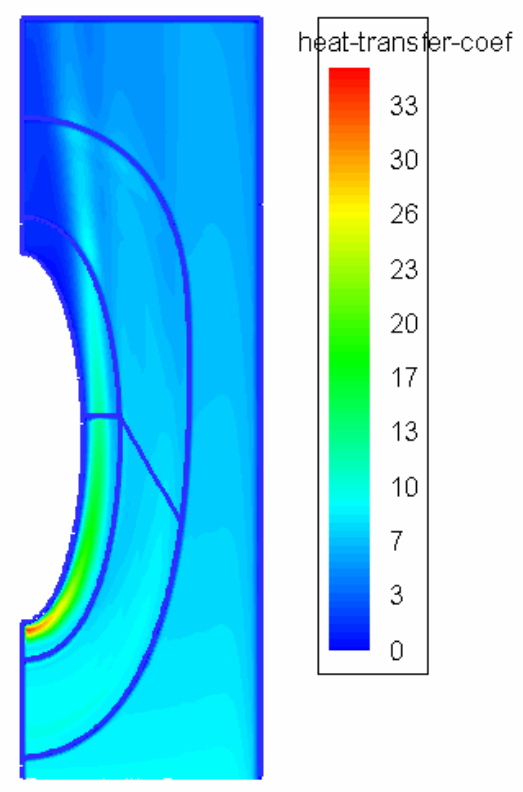

(b)

Figure 4-20. A baseline case without winglets: (a) velocity $(\mathrm{m} / \mathrm{s})$ pattern, (b) surface heat transfer coefficient $\left(\mathrm{W} / \mathrm{m}^{2} \cdot \mathrm{K}\right)$ distribution.
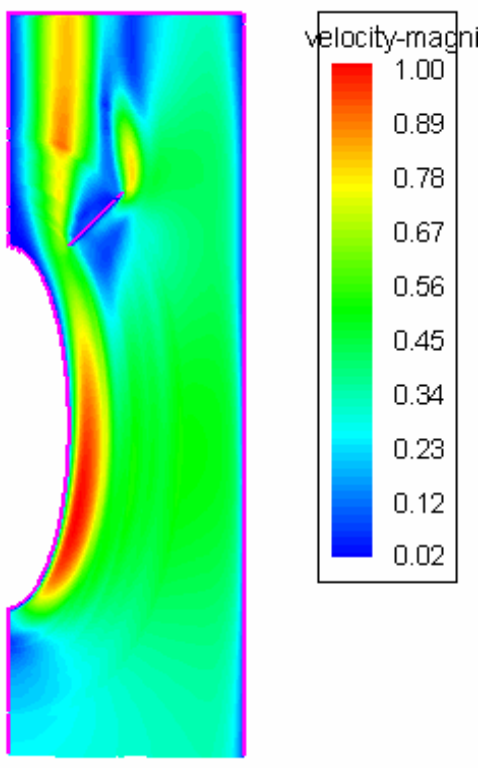

(a)
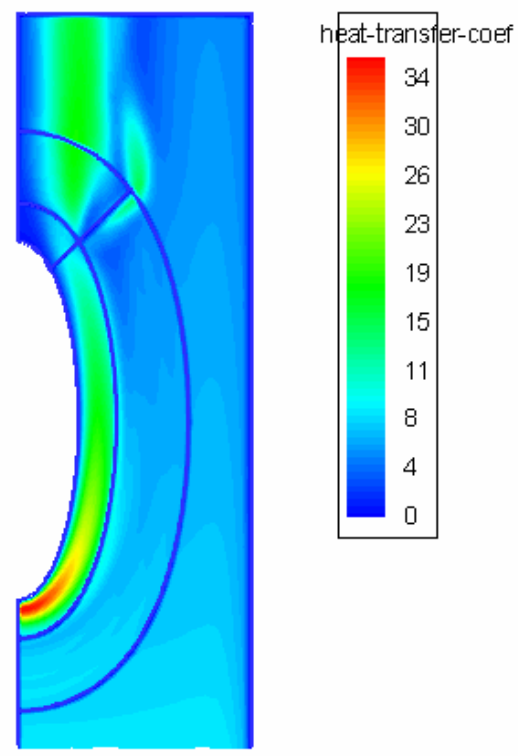

(b)

Figure 4-21. Configuration 1: (a) velocity $(\mathrm{m} / \mathrm{s})$ contours, (b) surface heat transfer coefficient $\left(\mathrm{W} / \mathrm{m}^{2} \cdot \mathrm{K}\right)$ distribution. 


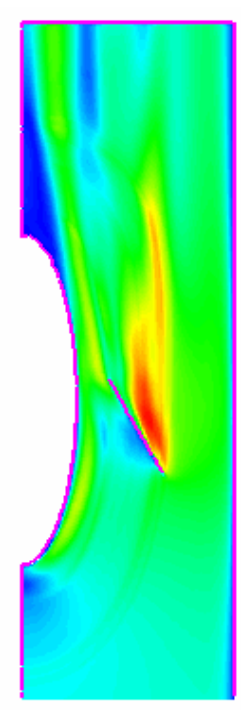

(a)

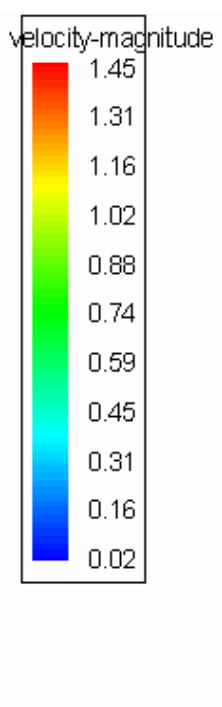

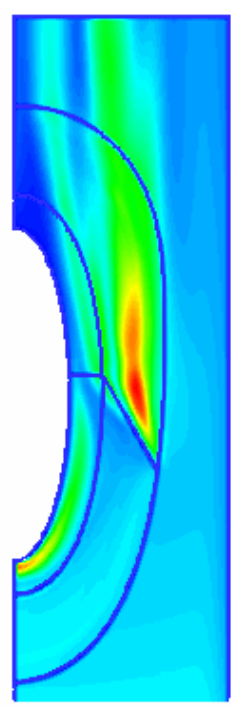

neat-transfer-coef

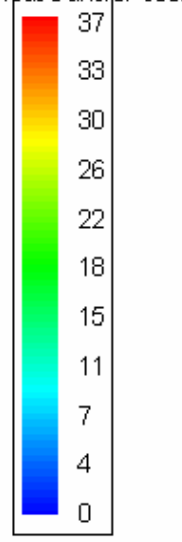

(b)

Figure 4-22. Configuration 2: (a) velocity $(\mathrm{m} / \mathrm{s})$ contours, (b) surface heat transfer coefficient $\left(\mathrm{W} / \mathrm{m}^{2} \cdot \mathrm{K}\right)$ distribution.

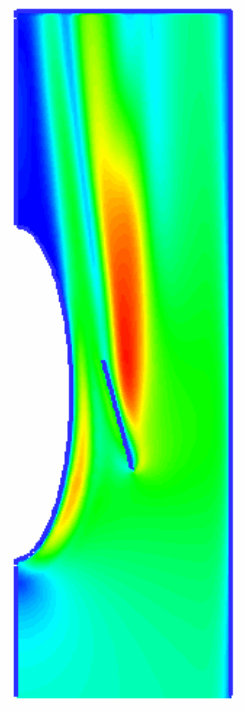

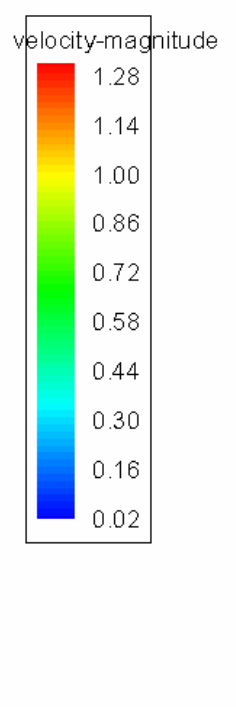

(a)
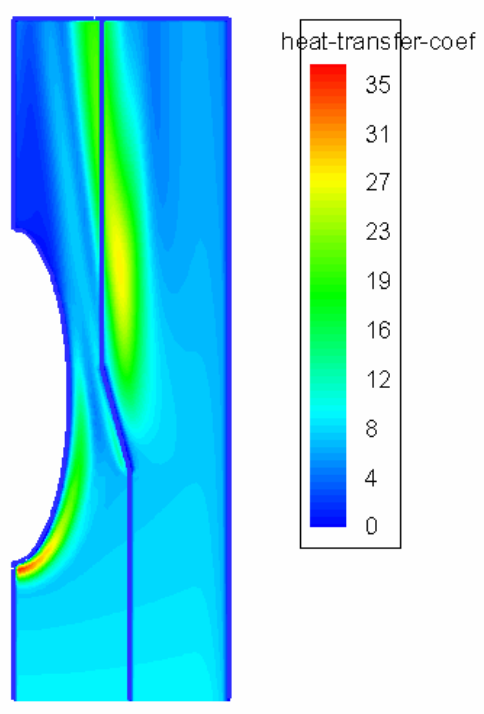

(b)

Figure 4-23. Configuration 3: (a) velocity $(\mathrm{m} / \mathrm{s})$ contours, (b) surface heat transfer coefficient $\left(\mathrm{W} / \mathrm{m}^{2} \cdot \mathrm{K}\right)$ distribution. 


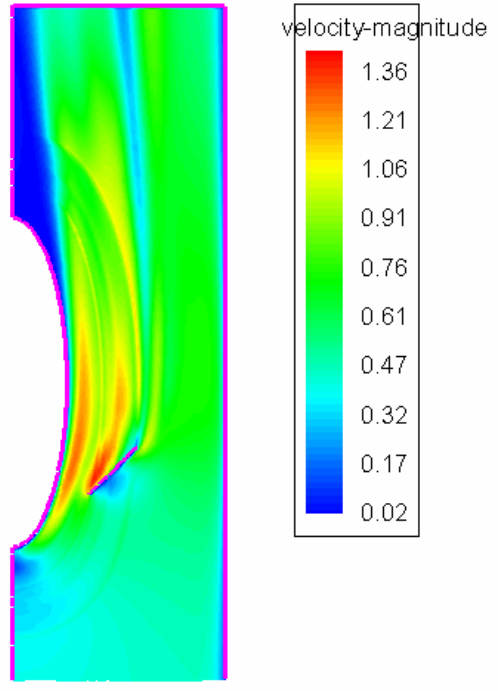

(a)

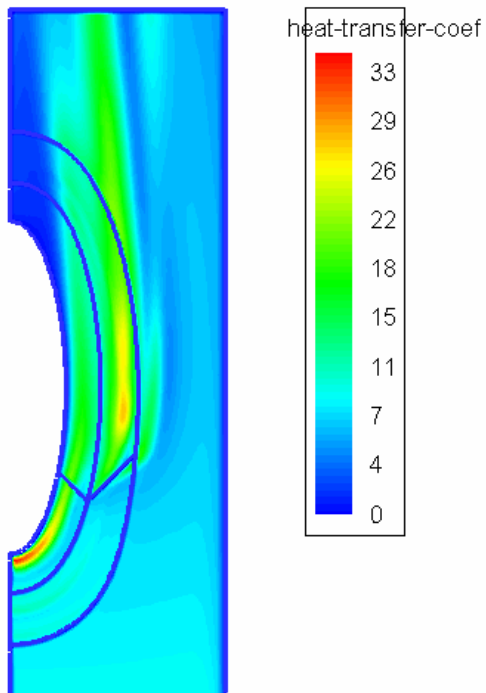

(b)

Figure 4-24. Configuration 4: (a) velocity ( $\mathrm{m} / \mathrm{s})$ contours, (b) surface heat transfer coefficient $\left(\mathrm{W} / \mathrm{m}^{2} \cdot \mathrm{K}\right)$ distribution.

This observation offers insight into why Configurations 2 and 4 have higher heat transfer enhancement and also higher pressure drop. Configuration 2 has an average heat transfer enhancement of $26.4 \%$ better than a tube without winglets, whereas for Configuration 4 , it is $19.8 \%$ higher over the range of ReH tested. Correspondingly, pressure loss for Configuration 2 is $44 \%$ higher than a tube without winglets, and for Configuration 4 it is $31 \%$ higher. This seems to indicate that although altering the primary flow patterns is good for heat transfer enhancement, it results in disproportionately higher pressure loss and an unfavorable performance ratio $(\mathrm{j} / \mathrm{j} 0) /(\mathrm{f} / \mathrm{f} 0)$, as shown in Figure $4-19$.

With winglets, flow behavior depends on winglet location and orientation, $\beta$. The location of the winglets relative to the tube is the same for Configurations 2 and 3; however, $\beta$ in Configuration 2 is 150 degrees, whereas it is 165 degrees for Configuration 3. Higher $\beta$ for Configuration 3 also results in primary vortices of higher strength and correspondingly higher fin surface heat transfer in the area immediately downstream of the winglets. However, in this configuration the impact on the primary flow patterns is relatively minor. Flow patterns and velocities for Configuration 2 in the immediate vicinity of the tube are very similar to flow patterns and velocities for the oval tube without winglets. Since the heat transfer enhancement and flow pattern-altering effects are only secondary, both the heat transfer enhancement (18\% over the range of $\mathrm{ReH}$ tested) and frictional pressure drop increase $(5 \%)$ are lower than the corresponding values for Configurations 2 and 4 . However, in this configuration the pressure drop increase is disproportionately lower than the heat transfer enhancement, resulting in a favorable $(\mathrm{j} / \mathrm{j} 0) /(\mathrm{f} / \mathrm{f0})$ ratio, as shown in Figure 4-19.

Figures 4-25 through 4-28 depict vector velocity plots in a plane immediately downstream of the winglets and in two planes further downstream ( $>4$ winglet lengths for the four tested configurations). The figures have been rotated as indicated by the axes orientation for illustration purposes. The oval tube surface and winglet are indicated in yellow. 


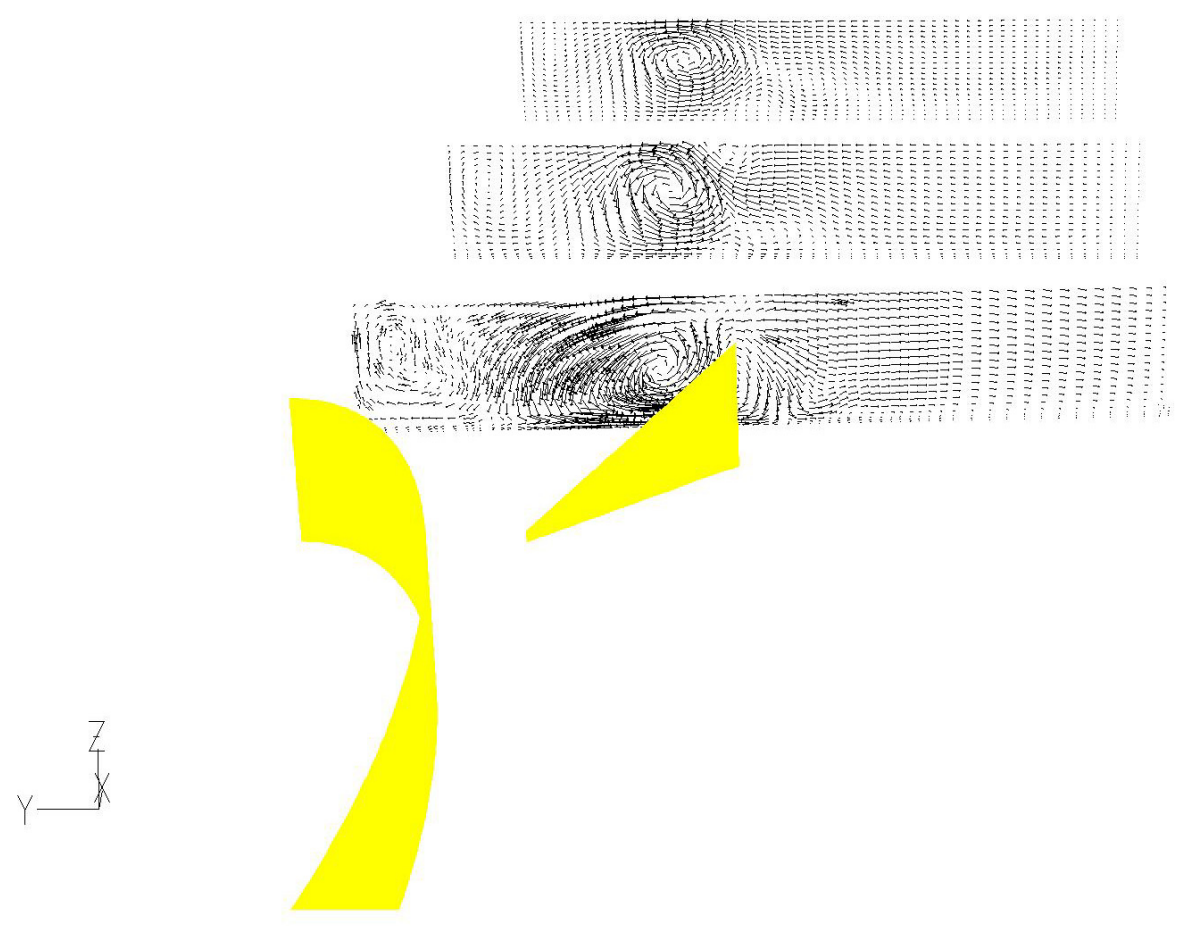

Figure 4-25. Velocity vectors for winglet Configuration 1.

Figure 4-25 reveals the nature of the vortex pattern created by the winglets in this configuration. In the plane immediately downstream of the winglet, the main longitudinal vortex is evident, as well as the corner and induced vortices, consistent with the winglet vortices flow patterns. The main vortex is by far the strongest of the three vortices and results in the most significant heat transfer enhancement of the three vortices. In the planes further downstream, only the main longitudinal vortex is evident, indicating the weaker corner, and induced vortices have dissipated due to viscous effects at these distances downstream. The downstream dissipation of the corner and induced vortices is also evident in Figure 4-21b, the surface heat transfer coefficient contour plot. Configuration 2 produces a stronger main vortex than Configuration 1, as can be evidenced by the higher heat transfer enhancement immediately downstream of the winglet, as indicated in Figure 4-22b. Figure 4-27 of Configuration 3 does not indicate the presence of corner and induced vortices, most likely due to the rectangular winglet used in this configuration. Configurations 2, 3, and 4 produce stronger main vortices compared to those produced by Configuration 1, most likely due to the winglets being in an area of higher flow in these configurations. In all four configurations, only the main vortex persists long distances downstream. 


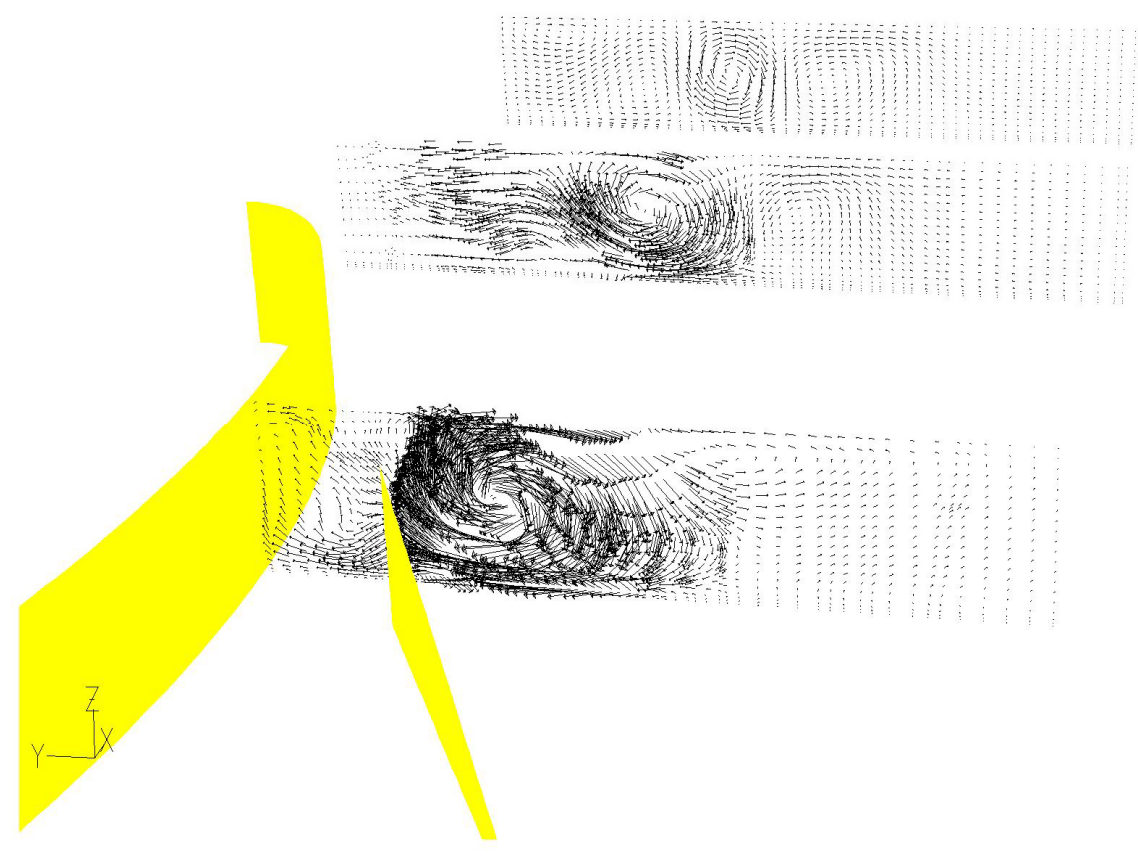

Figure 4-26. Velocity vectors for winglet Configuration 2.

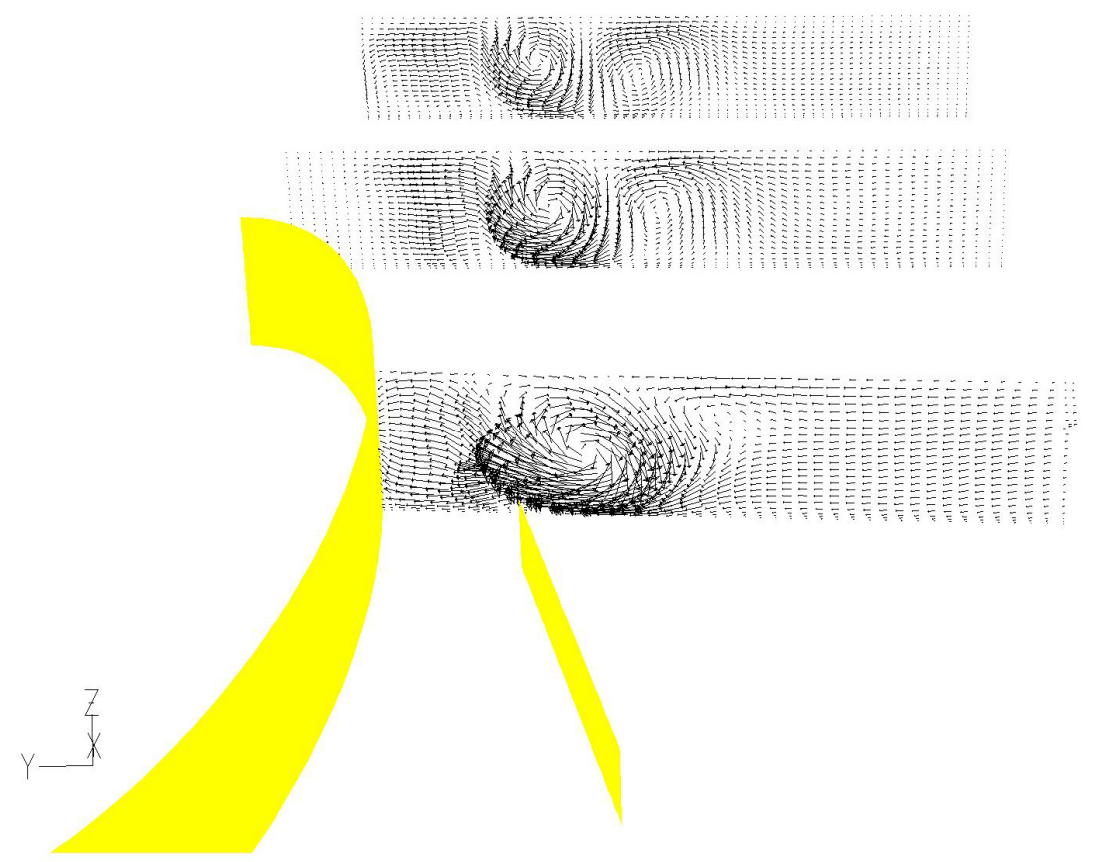

Figure 4-27. Velocity vectors for winglet Configuration 3. 


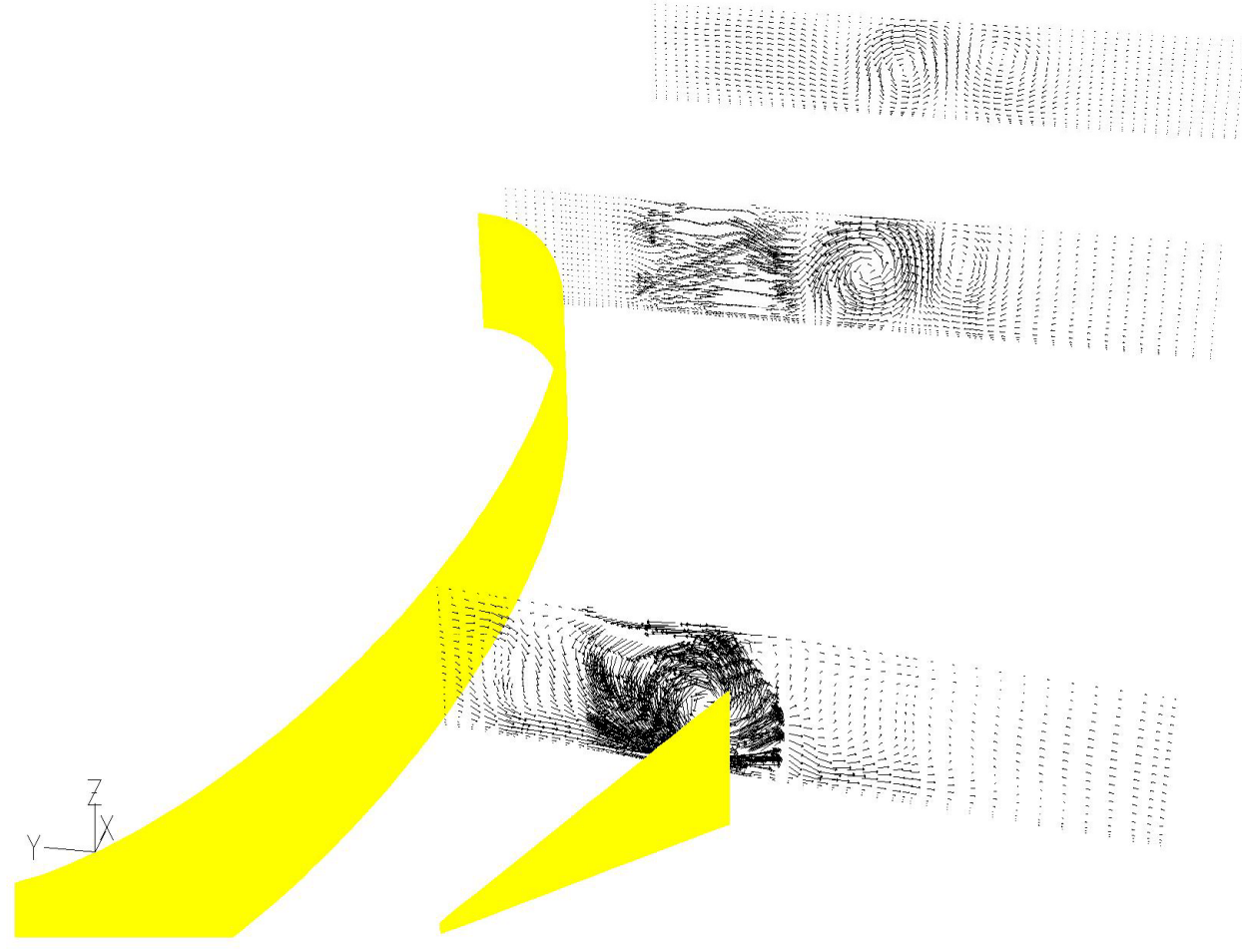

Figure 4-28. Velocity vectors for winglet Configuration 4

Although an exhaustive study on all possible winglet placements and orientations is not feasible or necessary, data from the tested configurations strongly suggest that the winglet effect on heat transfer and the flow field can be secondary, or primary and secondary. For the tested configurations, winglet placements and orientations that have primary as well as secondary influences on heat transfer and the flow field result in significant heat transfer enhancement, but at a disproportionately higher pressure drop increase, resulting in an unfavorable $(\mathrm{j} / \mathrm{j} 0) /(\mathrm{f} / \mathrm{f} 0)$ ratio. Configurations 2 and 4 fall into this category. Conversely, the winglet placements and orientations tested that have only a secondary influence on the flow field result in a lower heat transfer enhancement, but with a disproportionately lower pressure drop increase, yielding a favorable $(\mathrm{j} / \mathrm{j} 0) /(\mathrm{f} / \mathrm{f} 0)$ ratio. Configurations 1 and 3 fall into this category.

This analysis shows that for an optimal $(\mathrm{j} / \mathrm{j} 0) /(\mathrm{f} / \mathrm{f} 0)$ performance ratio for single tubes, winglet configurations should be selected that produce only secondary effects on heat transfer and flow patterns. This knowledge of the effects of different winglet configurations on flow and heat transfer patterns is very useful in predicting the expected $(\mathrm{j} / \mathrm{j} 0) /(\mathrm{f} / \mathrm{f} 0)$ ratio for different winglet configurations.

\subsection{Tube Bundle Analysis}

\subsubsection{Tube Bundle Computational Model}

The single-oval-tube analysis shows that Configuration 3 proposed by Torii et al. ${ }^{54}$ for a circular tube bundle and modified by this work for low axis ratio oval tubes produces the best $(\mathrm{j} / \mathrm{j} 0) /(\mathrm{f} / \mathrm{f} 0)$ results of the four tested configurations. This section reports on this oval tube with winglet configurations examined for both in-line and staggered oval tube bundles with one, two, and three rows of winglets. The in-line and staggered tube bundle configurations are shown in Figure 4-29. An oval tube with winglets of Configuration 3 design is shown in Figure 4-18c. Table 4-2 provides appropriate dimensions of the tube bundle arrangements. Again, as in the single-tube analysis, the conjugate heat transfer problem is solved 
numerically with the theoretical formulation and boundary conditions being the same as specified in earlier sections for a single tube analysis.

Table 4-2. Dimensions for in-line and staggered tube bundle configurations.

\begin{tabular}{cc} 
Variables & $\begin{array}{l}\text { Variable Values } \\
\text { for Both In-line } \\
\text { and Staggered } \\
\text { Tube Bundles }\end{array}$ \\
\hline I (= J ) & $13.4 \mathrm{H}$ \\
J (= I ) & $13.4 \mathrm{H}$ \\
W (= 2 J ) & $26.8 \mathrm{H}$ \\
L (= 4 J ) & $53.6 \mathrm{H}$ \\
\hline
\end{tabular}

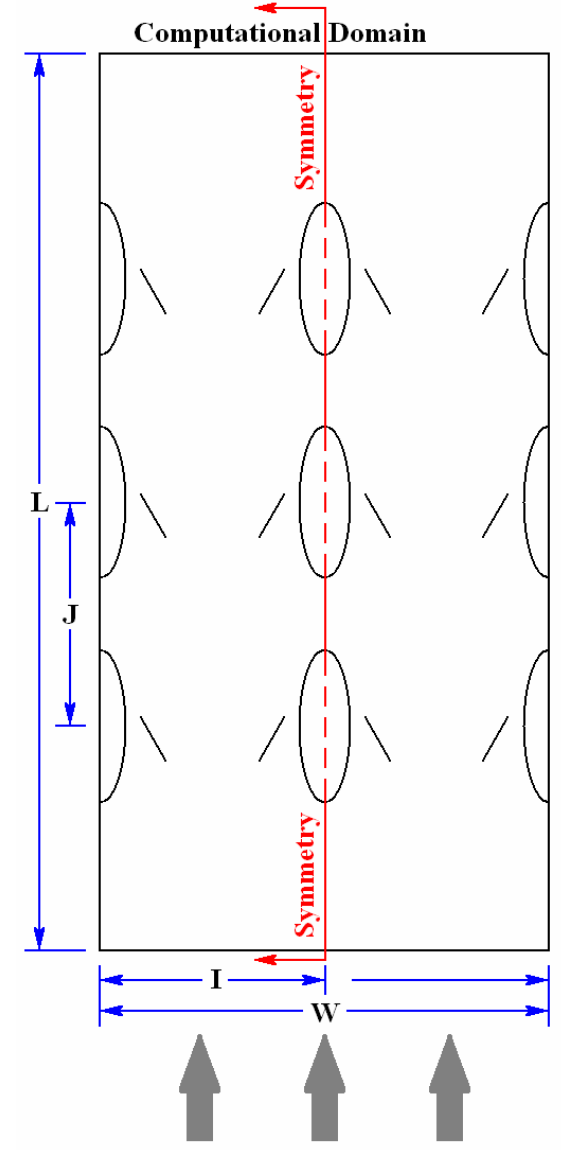

(a)

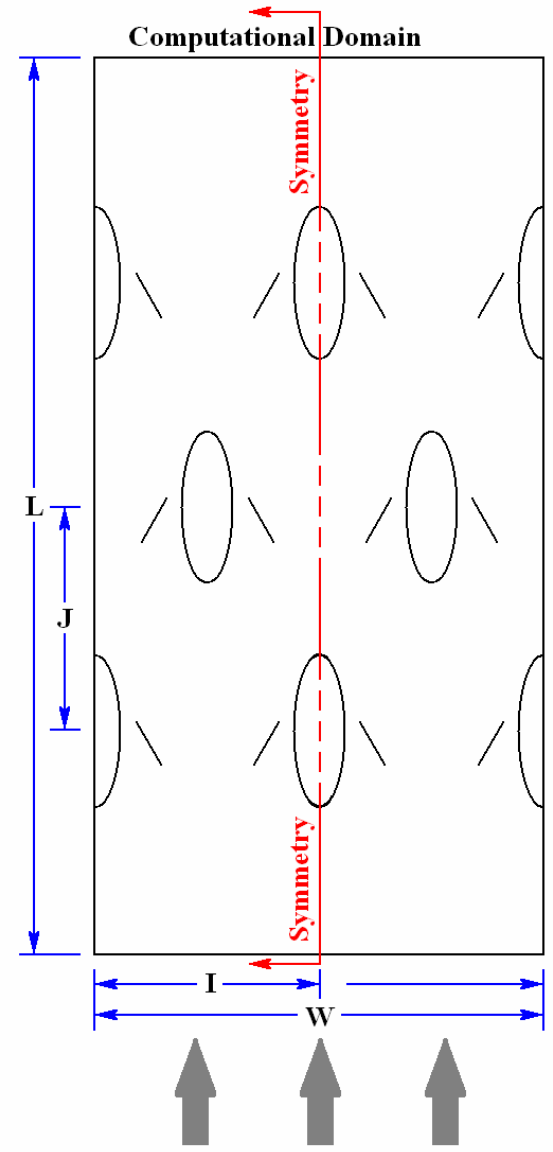

(b)

Figure 4-29. Tube bundle configurations: (a) in-line configuration, (b) staggered configuration.

\subsubsection{Tube Bundle Performance Results}

Figure 4-30 presents the results for the performance ratio, $\left(\mathrm{j} / \mathrm{j}_{0}\right) /\left(\mathrm{f} / \mathrm{f}_{0}\right)$, results for the in-line tube bundle arrangement. Here, note that the reference Colburn $j$-factor, $j_{o}$ and friction factor, $f_{o}$, values refer to the in-line tube bundle base case without winglets. 


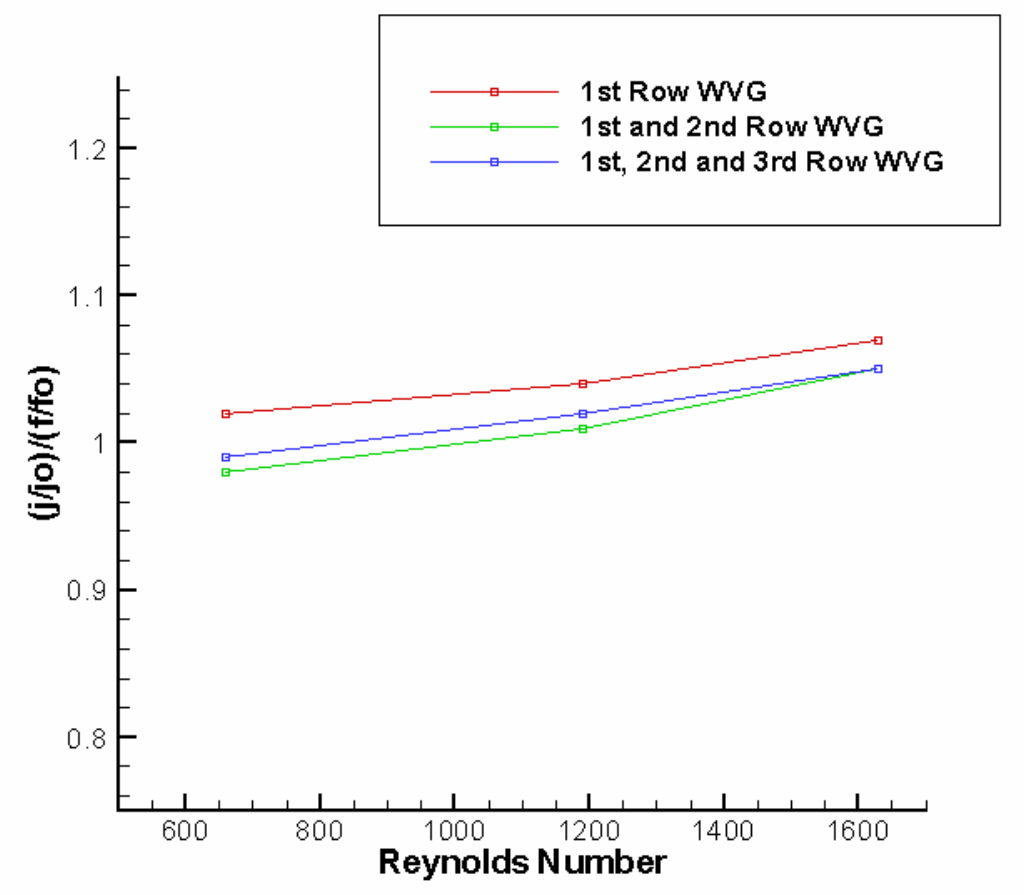

Figure 4-30. Performance ratio, $\left(\mathrm{j} / \mathrm{j}_{0}\right) /\left(\mathrm{f} / \mathrm{f}_{0}\right)$, results for in-line oval tube bundles with winglets in 1, 1-2, and 1-2-3 rows.

The in-line configuration with only one row of winglets on the first row of tubes yielded the best performance ratio, $(\mathrm{j} / \mathrm{j} 0) /(\mathrm{f} / \mathrm{f} 0)$, results of $1.02,1.04$, and 1.07 for $\mathrm{ReH}$ of 660,1190 , and 1630 , respectively. With two rows of winglets, the heat transfer enhancement values increase to 18.5, 23.6, and $24.6 \%$ for $\mathrm{ReH}$ of 660,1190 , and 1630 , respectively. With three rows of winglets, heat transfer enhancement values of 21.4, 26.2, and $26.1 \%$ were obtained for ReH of 660, 1190 and 1630, respectively. However, frictional pressure drop increased at a proportionately higher rate with additional winglet rows. Frictional pressure drop increased by $18.7,21.9$, and $18.8 \%$ for two rows of winglets and by $22.7,24.2$, and $19.8 \%$ for three rows of winglets for $\mathrm{ReH}$ equal to 660,1190 , and 1630 , respectively. These results indicate that additional rows of winglets are not justified for the in-line tube bundle arrangement.

Figure 4-31 presents the performance ratio, $(\mathrm{j} / \mathrm{j} 0) /(\mathrm{f} / \mathrm{f} 0)$, results for the staggered tube bundle arrangement. For this case, reference values jo and fo refer to the staggered tube bundle base case without winglets. The $(\mathrm{j} / \mathrm{j} 0) /(\mathrm{f} / \mathrm{f} 0)$ results for the staggered tube bundle arrangement are quite different than those for the in-line tube bundle arrangement. For the staggered tube bundle arrangement, the configuration with three rows of winglets provides the best results. For this case, heat transfer enhancement values of 38.6, 44.7, and 40.3\% were obtained at ReH of 660, 1190, and 1630, respectively. Frictional pressure drop increases of 26.2, 25.8, and 20.4\% were obtained for $\mathrm{ReH}$ equal to 660, 1190, and 1630 , respectively. These calculations yield a favorable performance ratio, $(\mathrm{j} / \mathrm{j} 0) /(\mathrm{f} / \mathrm{f} 0)$ values of $1.10,1.15$, and 1.16 for $\mathrm{ReH}$ of 660,1190 , and 1630, respectively. For the staggered tube bundle arrangement, adding additional rows of winglets increases heat transfer enhancement proportionately higher than corresponding increase in frictional pressure drop, resulting in a more favorable $(\mathrm{j} / \mathrm{j} 0) /(\mathrm{f} / \mathrm{f} 0)$ ratio as winglets rows are added.

These results, for both the staggered and in-line tube bundle arrangements, when compared with the corresponding results by Torii et al. ${ }^{54}$ for circular tubes show that present heat transfer enhancement values are slightly lower, and the present frictional pressure drop increases are slightly higher. Two 
possible reasons could be responsible for the winglets configurations being less effective in the oval tubes compared to circular tubes. First, due to the smaller wake and delayed flow separation in oval tubes compared to the same in circular tubes, the winglets in oval tubes are relatively less influential in enhancing heat transfer than in circular tubes. Second, by keeping the center-to-center spacing for oval tubes the same as that for circular tubes, the distance between the winglet pairs (Figure 64) becomes quite large.

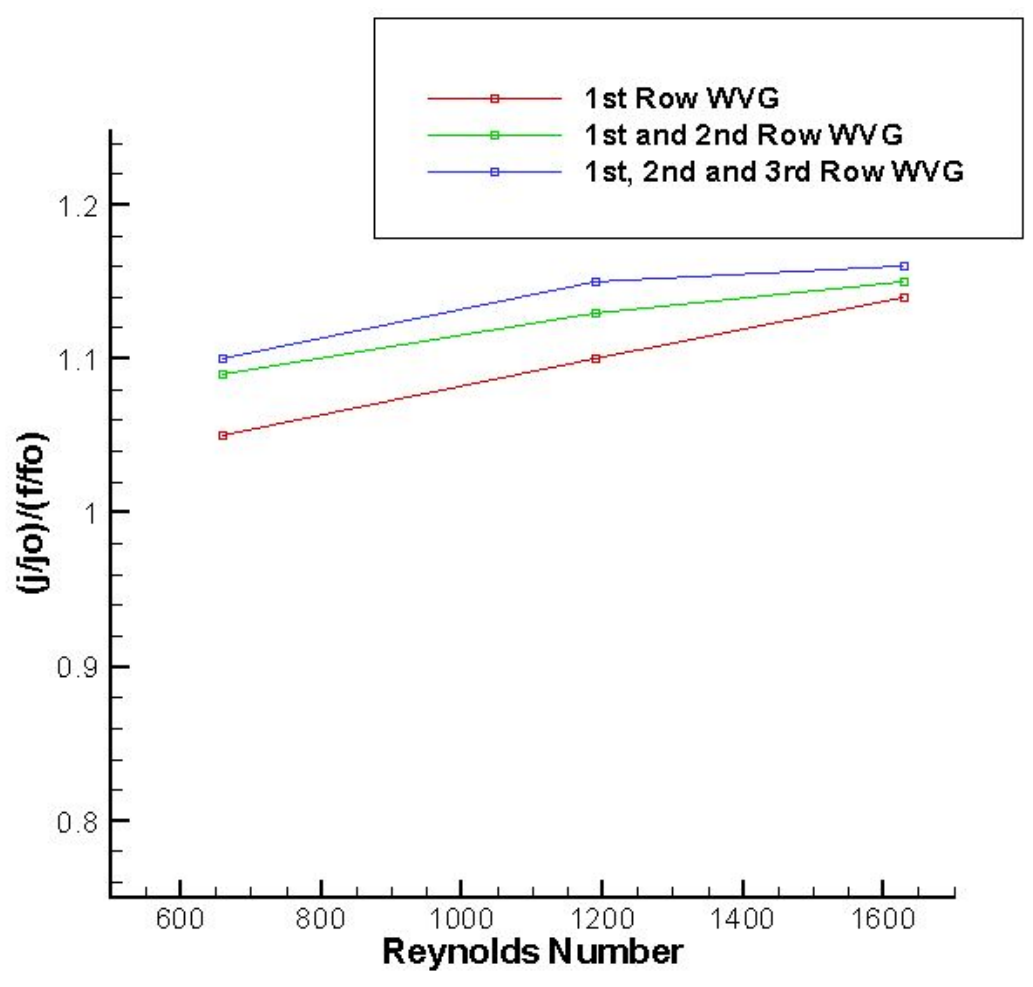

Figure 4-31. Performance ratio, $\left(\mathrm{j} / \mathrm{j}_{0}\right) /\left(\mathrm{f} / \mathrm{f}_{0}\right)$, results for staggered oval tube banks with winglets in $1,1-2$, and 1-2-3 rows.

Yanagihara and Torii ${ }^{44-46}$ studied the behavior of winglet pairs compared to a single winglet in both a co-rotating and counter-rotating configurations for both toe-in (common flow up) and toe-out (common flow down) orientations. They noted an improvement of 30\% in local heat transfer enhancement with winglet pairs when compared to a single winglet for the counter-rotating configuration in a toe-out (common flow down) orientation. Yanagihara and Torii ${ }^{44-46}$ attributed this result to a strong downward flow, which tends to push the main longitudinal vortices toward the fin surface, resulting in significant thinning of the boundary layer in the region between the main longitudinal vortices. This thinning of the boundary layer results in significant heat transfer enhancement in this region. On the upstream side of each winglet, the corner longitudinal vortex also results in heat transfer enhancement in this region. They noted that the smaller the distance between the winglets, the higher the downward movement of the flow in this region and the higher the heat transfer enhancement in the centerline region between two winglets.

Since the distance between the winglet pairs in both the in-line and staggered tube bank arrangements is very large, the winglets behave as individual winglets, not as winglet pairs, which may explain why lower heat transfer enhancement was observed in this study for the oval tube-bank arrangements. If the tubes were arranged with a tighter spacing for both the inline and staggered tube bank arrangements, better heat transfer enhancement could possibly be obtained from the closer spacing of the winglets between tubes. 


\subsubsection{Comparison of In-line versus Staggered Tube Bundle Performance}

Figure 4-32 presents a comparison of the performance ratio, $(\mathrm{j} / \mathrm{j} 0) /(\mathrm{f} / \mathrm{f} 0)$, results for the staggered tube bundle arrangement compared to the in-line tube bundle arrangement, where $\mathrm{j} 0$ and $\mathrm{f} 0$ are values for the in-line tube bundle arrangement. Thus, $(\mathrm{j} / \mathrm{j} 0) /(\mathrm{f} / \mathrm{f0})$ as shown on the ordinate of Figure $4-32$ will be the same as the ratio $[(\mathrm{j} / \mathrm{j} 0) /(\mathrm{f} / \mathrm{f0})]$ staggered $/[(\mathrm{j} / \mathrm{j} 0) /(\mathrm{f} / \mathrm{f} 0)]$ in-line, where $[(\mathrm{j} / \mathrm{j} 0) /(\mathrm{f} / \mathrm{f} 0)]$ staggered is from Figure 4-31 and $[(\mathrm{j} / \mathrm{j} 0) /(\mathrm{f} / \mathrm{f0})]$ in-line is from Figure 4-30.

In all configurations, the heat transfer is higher for the staggered tube bank arrangement compared to the in-line arrangement, but corresponding frictional pressure drop is higher. For the tube bundle without winglets case, the in-line arrangement yields better $(\mathrm{j} / \mathrm{j} 0) /(\mathrm{f} / \mathrm{f} 0)$ results. For all three winglet configurations, the staggered tube-bank arrangement gives a better performance ratio, with progressively better results for additional rows of winglets.

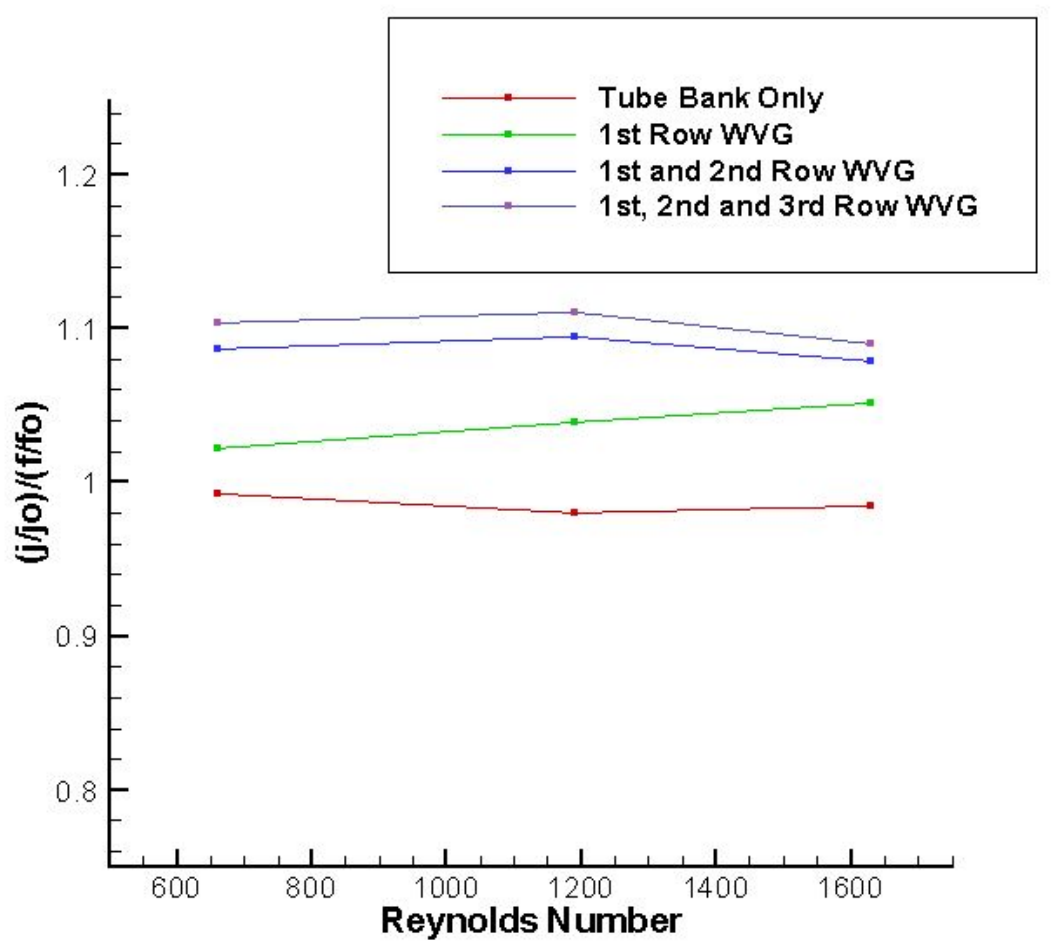

Figure 4-32. Comparison (ratio) of performances ratios, $\left(\mathrm{j} / \mathrm{j}_{0}\right) /\left(\mathrm{f} / \mathrm{f}_{0}\right)$, for staggered versus in-line tube bundles.

\subsection{Fluid Flow Contours and Heat Transfer Pattern for Tube Bundles}

\subsubsection{Contours for In-line Tube Bundles}

Figures 4-33a and -b present velocity magnitude contours and surface heat transfer patterns for the in-line tube-bank arrangement, $\mathrm{Re}_{\mathrm{H}}=1190$, for the following configurations: (a) baseline case without winglets, (b) one row of winglets, (c) two rows of winglets, and (d) three rows of winglets. Again, the solid dark blue lines are an artifact of the meshing and postprocessing techniques used in the numerical analysis and should be ignored. The figures reveal the correlation between the heat transfer and flow domains and provide insight into the HTE/FLP results for various winglet configurations. 


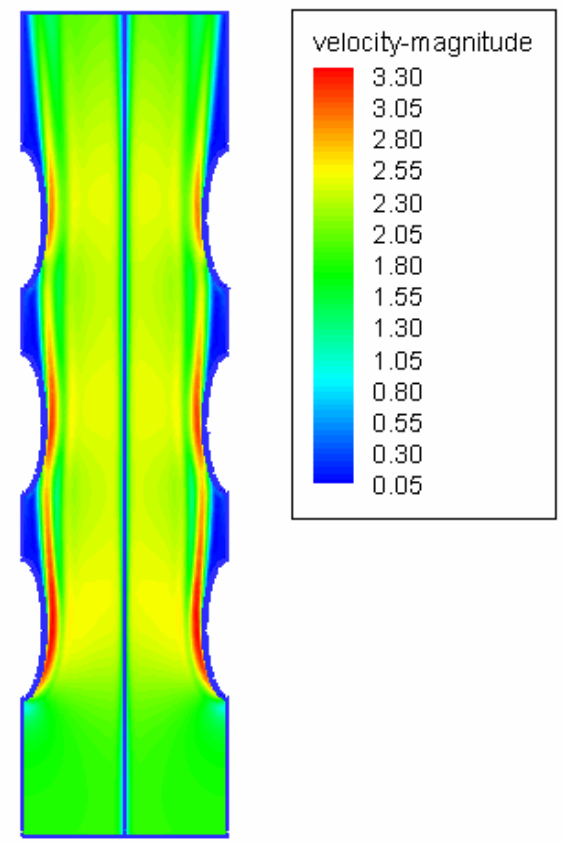

(a)

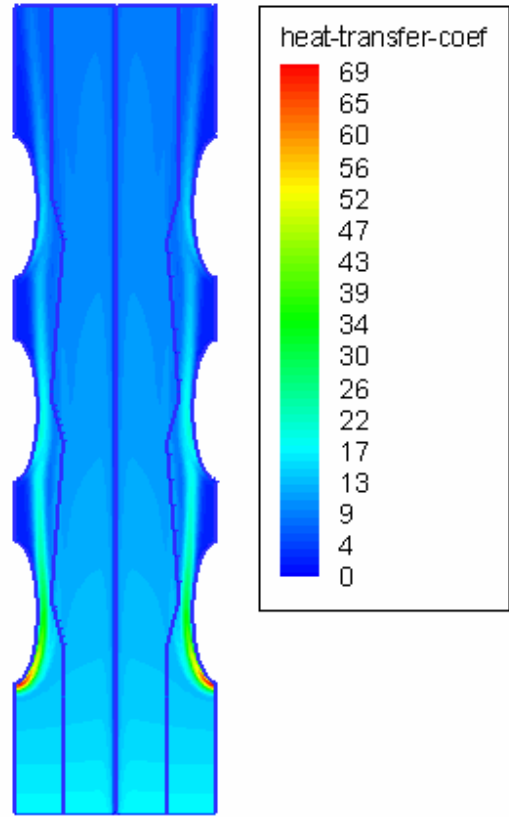

(b)

Figure 4-33a and -b. Base case with no winglets: (a) velocity $(\mathrm{m} / \mathrm{s})$ contours, (b) surface heat transfer coefficient $\left(\mathrm{W} / \mathrm{m}^{2} \cdot \mathrm{K}\right)$ distribution.

For the tube-only baseline case, an area of enhanced heat transfer is observed in the stagnation region of the first (leading) tubes due to the horseshoe vortices that get generated in this region. The longitudinal vortices formed when the horseshoe vortices separate from the tube persist over the length of the oval tube section. This longitudinal vortex interacts with the second and third downstream tubes and shows some weakening due to this interaction. Horseshoe vortices do not appear to form in the stagnation region of the downstream tubes (second and third), as is evident from the lack of enhanced surface heat transfer in these areas. This is due to the fact that these stagnation zones are located in the wake regions of the upstream tubes. Thus, it reduces the areas of enhanced heat transfer for the downstream tubes.

For the case of one row of winglets (winglet vortex generators, or WVGs) (Figure 4-33c and -d), the main longitudinal vortex off the trailing edge of the WVG persists over the entire length of the tube-bank section as it is swept downstream and results in significant HTE. The surface heat transfer and velocity magnitude contour plots bring to light the different mechanisms for HTE in the area far downstream of the WVG and in the area immediately downstream. Far downstream of the WVG, the longitudinal vortex is the primary mechanism for HTE. In the immediate downstream vicinity of the WVG, the interaction of the main, the corner, and the induced secondary vortices distort the flow and enhance heat transfer locally. As was shown in the single-oval-tube analysis, this WVG configuration has little to no effect on the primary flow for the in-line tube bank arrangement, and the flow and heat transfer mechanisms for the flow around the tubes remains essentially the same as the case with no WVGs.

The addition of second and third rows of WVGs ( Figures 4-33e, -f, -g, -h) only appears to affect heat transfer in the region in the immediate downstream vicinity of the WVGs. The embedded longitudinal vortex is only minimally affected by the additional rows of WVGs. The velocity magnitude plot shows that the downstream WVG rows interrupt this longitudinal vortex by the interaction of this vortex with the downstream WVG. However, the embedded longitudinal vortex is reestablished further downstream, and in this regard the effect on the strength and the position of the longitudinal vortex tends to be minor. 


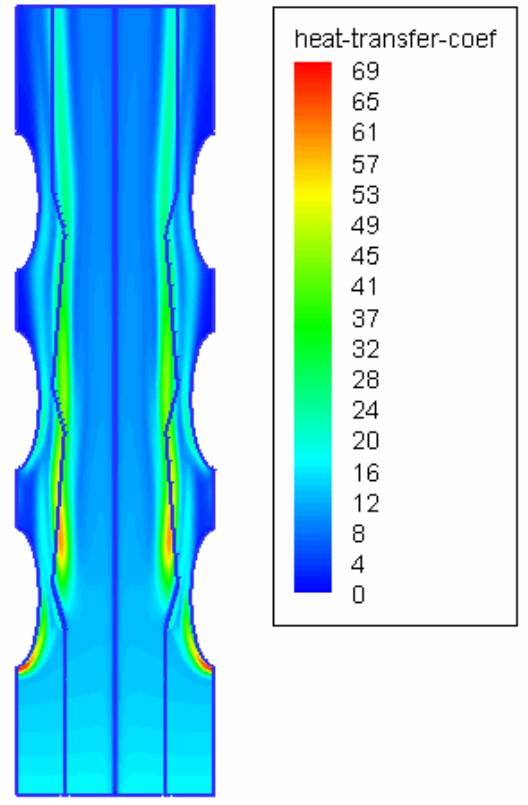

(c)

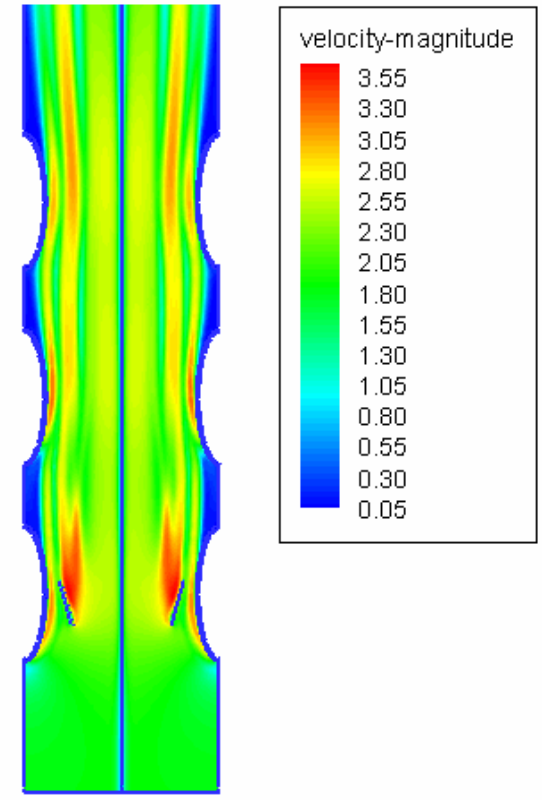

(d)

Figure $4-33 \mathrm{c}$ and d. First row WVGs: (c) surface heat transfer coefficient $(\mathrm{W} / \mathrm{m} 2 \cdot \mathrm{K})$ distribution, (d) contours of velocity magnitude $(\mathrm{m} / \mathrm{s})$.

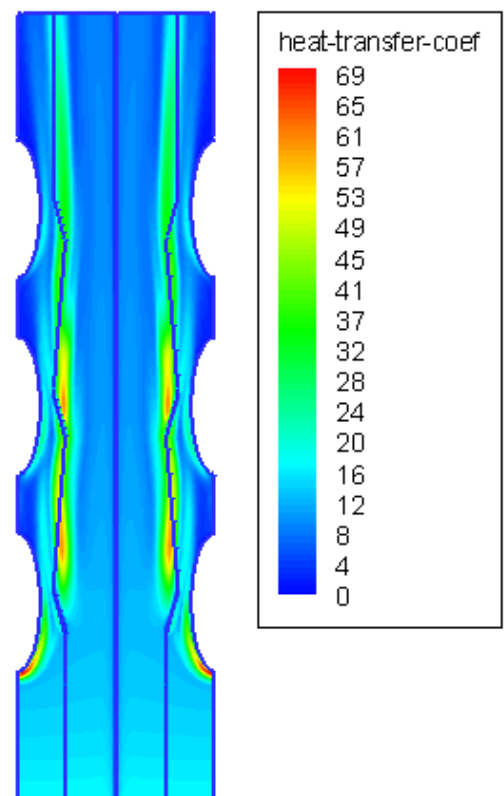

(e)

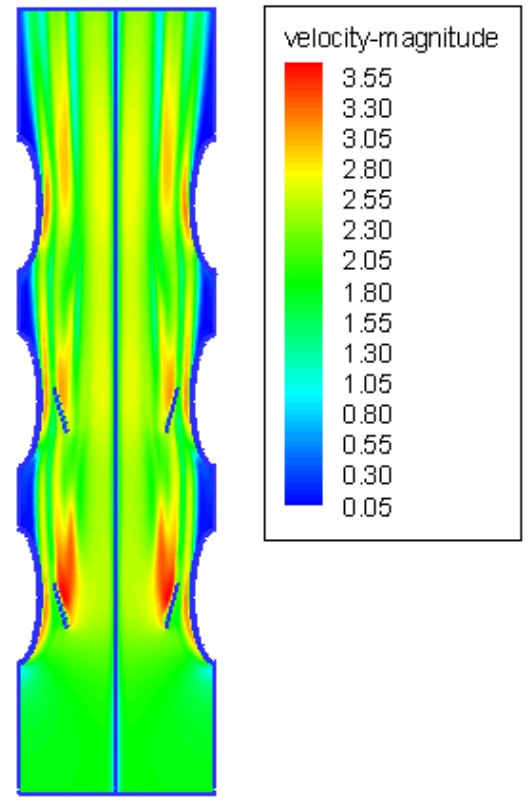

(f)

Figure 4-33e and f. First and second row WVGs: (e) surface heat transfer coefficient $\left(\mathrm{W} / \mathrm{m}^{2} \cdot \mathrm{K}\right)$ distribution, (f) contours of velocity magnitude $(\mathrm{m} / \mathrm{s})$. 


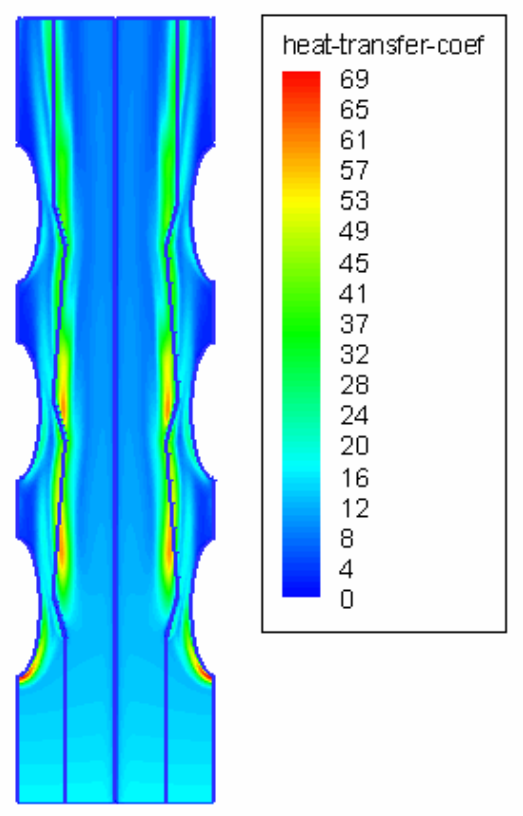

(e)

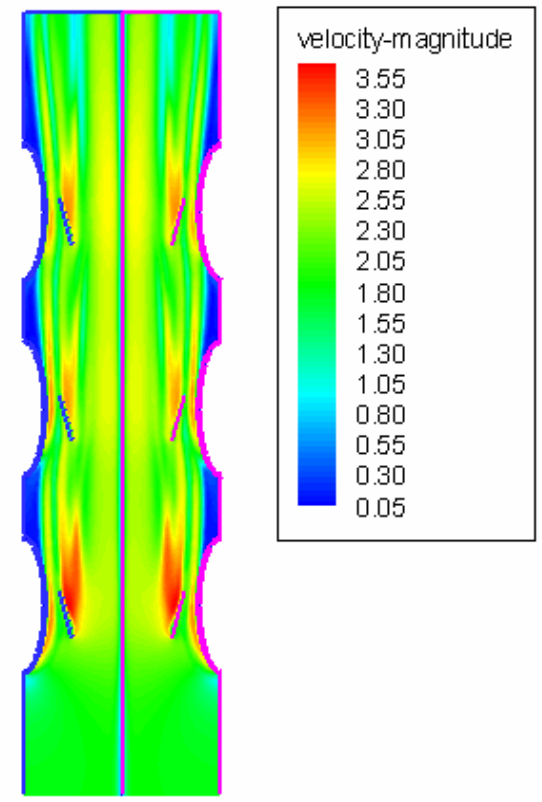

(f)

Figure 4-33g. First, second, and third row WVGs: (g) surface heat transfer coefficient $\left(\mathrm{W} / \mathrm{m}^{2} \cdot \mathrm{K}\right)$ distribution, $(\mathrm{h})$ contours of velocity magnitude $(\mathrm{m} / \mathrm{s})$.

Figure 4-34 presents dynamic pressure contours for the in-line tube bank arrangement. The pressure gradients in this in-line arrangement favor the vortices being swept downstream parallel to the $x$-axis in relative close proximity to the tubes, which is indicted in Figures 4-33a-f. The downstream tubes, since they are in the wake region of the upstream tubes, have minimal effect on the dynamic pressure gradients.

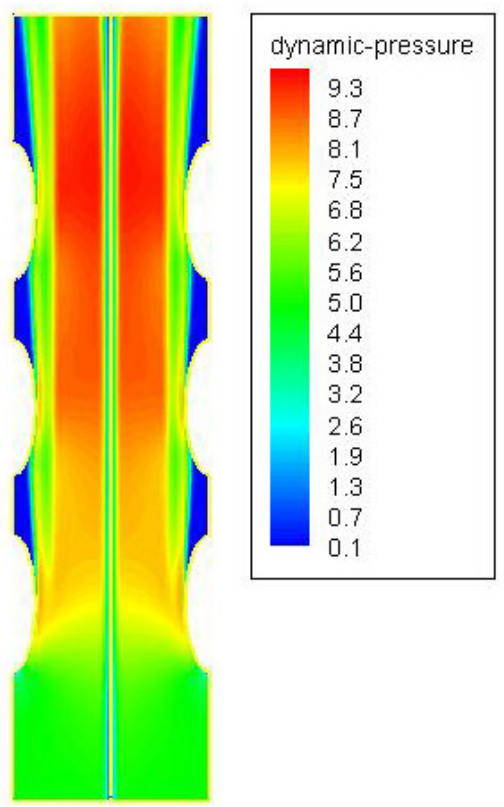

Figure 4-34. Dynamic-pressure gradients (pascals) for in-line tube bank arrangement. 
Figure 4-35 presents vector velocity plots for the in-line tube-bank arrangement for the following cases: (a) baseline case without WVGs, (b) 1 row of WVGs, (c) 2 rows of WVGs, and (d) 3 rows of WVGs for $\mathrm{ReH}=1190$.

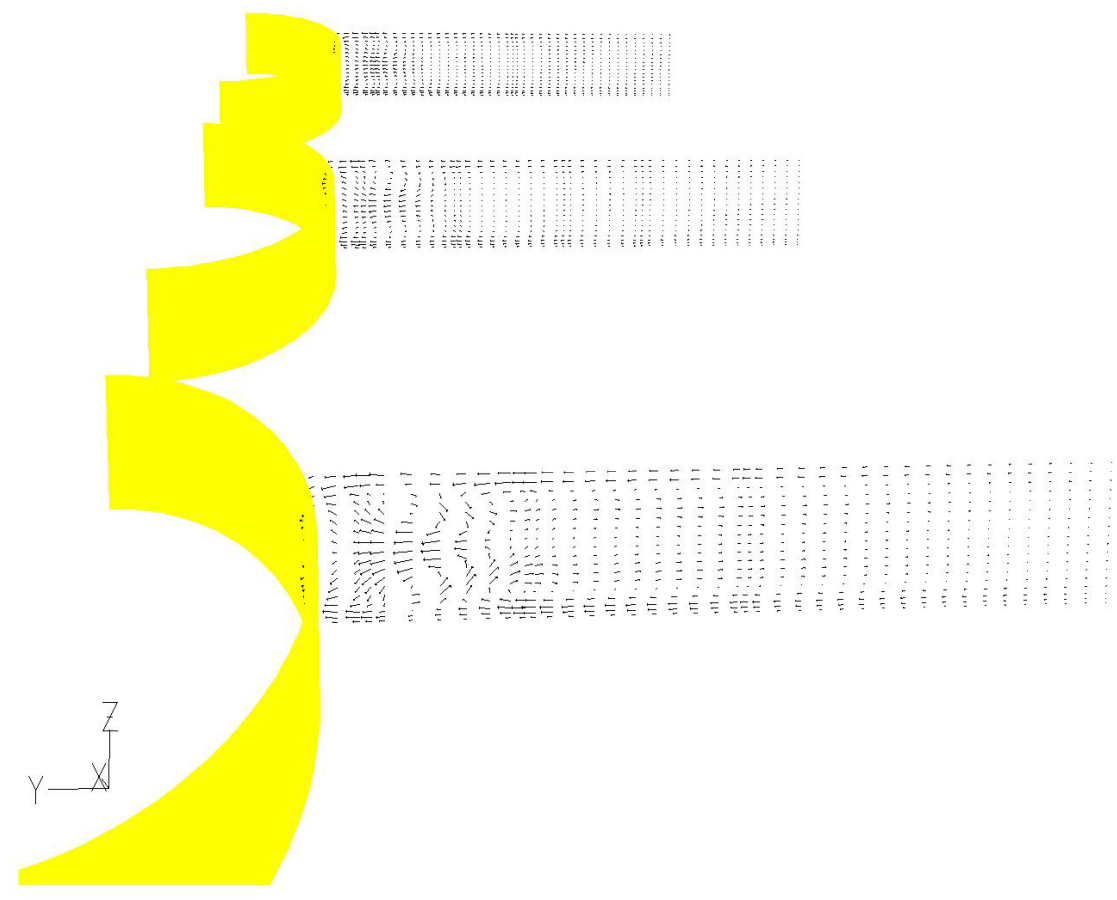

Figure 4-35a. Velocity vectors for in-line tube bank baseline case (no WVGs).

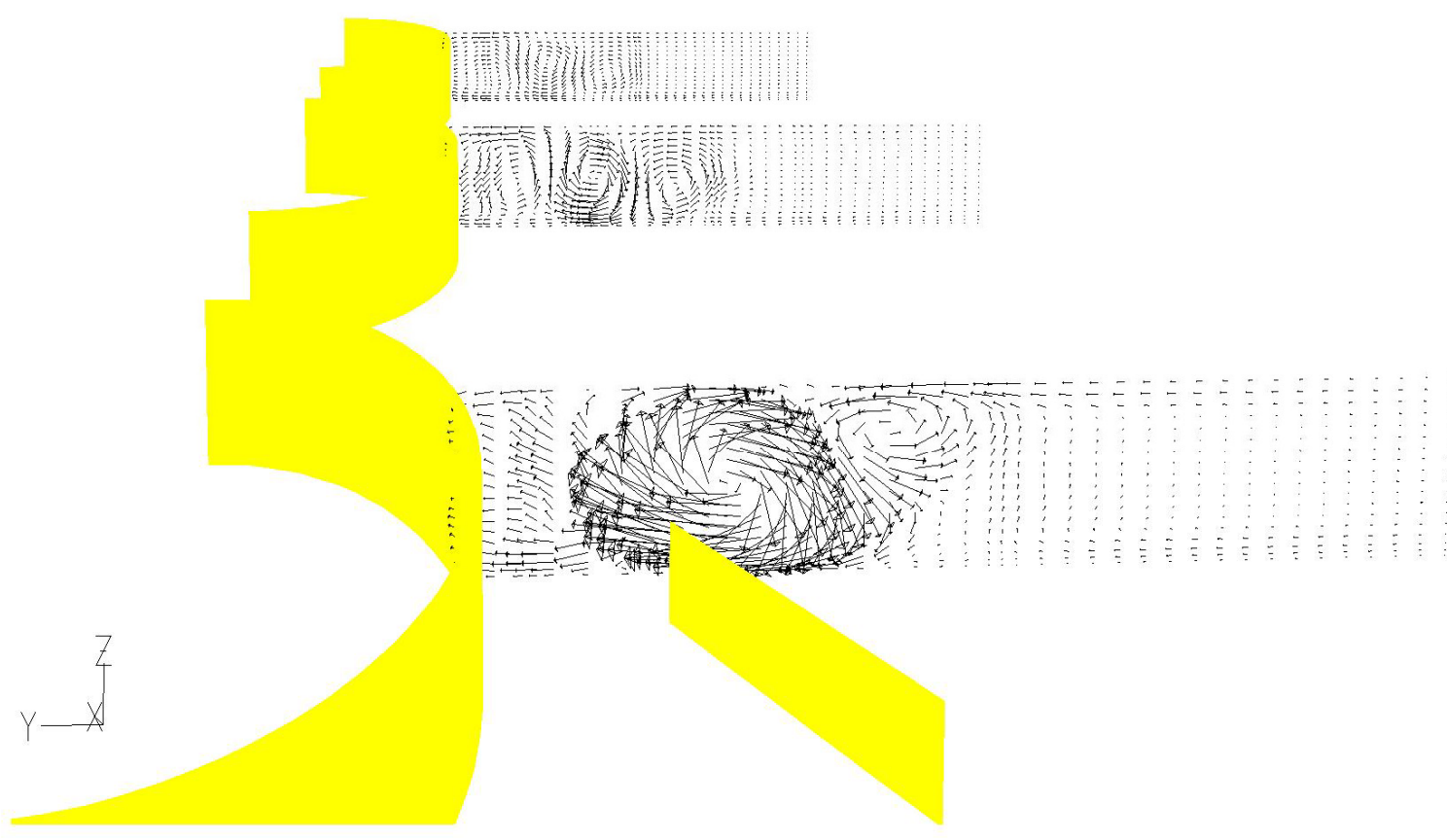

Figure 4-35b. Velocity vectors for in-line tube bank, 1 row of WVGs. 


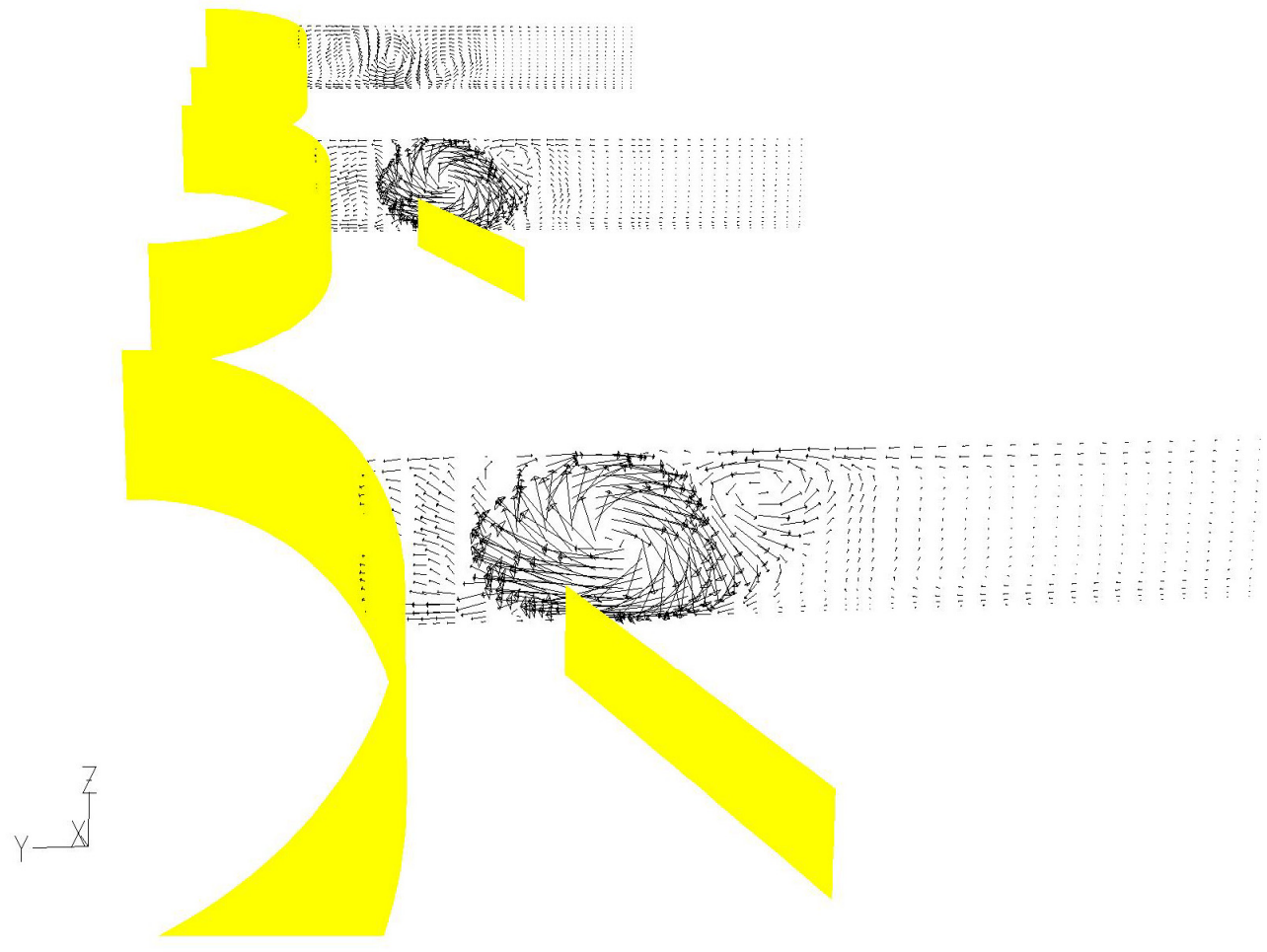

Figure 4-35c. Velocity vectors for in-line tube bank, 2 rows of WVGs.

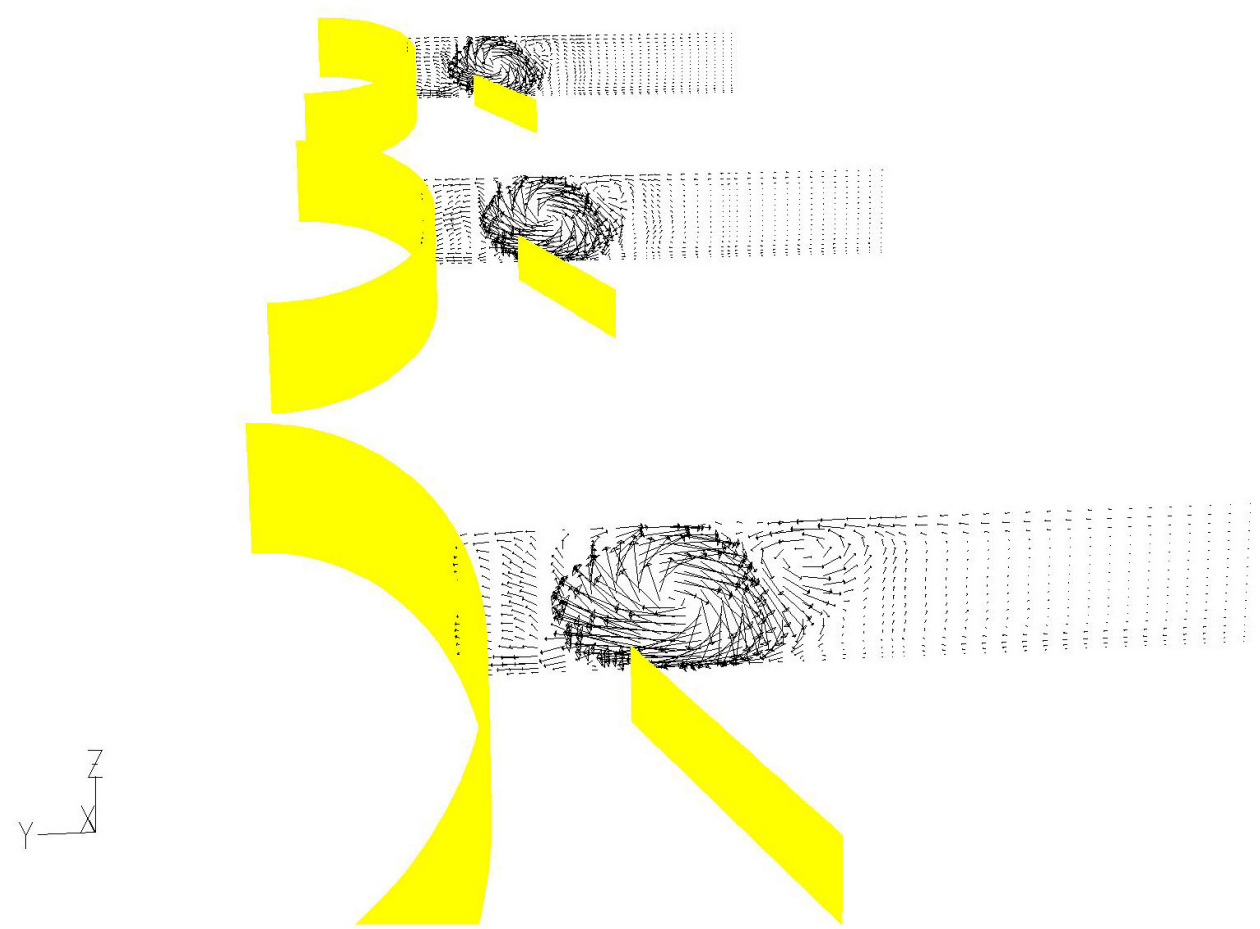

Figure 4-35d. Velocity vectors for in-line tube bank, 3 rows of WVGs. 
This plot provides evidence of the vortical motion in the analyzed cases. Vortical motion in the immediate downstream vicinity of the additional rows of WVGs is observed for the one-, two- and threerow case. This vortical motion and the enhanced area of surface heat transfer immediately downstream of the WVG explains why slight increases in overall HTE are observed with the addition of each row of WVG. Close inspection reveals that the magnitude of this vortical motion is reduced slightly for each successive row of WVGs, especially the corner vortex, which is noticeably weaker for WVG rows 2 and 3 than for row 1. A possible explanation of why this is the case is due to the interaction with the upstream-embedded longitudinal vortex, which may interact with the downstream WVG to weaken the corner vortex. This reduced vortical motion and area of enhanced heat transfer is most likely why smaller increases in HTE are observed with the addition of each successive row of WVGs. In addition, as in the case of one row of WVGs, the additional rows of WVGs do not have any significant affect on the flow around the tubes and confirm that the flow effects of this WVG configuration for the in-line tube-bank arrangement are secondary.

\subsubsection{Staggered Tube Bank Arrangement}

Figure 4-36 presents surface heat transfer and flow velocity magnitude plots for the staggered tube bank arrangement for the following cases: (a) baseline case without WVGs, (b) 1 row of WVGs, (c) 2 rows of WVGs, and (d) 3 rows of WVGs for $\mathrm{Re}_{\mathrm{H}}=1190$. Again, the solid dark blue lines are an artifact of the meshing technique used and should be ignored. Comparison of Figure 4-36a for the baseline case with no WVGS compared to the baseline case of Figure 4-36a reveals significant differences between the heat transfer and flow patterns of the staggered tube bank and the in-line arrangement. In contrast to the in-line arrangement, stronger horseshoe vortices are established in the $2^{\text {nd }}$ row middle tube than in the upstream tube row. In addition, compared to the in-line tube-bank arrangement, the interaction of the downstream tubes with the upstream tubes appears to be minor. Based on these differences in baseline heat transfer and flow patterns, the effects of the WVG rows would be expected to be quite different for the staggered tube bank arrangement compared to the in-line arrangement previously shown in Figure 4-32.

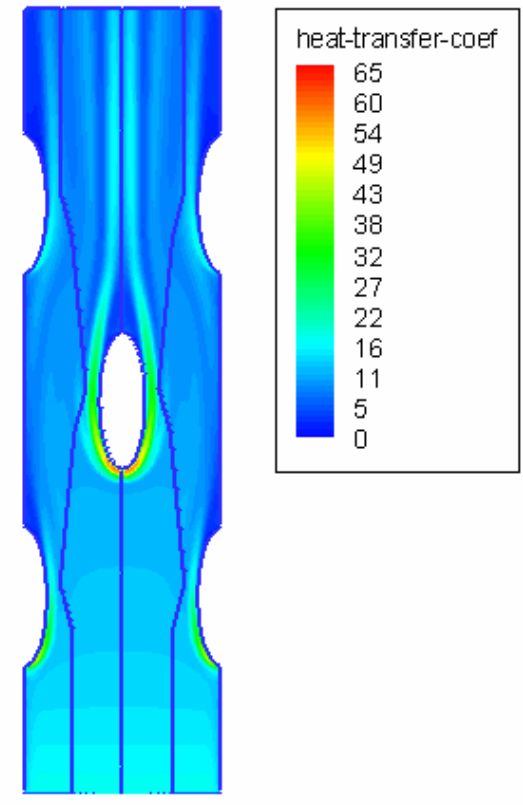

(a)

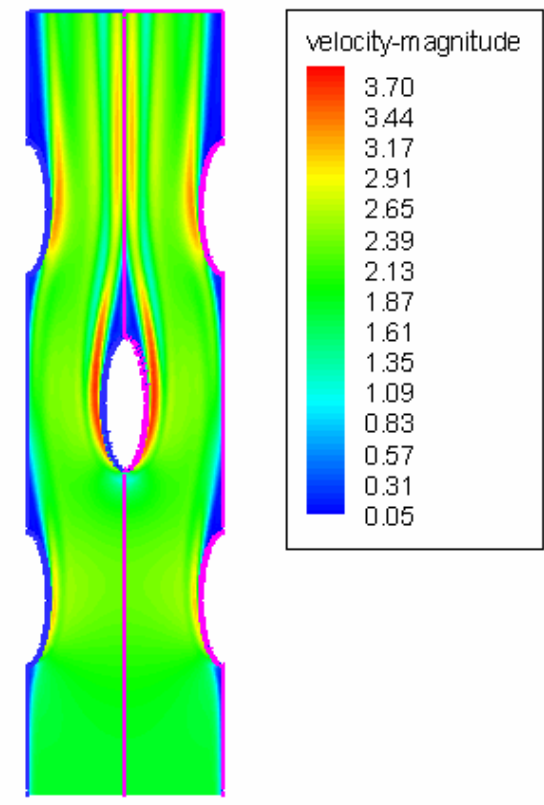

(b)

Figure 4-36a and b. Base case, no WVGs: (a) surface heat transfer coefficient (W/m $\mathrm{K}$ ) distribution, (b) contours of velocity magnitude $(\mathrm{m} / \mathrm{s})$. 


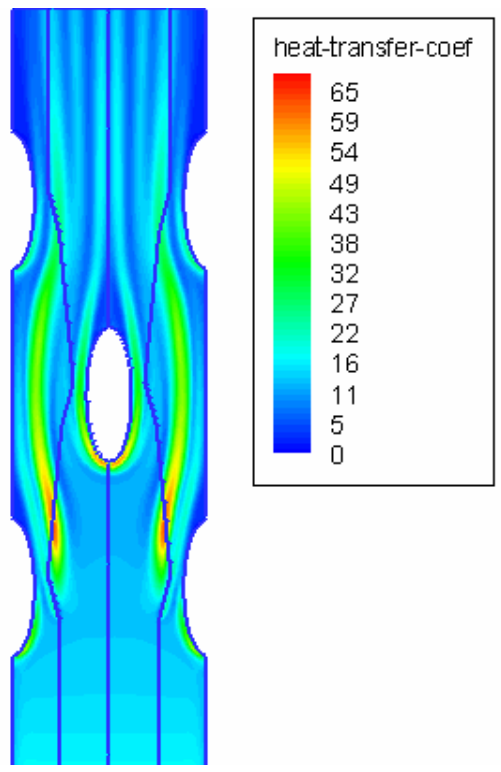

(c)

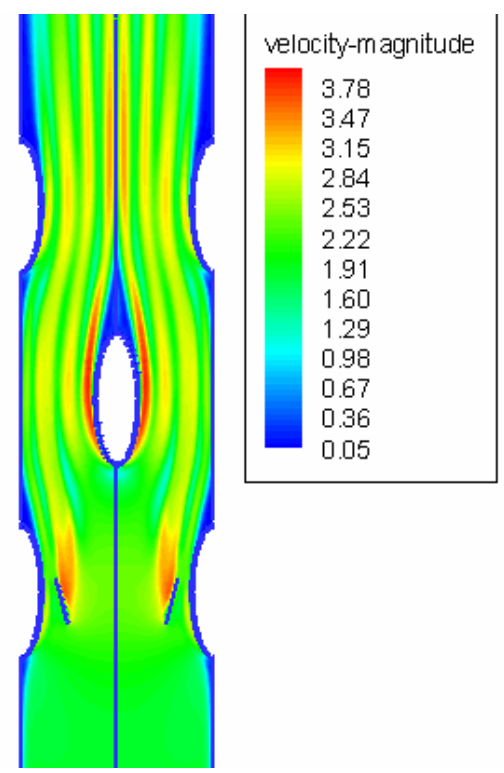

(d)

Figure 4-36c and d. First row WVGs: (c) surface heat transfer coefficient (W/m² K) distribution, (d) contours of velocity magnitude $(\mathrm{m} / \mathrm{s})$.

For the baseline case of no WVGs, an area of enhanced heat transfer is observed in the stagnation region of all tubes, due to the horseshoe vortices that are formed in this region. Instead of being swept straight downstream from the point of separation from the tube, as in the case of the single oval tube and the in-line tube-bank arrangement, the longitudinal heat transfer streaks associated with the horseshoe vortices follow a curving pattern around the downstream tubes as they are swept downstream. This results in a curving pattern for the longitudinal streaks from the front tube rows around the downstream tubes and a curving in and straight back for the longitudinal streaks from the middle tube.

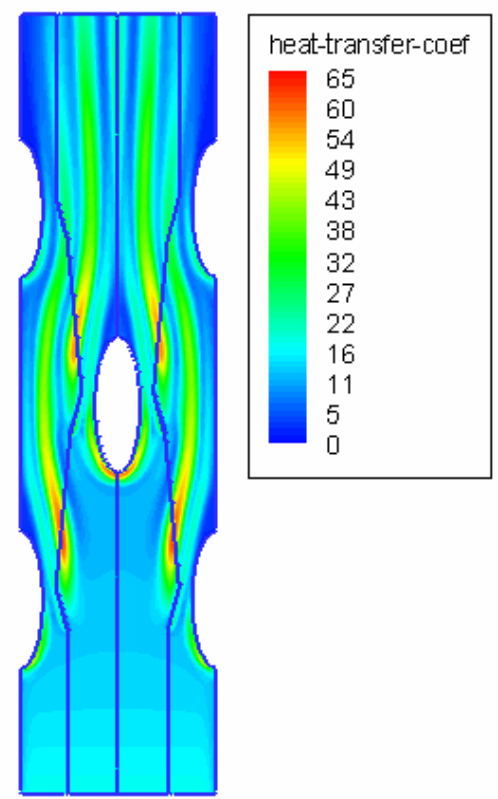

(e )

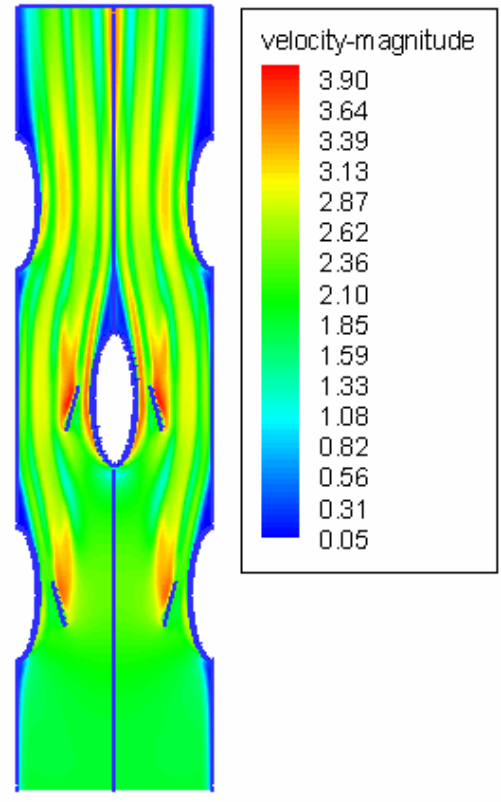

(f)

Figure 4-36e and f. First and second row WVGs: (e) surface heat transfer coefficient $\left(\mathrm{W} / \mathrm{m}^{2} \mathrm{~K}\right)$ distribution, (f) contours of velocity magnitude $(\mathrm{m} / \mathrm{s})$. 


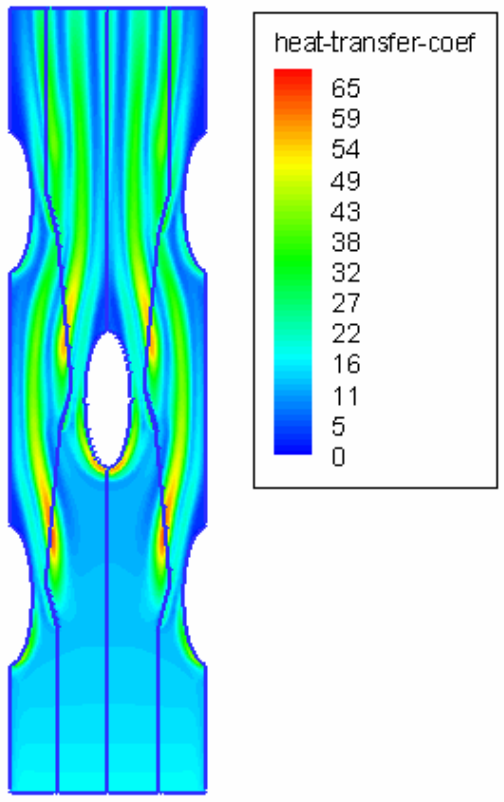

(g)

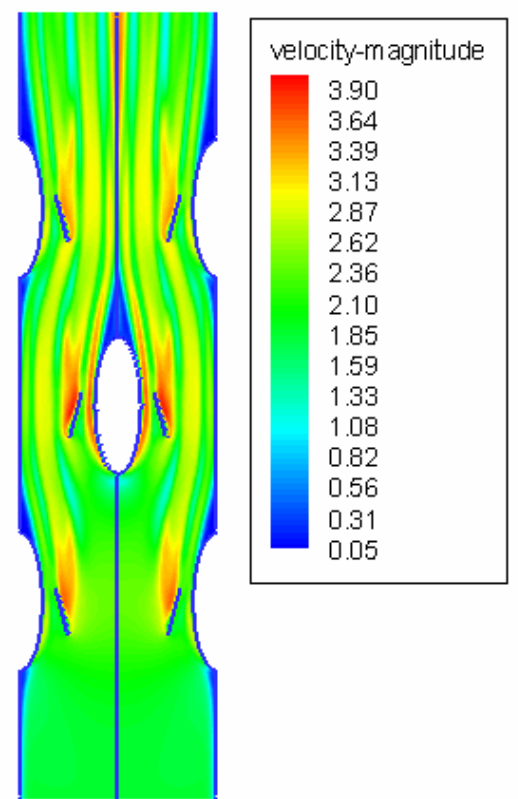

(h)

Figure 4-36g. First, second, and third row WVGs: (g) surface heat transfer coefficient $\left(\mathrm{W} / \mathrm{m}^{2} \mathrm{~K}\right)$ distribution, (h) contours of velocity magnitude $(\mathrm{m} / \mathrm{s})$.

Figure 4-37 presents dynamic pressure contours for the staggered tube bank arrangement. The pressure gradients favor pushing the flow in an outward normal direction from the downstream tubes. This provides an explanation as to why the vortices are curved around the downstream tubes, as they are swept downstream, as observed in Figures 4-36a-f. The highest outward normal dynamic pressure gradients are for the second and third rows of tubes. These pressure gradients appear to weaken the longitudinal vortex as it travels downstream as can be evidenced in Figure 4-36a- $\mathrm{h}$.

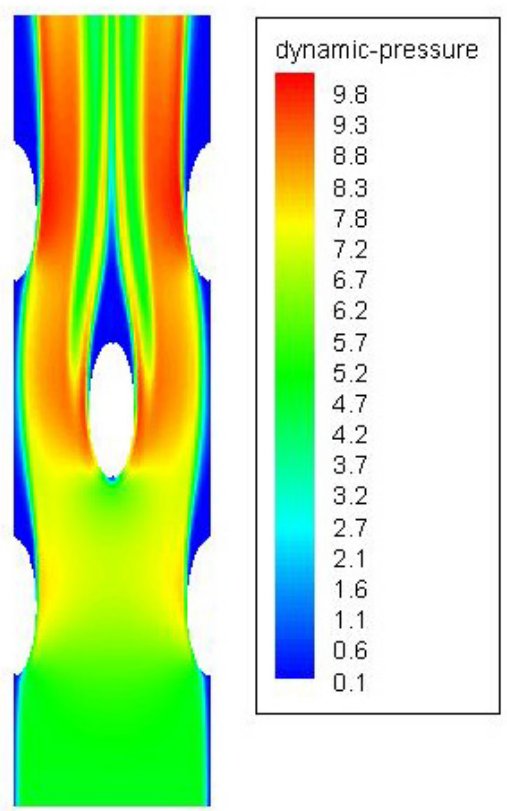

Figure 4-37. Dynamic-pressure gradients (pascals) for staggered tube bank arrangement. 
Most likely due to the increased distance between downstream and upstream tubes along the same centerline, the effects of the upstream tube wake on the downstream tube is significantly reduced from that of the in-line tube-bank arrangement. The outward dynamic pressure gradient from the middle tube also acts to delay the separation from the upstream tubes. This also acts to reduce the size of the wake region from the tubes in the first row. For the case of one row of WVGs (Figure 4-36c and -d), the main longitudinal vortex off the trailing edge of the WVG persists over the entire length of the tube-bank section and produces significant HTE. Again, as in the baseline case of no WVGs, the longitudinal streaks curve downstream around the downstream tubes due to the outward pressure gradient form the downstream tubes.

Inspection of Figure 4-36c and -d for one row of WVGs, reveals extra longitudinal streaks, which indicate that formation of an induced vortex is between the longitudinal vortex from the front row of WVGs and the horseshoe vortices on the middle tube. This vortex also persists over the entire length of the tube bank section, indicatingit is a longitudinal vortex. However, it appears to be weak, since it only results in minor HTE. In addition, the horseshoe vortices in the downstream tube row and the resultant local HTE appear to be stronger in this case than in the baseline case with no WVGs. It is difficult to explain why this would be the case; however, the plots seem to indicate that a very complex highly nonlinear flow pattern is established that results in induced vortices that interact with the downstream tubes and increase HTE in this region.

The horseshoe vortices around the middle tube and the downstream tube row do not appear affected by the first row of WVGs. Again, the downstream tube outward pressure gradient appears to weaken the strength of the vortices as they are swept downstream. As in the WVG plots for the in-line tube bank arrangement, the surface heat transfer and velocity magnitude contour plots illustrate the different mechanisms for HTE in the area far downstream of the WVG and in the area immediately downstream.

The addition of a second row of WVGs, Figure 4-36e and -f, results in enhancement of heat transfer in the area immediately downstream of the WVG and along the main longitudinal vortex off of the trailing edge that persists for the entire downstream length of the tube-bank section. Cross comparison of Figure 4-36e and -f with Figure 4-36c and -d reveals that the primary longitudinal vortex from the second row of WVGs around the middle tube occurs in about the same location as the induced vortex from the one-row WVG case. However, it appears to be a much stronger vortex, since it results in significantly higher HTE than the induced vortex for one row of WVGs. The primary longitudinal vortices from the first row of WVGs show significant weakening as they are swept downstream; however, conversely the primary longitudinal vortices from the second row of WVGs do not show significant weakening as they are swept downstream. Again, as is the case for one row of WVGs, the downstream tubes show an increased area of HTE compared to the baseline case of no WVGs, further evidence of induced vortices due to the complex highly nonlinear flow patterns that are established.

The main effect of adding a third row of WVGs on the third tube row is to strengthen the embedded longitudinal vortex from the first row of WVGs. Inspection of Figure 4-36g and -h reveals that complex vortex flow patterns are established, and these complex flow patterns are most likely why significant additional HTE is obtained by adding additional rows of WVGs in the staggered tube bank arrangement. In this configuration, the corner vortex from the third row of WVGs appears to persist further downstream than from the first two rows of WVGs, and it does show significant weakening as it is swept downstream.

Figure 4-38 presents vector velocity plots for the staggered tube-bank arrangement for the following cases: (a) baseline case without WVGs, (b) one row of WVGs, (c) two rows of WVGs and (d) three rows of WVGs for $\mathrm{Re}_{\mathrm{H}}=1190$. This figure provides further insight into the complex vortex flow patterns established for the staggered tube bank arrangement. Figure 4-38b, in the plane normal to the second row middle tube, shows the induced vortex between the longitudinal vortex from the first row WVG and the horseshoe vortex for the tube. As depicted in Figures 4-36c and -d, it is a weak vortex. Figures 4-38c and 
- $d$ show in the planes normal to tubes 2 and 3 the complex highly nonlinear vortex pattern established for rows 2 and 3 of WVGs. The longitudinal vortices and horseshoe vortices interact to induce additional longitudinal vortices. These vortices appear to be established as a function of the vorticity of the air, and are counter-rotating to the surrounding vortices. In contrast to the in-line tube bank arrangement, the vortex pattern established by the second and third row of WVGs is not weaker than for the first row of WVGs. The corner vortex for each successive row of WVGs is clearly evident and can be seen in the downstream planes.

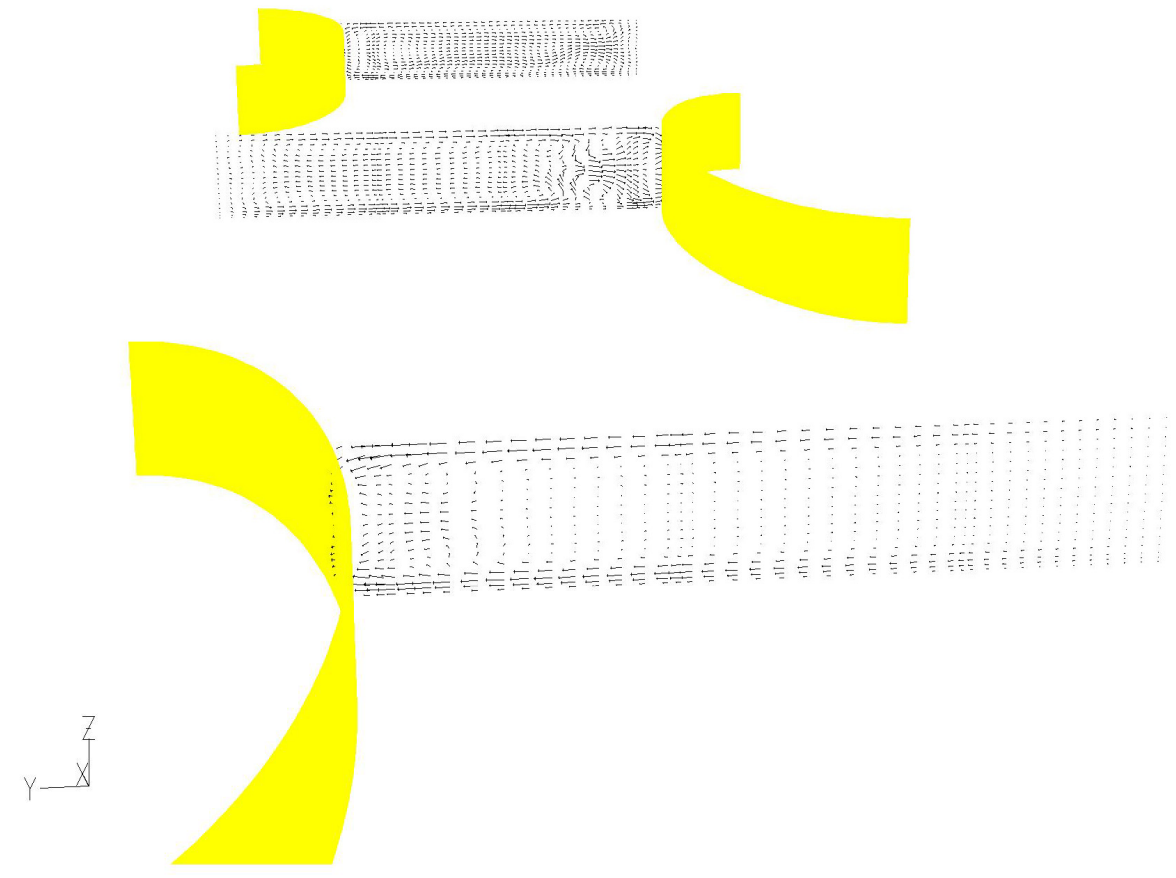

Figure 4-38a. Velocity vectors for staggered tube bank baseline case (no WVGs).

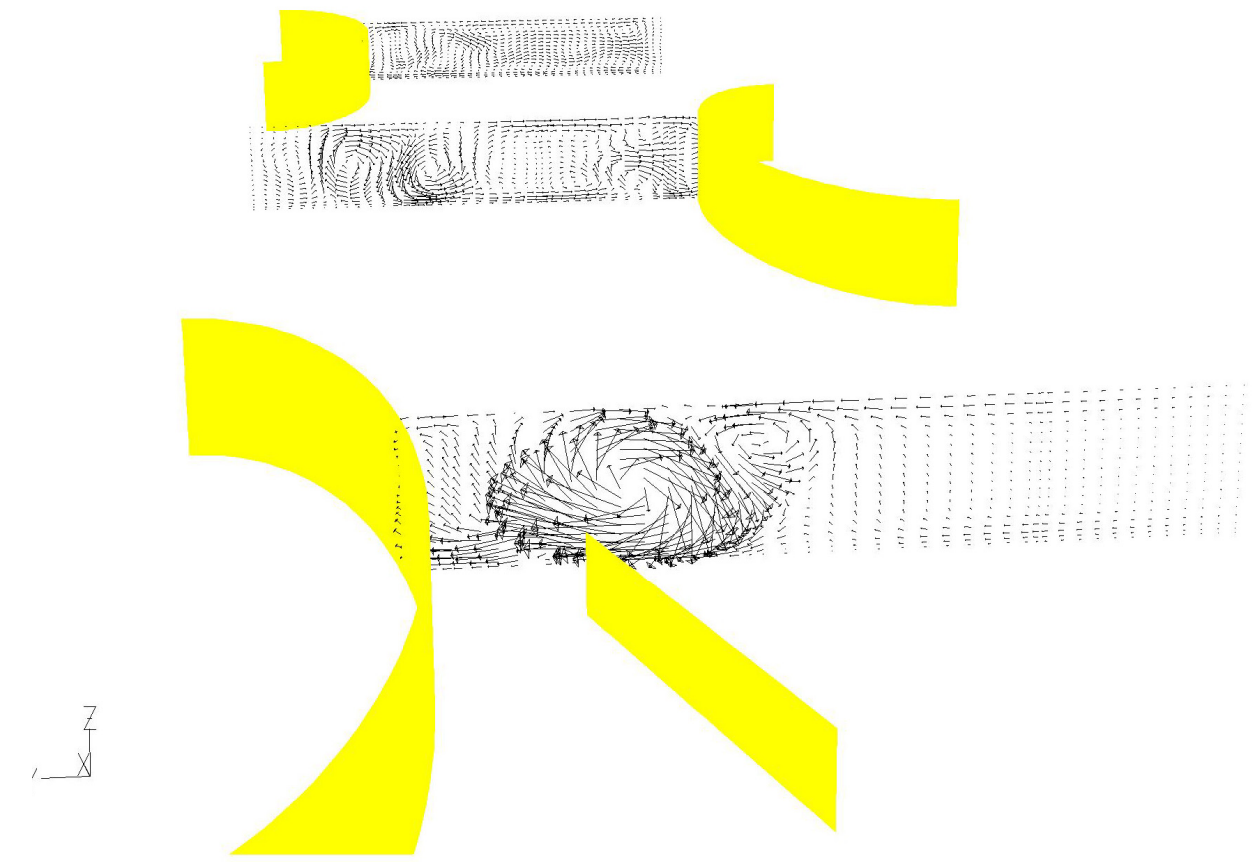

Figure 4-38b. Velocity vectors for in-line tube bank, one row of WVGs. 


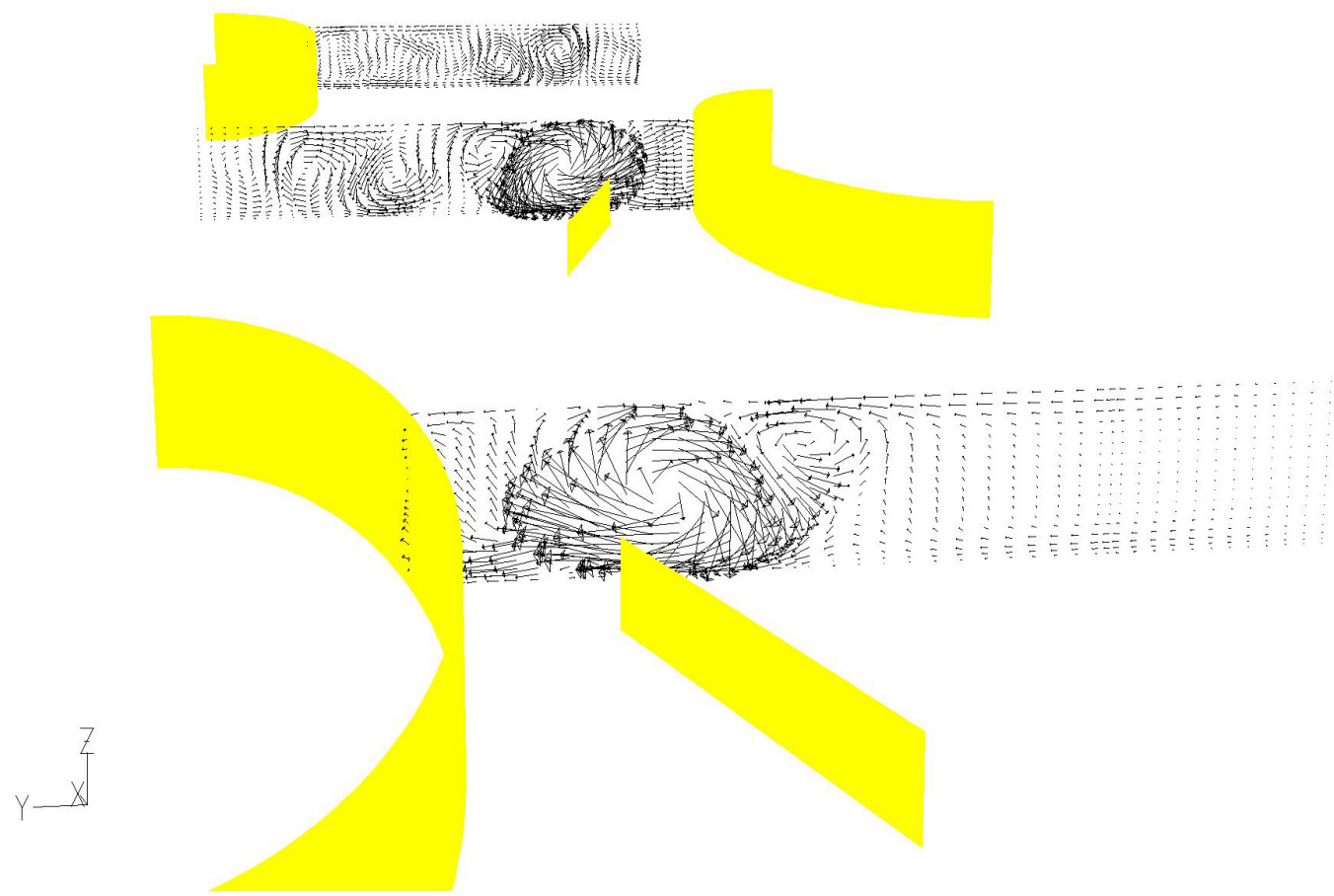

Figure 4-38c. Velocity vectors for in-line tube bank, two rows of WVGs.

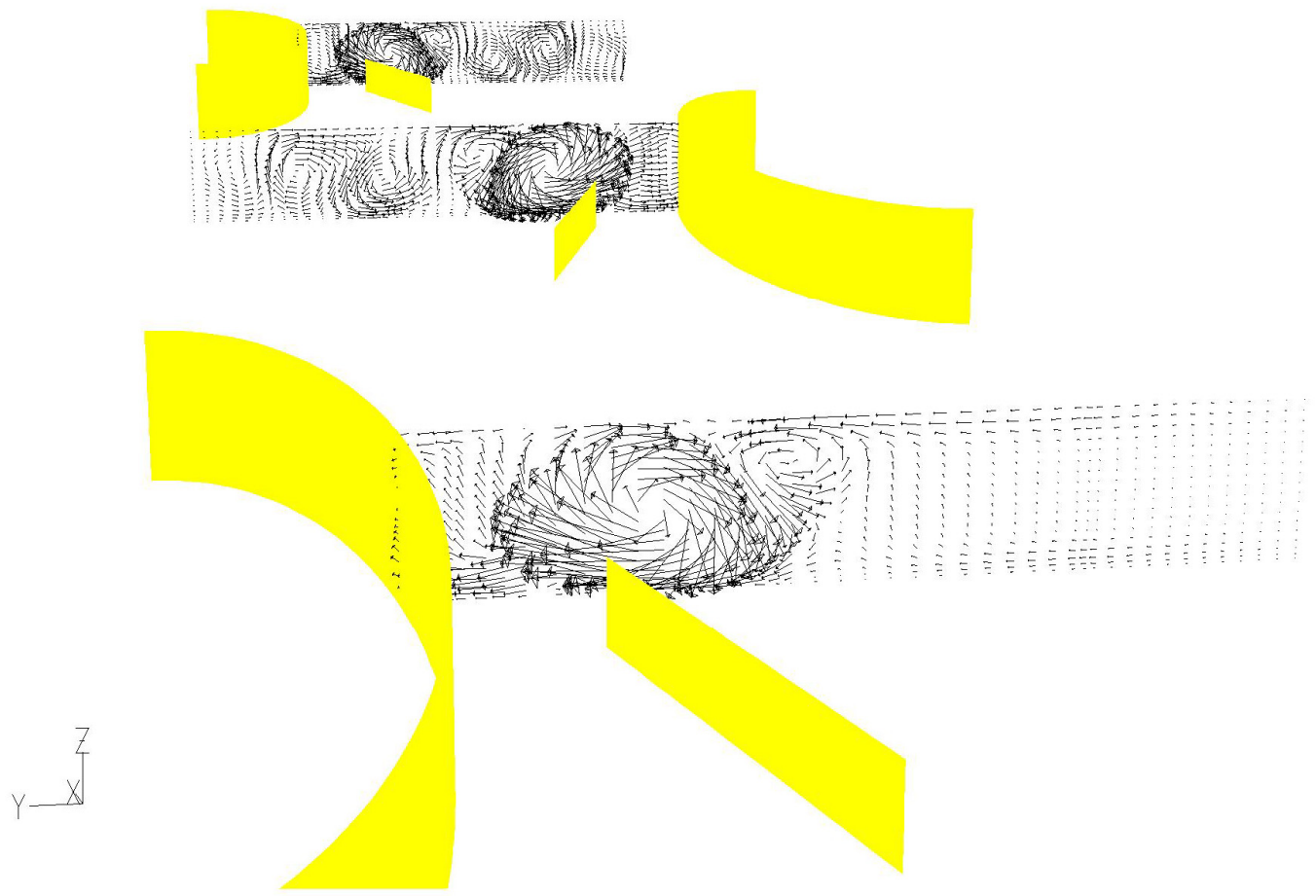

Figure 4-38d. Velocity vectors for in-line tube bank, three rows of WVGs. 


\subsection{Conclusions from the Analysis of the Tube Bank Arrangement}

For the in-line tube-bank arrangement with the analyzed WVG configuration, the best results were achieved with one row of WVGs. Favorable HTE/FLP results were achieved across the range of Reynolds numbers $\left(660<\mathrm{Re}_{\mathrm{H}}<1630\right)$ analyzed, validating the tested WVG configurations for in-line oval tube bank arrangements. Additional rows of WVGs were not justified in this case since less favorable HTE/FLP results were obtained with additional rows of WVGs. Closer tube spacing may yield improved results.

Significantly better results were obtained for the staggered tube bank arrangement with the analyzed WVG configuration. For the staggered tube bank arrangement, the best HTE/FLP results were obtained with three rows of WVGs. HTE/FLP values were very impressive at $1.10,1.15$, and 1.16 for $\mathrm{Re}_{\mathrm{H}} \mathrm{values}$ of 660,1190 , and 1630 respectively. Inspection of surface heat transfer, velocity magnitude plots, and velocity vector plots for the staggered tube bank arrangement revealed that the tested WVG configurations resulted in highly complex flow and heat transfer mechanisms with a series of primary and induced vortices. In this sense, the analyzed WVG configuration had both secondary and primary influences on the flow patterns for a staggered tube bank arrangement. As in the case for the in-line tube bank arrangement, closer tube spacings may yield improved results. 


\section{YOKOHAMA NATIONAL UNIVERSITY EXPERIMENTAL WORK}

The following experimental work was performed by $\mathrm{K}$. Torii, his coworkers, and students at Yokohama National University, Yokohama, Japan. The work was funded by the university.

\subsection{Experimental Method and Procedure}

\subsubsection{Experimental Apparatus}

The present experimental apparatus for a modified single-blow method was designed and built based on the design given by Mochizuki et al. ${ }^{21}$ The experiments were performed in a small wind tunnel of open-circuit, with a vertical test section of dimensions of $150 \times 100 \times 300 \mathrm{~mm}^{3}$ (width $\times$ depth $\times$ length), as shown in Figure 5-1. The blower fan is driven by a variable-speed, $1.5-\mathrm{kW}$ electric motor to control the air velocity. The mean flow velocity in the test section can be varied from 0.5 to $3.5 \mathrm{~m} / \mathrm{s}$. A heating screen is made of stainless-steel ribbon heated directly by ohmic Joule heating and is uniformly spread over an entire cross section at the inlet of the test section so that it can heat the flow quickly and uniformly. The heating rate is controlled by a slide regulator. A bulk temperature of the flow at the inlet or outlet is measured directly by a special sensor without any cross-sectional integration of the flow temperature. The quick response sensor is made of a single Platinum wire $0.03 \mathrm{~mm}$ in diameter that is woven diagonally and spread over an entire cross section at the inlet or outlet. Temperature is derived from its electric resistance.

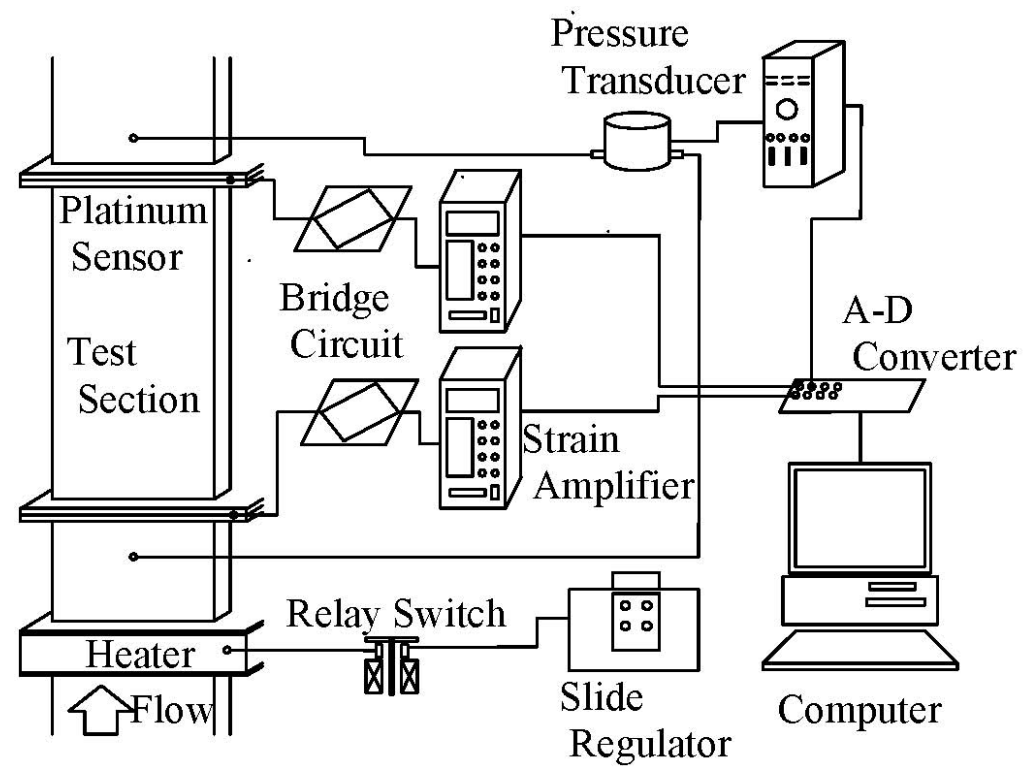

Figure 5-1. Schematic of YNU wind tunnel for transient method.

\subsubsection{Test Cores}

The geometrical parameters of the test-core simulate fin-tube heat exchangers, such as air-cooled condensers used in binary-cycle geothermal power plants. The test-cores of fin-tube bundles consist of 16 parallel plates representing the fins, and three rows of the circular tubes with in-line or staggered arrangements. The geometric arrangements of the test-cores are shown in Figure 5-2 and listed in Table 5-1. The fin pitch $\mathrm{H}$ is $5.6 \mathrm{~mm}$. Both streamwise and spanwise pitches of tube banks are equally set to $75 \mathrm{~mm}$. The vortex generators consist of delta winglet pairs made of 0.3-mm-thick Bakelite. The present configuration of the delta winglet pair is called a toe-in (common flow up) configuration. The base length, l, and height, $\mathrm{h}$, of the winglet are 30 and $5 \mathrm{~mm}$, respectively. In order to illustrate the 
favorable performance of the configuration proposed in the present study, the configuration with all three rows of winglet pairs shown in Figure 5-3 and Table 5-1 was also examined. This toe-out (common flow down) configuration of winglet pairs with a height/base length aspect ratio, $h / 1=1 / 2$, proposed by Fiebig et al. ${ }^{19}$ gave the best performance in their study.

Table 5-1. Summary of geometrical arrangements of test-cores and winglets.

\begin{tabular}{lcc}
\hline Variables & $\begin{array}{c}\text { Geometry proposed } \\
\text { by Torii et al. }\end{array}$ & $\begin{array}{c}\text { Geometry proposed } \\
\text { by Fiebig et al. }\end{array}$ \\
\hline $\mathrm{B} / \mathrm{H}$ & 13.39 & 13.39 \\
$\mathrm{~L} / \mathrm{H}$ & 13.39 & 13.39 \\
$\mathrm{D} / \mathrm{H}$ & 5.36 & 5.36 \\
$\mathrm{~L}_{\mathrm{1}} / \mathrm{D}$ & 1.25 & 1.25 \\
$\mathrm{D} / \mathrm{B}$ & 0.40 & 0.40 \\
$\mathrm{X}_{\mathrm{A}} / \mathrm{H}$ & 2.44 & 9.38 \\
$\mathrm{X}_{\mathrm{B}} / \mathrm{H}$ & 7.61 & 10.79 \\
$\mathrm{Y}_{\mathrm{A}} / \mathrm{H}$ & 1.19 & 4.02 \\
$\mathrm{Y}_{\mathrm{B}} / \mathrm{H}$ & 2.57 & 2.60 \\
$\mathrm{~h} / \mathrm{H}$ & 0.9 & 1.0 \\
$\mathrm{l} / \mathrm{D}$ & 1.0 & 0.37 \\
$\beta$ & $165^{\circ}$ & $135^{\circ}$ \\
\hline
\end{tabular}

a. In-line tube arrangement.

b. Staggered tube arrangement.
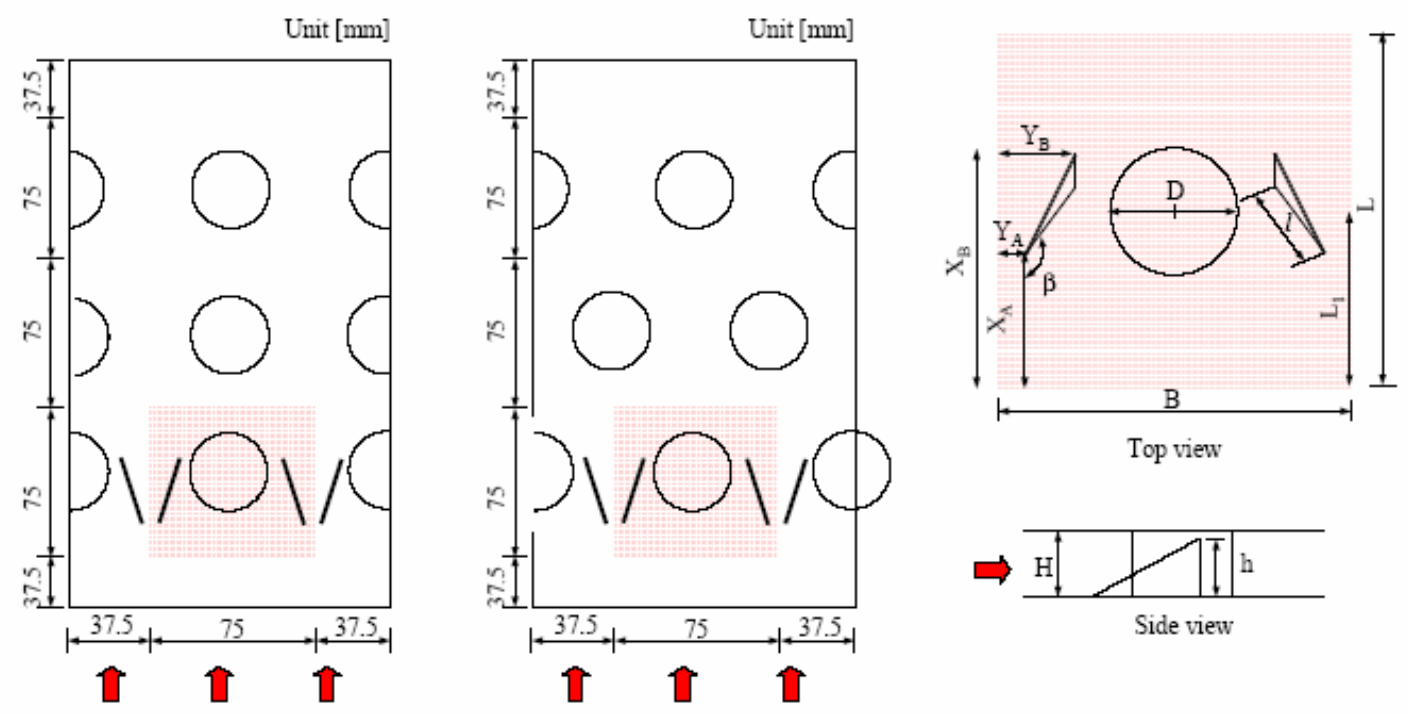

In-line tube bundle arrangement staggered tube bundle arrangement

Figure 5-2. Tube bundles and winglet arrangement proposed by Torii et al. ${ }^{54}$ 


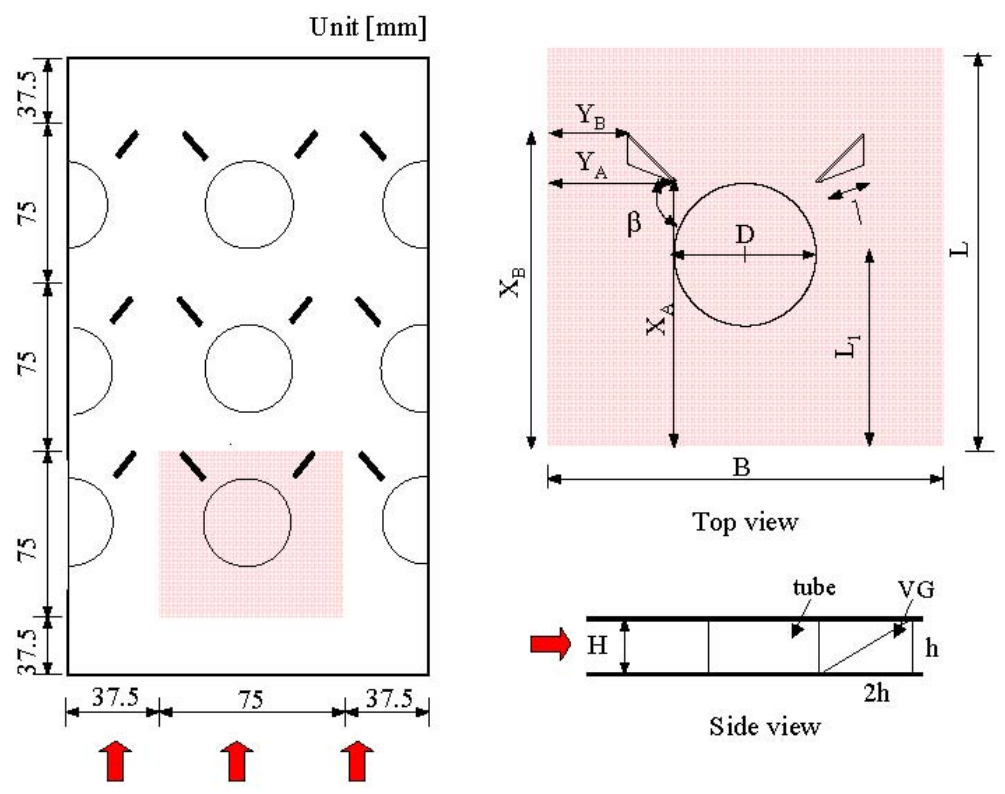

Figure 5-3. Tube bundle with winglet arrangement proposed by Fiebig et al. ${ }^{19}$

\subsubsection{Data reduction}

The experimental data are represented in terms of the Colburn factor, $\mathrm{j}$, and the Fanning friction factor, $\mathrm{f}$, as function of the Reynolds number, Re, as follows:

$j=\frac{N u}{\operatorname{Re} \cdot \operatorname{Pr}^{1 / 3}}, N u=\frac{h_{m} \cdot 2 H}{\lambda}, \operatorname{Re}=\frac{U_{i n} \cdot 2 H}{v}, f=\frac{2 H}{4 X}\left\{\frac{\Delta P}{\rho \mathrm{U}_{\text {in }}^{2} / 2}-\left(K_{c}+K_{e}\right)\right\}$

For determining heat transfer performance of a heat exchanger unit or surface, Liang and Yang ${ }^{24}$ developed the energy equations between the heat transfer surface and the fluid that vary with time and position along with flow passage. They determined experimentally the boundary condition that describes the time-wise change of the inlet fluid temperature, as follows.

$T_{f}^{*}\left(\theta^{*}, 0\right)=1-e^{-\theta^{*} / \tau^{*}}$

$\mathrm{Tf}^{*}$ is the dimensionless fluid temperature, and dimensionless time, $\theta^{*}$, is defined as the ratio of the physical time, $\theta$, to the time-constant, $\tau$ sys $=(\mathrm{mscs} / \mathrm{hA})$ of the solid-fluid system, where $\mathrm{ms}, \mathrm{cs}, \mathrm{h}$, and A are mass of solid, specific heat of solid, average heat transfer coefficient, and heat transfer area, respectively. $\tau^{*}$ is defined as $\tau$ in/ $/$ sys, in which $\tau$ in is the time-constant of the measured inlet fluid temperature to be determined experimentally. Employing the Laplace transform method, they obtained the following expression for fluid outlet temperature in two time-domains:

(i) when $\theta^{*}<\mathrm{t}^{*}$, or equivalently $\theta<\mathrm{L} / \mathrm{U}_{\mathrm{c}}$,

$\bar{T}_{f}^{*}\left(\theta^{*}, N T U\right)=0$ 
(ii) when $\theta^{*} \geq t^{*}$, i.e., $\theta \geq \mathrm{L} / \mathrm{U}_{\mathrm{c}}$,

$$
\begin{aligned}
& T_{f}^{*}\left(\theta^{*}, N T U\right)=\frac{1}{\tau^{*}} \int_{t^{*}}^{\theta^{*}} e^{-\left(\theta^{*}-\eta\right) / \tau^{*}-b_{2} t^{*}} \\
& \times\left\{e^{-\left(\eta-t^{*}\right)} J_{0}\left[2 \sqrt{b_{2} t^{*}\left(\eta-t^{*}\right)}\right]+\psi_{2}(\eta, N T U)\right\} d \eta
\end{aligned}
$$

where

$\mathrm{J}_{0}=$ the zero-order Bessel function of the first kind,

$$
\begin{aligned}
& \mathrm{t}^{*}=N T U / b_{1} \\
& \psi_{2}(\eta, N T U)=\int_{0}^{\eta-t^{*}} e^{-\xi} J_{0}\left[2\left(b_{2} t^{*} \xi\right)^{1 / 2}\right] d \xi \\
& \mathrm{t}^{*}=\text { dimensionless time defined as NTU/b1 } \\
& \mathrm{b} 1=U\left(m_{s} c_{s}\right) /\left(\dot{m}_{f} c_{f} L\right) .
\end{aligned}
$$

$\mathrm{U},(\mathrm{mscs}), \dot{m}_{f}$, cf, and L are mean velocity of fluid in test core, heat capacity of solid, mass flow rate of fluid, specific heat of fluid at constant pressure, and length of flow channel (heat transfer surface), respectively. $\mathrm{b} 2$ is the heat capacity ratio between the solid and the fluid defined as $\{(\mathrm{mscs}) /(\mathrm{mfcf})\}$. Uc is mean velocity based on the minimum cross-sectional area. Ac, $\xi$, and $\eta$ are dummy variables.

First, a measured inlet air temperature is fitted with Equation (5-2) at three periods of time: $\theta 1$, $\theta 2=2 \theta 1$, and $\theta 3=3 \theta 1$ to determine the values of the final steady inlet temperature, Tfinal, and $\tau$ in. The inlet temperature is recorded with a time step of $0.02 \mathrm{~s}$ for 1500 steps corresponding to $30 \mathrm{~s}$ after it starts rising. During the early period of time steps, it cannot follow Equation (5-2) but shows a response with a time lag, $\theta 0$. In order to avoid this period and find the appropriate period automatically, the following procedure is taken.

The time, $\theta 1$, is assumed to be $3 \mathrm{~s}$ as a first approximation and is increased by each time-step until the measured inlet temperatures can be fitted well with Equation (5-2) without introducing the time lag, $\theta 0$, within one time step. Hence, the measured inlet temperatures, Tf1, Tf 2 , and $\mathrm{Tf} 3$, at $\theta 1, \theta 2=2 \theta 1$, and $\theta 3=3 \theta 1$, respectively, satisfy Equation (5-2) and give the final temperature Tfinal, and the time constant tin by the following equations:

$T_{\text {final }}=T_{f 1}+\left(T_{\text {final }}-T_{f 2}\right)\left(\frac{T_{\text {final }}-T_{f 2}}{T_{\text {final }}-T_{f 3}}\right)^{\frac{\theta_{2}-\theta_{1}}{\theta_{3}-\theta_{2}}}, \quad \tau_{\text {in }}=\frac{\theta_{2}-\theta_{1}}{\ln \frac{T_{\text {final }}-T_{f 1}}{T_{\text {final }}-T_{f 2}}}$.

The obtained value of $\tau$ in ranges from 6.6 to $23 \mathrm{~s}$, and the values of $\theta 1 /$ in were found to be 0.16 to 0.53 for the present experiments.

By substituting the values of $\tau$ in, Tfinal, geometrical and physical properties of the flow channel, heat transfer surfaces, and an assumed value of heat transfer coefficient $h$, into theoretical Equation (5-4), the exit fluid temperature, Tf,th-at exit, is calculated and then compared with the measured value of fluid temperature at the exit, Tf,exp-at exit. If the difference between Tf,th-at exit and Tf,exp-at exit is within 
an acceptable degree of accuracy less than $0.1 \%$, then the assumed value of $\mathrm{h}$ is considered to be correct. However, if the theory fails to agree with the measured response, a new value of $h$ is picked, and the procedure is repeated until the correct value of $h$ is found. These calculations are performed to get the average value of the heat transfer coefficient, $h$, at ten points of time in the neighborhood of $\tau$ in for each experiment. The assumption that a temperature gradient is negligible inside the fin is acceptable, since the Biot number, $\mathrm{hm} \delta / 2 \lambda \mathrm{f}$, based on the measured, $\mathrm{hm}$, and fin thickness, $\delta$, is of the order of $10^{-6}$.

\subsubsection{Experimental Results and Discussion}

The Reynolds number, $\mathrm{Re}=(\mathrm{Uin} 2 \mathrm{H}) / v$, based on the hydraulic diameter of the test-core inlet varies from 350 to 2400 . The average heat transfer coefficient of the test-core is defined by using its heat transfer area, excluding the surface area of tube and vortex generators. Figure 5-4 shows the performance of an in-line tube-bundle with the toe-out (common flow down) winglet configuration proposed by Fiebig et al. ${ }^{19}$ and shown in Figure 5-3. Heat transfer enhancement, $\mathrm{j} / \mathrm{j}$ Go, where the subscript G0 denotes inline tube-bundle without vortex generator, and the pressure-loss penalty, $\mathrm{f} / \mathrm{fGo}$, are shown with respect to a range of Reynolds number. The vortex generators cause 10 to $25 \%$ heat transfer enhancement and also 20 to $35 \%$ increase in the pressure-loss penalty in comparison with a tube-bundle without vortex generators. In addition, this configuration is found to be not so effective in heat transfer enhancement in a low Reynolds number range as in a high Reynolds number range. Because geothermal air-cooled condensers operate at a low Reynolds number $(\sim 1000)$, heat transfer enhancement in a laminar flow of a low Reynolds number necessitates an innovative concept being somewhat different from a concept effective in a high Reynolds number flow. O'Brien and Sohal ${ }^{22}$ investigated the local heat transfer in a single passage of narrow rectangular duct fitted with a circular tube with and without a delta-winglet pair using imaging infrared camera. The location and aspect ratio of the winglets were the same as shown in Figure 5-3. The present concept is to create a constricted passage between the tube and the winglets with a toe-in (common flow up) configuration (Figure 5-2), which induces flow acceleration as well as vortices and turbulence,. This flow acceleration brings about separation delay from the tube, reduces form drag across the tube, and finally reduces the zone of poor heat transfer from the wake zone of the tube, without an excessive amount of pressure loss penalty. As shown in Figure 5-5, the delta winglets with toe-in (common flow up) configuration in a fin-tube bundle in an in-line tube arrangement successfully increase the average heat transfer by 10 to $20 \%$, and simultaneously decrease the pressure drop by 8 to $15 \%$. It is worth noting that such a remarkable performance can be brought about by a single front row of the winglet pairs in three rows of tube banks, comparing that the performance shown in Figure 5-4 is achieved by means of all three rows of winglet pairs.

A much better performance (Figure 5-6) was achieved in a

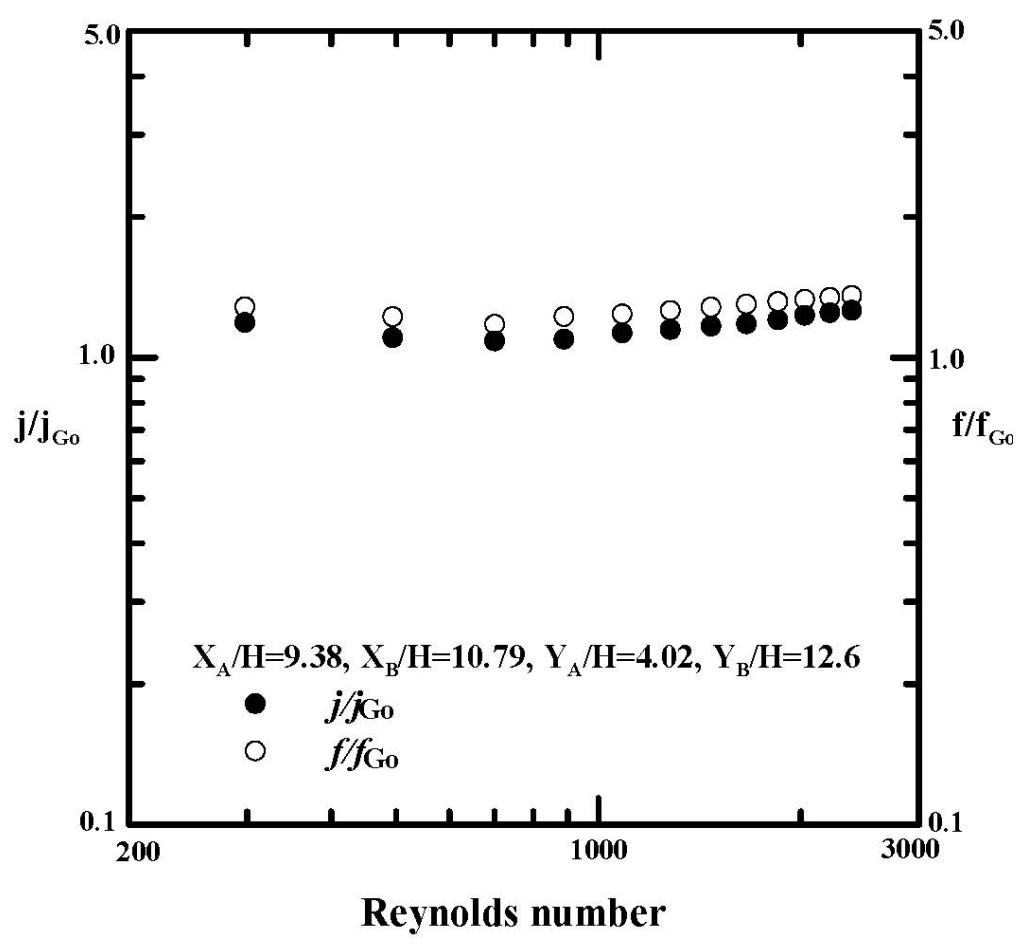

Figure 5-4. The comparison of $\mathrm{j} / \mathrm{j}_{\mathrm{Go}}$ and $\mathrm{f} / \mathrm{f}_{\mathrm{Go}}$ with respect to Reynolds number for the winglet configuration suggested by Feibig et al. ${ }^{19}$ (Figure 5-3). 
staggered tube bundle arrangement with the same winglet configuration as in the in-line tube bundle arrangement.

The heat transfer enhancement $(\mathrm{j} / \mathrm{jGo})$ of 10 to $30 \%$ is achieved together with a pressure-loss reduction ( 1 - $\mathrm{f} / \mathrm{fGo}$ ) of 34 to $55 \%$. It is a favorable performance that the pressure-loss reduction gets better with a lower Reynolds number. For a Reynolds number of 350, the pressure-loss reduction of 55\% is achieved together with the heat transfer enhancement of $30 \%$. This may be explained as follows. The nozzle-like flow passages created by the delta winglet pair and the aft region of the tube promote flow acceleration to bring about delay in flow separation and thereby removes the zone of poor heat transfer from the near wake region. This flow structure has been confirmed by visualizing the flow by means of Particle Image Velocimetry. ${ }^{56}$ In the absence of any winglet, a wake behind the tube gets weaker in vortex motion and develops a wider, longer zone of poor heat transfer as the Reynolds number decreases. When the wake can travel down to the next row, its length in the staggered arrangement gets twice as long as in the in-line one. Hence, the present configuration delta winglet pair improves the performance for a low Reynolds number more for the staggered tube bundle case than for the in-line tube bundle case.

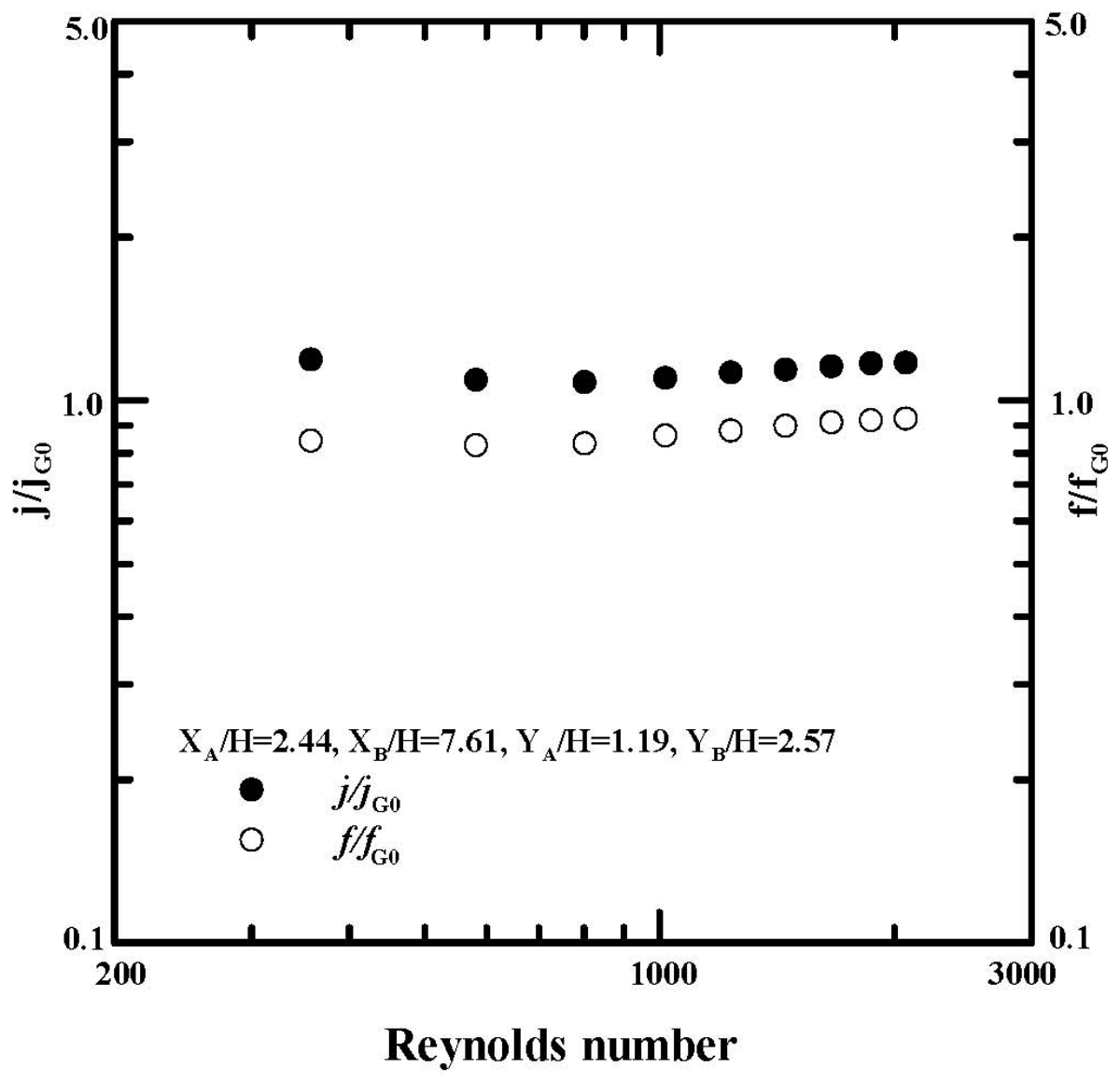

Figure 5-5. Comparison of $\mathrm{j} / \mathrm{jGo}$ and $\mathrm{f} / \mathrm{fGo}$ with respect to the Reynolds number for the in-line tube bundle and a winglet configuration suggested by Torii et al. ${ }^{54}$ (Figure 5-2).

\subsubsection{Concluding Remarks}

The present experiments verify that the present technique combining a circular tube with the delta winglet pair of the toe-in (common flow up) configuration enables enhancing the heat transfer together with a significant amount of pressure loss reduction. The winglet configuration is different from a 
conventional winglet design. The nozzle-like flow passages created by the delta winglet pair and the aft region of the circular tube promote acceleration to bring about a separation delay and form drag reduction of the tube, and remove the zone of poor heat transfer from the wake. In case of staggered tube bundle, the heat transfer was augmented by 30 to $10 \%$, with the winglet pairs of the present configuration, and yet the pressure loss was reduced by 55 to $34 \%$, for the Reynolds number (based on two times the channel height) ranging from 350 to 2100 . In case of in-line tube bundle, the heat transfer was augmented by 20 to $10 \%$ together with the pressure loss reduction of 15 to $8 \%$ in the same range of Reynolds number. Applying the configuration proposed by Fiebig et al., ${ }^{19}$ the heat transfer was augmented by 25 to $10 \%$, but the pressure loss was also increased by 35 to $20 \%$ in the same Reynolds number range.

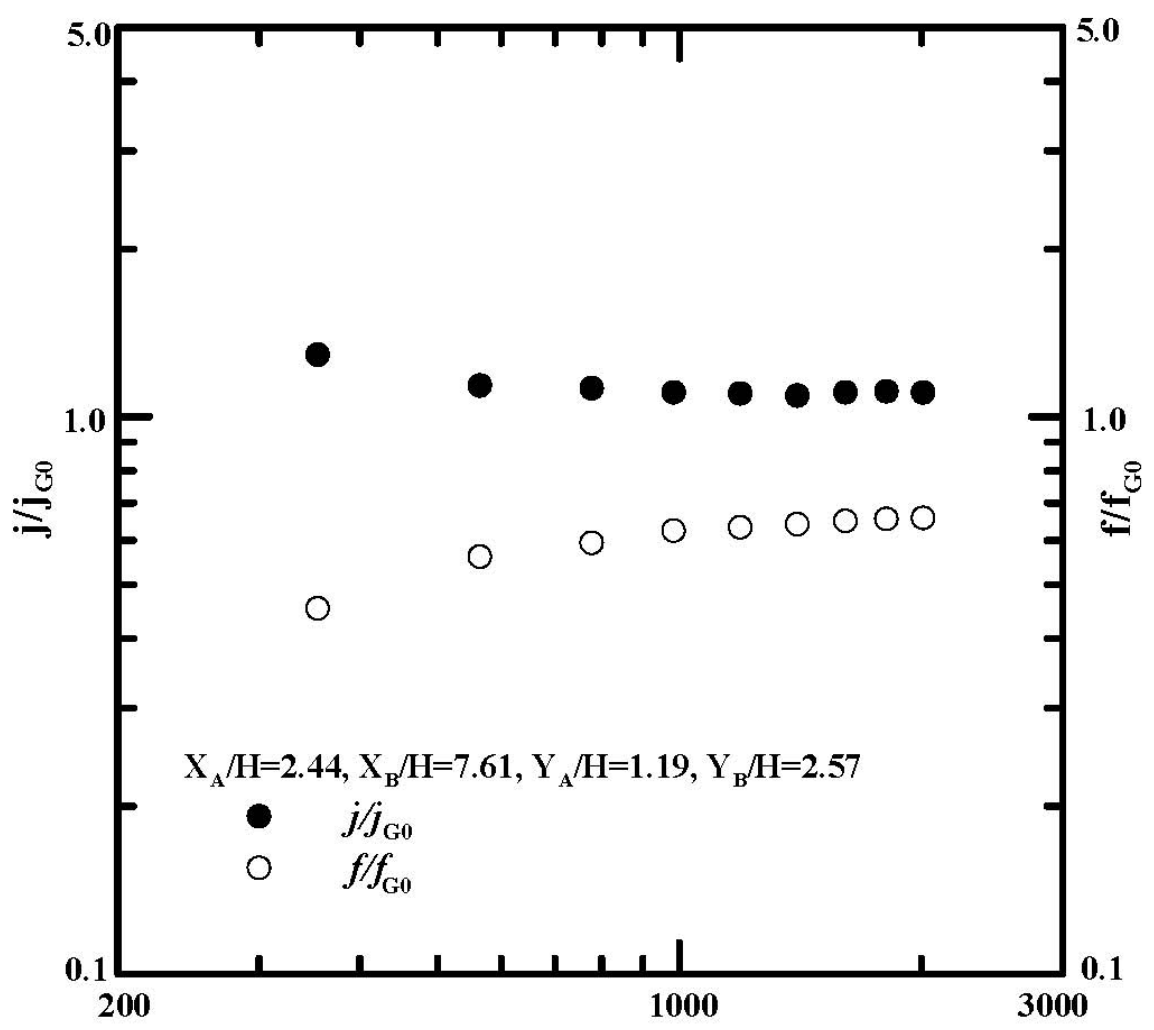

\section{Reynolds number}

Figure 5-6. Comparison of $\mathrm{j} / \mathrm{j}_{\mathrm{Go}}$ and $\mathrm{f} / \mathrm{f}_{\text {Go }}$ with respect to the Reynolds number for the staggered tube bundle and a winglet configuration suggested by Torii et al. ${ }^{54}$ (Figure 5-2).

\subsection{Local Heat Transfer Measurements}

\subsubsection{Introduction}

The purpose of measurement by imaging infra-red (IR) camera is to grasp the heat transfer coefficient distribution of the fin-tube bundles with vortex generators and to make use of results of visualization to find the optimal configuration of fin-tube bundles and vortex generators. This experiment reveals visual and quantitative details of local fin-surface heat transfer distributions in the vicinity of a circular tube and a combination of a circular tube and a delta-winglet pair. Comparisons of local heat transfer distributions for the circular tube with and without winglet are provided. The experimental technique used here is similar to the one used by INL researchers described in Section 2. 


\subsubsection{Experiment apparatus}

A transient heat transfer measurement technique was employed for obtaining detailed local heat transfer measurements on the model fin surface. The transient heat transfer experiments were performed in a narrow rectangular duct designed to simulate a single passage of a fin-tube heat exchanger. A drawing of the test section is shown in Figure 5-7 with three rows of tube-banks and a single row of vortex generators in place. Dimensions of the duct are, width, $\mathrm{W}=150 \mathrm{~mm}, \mathrm{H}=6 \mathrm{~mm}$. The duct was fabricated primarily out of acrylic resin. The test section length was $450 \mathrm{~mm}$, yielding $\mathrm{L} / \mathrm{H}=75$. The thickness of the acrylic resin wall is $10 \mathrm{~mm}$. A flow development section with $\mathrm{L} / \mathrm{H}=30$ was located upstream of the test section. Consequently, depending on Reynolds number, the flow is about hydrodynamically fully developed as it enters the test section. In order to enable thermal visualization of the test section, the bottom duct surface (representing the fin surface), using an imaging infrared camera, the top wall of the duct in the vicinity of the tube banks was formed by a $\mathrm{ZnSe}$ window, $100 \times 100 \mathrm{~mm}$ $\times 5 \mathrm{~mm}$. The transmissivity of the $\mathrm{ZnSe}$ window is nearly over $90 \%$ (in the wavelength range of 8 to $14 \mu \mathrm{m})$. The test section bottom surface was painted black in order to achieve a surface emissivity very close to 1.0. Temperatures indicated by the IR camera were calibrated with the surface temperature measured using a thermocouple embedded in a black-painted test surface.

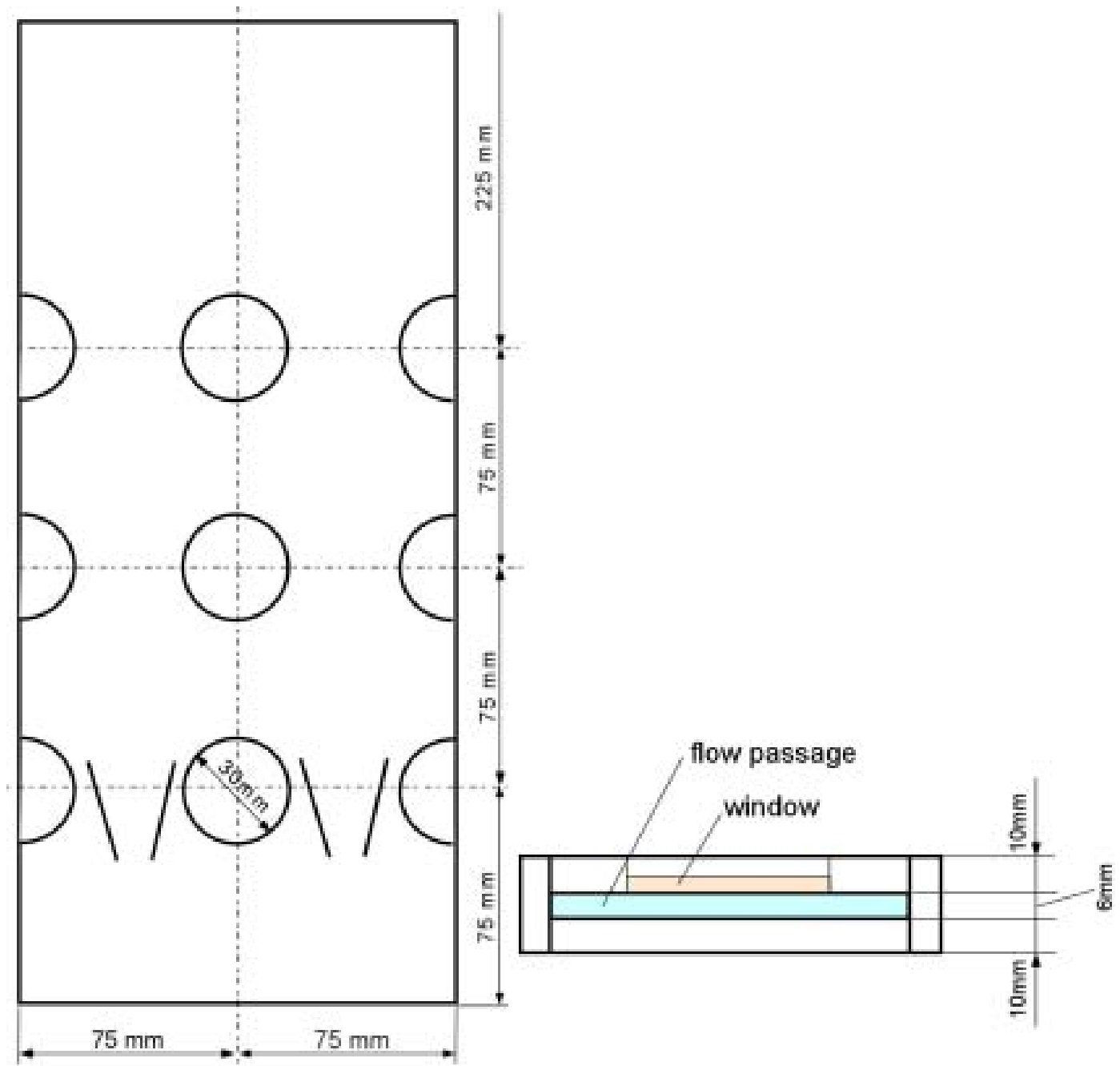

TOP VIEW

SIDE VIEW

Figure 5-7. Test section for transient heat transfer measurements. 
Figure 5-8 is a schematic diagram of the experimental apparatus for measuring local heat transfer coefficient by a transient technique. Inlet air is suddenly heated to a desired setpoint temperature using the same heater as in the transient measurements of overall heat transfer. The air temperature is measured with the thermocouple installed in the center of flow development section. Using this apparatus, the room-temperature fin-tube model is suddenly exposed to a uniformly heated airflow. Local surface temperatures on the model surfaces increase at a rate that depends on the value of the local heat transfer coefficient. This transient local heating is quantitatively recorded using an imaging infrared camera. The value of local heat transfer coefficients can then be determined from an inverse heat conduction analysis. Air temperature uniformity across the duct was verified by the images of the surface temperature obtained by IR camera during preliminary tests with no tube in place.

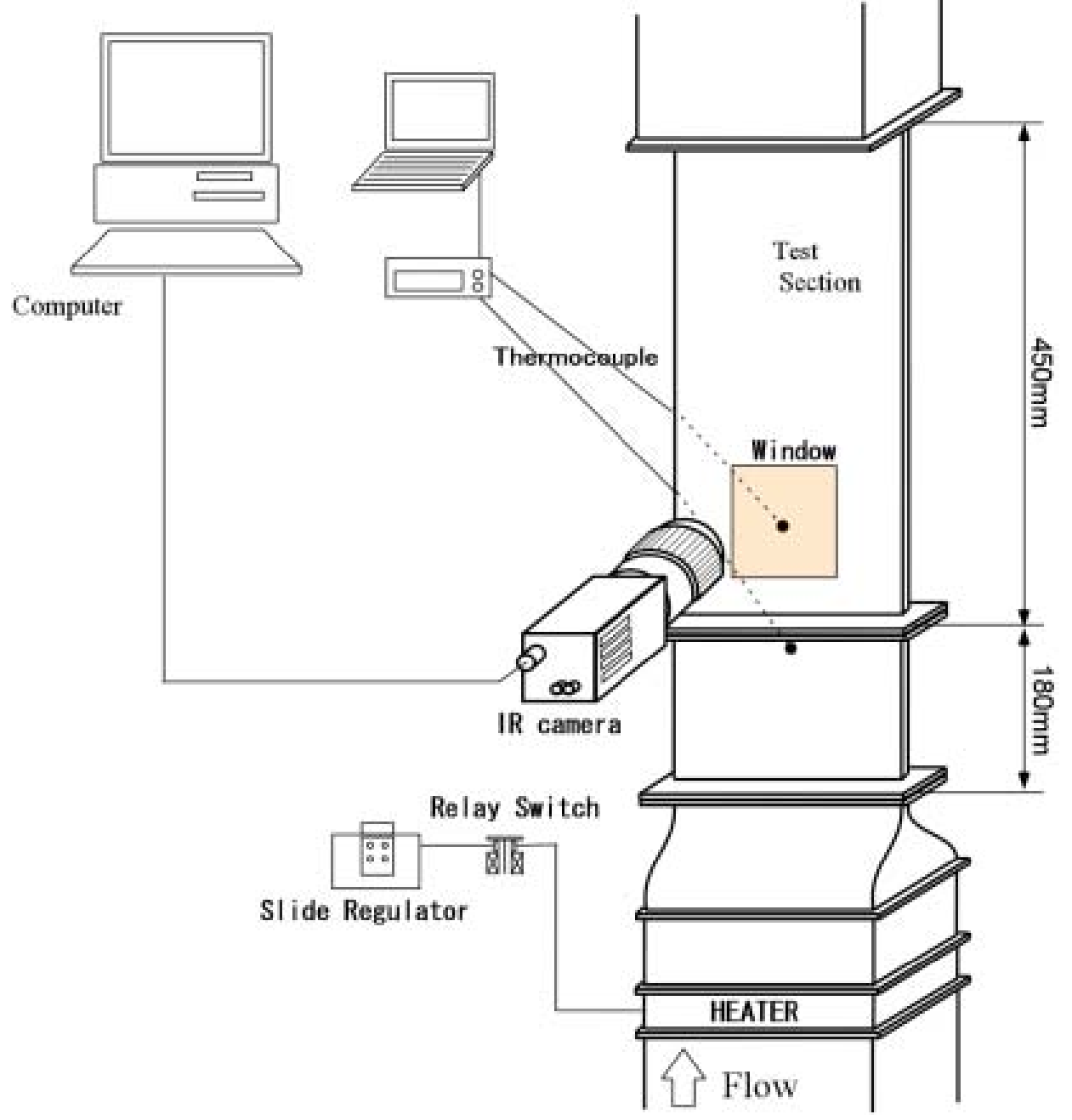

Figure 5-8. Schematic of the experimental apparatus for measuring local heat transfer.

Quantitative thermal visualization images were obtained using a precision imaging infrared camera (Nippon Avionics Co., Ltd., NeoThermo TVC-610). This camera uses a noncooling two-dimensional micro-bolometer array detector, $320 \times 240$ pixel. The camera can be used to measure infrared intensities, corresponding to temperature in the -20 to $150^{\circ} \mathrm{C}$ range, with a minimum discernible temperature difference of $0.1^{\circ} \mathrm{C}$ at $30^{\circ} \mathrm{C}$. The binary data of radiometric information from the camera is stored in the hard disk of PC via the IEEE 1394 bus. The thermal image data files created by the camera onboard 
processor are in a special TVS format. These files can also be outputted in a BMP file format, being divided into each frame.

\subsubsection{Experimental Procedure}

After the IR camera is powered up, it is positioned above the center of the observation window. In order to avoid IR reflections of the warm camera body off the $\mathrm{ZnSe}$ window, the camera is positioned at a small angle off the vertical. As shown in Figure 5-8, two thermocouples for the flow and model surface temperatures are connected to data acquisition system module (KEYENCE, NR-250). Flow is initiated by adjusting the blower speed until the desired flow rate is observed. At this time, the data acquisition system of the IR camera and thermocouples is set to begin writing data to the hard disk of the PC. The heater is then switched on to heat the air flow quickly up to a constant temperature of about $50^{\circ} \mathrm{C}$ within $3 \mathrm{~s}$. A number of thermal images of the test section (typically 1800 frames) are acquired during the first 180 seconds of the transient.

\subsubsection{Data reduction}

In order to obtain local heat transfer coefficients from the surface temperatures measured during the transient heat-up of the test section, the bottom surface of the test section is assumed to behave locally as a one-dimensional semi-infinite solid undergoing a step change in the flow temperature. Initially, the wall is at a uniform temperature, $\mathrm{Ti}$, and at time zero the surface is suddenly exposed to the flow temperature of $\mathrm{T}=\mathrm{T} \infty$. For constant properties, the differential equation for the temperature distribution is

$$
\frac{\partial^{2} T}{\partial x^{2}}=\frac{1}{\alpha} \frac{\partial T}{\partial \tau} .
$$

The boundary condition is

$h A\left(T_{\infty}-T\right)_{x=0}=-k A{\frac{\partial T}{\partial x_{x=0}}}$.

The equations given here are identical to Equations (2-1) through (2-4) in Section 2. The solution for these equations is similar to the same equations. The result is

$$
\frac{T-T_{i}}{T_{\infty}-T_{i}}=1-\operatorname{erf}(X)-\left[\operatorname{Exp}\left(\frac{h x}{k}+\frac{h^{2} \alpha \tau}{k^{2}}\right)\right] \times\left[1-\operatorname{erf}\left(X+\frac{h \sqrt{\alpha \tau}}{k}\right)\right]
$$

where

$$
\mathrm{Ti}=\text { initial temperature of solid }
$$

T $\infty=$ environment temperature

$X=x /(2 \sqrt{\alpha \tau})$.

Therefore, the time-dependent surface temperature is

$$
\frac{T-T_{i}}{T_{\infty}-T_{i}}=1-\left[\operatorname{Exp}\left(\frac{h^{2} \alpha \tau}{k^{2}}\right)\right] \times\left[1-\operatorname{erf}\left(\frac{h \sqrt{\alpha \tau}}{k}\right)\right] .
$$


This equation represents the relationship between heat transfer coefficient and surface temperature measured at a specific time after the start of the test. It solved by original program of iterative calculation for $h$.

\subsubsection{Preliminary Test}

The IR camera was calibrated over a wide temperature range by comparing camera-indicated temperatures with surface temperatures measured using a precision thermocouple embedded in a blackpainted test surface, as shown in Figure 5-9. The calibrated temperature is given by the following equation represented by a solid line in Figure 5-9 as

$T_{I R}^{*}=1.34 T_{I R}-96.99$

$T_{I R}^{*}:$ Actual temperature calculated using calibration curve $(\mathrm{K})$

$T_{I R}:$ Temperature reading obtained by the IR camera $(\mathrm{K})$

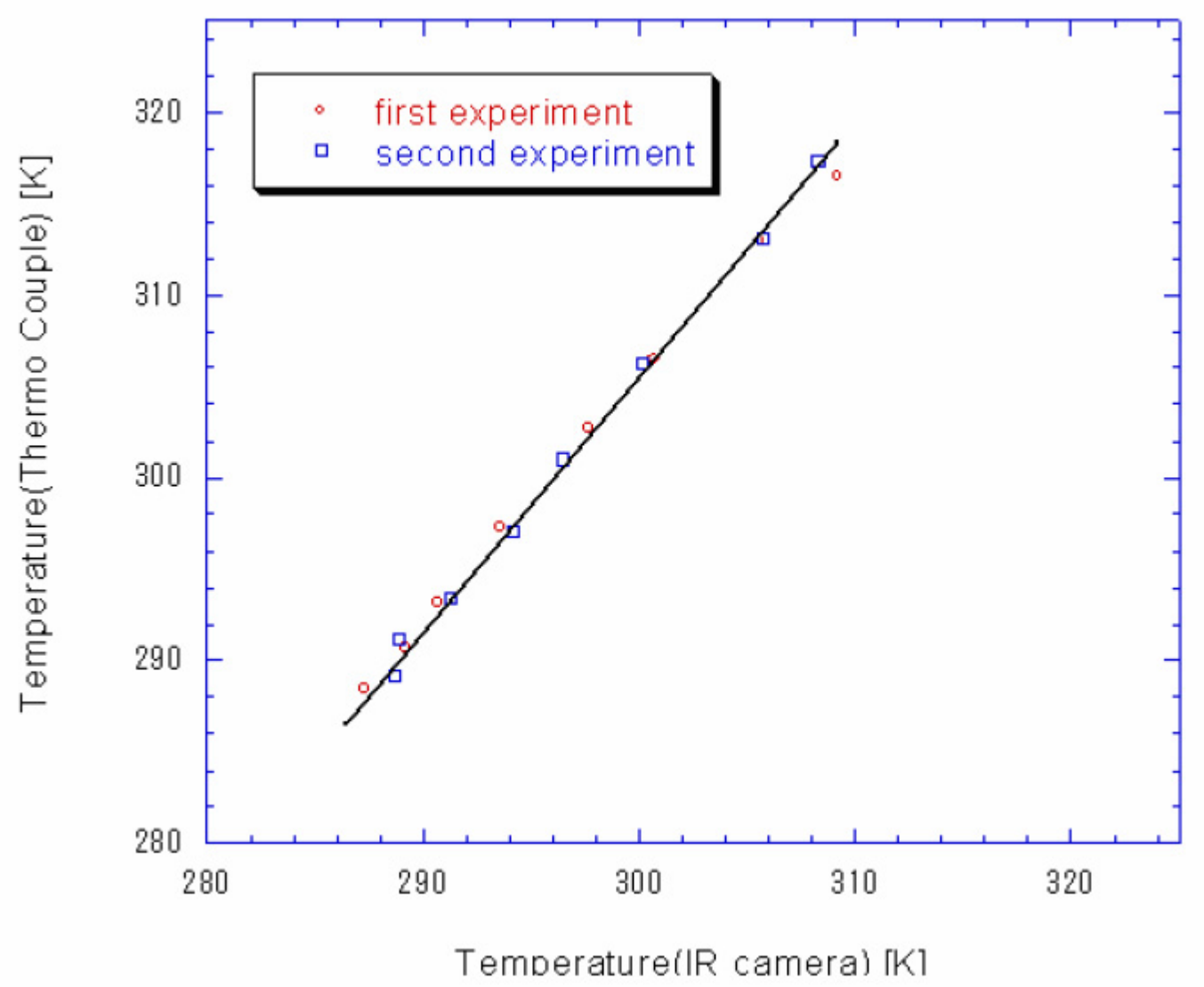

Figure 5-9. Calibration curve for calculating the temperature from an IR camera measurement.

\subsubsection{Results and Discussion}

In order to elucidate the effect of the proposed type of vortex generator on heat transfer around tube bundles, first a single row of tube bundles with winglet pairs was tested, the geometrical conditions of which are illustrated in Figure 5-10. Figure 5-11 compares the experimental results with the 
computational results of G. Biswas, a member of the NEDO project team of the Indian Institute of Technology, Kanpur, India.
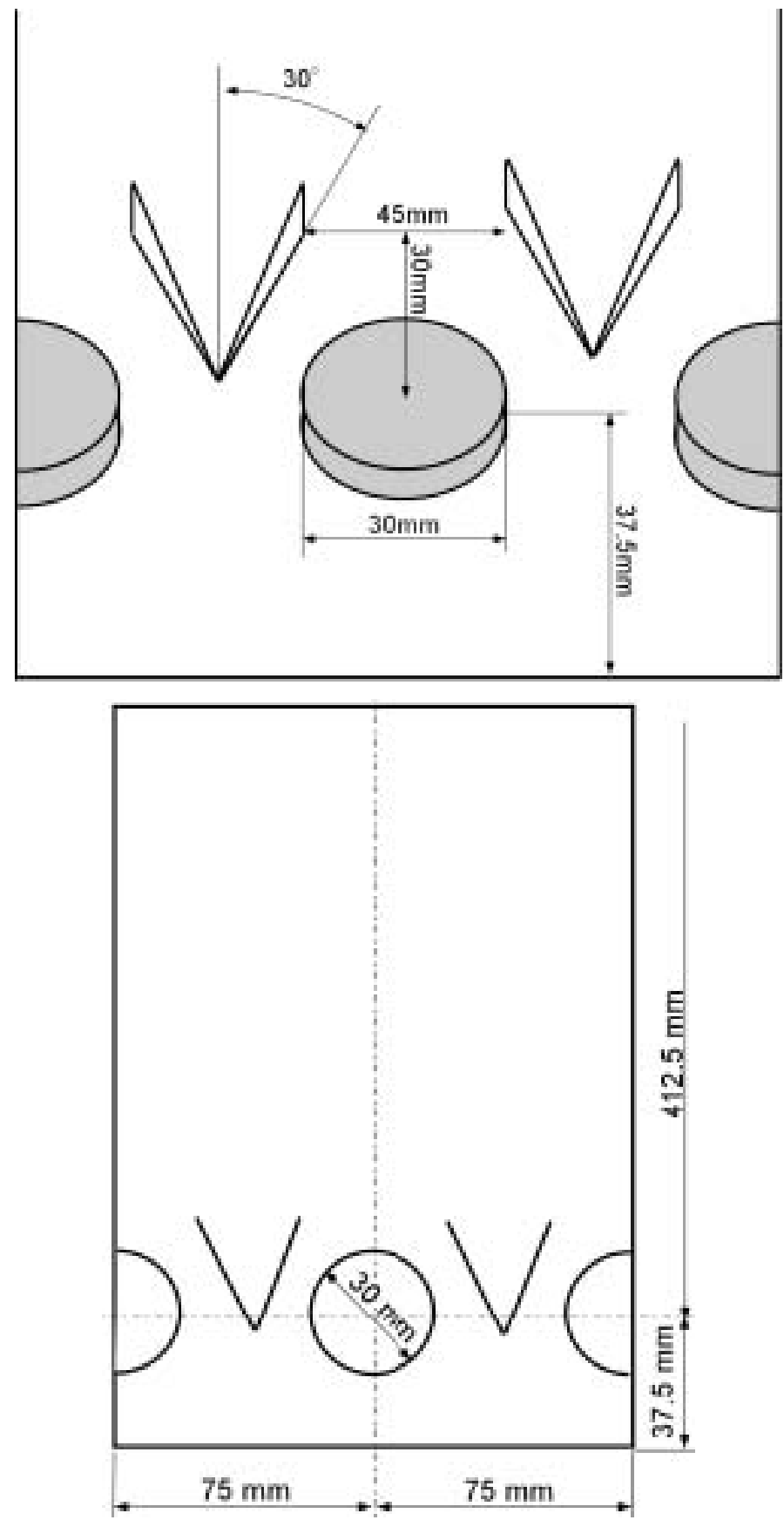

Figure 5-10. Geometrical details of test section with finned tubes and winglets.

The enhancement is expressed in terms of the ratio of $\overline{N u_{S}}$ and $\overline{N u_{S 0}}$. Here, $\overline{N u_{S}}$ is the spanaveraged Nusselt number at any axial location $\mathrm{x}$ for the case of a channel with built-in circular tubes and vortex generators. Similarly, $\overline{N u_{S 0}}$ is the span-averaged Nusselt number at any axial location $\mathrm{x}$ for the case of a channel with circular tubes without vortex generators. The maximum heat transfer enhancement 
takes place at the trailing edge of the winglet. In general, the computation and experimental results compare well, especially at the downstream region of the trailing edge.

Second, a three-row tube bundle with built-in winglet pairs only in the (front) first row (Figure 5-12) was examined. This configuration was found to give the best performance for heat transfer enhancement in the all the experiments presented in Section 5.1. The winglet pair is located where the spanwise distance between the trailing edge of the winglet and the cylindrical surface, $\mathrm{s}$, is $9 \mathrm{~mm}$.

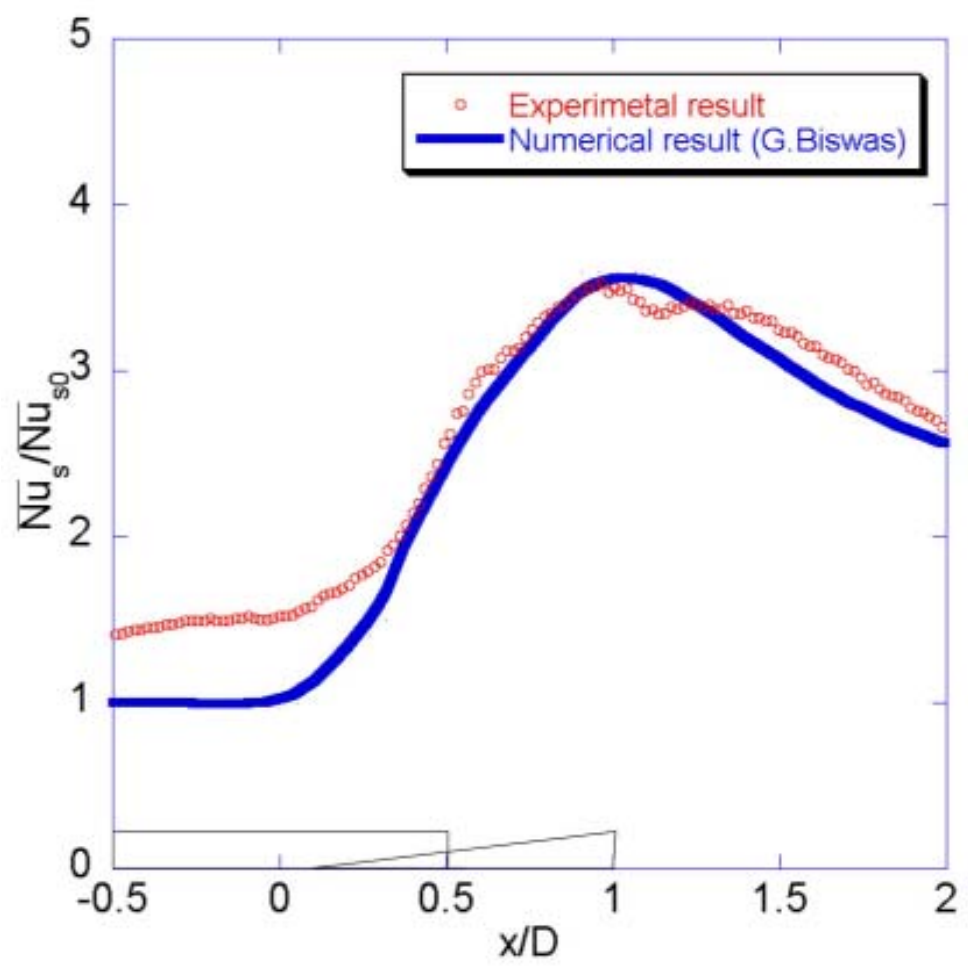

Figure 5-11. The ratio of span-averaged Nusselt number distributions with and without vortex generators for $\mathrm{Re}_{\mathrm{H}}=1320$.

The angle of attack is $\alpha=15$ degrees, and the tube center angle is $\beta=110$ degrees. Figure $5-12$ shows the distributions of heat transfer on the bott,om surface of the test channel for $\mathrm{ReH}=2300$, based on channel height, $\mathrm{H}$. The enhancement is expressed in terms of the ratio of the local Nusselt numbers, $\mathrm{Nu} / \mathrm{Nu} 0$, for with and without winglets.

Figure 5-13 shows the enhancement distribution of span-averaged Nusselt number on the bottom surface of the channel with vortex generators, $\overline{N u_{S}} / \overline{N u_{S 0}}$. The heat transfer enhancement reaches the maximum value near the middle between the first and second rows of the tube and persists far downstream up to the third row.

The spanwise variations of the enhancement of local Nusselt number with vortex generators, $\mathrm{Nu} / \mathrm{Nu} 0$, are presented in Figures 5-14 through 5-16. At $\mathrm{x} / \mathrm{D}=0.7$ behind the first row of tube, four very high peaks of heat transfer enhancement are attributed to main vortices induced by vortex generators. At $\mathrm{x} / \mathrm{D}=3.2$ behind the second row of tubes, the four peaks caused by the winglet pairs of the first tube-row have merged into two broader peaks, resulting from the formation of two longitudinal vortices, that enhance the span-averaged heat transfer about 50\%. At $\mathrm{x} / \mathrm{D}=5.7$ behind the third row, the heat transfer 
of the so-called dead zone is still enhanced well between $y / D=-0.4$ and 0.4 by the winglet pairs of the first row.

\subsubsection{Conclusions}

Local Nusselt number distributions on the bottom surface of the channel with built-in tube-bundles with winglet-pairs simulating a single passage of a fin-tube heat exchanger were obtained using an infrared camera by a transient heat transfer measurement technique. For the case of the channel with built-in a single row of tube bundles with winglet pairs, the experimental results show good agreement with the computation made by G. Biswas. For three rows of tube bundles with a single front row of winglet pair, the details of the heat transfer enhancement distribution were obtained. The heat transfer enhancement of span-averaged Nusselt number reaches the maximum value near the middle between the first and second rows of the tube, and persists far downstream up to the third row. Even behind the third tube-row, the heat transfer of the so-called dead zone, was enhanced well by the winglet pairs.
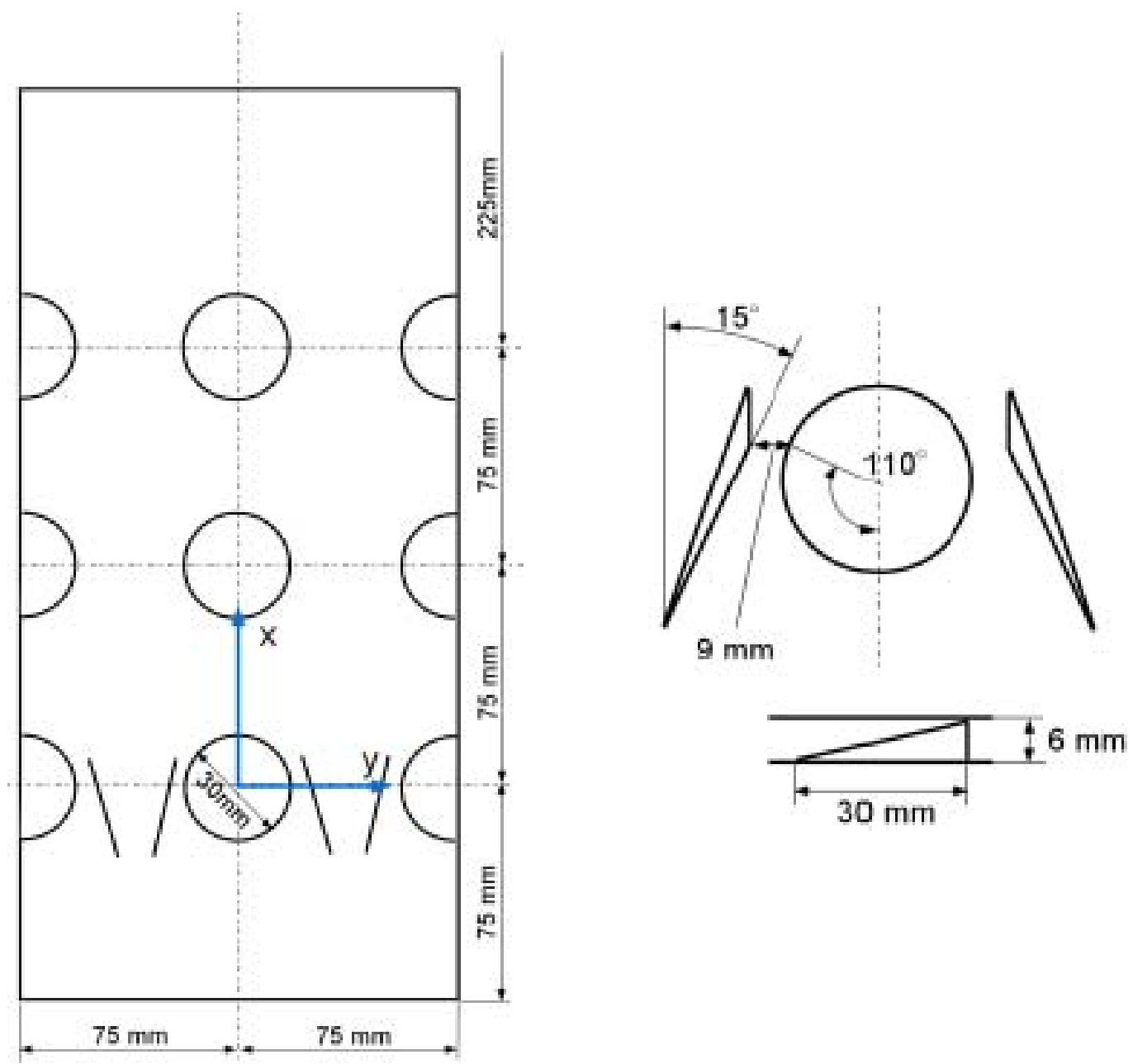

Figure 5-12. Geometrical details of a test section with finned tubes and proposed winglets. 


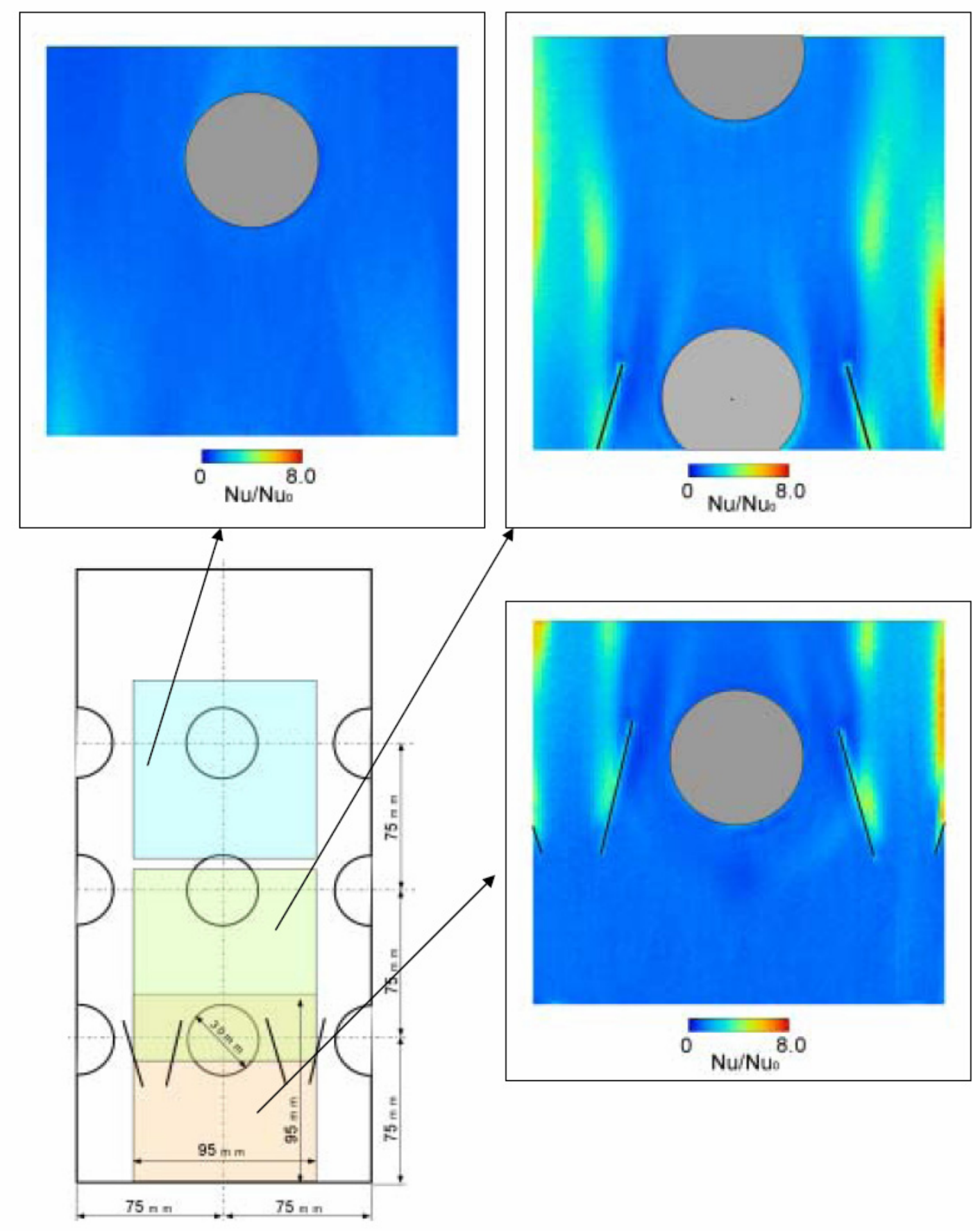

Figure 5-13. The ratio of local Nusselt numbers with and without vortex generators on the bottom surface of the channel for $\mathrm{Re}_{\mathrm{H}}=2300$. 


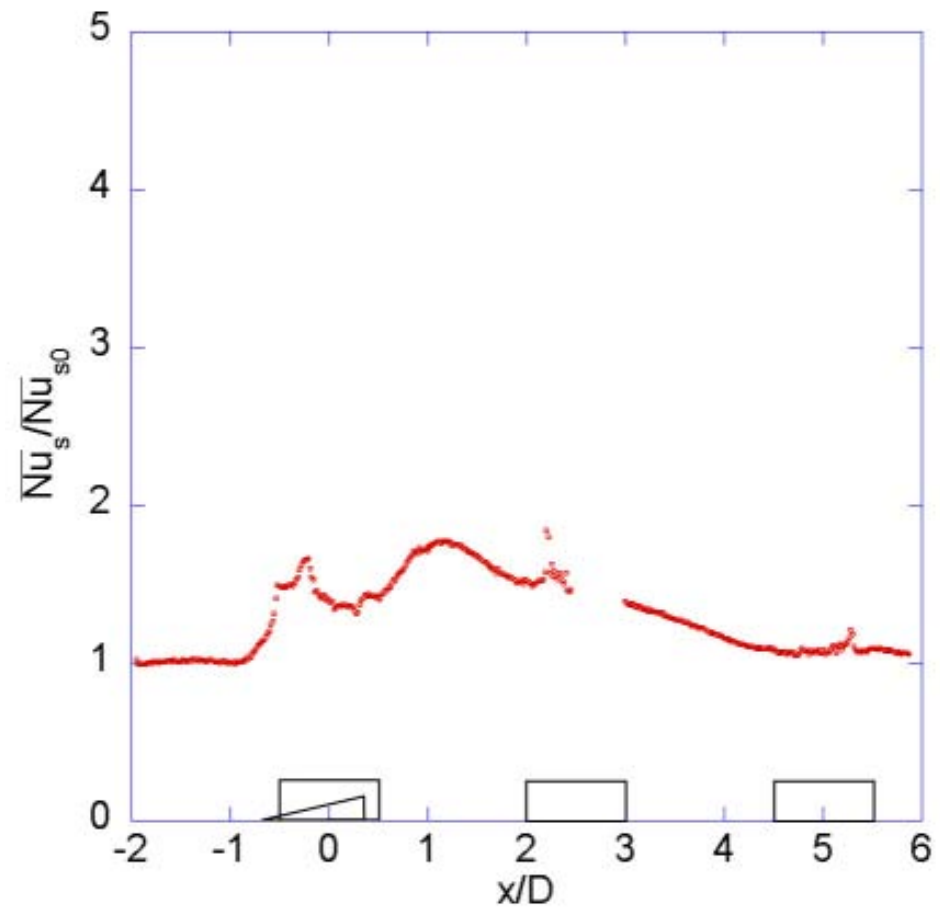

Figure 5-14. Longitudinal variation of the heat transfer enhancement in terms of the Nusselt number ratio for $\mathrm{Re}_{\mathrm{H}}=2300$.

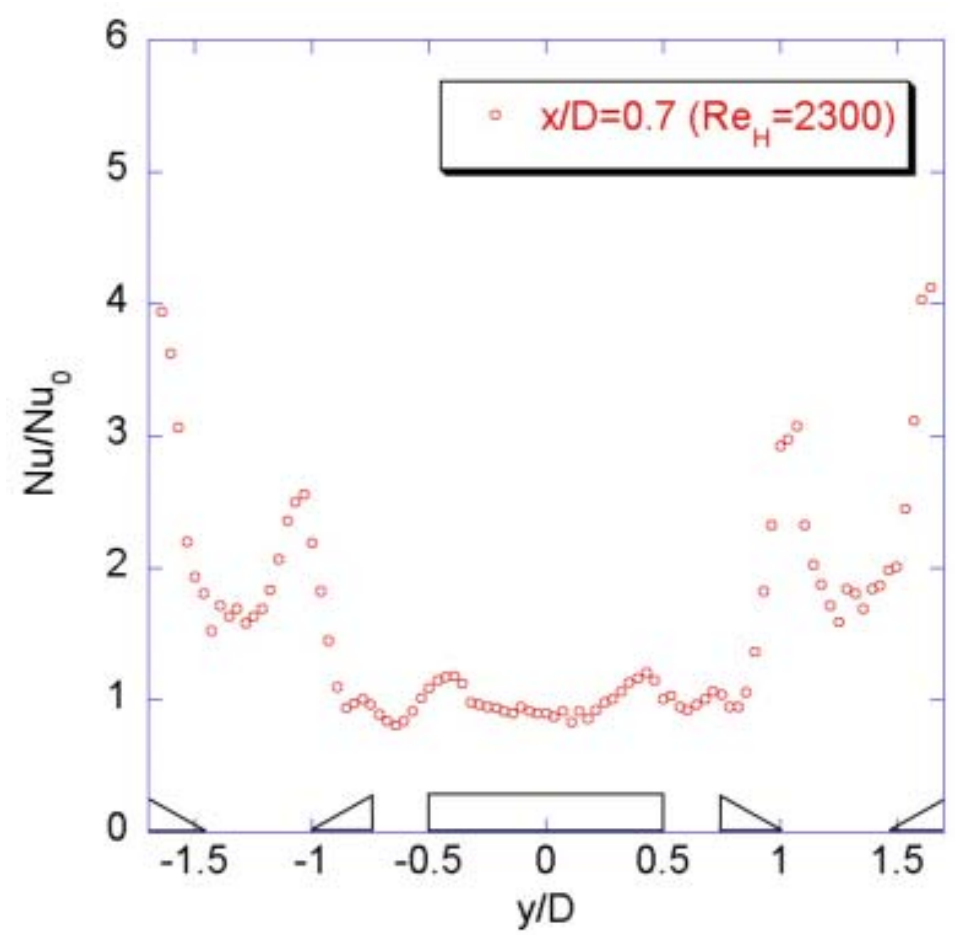

Figure 5-15. Spanwise variation of the heat transfer enhancement in terms of the Nusselt number ratio at $\mathrm{x} / \mathrm{D}=0.7$ for $\mathrm{ReH}=2300$. 


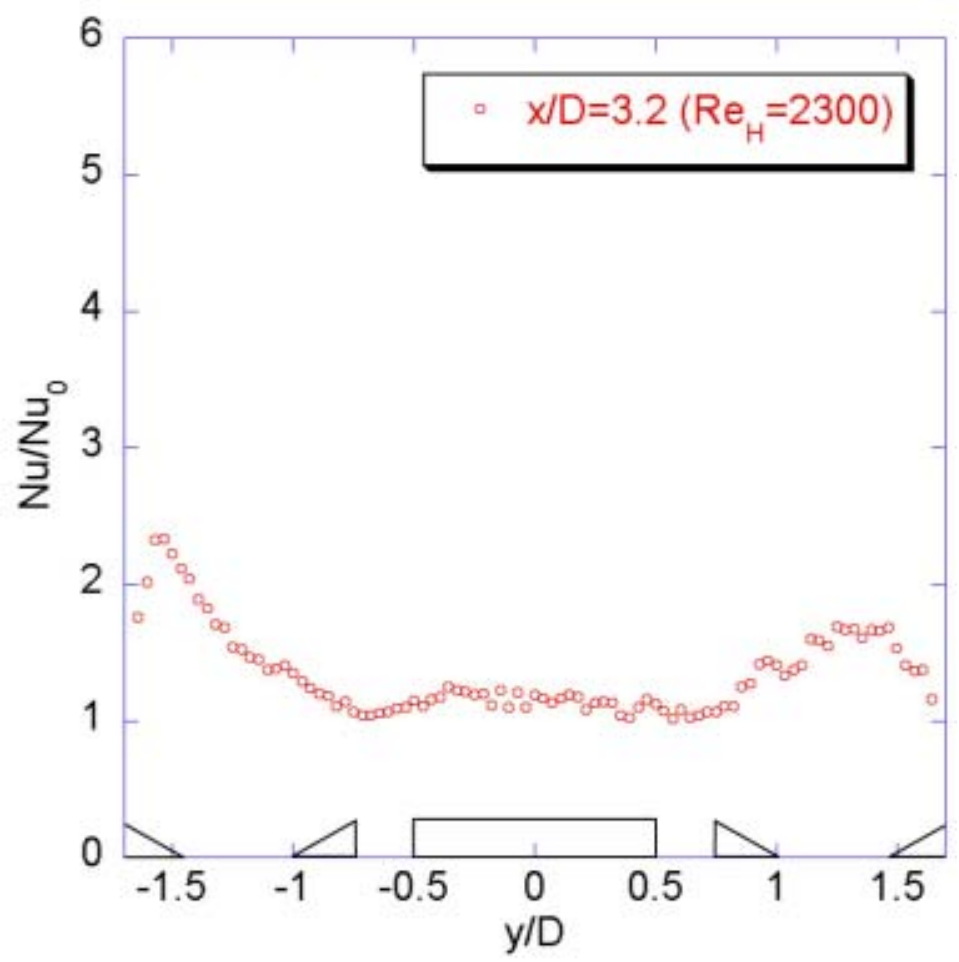

Figure 5-16. Spanwise variation of the heat transfer enhancement in terms of the Nusselt number ratio at $\mathrm{x} / \mathrm{D}=3.2$ for $\mathrm{ReH}=2300$.

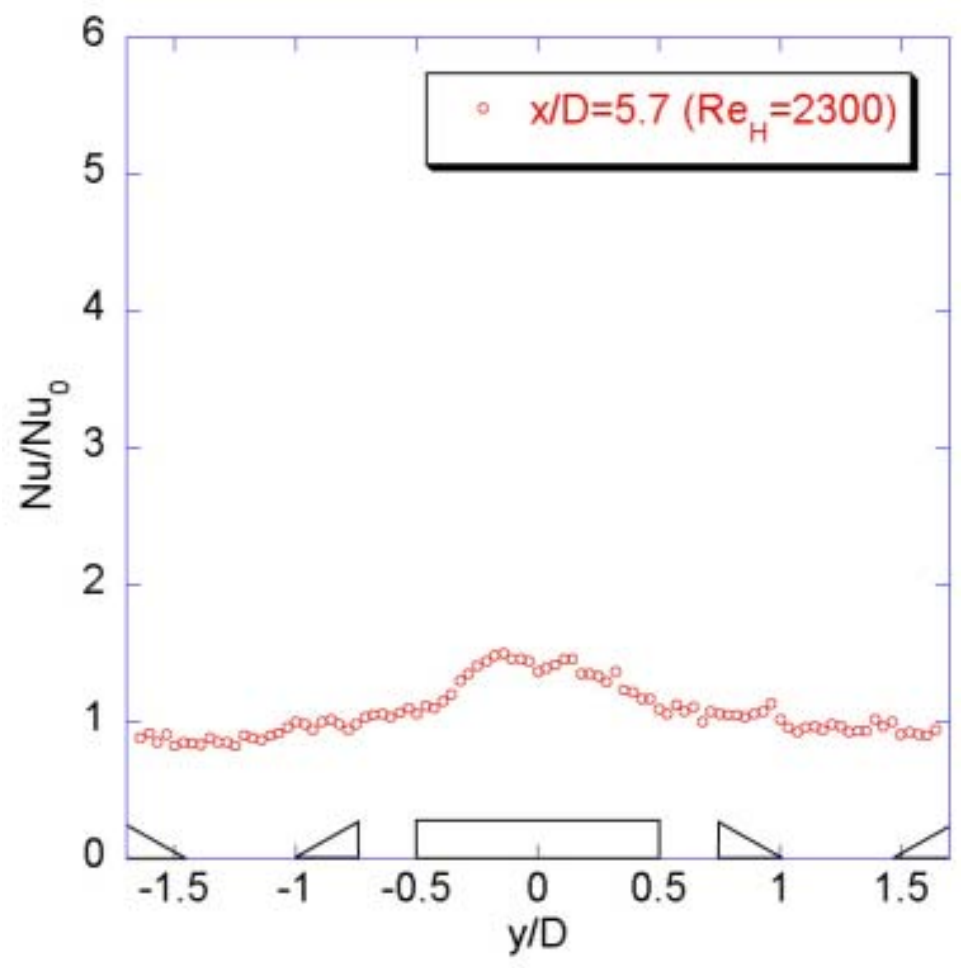

Figure 5-17. Spanwise variation of the heat transfer enhancement in terms of the Nusselt number ratio at $\mathrm{x} / \mathrm{D}=5.7$ for $\mathrm{ReH}=2300$. 


\subsection{Flow Measurement}

\subsubsection{Introduction}

As described in Section 5.1, the installation of vortex generators (winglets) on the fin surface can considerably enhance the rate of heat transfer while achieving the net reduction in pressure loss. This finding is very encouraging for the design of heat exchangers in air-cooled condensers for geothermal power plants. It is reasonably expected that the overall heat-transfer performance of finned-tube bundles with winglets can be optimized once the detailed heat transfer and fluid flow mechanisms responsible for the experimentally observed heat-transfer enhancement are clarified. To this aim, extensive flow measurements have been carried out in a water channel facility at Yokohama National University. This section describes the method used, the results obtained, and some conclusions drawn from the results.

The facility was constructed in the first year of the project and used for hydrogen-bubble flow visualization in the second year. The particle image velocimetry (PIV) is employed to measure highly three-dimensional flow fields around finned-tube bundles with winglets. As PIV measures velocity distributions in a two-dimensional plane of light sheet, the position of light sheet relative to the fin surface is displaced so that an entire flow domain between neighboring fins is covered with a stack of parallel measurement planes displaced at a small distance (Figure 5-25). The detailed flow characteristics revealed thus are used to understand the role of winglets for heat-transfer enhancement observed in the overall heat-transfer measurements. The whole-field velocity data obtained thus are further analyzed to evaluate wall-shear-stress distribution, which forms the basis for identifying the reasons for the heattransfer enhancement.

\subsection{Experimental Methods}

\subsubsection{Flow Facility and Test Core}

The flow facility is a re-circulating open water channel (Figure 5-18). The flow is driven by a constant head maintained in the head tank. After passing through the settling chamber consisting of a packed-bead section followed by a honeycomb section, the flow enters the test section whose crosssectional area is $400 \mathrm{~mm} \times 250 \mathrm{~mm}$. The flow rate is monitored with an electromagnetic flow meter to ensure the constancy of the flow rate during experiment. The water is de-aerated by heating before each experiment and then seeded with nylon particles of 28-32 $\mu \mathrm{m}$ in diameter and 1.02-1.03 in specific density. To achieve perfect density matching between the water and particles, an adequate amount of sodium chloride $(\mathrm{NaCl})$, i.e., $20 \mathrm{~g} /$ liter of water, is added to the water. This density matching is found to be essential for successful PIV measurements of stagnant region behind the tubes, because even a slight density difference will end up with considerable sedimentation of the particles onto the bottom fin surface.

The details of the test cores, both in-line and staggered tube arrangements, are given in Figure 5-19. Each consists of two glass plates, $1.4 \mathrm{~mm}$ thick, forming a horizontal channel having a wall-to-wall distance of $9 \mathrm{~mm}$. This flow channel simulates a heat-transfer passage formed by two neighboring fin plates. Transparent circular tubes, $50 \mathrm{~mm}$ in outer diameter and $0.1 \mathrm{~mm}$ in thickness, are placed in the channel in order to mimic heat-transfer tubes in air-cooled condensers. The spanwise and longitudinal distances between the tubes are $125 \mathrm{~mm}$, as shown in Figure 5-19. Note that the interior of the tubes is filled with water during experiments, thus minimizing the effect of light reflection at the curved tube surface when it is illuminated with a laser light sheet from the side of the channel. The bulk mean velocity in the channel is $16-20 \mathrm{~mm} / \mathrm{s}$, giving a bulk-mean Reynolds number of 300-350, based on the hydraulic diameter of the channel and the kinematic viscosity evaluated for the $\mathrm{NaCl}$ solution used in the experiment. Other details of the experimental conditions are given below. 

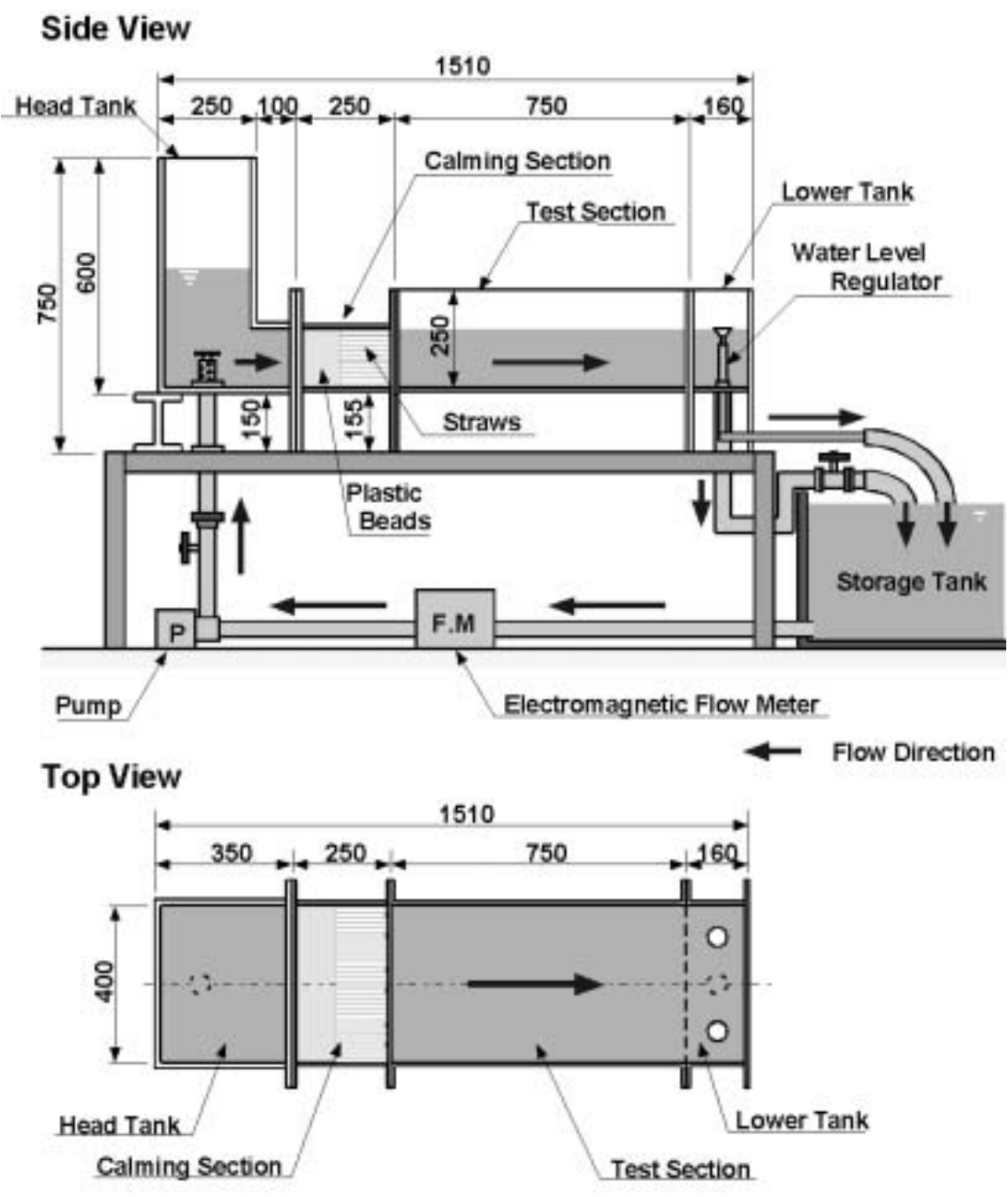

Figure 5-18. Re-circulating water channel facility at Yokohama National University.

Four basic arrangements of tubes and winglets are considered here. They are single-row tubes with and without winglets (Arrangement I), three-row in-line tube bundle with and without single-row winglets (Arrangement II), three-row in-line tube bundle with three-row winglets (Arrangement III), and three-row staggered tube bundle with and without single-row winglets (Arrangement IV). These arrangements are shown in Figure 5-20. 


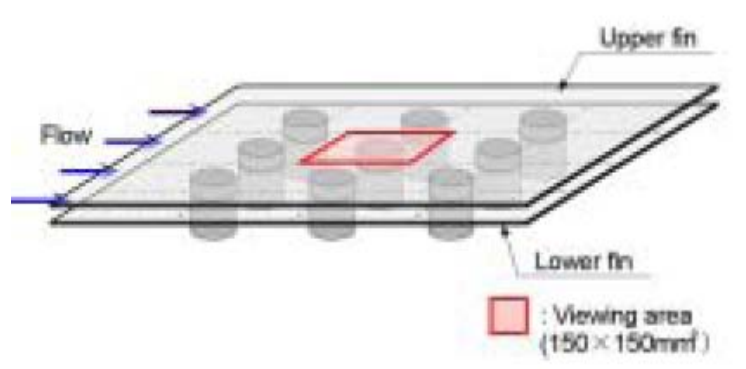

(a) in-line tube arrangement

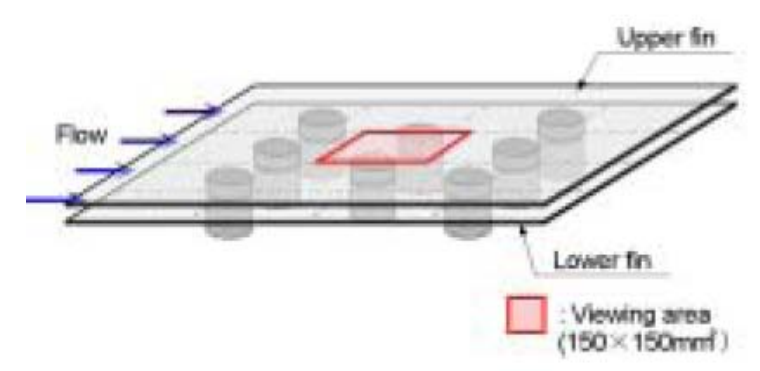

(b) staggered tube arrangement
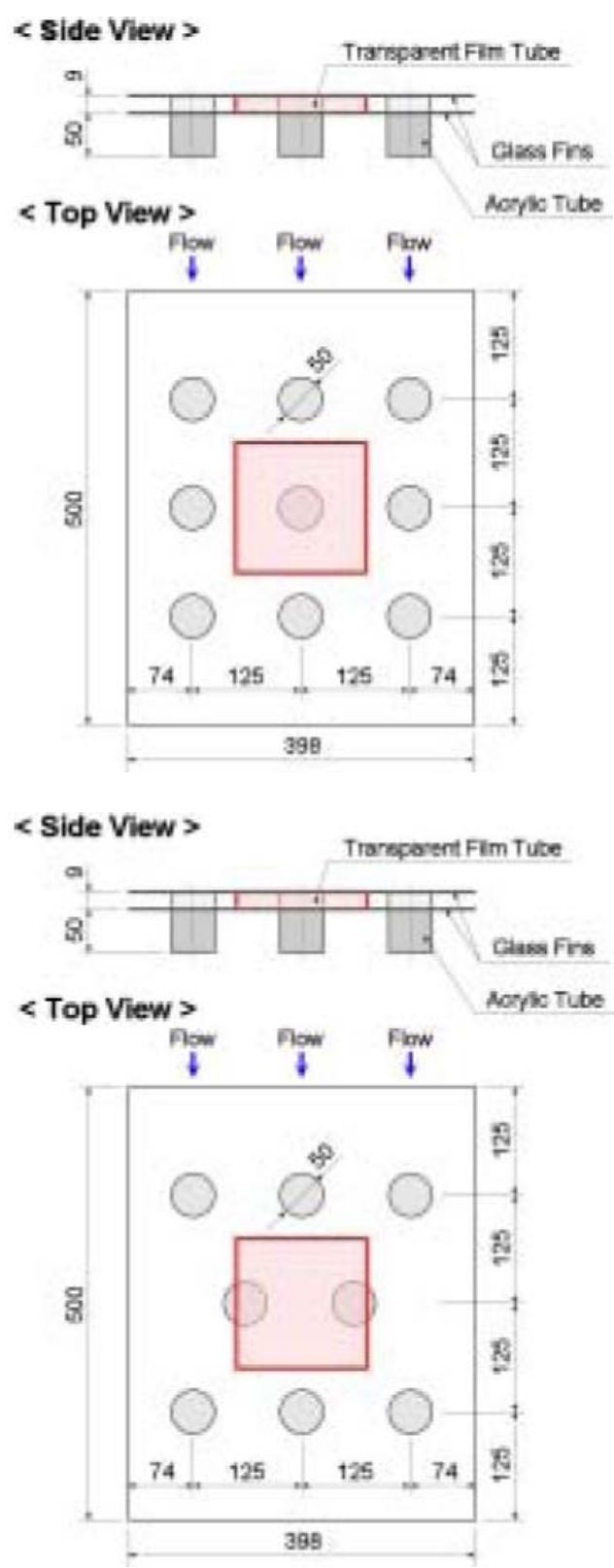

Figure 5-19. Test core tube bundle arrangement, (a) in-line tubes, (b) staggered tubes.

Each arrangement is further classified into Type A, Type B, Type C, and Type D, depending on the absence or presence of winglets and on the geometry of the winglets with respect to the tubes. Types $\mathrm{C}$ and $\mathrm{D}$ are the arrangements that are shown to have excellent overall heat-transfer performance. In particular, Type $\mathrm{C}$ of Arrangement IV can achieve 10\% increase of overall heat-transfer coefficient while realizing 50\% decrease of pressure loss, as described earlier in Section 5.1. The details of the configurations of tubes and winglets are shown in Figure 5-21. Type B is the configuration studied by Fiebig et al. ${ }^{19}$ They proposed to place a pair of winglets downstream of each tube so that the longitudinal vortices generated by the winglets can enhance heat transfer in the stagnation region behind the tube (see Figure 5-20). 
They reported that the overall heat-transfer enhancement was 55-65\%, with 20-45\% increase of pressure loss at a Reynolds number range of 1200-5400. Types C and D are the configurations proposed in the present study. They are mainly different in the angle of attack, $\alpha$, and in the spanwise gap between the winglet and the tube surface, $d$ (see Figure 5-21). Type $\mathrm{C}$ and $\mathrm{D}$ are completely different from Type $\mathrm{B}$ in the sense that the winglets in Type $\mathrm{C}$ and $\mathrm{D}$ act not only as vortex generators but also as guide vanes that effectively introduce high-momentum stream into the stagnation region behind the tube.

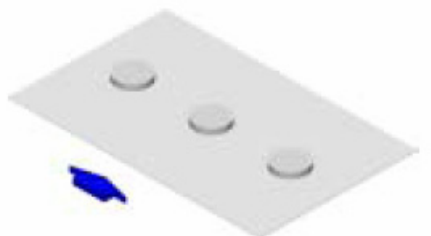

Type A

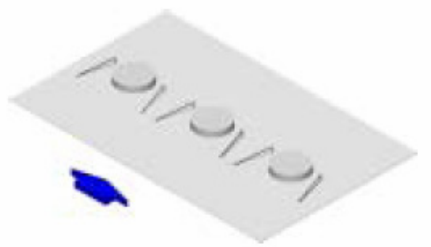

Type C

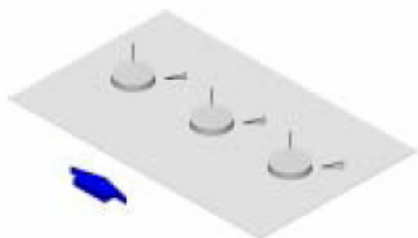

Type B

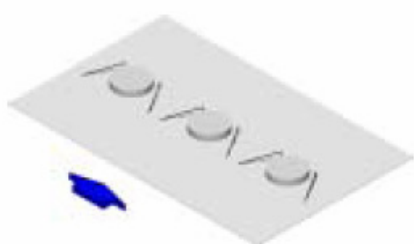

Type D

Arrangement I

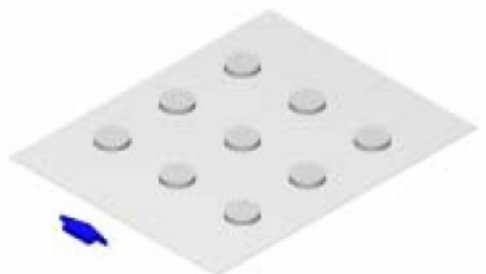

Type A

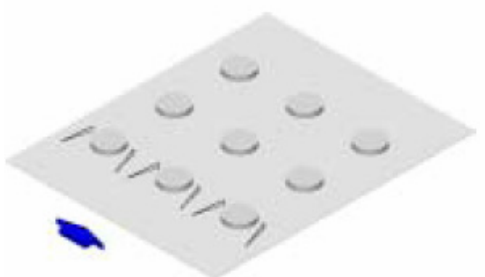

Type C

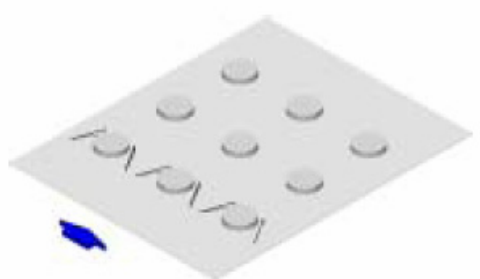

Type D

Arrangement II

Figure 5-20. Various arrangements of tube bundles and winglets studied. 


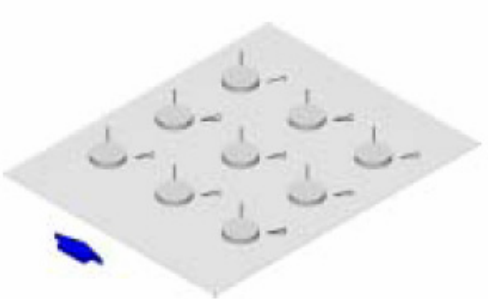

Type B

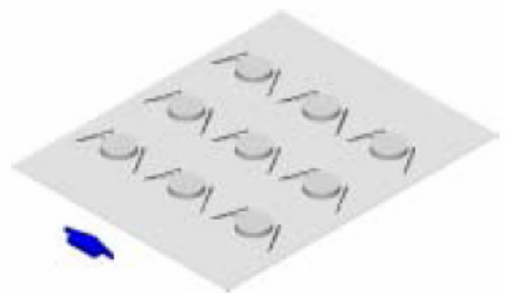

Type D

Arrangement III

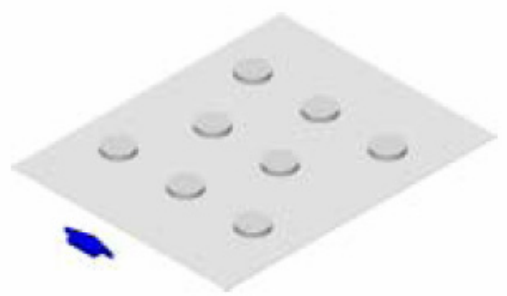

Type A

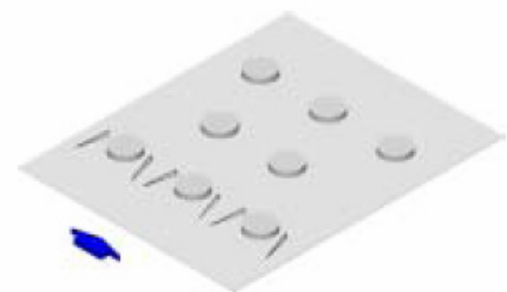

Type C

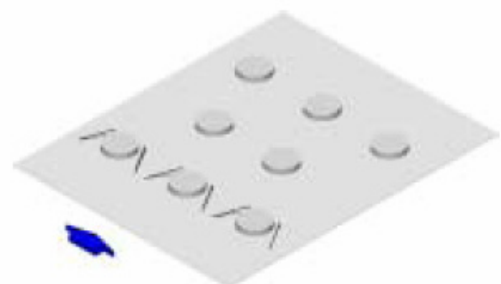

Type D

Arrangement IV

Figure 5-20 (continued). Various arrangements of tube bundles and winglets studied. 


\section{$<$ Top View>}
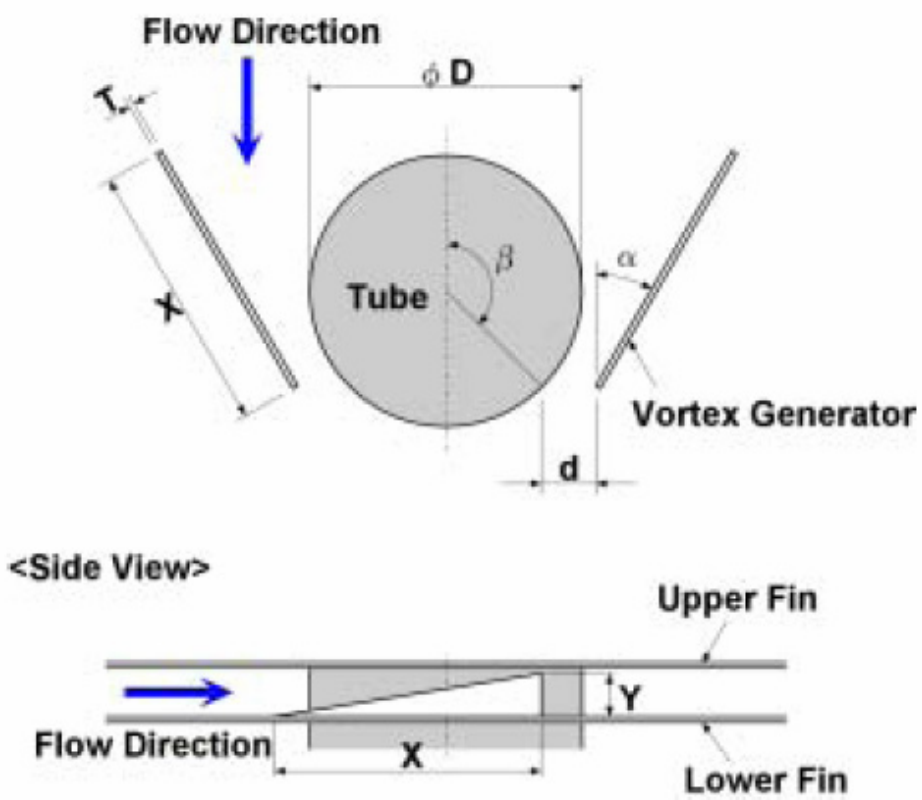

\begin{tabular}{|c|c|c|c|}
\hline $\mathrm{X}[\mathrm{mm}]$ & Type B & Type C & Type D \\
\hline $\mathrm{Y}[\mathrm{mm}]$ & 16 & 50 & 50 \\
\hline $\mathrm{T}[\mathrm{mm}]$ & 8 & 8 & 8 \\
\hline$\alpha[$ degree] & 1 & 1 & 1 \\
\hline$\beta[$ degree $]$ & $*$ & 15 & 30 \\
\hline $\mathrm{d}[\mathrm{mm}]$ & $*$ & 110 & 135 \\
\hline
\end{tabular}

Figure 5-21. Details of tube and winglet arrangements.

This function of the winglets, coupled with the function of generating longitudinal vortices, is anticipated to bring about higher heat transfer with lower pressure loss that would otherwise be caused by the form drag of the tube.

\subsubsection{PIV Technique}

Figure 5-22 shows the configuration of the present PIV measurement. A digital CCD camera $(1024 \times 1024$ cells $)$ is used to observe the flow from the top of the channel. The size of the viewing area is $150 \mathrm{~mm} \times 150 \mathrm{~mm}$, as indicated by each hatched square in Figure 5-19. A long working distance between the viewing area and the camera (about $1 \mathrm{~m}$ ) is allowed to minimize perspective errors included in two-component planar PIV measurement. The perspective error estimated is $6 \%$ or less at the periphery of the viewing area. 

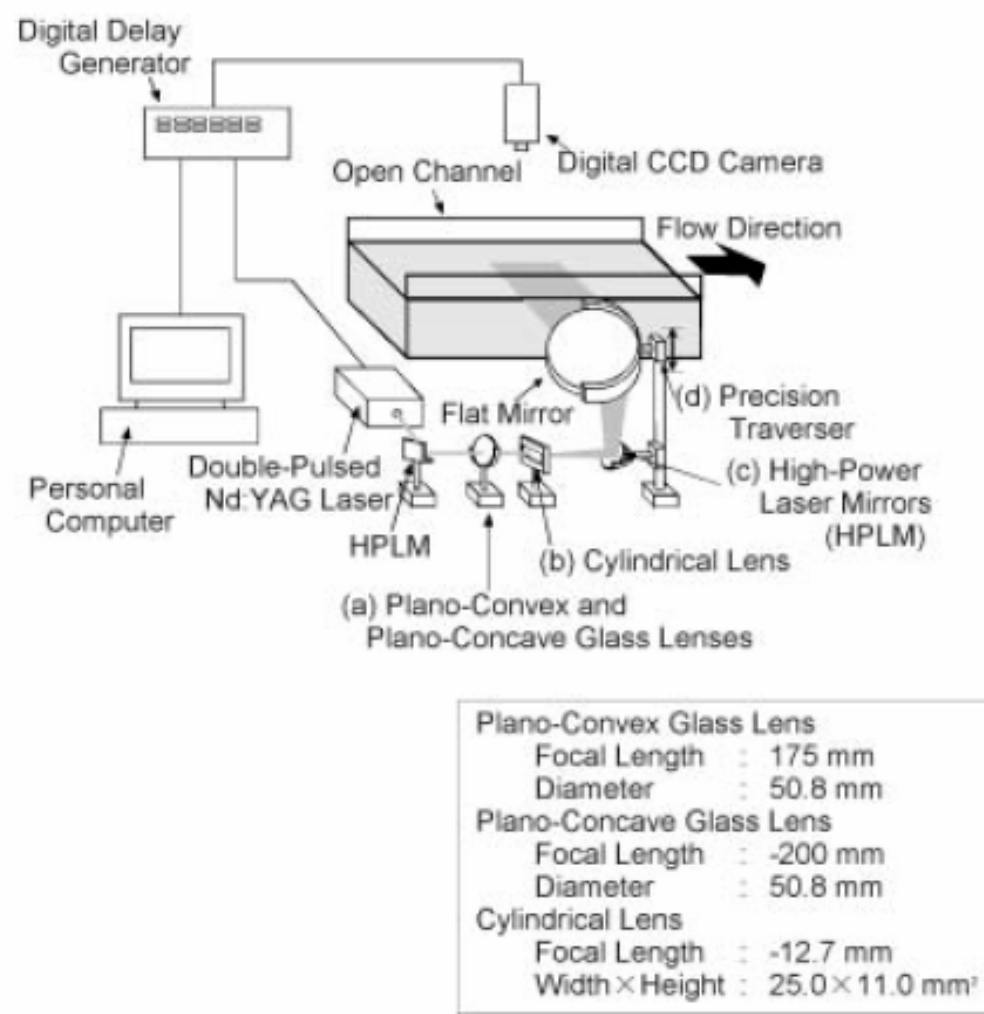

Figure 5-22. Details of PIV setup.

Illumination is provided by a double-pulsed Nd:YAG laser, which emits beams $532 \mathrm{~nm}$ in wave length and $20 \mathrm{~mJ} /$ pulse in output energy. As the flow velocity is low, the time interval between the pulses is set at $33.3 \mathrm{~ms}$, which results in less than 20-pixel displacement of consecutive particle images. By using a series of lenses, shown in Figure 5-23, the laser beam is shaped into a thin horizontal sheet about $0.6 \mathrm{~mm}$ thick and $240 \mathrm{~mm}$ wide at the viewing area. The light sheet is guided into the channel formed by the two glass plates of the test core. Great care is paid to ensure that the light sheet propagates parallel to the channel wall. The method for light-sheet adjustment is depicted in Figure 5-24. An acrylic block having two horizontal lines on its side surface is machined in such a way that the lines become precisely parallel to the bottom surface of the block. The block is placed on the channel bottom wall and the orientation of the light sheet is adjusted using a CCD camera and a monitor so that the light sheet and the lines are aligned satisfactorily. Inasmuch as the lines are parallel to the channel wall, this alignment guarantees the orientation of the light sheet to be as desired. The whole-field velocity measurement in the channel is performed by traversing the light sheet in the direction normal to the channel wall. A total of 17 cuts at a $0.5-\mathrm{mm}$ interval are made to encompass the region from $0.5 \mathrm{~mm}$ above the bottom wall to $0.5 \mathrm{~mm}$ below the top wall, as indicated in Figure 5-25.

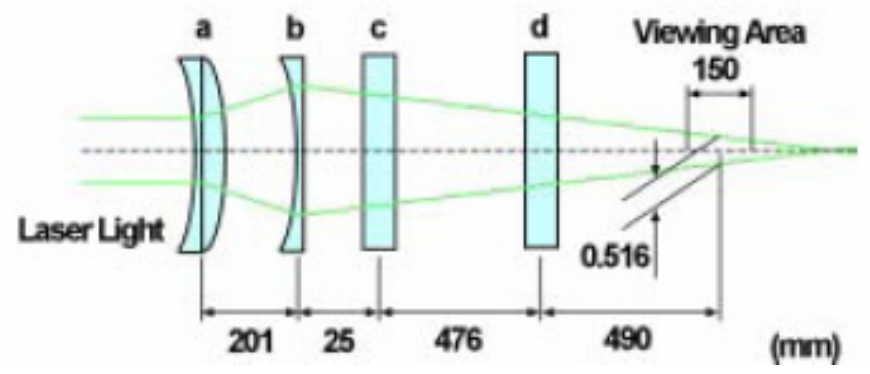

Figure 5-23. Layout and combination of lenses for producing a sheet of laser light 


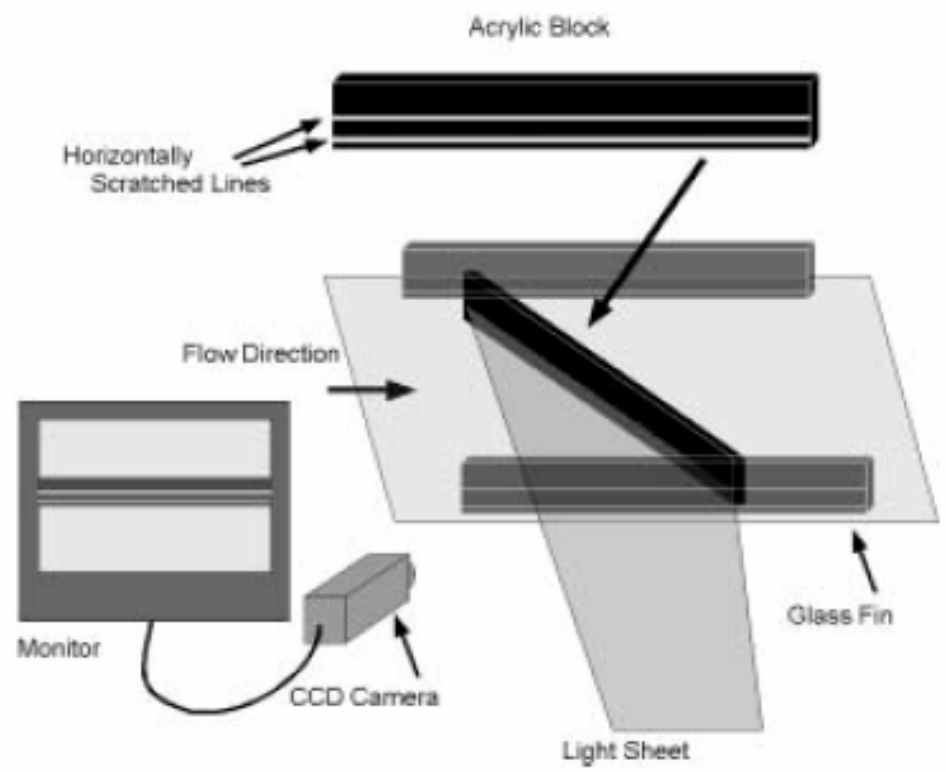

Figure 5-24. A method to adjust sheet of laser light.

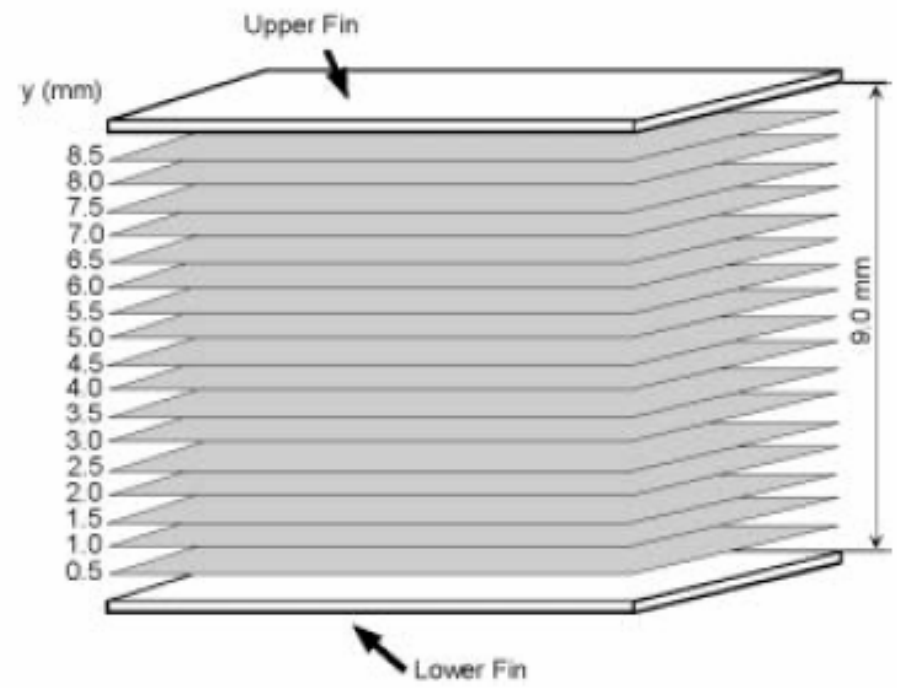

Figure 5-25. Position of laser light sheet in the channel.

A direct cross-correlation PIV technique developed in the Heat Transfer Laboratory at Yokohama National University is used to analyze particle images. The interrogation window size is $32 \times 32$ pixels, and a $50 \%$ overlap is chosen. This interrogation window size corresponds to a $4.8 \mathrm{~mm} \times 4.8 \mathrm{~mm}$ spot in the $150 \mathrm{~mm} \times 150 \mathrm{~mm}$ viewing area. The lateral (or spanwise) search distance is set to be \pm 5 pixels, while the longitudinal (or streamwise) one is set to be from -5 pixels to +20 pixels by considering that the magnitude of flow reversal is not large under the present conditions, as confirmed in a preliminary hydrogen-bubble flow visualization. The threshold for valid correlation-peak detection is set to be 0.4 , and any correlation-peak values below this are simply discarded. In order to remove erroneous vectors and replace them with correct ones, the median filtering proposed by Westerweel ${ }^{57-58}$ are implemented in the laboratory-made PIV software. Also, the algorithm proposed by Meinhart et al. ${ }^{59}$ for replacement of erroneous first peaks with correct 2 nd or 3rd ones is implemented. Figure 5-26 is an example of a pair of PIV images acquired and an instantaneous velocity field obtained. A total of 1000 pairs of PIV images are acquired at each horizontal cut to obtain an average velocity distribution. 


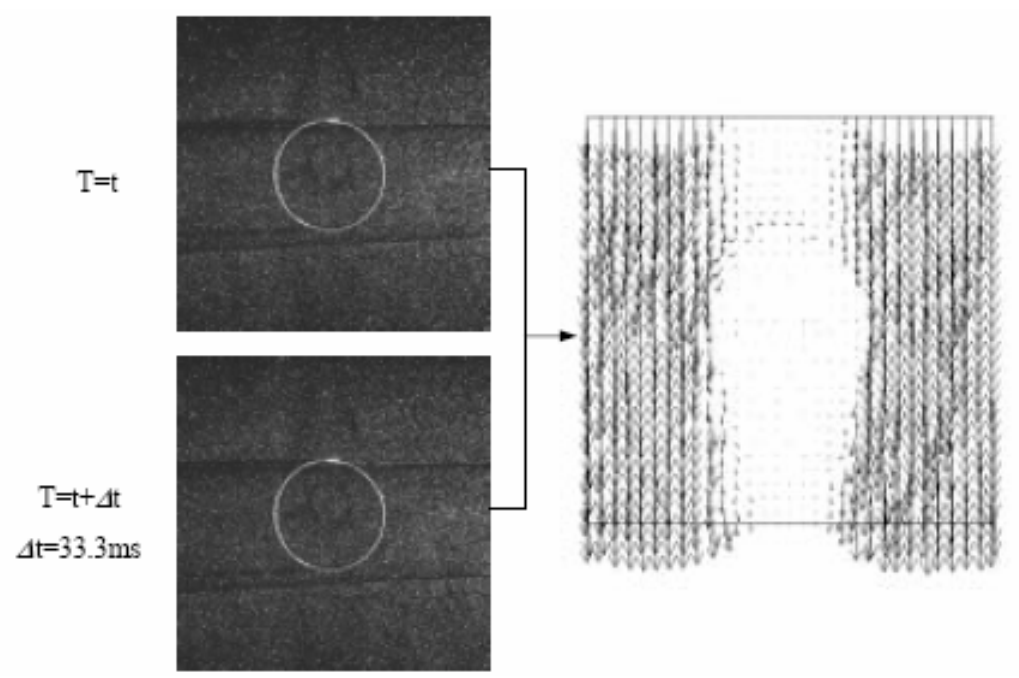

Figure 5-26. Example of a pair of PIV images and a result of velocity distribution.

\subsubsection{Experimental Conditions}

Experimental conditions are summarized in Table 5-2. The mean velocities, $\mathrm{U}_{\mathrm{m}}$, are in the range of $16.0-20.3 \mathrm{~mm} / \mathrm{s}$, giving the bulk Reynolds numbers, Re, of 290-366. The Reynolds number is defined as

$R e=\frac{U_{m} \cdot 2 h}{v}$

where $\mathrm{h}$ is the wall-to-wall distance of the channel, and $v$ is the kinematic viscosity of the working fluid. The hydraulic diameter of the channel, i.e., $2 \mathrm{~h}$, is used as a length scale.

Table 5-2. Summary of experimental conditions

\begin{tabular}{|l|c|c|c|c|}
\hline Arrangement I & Type A & Type B & Type C & Type D \\
\hline Flow rate[1/min] & 90.0 & 90.0 & 90.0 & 90.0 \\
\hline Water tenperature[ $\left.{ }^{\circ} \mathrm{C}\right]$ & 20.0 & 20.0 & 20.0 & 20.0 \\
\hline Vater depth[mm] & 105 & 105 & 105 & 105 \\
\hline Hean velocity [ni/s] & 20.32 & 20.04 & 19.18 & 19.33 \\
\hline Reynolds number & 366 & 361 & 345 & 348 \\
\hline
\end{tabular}

\begin{tabular}{|l|c|c|c|}
\hline Arrangement II & Type A & Type C & Type D \\
\hline Flow rate[1/min] & 90.0 & 90.0 & 90.0 \\
\hline Water tenperature[ $\left.{ }^{\circ} \mathrm{C}\right]$ & 27.0 & 27.0 & 27.0 \\
\hline Water depth[mm] & 103 & 103 & 103 \\
\hline Hean velocity[ma/s] & 16 & 16 & 16 \\
\hline Reynolds number & 290 & 290 & 290 \\
\hline
\end{tabular}

\begin{tabular}{|l|c|c|}
\hline Arrangement III & Type B & Type D \\
\hline Flow rate[1/min] & 89.8 & 89.8 \\
\hline Vater tenperature [ $\left.{ }^{\circ} \mathrm{C}\right]$ & 26.0 & 26.0 \\
\hline Vater depth[mm] & 105 & 105 \\
\hline Hean velocity [nn/s] & 20 & 20 \\
\hline Reynolds number & 350 & 350 \\
\hline
\end{tabular}

\begin{tabular}{|l|c|c|c|}
\hline Arrangement IV & (8) Type A & (9) Type C & (10) Type D \\
\hline Flow rate[1/min] & 90.0 & 90.0 & 90.0 \\
\hline Water tenperature[ $\left.{ }^{\circ} \mathrm{C}\right]$ & 28.0 & 28.0 & 28.0 \\
\hline Water depth[mm] & 103 & 103 & 103 \\
\hline Hean velocity[ma/s] & 16 & 16 & 16 \\
\hline Reynolds number & 290 & 290 & 290 \\
\hline
\end{tabular}


The kinematic viscosity is evaluated for the working fluid used as, e.g., $1.19 \times 10^{-6} \mathrm{~m}^{2 / \mathrm{s}}$ for $2 \mathrm{wt} \%$ $\mathrm{NaCl}$ solution at $16^{\circ} \mathrm{C}$.

Figure 5-27 presents inlet velocity profiles measured at $75 \mathrm{~mm}$ upstream of the tube row in Types A$\mathrm{D}$ of Arrangement I (i.e., single-row tube arrangement). Each profile is plotted as function of height above the bottom channel wall.
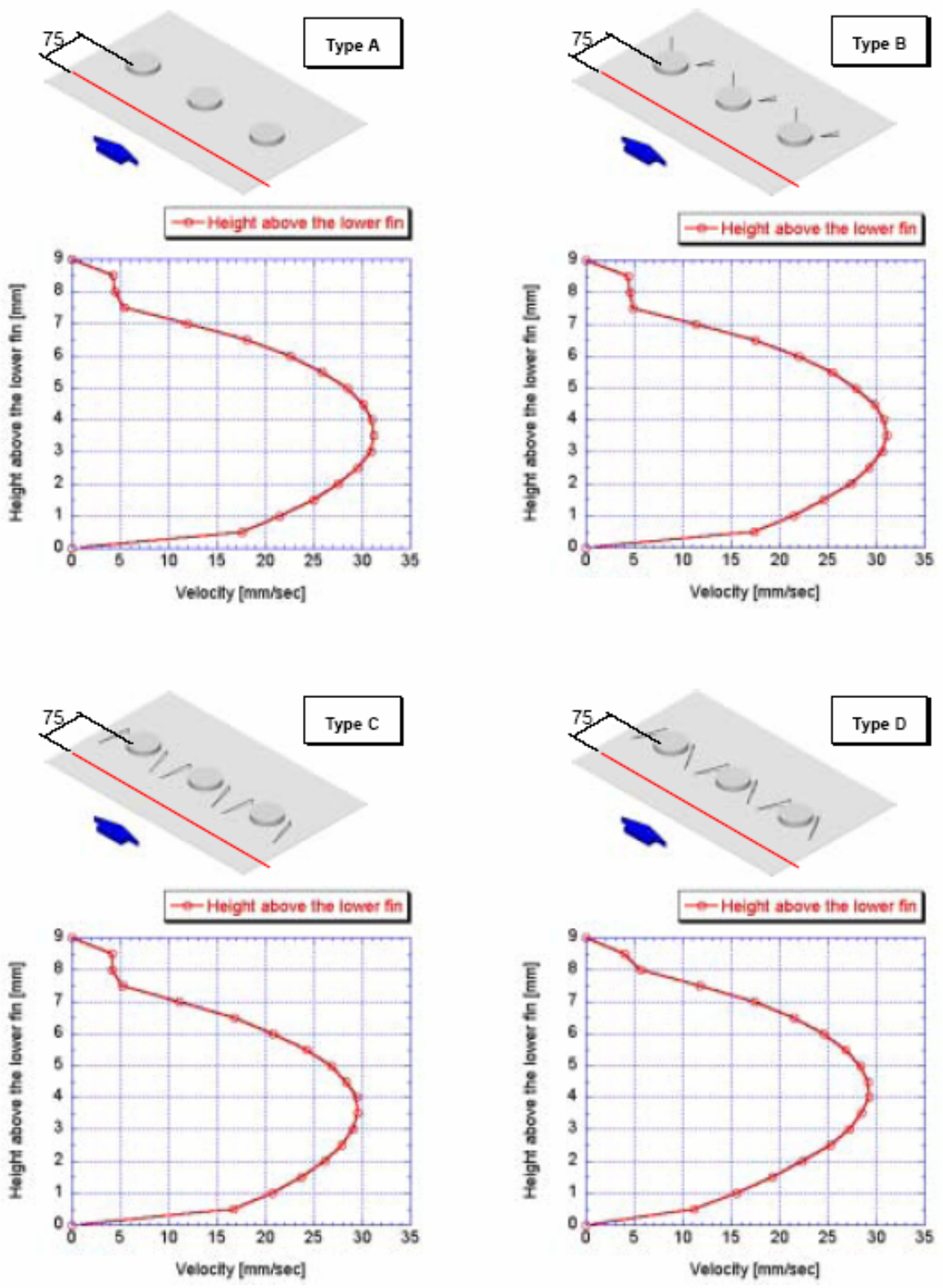

Figure 5-27. Velocity profiles for Types A-D of tube bundle Arrangement I.

Noticed that the profiles are close to a parabolic one, except for the region close to the top channel wall, where a jump of the profile is seen. Also, notice a slight shift of the maximum velocity location toward the bottom channel wall. It is conjectured that this nonsymmetry is due to (a) insufficient entry length $(50 \mathrm{~mm})$ and $(\mathrm{b})$ nonuniform velocity distribution near the suction pipe of the channel facility. 


\subsection{Results and Discussion}

\subsubsection{Flow Characteristics in Arrangement I (Single Row Tubes)}

Figure 5-28 shows the flow patterns in horizontal planes for Type A. The symmetry of the flow patterns with respect to the tube is an evidence of the good alignment of the present laser light sheet. There is a large stagnant region behind the tube, where the heat transfer gets deteriorated. The reduction in size (or even elimination) of this stagnant region is an important goal of the heat-transfer enhancement of finned-tube bundles.

Figure 5-29 shows the flow patterns for Type B, where a pair of winglets is placed downstream of the tube in an arrangement as recommended by Fiebig et al. ${ }^{19}$ Each flow pattern exhibits the presence of a pair of slender high-speed regions extending from the leading edge of each pair of winglets. Their presence is due to the downwash action of longitudinal vortices induced by winglets. As the downwash action carries high-speed fluid toward the bottom channel wall, the heat transfer in that area gets considerably enhanced. Fiebig et al. ${ }^{19}$ reported that the overall heat-transfer enhancement was $55-65 \%$ at a cost of $20-45 \%$ increase in pressure loss for the Reynolds number range of $1200-5400$. The overall performance test described in Section 5.1 has shown that the heat transfer is enhanced by $66 \%$ at a penality of $6 \%$ increase in pressure-loss at a Reynolds number of 350.

Figure 5-30 presents the flow patterns for Type C, where a pair of winglets is mounted beside the tube in a manner proposed by Torii et al. ${ }^{54}$ The fluid is expected to be guided into the stagnant region behind the tube through a pair of narrow passages formed between tube and the winglets, reducing the size of the stagnant region behind the tube. However, this angle of attack ( 15 degrees) reduces the stagnant region behind the winglets and causes little penalty of the pressure loss. This observation is supported by the heat-transfer test, which shows that this configuration achieves $45 \%$ increase of heat transfer without additional pressure loss.

Figure 5-31 shows the flow patterns for Type D. The differences between Types C and D are the angle of attack ( 15 versus 30 degrees) and the width of the gap between the tube and winglets $(15 \mathrm{~mm}$ versus $5 \mathrm{~mm}$ ). It is seen that the flow of high-speed fluid guided by the winglets effectively reduces the size of the stagnant wake region behind the tube. The overall heat-transfer test for Type D indicates that the heat transfer is significantly enhanced ( $136 \%$ increase), with moderate increase in pressure loss (35\%). Thus, for single row tubes at a low Reynolds number range, the proposed configurations are able to achieve either (1) a substantial heat-transfer enhancement with negligible pressure loss increase (Type C) or (2) a drastic heat-transfer enhancement with reasonable pressure-loss increase (Type D).

The profiles of streamwise and spanwise mean velocities are plotted along a horizontal line located $0.5 \mathrm{~mm}$ above the bottom channel wall and $26 \mathrm{~mm}$ downstream of the tube in Figure 5-32 (see the insets for the position of the line). The profiles exhibit the presence of both stagnant region behind tube and high-speed regions formed by the influence of winglets. The results shown here are consistent with the interpretation of flow patterns seen in Figures 5-28 through 5-31. In fact, the profile of streamwise mean velocity for Type $\mathrm{D}$ has a considerably decreased width of velocity deficit, which is brought about by a pair of positive peaks of streamwise component at $\mathrm{z}= \pm 10 \mathrm{~mm}$ accompanying positive and negative peaks of spanwise component. Although similar peaks are seen in Types B and C, the reduction of the size of stagnant region is not as remarkable as in Type $\mathrm{D}$. 


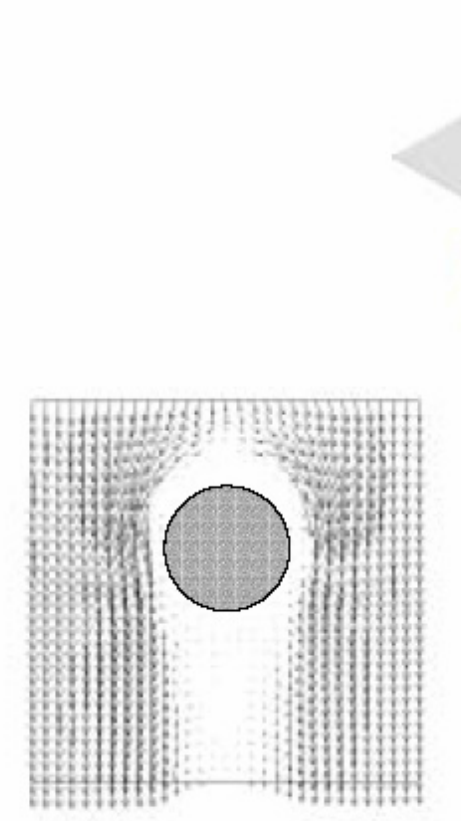

$$
y=0.5 \mathrm{~mm}
$$
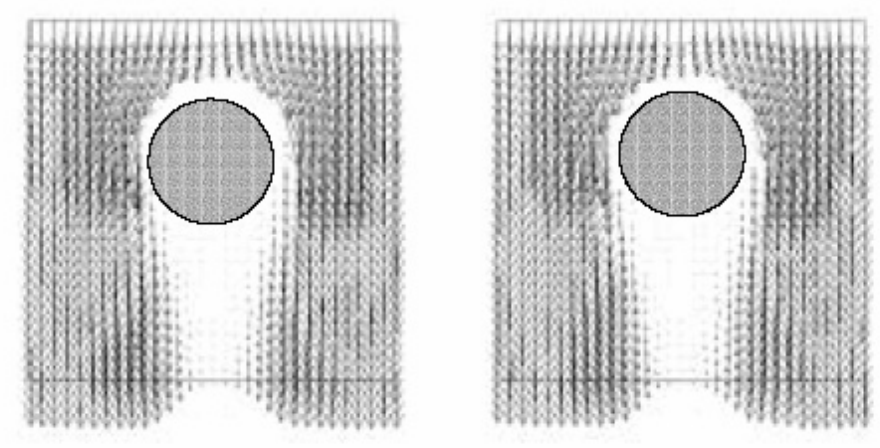

$$
y=3.5 \mathrm{~mm}
$$

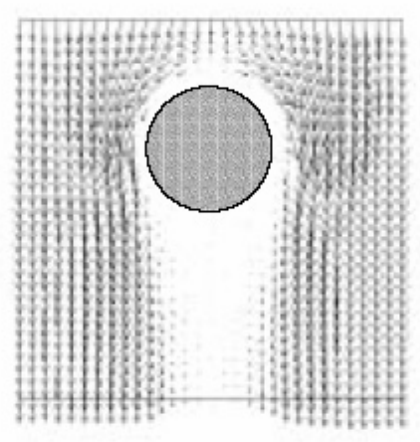

$$
y=6.5 \mathrm{~mm}
$$

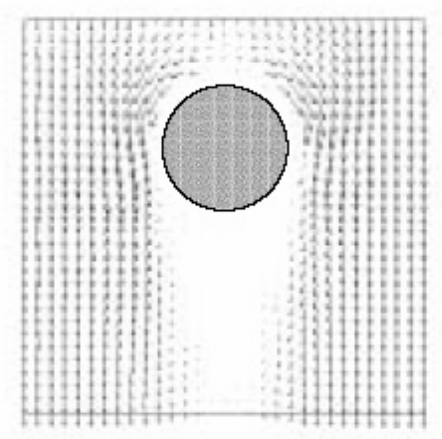

$y=7.5 \mathrm{~mm}$

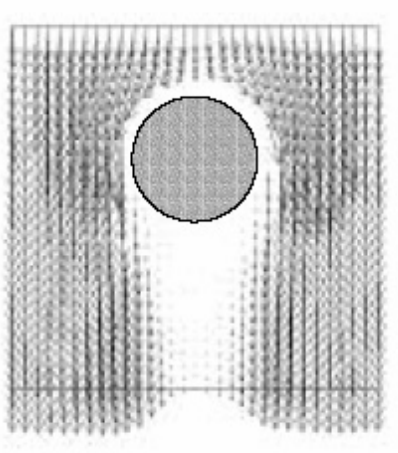

$y=2.5 \mathrm{~mm}$

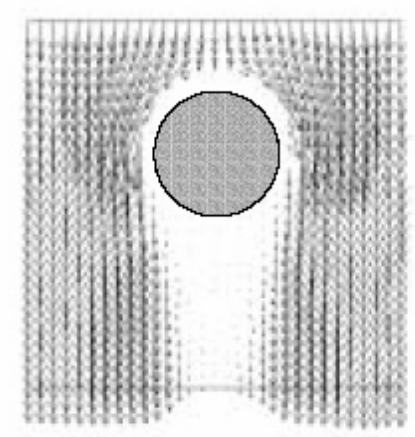

$y=5.5 \mathrm{~mm}$

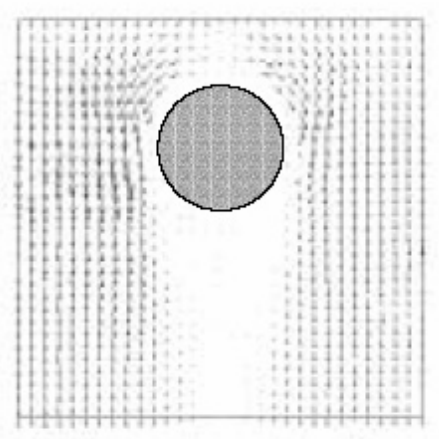

$y=8.5 \mathrm{~mm}$

Figure 5-28. Flow patterns for Type A of Arrangement I (single row tubes). 


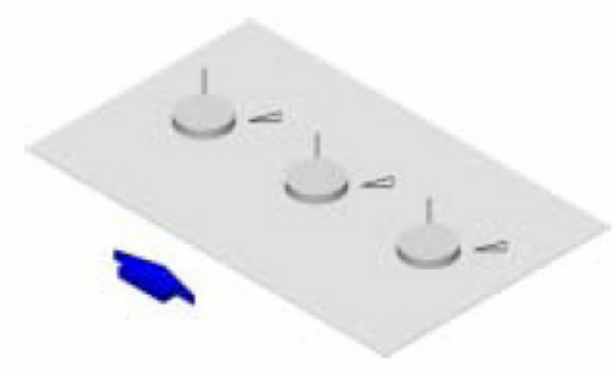

$\downarrow: 30 \mathrm{~mm} / \mathrm{sec}$
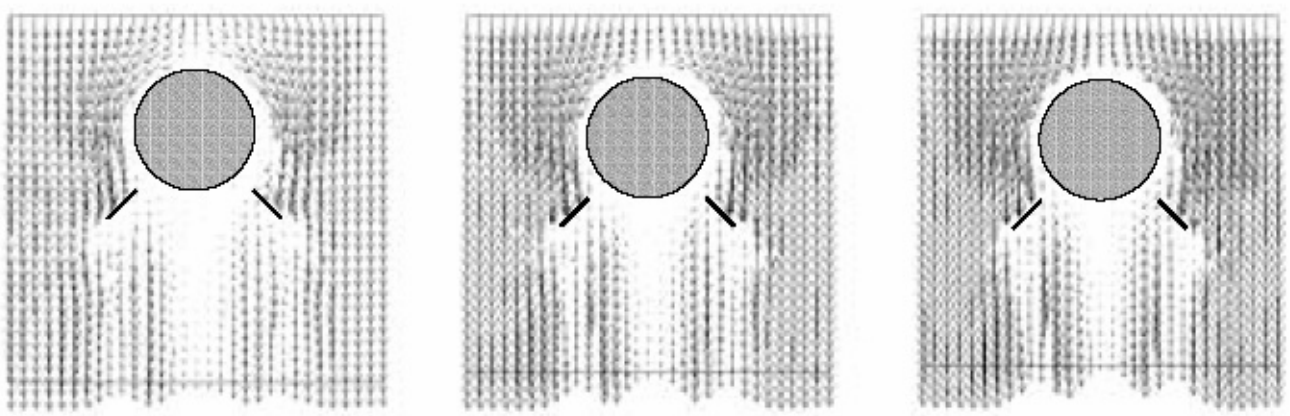

$y=0.5 \mathrm{~mm}$

$y=1.5 \mathrm{~mm}$

$y=2.5 \mathrm{~mm}$
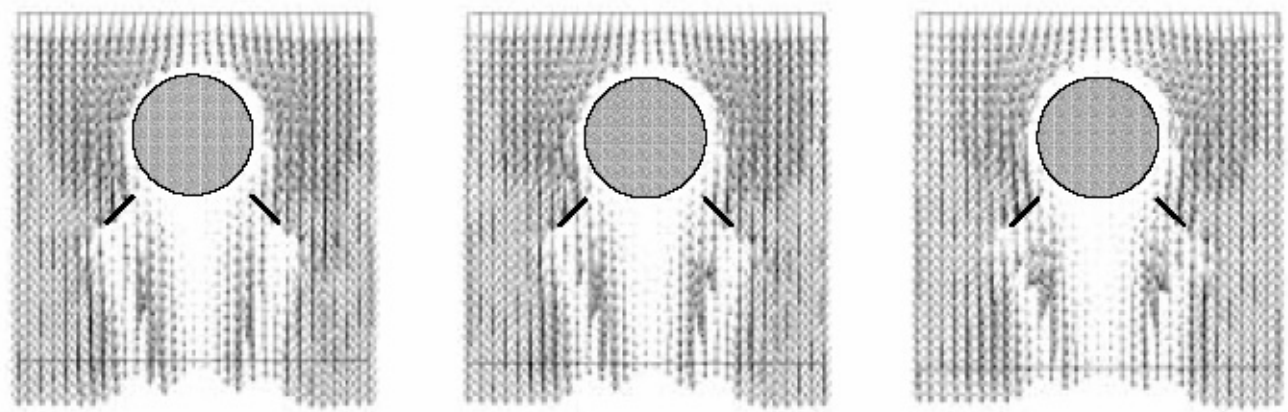

$y=3.5 \mathrm{~mm}$

$y=4.5 \mathrm{~mm}$

$y=5.5 \mathrm{~mm}$
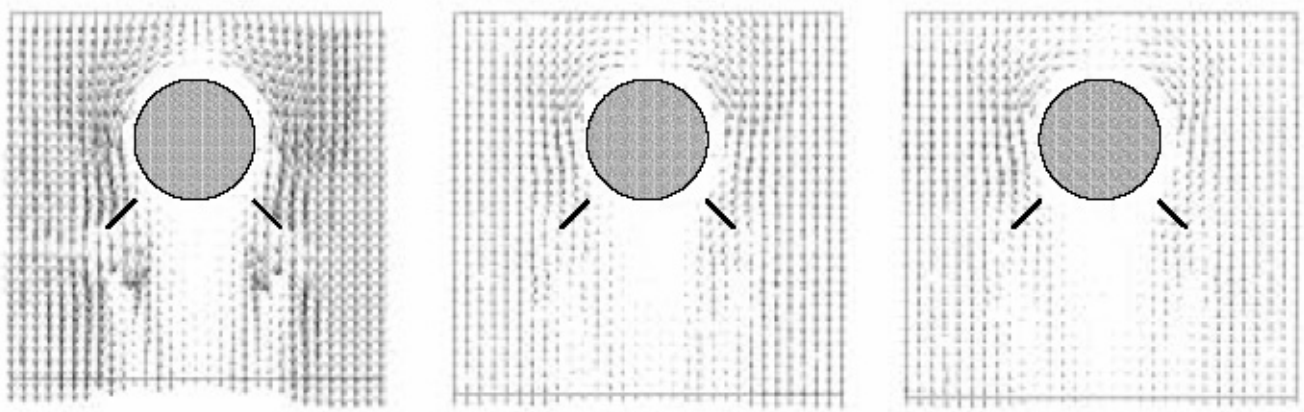

$y=6.5 \mathrm{~mm}$

$y=7.5 \mathrm{~mm}$

$y=8.5 \mathrm{~mm}$

Figure 5-29. Flow patterns for Type B of Arrangement I (single row tubes). 


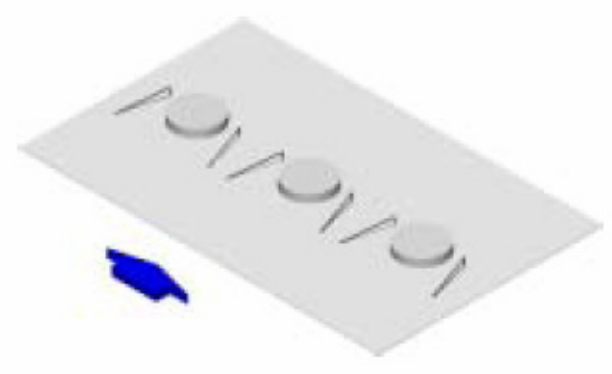

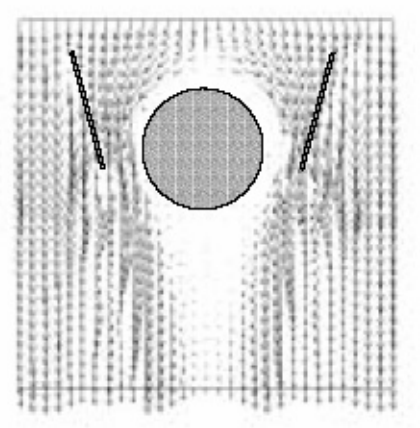

$y=0.5 \mathrm{~mm}$

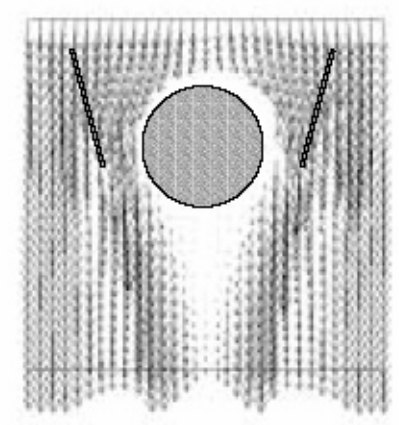

$y=3.5 \mathrm{~mm}$

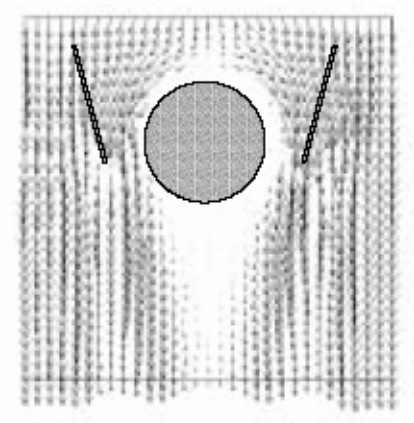

$y=6.5 \mathrm{~mm}$

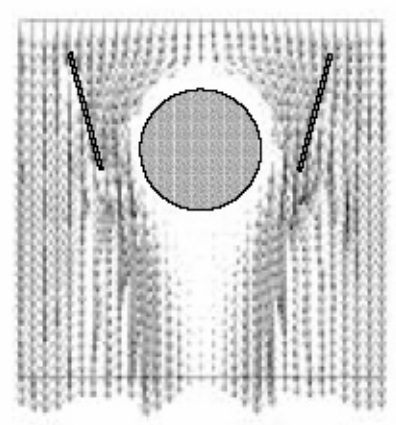

$y=1.5 \mathrm{~mm}$

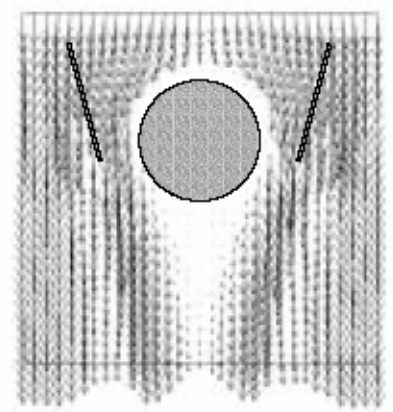

$y=4.5 \mathrm{~mm}$

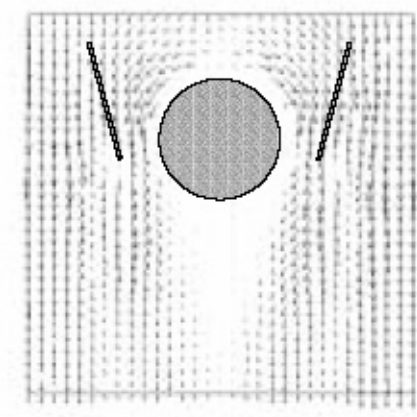

$y=7.5 \mathrm{~mm}$

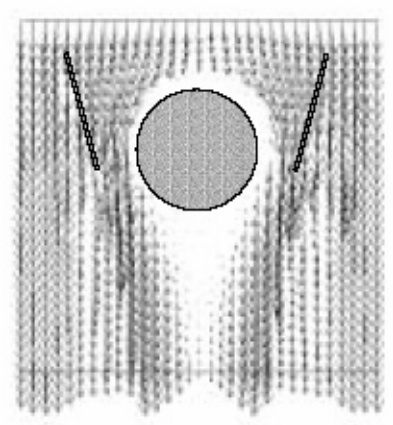

$y=2.5 \mathrm{~mm}$

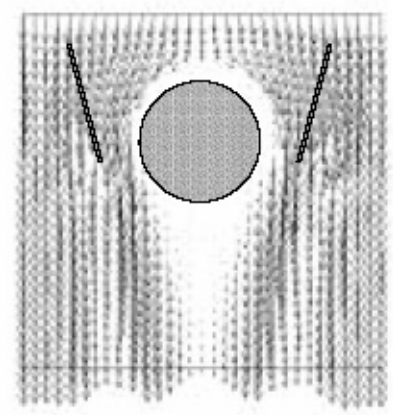

$y=5.5 \mathrm{~mm}$

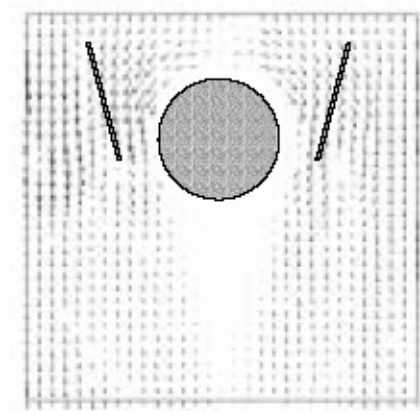

$y=8.5 \mathrm{~mm}$

Figure 5-30. Flow patterns for Type C of Arrangement I (single row tubes). 

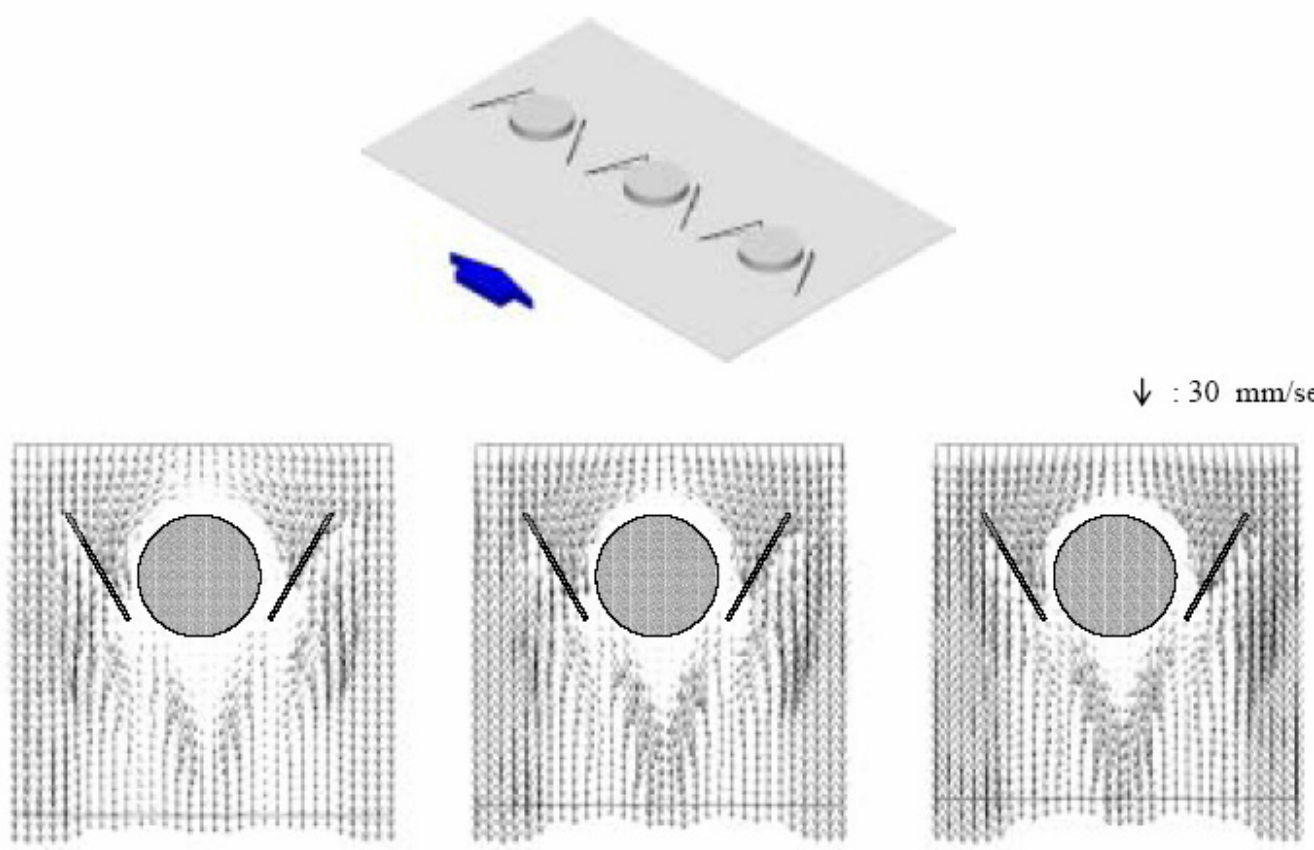

$$
y=0.5 \mathrm{~mm}
$$

$$
y=1.5 \mathrm{~mm}
$$

$$
y=2.5 \mathrm{~mm}
$$
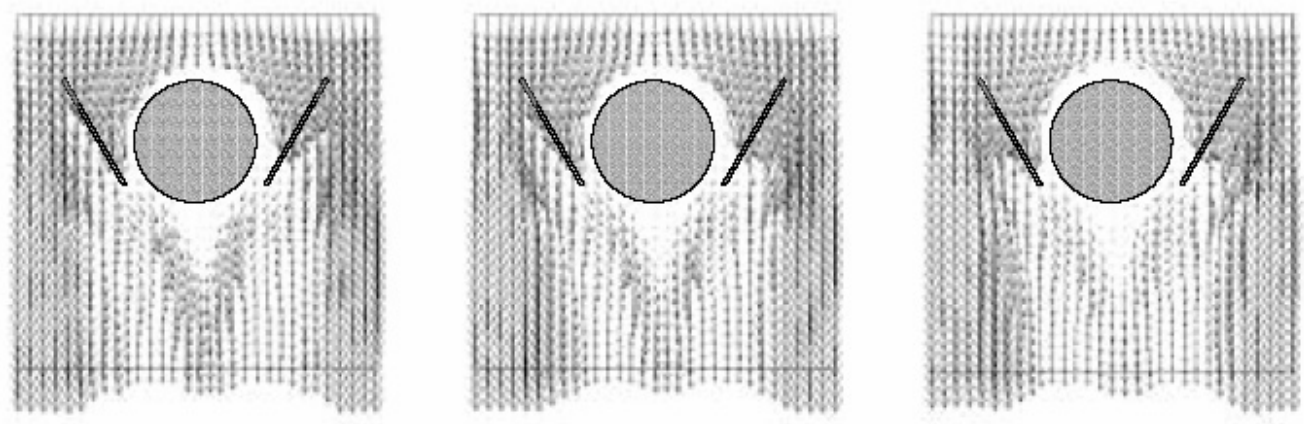

$y=3.5 \mathrm{~mm}$

$y=4.5 \mathrm{~mm}$

$y=5.5 \mathrm{~mm}$
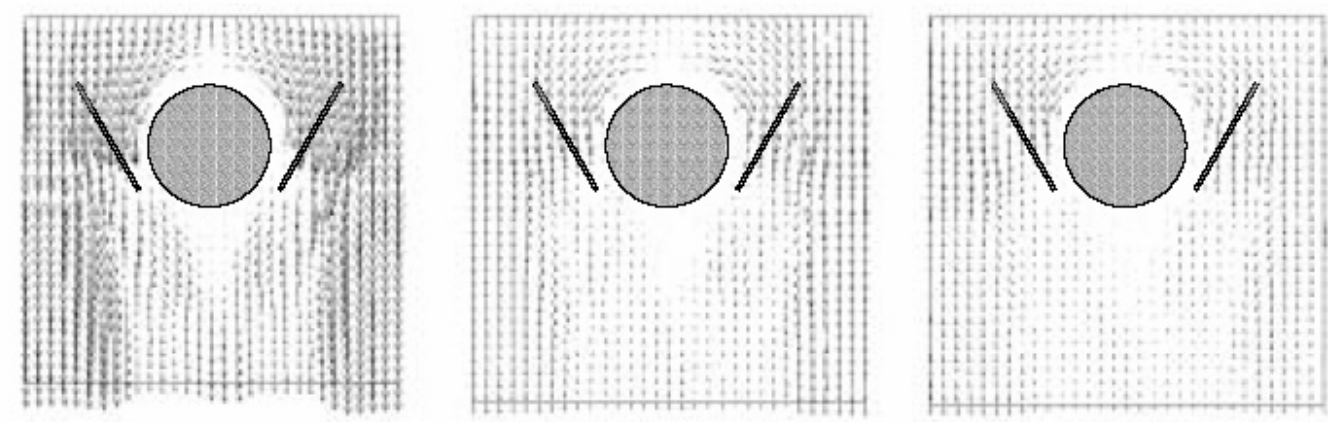

$y=6.5 \mathrm{~mm}$

$y=8.5 \mathrm{~mm}$

Figure 5-31. Flow patterns for Type D of Arrangement I (single row tubes). 

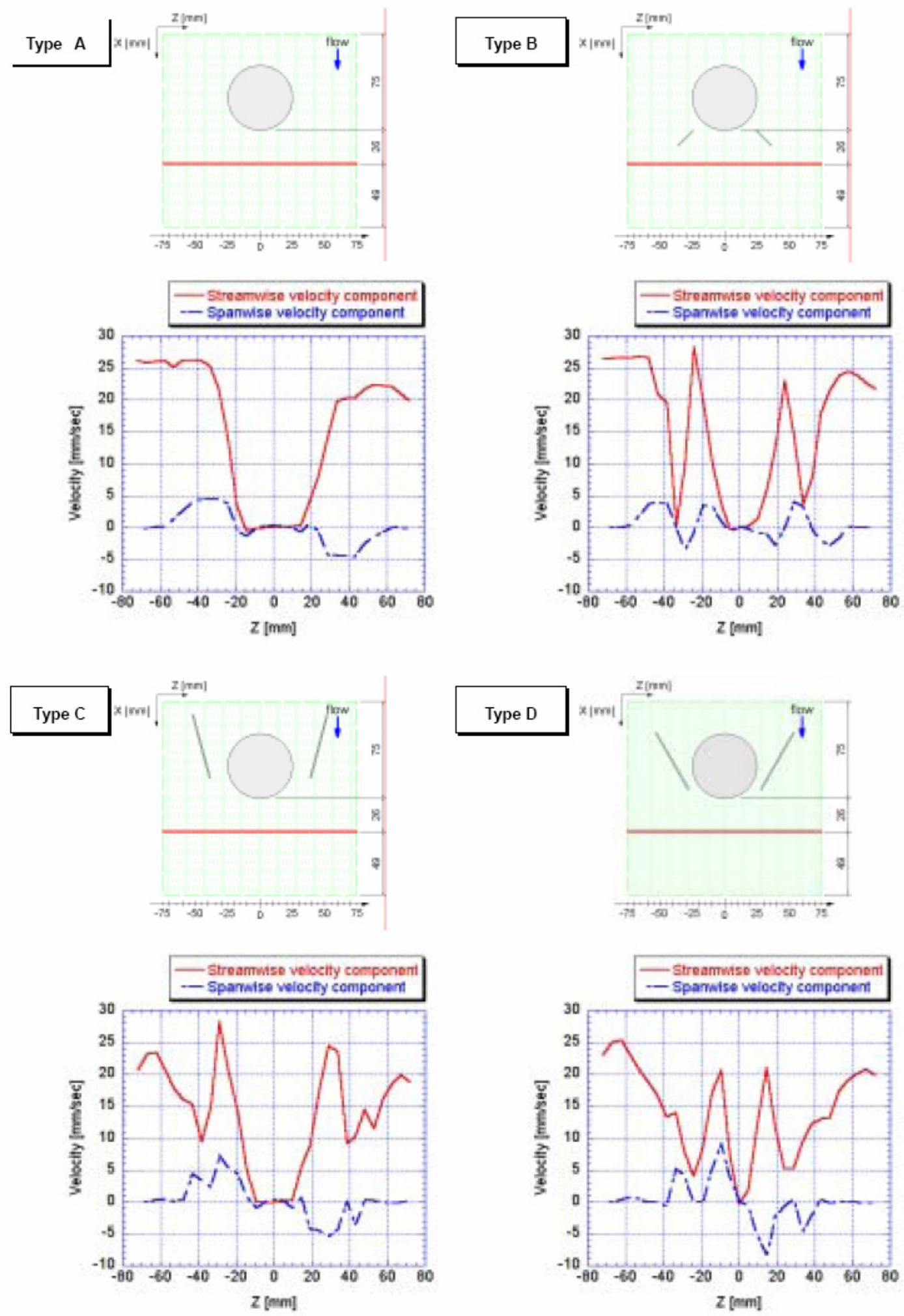

Figure 5-32. Mean velocity profile along a horizontal line for Arrangement I. 
Flow visualization using the hydrogen-bubble technique has indicated the presence of unsteadiness of flow patterns. The profiles of the root-mean-square (RMS) values of velocity fluctuations are shown in Figure 5-33, where the streamwise component, urms, the spanwise component, wrms, and their sum, (urms $2+$ wrms2)1/2, are plotted. The profiles are those along a horizontal line located $0.5 \mathrm{~mm}$ above the bottom channel wall. Its streamwise position with respect to the tube is given in the insets of the figure. Notice that the magnitude of velocity fluctuations is not negligible in comparison with the bulk mean velocities (16.0-20.3 mm/s). The RMS values of streamwise velocity fluctuations are particularly high in the region downstream of winglets for Type $\mathrm{B}(\mathrm{z}=25 \mathrm{~mm})$ and in the region of high-speed fluid inrush for Type $\mathrm{C}(\mathrm{z}=30 \mathrm{~mm})$. This result suggests that the fluid motions responsible for the heat-transfer enhancement in the stagnation region have appreciable magnitude of unsteadiness and therefore that any interpretation or prediction assuming steadiness of flow fields will fail to provide reliable results. The distributions of urms and wrms in a horizontal plane located $0.5 \mathrm{~mm}$ above the bottom channel wall for Type $\mathrm{C}$ are shown in Figure 5-34. As expected, a slender region of large RMS values of streamwise velocity fluctuations extends from the trailing edge of each winglet.

\subsubsection{Advanced Analysis of Flow Fields in Arrangement I (Single Row Tubes)}

In order to understand the flow field in more detail, the out-of-plane velocity component (or the wallnormal velocity component) is calculated using the continuity equation from a set of in-plane mean velocity data measured in a total of 17 horizontal planes. The continuity equation for incompressible fluid is

$$
\frac{\partial U}{\partial x}+\frac{\partial V}{\partial y}+\frac{\partial W}{\partial z}=0
$$

where U, V, W are the streamwise, wall-normal and spanwise mean velocity components, respectively. The wall-normal velocity component is calculated from Equation (5-9). 

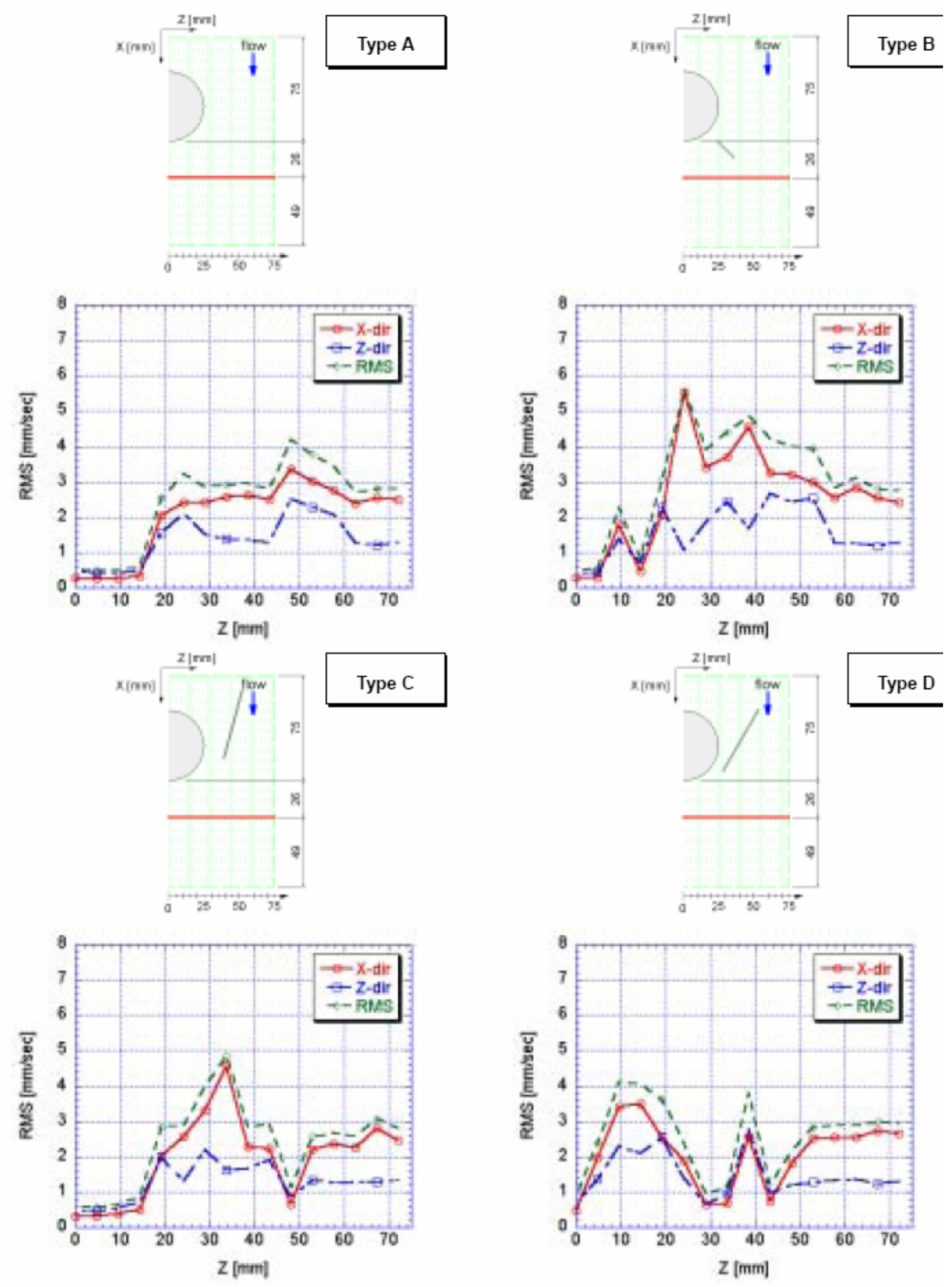

Figure 5-33. RMS of velocity variation along a horizontal line for Arrangement I. 


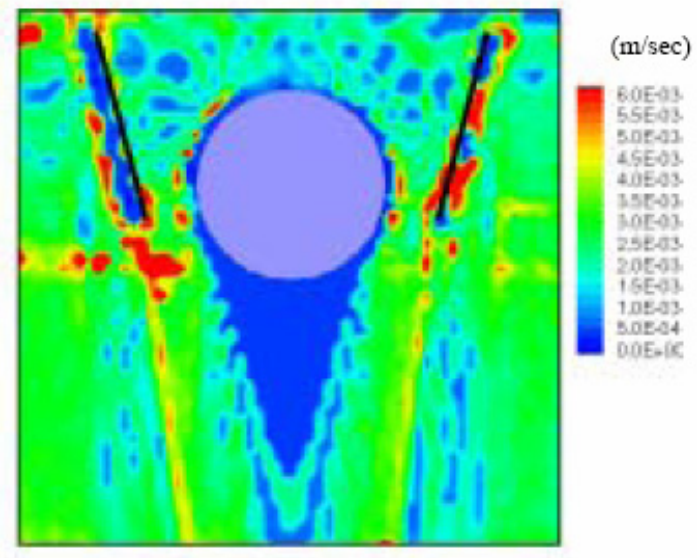

Streamwise

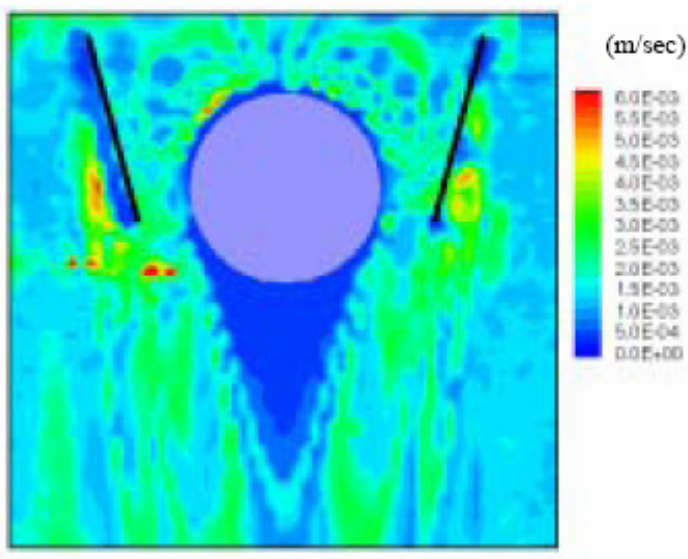

Spanwise

Figure 5-34. Distribution of RMS values of streamwise and spanwise velocity fluctuations in a horizontal plane $0.5 \mathrm{~mm}$ above the bottom channel wall: Type C of Arrangement I.

$V=\int\left\{-\frac{\partial U}{\partial x}-\frac{\partial W}{\partial z}\right\} d y$

Numerical integration using the trapezoidal formula is applied to evaluate the value of $\mathrm{V}$ at $(\mathrm{i}, \mathrm{j}, \mathrm{k})$, where $\mathrm{i}, \mathrm{j}$, and $\mathrm{k}$ denote the grid number in the streamise, wall-normal and spanwise directions, respectively. The resultant discretized equation is

$$
V_{i, j, k}=V_{i, j-1, k}+\left(f_{i, j, k}+f_{i, j-1, k}\right)\left(y_{i, j, k}-y_{i, j-1, k}\right) / 2
$$

where fi, j, $\mathrm{k}=[-\partial \mathrm{U} / \partial \mathrm{x}-\partial \mathrm{W} / \partial \mathrm{z}] \mathrm{I}, \mathrm{j}, \mathrm{k}$. Note that the values of $\mathrm{V}$ at the wall, i.e., Vi,1,k and Vi,M,k are zero from the nonslip condition. The velocity derivatives in Equation (5-11) are evaluated from the central differentiation of the quantities as follows:

$$
\begin{gathered}
{\left[\frac{\partial U}{\partial x}\right]_{i, j, k} \simeq \frac{U_{i+1, j, k}-U_{i-1, j, k}}{x_{i+1, j, k}-x_{i-1, j, k}}=\frac{U_{i+1, j, k}-U_{i-1, j, k}}{2 \Delta x},} \\
{\left[\frac{\partial W}{\partial z}\right]_{i, j, k} \simeq \frac{W_{i, j, k+1}-W_{i, j, k-1}}{z_{i, j, k+1}-z_{i, j, k-1}}=\frac{W_{i, j, k+1}-W_{i, j, k-1}}{2 \Delta z},}
\end{gathered}
$$

The last equality of the above equations is valid when an equidistant grid system is used.

The calculated wall-normal mean velocities for Type $\mathrm{C}$ are shown in Figure 5-35 in the form of velocity vector plots in cross-sectional $(\mathrm{y}-\mathrm{z})$ planes. The position of each plane is given in the inset of the figure. Similarly, the mean velocity vectors on x-y planes are presented in Figure 5-36. The wall-normal mean velocities near the winglets in plane $G$ of Figure 5-36 exhibit upward motion in front of the winglets and downward motion behind it. Such wall-normal motions are associated with longitudinal vortical motions that are shown in Plane B of Figure 5-35. The vortical motions are, however, not as evident as expected, and it is certainly due to their unsteadiness which will make the mean vortical 
motions obscured. The results for Type D are presented in Figures 5-37 and 5-38, from which the same observations as for Type $\mathrm{C}$ can be made.
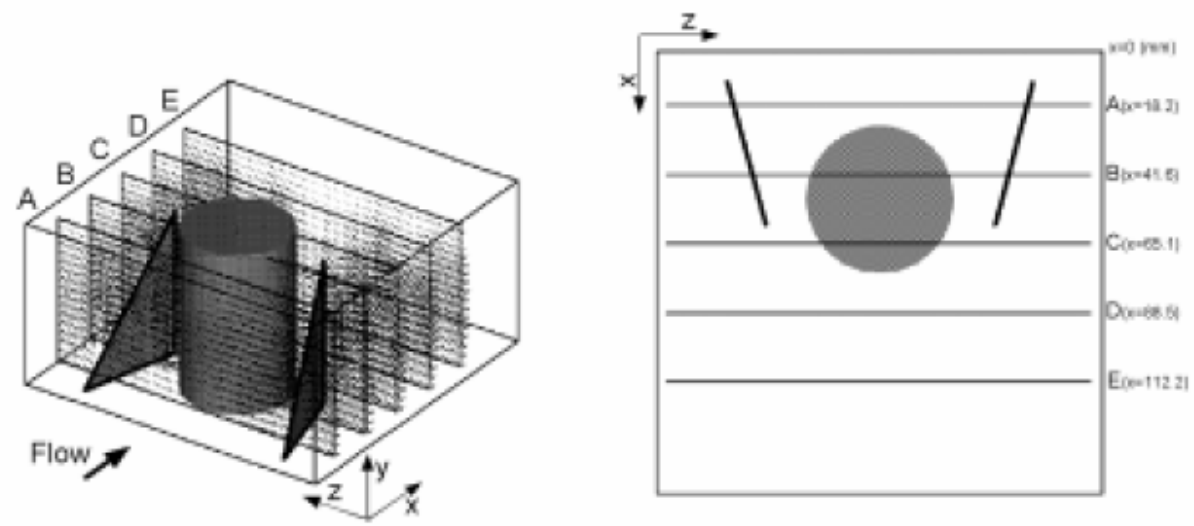

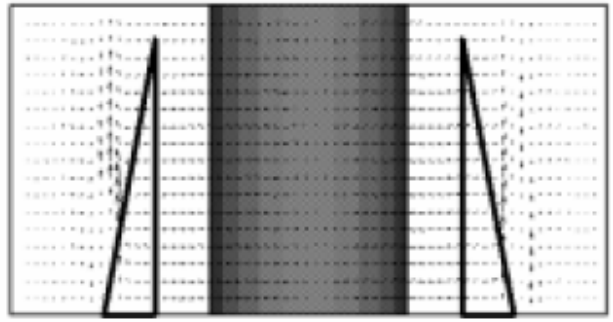

Cut A

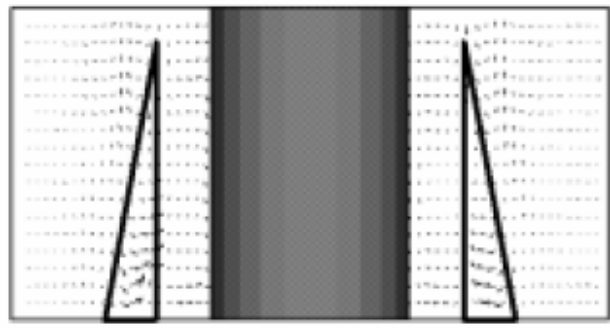

Cut $\mathrm{C}$

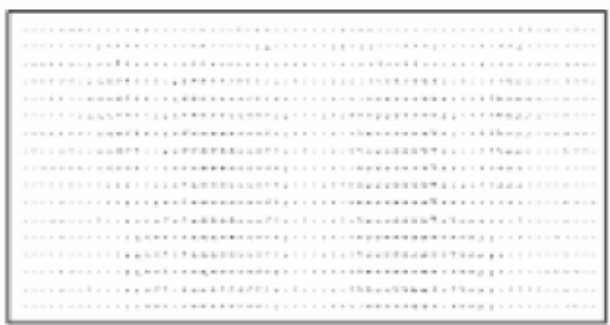

Cut E

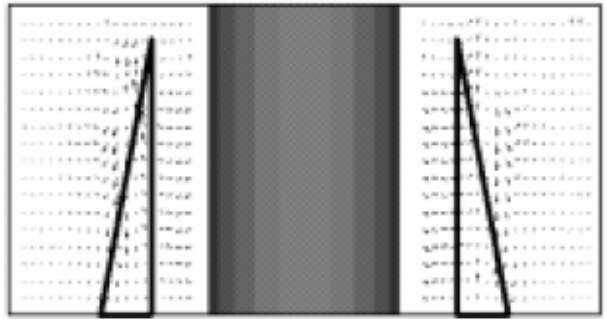

Cut B

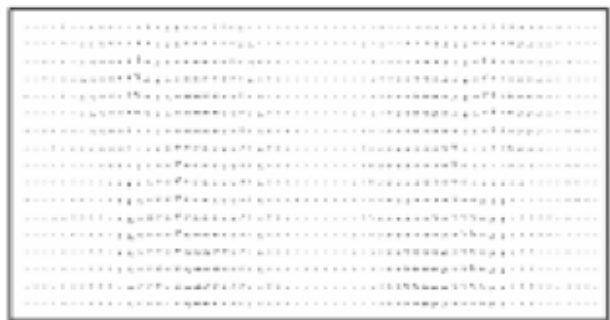

Cut D

$1.5 \mathrm{~mm} / \mathrm{sec}$

$\overleftarrow{30} \mathrm{~mm} / \mathrm{sec}$

Figure 5-35. Mean velocity vectors in Y-Z planes for Type C of Arrangement I. 

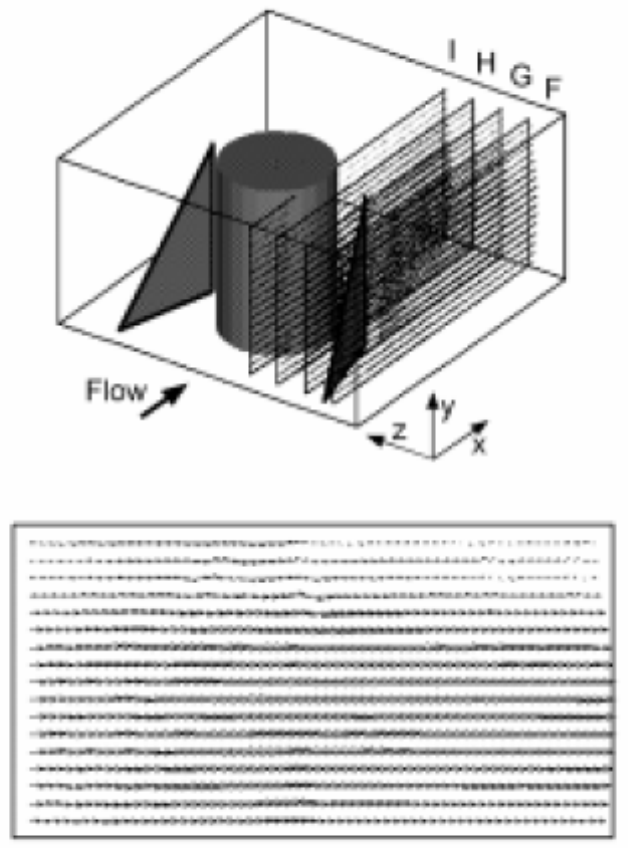

Cut F

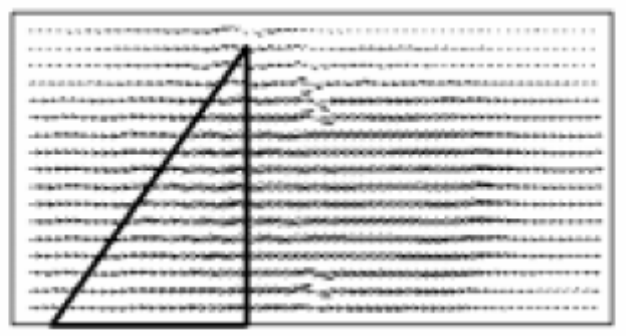

Cut $\mathrm{H}$
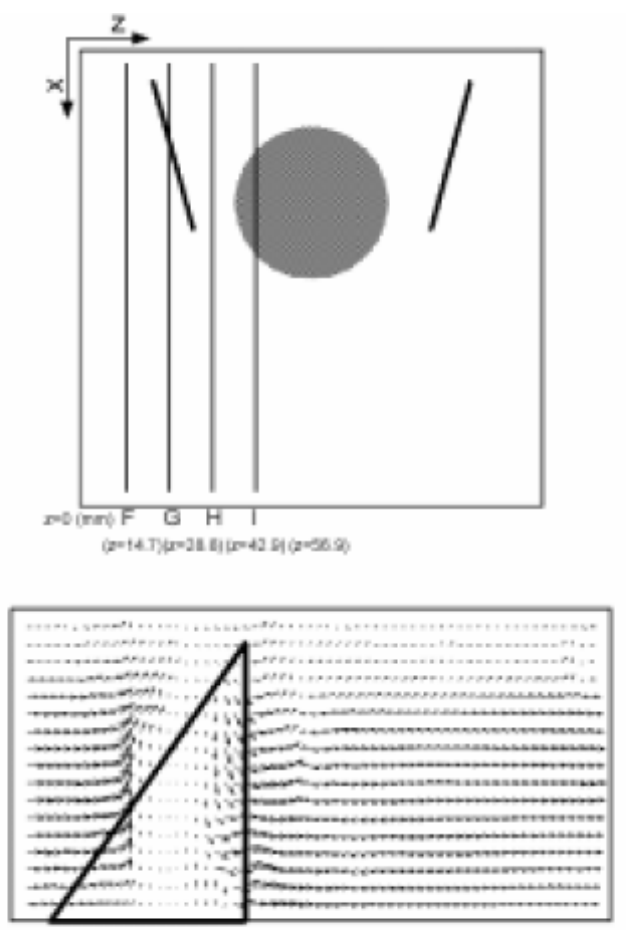

Cut G

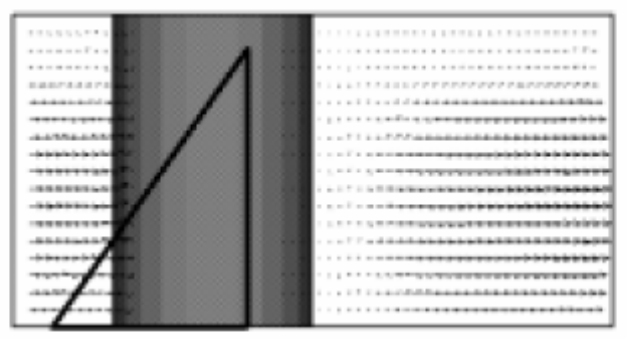

Cut I

Figure 5-36. Mean velocity vectors in X-Y planes for Type C of Arrangement I.

To provide a physical link between the velocity fields obtained with PIV and the overall heat-transfer enhancement measured in the performance test, the wall shear stress (WSS) distributions on the bottom channel wall are evaluated from the velocity fields. The streamwise and spanwise wall shear stresses, $\tau \mathrm{x}$ and $\tau z$, on the bottom channel wall are defined as

$\tau_{x}=\mu\left\lfloor\frac{\partial U}{\partial y}\right\rfloor_{y=0} \quad$ and $\quad \tau_{z}=\mu\left\lfloor\frac{\partial W}{\partial y}\right\rfloor_{y=0}$

where $\mu$ is the dynamic viscosity of working fluid. The velocity gradient at the wall is evaluated by assuming that the streamwise mean velocities, $\mathrm{U}$ and $\mathrm{W}$, are expressed by a 2 nd-order polynomial function as follows:

$U=a_{x} y+b_{x} y^{2} \quad$ and $\quad W=a_{z} y+b_{z} y^{2}$ 
Consequently, $\tau \mathrm{x}=\mathrm{ax} \mu$ and $\tau \mathrm{z}=\mathrm{az} \mu$. The coefficients au, bu, aw and bw are determined by mean of the least-square-method using velocity data taken at three points adjacent to the wall $(\mathrm{y}=0.5,1.0$, and 1.5 $\mathrm{mm}$ ). It is true that the locations of these data points are not sufficiently close to the wall for accurate evaluation of wall shear stress and therefore, the evaluation here can provide only qualitative information. Nevertheless, the wall shear stress distributions reveal some interesting patterns as described below.
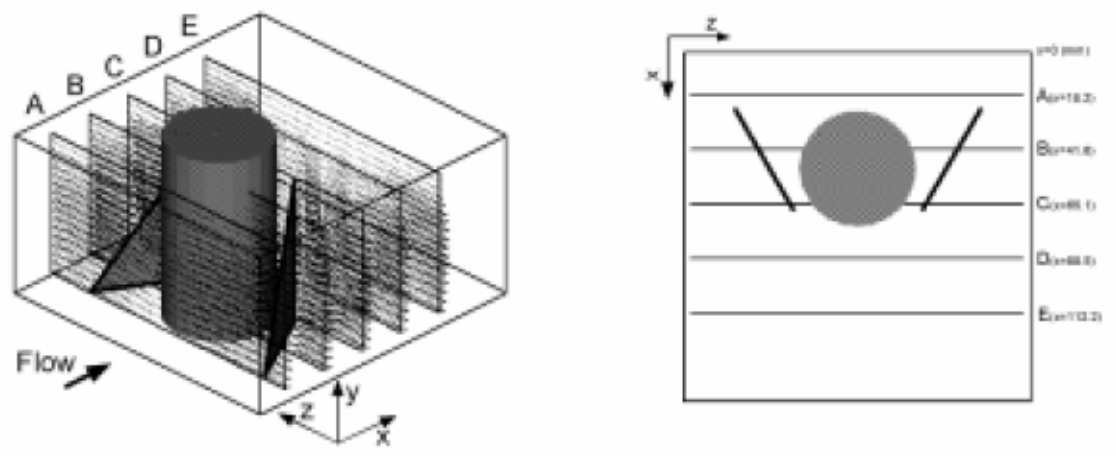

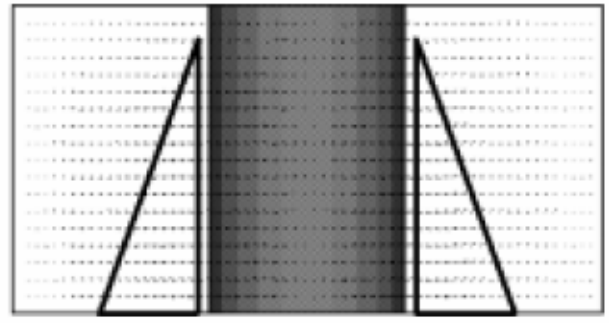

Cut A

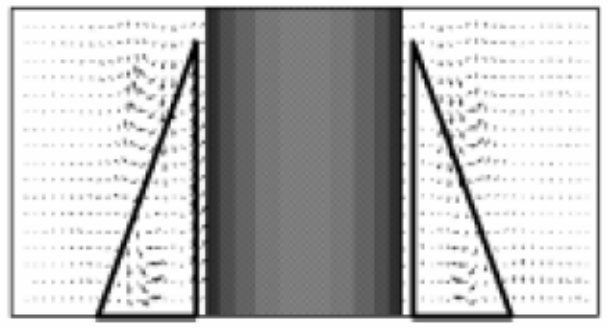

Cut C

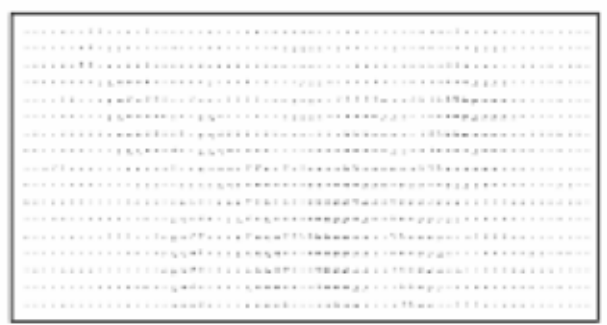

Cut E

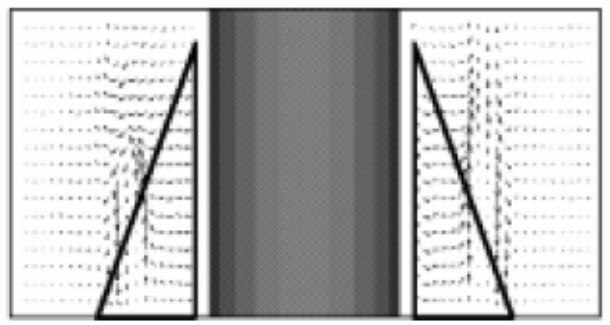

Cut B

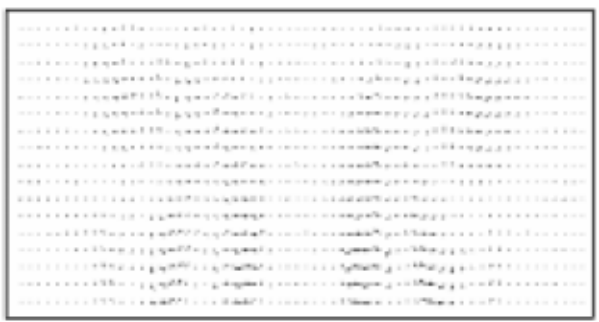

Cut D

11.5 mmises

30 mmisec

Figure 5-37. Mean velocity vectors in Y-Z planes for Type D of Arrangement I. 

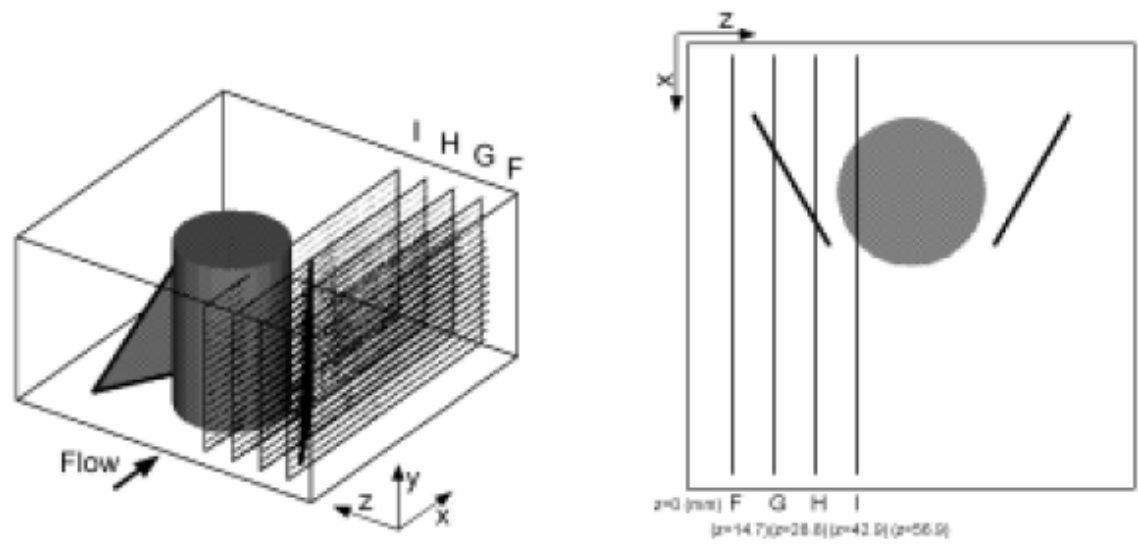

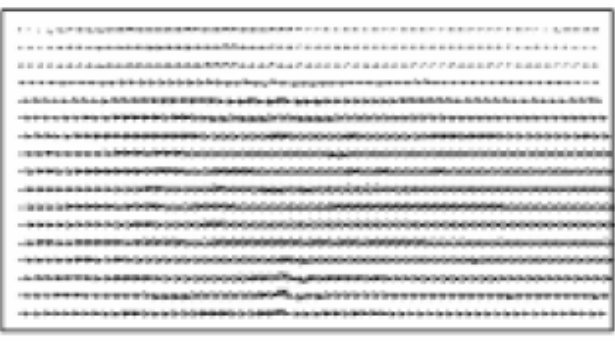

Cut $\mathrm{F}$

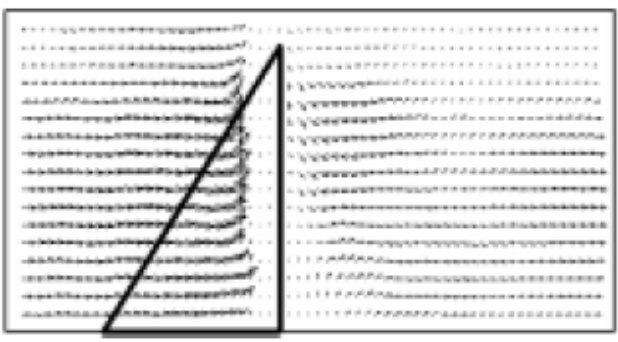

Cut $\mathrm{H}$

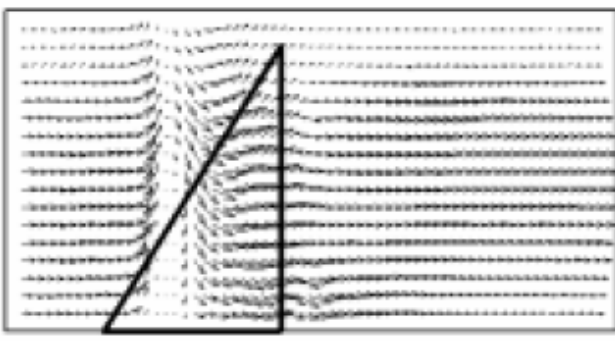

Cut $\mathrm{G}$

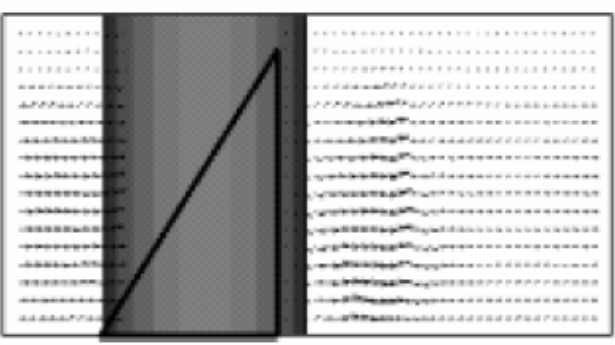

Cut I
$11.5 \mathrm{~mm} / \mathrm{ses}$

30 mmisec

Figure 5-38. Mean velocity vectors in X-Y planes for Type D of Arrangement I.

Figure 5-39 shows the distributions of total wall shear stres, $\sqrt{\tau_{x}^{2}+\tau_{z}^{2}}$, , for Types A-D. A pair of high shear stress regions appears near both sided of the tube of Types A and B. This is due to the fluid acceleration in that region. Types $\mathrm{C}$ and $\mathrm{D}$ display similar high shear stress regions beside the winglets, but the regions are comparatively elongated, suggesting the effect of longitudinal vortical motions created by the winglets. A pair of elongated high wall shear stress regions also appears downstream of the winglets in Type B. Comparison between Types C and D clearly indicates that the shrinkage of the stagnant region behind the tube is more pronounced in Type $\mathrm{D}$ than in Type $\mathrm{C}$. This is consistent with higher heat-transfer enhancement in Type D than in Type C (136\% versus $45 \%)$. In comparison, the reduction of the stagnant region of Type $\mathrm{B}$ is larger than that of Type $\mathrm{C}$ but smaller than that of Type $\mathrm{D}$. This is also consistent with the rate of heat-transfer enhancement of Type B (66\%). The results presented here demonstrate that the heat-transfer enhancement can be estimated, at least qualitatively, by examining wall shear stress distributions. This is quite useful for optimization of configurations of tubes and winglets. 


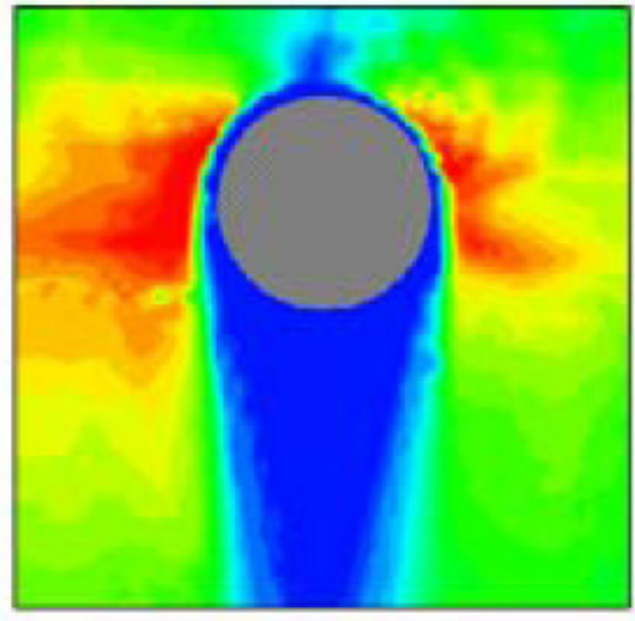

Type A

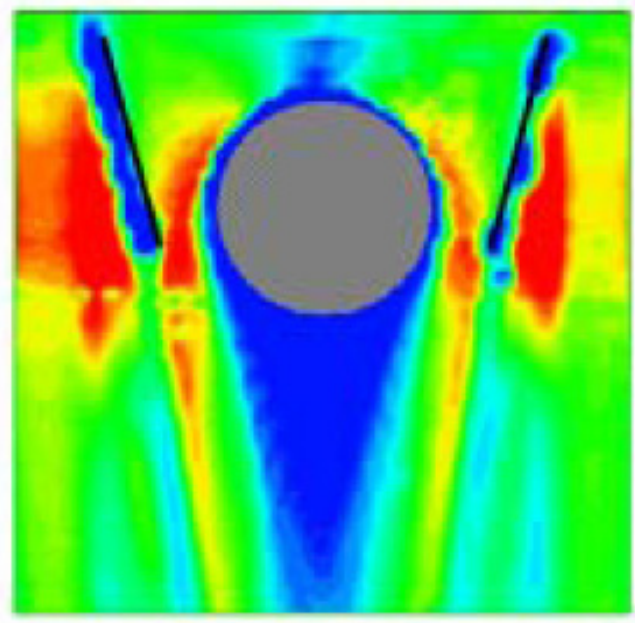

Type C

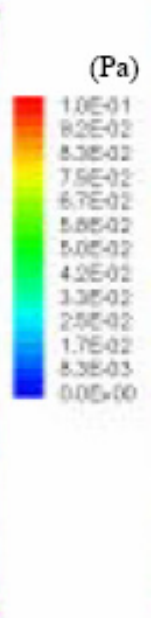

$(\mathrm{Pa})$

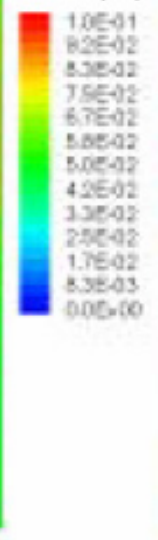

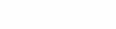

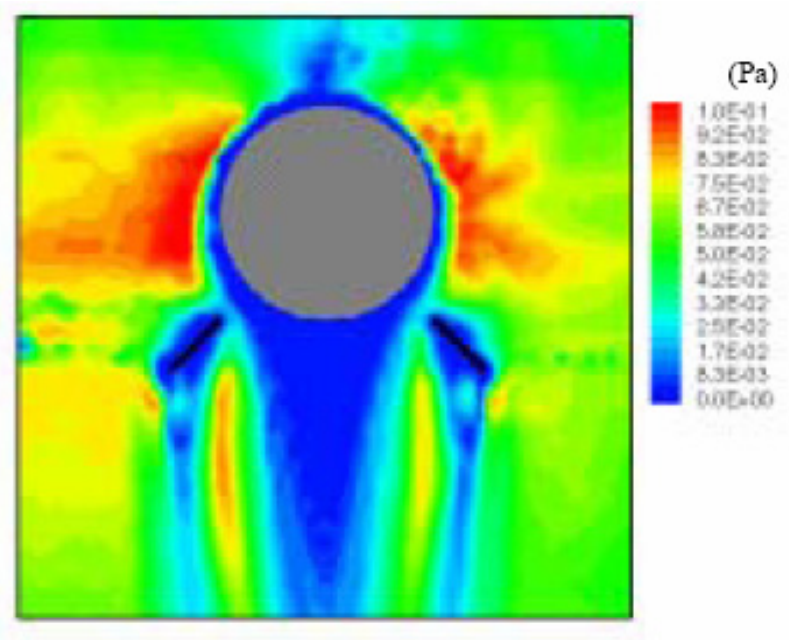

Type B

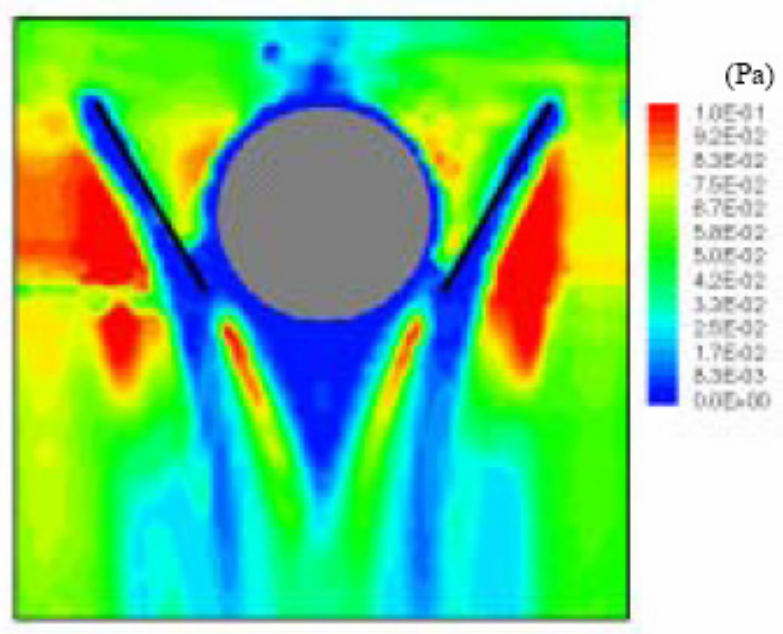

Type D

Figure 5-39. Total wall shear stress distribution for Types A-D of Arrangement I.

\subsubsection{Flow Characteristics in Arrangement II (Three-Row In-Line Tube Bundle with Single Row Winglets)}

Figures 5-40 through 5-42 present the flow patterns in horizontal planes for Types A, C and D, respectively. Note that only the tubes in the first row are mounted with winglets, and those in the second and third rows do not have winglets. The measurements are taken in the region around the center tube in the second row. In Figure 5-40, an elongated stagnant region is seen to occupy almost 1/3 of the domain. A similarly elongated region is seen in Type C (Figure 5-39). The overall performance test (see Section 5.1) has shown that Type $C$ increases heat transfer by $10 \%$ and decreases pressure loss by $8 \%$ in comparison with Type A. In contrast, Type D has a stagnant region only downstream of the tube, caused by the winglets in the first row (Figure 5-40). 


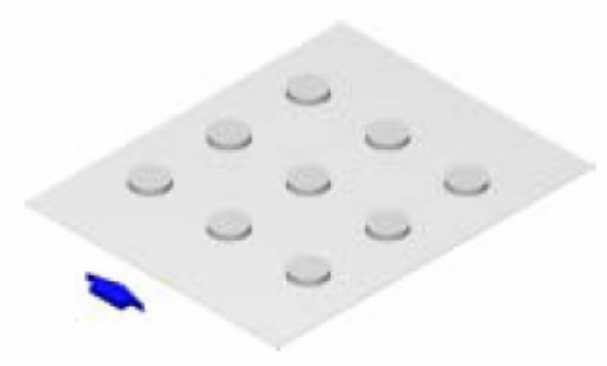

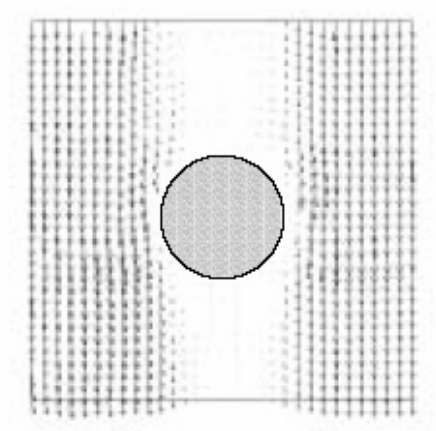

$y=0.5 \mathrm{~mm}$

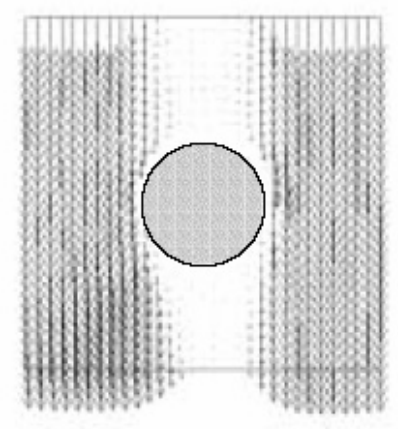

$y=3.5 \mathrm{~mm}$

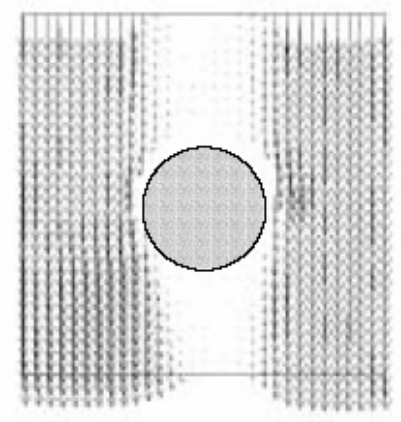

$y=6.5 \mathrm{~mm}$

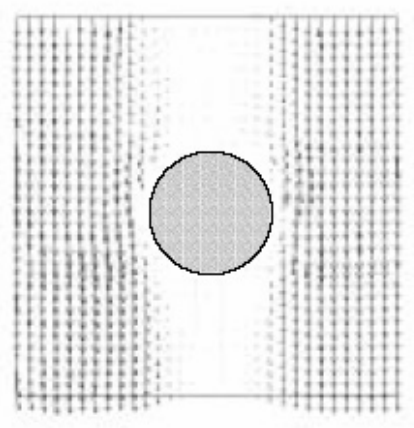

$y=1.5 \mathrm{~mm}$

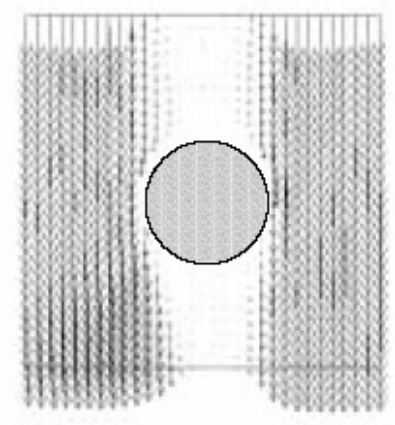

$y=4.5 \mathrm{~mm}$

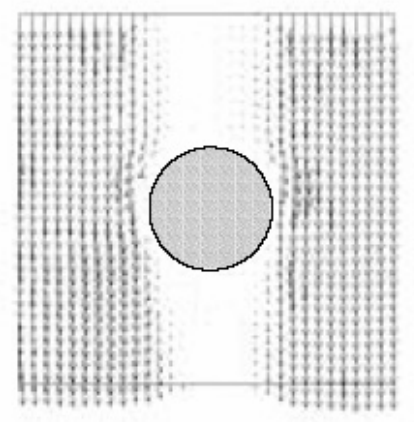

$y=7.5 \mathrm{~mm}$
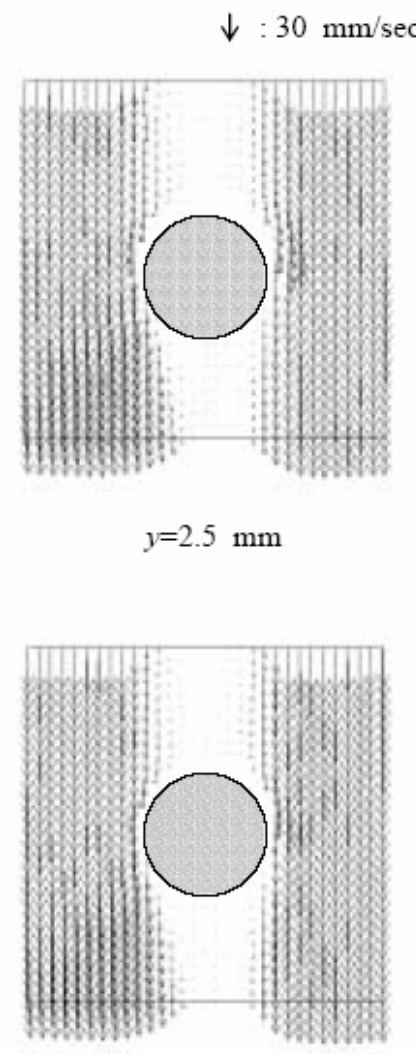

$y=5.5 \mathrm{~mm}$

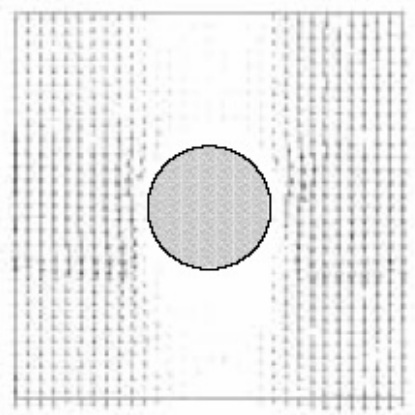

$y=8.5 \mathrm{~mm}$

Figure 5-40. Flow patterns of Type A of Arrangement II (three row in-line tube bundle). 


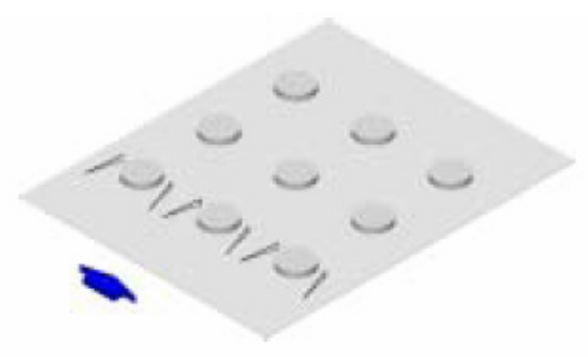

$\downarrow: 30 \mathrm{~mm} / \mathrm{sec}$

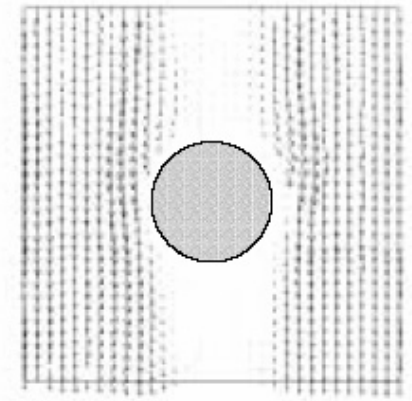

$y=0.5 \mathrm{~mm}$

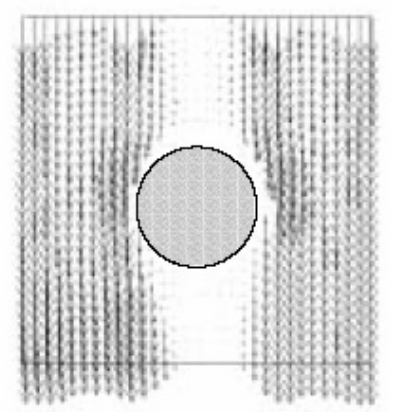

$y=3.5 \mathrm{~mm}$

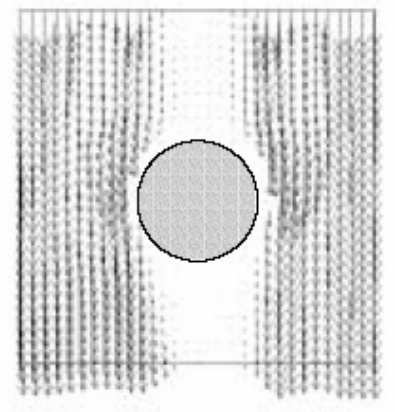

$y=6.5 \mathrm{~mm}$

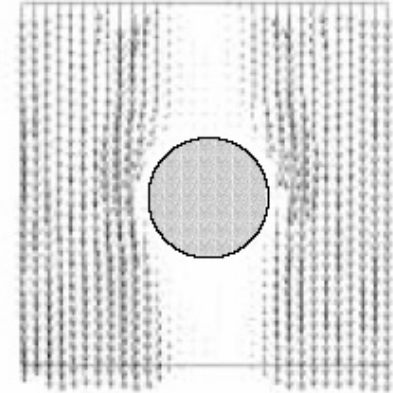

$y=1.5 \mathrm{~mm}$

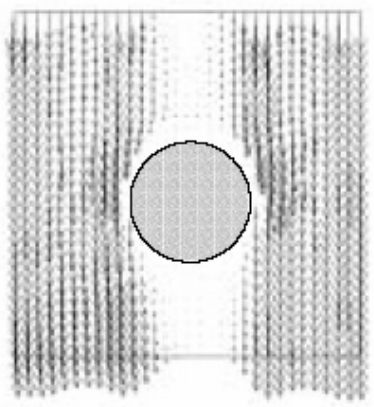

$y=4.5 \mathrm{~mm}$

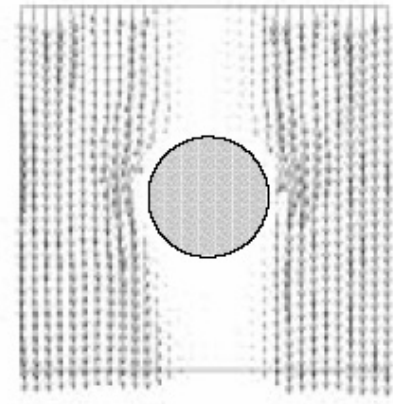

$y=7.5 \mathrm{~mm}$

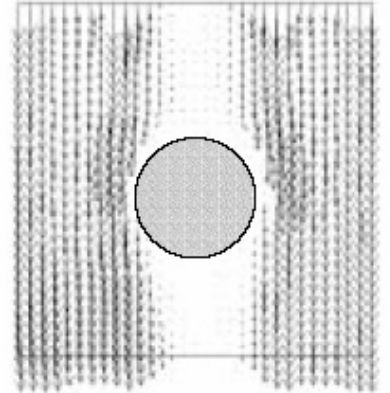

$y=2.5 \mathrm{~mm}$

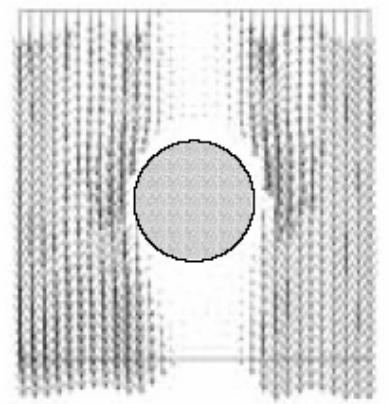

$y=5.5 \mathrm{~mm}$

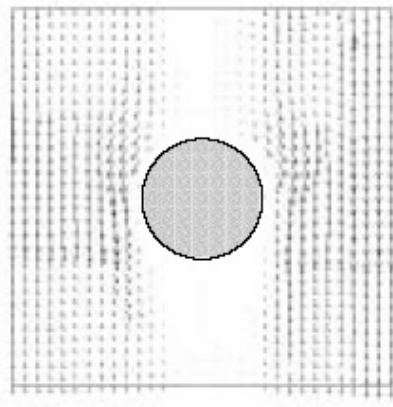

$y=8.5 \mathrm{~mm}$

Figure 5-41. Flow patterns of Type B of Arrangement II (three row in-line tube bundle). 


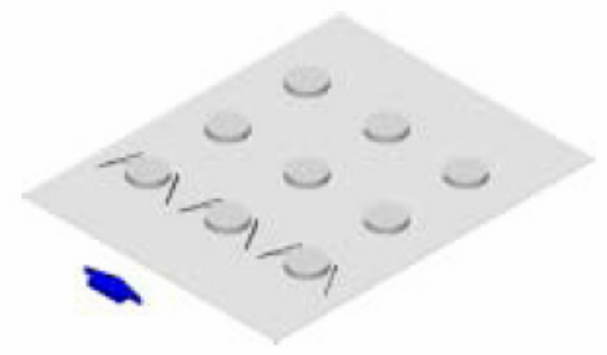

$\downarrow: 30 \mathrm{~mm} / \mathrm{sec}$

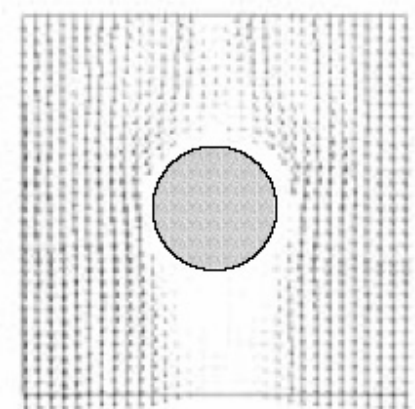

$y=0.5 \mathrm{~mm}$

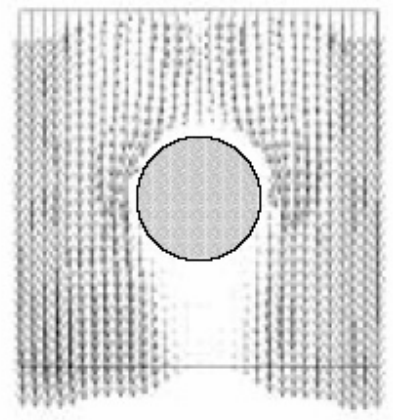

$y=3.5 \mathrm{~mm}$

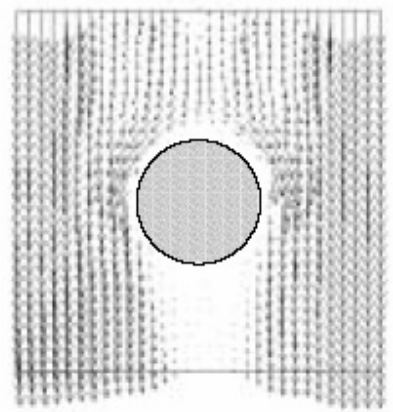

$y=6.5 \mathrm{~mm}$

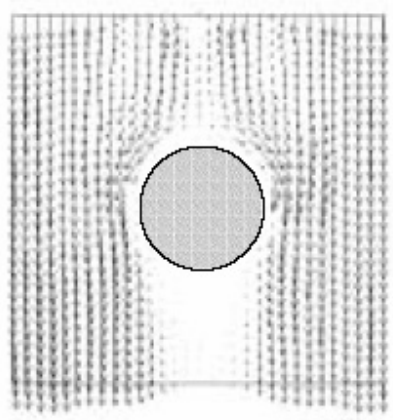

$y=1.5 \mathrm{~mm}$

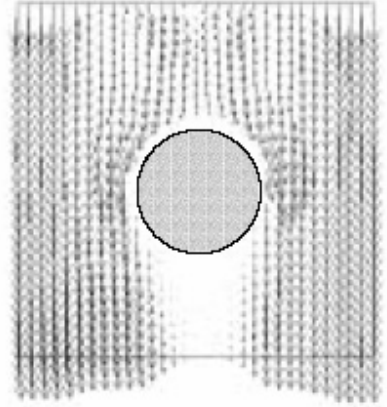

$y=4.5 \mathrm{~mm}$

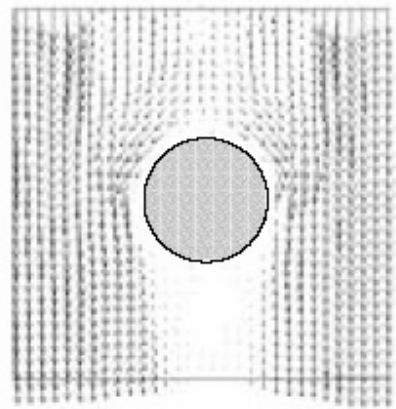

$y=7.5 \mathrm{~mm}$

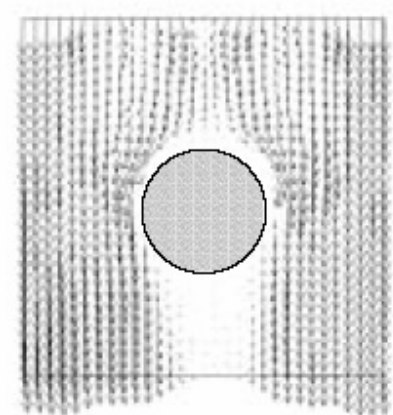

$y=2.5 \mathrm{~mm}$

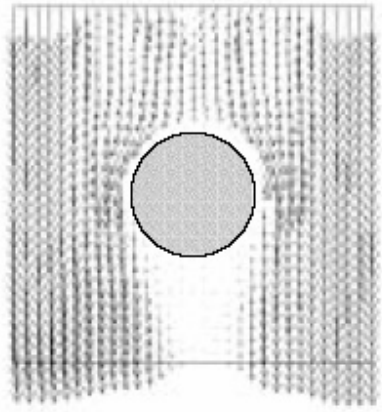

$y=5.5 \mathrm{~mm}$

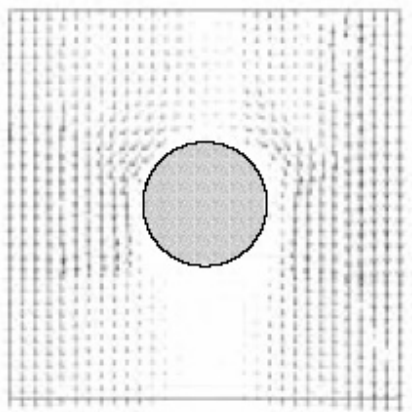

$y=8.5 \mathrm{~mm}$

Figure 5-42. Flow patterns of Type $\mathrm{C}$ of Arrangement II (three row in-line tube bundle). 


\subsubsection{Flow Characteristics in Arrangement III (Three-Row In-Line Tube Bundle with Three-Row Winglets)}

Winglets are mounted with every row of tubes in Types B and D, as shown in Figures 5-43 and 5-44, respectively. It is seen that Type D reduces (or nearly eliminates) the stagnant region effectively. Such effect is less pronounced in Type B than in Type D. Judging from the flow patterns, these configurations are anticipated to achieve the most remarkable heat-transfer enhancement at the highest cost of pressure loss.

\subsubsection{Flow Characteristics in Arrangement IV (Three-Row Staggered Tube Bundle)}

Figures 5-45 through 5-47 present the flow patterns in horizontal planes for Types A, C, and D. In general, the rate of heat transfer on the fin surface of staggered tube bundle is higher than that of in-line tube bundle. The reason is seen in Figure 5-46, where the stagnant region created by the upstream tubes is suppressed by the flow acceleration in the gap between the downstream tubes, thus limiting the streamwise extent of the stagnant region to relatively small value. This effect appears to be exploited both in Types $\mathrm{C}$ and $\mathrm{D}$, so that the flow guided into the stagnant region by the winglets in the first row smoothly goes through the gap between the tubes in the second row. This mechanism also appears to contribute to the reduction of the stagnant region behind the second tubes. These observations are supported by the remarkable performance confirmed by the overall heat-transfer experiment: Type $\mathrm{C}$ has an increase of heat transfer by $10 \%$, along with a decrease of pressure loss by $55 \%$, both in comparison with Type A.

\subsubsection{Conclusions of PIV Study}

Conclusions drawn from the results presented in the previous sections are summarized below.

1. The configurations of tubes and vortex generators proposed in this report, i.e., Types $\mathrm{C}$ and $\mathrm{D}$, have a favorable effect of introducing high-momentum fluid into the stagnant region behind tubes. This effect of winglets coupled with their capability of creating longitudinal vortical motions explains remarkable heat-transfer enhancement, particularly in Type $\mathrm{D}$, as revealed by the overall performance test described in the previous sections of this report.

2. The detailed flow patterns guided into the stagnant region are observed to differ considerably, depending on the configuration parameters, such as the angle of attack of winglets and the gap between a tube and corresponding winglets. Inasmuch as the pressure loss in tube-bundle arrangements is largely due to form drag of the tubes, pressure-loss characteristics can be assessed from the flow patterns, particularly in the stagnation region. In this respect, the present flow measurements are successful in providing physical evidence for explaining some pressure-loss characteristics measured in the overall performance test.

3. The wall shear stress distributions evaluated from the whole-field velocity data are consistent, at least qualitatively, with the observed heat-transfer enhancement for various configurations in singlerow tube arrangement. It can be concluded that the use of PIV is helpful for searching for the optimum configuration in terms of heat-transfer enhancement and pressure-loss penalty.

4. The present flow fields exhibit significant magnitude of unsteadiness in the regions where vortical motions exist. This is verified by the large root-mean-square (RMS) values of velocity fluctuations. This feature is shown to be the reason for misinterpretation about the effects of vortical motions behind the winglets in the reconstructed three-dimensional velocity plots. The presence of unsteadiness implies that any interpretation and prediction based on the assumption of steadiness will fail, in quantitative manner, to capture the true mechanisms happening in the flow fields. 

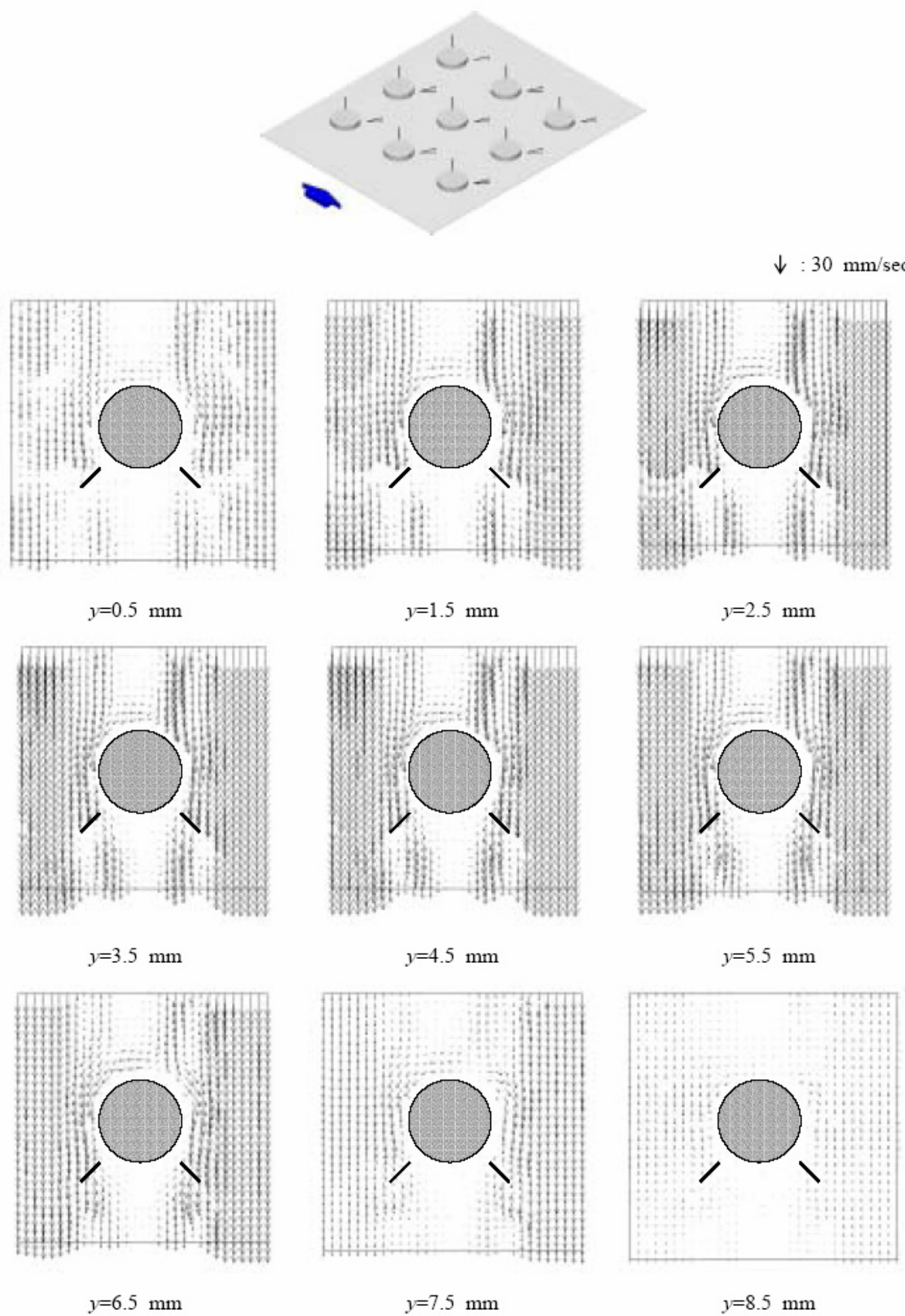

Figure 5-43. Flow patterns for Type B of Arrangement III (three-row in-line tube bundle with three-row winglets). 

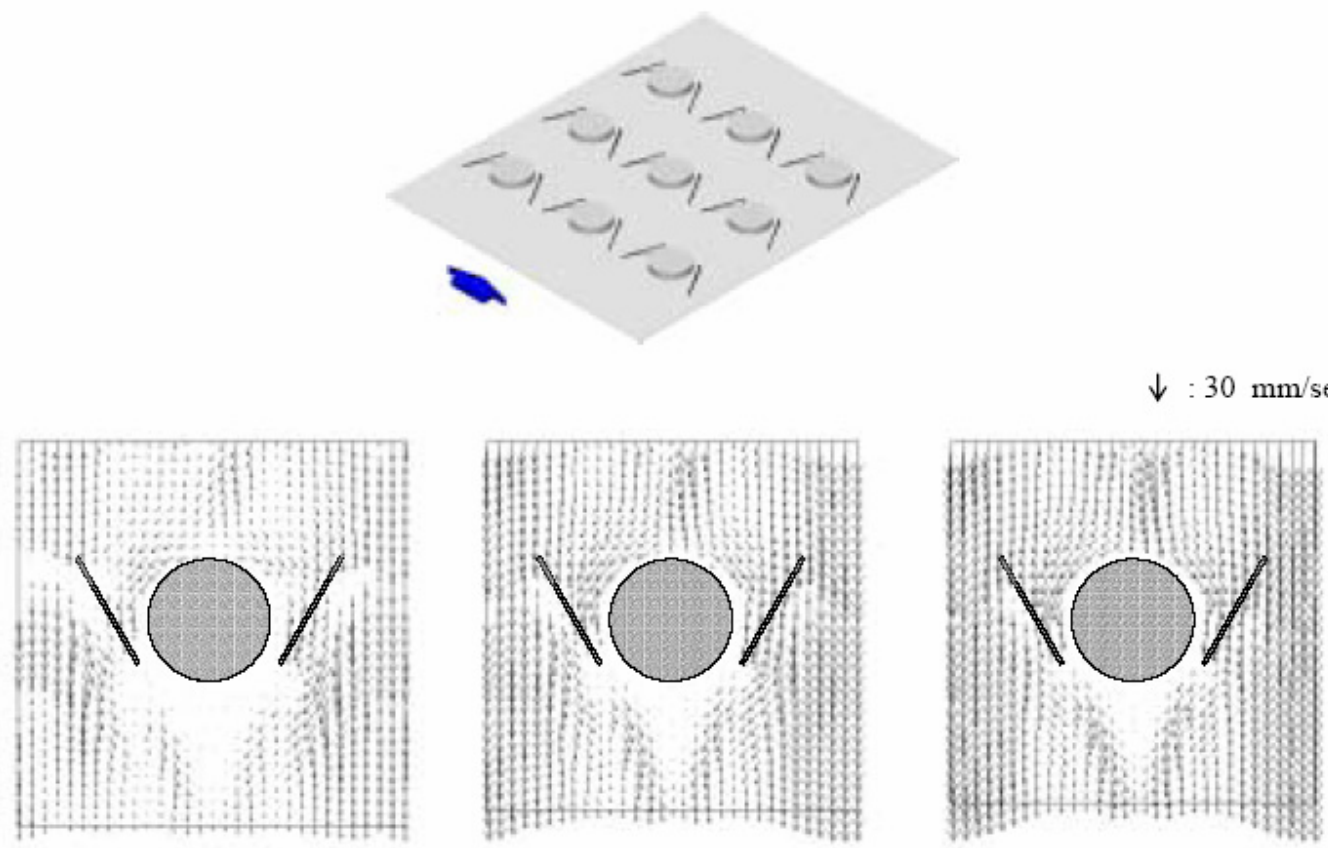

$y=0.5 \mathrm{~mm}$

$y=1.5 \mathrm{~mm}$
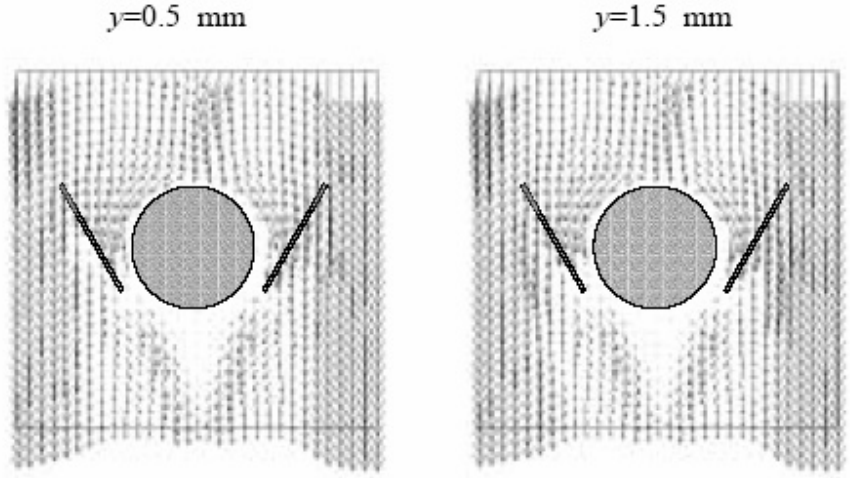

$y=2.5 \mathrm{~mm}$

$y=3.5 \mathrm{~mm}$

$y=4.5 \mathrm{~mm}$
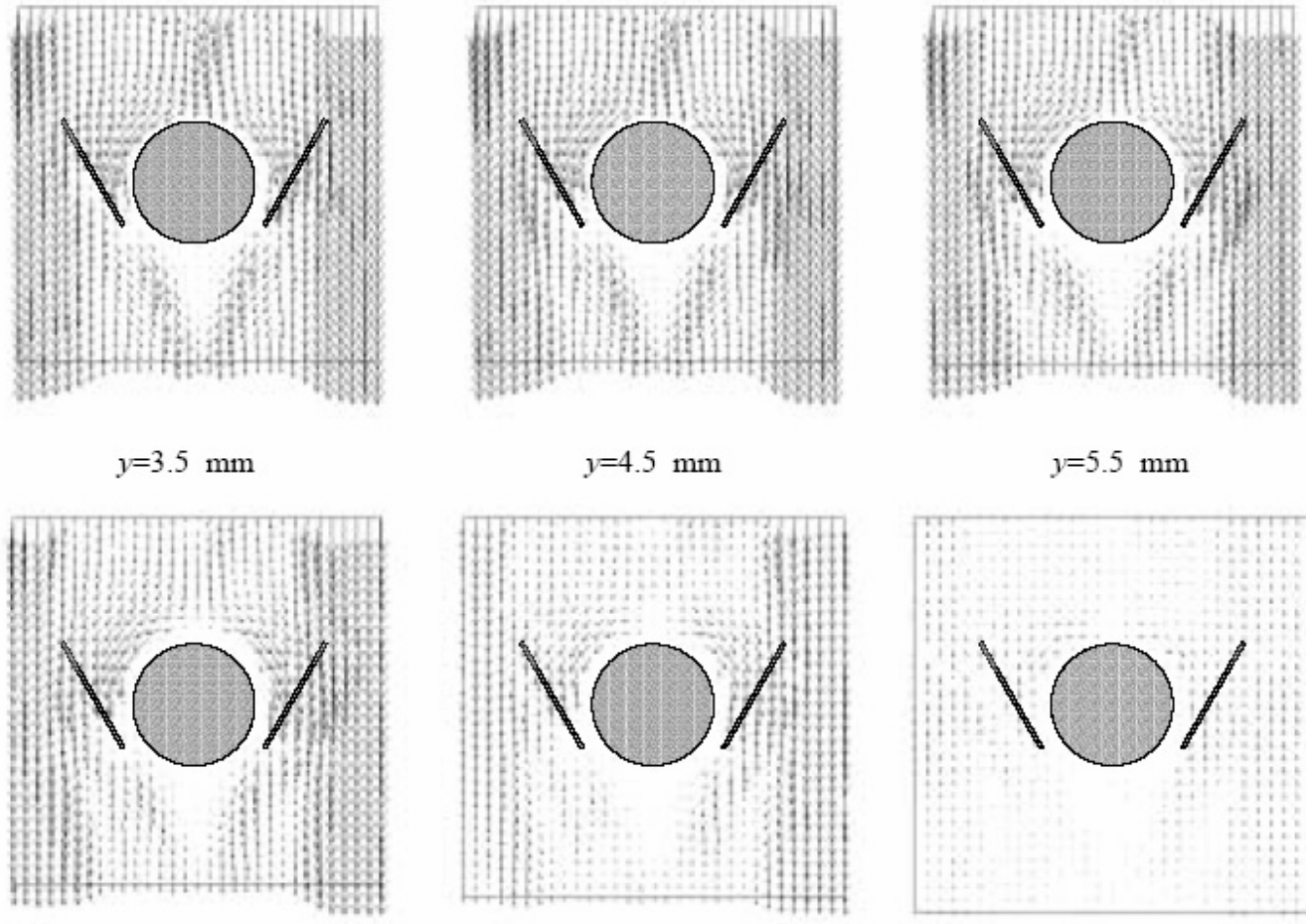

$y=6.5 \mathrm{~mm}$

$y=7.5 \mathrm{~mm}$

$y=8.5 \mathrm{~mm}$

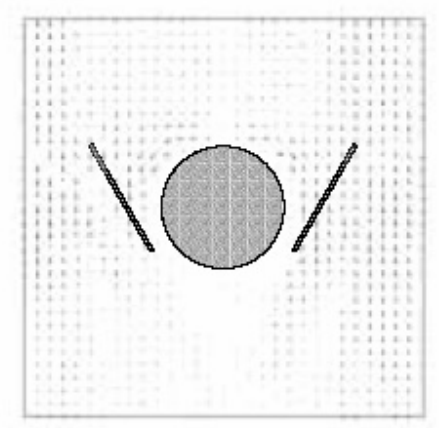

Figure 5-44. Flow patterns for Type D of Arrangement III (three-row in-line tube bundle with three-row winglets). 

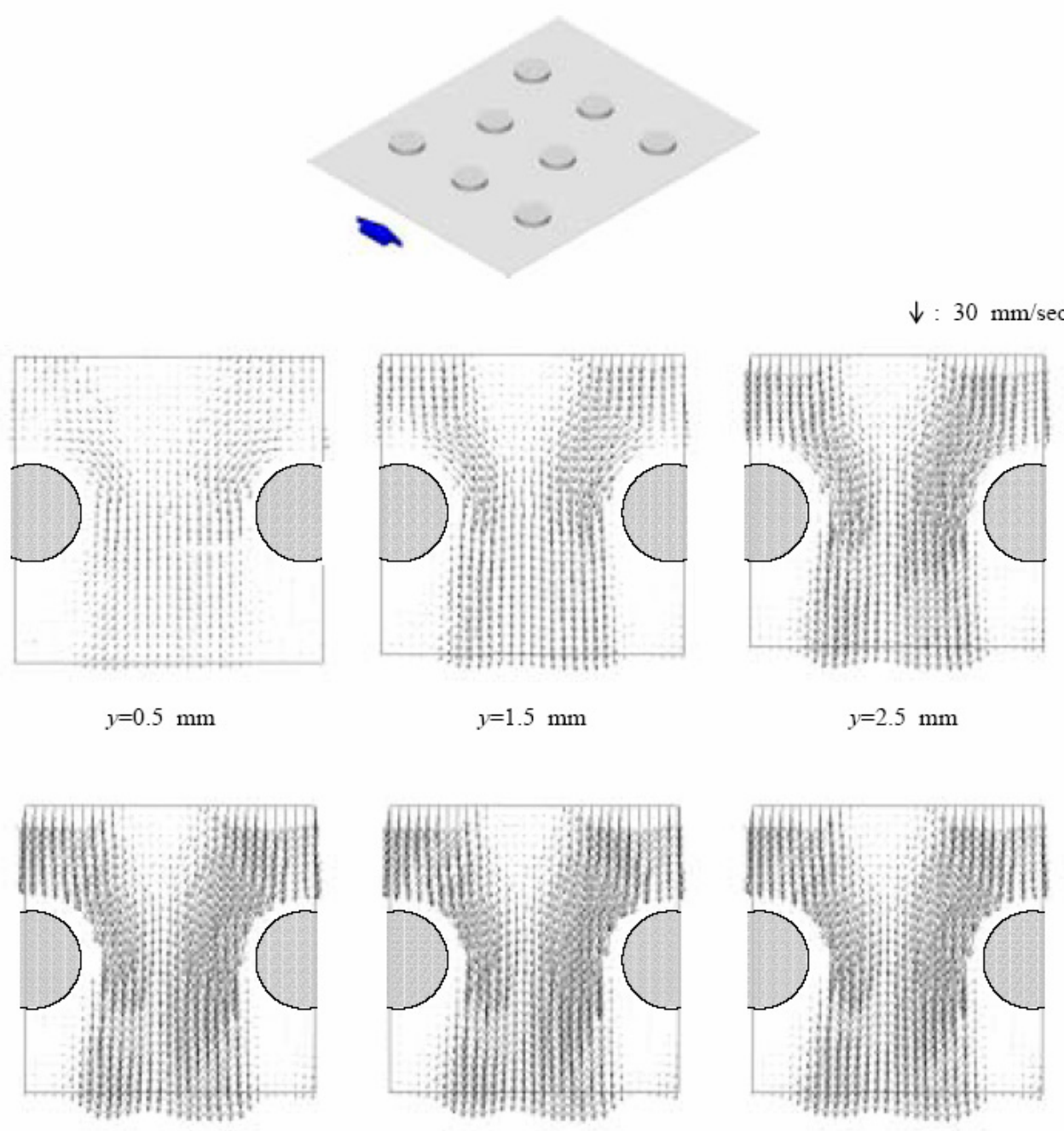

$y=3.5 \mathrm{~mm}$

$y=4.5 \mathrm{~mm}$

$y=5.5 \mathrm{~mm}$
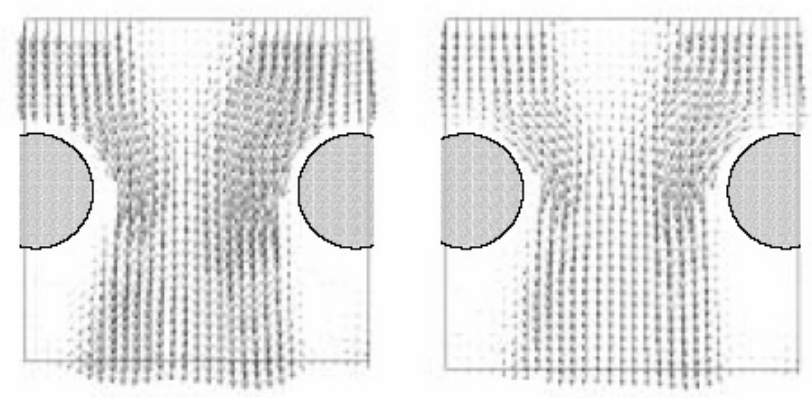

$y=6.5 \mathrm{~mm}$

$$
y=7.5 \mathrm{~mm}
$$

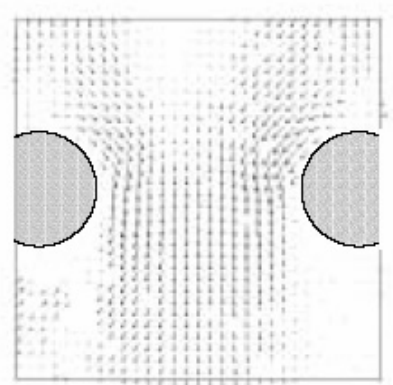

$y=8.5 \mathrm{~mm}$

Figure 5-45. Flow patterns for Type A of Arrangement IV (three-row staggered tube bundle with no winglets). 


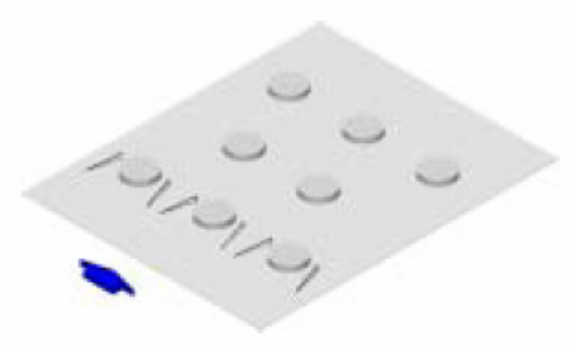

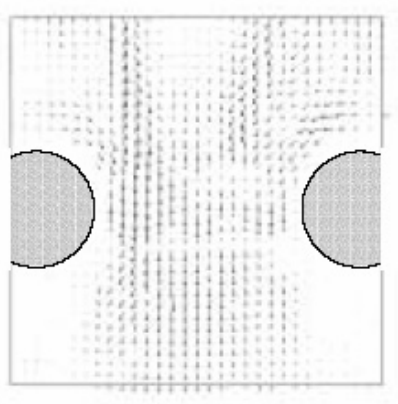

$y=0.5 \mathrm{~mm}$

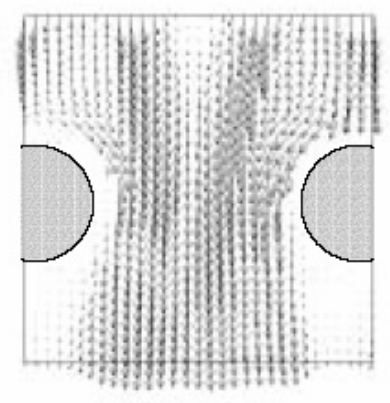

$y=3.5 \mathrm{~mm}$

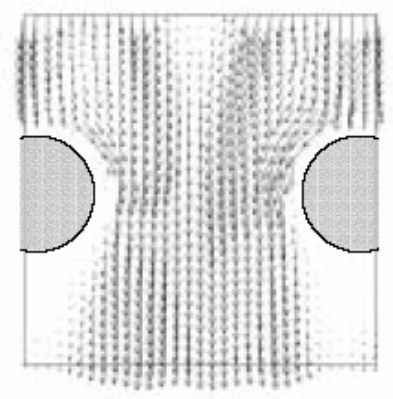

$y=6.5 \mathrm{~mm}$

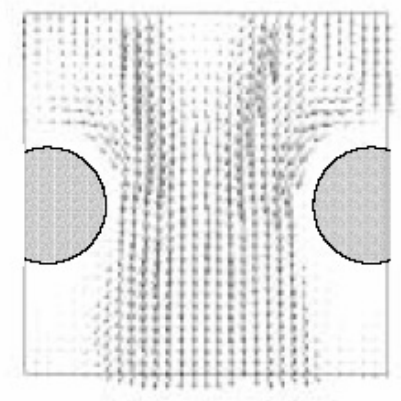

$y=1.5 \mathrm{~mm}$

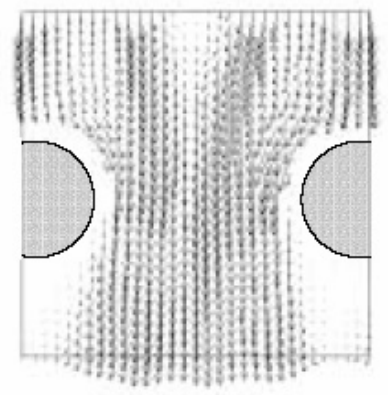

$y=4.5 \mathrm{~mm}$

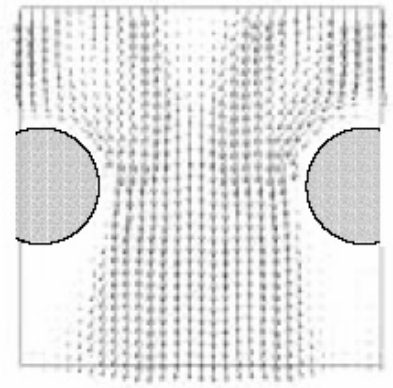

$y=7.5 \mathrm{~mm}$

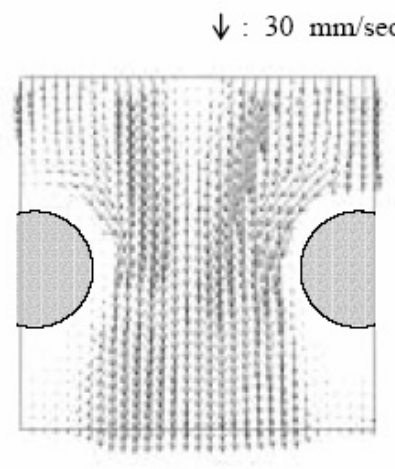

$y=2.5 \mathrm{~mm}$

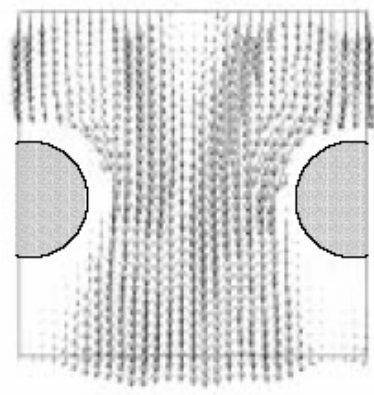

$y=5.5 \mathrm{~mm}$

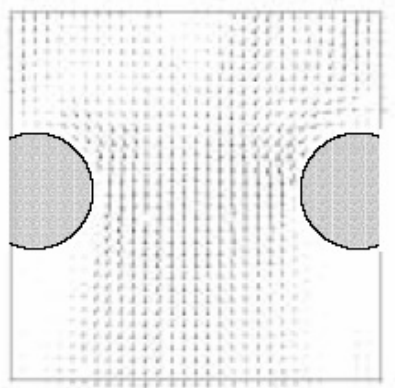

$y=8.5 \mathrm{~mm}$

Figure 5-46. Flow patterns for Type C of Arrangement IV (three-row staggered tube bundle with one row of winglets). 


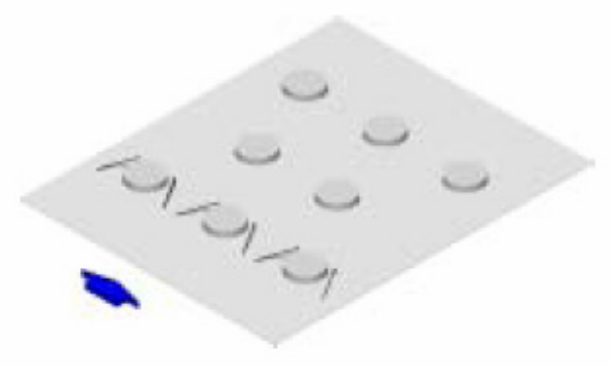

$\downarrow: 30 \mathrm{~mm} / \mathrm{sec}$

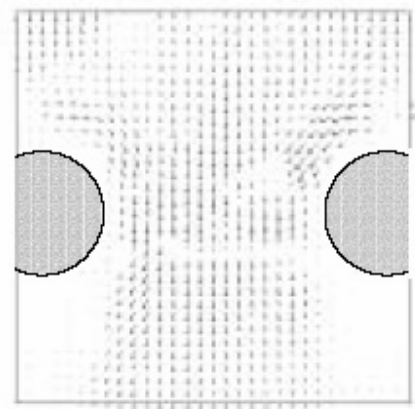

$y=0.5 \mathrm{~mm}$

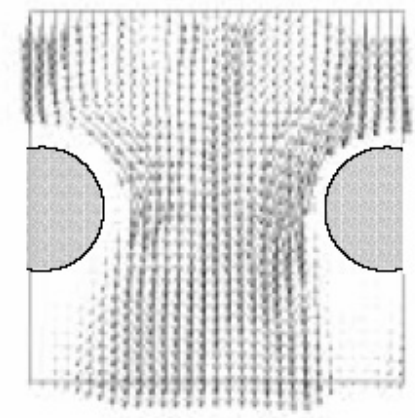

$y=3.5 \mathrm{~mm}$

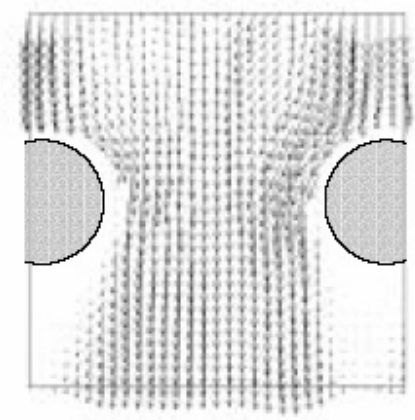

$y=6.5 \mathrm{~mm}$

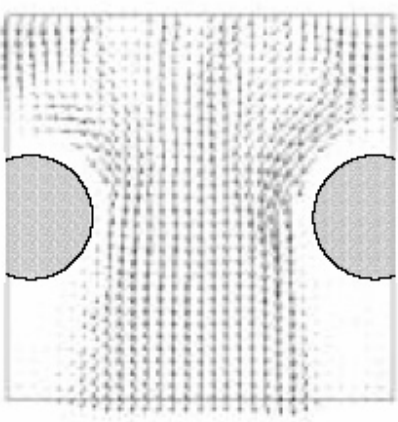

$y=1.5 \mathrm{~mm}$

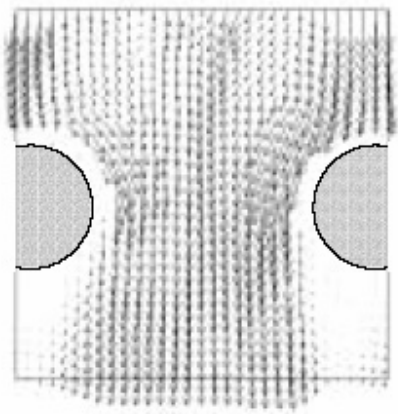

$y=4.5 \mathrm{~mm}$

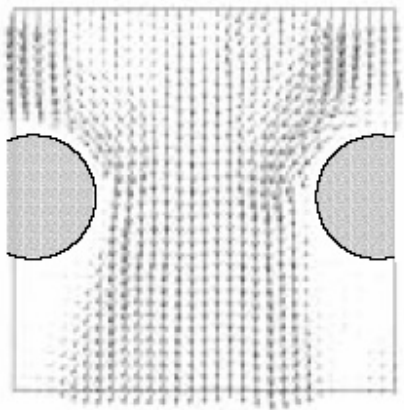

$y=7.5 \mathrm{~mm}$

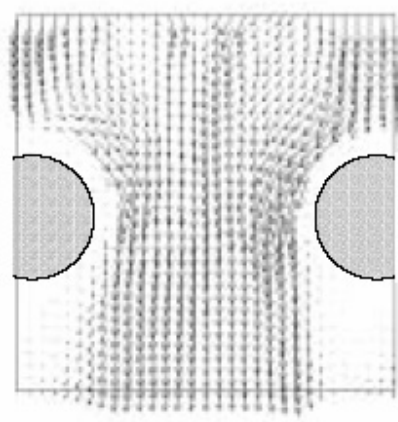

$y=2.5 \mathrm{~mm}$

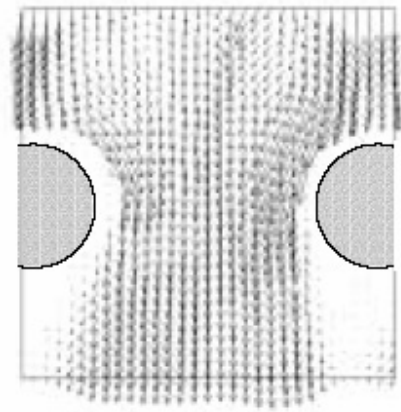

$y=5.5 \mathrm{~mm}$

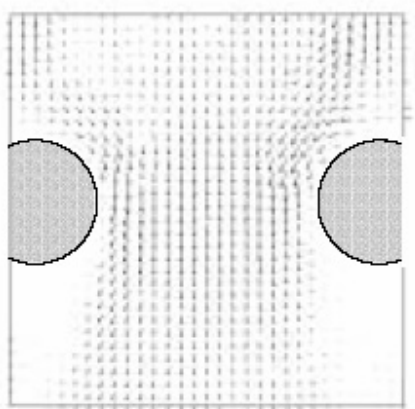

$y=8.5 \mathrm{~mm}$

Figure 5-47. Flow patterns for Type D of Arrangement IV (three-row staggered tube bundle with one row of winglets). 


\section{INDIAN INSTITUTE OF TECHNOLOGY NUMERICAL WORK (NEDO)}

The following numerical work was performed by G. Biswas and his students at the Indian Institute of Technolgy, Kanpur, India and was funded by NEDO, Japan. This work reported here is from five papers (listed below) published by Biswas and co-workers. A sixth paper, although completed under NEDO grant, is not reproduced here as it does not directly relate to the subject matter of this report.

1. S. Tiwari, G. Biswas, P. L. N. Prasad, and S. Basu, "Numerical Prediction of Flow and Heat Transfer in a Rectangular Channel with a Built-in Circular Tube," Journal of Heat Transfer, Vol. 125, 2003, pp. 413-421.

2. A. Jain, G. Biswas, and D. Maurya, "Winglet-type Vortex Generators with Common-flow-up Configuration for Fin-tube Heat Exchangers," Numerical Heat Transfer, Part A, Vol. 43, 2003, pp. 201-219.

3. V. Prabhakar, G. Biswas, and V. Eswaran, "Numerical Prediction of Heat Transfer in a Channel with a built-in Oval Tube and Two Different Shaped Vortex Generators," Numerical Heat Transfer, Part A, Vol. 41, 2002, pp. 201-219.

4. S. Tiwari, D. Maurya, G. Biswas, and V. Easwaran, "Heat Transfer Enhancement in Cross-flow Heat Exchangers using Oval Tubes and Multiple Delta Wings," International Journal of Heat and Mass Transfer, Vol. 46, 2003, pp. 2841-2856.

5. V. Prabhakar, G. Biswas, and V. Eswaran, "Numerical Prediction of Heat Transfer in a Channel with a built-in Oval Tube and Various Arrangements of the Vortex Generators," Numerical Heat Transfer, Part A, Vol. 44, 2003, pp. 315-333.

\subsection{Numerical Prediction of Flow and Heat Transfer in a Rectangular Channel with a Built-in Circular Tube}

Existing air-cooled condensers in geothermal power plants consist of the same fin-tube arrangement as shown in Figure 6-1. In order to analyze the mechanism involved in the heat transfer and flow behavior in such heat exchangers, a detailed investigation on a heat exchanger module is necessary. Such a module is shown in Figure 6-2. In the numerical model, the Navier-Stokes equations, together with the governing equation of energy, were solved in a rectangular channel with a built-in circular tube

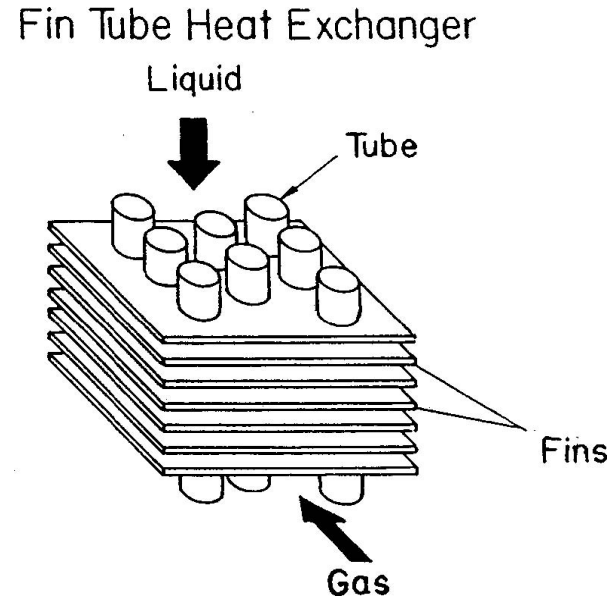

Figure 6-1. Schematic of the core region of a fin-tube heat exchanger. 


\subsubsection{Formulation of the Problem}

Figure 6-2 also represents the computational domain. Two neighboring fins form a channel of height $\mathrm{H}$, width $\mathrm{B}=11.25 \mathrm{H}$, and length $\mathrm{L}=25 \mathrm{H}$. Circular tubes of various diameters are located at a distance $\mathrm{L} 1=\mathrm{L} / 4$ from the inlet. The blockage ratio, $\mathrm{D} / \mathrm{B}$, was varied using the values of $0.267,0.444$, and 0.533 . Air was used as the working fluid; hence, the Prandtl number of the study is 0.7 .

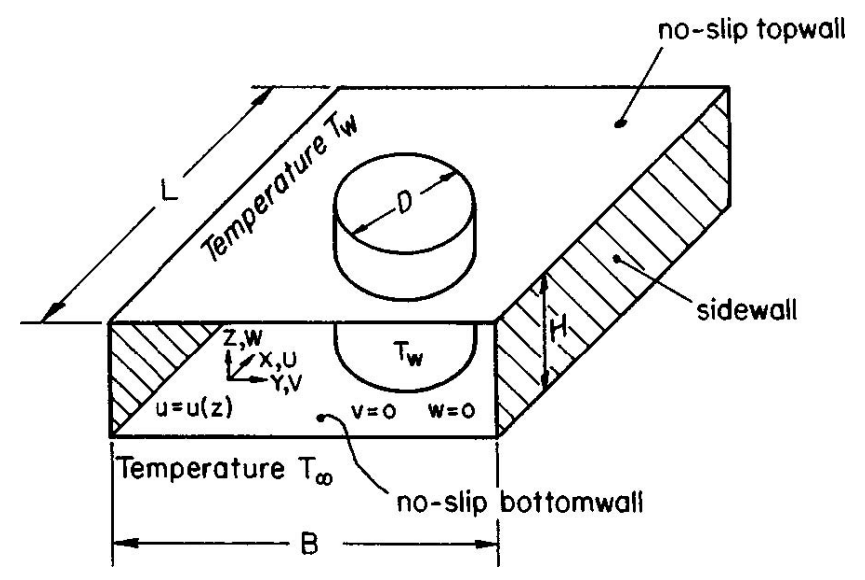

Figure 6-2. Module of the heat exchanger.

The three-dimensional Navier-Stokes equations for laminar flow of an arbitrary spatial control volume $\mathrm{V}$, bound by a closed surface $\mathrm{S}$, can be expressed in the following general convection-diffusionsource integral form:

$$
\frac{\partial}{\partial t} \int_{V} \rho \phi d V+\int_{S}\left(\rho \vec{u} \phi-\Gamma_{\phi} \nabla \phi\right) \cdot d \vec{S}=\int_{V} S_{\phi} d V
$$

where $\rho$ represents the fluid density, $\bar{u}$ is the fluid velocity, $\varphi$ is any vector component or scalar quantity, and $S_{\varphi}$ is the volumetric source term. For incompressible flow of a Newtonian fluid, the equation takes the form, and the source term for the momentum equation becomes $-1 / \rho \int p I$. $d \vec{S}$, where $I$ is the unit tensor. In this formulation, components of velocity are in Cartesian coordinates. The quantity $\varphi$ can be the three Cartesian components of velocity $u, v$, and $w$ and also any scalar, e.g., temperature $T$, that needs to be determined. The variables of the general transport Equation (6-2) are given in Table 6-1.

$$
\frac{\partial}{\partial t} \int_{V} \phi d V+\int_{S}\left(\vec{u} \phi-\frac{\Gamma_{\phi}}{\rho} \nabla \phi\right) \cdot d \vec{S}=\frac{1}{\rho} \int_{V} S_{\phi} d V
$$

Table 6-1. The variables for Equation (6-2).

\begin{tabular}{lccc}
\hline Equation & $\varphi$ & $\Gamma_{\varphi}$ & $S_{\varphi}$ \\
\hline Continuity & 1 & 0 & 0 \\
Momentum & $\mathrm{u}, \mathrm{v}, \mathrm{w}$ & $\mu$ & $\partial p / \partial x, \partial p / \partial y, \partial p / \partial z$ \\
Energy & $\mathrm{T}$ & $k / C p$ & 0 \\
\hline
\end{tabular}

\subsubsection{Boundary Conditions}

- $\quad$ Top and bottom plates

$\mathrm{u}=\mathrm{v}=\mathrm{w}=0$ (no-slip boundary condition)

$\partial \mathrm{p} / \partial \mathrm{z}=0$

$\mathrm{T}=\mathrm{Tw}(\mathrm{Tw}$ represents wall temperature $)$ 
- $\quad$ Side walls

$\partial \mathrm{u} / \partial \mathrm{y}=\partial \mathrm{w} / \partial \mathrm{y}=0, \mathrm{v}=0$ (free-slip boundary condition)

$\partial \mathrm{p} / \partial \mathrm{y}=0$

$\partial \mathrm{T} / \partial \mathrm{y}=0$

- Channel inlet

$\mathrm{u}=\mathrm{U} \infty, \mathrm{v}=\mathrm{w}=0$

$\partial \mathrm{p} / \partial \mathrm{x}=0$

$\mathrm{T}=\mathrm{T} \infty$

- $\quad$ Channel exit

$\partial \varphi / \partial \mathrm{t}+\operatorname{Uav} \partial \varphi / \partial \mathrm{x}=0$ (Orlanski60 boundary condition)

(where $\varphi$ represents either of $\mathrm{u}, \mathrm{v}, \mathrm{w}$ or $\mathrm{T}$ )

$\mathrm{P}=\partial \mathrm{p} \infty$

- Circular tube surface

$\mathrm{u}=\mathrm{v}=\mathrm{w}=0$

$\partial \mathrm{p} / \partial \mathrm{n}=0$ (where $\mathrm{n}$ signifies the normal direction)

$\mathrm{T}=\mathrm{Tw}$ Numerical Solution Technique

\subsubsection{Numerical Solution Technique}

Figure 6-3 is a schematic representation of the three-dimensional grid used for the present computation. The initial grid is generated by the method of transfinite interpolation. This method essentially uses a linear interpolation scheme to compute the interior points by using the coordinate values from the boundaries. The grid obtained by algebraic mapping is further improved by the use of the partial differential equations technique. The two-dimensional grids on the $x-y$ plane are stacked in the $z$ direction, with constant $\Delta z$. A finite-volume method from Eswaran and Prakash ${ }^{61}$ has been used to discretize and solve the governing conservation equations. The pressure-velocity iterations follow the method from Harlow and Welch. ${ }^{62}$ A $69 \times 49 \times 23$ grid-mesh is used in the present computation. The divergence-free criterion is satisfied using an upper bound of $10^{-4}$. Computations are carried out for three blockage ratios, $0.267,0.444$, and 0.533 , and three Reynolds numbers, 500, 1000, and 1400. Air is used as the working fluid; hence, the Prandtl number is 0.7 . The span-averaged Nusselt number is calculated on the basis of the bulk-mean temperature.

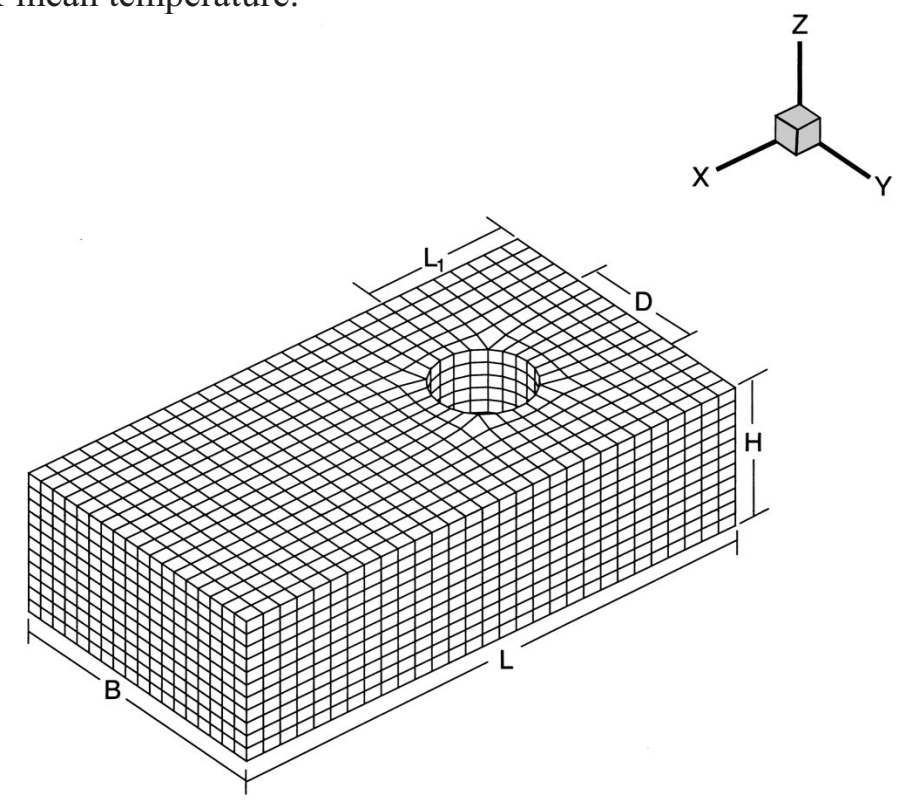

Figure 6-3. Schematic of the three-dimensional grid. 


\subsubsection{Flow Characteristics}

In this section, a detailed investigation of the flow structure in the passage of a fin-tube element is presented. For a three-dimensional flow simulation, it becomes difficult to handle the huge number of data, and only the effective use of some approaches proposed in the literature can depict the flow structure. Of the possible methodologies that enable exploring the kinematics of complex flows, the method of topological study of limiting streamlines is the most preferred, because the flow structure is best described by the topological properties inferred from computed streamlines. Figure 6-4 is a schematic representation of various singular points that may appear in a flow field. At the singular points in a flow field, the velocity vectors have zero magnitude but indeterminate direction. In the vicinity of these singular points, the dynamic characteristics of the flow depend on the eigenvalues of the Jacobian of the field. Corresponding to each eigenvalue, there exists an eigenvector specifying the principal value of the field component, with vanishing cross components. The dynamic character of the singular points, defined as saddle points or nodal points, also depends on the divergence of the shear-stress vector. Thus, the field Jacobian and the divergence of the shear stress vector together establish the criteria for complete characterization of the singular points or critical points associated with the flow field. A focus is a spiral node, and a sink is a stable node. An infinite number of shear-stress lines pass through the nodal points. Nodal points of attachment act as sources of skin-friction lines from the point, and the nodal points of separation act as sinks, where the skin-friction lines meet. For a singular point, defined as a spiral node, all shear stress lines spiral onto or out of this point. Only two sets of shear-stress lines pass through a saddle point. On each of these lines, the skin-friction behaves as a vector field. Shear stress in a threedimensional flow field is brought about by each of the two independent flow velocity components. On a two-dimensional plane, in the vicinity of a solid surface, say on either of the no-slip surfaces of the channel walls, the direction of the skin-friction vector field is dictated by its associated direction ratios $(\mu \partial u / \partial z, \mu \partial v / \partial z)$. The direction keeps on changing, depending on the relative magnitudes of the two direction ratios. Here, the direction $z$ is the direction normal to the surface containing the skin-friction lines of interest. For any general surface, we may use the symbol $S$ to show the perpendicular direction to the plane of interest.

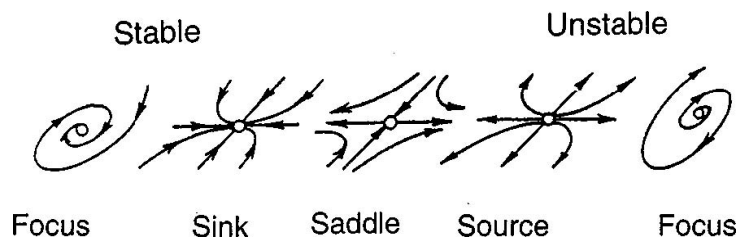

Figure 6-4. Schematic representation of the singular points.

The saddle points describe either separation or attachment. For a saddle point of separation, the skin-friction directions are toward the critical point, and for a saddle point of attachment, these directions are away from the point. In a two-dimensional flow, there exist special streamlines, called separation lines, which conveniently divide the flow into two distinct regions. Such streamlines originate at the saddle points. The three-dimensional counterparts to these separation lines are special stream surfaces.

\subsubsection{Flow Topology on the Horizontal and the Vertical Planes}

Figure 6-5 presents the streamline plots on the horizontal mid-plane of the channel corresponding to the instantaneous flow field and the time-averaged flow field. Observe that the instantaneous flow field illustrates vortex shedding. However, this asymmetry is suppressed when the time-averaged flow field is viewed on the horizontal mid-plane. The asymmetry in the wake zone of Figure 6-5a is time dependent and possesses an unsteady periodic nature. Such a flow structure may be observed in a flow field only beyond a critical Reynolds number. Below this Reynolds number, the flow field is steady in nature. For the present case, the shedding pattern corresponds to $R e=1000$. The flow field, shown in Figure 5-5b, is averaged over a large span of time (typically a few shedding cycles), so that the asymmetry in the wake 
zone disappears and a symmetrical field pattern is discerned. It may be mentioned that the averaged flow field is an abstract construct that reveals a pseudo-steady-state behavior.

$$
\mathrm{Re}=1000 \mathrm{Pr}=0.7 \mathrm{D} / \mathrm{B}=0.444
$$
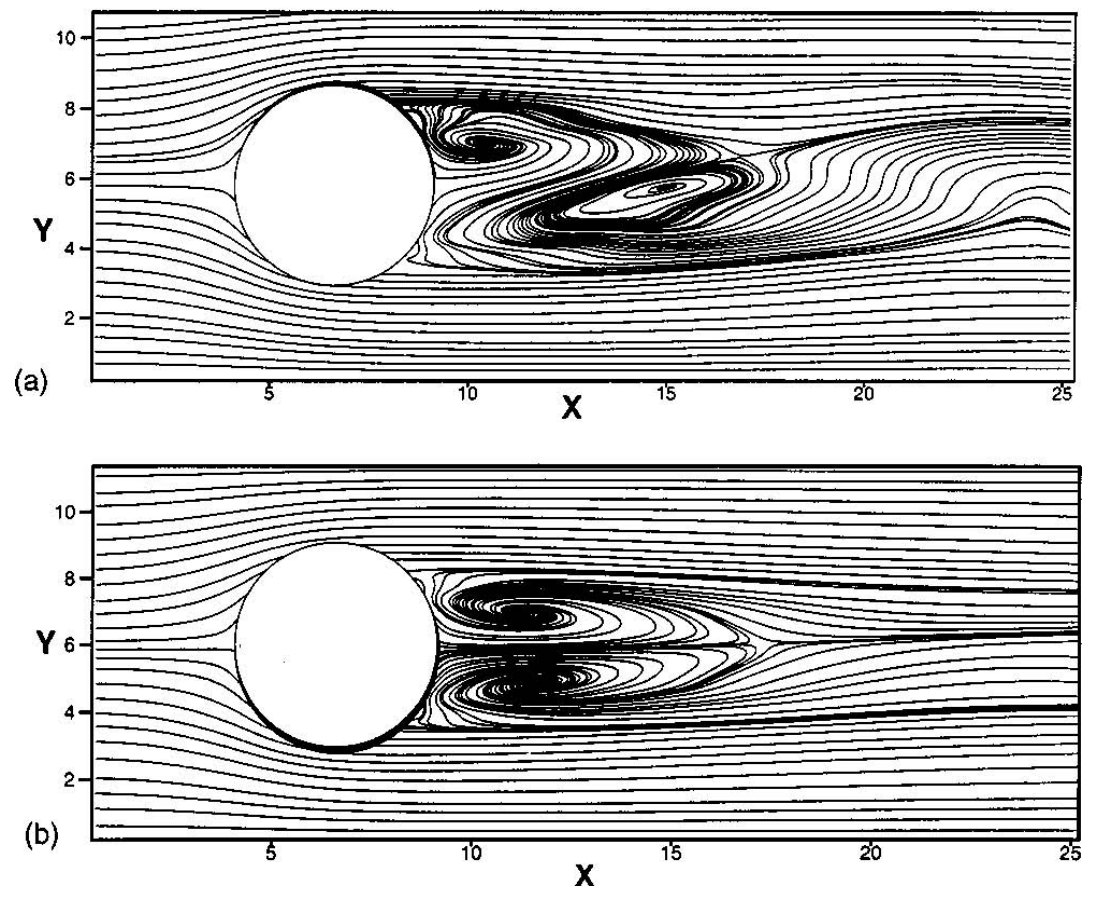

Figure 6-5. Streamline representation of the flow past a circular tube placed in a channel on the horizontal mid-plane for (a) instantaneous flow field and (b) time-averaged flow field.

Figure 6-6 illustrates the particle traces for an instantaneous flow field, showing the paths followed by the fluid particles during the course of their travel through the channel. The flow pattern clearly shows the existence of a screw-like helical vortex motion in the region close to the circular tube. The wake zone of the tube exhibits a strong three-dimensional behavior. The significant normal velocity component in the wake zone, $w$, is due to the pressure gradient in the direction normal to the no-slip walls toward the mid-plane.

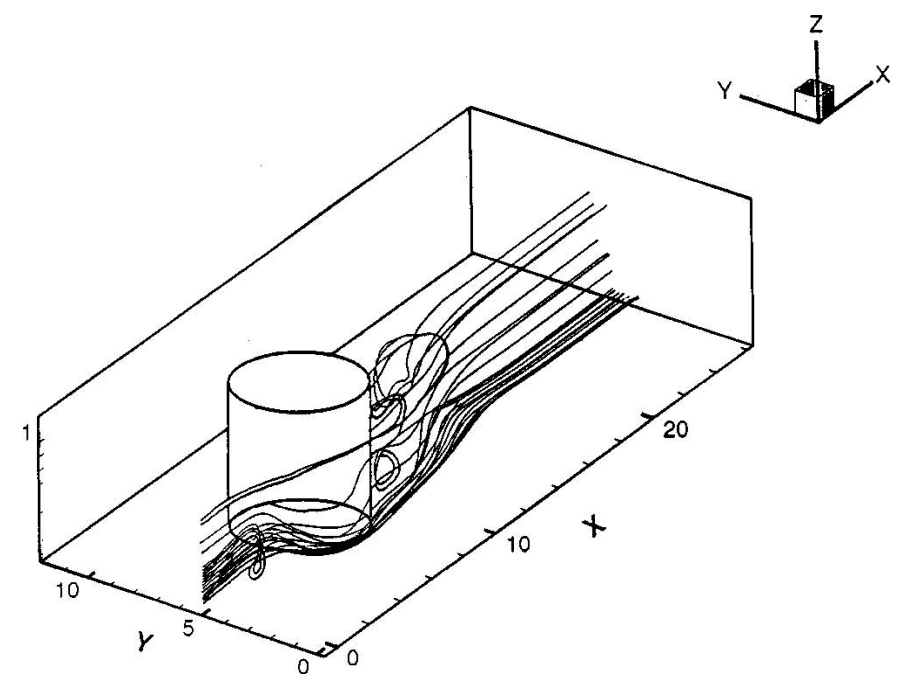

Figure 6-6. Structure of three-dimensional flow (particle path). 


\subsubsection{Limiting Streamlines on the Tube Surface and the Bottom Plate}

The structure of the wall streamlines or limiting streamlines on the tube surface was investigated. These streamlines consist of only the tangential component of velocity near the surface of the tube. Figure 6-7 shows such streamlines corresponding to the time-averaged velocity field. It is seen that the surface streamlines are symmetrically distributed about the mid-plane of the channel. These surface streamlines reveal bifurcation. The bifurcation may be either a positive bifurcation or a negative bifurcation. A streamline that eventually bifurcates into two or more streamlines follows a positive bifurcation (Figure 6-8). On the other hand, two or more streamlines merging into a single streamline show the behavior of a negative bifurcation (Figure 6-8). In Figure 6-7, a positive bifurcation is observed near the forward stagnation line of the tube surface $(\theta=0$ or $2 \pi)$. The line of reattachment at $\theta=\pi$ is also clearly observed in this figure. Two other lines are also seen having their locations at an angle $0.7 \pi<\theta<$ $0.8 \pi$ and $1.2 \pi<\theta<1.3 \pi$, respectively. Near these lines, the tangential components of the shear stress vector vanish. Hornung and Perry ${ }^{61}$ called this a negative stream surface bifurcation. The streamlines combine to form a single streamline along the negative bifurcation line. These lines can be termed separation lines, along which the boundary layer separates from the tube surface. In Figure 6-7, the separation angle shows a symmetric variation about the mid-plane. The values of the separation angle at four different heights from the bottom plane, $z=0.1 \mathrm{H}, 0.2 \mathrm{H}, 0.43 \mathrm{H}$, and $0.5 \mathrm{H}$ are $\theta=0.75 \pi, 0.74 \pi$, $0.68 \pi$, and $0.73 \pi$, respectively. This shows that the separation angle is large in the vicinity of the midplane of the tube and near the channel walls, and is relatively smaller at an intermediate height close to the mid-plane.
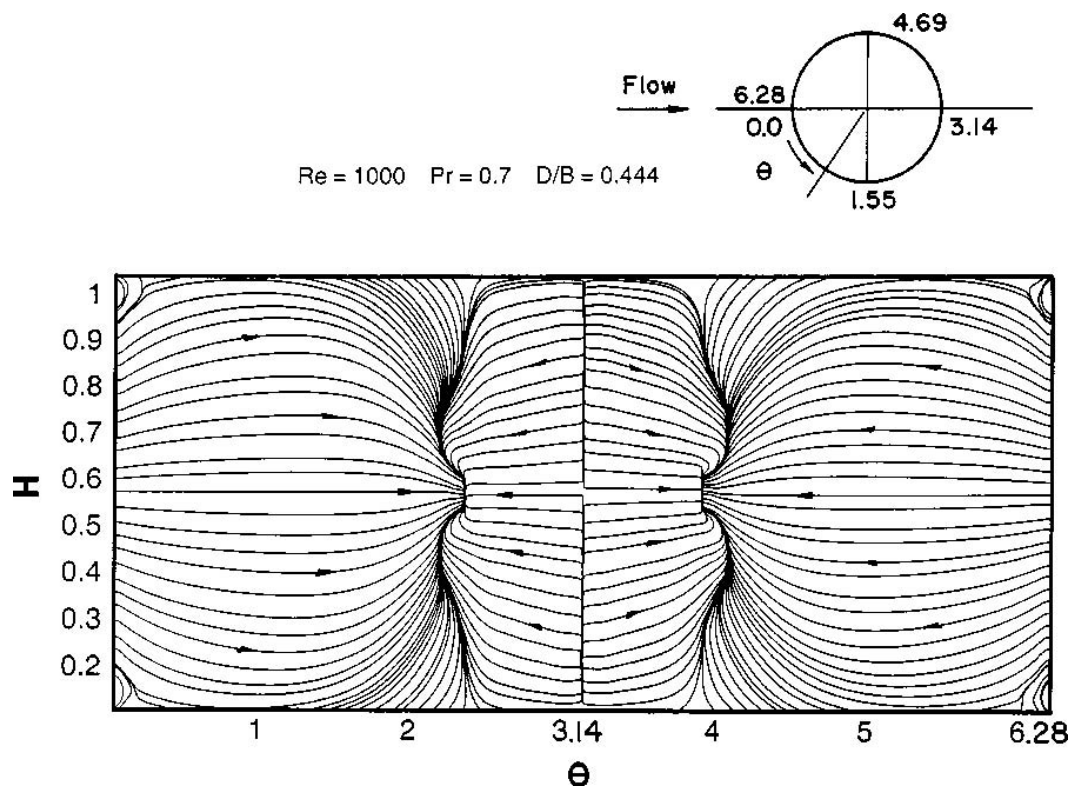

Figure 6-7. Limiting streamlines on the tube surface.

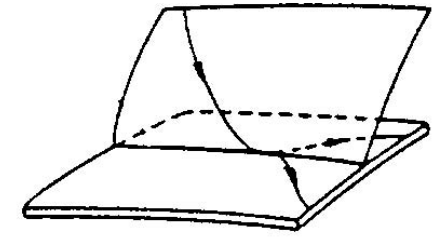

Positive bifurcation

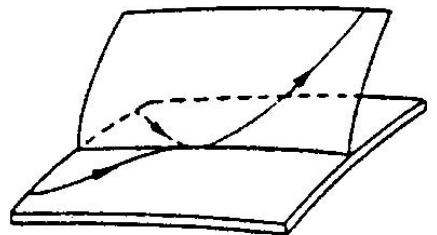

Negative bifurcation

Figure 6-8. Positive and negative bifurcation lines. 
Figure 6-9 shows the streamlines in the region close to the bottom wall corresponding to the instantaneous field and the time-averaged field. A great deal of information about the flow and heat transfer characteristics can be extracted from these figures. The asymmetry due to vortex shedding is apparent in the instantaneous field (Figure 6-9a), while the time-averaged field (Figure 6-9b) is almost symmetrical. A saddle point of separation and a horseshoe vortex system are seen in Figure 6-9b. The incoming flow does not separate in the traditional sense but reaches a stagnation or saddle point of separation (A) and goes around the body. The nodal point of attachment (C) and the separation lines that form circular arcs across the tube are also shown. The flow away from no-slip top and bottom walls, after it hits the forward stagnation line of the tube, moves toward the walls due to normal pressure gradient and creates a small region of reversed flow. On each side of the tube, one finds a region of converging streamlines $(\mathrm{G})$. These are the foot prints of the horseshoe vortex system. Behind the tube, in the wake zone, two areas of swirling flow (E) are seen. A wake stagnation point (F) downstream of the tube is also shown, which, together with the swirls E, constitutes a saddle zone.
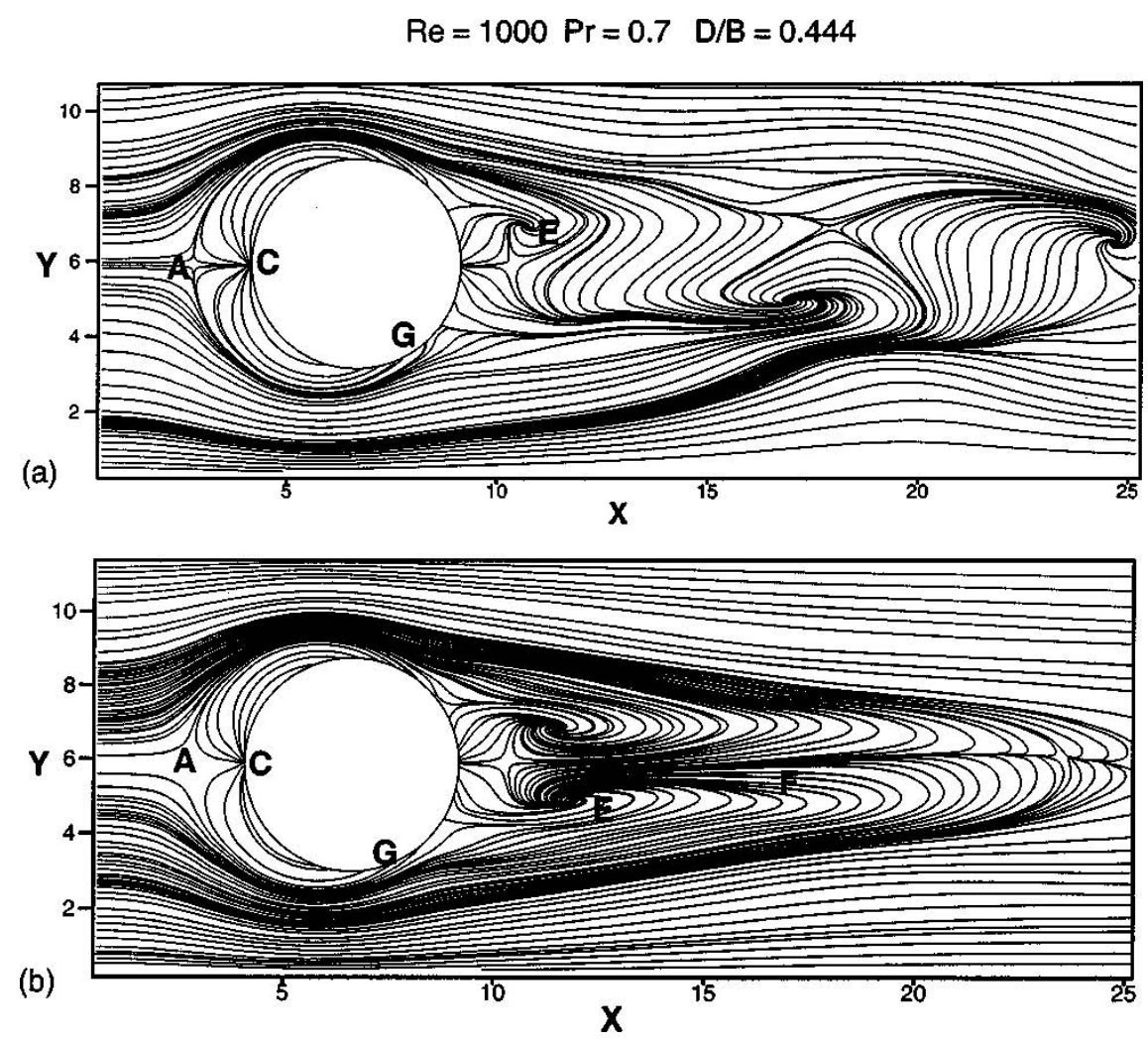

Figure 6-9. Limiting streamlines of the flow past a built-in circular tube in a channel at the bottom plate corresponding to (a) instantaneous flow field and (b) time-averaged flow field.

\subsubsection{Heat Transfer Characteristics}

Figure 6-10 shows the isolines of local Nusselt number obtained from the time-averaged flow field and temperature field on the bottom plate. Several interesting features may be observed from the figure. At the leading edge of the channel walls, the Nusselt number has a large value and then decreases gradually. At the leading edge, the cooler fluid comes in contact with the hot solid wall for the first time, and as a result of which the convection heat transfer is large. A gradual decrease in the Nusselt number is attributed to the development of the thermal boundary layer on the channel wall. In fact, the inlet condition is one of uniform velocity as well as uniform temperature. Therefore, both the velocity and the 
thermal boundary layers are developing at the channel leading edge. The region that follows the leading edge of the channel is the combined-entrance region. A significant increase in Nusselt number is observed in front of the tube, which results from the formation of a horseshoe vortex system that consists of two counter-rotating longitudinal vortices. As the fluid approaches the stagnation line of the circular tube, it slows and its pressure increases. The smaller velocity in the boundary layer, in the vicinity of the channel walls, leads to a smaller pressure increase compared with the mid-plane. This induces a pressure gradient normal to the no-slip walls, and the pressure gradient-driven flow is directed from the mid-plane toward the wall. Hence, the induced pressure gradient along the stagnation line induces the flow toward the noslip walls that interacts with the main stream. The fluid rolls up, forming vortices, which finally wrap around the front half of the tube and extend to the rear of the tube. ${ }^{16}$ Figure 6-6 explains the formation of a critical point and initiation of the spiraling motion adjacent to the critical point. The spiraling motion of the horseshoe vortices brings about better mixing, and the heat transfer in this region is significantly enhanced. The Nusselt number is low in the wake region, as shown in Figure 6-10. The poor heat transfer in this region is attributed to the separated dead zone with fluid recirculating at a low velocity. This trend matches qualitatively well the experimental results of O'Brien and Sohal. ${ }^{11}$

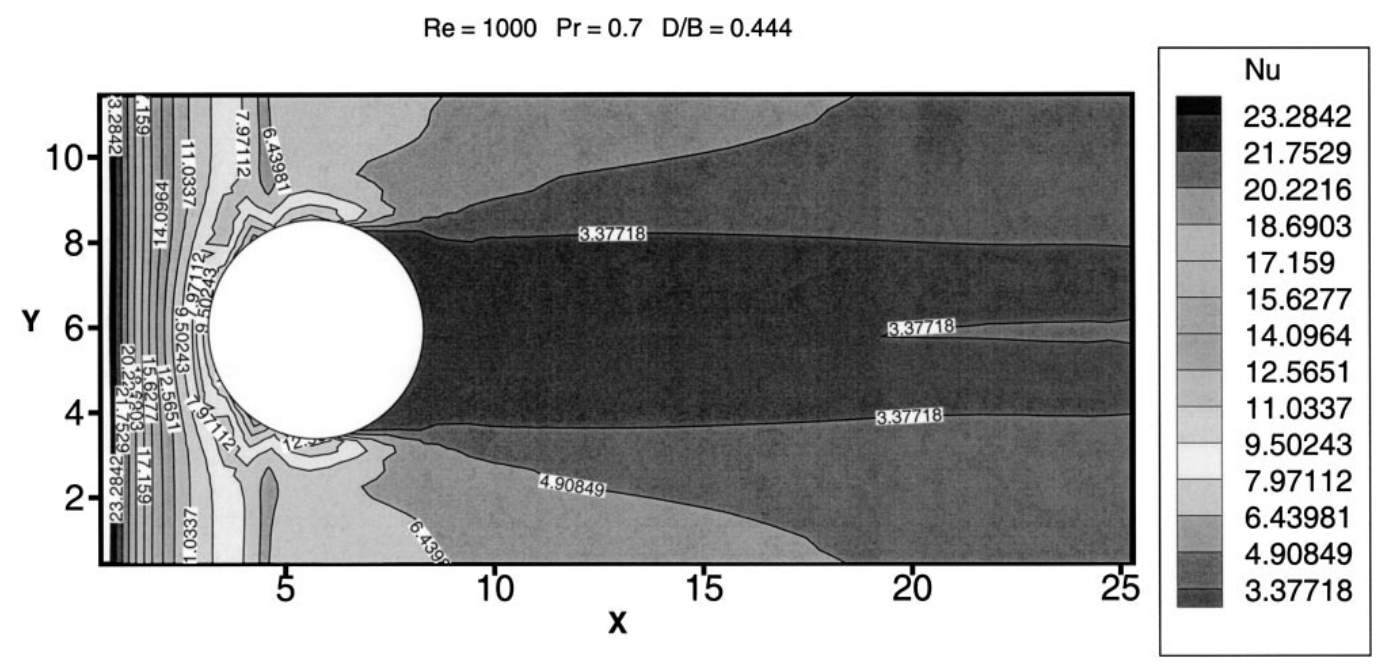

Figure 6-10. Iso-Nusselt number distribution on the bottom wall.

Figure 6.1-11 shows the distribution of the time-averaged and span-averaged Nusselt number in the streamwise direction for three Reynolds numbers, 600, 1000, and 1400, corresponding to a blockage ratio of 0.444 . In each case, at the leading edge of the fin, the Nusselt number is high and then decreases gradually, showing a local maximum in front of the tube. The reasons for this increase in the Nusselt number in front of the tube have been explained earlier. In the developing region of the flow, the spanaveraged Nusselt number shows a significant increase in value with the increasing Reynolds number. As the flow develops downstream, this increase is not very significant. 


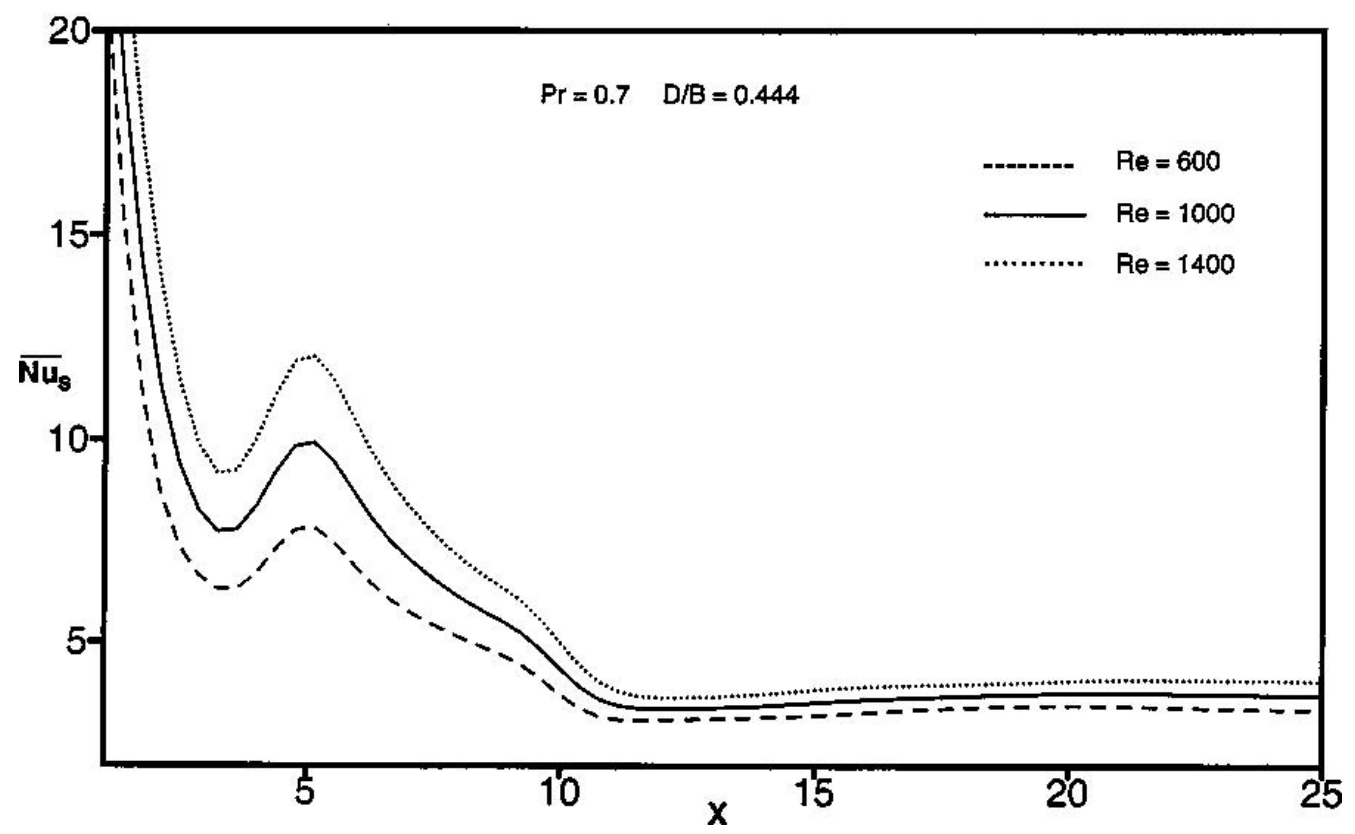

Figure 6-11. Variation of span-averaged $\mathrm{Nu}$ with Reynolds number $(\mathrm{D} / \mathrm{B}=0.444)$.

The variation of the span-averaged Nusselt number along the length of the channel does not convey any information about the spanwise variation of local Nusselt number on the channel walls. For the timeaveraged field, the variation of local Nusselt number in the spanwise direction at any axial location is expected to be symmetric about the midpoint on the span of the channel. Figure 6-12 shows the spanwise variation of the local Nusselt number in the wake region at an axial distance of $\mathrm{x} / \mathrm{R}=1.2$ from the center of the tube. The two peaks in the figure result from almost symmetric swirls generated by the longitudinal horseshoe vortices. The poor heat transfer in the dead wake region $(-0.5<\mathrm{y} / \mathrm{R}<0.5)$ is evident.

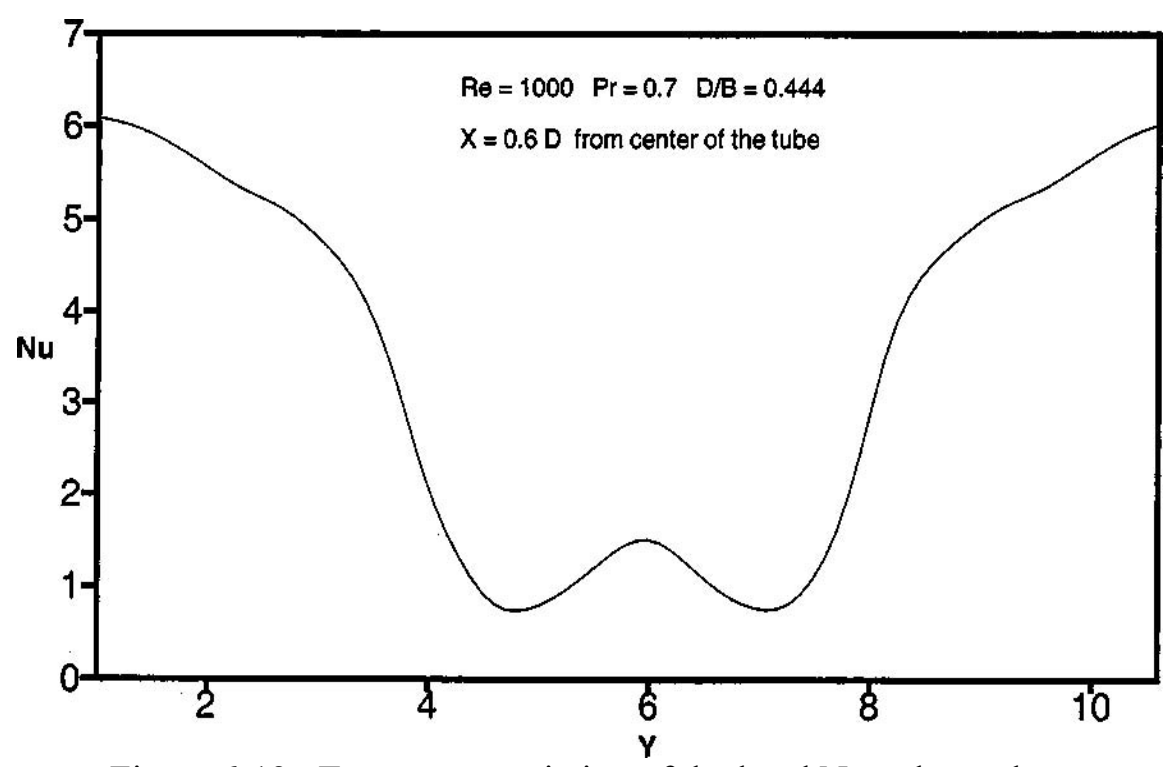

Figure 6-12. Transverse variation of the local Nusselt number. 
Figure 6-13 shows variation of the time-averaged and span-averaged Nusselt number along the channel length for three blockage ratios (D/B), 0.267, 0.444, and 0.533, at a Reynolds number of 1000 . Observe that with increasing blockage ratio, the location of the maximum value of the span-averaged Nusselt number shifts more toward the upstream side. This may be attributed to earlier onset of formation of the horseshoe vortices, with increasing radius of the circular tube, for a fixed location of the center of the tube. Observe also that he maximum value of the span-averaged Nusselt number is higher for higher blockage ratios. The increased constriction in the passage causes more acceleration on the flow, and the acceleration-driven steeper temperature gradients contribute to a larger span-averaged Nusselt number.

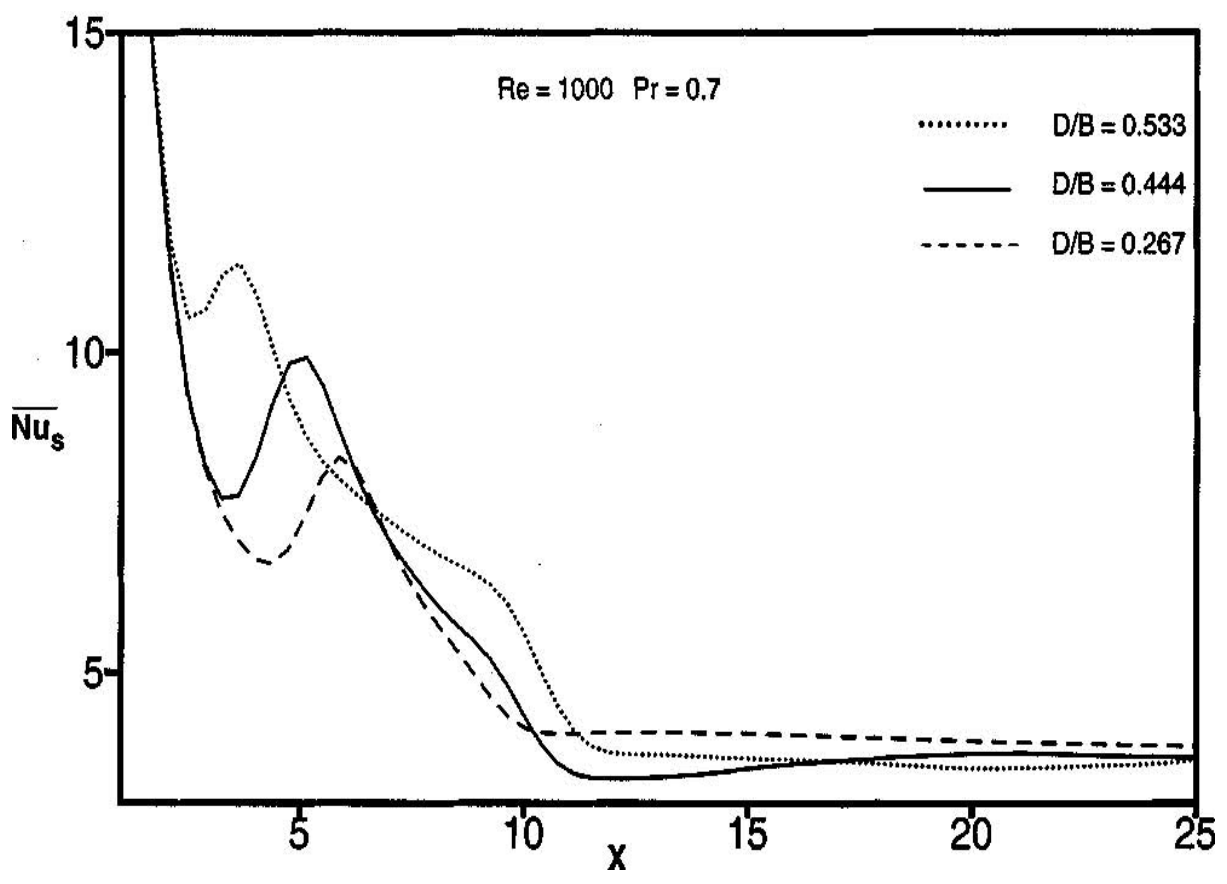

Figure 6-13. Variation of span-averaged $\mathrm{Nu}$ with blockage ration $(\operatorname{Re}=1000)$.

In addition to the heat transfer behavior corresponding to the time-averaged field, the heat transfer characteristics corresponding to the instantaneous field bear special significance. In design considerations, this aspect plays a very important role. Figure 6-14 shows the distribution of the spanaveraged Nusselt number on the bottom wall at three arbitrary times, say nondimensional times of 525.97, 650.20 , and 847.50 units. The Reynolds number for this computation is 1000 , and the blockage ratio is $D / B=0.444$. This figure shows very little difference in the distribution pattern. The flow fluctuates with a low-frequency oscillation. The difference appears mainly in the wake zone, as expected. This figure also reveals that the amplitude of the oscillations is not very large. The increase in the value of the spanaveraged Nusselt number in the downstream section, at larger time instants, is mainly attributable to the development of the flow in that section with passage of time. The effect of mild oscillations on heat transfer becomes apparent when the results are compared with those for the time-averaged field presented in the same plot. 


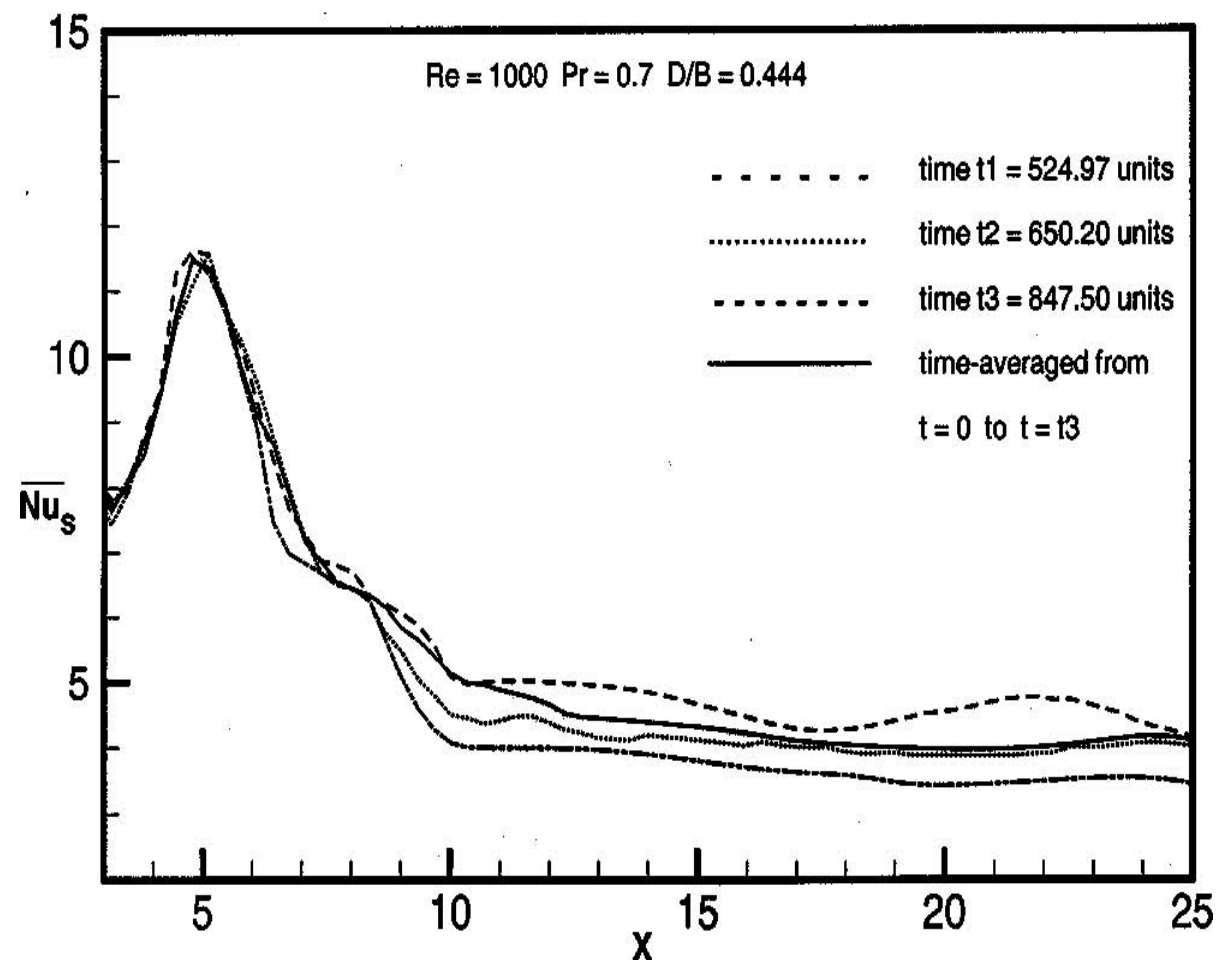

Figure 6-14. Comparison of the span-averaged Nusselt number at three different times with the time-averaged span-averaged Nusselt number.

Figure 6-15 represents the pressure variation along the length of the channel. The variation of pressure is considered for three Reynolds numbers, 600, 1000, and 1400, with a blockage ratio of 0.444 . The cross-stream averaged pressure distribution, $\left(p / \rho U_{\infty}{ }^{2}\right) \mathrm{Re}$, along the channel length is shown as a function of the Reynolds number. The dimensionless pressure drop in Figure 6-15 has been scaled with the Reynolds number, entailing the matching points at the entry plane of the channel over the entire range of the Reynolds number.

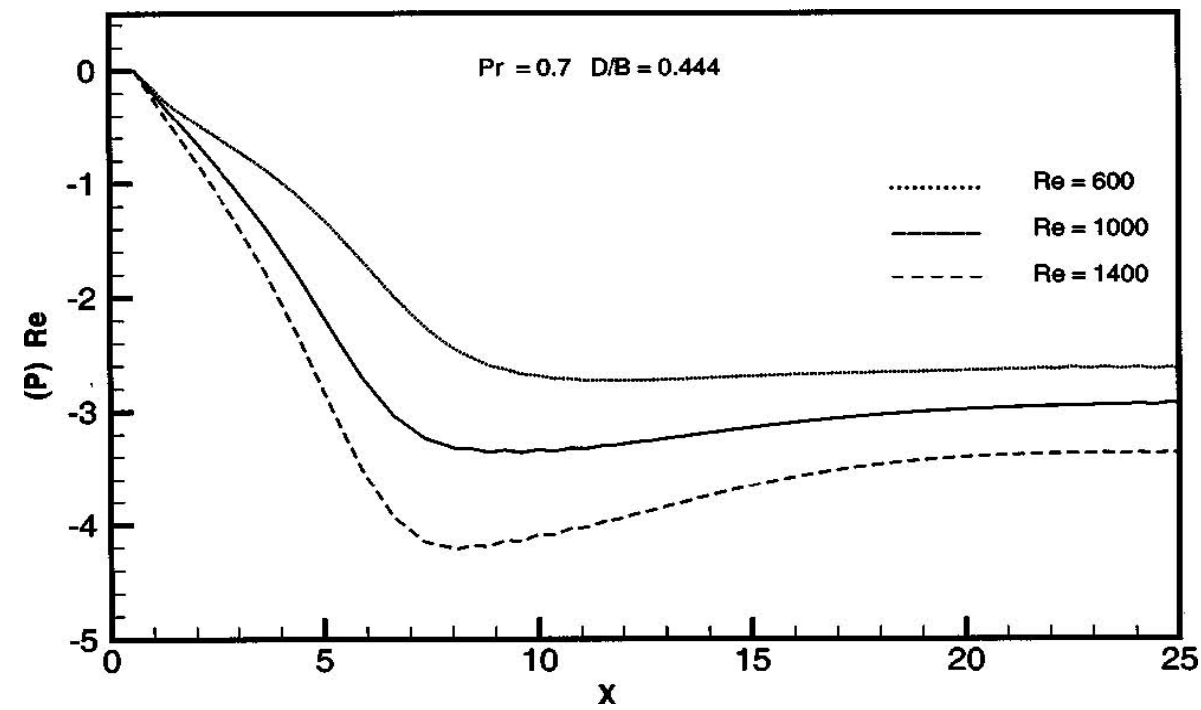

Figure 6-15. Pressure variation along the length of the channel for different values of the Reynolds number. 


\subsubsection{Validation of Results and Grid Independence}

The model validation was performed by comparing it with the experimental results of O'Brien and Sohal. ${ }^{11}$ Figure 6-16 shows a comparison between the predicted and the experimentally obtained variation of the local Nusselt number in the spanwise direction at an axial location $\mathrm{x} / \mathrm{D}=1.38$ from the center of the tube. The geometric parameters were described by O'Brien and Sohal. ${ }^{11}$ In the experiment, an unheated channel length was provided to ensure a hydrodynamically developed condition at the test section. In the numerical simulation used for the comparison, a hydrodynamically fully developed velocity profile was used at the inlet of the channel. O'Brien and Sohal11 used infrared thermography to measure the heat transfer characteristics. The predicted values compare fairly well with their experimental counterparts.

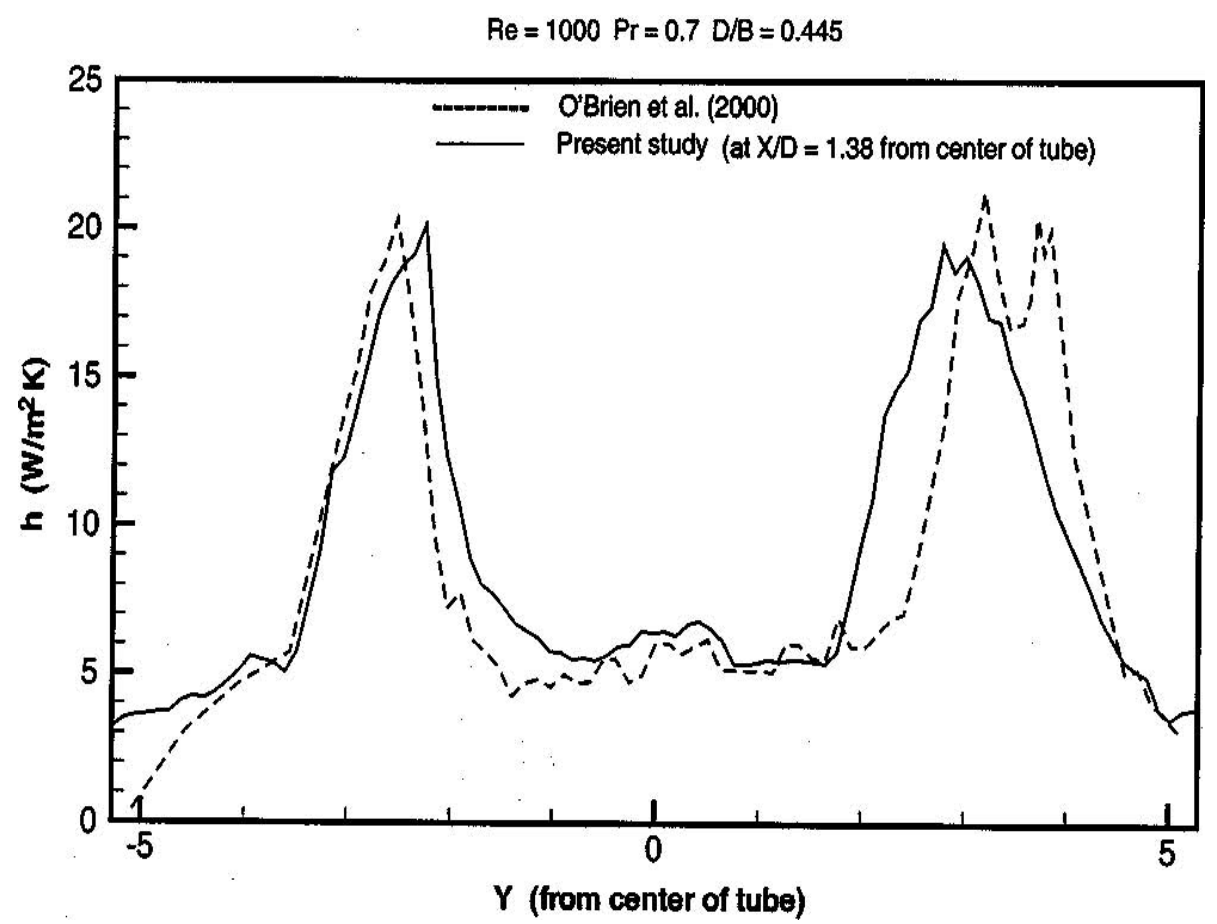

Figure 6-16. Comparison of the transverse heat transfer coefficient in the wake region.

The grid independence was confirmed rigorously. The variation of the span-averaged Nusselt number for two grid sizes, $79 \times 57 \times 27$ and $69 \times 49 \times 23$, corresponding to $\operatorname{Re}=1000$ and $D / B=0.444$, is shown in Figure 6-17. Observed that the time and space averaged Nusselt number for the channel differs from the projected grid-independence situation by less than $3 \%$. All computations in the present case were conducted on a grid-mesh of $69 \times 49 \times 23$ to save some computation time. 


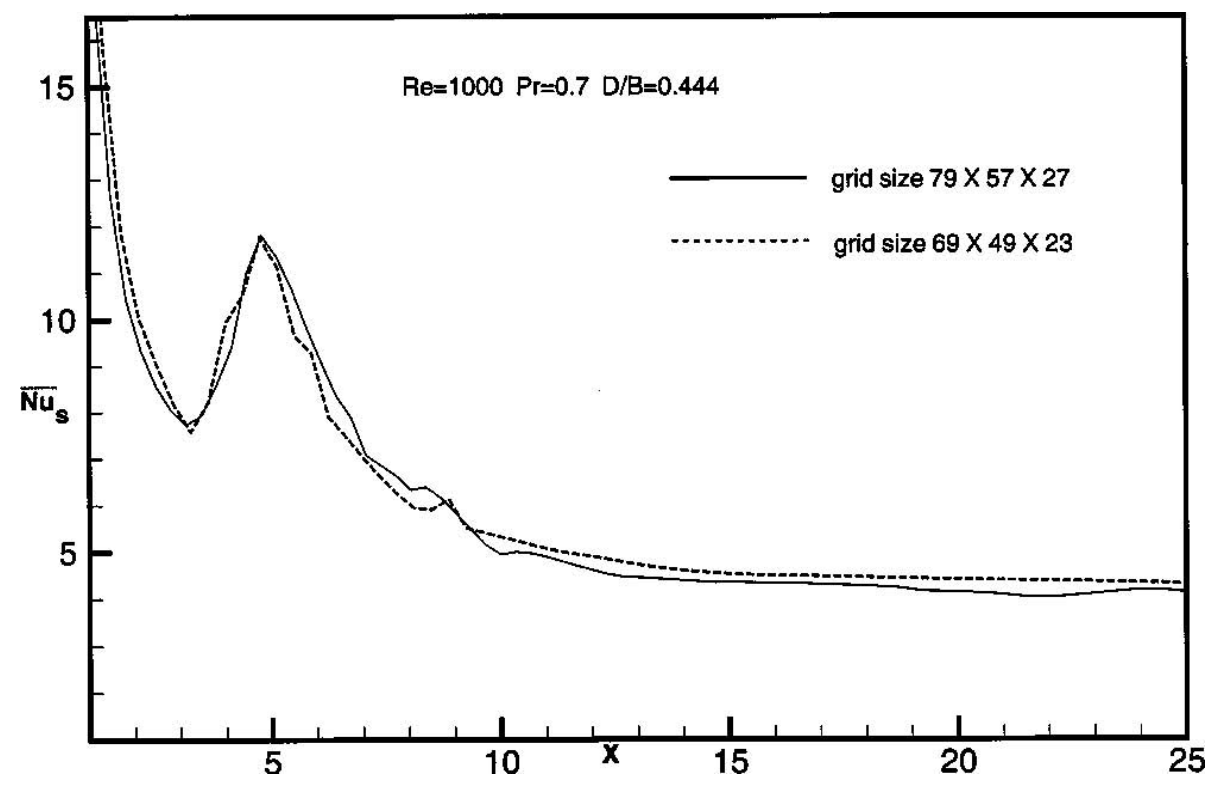

Figure 6-17. Comparison of the span- and time-averaged Nusselt number for two different grids.

\subsubsection{Conclusions}

A three-dimensional numerical study has been performed on the flow and heat transfer characteristics in a narrow rectangular duct with a built-in circular tube in a cross-flow configuration. The duct was designed to simulate a passage formed by any two neighboring fins in a fin-tube heat exchanger. The flow-field is different than two-dimensional flows. At the rear of the tube, it leads to a screw-like motion of a helical vortex tube. Limiting streamlines on the tube surface showed typical separation lines. Limiting streamlines on the bottom wall clearly established the presence of a saddle point of separation, a nodal point of attachment in front of the tube and bottom wall junction, and a horseshoe vortex system that wraps around the tube and extends to the rear of the tube. The spanaveraged Nusselt number distribution and iso-Nusselt number distribution clearly establish the high heat transfer near the leading edge of the fins and in the region influenced by the horseshoe vortex system. Poor heat transfer in the dead wake region is observed. The heat transfer enhancement caused by the horseshoe vortices around the fore-root of the tube is unable to offset the poor heat transfer in the nearwake region. The zone of poor heat transfer can possibly be removed by inducing a swirling motion in the wake region. This study confirmed that onset of turbulence is not brought about in the range of Re considered. As a consequence, the predicted heat transfer results, without using any turbulence model, are a meaningful numerical approximation of the physical situation.

\subsection{Winglet-Type Vortex Generators with Common-flow-up Configuration for Fin-Tube Heat Exchangers}

Biswas et al. ${ }^{52}$ numerically investigated the characteristics of vortex generators in a channel. In the investigation, enhancement of heat transfer from the fin surfaces is achieved by placing delta-winglet-type vortex generators on the flat fin surfaces in the neighborhood of the tubes. In order to achieve this desired objective, a three-dimensional numerical model has been formulated to provide better understanding of the flow physics. In the numerical model, the complete Navier-Stokes equations, together with governing equations of energy, are solved in an element of a heat exchanger, as shown in Figure 6-18. The heat exchanger element consists of a rectangular channel with a built-in circular tube and a winglet pair. A detailed analysis of the flow structure along with heat transfer characteristics in such an element was studied in this investigation. 


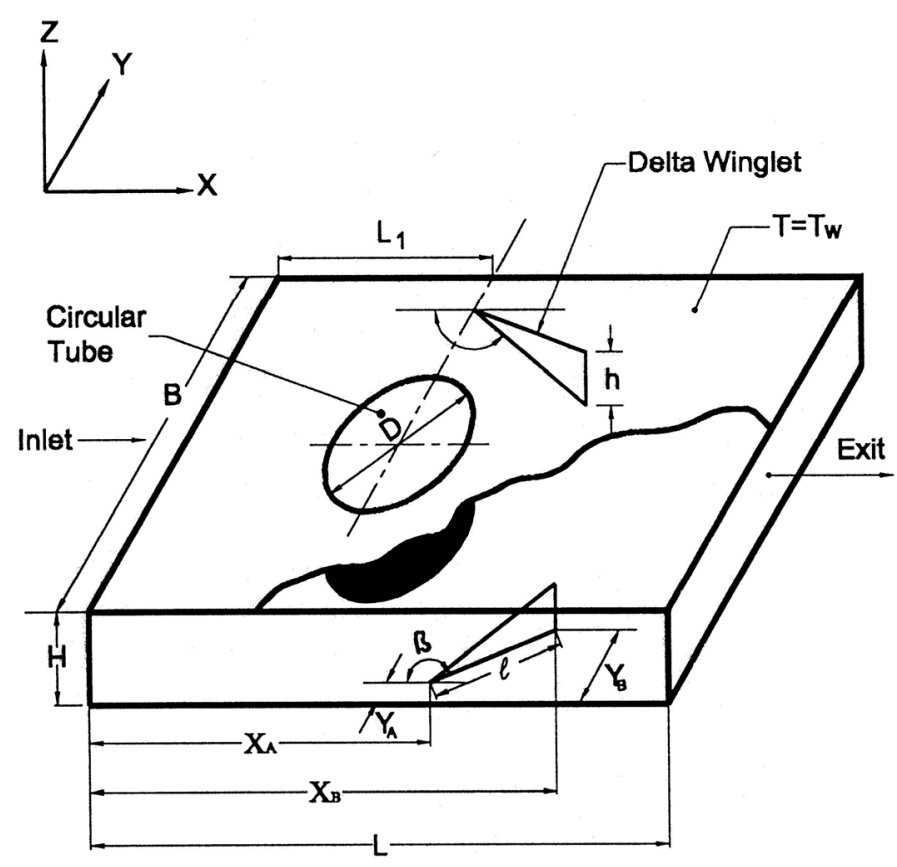

Figure 6-18. Heat exchanger module considered in the present investigation.

In general, delta winglet type vortex generators can be mounted on the flat surfaces with a toe-out (common flow down) configuration. For such a configuration, the transverse distance between the leading edges of the winglet pair is less than the transverse distance between the trailing edges of a winglet pair. In a toe-in (common flow up) configuration, the transverse distance between the leading edges of the winglet pairs is more than that between the trailing edges. Torii et al. ${ }^{54}$ have indicated that the delta winglet pair with a toe-out (common flow up) configuration creates constricted passages in the aft region of the tube, which brings about separation delay. The fluid is accelerated in the constricted passages, and, as a consequence, the point of separation travels downstream. Narrowing of the wake and suppression of vortex shedding are the obvious outcomes of such a configuration. Since the fluid is accelerated in this passage, the zone of poor heat transfer is also removed. The poor heat transfer zone is created in the near wake of the tube in the absence of any vortex generators. The common flow up configuration of the delta winglet pair emerges as the most attractive enhancement technique for the air-cooled condensers.

\subsubsection{Statement of the Problem}

Figure 6-18 also represents the computational domain. Two neighboring fins form a channel of height $\mathrm{H}$, width $\mathrm{B}=11.25 \mathrm{H}$, and length $\mathrm{L}=20 \mathrm{H}$. The circular tube of diameter $\mathrm{D}=4.0 \mathrm{H}$ is located at a distance $\mathrm{L} 1=1.6 \mathrm{D}$ from the inlet. The blockage ratio, $\mathrm{D} / \mathrm{B}$, has been kept at 0.36 . The position of the winglet is given by $\mathrm{XA}=7: 6 \mathrm{H}, \mathrm{XB}=9: 8 \mathrm{H}, \mathrm{YA}=1: 7 \mathrm{H}$, and $\mathrm{YB}=3: 66 \mathrm{H}$. The height of the winglet is $\mathrm{h}=0.347 \mathrm{H}$; the angle of attack is $\beta=138.61$. Air has been used as the working fluid; hence, the Prandtl number of this study is 0.7 .

\subsubsection{Governing Equations and Numerical Scheme}

The three-dimensional Navier-Stokes equations for laminar flow of an arbitrary spatial control volume $\mathrm{V}$ bounded by a closed surface $\mathrm{S}$ are given by Equations (6-1) and (6-2) in Section 6.1.1 in a general convection-diffusion source integral form. The variables of the general transport equation are given in Table 6-1. The boundary conditions are the same as given in Section 6.1.2. The grid generation is given in Section 6.1.3. The finite volume numerical scheme is based on the technique described by 
Easwaran and Prakash. ${ }^{61}$ Figure 6-3 is a schematic of the three-dimensional grid used for the present computation. The initial grid is generated by the method of transfinite interpolation. This method essentially uses a linear interpolation scheme to compute the interior points by using the coordinate values from the boundaries. The algebraic grid so generated in general is not smooth because the slope discontinuities at the boundary propagate in the interior of the domain. So the mesh obtained by algebraic mapping is further improved by using partial differential equations (PDEs). In this technique, a system of elliptical PDEs is solved for obtaining the location of the grid points in the physical space, whereas the computational space is a rectangular parallel piped domain with uniform spacing.

The conservation equations are discretized employing the finite-volume approach of Eswaran and Prakash. ${ }^{61}$ The solution domain is divided into a number of contiguous (finite) control volumes (CVs). The coordinates of the $\mathrm{CV}$ vertices are calculated by the grid generation procedure. These vertices, which are assumed to be connected by straight lines, define the CV. A collocated grid arrangement is employed and all dependent variables ( $\mathrm{u}, \mathrm{v}, \mathrm{w}, \mathrm{p}$, and $\mathrm{T}$ ) are defined at the same location, the centroid of the control volume (Figure 6-19). Cartesian velocity components have been used. The symbols, E, W, N, S, T, and B indicate the six neighboring CV centers for the east, west, north, south, top, and bottom neighbors. The face center points $\mathrm{e}, \mathrm{w}, \mathrm{n}, \mathrm{s}, \mathrm{t}$, and $\mathrm{b}$ are located at the intersection of the lines joining the midpoints of the opposite edges. Edge centers te, be, ne, and se are the midpoints of the edges that form the east face, with e as the center of the cell face. Similarly, tw, bw, nw, sw, tn, bn, ts, and bs are located at the corresponding center of edges that form the west, north, and south faces. The finite volume vertices are numbered 1 to 8 in the manner shown in

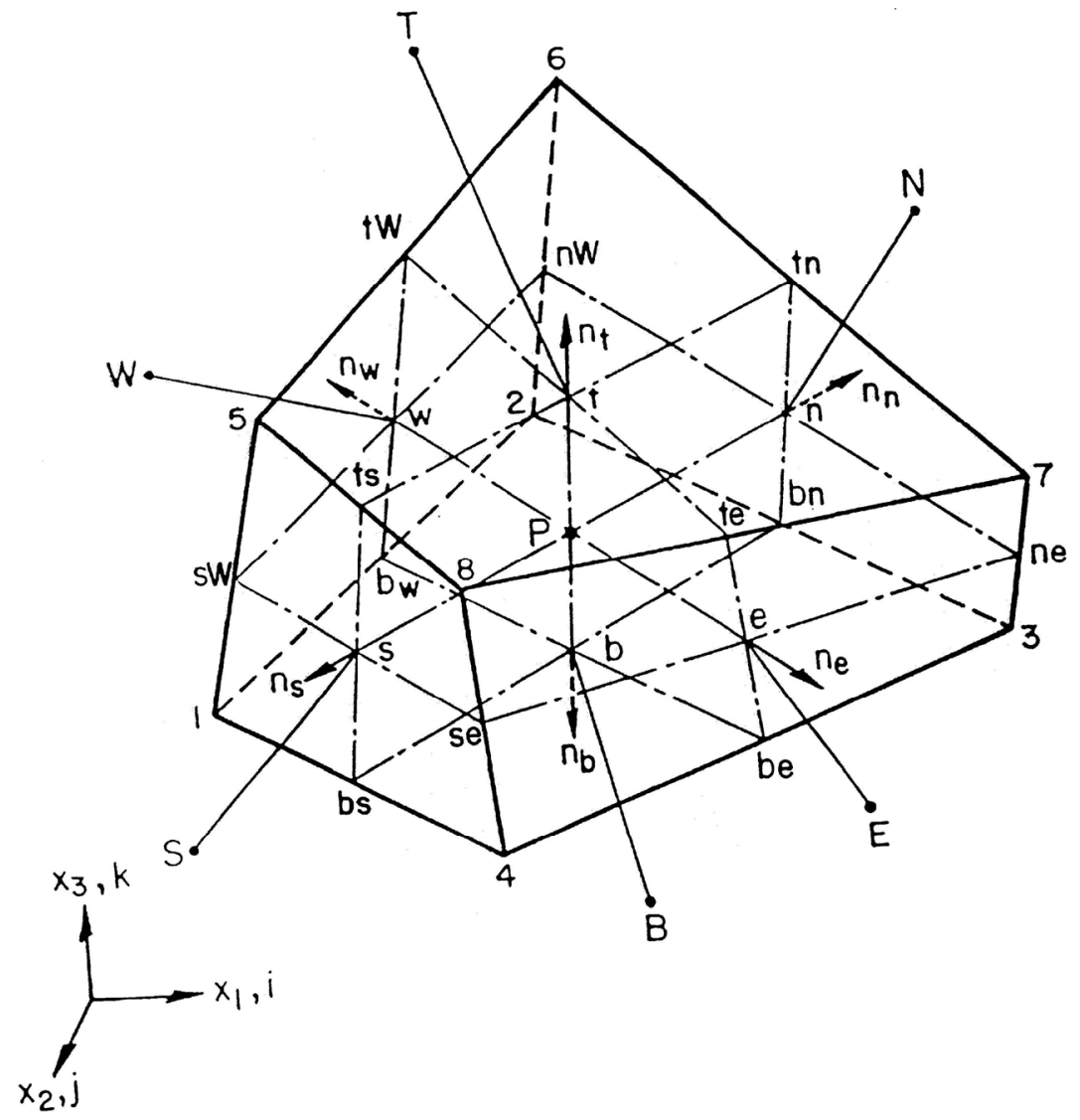

Figure 6-19. Three-dimensional finite volume cell.

Figure 6-19. The outward surface normals and volume can be found in the manner suggested by Kordulla and Vinokur. ${ }^{63}$ The rates of change and source terms are integrated over the cell volume, whereas the 
convection and the diffusion terms form the sum of fluxes through the faces of the CV. The value of the dependent variable at the centroid of the $\mathrm{CV}$ (the geometric center) represents an average over the $\mathrm{CV}$ as a whole. Peric64 developed the expression for the diffusion fluxes for the finite volume. Later, Ferziger and Peric65 and Eswaran and Prakash61 also explained the means of expressing diffusion fluxes on different faces of arbitrary-shaped differential CVs. To obtain the velocity and pressure field satisfying the mass and momentum conservation laws, a procedure akin to the marker and cell (MAC) method given by Harlow and Welch ${ }^{62}$ is used. This method offers an efficient and easy way of pressure-velocity coupling. It is basically a semiexplicit method. The momentum equations are discretized explicitly, with the exception of the pressure gradient terms, which are treated implicitly, as are also the continuity equations. Further details of the discretization procedure, convection fluxes diffusion fluxes, and pressure-velocity coupling can be obtained from Jain et al. ${ }^{55}$

\subsubsection{Results and Discussion}

A74 $\times 48 \times 23$ grid mesh is used in this computation. The divergence-free criterion is satisfied using an upper bound of $10^{-4}$. Computations have been carried out for Reynolds numbers of 1000. Air has been taken as the working fluid; hence, the Prandtl number of the present study is 0.7 . The Nusselt number is calculated on the basis of bulk mean temperature. For spatial grid-independent results, computations were done on a $60 \times 48 \times 20$ grid mesh. The span-averaged Nusselt number at any axial location differed from the projected grid-independent value by less than $3 \%$.

Figure 6-20 shows limiting streamlines for the velocity field on a horizontal plane close to the bottom wall for a Reynolds number of 1000. If one considers the flow passage between one of the winglets and the curved surface of the tube, the geometrical shape resembles a nozzle-like configuration. The fluid layers do not face an adverse pressure gradient aft of the circular tube.

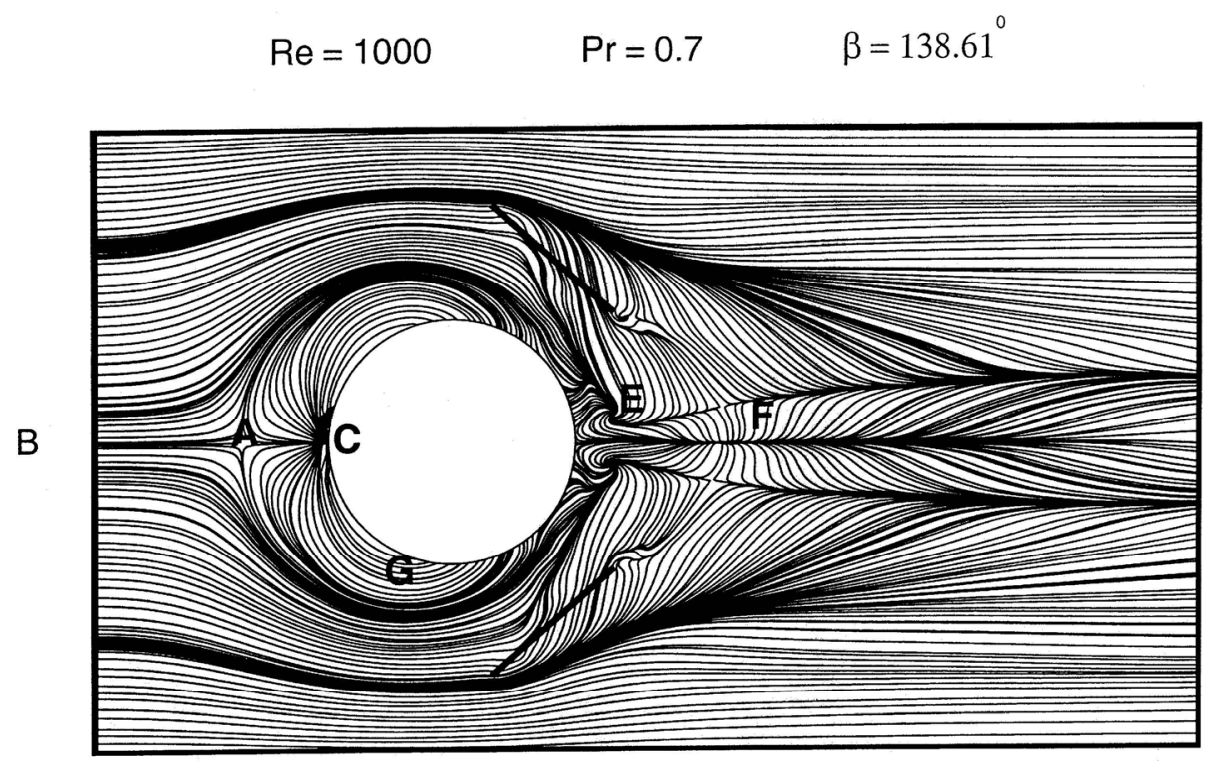

$\mathrm{L}$

Figure 6-20. Limiting streamlines on a plane close to the bottom plate of a channel with a built-in circular tube and a pair of delta winglets in a toe-in (common flow up) configuration.

Instead, the fluid accelerates in the constricted passage and brings about a significant separation delay. The wake region is indeed quite narrow. The winglet pair causes a transverse motion, which in turn delays the separation. A saddle point of separation and a horseshoe vortex system is observed in the figure. The incoming flow reaches a stagnation or saddle point of separation (marked by A) and goes 
around the body. The nodal point of attachment (marked C) and the separation lines are also visible. On each side of the tube, one finds a region of converging streamlines (marked $\mathrm{G}$ ). These are the traces of the horseshoe vortices. Behind the tube one finds two zones of swirling flow (marked E). The wake stagnation point $(\mathrm{F})$ is located at a distance slightly more than one diameter downstream.

Figure 6-21 shows cross-stream velocity vectors at various axial locations in the channel with the circular tube and the winglet pair shown in Figure 6-21. The axial location of Figure 6-21a is near the trailing edge of the winglet pair. The velocity vectors depict the secondary flow pattern on various crossstream planes in the channel. The results correspond to a Reynolds number of 1000 and an angle of attack of 138.61 degrees. The pressure difference between the front surface, facing the flow, and the suction surface of the winglet generates a swirling motion. The swirling motion is described by the longitudinal vortices. This figure clearly reveals that the cross flow pattern depicts a common-flow-up situation. The strength of the vortex motion decreases with the downstream distance from the winglet. The decrease in vortex strength can be attributed to viscous diffusion.

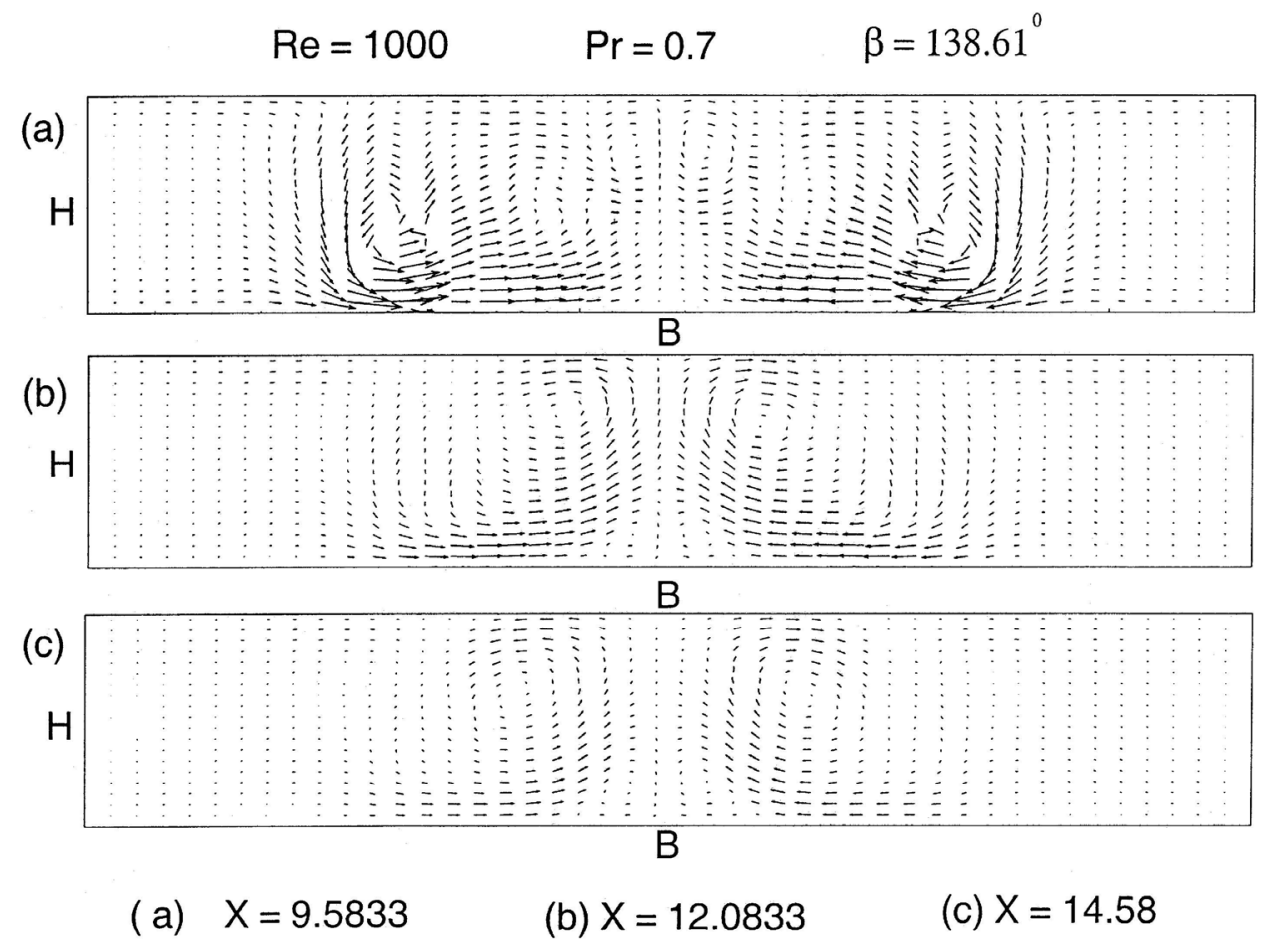

Figure 6-21. Cross-stream velocity vectors at different axial (X) locations.

Figure 6-22 represents the streamlines on the cross-stream plane at the nondimensional axial locations of $9.5833,12.0833$, and 14.58 from the leading edge of the channel. The streamlines confirm existence of a strong vortical motion that becomes clear examining Figure 6-21. The centers of the main vortices are located at a distance of $\pm 0.24 \mathrm{~B}$ from the vertical mid-plane. Induced vortices become prominent as the pair of longitudinal vortices travel in the downstream. 


$$
\operatorname{Re}=1000 \quad \operatorname{Pr}=0.7 \quad \beta=138.61^{\circ}
$$
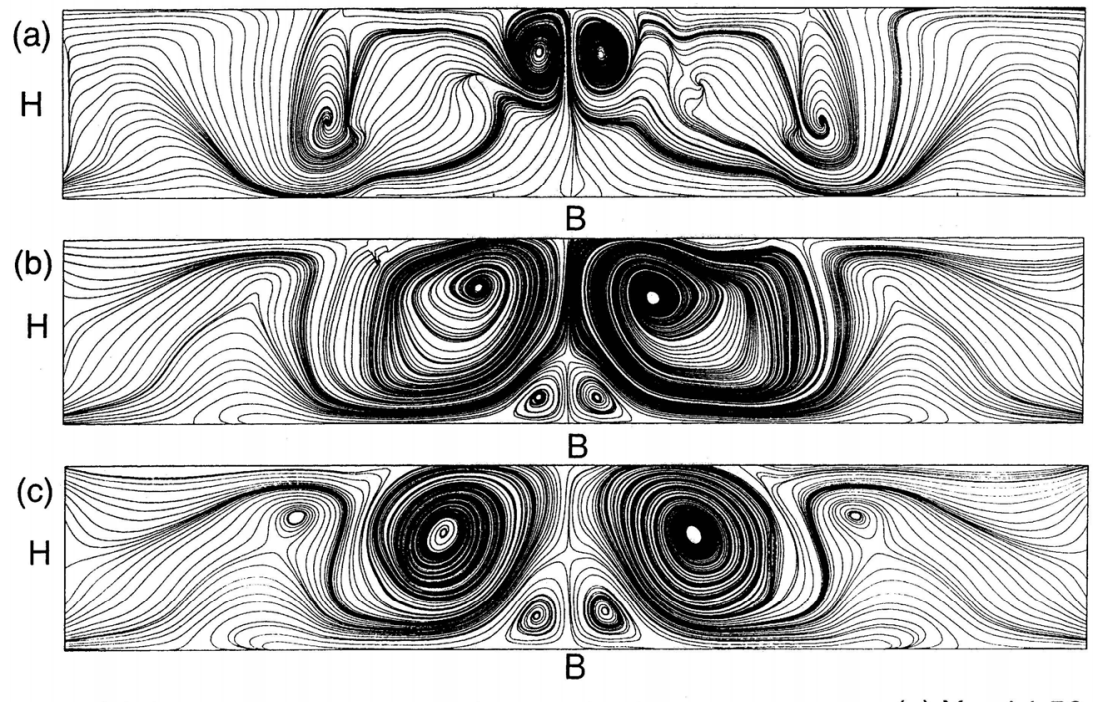
(a) $\mathrm{X}=9.5833$
(b) $X=12.0833$
(c) $X=14.58$

Figure 6-22. Streamlines on the cross-stream planes at different axial (X) locations.

Figure 6-23 represents the Nusselt number distribution on the bottom plate of the channel for the Reynolds number of 1000. The following regimes may be distinguished. At the leading edge of the bottom plane, the Nusselt number starts with a very high value and decreases gradually. At the leading edge of the plate, the cooler fluid comes in contact with the hot fin for the first time; hence, the heat transfer is very high. The gradual decrease in the Nusselt number is attributed to the development of a thermal boundary layer on the channel walls. In front of the tube, a zone of high Nusselt number is observed. This results from the formation of the horseshoe vortex system, which consists of two counterrotating longitudinal vortices. As the fluid approaches the stagnation line of

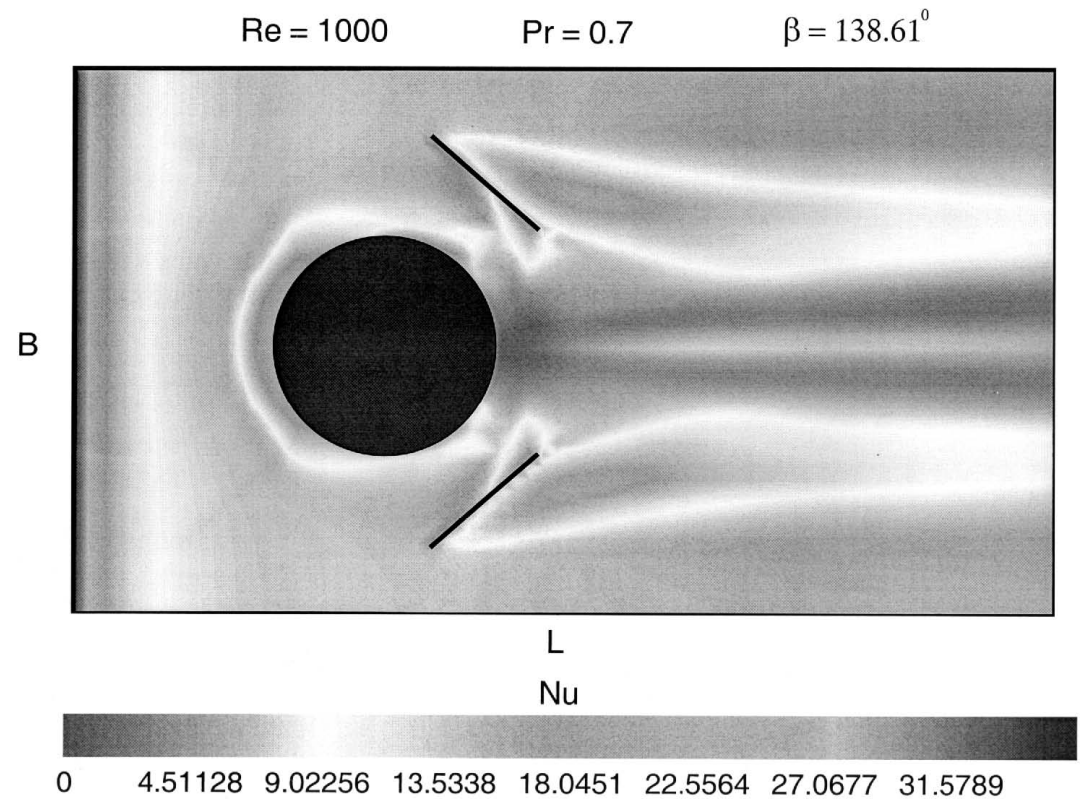

Figure 6-23. Nusselt number distribution on the bottom plate of a channel with built-in circular tube and delta winglet pair in toe-in (common-flow-up) configuration. 
the circular tube, it slows Down, and its pressure increases. The smaller velocity within the boundary layer in the vicinity of the bottom plate leads to a smaller pressure increase. Thus, the induced pressure gradient on the stagnation line causes the flow toward the bottom wall that interacts with the main stream. The fluid rolls up, forming vortices that finally wrap around the front half of the tube and extend to the rear of the tube (Goldstein and Karni). ${ }^{16}$ An abrupt increase of the Nusselt number is observed near the location of the trailing edge of the winglets. This enhancement is attributed to the formation of a complex vortex system that consists of two counter-rotating longitudinal vortices. The formation of longitudinal vortices brings about better mixing, and the Nusselt number becomes high near the location of the winglets.

Figure 6-24 compares the span-averaged Nusselt number distribution on the bottom wall in a channel for the cases of a built-in circular tube, without and with a winglet pair. The Reynolds number of interest is 1000 . The span-averaged Nusselt number reaches the maximum value near the axial location of the leading edge of the circular tube. This is primarily governed by the formation of horseshoe vortices at the tube and bottom wall junction, as explained earlier. The peak value of the span-averaged Nusselt number is about 11.18. Up to the location of the leading edge of the winglets, the distribution of the spanaveraged Nusselt number is almost the same for the cases with and without winglets. However, beyond that location, the Nusselt number starts increasing for the case of a built-in winglet pair. The maximum enhancement in heat transfer (96\%) takes place immediately upstream from the trailing edge of the winglet pair $(X=9.7)$. At the exit of the channel, the span-averaged value of the Nusselt number is 5.7. The span-averaged Nusselt number value at the exit $(X=20)$ of a plane channel is 3.98 .

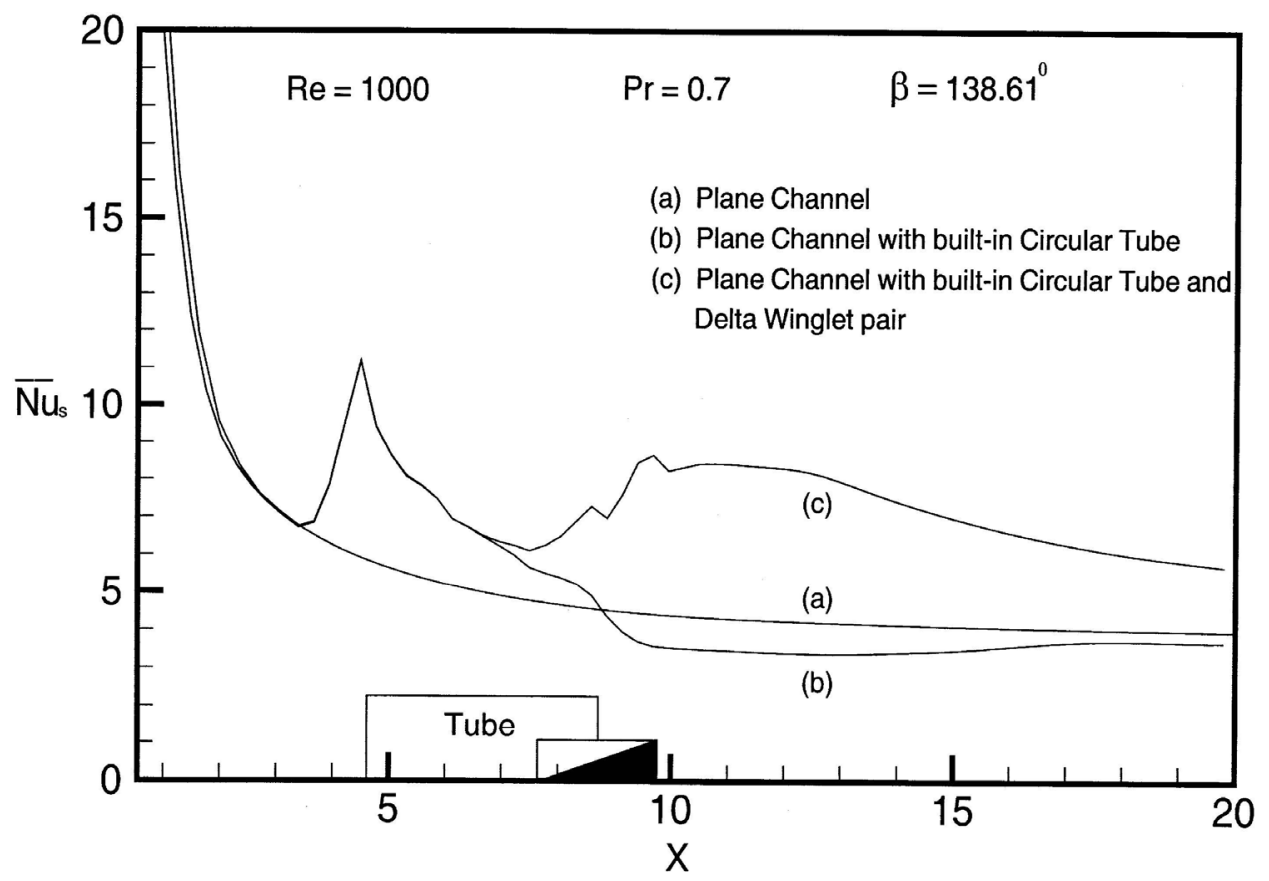

Figure 6-24. Span-averaged Nusselt number distribution on the bottom plate of the channel for $\mathrm{Re}=1000$.

Figure 6-25 compares the distribution of a span-averaged Nusselt number on the bottom wall of a channel for two Reynolds numbers, namely, $R e=1000$ and 1350. As expected, the Nusselt number distribution is higher for the higher Reynolds number. The angle of the attack of the winglet pair is kept as 151.27 degrees. 


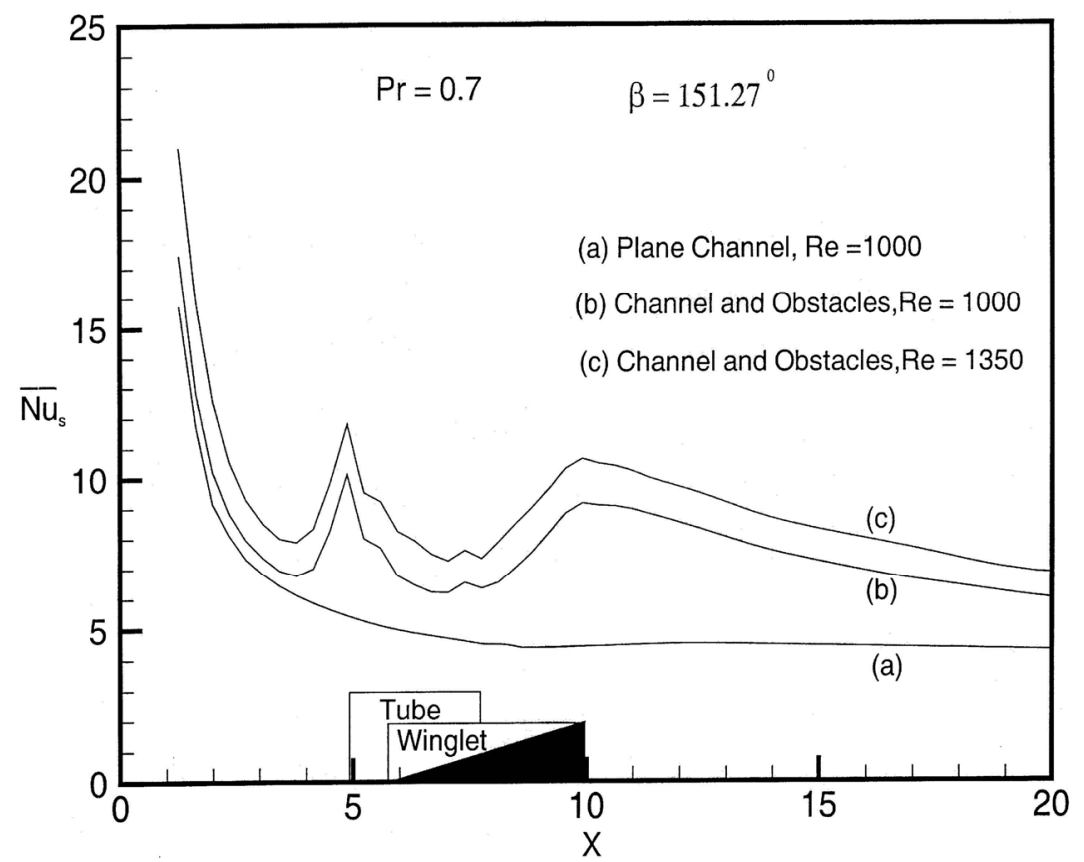

Figure 6-25. Span-averaged Nusselt number distribution for two configurations and two Reynolds numbers.

The numerical results were validated by comparing these with the experimental data of Torii et al. given in Section 4, as shown in Figure 6-26. The enhancement is expressed in terms of the ratio of $\mathrm{Nu}_{\mathrm{s}}$ and $\mathrm{Nu}_{\mathrm{so}}$. Here, $\mathrm{Nu}_{\mathrm{s}}$ is the span-averaged Nusselt number at any axial location for the case of a channel with a built-in circular tube and winglet pair, and $\mathrm{Nu}_{\mathrm{so}}$ is the span-averaged Nusselt number at any axial location for a plane channel. The angle of attack of the winglets has been taken as 151.27

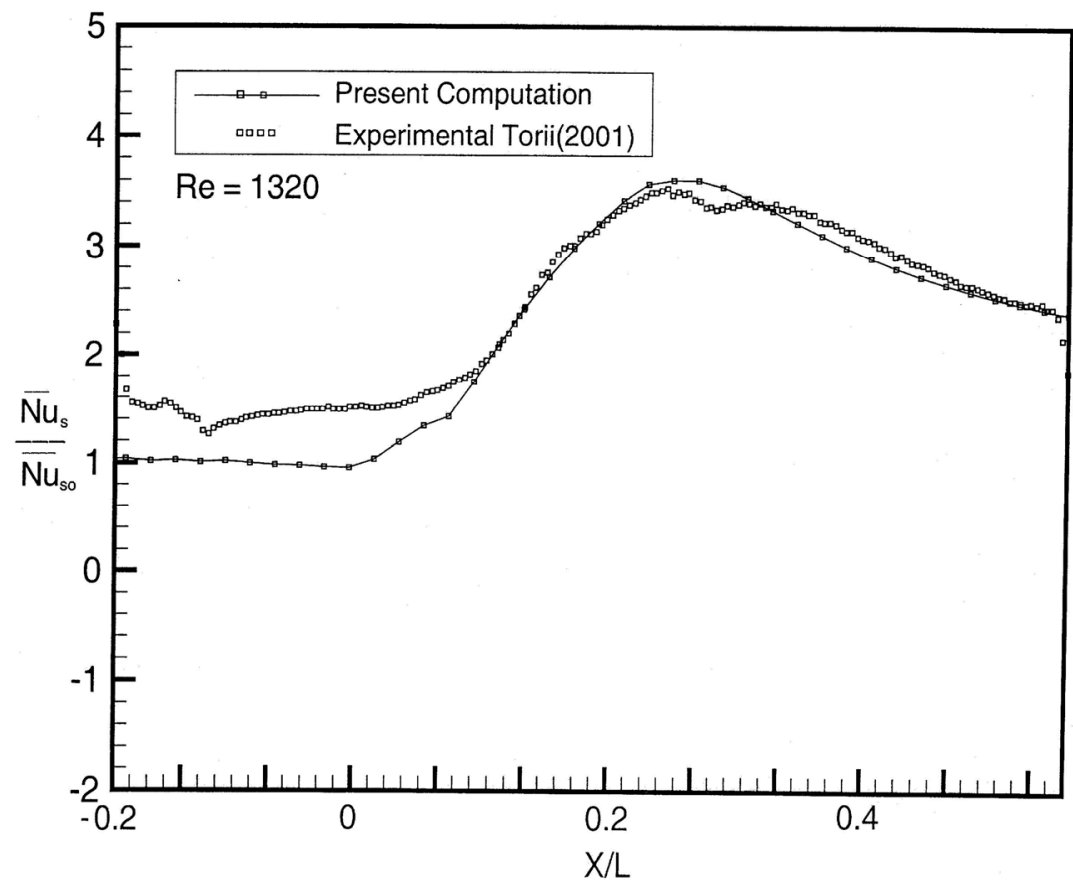

Figure 6-26. Comparison between the numerical results and experimental data of Torii et al. (Section 4 of this report). 
degrees. The axial location of the center of the tube has been taken as the reference coordinate point in this figure. The Reynolds number of interest is 1320, and the experimental technique of heat transfer measurement is based on the experimental study of Mochizuki et al. ${ }^{21}$ The computation and experimental results compare well in general, and downstream of the trailing edge in particular.

\subsubsection{Concluding Remarks}

A computational study has been accomplished to determine flow structure and heat transfer in a rectangular channel with a built-in circular tube and delta-winglet-type vortex generators. The horseshoe vortices are generated at the junction of the forward stagnation line and the bottom plate. The horseshoe vortices travel downstream, inducing a flow structure that is primarily a longitudinal vortex system. However, this natural horseshoe vortex system does not persist downstream. Longitudinal vortices can be created by winglet-type vortex generators. The present analysis reveals that the combination of circular tube and delta winglet pair with toe-in (common-flow-up) configuration improves the heat transfer performance even more. The nozzle-like flow passages created by the delta winglet pair and the aft region of the circular tube promote acceleration and thereby remove the zone of poor heat transfer from the near wake. At the end of a channel $(X=20)$ the span-averaged value of Nusselt number (based on channel height) is 5.7 for a Reynolds number of 1000 . It may be mentioned that the span-averaged Nusselt number (based on channel height) near the exit of a plane channel is 3.98 for the same Reynolds number. Enhancement of heat transfer is remarkable even far downstream. The average Nusselt number of the entire bottom plate for the case of a channel with a built-in circular tube is 5.37 for a Reynolds number of 1000. The average Nusselt number of the plate improves to a value of 7.27 for the same Reynolds number when the winglet pair is added following the toe-in (common-flow-up) configuration.

\subsection{Numerical Prediction of Heat Transfer in a Channel with a Built-in Oval Tube}

One heat transfer enhancement strategy involves use of an oval tube in place of a circular tube. ${ }^{29}$ This strategy results in a more streamlined air flow pattern around the tube and less airside pressure drop for the same flow area inside the tube. It also reduces the size of the wake region downstream of the tube, which is a region of poor heat transfer. In their experimental study, O'Brien and Sohal ${ }^{11}$ have shown that performance of oval tubes is indeed promising. The second strategy involves placing vortex generators on the fin surfaces in order to increase overall fin heat transfer rates by producing longitudinal vortices similar to horseshoe vortices. This section reports the influence of two different shaped winglet pairs, such as the delta winglet pair and the rectangular winglet pair. To analyze the mechanisms involved in the heat transfer and flow behavior in such heat exchangers, a detailed investigation on a heat exchanger module is necessary. Figure 6-27 is a schematic diagram of such a module. The gap between the two neighboring fins forms the domain of interest in this study. One can also call it an element of a heat exchanger.

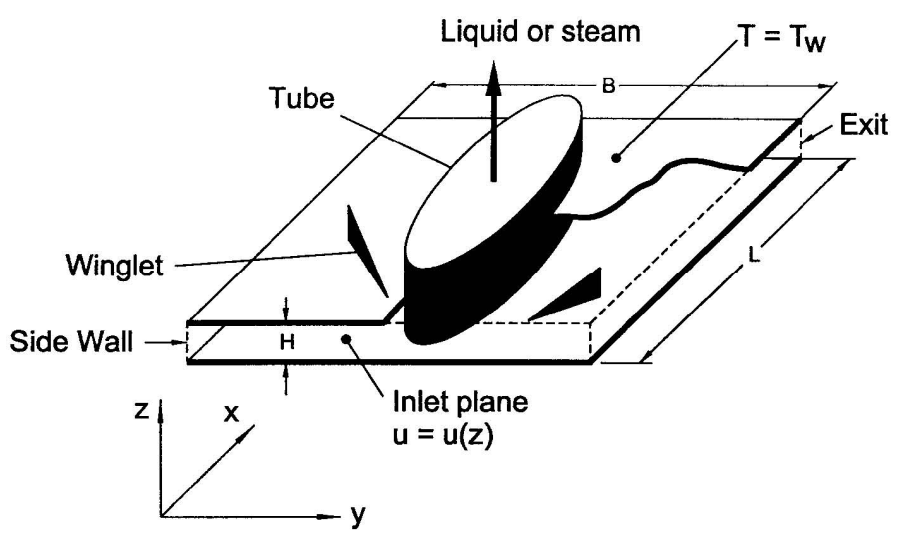

Figure 6-27. Heat exchanger module with oval tube and vortex generators. 


\subsubsection{Formulation of the Problem}

The computational domain is shown in Figure 6-27. Two flat plate fins form a channel of height $H$, width $B=11: 25 H$, and length $L=13: 75 H$. The oval tube of major diameter $2 a=8.66 \mathrm{H}$ and minor diameter $2 b=2.88 \mathrm{H}$ is located at a distance $L_{l}=6.875 \mathrm{H}$ from the inlet. Coordinates of the center of the tube are $X=6.875, Y=5.625$. The axial distance between the leading edge of the winglet and the channel inlet is $1.88 \mathrm{H}$. The transverse distance between the channel centerline and the leading edge of the winglet is $2.38 \mathrm{H}$. Similarly, the axial distance between the trailing edge of the winglet and channel inlet is $3.38 \mathrm{H}$. The transverse distance between the channel centerline and the trailing edge of the winglet is $3.25 \mathrm{H}$. The two winglets are symmetrical about the channel centerline. The angle of attack, $\beta$, is 30 degrees. The length of each winglet is $L=1: 73 \mathrm{H}$, and height is $h=0: 857 \mathrm{H}$. So, aspect ratio is $\Lambda=2$. The position and the length of the rectangular winglets are the same as the delta winglets. The height of the rectangular winglet is half of the height of the delta winglet in order to keep the surface areas of two different types of vortex generators the same. The computations are also performed for varying angles of attack of the winglet pair, such as $\beta=35$ and 45 degrees. Leading edges of all the winglets are located at the same point.

The governing equations, the grid (shown in Figure 6-28), solution technique, boundary conditions, discretization procedure, and pressure-velocity coupling are similar to those given in Sections 6.1 and 6.2. For details, appropriate journal references are also cited.

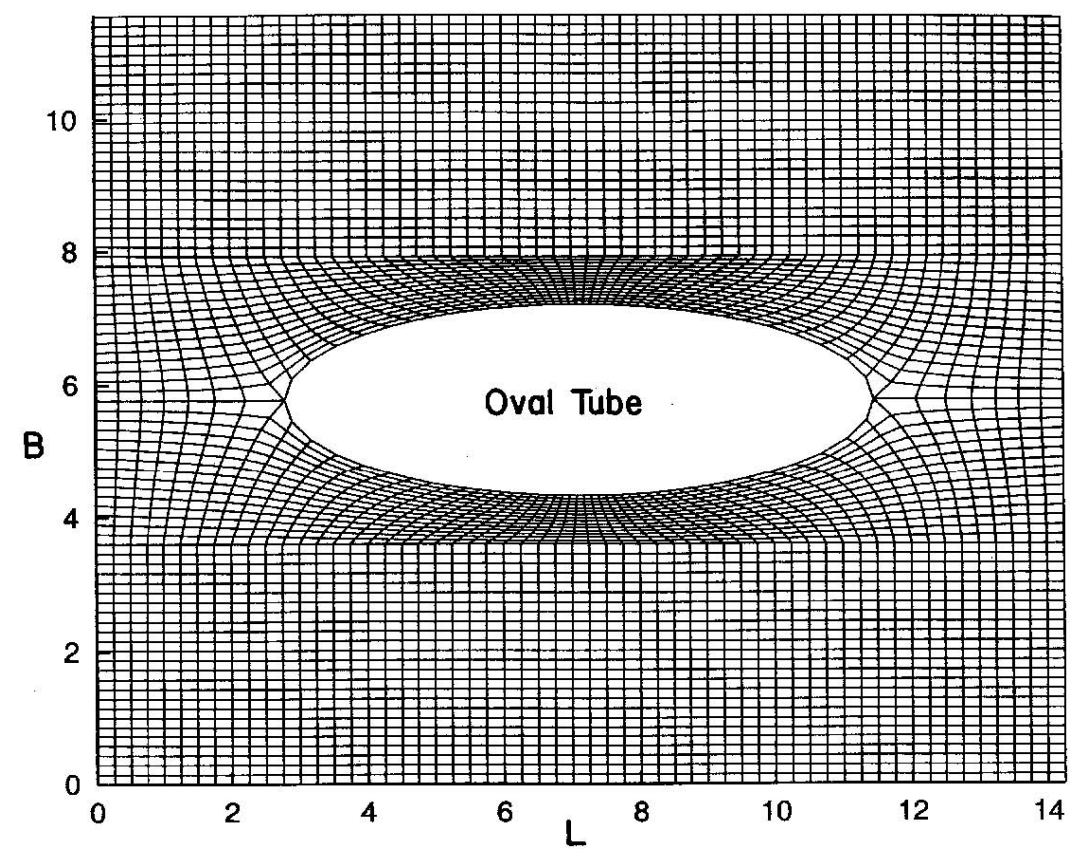

Figure 6-28. The schematic of the grid system on a two-dimensional plane.

\subsubsection{Results and Discussion}

A $57 \times 80 \times 16$ grid mesh is used in the present computation. The flow direction consists of 57 grid points, while 80 and 16 grid points have been deployed in the $\mathrm{Y}$ and $\mathrm{Z}$ directions, respectively. The divergence free criterion is satisfied using an upper bound of $10^{-4}$. Computations were carried out for Reynolds numbers of 560, 1300, 2700, and 3500. Air was taken as the working fluid; hence, the Prandtl number is 0.7 .

Figure 6-29 shows the stream lines for the time -averaged velocity field on the horizontal mid-plane for a Reynolds number of 1300. This figure clearly shows two vortices in the wake region. The flow 
separation and wake formation are evident in the figure. However, being that the oval tube is a more streamline body than a circular tube, there is considerable separation delay, and the wake is quite narrow.

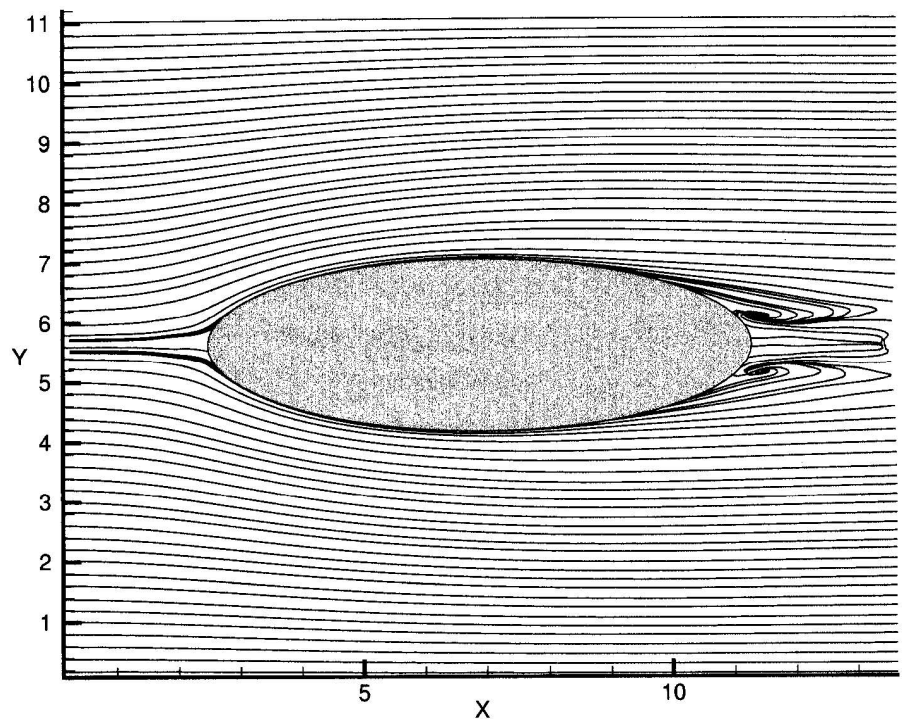

Figure 6-29. Steamlines on the horizontal mid-plane of the channel.

Figure 6-30 shows the stream lines of the time-averaged flow in the region close to the plate for the oval tube and winglet pair. The incoming flow does not separate in the traditional sense but reaches a stagnation or saddle point of separation (A on the figure) and goes around the tube. The nodal point of attachment (C) is also clearly visible in the figure. On each side of the tube, one finds a region of converging streamlines $(\mathrm{F})$. These are traces of horseshoe vortices. Almost behind the body, are the footprints of an arch vortex (E).

Figures 6-31 show cross-stream velocity vectors at $X=6.125$ in the channel for the case of the oval tube as the built-in obstacle and for the oval tube and the winglet pair as the built-in obstacle s with $\beta=30$ degrees. Fluid in the upper half of the channel has a tendency to go downward, while the lower half of the fluid moves upward in the channel due to the formation of horseshoe vortices (Figure 6-31a). The longitudinal vortex generated by the winglet suppresses the upward motion of fluid in the lower part while it reinforces the downward motion in the upper half. Due to this interaction of vortices, heat transfer characteristics change.

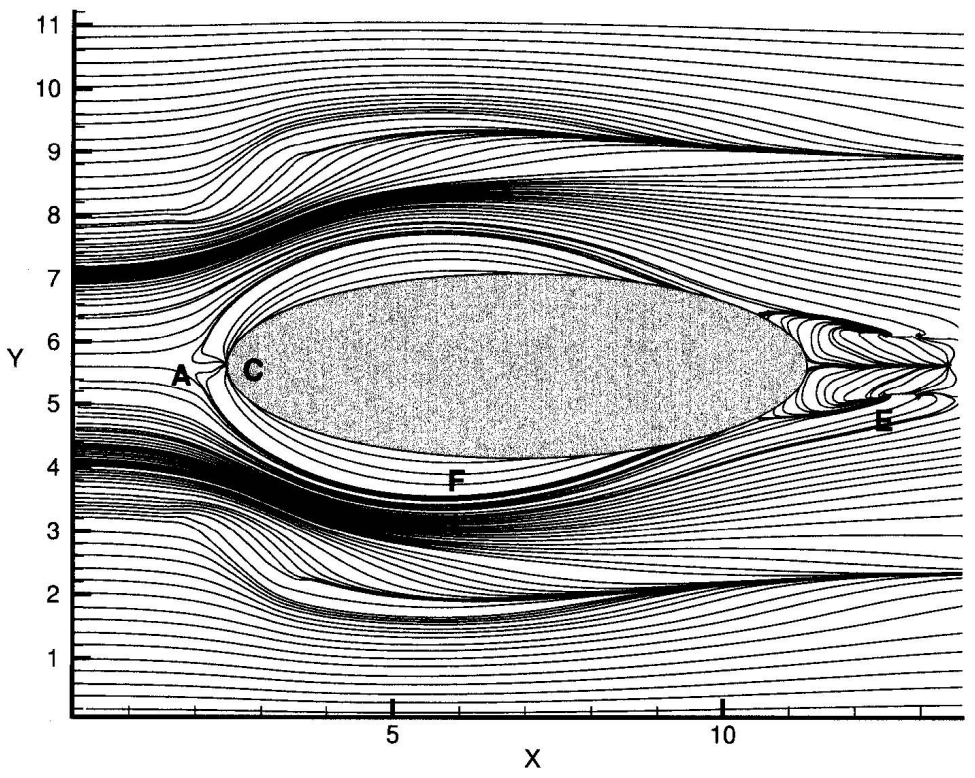

Figure 6-30. Limiting streamlines on the bottom plate. 

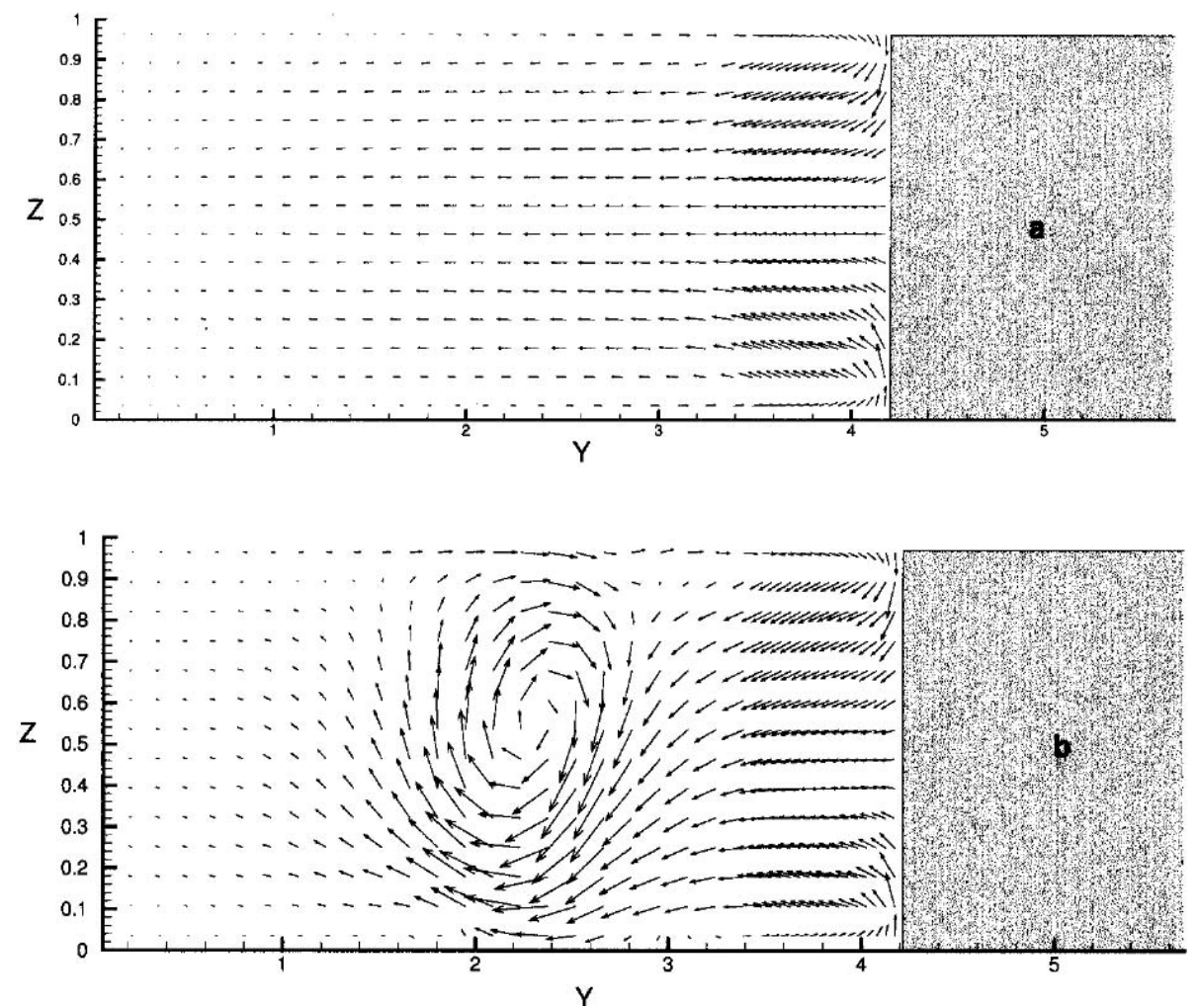

Figure 6-31. Cross stream velocity vectors at axial location $X=6: 125$ : (a) oval tube, (b) oval tube and winglet pair.

Figure 6-32 represents the local Nusselt number distribution on the bottom plate of the channel for the Reynolds number of 1300 . The following regimes may be distinguished. At the leading edge of the bottom plane, the Nusselt number begins with a very high value and decreases gradually. At the leading edge, the cooler fluid comes in contact with a hot fin for the first time, hence the heat transfer is very high. The gradual decrease in the Nusselt number is attributed to the development of thermal boundary layer on the channel walls. An abrupt increase of the Nusselt number in front of the tube is observed. This results from the formation of a horseshoe vortex system that consists of two counter-rotating longitudinal vortices. As the fluid approaches the stagnation line of the oval tube, it slows down, and its pressure increases. The smaller velocity in the boundary layer in the vicinity of the bottom plate, which supports the oval tube, leads to a smaller pressure increase. Thus, the induced pressure gradient on the stagnation line causes the flow toward the bottom wall, which interacts with the main stream. The fluid rolls up, forming vortices that finally wraps around the front half of the tube and extends to the rear of the tube (Goldstein and Karni). ${ }^{16}$

The local heat transfer distribution, associated with the horseshoe vortices, is resolved accurately. The distribution of local Nusselt number qualitatively matches the results of Foust et al. ${ }^{66}$ The Nusselt number is low in the near wake region. The poor heat transfer in this region is attributed to the separated dead wake zone with recirculating fluid at a low velocity. 


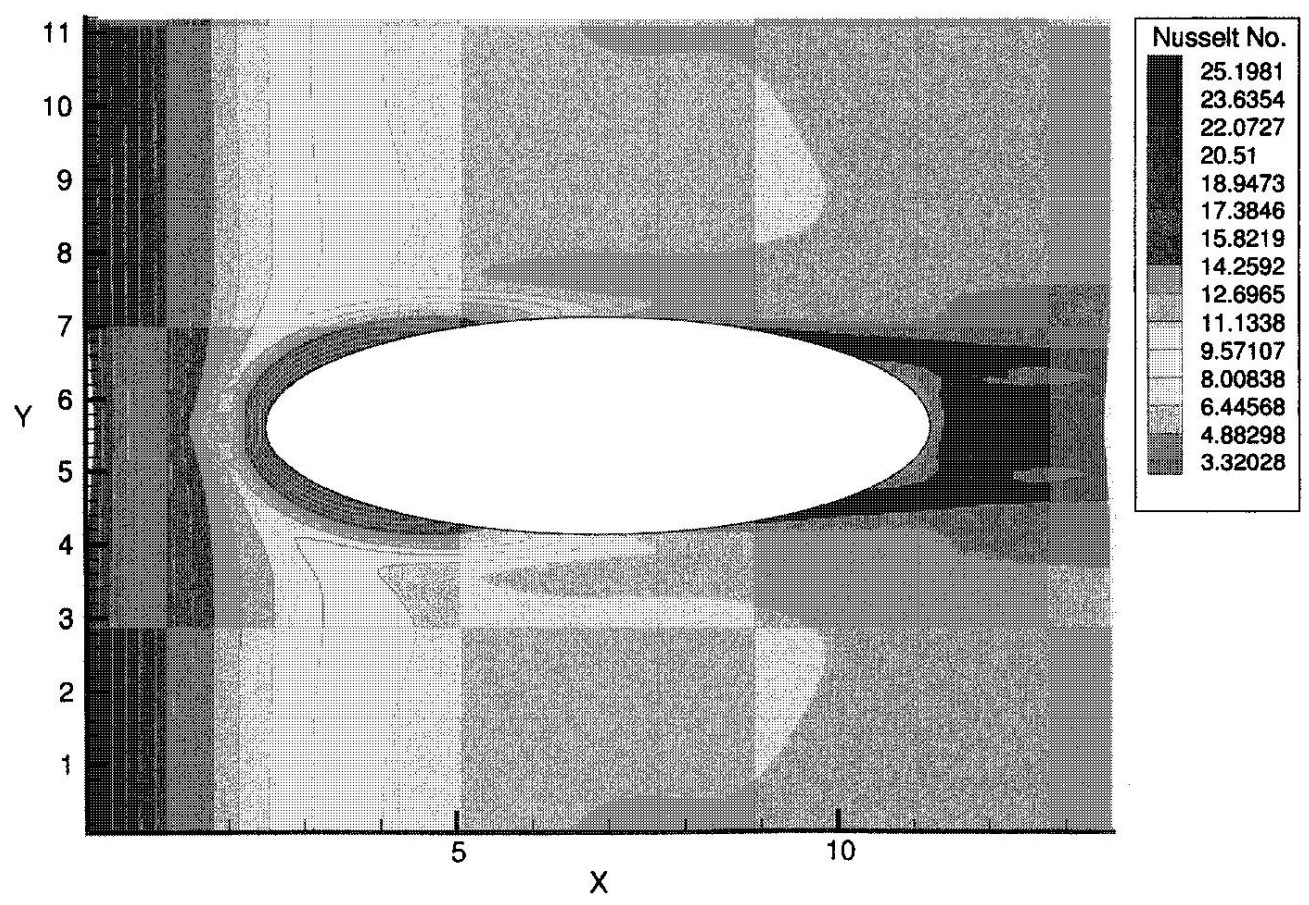

Figure 6-32. Nusselt number distribution on the bottom plate for $\operatorname{Re}=1300$.

Figure 6-33 compares the experimentally obtained span-averaged Nusselt number distribution, based on the fluid temperature at the entrance (Hagedorn), ${ }^{67}$ with the computed span-averaged Nusselt number distribution for the finned oval tube, and with constant fin temperature. In the experimental study, the Nusselt numbers were deduced from the heat and mass transfer analogy. The ammonia absorption measurements ${ }^{67}$ were performed on a finned oval tube model in a wind tunnel for a Reynolds number of 1090. Starting with $X=4$, it can be seen that the measured and the computed results agree quite well. But there is discrepancy in the earlier part prior to $X=4$. The reason for this discrepancy may be the difference of the velocity distribution at the entrance. For the computation, a uniform velocity distribution was employed at the entrance. In the experiment, great effort was put into realizing a uniform velocity field at the inlet. However, the influence

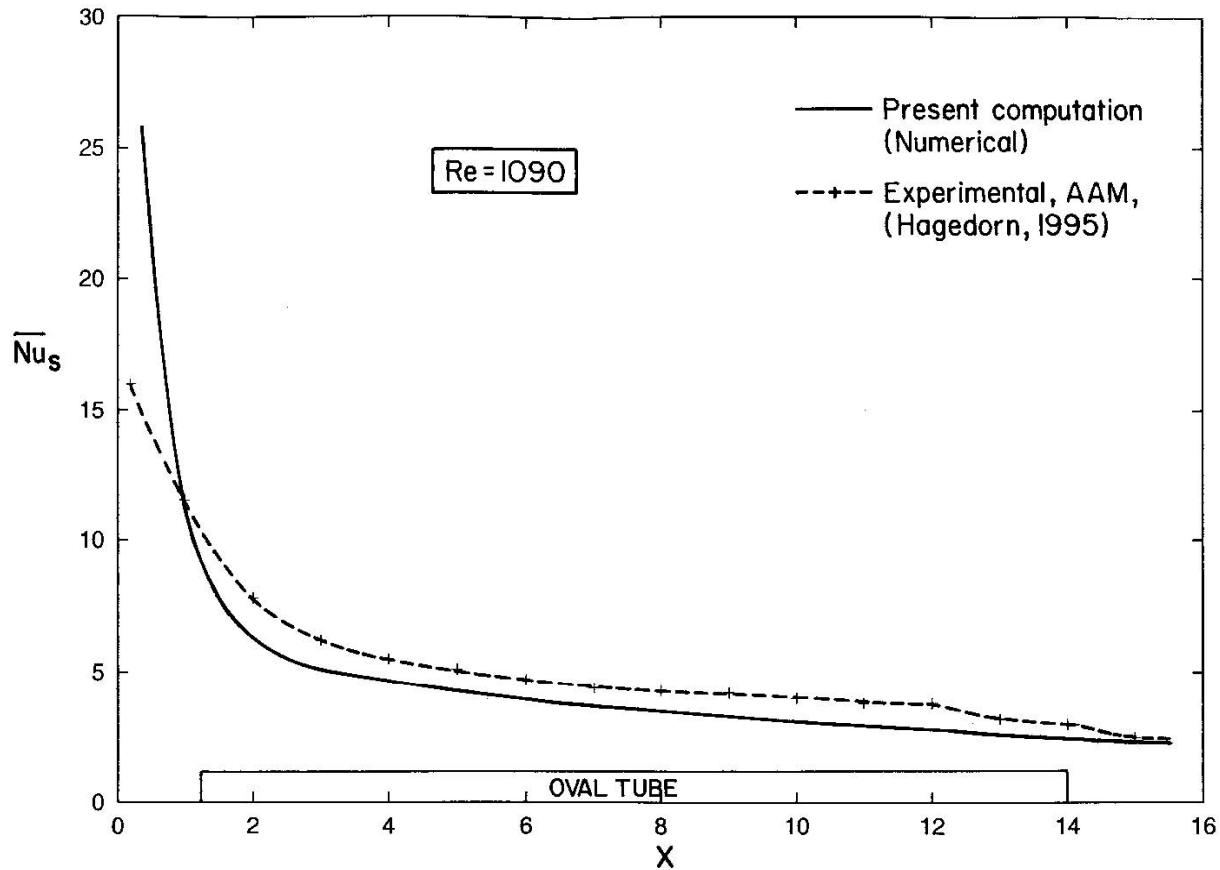

Figure 6-33. Comparison of experimental and numerical span-averaged Nusselt number for $\mathrm{Re}=1090$. 
of the side walls and the blockage at the leading edge of the test section cannot be avoided. Besides, an exact measurement of the mass transfer at the leading edge by the ammonia absorption technique is nearly impossible.

Figure 6-34 shows the local Nusselt number distribution on the bottom wall in a channel with the built-in oval tube and the winglet pair for the Reynolds number of 1300. Each winglet produces two vortices, as described by Biswas et al. ${ }^{68}$ The primary vortex located directly downstream of the vortex generator is formed by flow separation along the top edge of the winglet. The corner vortex, located outside of the main vortex, develops like a horseshoe vortex on the upstream facing pressure side of the winglet. The heat transfer enhancement associated with the primary vortex is visible clearly in the figure directly downstream of each winglet. The heat transfer enhancement associated with the corner vortex of each winglet is also visible in the figure beginning on the upstream side of each winglet and is swept to the outside and downstream.

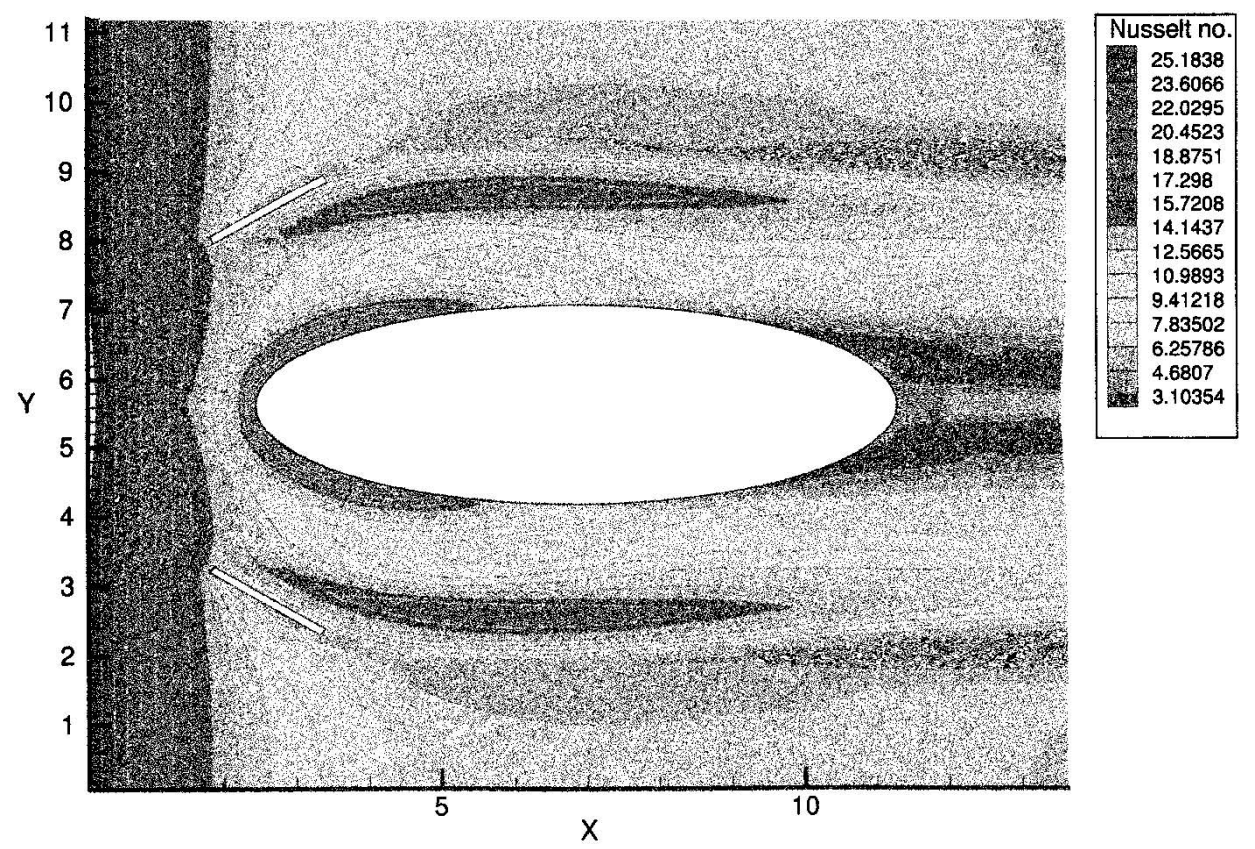

Figure 6-34 Nusselt number distribution on the bottom plate with an oval tube and a winglet pair for $\operatorname{Re}=1300$.

Figure 6-35 compares the span-averaged Nusselt number for the above mentioned geometrical configuration for Reynolds numbers, $R e=560,1300,2700$, and 3500. Table 6-2 shows the effect of the Reynolds number on the average Nusselt number on the bottom plate of the channel. Figure 6-35 compares the local Nusselt number distribution at $X=5.125$ for two cases of a channel with an oval tube alone and a channel with an oval tube and a winglet pair. Consider the Nusselt number distribution on one side of the tube for the case of a channel with an oval tube and the winglet pair. The first peak adjacent to the oval tube corresponds to a horseshoe vortex, while the second peak signifies the primary vortex due to the winglet, and the third peak corresponds to the existence of the corner vortex due to the winglet. The Nusselt number near the tube for the case of an oval tube and a delta winglet pair is slightly greater than that of the case without a winglet pair. The overall effect of interaction between the horseshoe vortex due to the oval tube and the primary vortex due to the winglet pair is enhancement of heat transfer near the tube. 


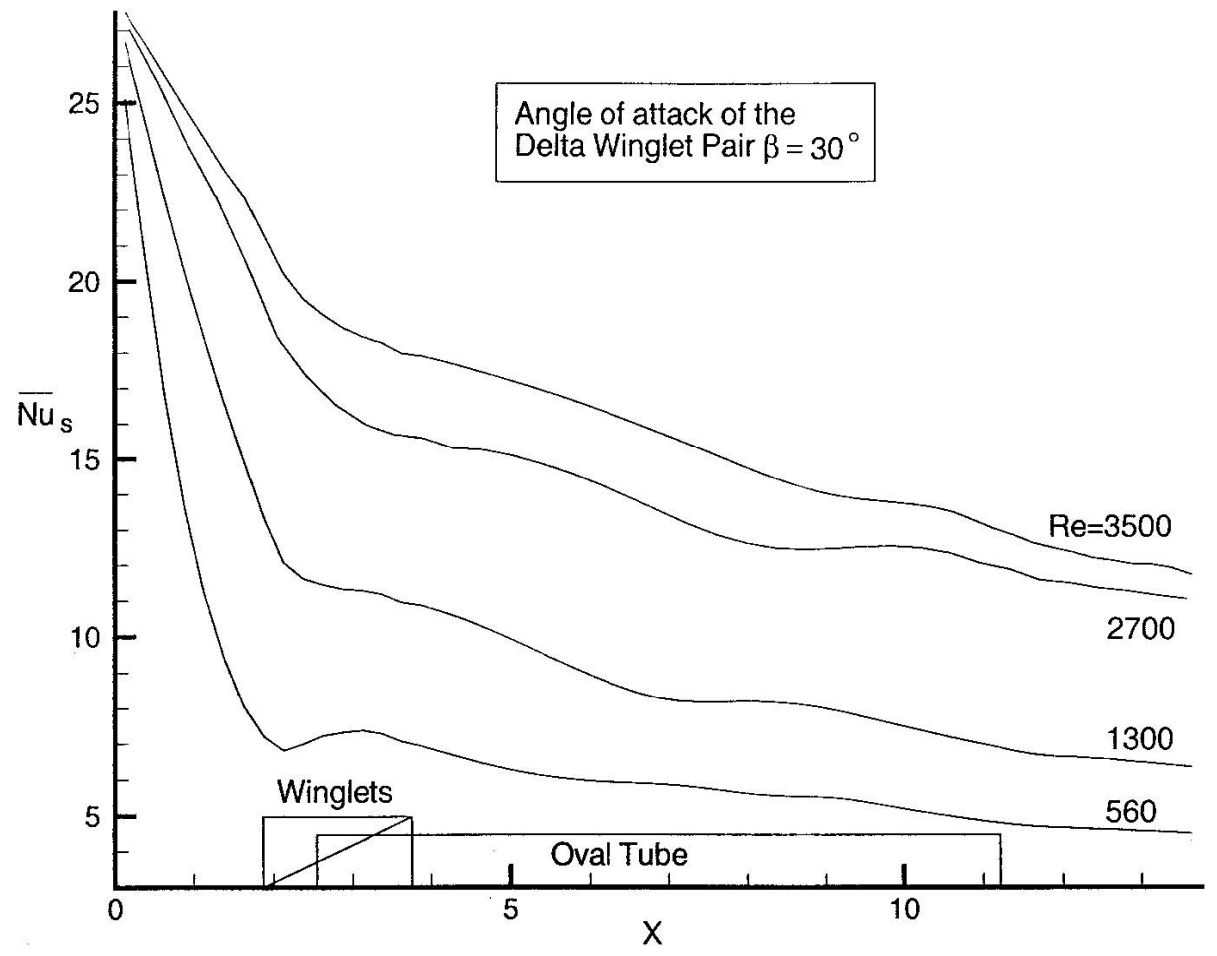

Figure 6-35. Span-averaged Nusselt number distribution for various Re in a channel with an oval tube and a winglet pair.

Table 6-2. Effect of the Reynolds number on $\mathrm{Nu}_{\mathrm{m}}$.

\begin{tabular}{ccc}
\hline $\mathrm{Re}$ & $\mathrm{Nu}_{\mathrm{m}}$ & $\begin{array}{c}\text { Percentage of } \mathrm{Nu}_{\mathrm{m}} \\
\text { Enhancement }\end{array}$ \\
\hline 560 & 6.94 & - \\
1300 & 10.17 & 46.54 \\
2700 & 14.95 & 115.41 \\
3500 & 16.56 & 138.61 \\
\hline
\end{tabular}

The angle of attack of a winglet influences the strength and the form of the vortices. Figure 6-37 compares the span-averaged Nusselt number for three angles of attack: $\beta=30,35$, and 45 degrees. Winglets with a higher angle of attack produce vortices with higher strength, which results in a better heat transfer. Table 6-3 summarizes the effect of angles of attack on the average Nusselt number on the bottom plate of the channel.

A rectangular winglet pair can also be used instead of a delta winglet pair for enhancement of heat transfer. Figure 6-38 compares the local Nusselt number distribution on the bottom plate of the channel for the cases of (a) a delta winglet pair and (b) a rectangular winglet pair for a Reynolds number of 500 and angle of attack, $\beta=25$ degrees. Note that the effect of the oval tube has not been considered here. 


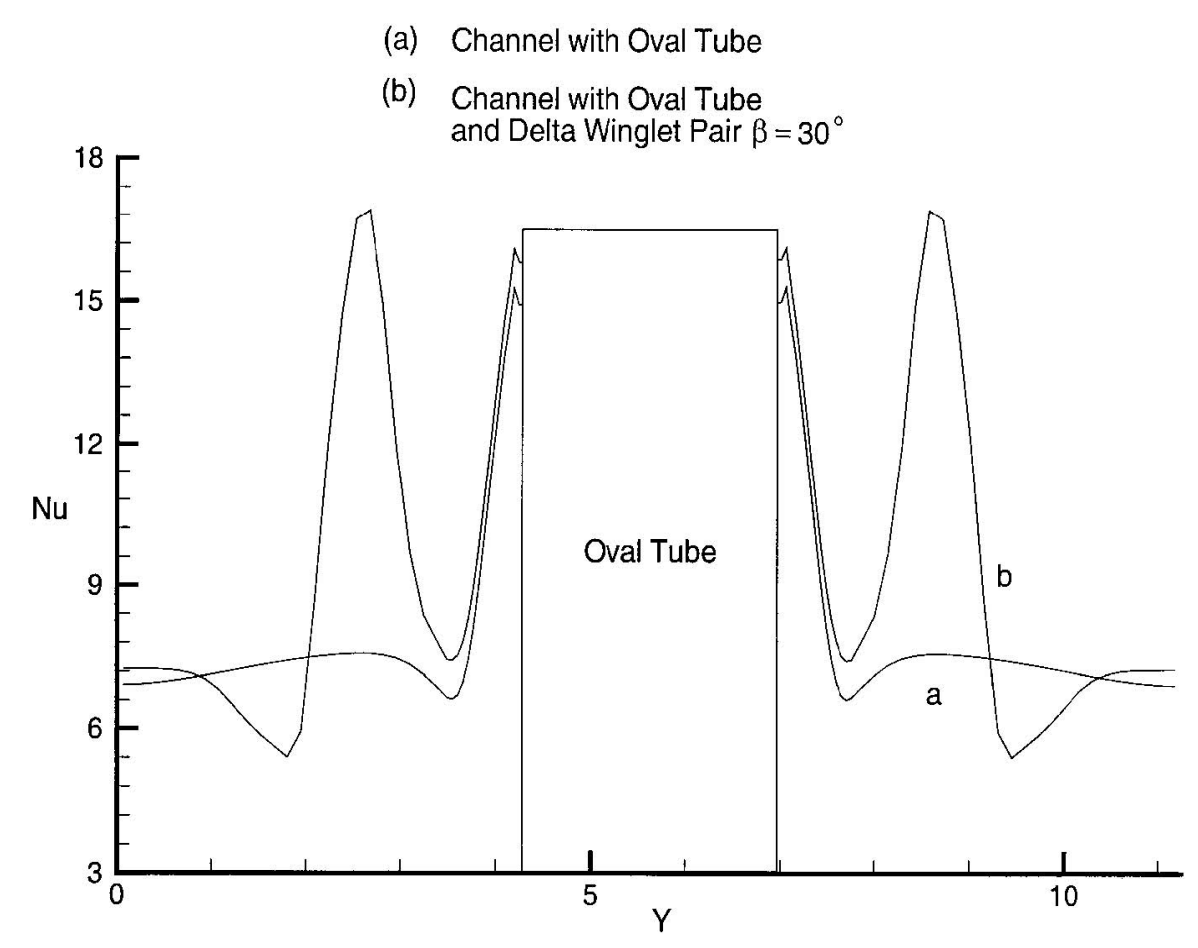

Figure 6-36. Transverse variation of local Nusselt number for $\mathrm{Re}=1300$.

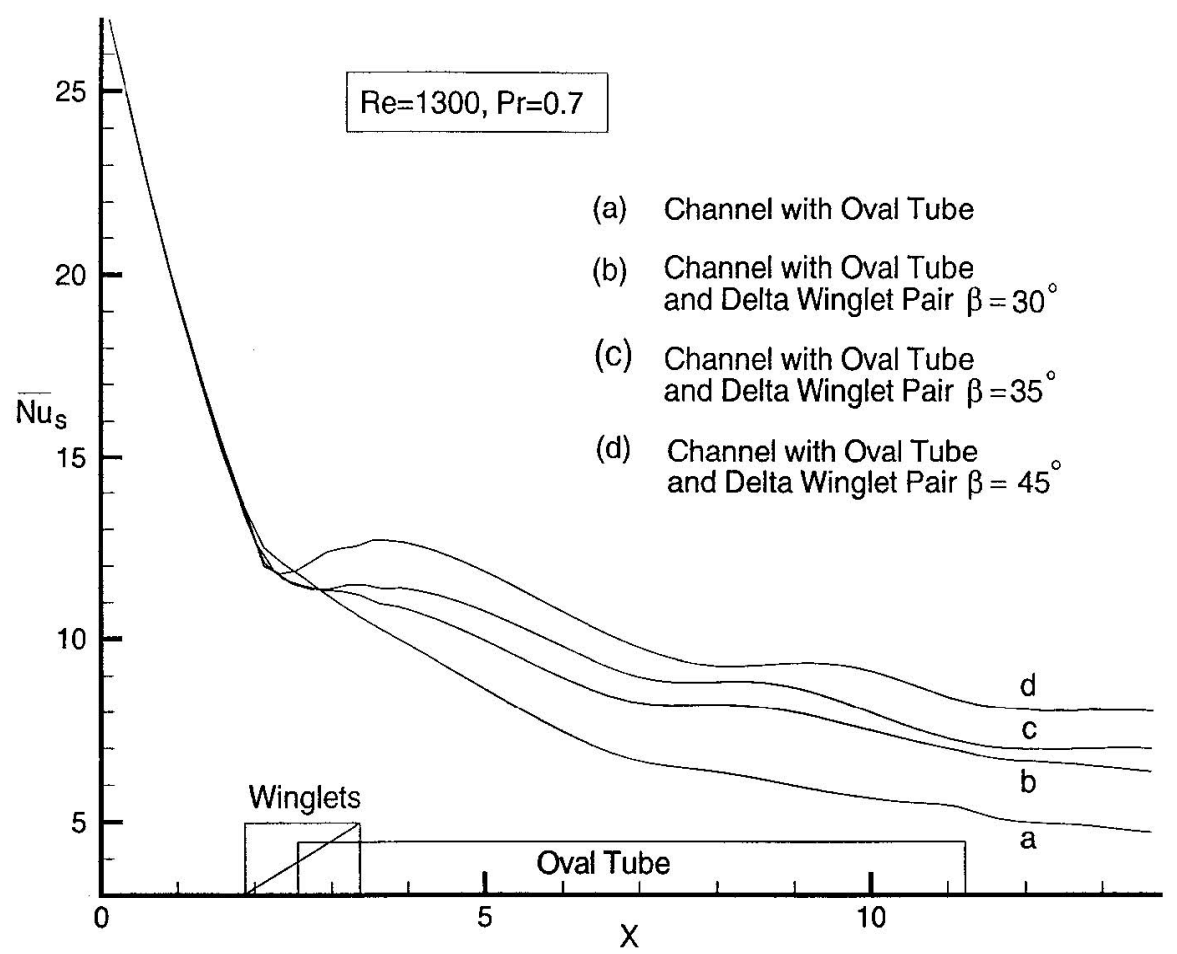

Figure 6-37. Span-averaged Nusselt number distribution for various angles of attack for $\operatorname{Re}=1300$. 
Table 6-3. Effect of angles of attack on $\mathrm{Nu}_{\mathrm{m}}$.

\begin{tabular}{ccc}
\hline Configuration & $\mathrm{Nu}_{\mathrm{m}}$ & $\begin{array}{c}\text { Percentage of } \mathrm{Nu}_{\mathrm{m}} \\
\text { Enhancement }\end{array}$ \\
\hline Without winglets & 8.99 & - \\
Winglet pair, $\beta=30$ degrees & 10.17 & 13.12 \\
Winglet pair, $\beta=35$ degrees & 10.60 & 17.90 \\
Winglet pair, $\beta=45$ degrees & 11.41 & 26.91 \\
\hline
\end{tabular}

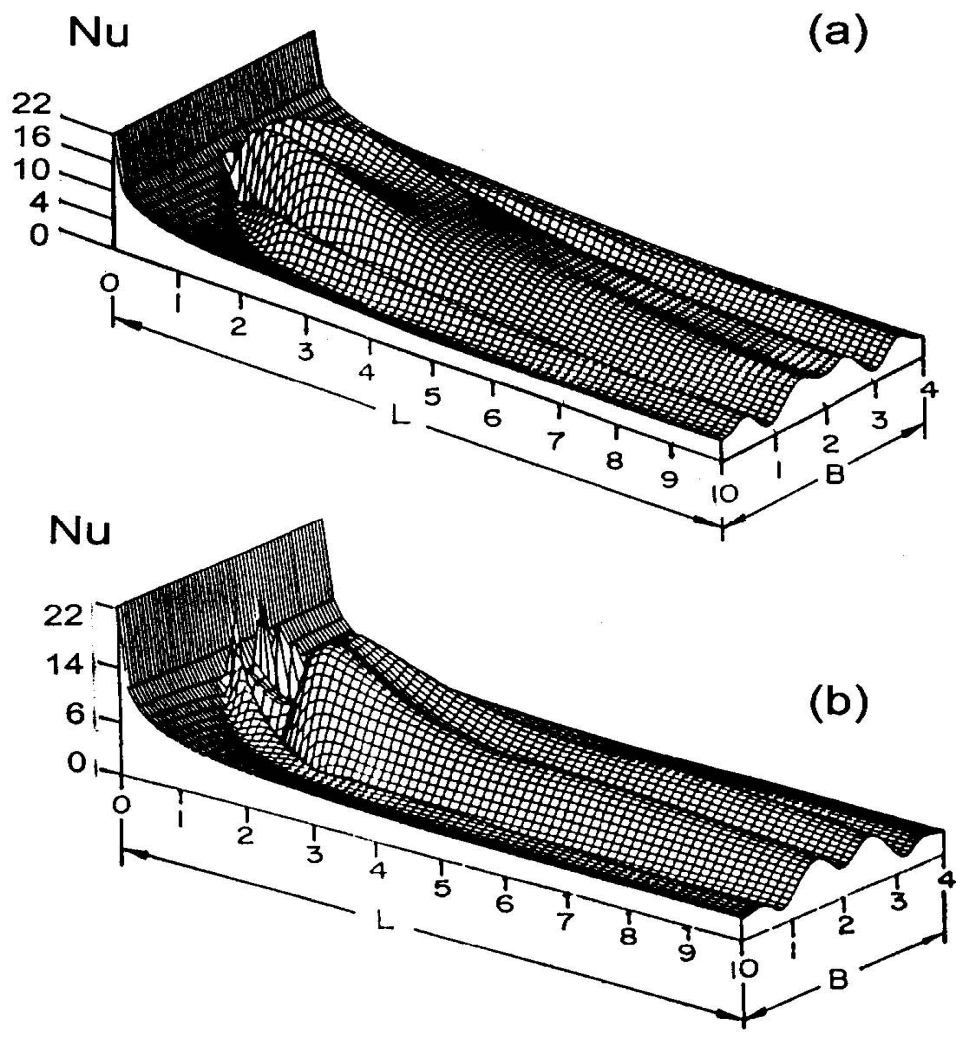

Figure 6-38. Local Nusselt number distribution on the bottom plate of a channel for (a) a delta winglet pair and (b) a rectangular winglet pair.

Figure 6-39 compares the span-averaged Nusselt number distribution on the bottom wall in a channel for the cases of (a) a built-in oval tube, (b) a built-in oval tube and a delta winglet pair, and (c) a built-in oval tube and a rectangular winglet pair. The Reynolds number of interest is 1300 . Up to the location of the leading edge of the vortex generator, all three curves coincide. Beyond that location, the Nusselt number distribution changes. The span-averaged Nusselt number distribution for the case of a channel with a built-in oval tube and the delta winglet pair appears to be the most promising. Table 6-4 shows the effect of the shape of the winglet on the average Nusselt number on the bottom plate of the channel.

Two different grid meshes of $99 \times 40 \times 16$ and $109 \times 34 \times 16$ were used to test the grid sensitivity. The difference between the average Nusselt number due to present grid mesh $(57 \times 80 \times 16)$ and that of the projected grid-independent conditions were less than $2 \%$. 


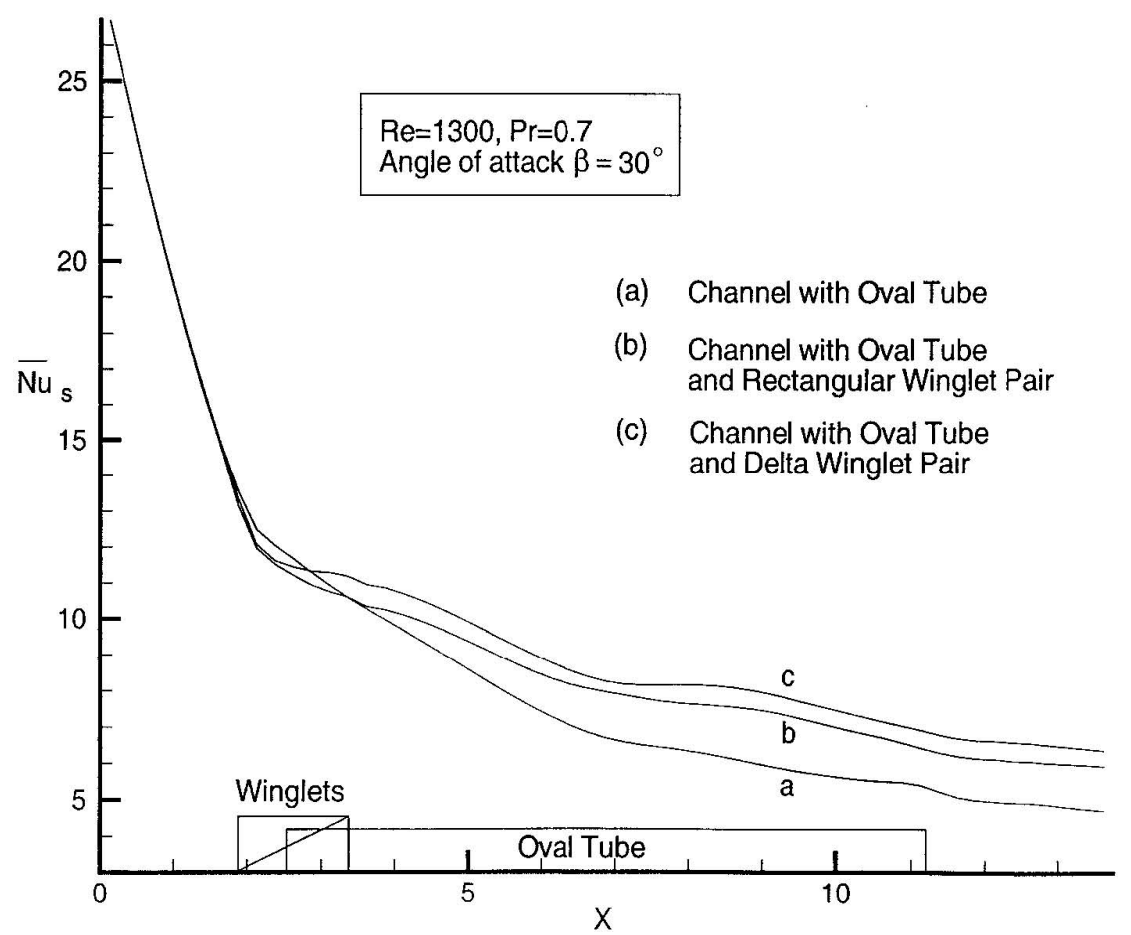

Figure 6-39. Span-averaged Nusselt number distribution on the bottom plate.

Table 6-4. Effect of winglet shape on $\mathrm{Nu}_{\mathrm{m}}$.

\begin{tabular}{lcc}
\hline \multicolumn{1}{c}{ Configuration } & \multicolumn{2}{c}{$\begin{array}{c}\text { Percentage of } \\
\mathrm{Nu}_{\mathrm{m}}\end{array}$} \\
\hline Without winglets & 8.99 & - \\
Delta winglet pair & 10.17 & 13.12 \\
Rectangular winglet pair & 9.78 & 8.78 \\
\hline
\end{tabular}

\subsubsection{Concluding Remarks}

A computational study was performed to determine flow structure and heat transfer in a rectangular channel for the cases of (a) a built-in oval tube, (b) a built-in oval tube and a delta winglet pair, and (c) a built-in oval tube and a rectangular winglet pair. O'Brien and Sohal ${ }^{11}$ have shown that local stagnationregion fin surface heat transfer is higher for the oval tube case than for the circular tube. This study reveals that the combination of an oval tube and delta winglet pair improves the heat transfer performance significantly. Since the oval tube and the winglet pair both are streamline bodies, the pressure drop due to the combination of the oval tube and the winglet pair vis-à-vis the pressure drop due to the circular tube are not expected to differ much. The performance of a heat exchanger module with the oval tube and the delta winglet pair appears to be the best of the three configurations examined in this investigation.

\subsection{Heat Transfer Enhancement using Oval Tubes and Multiple Delta Winglets}

In order to achieve significant heat transfer enhancement on the gas side, the present numerical study investigated the use of oval tubes in place of circular tubes, with delta-winglets mounted in front of the oval tubes, as shown in Figure 6-27. Vortex generators can be mounted in the fin-tube heat exchangers using the following two common configurations: (1) toe-out (common-flow-down) and (2) toe-in 
(common-flow-up). Figure 6-27 is a schematic diagram of one possible configuration. However, in this section several other configurations will also be considered.

\subsubsection{Problem Formulation}

The plan-view of the computational domain is shown in Figure 6-40. The oval tube and channel dimensions used here are the same as those given in Section 6.3.1. The winglets are thin triangular devices (shown top right in Figure 6-40) placed vertically on the fin surface, with their horizontal axis (base edge) from the tip either angled outward or inward from the oval tube centerline. The positions of the winglets are shown as W1 and W2. When angled out (as W1 in Figure 6-40), it is called a toe-out (common-flow-down) configuration, and when angled in (as W2 in Figure 6-40), it is called a toe-in (common-flow-up) configuration. The axial distance (X11) between the leading edge of the first winglet pair in a toe-out (common-flow-down) configuration and the channel inlet is $1.63 \mathrm{H}$. The transverse distance (Y11) between the channel centerline and the leading edge of either winglet is $2.23 \mathrm{H}$. The axial distance (X12) between the trailing edge of the either winglet and the channel inlet is $3.38 \mathrm{H}$. The transverse distance (Y12) between the channel centerline and the trailing edge of either winglet is $3.69 \mathrm{H}$. The two winglets of the first winglet pair are placed symmetrically about the channel centerline. The axial distance (X21) of the leading edge of the second pair of winglets in the toe-in (common-flow-up) configuration from the inlet of channel is $3.96 \mathrm{H}$, and the transverse distance (Y21) from the centerline of the channel is $5.33 \mathrm{H}$. The axial distance (X22) between the trailing edge of the second pair of winglets and channel inlet is $5.71 \mathrm{H}$, and the distance (Y22) of it from the channel centerline is $3.88 \mathrm{H}$. The length of all the winglets is $2.27 \mathrm{H}$; their height is $\mathrm{h}=0: 5 \mathrm{H}$. Figure $6-41$ shows a layout of various winglet configurations analyzed in the present study. Computations were performed for each configuration. Air was considered as the working fluid; hence, the Prandtl number is taken as 0.7. The winglets and oval tube are assumed to be at the same temperature as the channel wall.

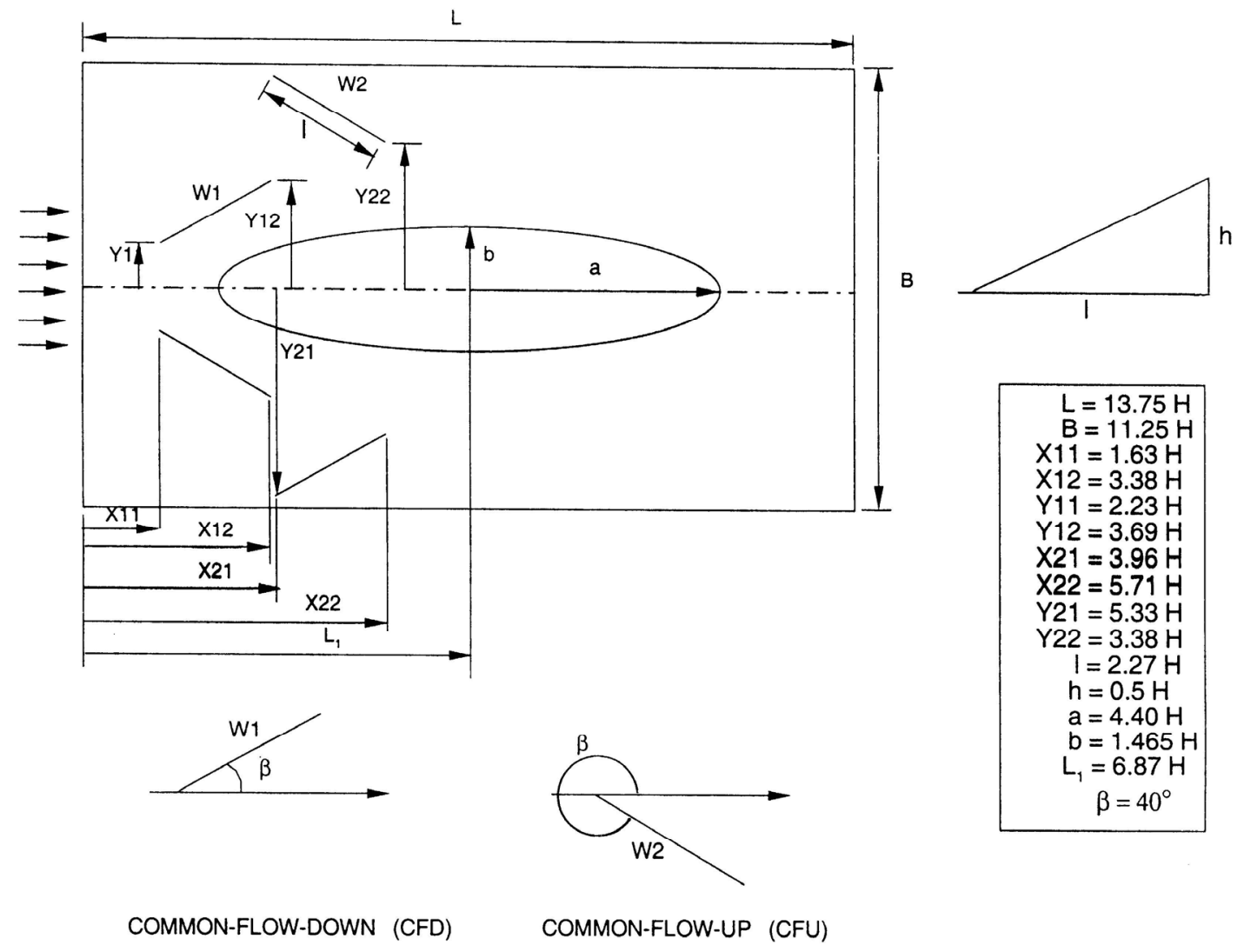

Figure 6-40. Two-dimensional representation of the computational domain. 


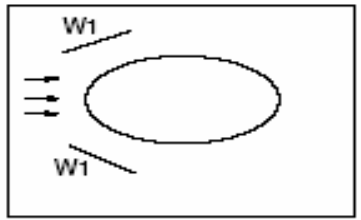

(a)

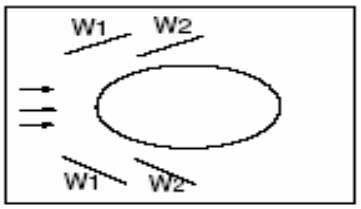

(c)

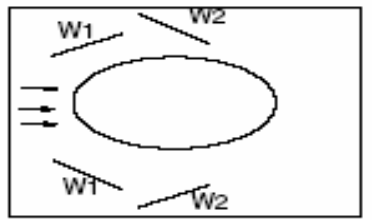

(b)

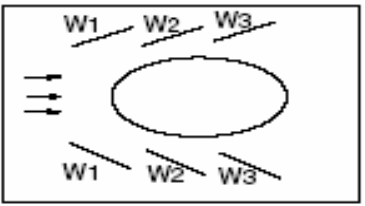

(d)

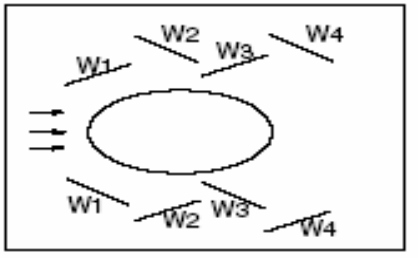

(e)

Figure 6-41. Various configurations of the winglet pairs: (a) one winglet pair (W1) toe-out, (b) two winglet pairs (W1 toe-out; W2 toe-in, (c) two winglet pairs (W1 and W2 both toe-out), (d) three winglet pairs (W1, W2 and W3 toe-out), and (e) four winglet pairs (W1 and W3 toe-out, and W2 and W4 toe-in).

The governing equations, the grid (Figure 6-28), solution technique, boundary conditions, discretization procedure, and pressure-velocity coupling are similar to those given in Sections 6.1 and 6.2. Further details can be obtained from the references cited in these Chapters.

\subsubsection{Results and Discussion}

A $62 \times 61 \times 19$ three-dimensional grid mesh was used for the numerical study of flow and heat transfer in a channel with built-in oval tube and delta-winglet type vortex generators in both toe-out (W1) and toe-in (W2) configurations, as shown in Figure 6-40. The present computations were carried out for various configurations of winglet pairs, as shown in Figure 6-41. In the Figure 6-41 configuration, different axial positions of one delta winglet pair are considered for an angle of attack of 40 degrees. In the Figure 6.4-2b configuration, the investigations are carried out for three different angles of attack of 30,35 and 40 degrees. The blockage ratio (of the minor diameter of oval tube to the width of the channel) is 0.26 . The Reynolds number in the present study is 1000 , based on the incoming average fluid velocity and the channel height. The span-averaged Nusselt number $\left(\overline{N u_{s}}\right)$, based on the bulk-mean temperature, was used for comparing the heat transfer performance. The delta-winglets were taken to be nonconductors of heat, and so conjugate heat transfer analysis was not required in the present problem. Considering a possible practical scenario, the winglets are likely to be punched out from the fins. Therefore, creation of a winglet can result in a punched hole on the fin and, the winglet being of the same material as that of the fin, will be a conductor. The cumulative effect of these two variations may not be trivial. However, the winglets are assumed to be the nonconductors in this study, and the focus is primarily on the transport enhancement due to the swirling motion.

Figure 6-42a and -b show the instantaneous streamline plots at the mid-plane of the channel for flow past a circular tube and an oval tube (both with the same cross-sectional perimeter) placed in a channel. In Figure 6-42a, the flow past the circular tube, clearly exhibits the phenomenon of vortex shedding, which is not seen in the case of flow past the oval tube (Figure 6-42b). Such a distinguishing behavior 
can be attributed to the streamlined nature of the flow past an oval tube. The streamline plot for the timeaveraged flow at the mid-plane of the channel, shown in Figure 6-42c, is seen to be nearly identical to that of the instantaneous field shown in Figure 6-42b. Thus, the flow past an oval tube gives rise to a steady state solution unlike the time-periodic dynamic response in the case of a circular tube. This lack of vortex shedding lowers the pressure drop for the oval tube case. For various configurations of the vortex generators analyzed here, the analysis is carried out using the time-averaged flow.

$\operatorname{Re}=1000 \operatorname{Pr}=0.7 \quad \mathrm{BR}=0.5213$

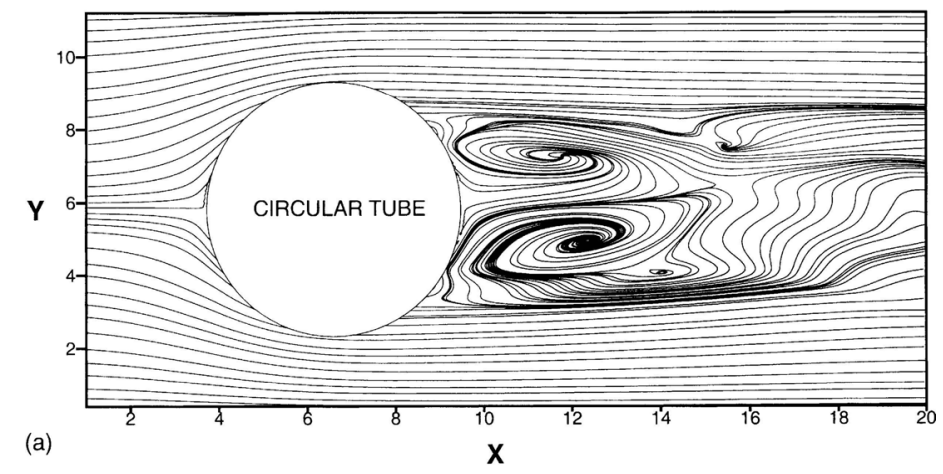

$\mathrm{Re}=1000 \mathrm{Pr}=0.7 \mathrm{BR}=0.260$

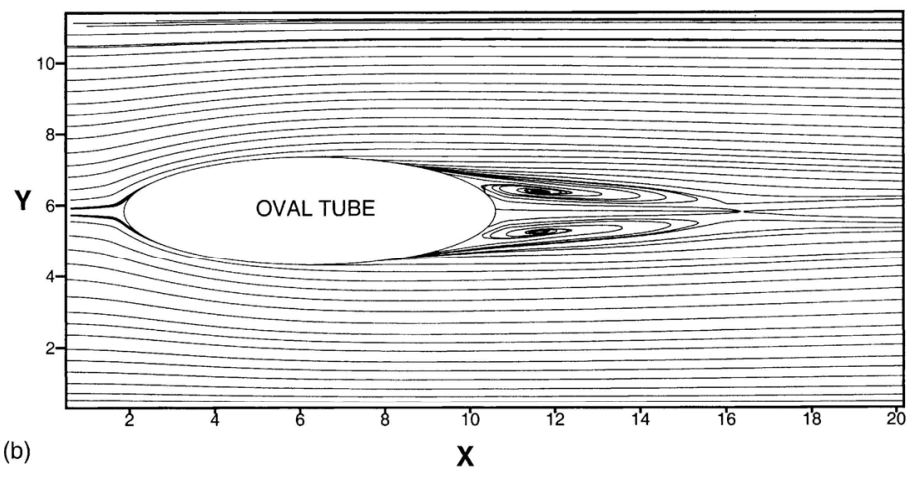

$\mathrm{Re}=1000 \mathrm{Pr}=0.7 \mathrm{BR}=0.260$

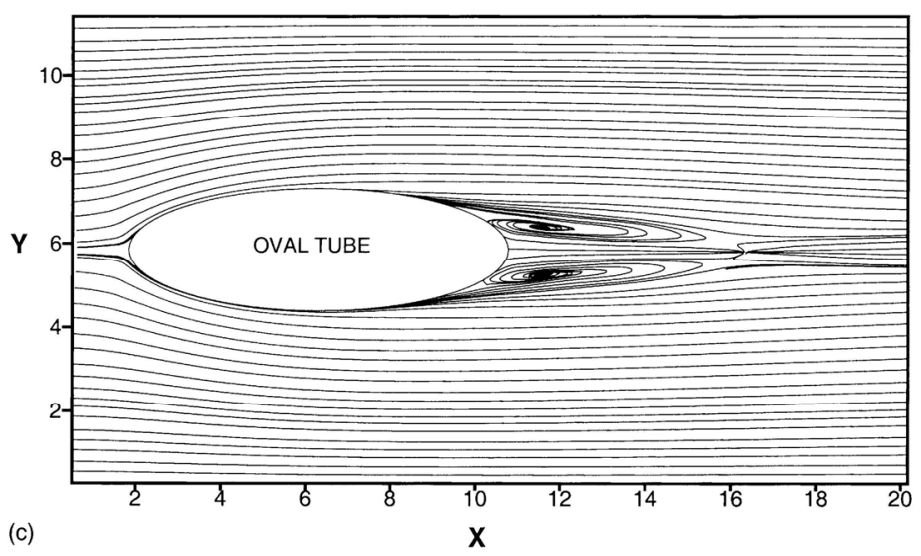

Figure 6-42. (a) Streamline plot of the instantaneous flow field at the mid-plane, showing vortex shedding (circular tube without winglets). (b) Streamline plot of the instantaneous field at the mid-plane (oval tube without winglets and having the same perimeter as the circular tube). (c) Streamline plot of the timeaveraged field at the mid-plane (oval tube without winglets). 
Figure 6-43a shows the limiting streamlines for the time-averaged flow in the region close to the bottom plate for an oval tube. The incoming flow does not separate in the usual manner of circular tubes but reaches a stagnation or saddle point of separation (marked A). The nodal point of attachment or stagnation line is marked B in Figure 6-43a. The separation lines form elliptical arcs along the tube. Point $\mathrm{C}$ indicates where horseshoe vortices graze the channel walls in the time-averaged flow field. These vortices are formed in the region between $\mathrm{B}$ and $\mathrm{C}$ at the top and bottom walls of the channel. Figure 6$43 \mathrm{~b}$ shows the local Nusselt number distribution on the bottom wall of the channel. At the leading edge of the channel, cool incoming fluid comes in contact with the fin surface for the first time, so the local Nusselt number is high everywhere near the leading edge. The gradual decrease in the Nusselt number away from the leading edge is due to the growth of the boundary layer on the channel walls. However, observe the abrupt increase in the Nusselt number in front of the oval tube. This is due to the swirling action of the horseshoe vortices, which brings about a better mixing, due to which the heat transfer in the neighborhood is enhanced significantly. The Nusselt number is again low in the wake region, as shown in the figure. This is due to the separated dead water zone with low recirculating fluid velocity.

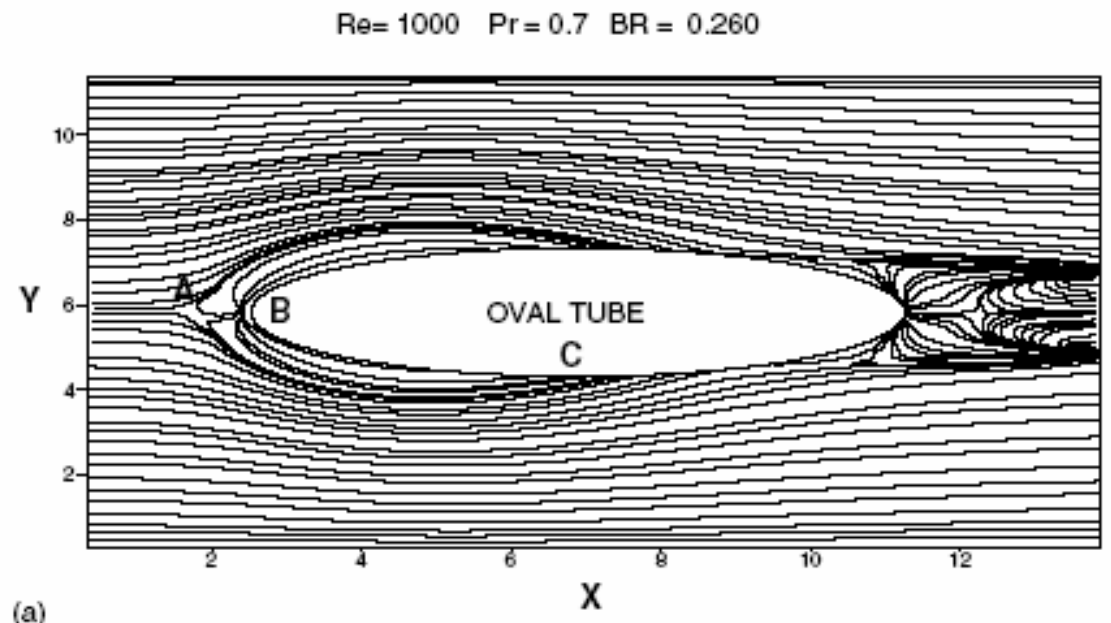

(a)

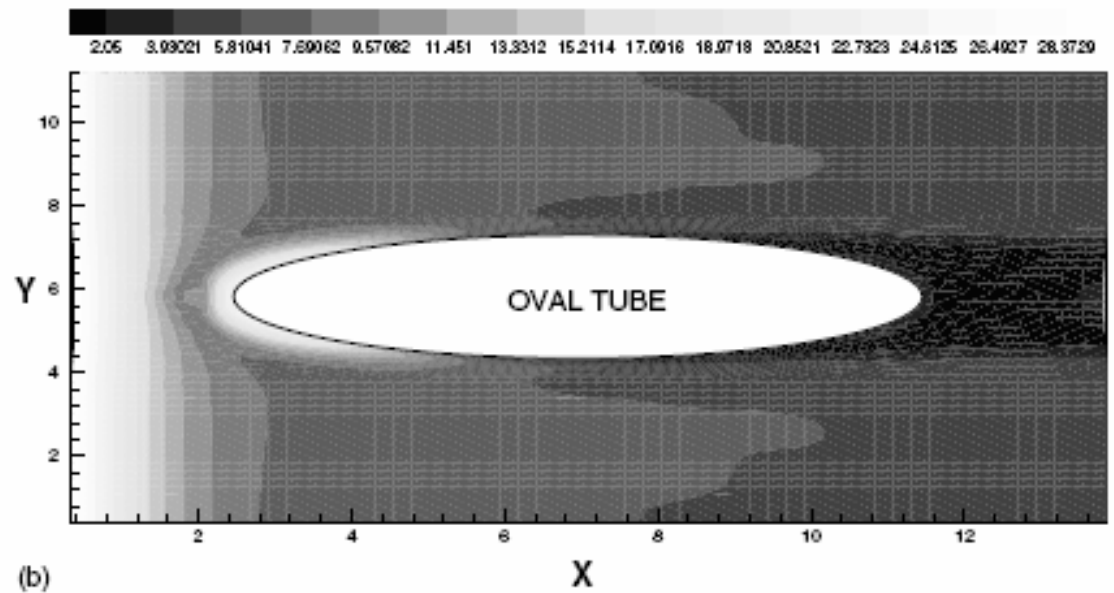

Figure 6-43. (a) Streamline plot of the time-averaged field on the bottom plate (in the case of oval tube without winglets), (b) iso-Nusselt number distribution on the bottom plate (in the case of oval tube without winglets).

Figure 6-44a shows limiting streamlines on a plane close to the bottom plate for the flow through the rectangular channel, with a built-in oval tube and one pair of winglets in toe-out (common-flow-down) configuration (W1 pair, the other pair, W2, in Figure 6-44 is removed). This geometry is for the 
configuration shown in Figure 6-41a. The winglets generate longitudinal vortices downstream. The saddle point of separation and the nodal point of attachment are visible in the figure. The time-averaged wake is symmetrical. The twisted streamlines near the winglets are the footprints of the swirling motion in the $\mathrm{x}-\mathrm{y}$ plane, which has a dominant component in the transverse direction. The transverse momentum transfer to the near field boundary layer of the aft region of the oval tube delays the separation. Figure $6-44 \mathrm{~b}$ shows the distribution of the local Nusselt number on the bottom plate of the channel corresponding to the flow field of Figure 6-44a. At the leading edge of the channel and near the oval tube, the local Nusselt number distribution has the same upward trend as in the earlier case for the reasons explained earlier. The heat transfer enhancement associated with the winglet pair in this case is evident in the figure. A strong influence of the swirling motion exists in the downstream region of each winglet pair, and its effect persists over a nondimensional distance of 8 . The heat transfer enhancement due to the corner (horseshoe) vortex of each winglet appears as a streak in the figure that begins on the upstream side of the winglet and is swept downstream, wrapping the winglet.
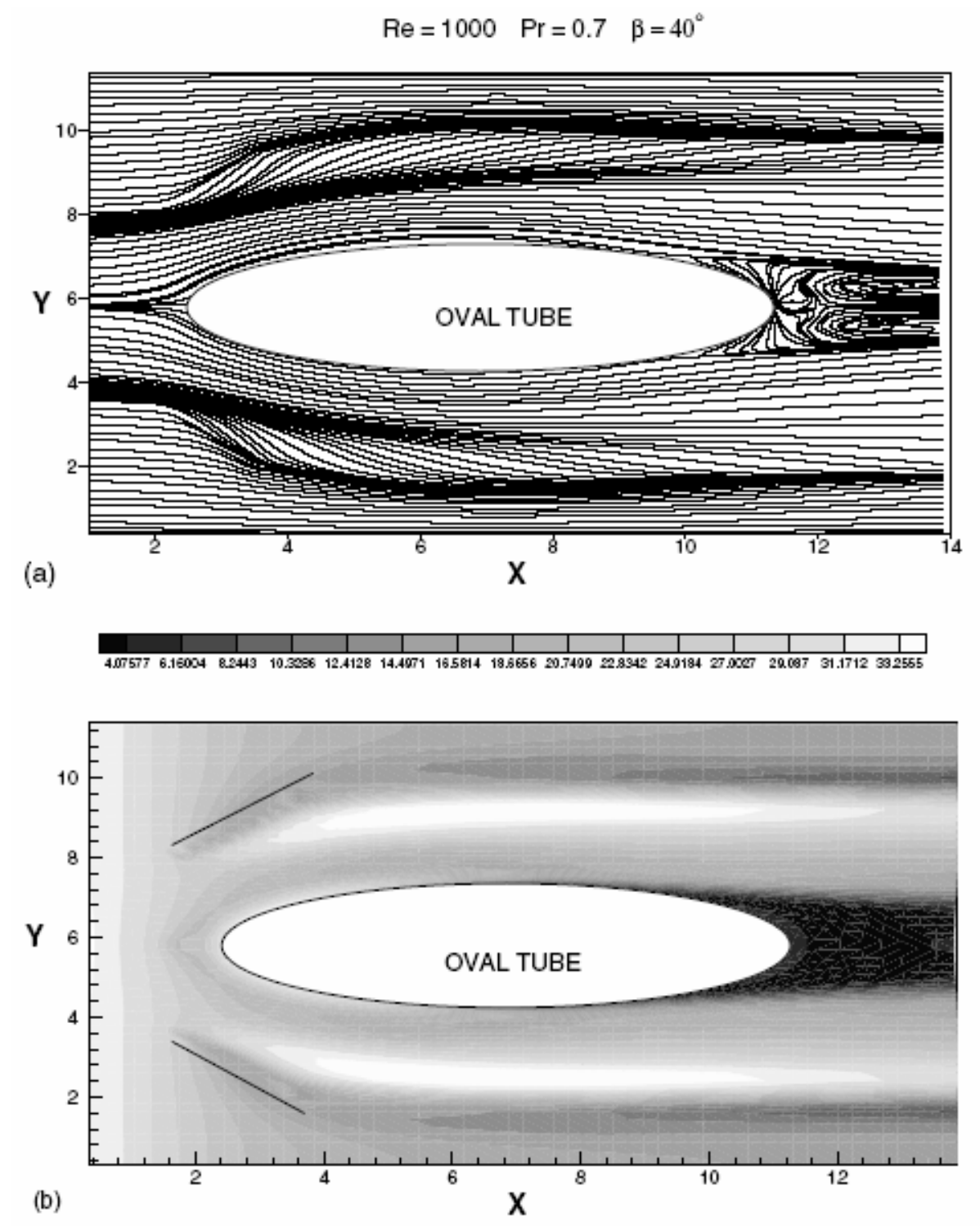

Figure 6-44. (a) Limiting streamlines on the bottom plate for flow past a built-in oval tube with one pair of winglets, (b) Iso-Nusselt number distribution on the bottom plate for flow past a built-in oval tube with one winglet pair. 
Figure 6-45 shows the iso-Nusselt number distribution on the bottom plate of the channel for the above-mentioned configuration (Figure 6-41b) of two winglet pairs. Local regions of high heat transfer associated with the tube stagnation region, the horseshoe vortex system due to the tube, and the main (primary) and corner (horseshoe) vortices produced by each winglet are visible in the figure. In this context, it is worth mentioning that the horseshoe vortices are created due to variation in total pressure along the stagnation line on the pressure surface of the winglet. The smaller velocity in the boundary layer on the flat bottom wall which is attached to the side of the winglet leads to a smaller pressure increase on the stagnation line. Thus the induced pressure gradient on the stagnation line causes a flow towards the bottom wall, which interacts with the mainstream. The fluid rolls up forming vortices looking like a half horseshoe.

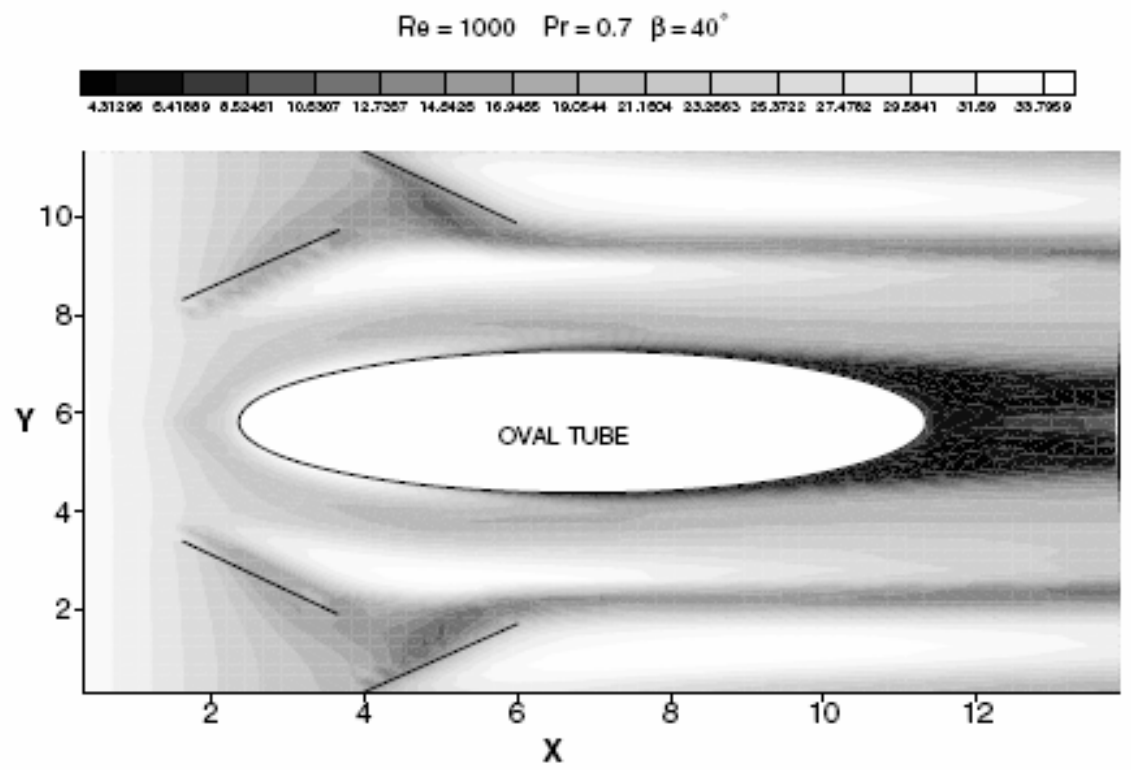

Figure 6-45. Iso-Nusselt number distribution on the bottom plate for flow past a built-in oval tube with two winglet pairs (configuration shown in Figure 6-44b).

Figure 6-46 shows the iso-Nusselt number distribution on the bottom plate of the channel for the parallel configuration of two winglet pairs (both W1 and W2 in the toe-out configuration, as shown in Figure 6-41c). The local regions of high heat transfer associated with the above-mentioned attributes can be seen to be modified when compared to the previous case. For example, the region close to the wake zone and downstream to the winglet pairs shows improved heat transfer behavior in the latter configuration, although the region of enhanced heat transfer is greater in the former case.

Figure 6-47 shows the iso-Nusselt number distribution on the bottom plate of the channel for the parallel configuration of three winglet pairs (all W1, W2, and W3 in toe-out configuration, as shown in Figure 6-41d). The poor heat transfer associated with the wake zone is significantly reduced, and the zone of enhanced heat transfer gets appreciably broadened.

Figure 6-48 shows the iso-Nusselt number distribution on the bottom plate of the channel for the configuration of four winglet pairs mounted simultaneously (W1 and W3 in toe-out configuration; W2 and W4 in toe-in configuration, as shown in Figure 6-41e). Here again, the poor heat transfer associated with the wake zone is significantly reduced, and the zones of enhanced heat transfer get more broadly distributed in the spanwise direction. 


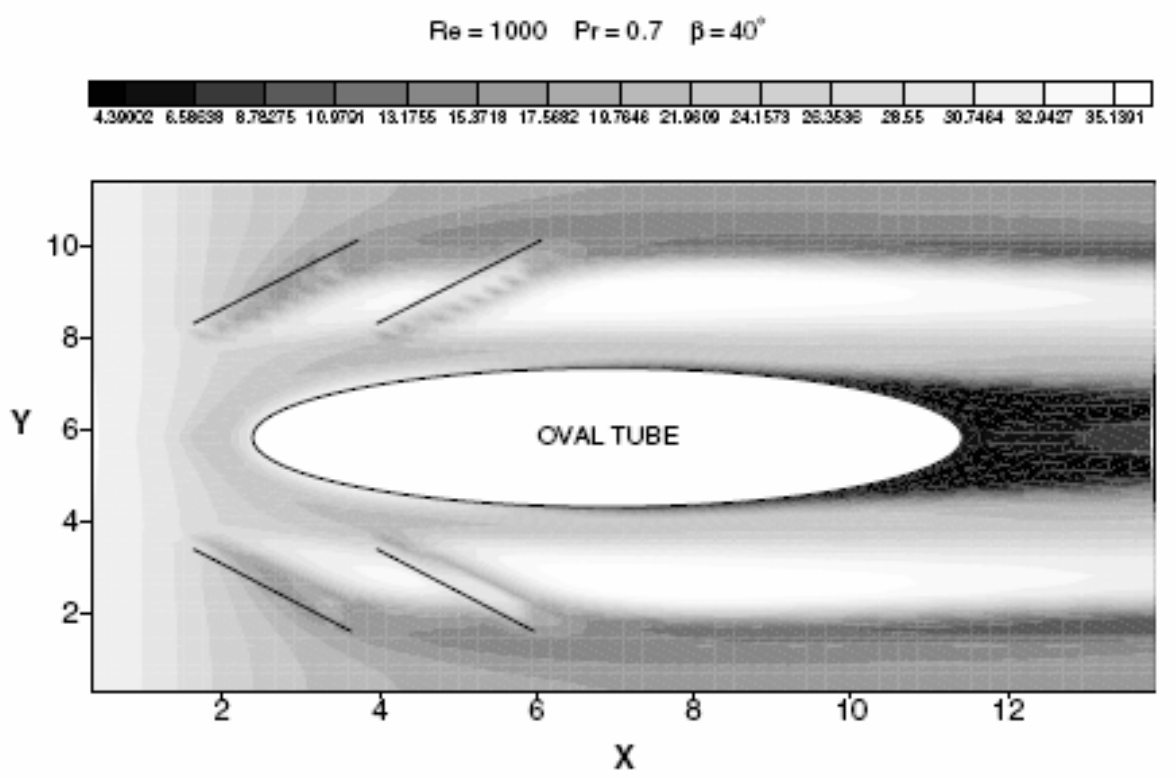

Figure 6-46. Iso-Nusselt number distribution on the bottom plate for flow past a built-in oval tube with two winglet pairs (configuration shown in Figure 6-41c).

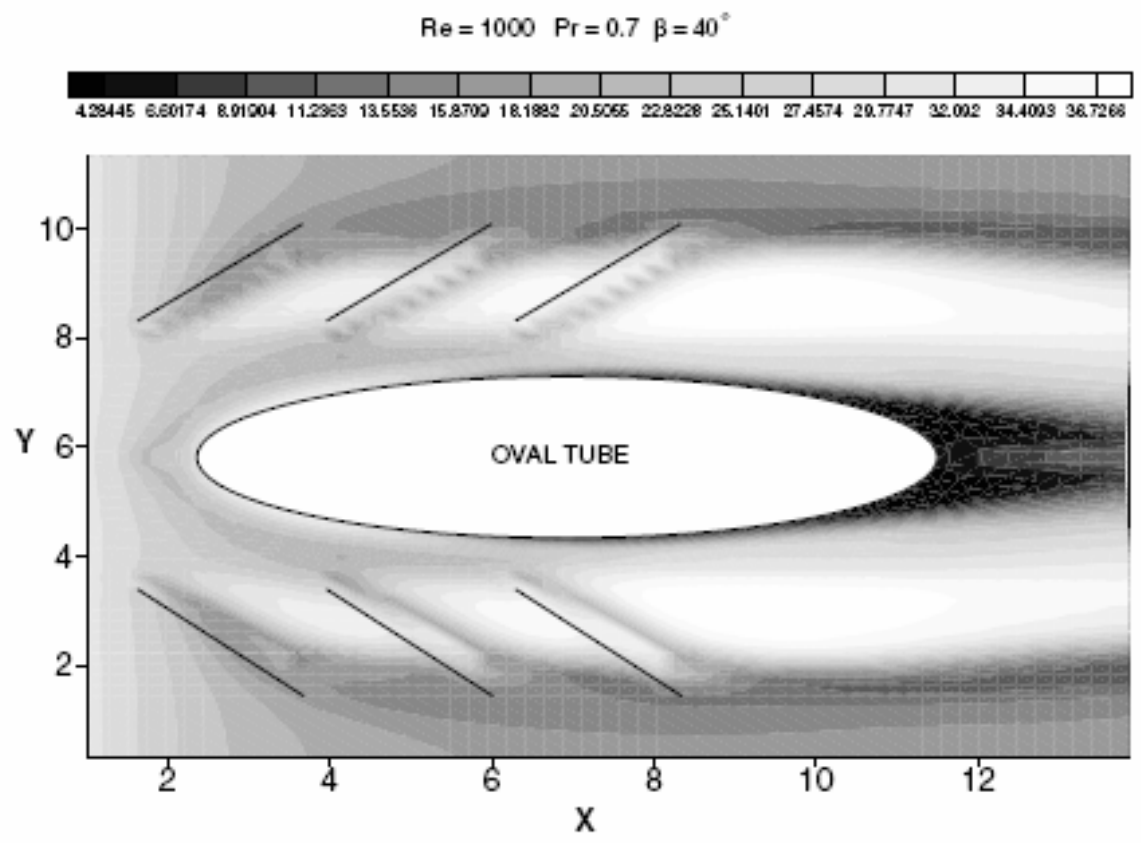

Figure 6-47. Iso-Nusselt number distribution on the bottom plate for flow past a built-in oval tube with three winglet pairs (configuration shown in Figure 6-41d). 


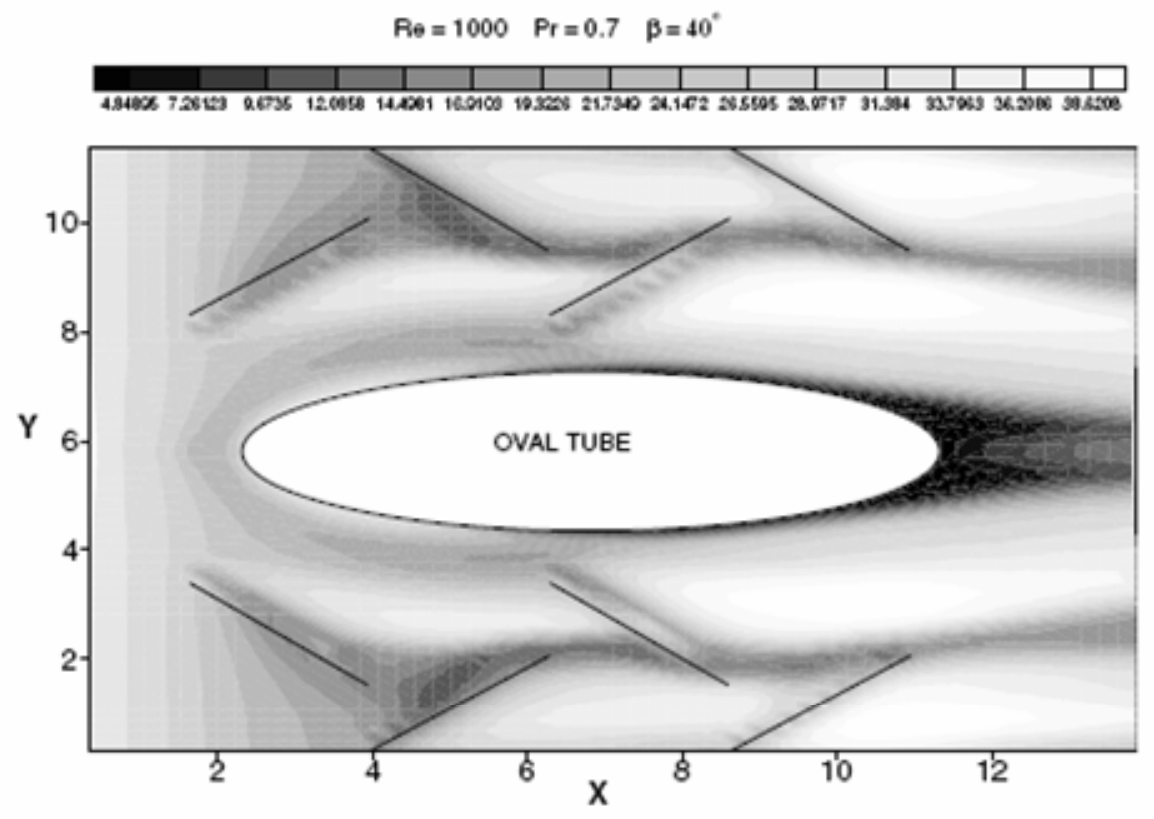

Figure 6-48. Iso-Nusselt number distribution on the bottom plate for flow past a built-in oval tube with four winglet pairs (configuration shown in Figure 6-41e).

Figure 6-49 compares span-averaged Nusselt number distribution on the bottom wall of the channel for the cases of (a) a rectangular channel, (b) channel with built-in oval tube, (c) channel with built-in oval tube and one winglet pair (W1 in Figure 6-41a) in toe-out configuration, and (d) channel with builtin oval tube and two winglet pairs (both W1 and W2 in Figure 6-41b) in toe-out and toe-in configurations, respectively. The locations of the oval tube and winglets are also indicated at the bottom of Figure 6-49. For the case of a plane channel, the span-averaged Nusselt number decreases in the downstream direction due to the growth of the wall boundary layer, and finally becomes almost constant (4.17) at the exit (curve marked a). With a built-in oval tube placed in the plane channel, there is an increase in the span-averaged Nusselt number at the axial location that corresponds to the forward stagnation zone (curve b). This is due to the formation of horseshoe vortices in this zone. The value of the span-averaged Nusselt number at this location is 10.58. Curve $\mathrm{c}$ shows the effect of a winglet pair (W1) in toe-out configuration. At the axial location corresponding to the location of trailing edge of the winglet $(X=3: 61)$, the span-averaged Nusselt number reaches a high value (11.18). The span-averaged Nusselt number is about $81 \%$ higher

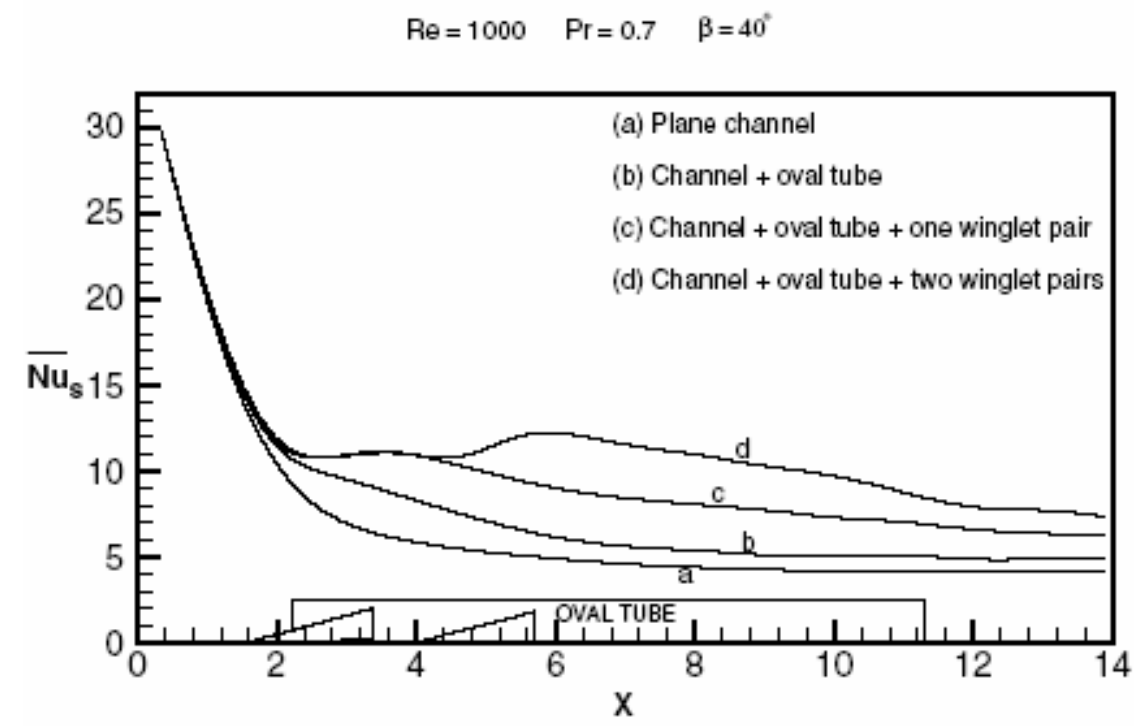

Figure 6-49. Comparison of span-averaged Nusselt number for a plane channel, channel with oval tube, channel with oval tube, and one and two winglet pairs. 
than that of a plane channel at the same axial location. Further improvement is shown when the twowinglet pairs (W1 and W2) are present simultaneously, as indicated by curve d. Here, the maximum value of the span-averaged Nusselt number (12.22) is observed at the axial location corresponding to the location of the trailing edge of the second winglet pair (W2). This is at $X=5: 84$, an enhancement of about $147 \%$ compared to the span-averaged Nusselt number value for the plane channel at the same location. Table 6.5 compares the mean Nusselt number (global mean) for different configurations used in this study. Percentage enhancement for the configuration of oval tube with winglets has been calculated by comparing it with the baseline case of a plane channel. The oval tube is also considered the essential part of the baseline configuration for a fin-tube heat-exchanger. On the basis of the finned oval tube as the baseline case, the enhancement in the last two configurations would be 24.16 and $43.86 \%$, respectively.

Table 6-5. Comparison of mean Nusselt number for different configurations

\begin{tabular}{lcc}
\hline \multicolumn{1}{c}{ Configuration } & $\begin{array}{c}\text { (Global) Mean Nusselt } \\
\text { Number, } \mathrm{Nu}_{\mathrm{m}}\end{array}$ & $\begin{array}{c}\text { Percentage } \mathrm{Nu}_{\mathrm{m}} \\
\text { Enhancement }\end{array}$ \\
\hline Plane channel & 6.67 & - \\
$\begin{array}{l}\text { Channel with built-in oval tube } \\
\text { Channel with built-in oval tube }\end{array}$ & 7.82 & 17.24 \\
$\begin{array}{l}\text { and one winglet pair } \\
\text { Channel with built-in oval tube } \\
\text { and two winglet pairs }\end{array}$ & 9.71 & 45.58 \\
\hline
\end{tabular}

Figure 6-50 compares the span-averaged Nusselt number distribution in the channel with a built-in oval tube and only one winglet pair (with $\beta=40$ degrees) for various positions of a one winglet pair (corresponding to the configuration in Figure 6-41a). The (global) mean Nusselt number, $\mathrm{Nu}_{\mathrm{m}}$, is 9.71 for the location of the leading edge of the winglet pair at $\mathrm{X} / \mathrm{L}=0: 119$. Likewise, $\mathrm{Nu}_{\mathrm{m}}$ is 9.22 for the location of the leading edge of the winglet pair at $\mathrm{X} / \mathrm{L}=0: 186$, and it is 9.23 for $\mathrm{X} / \mathrm{L}=0: 254$. This shows that for better heat transfer the single winglet pair must be optimally mounted on the fin surface near the leading edge of the oval tube. The angle of attack, $\beta$, was varied up to $\beta=40$ degrees, since there exists a possibility of vortex breakdown beyond $\beta=45$ degrees [see reference (69)]. Admittedly, the estimated upper limit of $\beta=40$ degrees is conservative. In the present study, no analysis was performed for $\beta>$ 40 degrees, although in similar applications the Nusselt number starts to decrease when $\beta$ approaches 45 degrees.

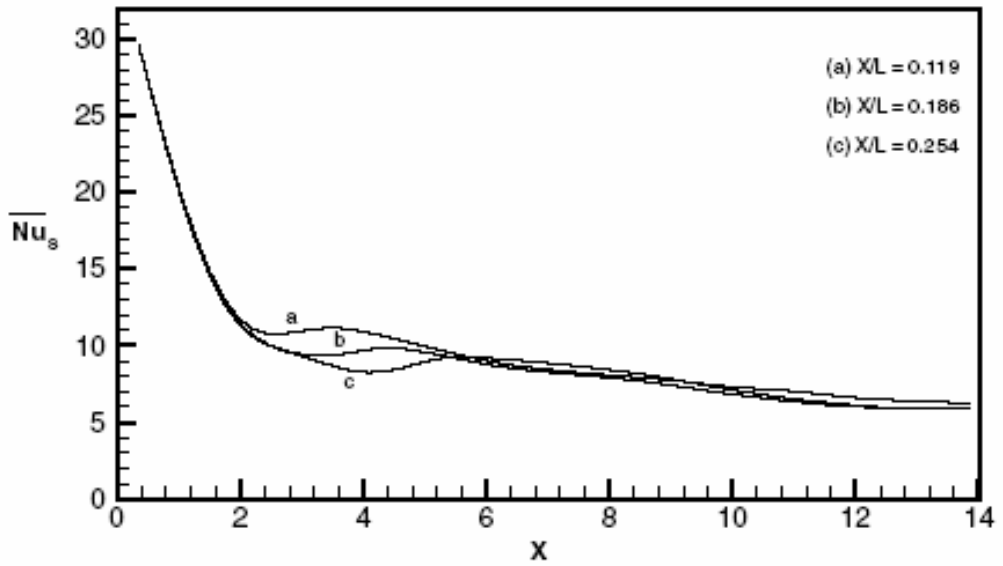

Figure 6-50. Comparison of the span-averaged Nusselt number for different axial locations of one winglet pair (configuration shown in Figure 6-41a). 
Figure 6-51 compares the span-averaged Nusselt number distribution in the channel for the case of two winglet pairs mounted in staggered mode (the configuration shown in Figure 6-41b) for different winglet angles of attack. The curves show a similar qualitative trend, but the winglet configuration with a larger angle of attack shows a higher value of span-averaged Nusselt number over a wide range. This is because winglets with larger angles of attack produce vortices of higher strengths, which result in better heat transfer. There always exists an optimum angle of attack beyond which vortex breakdown may occur. This may destroy the desirable effect of the longitudinal vortices on heat transfer. Table 6-6 summarizes the effect of angle of attack on (global) mean Nusselt number, $\mathrm{Nu}_{\mathrm{m}}$ on the bottom plate of the channel. Table 6-7 shows the variation of average pressure drop in the duct, in the finned oval tube, and in the duct with oval tube and winglets of varying orientation. The average pressure at any section was determined through the ratio of the area integral of pressure at that section to the cross-sectional area. The dimensionless pressure drop in Table 6-7 has been scaled with the Reynolds number entailing the matching points at the entry plane of the channel. On the basis of a plane channel as the baseline case, the additional pressure penalty for the subsequent configurations are $68.61,73.31$, and $82.25 \%$, respectively.

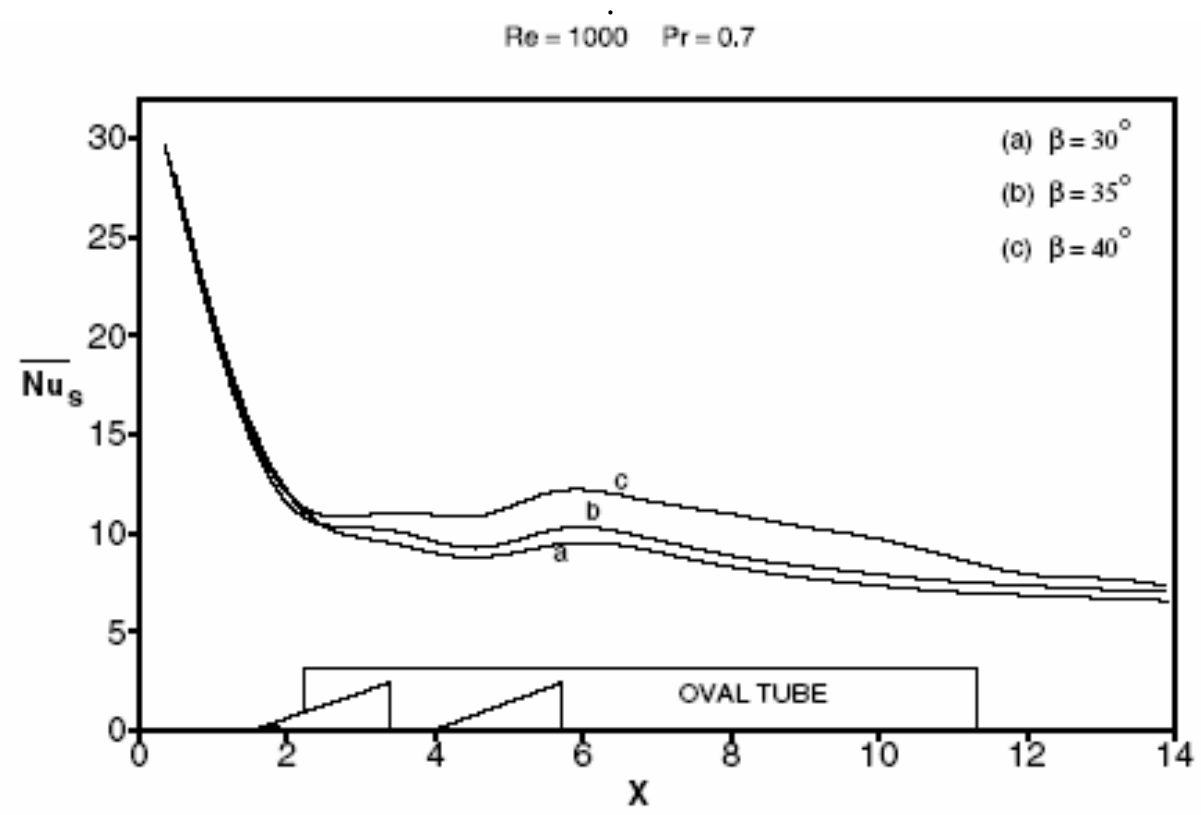

Figure 6-51. Comparison of span-averaged Nusselt number for different angles of attack for flow past a built-in oval tube with two winglet pairs (configuration shown in Figure 6-41b).

Table 6-6. Comparison of mean Nusselt number for different angles of attack.

\begin{tabular}{lcc}
\hline \multicolumn{1}{c}{ Configuration } & $\begin{array}{c}\text { (Global) Mean Nusselt } \\
\text { Number }\left(\mathrm{Nu}_{\mathrm{m}}\right)\end{array}$ & $\begin{array}{c}\text { Percentage } \mathrm{Nu}_{\mathrm{m}} \\
\text { Enhancement }\end{array}$ \\
\hline Without winglet pairs & 7.82 & - \\
$\beta=30$ degrees & 9.37 & 19.82 \\
$\beta=35$ degrees & 10.00 & 27.88 \\
$\beta=40$ degrees & 11.25 & 13.86 \\
\hline
\end{tabular}


Table 6-7. Pressure drop in the duct for various configurations.

\begin{tabular}{lrc}
\hline \multicolumn{1}{c}{ Configuration } & $(\Delta \mathrm{p}) \cdot \mathrm{Re}$ & $\begin{array}{c}\text { Percentage Increase } \\
\text { in }(\Delta \mathrm{p}) \cdot \mathrm{Re}\end{array}$ \\
\hline Plane channel & 870.57 & - \\
Channel with built-in oval tube & 1467.86 & 68.61 \\
$\begin{array}{l}\text { Channel with built-in oval tube } \\
\text { and one winglet pair }\end{array}$ & 1508.23 & 73.31 \\
$\begin{array}{l}\text { Channel with built-in oval tube } \\
\text { and two winglet pairs }\end{array}$ & 1586.60 & 82.25 \\
\hline
\end{tabular}

Figure 6-52 compares the variation of the span-averaged Nusselt number corresponding to the parallel configurations of one, two, and three pairs of winglets (configured as in Figure 6-41a, -c and -d). Here, each extra-added pair of the winglet is in toe-in (common-flow-up) configuration and can be seen to enhance the heat transfer effectively, especially in the dead wake zone. The only constraint of adding an extra pair of winglets to enhance heat transfer appears to be control of the pressure loss penalty. The enhanced heat transfer is almost on the order of $100 \%$ near the dead wake zone in the present case, compared to the flow past an oval tube in the absence of winglet pairs.

Figure 6-53 compares the span-averaged Nusselt number corresponding to the winglet configurations shown in Figure 6-41b and -e. Here, the first case has a staggered arrangement of two pairs of winglets (W1 and W2 in common-flow-down and common-flow-up configurations, respectively) and the second has two such arrangements, one after the other along the length. In both the cases, the varia-tions of the span-averaged Nusselt number from channel inlet until the end location of W2 (Figure 6-41e) is same, but there is a strong increase in the value of $\overline{N u_{s}}$ beyond this location for the second case. There is an increment in heat transfer to nearly $180 \%$ in the dead wake zone, compared to the case of flow past an oval tube in absence of winglet pairs.

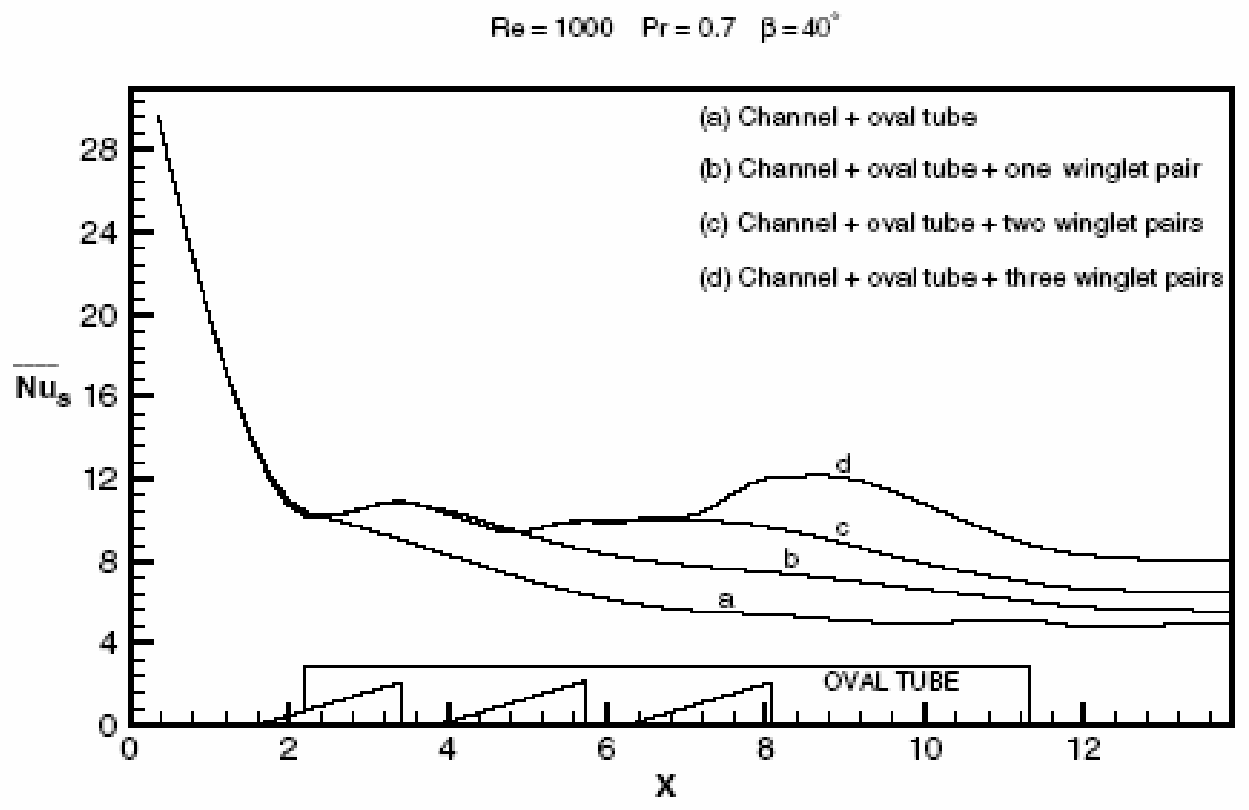

Figure 6-52. Comparison of the span-averaged Nusselt number for flow past a built-in oval tube with one, two, and three winglet pairs (the configurations in Figures 6-41a, -c, and -d, respectively). 


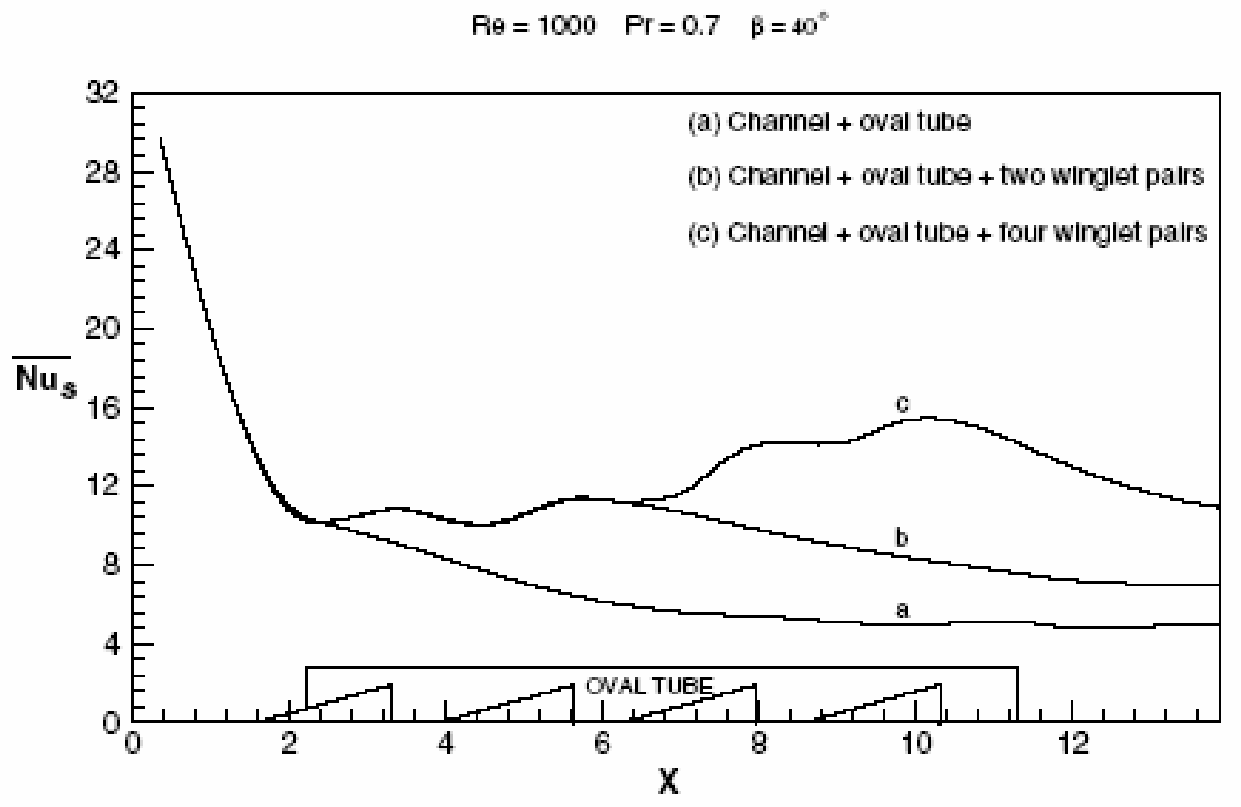

Figure 6-53. Comparison of span-averaged Nusselt number for flow past a built-in oval tube with two and four winglet pairs (the configurations in Figures 6-41b and -e, respectively).

Figure 6-54 shows the variation of span-averaged Nusselt number in the flow direction for three different Reynolds numbers, $R e=500,1000$, and 1500. with one pair of winglet mounted in the toe-out (common-flow-down) configuration. An increase in $\overline{N u_{s}}$ is observed all along the channel length with increasing values of the Reynolds number. This may be expected, owing to the following reason. At higher values of the Reynolds number, the thermal boundary layer thickness decreases, and the degree of fluid mixing increases. As a consequence, a global enhancement in heat transfer is observed with increasing values of the Reynolds number.

$\operatorname{Pr}=0.7 \quad \beta=40^{\circ}$

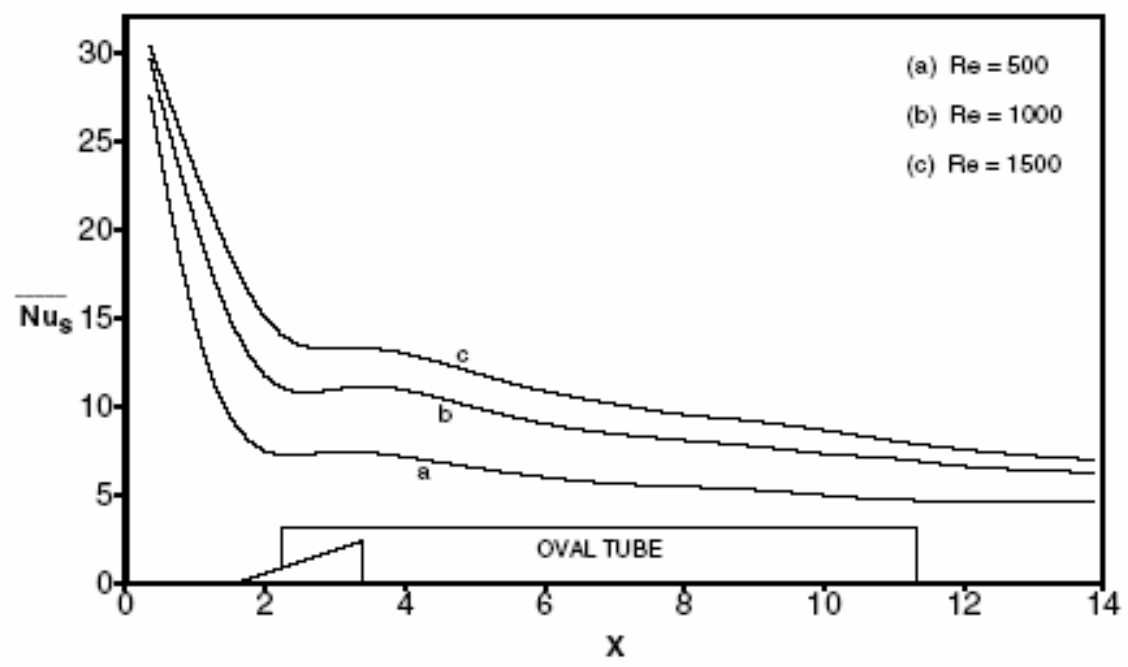

Figure 6-54. Comparison of span-averaged Nusselt number for different Reynolds numbers for flow past a built-in oval tube with one winglet pair (configuration shown in Figure 6-41a). 
Figure 6-55 compares the span-averaged Nusselt number distribution on the bottom wall of the channel for three different grids for the flow through a channel with built-in oval tube and one winglet pair in toe-out (common-flow-down) configuration (Figure 6-41a). Three different grid-meshes, namely $62 \times 61 \times 19,72 \times 71 \times 21$, and $82 \times 81 \times 25$ were used to test the grid sensitivity. The results reveal minor changes (less than $4 \%$ ) in span-averaged value of the Nusselt number, at any axial location in the channel for the three different grids.

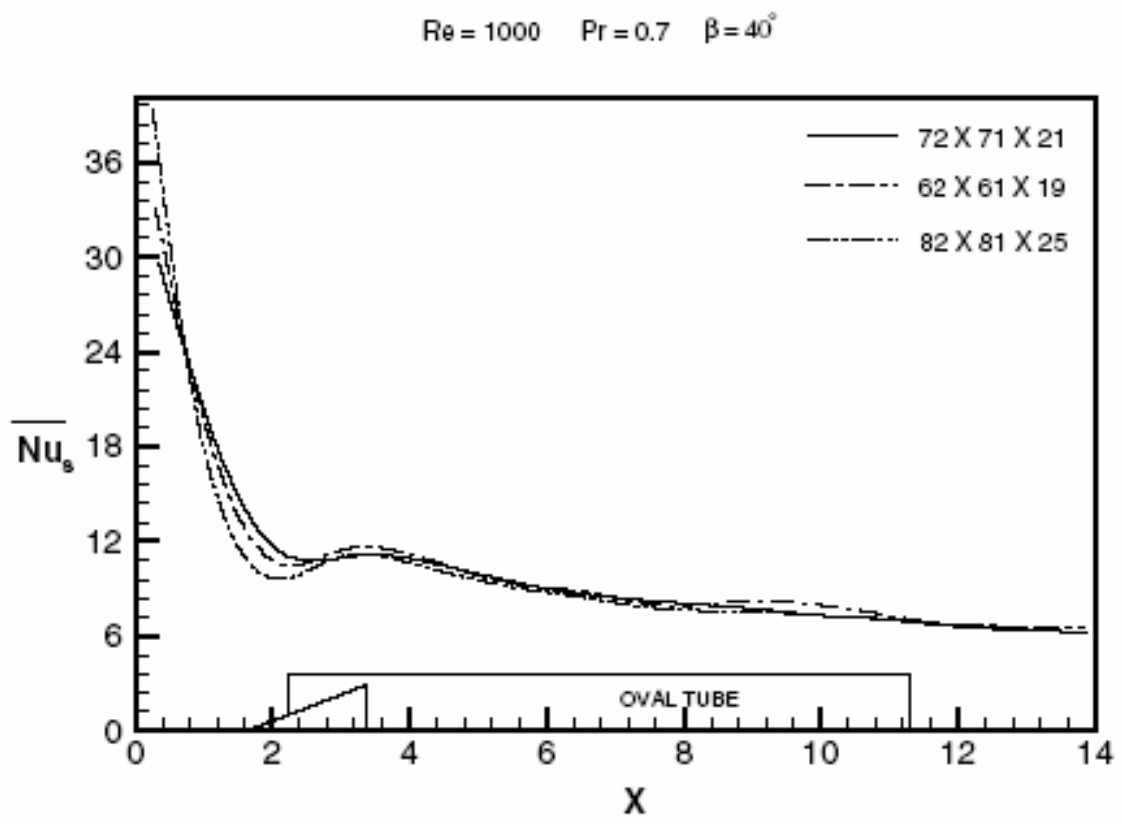

Figure 6-55. Comparison of the span-averaged Nusselt number for three different grid sizes (configuration shown in Figure 6-41a).

\subsubsection{Conclusions}

A three-dimensional computational study of forced convection heat transfer has been completed to determine the flow structure and heat transfer in a rectangular channel with a built-in oval tube and deltawinglet type vortex generators in various configurations. The duct was designed to simulate a passage formed by two neighboring fins in a fin-tube heat exchanger. The present study reveals that combinations of oval tube and the winglet pairs improve the heat transfer significantly, especially in the dead wake region. The mean span-averaged Nusselt number for the case of four winglet pairs, each two in sequence having a staggered configuration (inner pair in toe-out or common-flow-down and outer pair in toe-in or common-flow-up arrangement) is about $100 \%$ higher compared to the no-winglet case at a Reynolds number of 1000. The enhancement in heat transfer, on the basis of finned oval tube as the base line case, is $43.86 \%$ for the case of two winglet pairs in staggered mode. Comparison of heat transfer for the cases of one, two, and three winglet pairs (all in toe-out or common-flow-down configuration) confirms that the addition of each extra winglet pair causes further enhancement of heat transfer. The enhancement of heat transfer is marked even at far downstream locations. The winglets, at their moderate angle of attack, have quite streamlined like behavior and so are not expected to contribute much toward pressure losses. On the other hand, the contribution toward enhancement in heat transfer due to the winglet pairs is undoubtedly significant. 


\section{INDEPENDENT TESTING OF TUBE BUNDLES BY INTERTEK TESTING SERVICES}

After completing laboratory-scale experiments at INL, a small tube bundle with an enhanced finned tube was designed and then tested independently by Intertek Testing Services, Philadelphia to determine the performance of enhancement devices. The enhancement configuration selected for testing considered the following primary objectives, the results of the laboratory-scale experiments and fabrication limitations. (These objectives incorporated comments of manufactures of the air-cooled condenser.)

- The enhancement design should be kept as close as possible to the existing industrial practice.

- The enhancement device should be favorably accepted by the industrial users.

- $\quad$ The manufacturing cost of the enhanced finned tubes should be kept the same or have minimum increase so as not to offset or negate any performance gain of the enhancement device.

- Introduction of any enhancement device should result in no or minimum pressure drop increase compared to the existing condenser tubes.

- Keeping the enhancement device clean and retain its cleaning ability as it is exposed to dirty environmental conditions.

The numerical analysis by INL, Indian Institute of Technology, Kanpur India, and experimental investigations by Yokohama National University, Yokohama, Japan were performed independently with funding provided by NEDO, Japan. Results of this on-going work were incorporated into the selection of winglet configurations for INL's laboratory testing. However, the complete results of this independent work were not available at the time of selection of the configuration for testing at Intertek.

Considering these objectives, it was decided that oval tubes would not be considered for this stage of the testing, though the NEDO-funded numerical work suggested that the oval tubes might perform better. Once this decision was made, INL pursued a collaborative effort with McElroy Manufacturing Company, Tulsa, Oklahoma to fabricate finned circular tubes having the winglet enhancement. At the time INL was ready to select a configuration for testing at Interteck, McElroy had only limited success in incorporating the winglet onto the fin surfaces during tube fabrication. With limited resources available to INL, one of the configurations already tested by INL in laboratory-scale experiments toe-in (Concept 2, common flow up), was selected for fabricating the test tube bundle. The toe-in (Concept 2, common flow up) design of the winglet is shown in Figure 7-1. This configuration was incorporated on the fin with an OD diameter of 2.25, which is mounted on 1-in. OD tube.

\subsection{Test Tube Bundle Fabrication and Test Conditions}

Figure 7-2 is a schematic drawing of a 6 (rows) $\times 3$ (columns) staggered tube bundle with a total of 17 tubes. Each outer column has six tubes; the middle column has only five tubes. However, the tube bundle was fabricated with each plenum being of $6 \times 6$-in. square cross-section instead of a 4 -in.-diameter circular pipe, as shown in Figure 7-2. Also, the 3.00-in. gap between the plenum and the last (or first) fin was reduced to a minimum of $\sim 0.1$ in. to minimize errors during air flow over the finned heat transfer area. Super Radiator Coils (SRC), Richmond, Virginia punched out individual circular fins. On these fins, INL punched out the toe-in (common flow up) winglets, as shown in Figure 7-1. SRC mounted and aligned these fins with winglets on the tubes, and the remainder of the test apparatus was also fabricated by SRC. A photograph of the finished test apparatus is shown in Figure 7-3. SRC pressurized and stretched the tubes to improve the thermal contact between the fins and the tube. A close-up of the finned tube is shown in Figure 7-4. In order to calculate relative improvement in performance, the SRC also fabricated an identical baseline finned tube bundle, but without the winglets. 


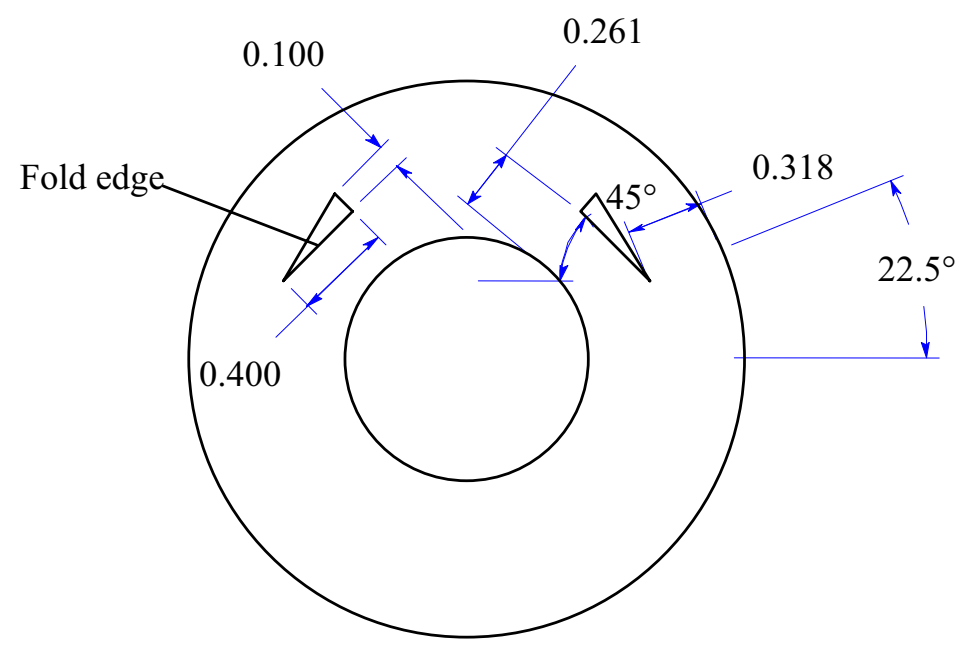

Figure 7-1. Schematic of Concept 2, toe-in (common flow up) winglet design (all dimensions are in inches).

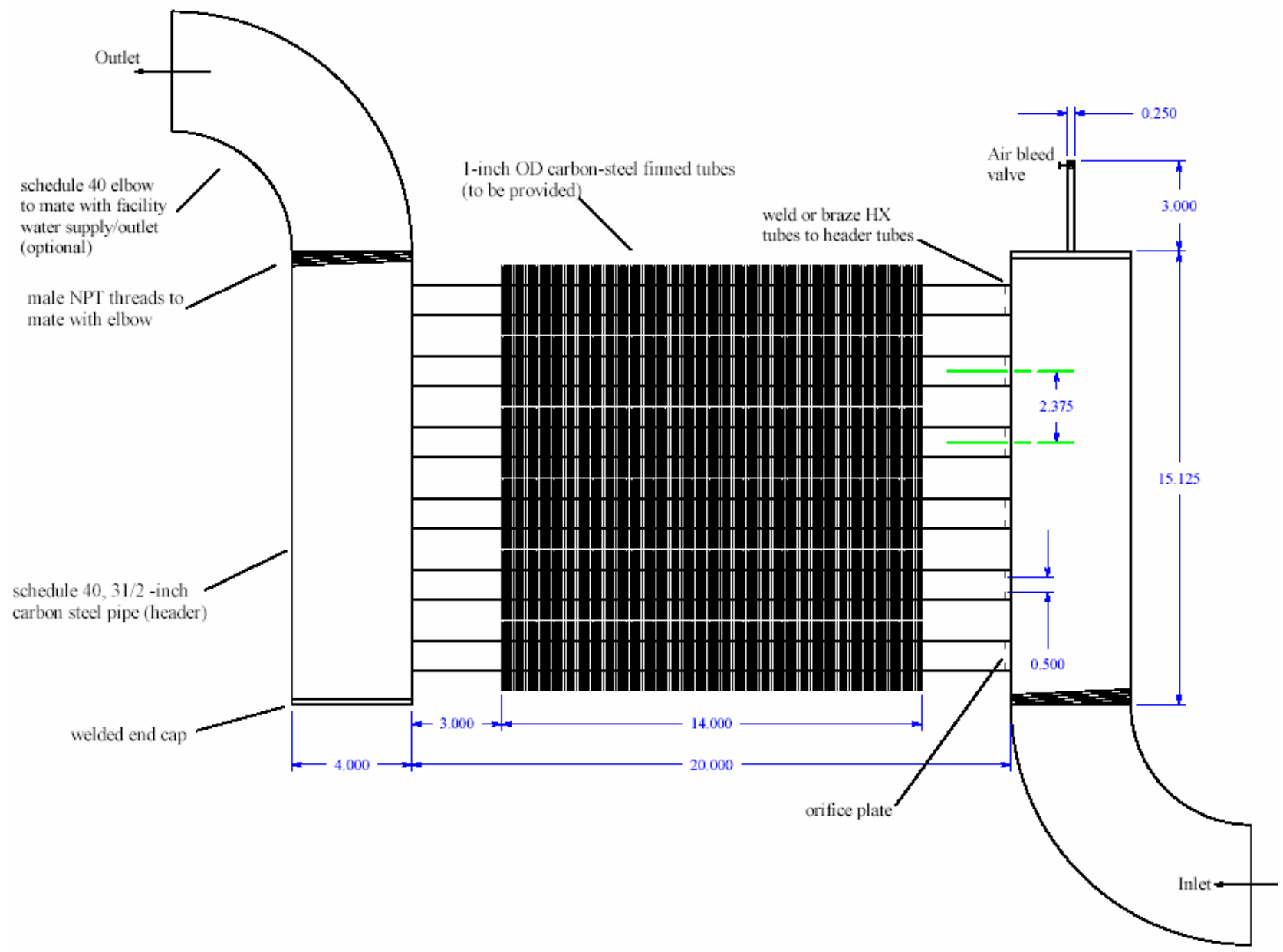

Figure 7-2. A (6 rows $\times 3$ column) staggered tube bundle. (All dimensions are in inches.) 


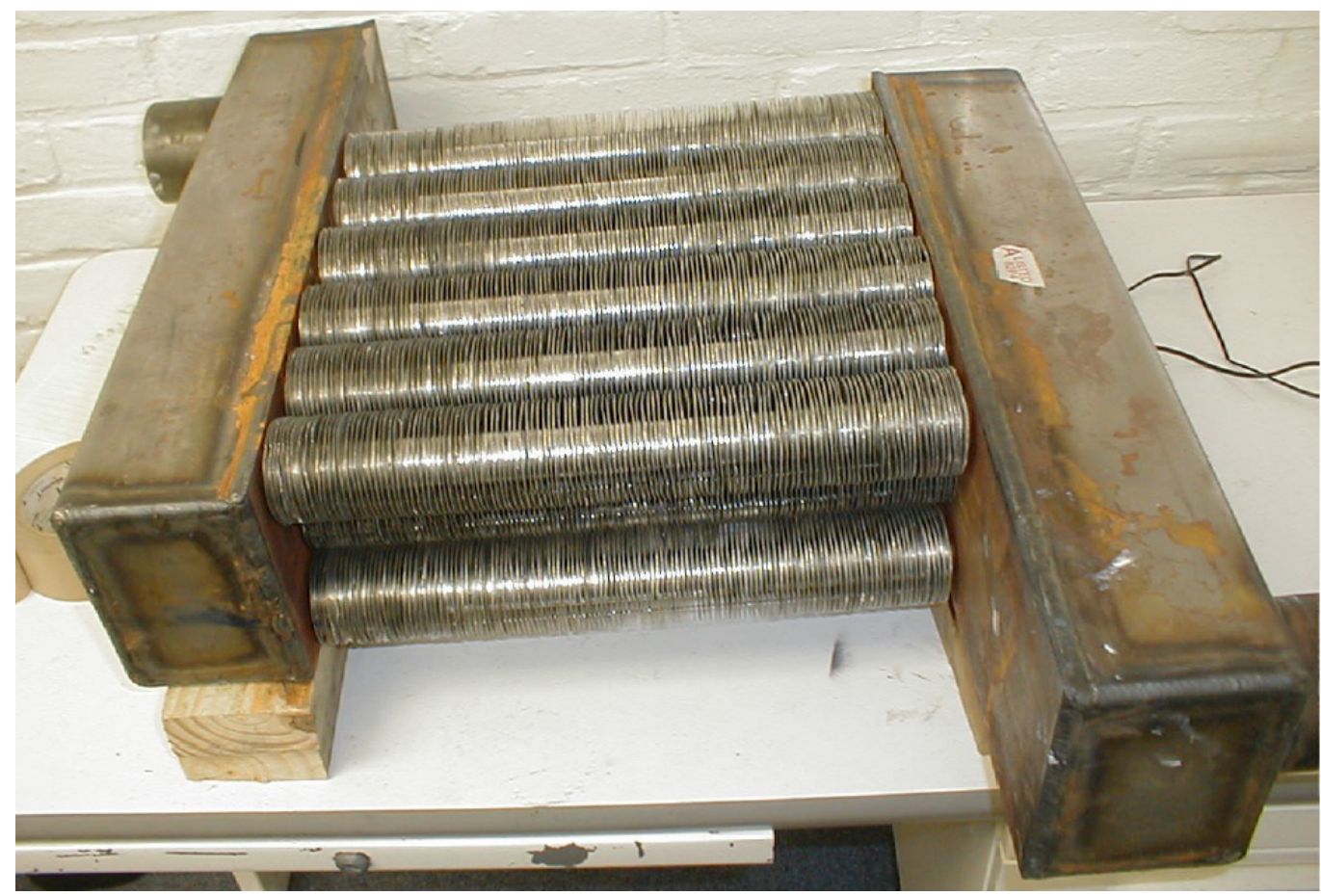

Figure 7-3. Photograph of the test apparatus with enhanced finned tubes and inlet and outlet plena.

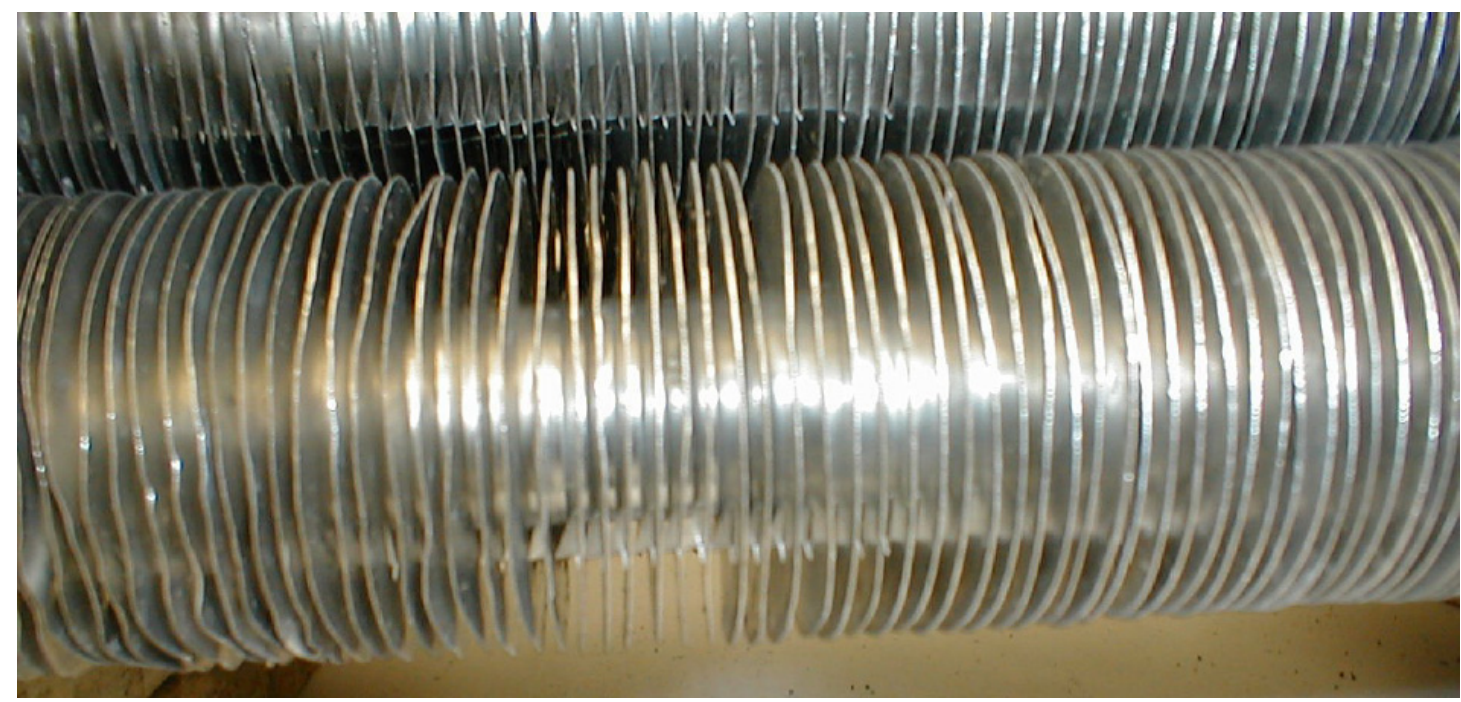

Figure 7-4. Close-up of the finned tubes with toe-in (common flow up) winglets.

The INL/Super Radiator Coils tube bundles were tested at the flow and temperature conditions shown in Table 7-1. The data obtained are shown in Table 7-2. During the test, it was decided not to take any data at $800 \mathrm{ft} / \mathrm{min}$ face air velocity because the other three sets of data gave enough desired information about the performance of the tube bundle. 
Table 7-1. Experimental test matrix.

\begin{tabular}{ccccc}
\hline $\begin{array}{c}\text { Face Velocity } \\
\mathrm{ft} / \mathrm{min}(\mathrm{m} / \mathrm{s})\end{array}$ & $\begin{array}{c}\text { Air Inlet Temperature } \\
{ }^{\circ} \mathrm{F}\left({ }^{\circ} \mathrm{C}\right)\end{array}$ & $\begin{array}{c}\text { Water Flow Rate } \\
\mathrm{gal} / \mathrm{min}(\mathrm{kg} / \mathrm{s})\end{array}$ & $\begin{array}{c}\text { Water Inlet Temperature } \\
{ }^{\circ} \mathrm{F}\left({ }^{\circ} \mathrm{C}\right)\end{array}$ \\
\hline $400(2.032)$ & $60(15.56)$ & $7(0.42847)$ & $180(82.22)$ \\
$600(3.048)$ & $60(15.56)$ & $7(0.42847)$ & $180(82.22)$ \\
$800(4.064)$ & $60(15.56)$ & $7(0.42847)$ & $180(82.22)$ \\
$1,000(5.08)$ & $60(15.56)$ & $7(0.42847)$ & $180(82.22)$ \\
\hline
\end{tabular}

\subsection{Test Results and Discussion}

In September 2004, Intertek Testing Services, Philadelphia performed the test per the conditions given in Table 7-1. The data as obtained for a baseline and the INL-enhanced bundles under nearly identical conditions are shown in Table 7-2. The last two rows give calculated heat transfer enhancement and pressure drop increase, respectively.

Table 7-2. Test data for a baseline and the INL enhanced finned tube bundles.

\begin{tabular}{|c|c|c|c|c|c|c|}
\hline & \multicolumn{6}{|c|}{ Tube Bundle Face Air Velocity (ft/min.) } \\
\hline & \multicolumn{2}{|c|}{400} & \multicolumn{2}{|c|}{600} & \multicolumn{2}{|c|}{1000} \\
\hline & Baseline & INL & Baseline & INL & Baseline & INL \\
\hline Barometer (in. of water) & 30.26 & 30.20 & 30.26 & 30.18 & 30.34 & 30.17 \\
\hline Entering dry bulb $\left({ }^{\circ} \mathrm{F}\right)$ & 59.70 & 60.00 & 60.12 & 59.98 & 60.13 & 60.02 \\
\hline Entering wet bulb $\left({ }^{\circ} \mathrm{F}\right)$ & 55.63 & 55.89 & 57.79 & 57.49 & 58.02 & 57.58 \\
\hline Leaving dry bulb $\left({ }^{\circ} \mathrm{F}\right)$ & 114.14 & 114.19 & 102.49 & 102.20 & 91.01 & 90.42 \\
\hline $\begin{array}{l}\text { Air pressure drop across the bundle } \\
\text { (in. of water) }\end{array}$ & 0.16 & 0.17 & 0.32 & 0.33 & 0.71 & 0.73 \\
\hline Entering water temp $\left({ }^{\circ} \mathrm{F}\right)$ & 180.02 & 179.96 & 180.00 & 179.99 & 180.04 & 179.99 \\
\hline Leaving water temp $\left({ }^{\circ} \mathrm{F}\right)$ & 174.93 & 174.92 & 173.98 & 173.98 & 172.88 & 172.84 \\
\hline Water temp. drop $\left({ }^{\circ} \mathrm{F}\right)$ & 5.08 & 5.04 & 6.02 & 6.02 & 7.16 & 7.14 \\
\hline Water flow rate (gal./min.) & 14.01 & 14.09 & 14.02 & 13.99 & 13.99 & 13.96 \\
\hline Adjusted heat lost by water (BTU/hr) & 34,672 & 34,566 & 41,065 & 40,964 & 48,780 & 48,524 \\
\hline Heat gained by air (BTU/hr) & 33,589 & 33,478 & 39,449 & 39,280 & 47,897 & 46,883 \\
\hline Average heat transfer rate (BTU/hr) & 33,860 & 34,022 & 39,853 & 40,122 & 48,118 & 47,704 \\
\hline Heat transfer imbalance & $-3.17 \%$ & $-3.20 \%$ & $-4.02 \%$ & $-4.20 \%$ & $-1.83 \%$ & $-3.44 \%$ \\
\hline Actual airflow ( $\left.\mathrm{ft}^{3} / \mathrm{min}.\right)$ & 606 & 608 & 900 & 901 & 1,468 & 1468 \\
\hline Airflow (standard $\mathrm{ft}^{3} / \mathrm{min}$.) & 567 & 567 & 855 & 854 & 1,424 & 1416.02 \\
\hline \multicolumn{7}{|l|}{ Standard air velocity } \\
\hline$($ Standard $\mathrm{ft} / \mathrm{min})$ & 405 & 405 & 610 & 610 & 1017 & 1011 \\
\hline Log mean temp. diff., LMTD $\left({ }^{\circ} \mathrm{F}\right)$ & 88.268 & 88.071 & 94.523 & 98.073 & 100.424 & 100.748 \\
\hline $\begin{array}{l}\text { Overall heat transfer coeff., } \mathrm{U} \\
\left(\mathrm{BTU} / \mathrm{hr} \cdot \mathrm{ft}^{2} \cdot{ }^{\circ} \mathrm{F}\right)\end{array}$ & 3.8236 & 3.8505 & 4.2025 & 4.2164 & 4.7759 & 4.7196 \\
\hline Enhancement, based on calculated U & & $0.7 \%$ & & $0.33 \%$ & & $-1.18 \%$ \\
\hline $\begin{array}{l}\text { Enhancement, based on heat transfer } \\
\text { rate data }\end{array}$ & & $0.48 \%$ & & $0.675 \%$ & & $-0.86 \%$ \\
\hline Pressure drop increase & & $6.3 \%$ & & $3.1 \%$ & & $2.8 \%$ \\
\hline
\end{tabular}


Examining the test data, especially the heat transfer enhancement, it is quite obvious that the results did not come out as had been expected per single blow test results, as shown in Section 3.5. Corresponding to the three face velocities of 400,600 , and $1000 \mathrm{ft} / \mathrm{min}$, the heat transfer enhancement by INL winglet finned tubes is $0.48,0.675$, and $-0.86 \%$ (based on the mean of heat transfer rates for air cooling and water heating), and the enhancement of the overall heat transfer coefficient is $0.7,0.33$, and $-1.18 \%$, respectively. The corresponding pressure drop increase for the three air flow rates is $6.3,3.1$, and $2.8 \%$, respectively.

During tests by the INL Single Blow Test Facility, the toe-in (Concept 2) configuration gave a Colburn j-factor enhancement of $\sim 33 \%$ and a friction factor increase of $\sim 25 \%$ at $\operatorname{Re}_{\mathrm{Dh}}=1000$. Thus, on the surface, both $\mathrm{j}$-factor enhancement and friction factor increase are not what might have been expected. For this reason, it became pertinent to re-examine both Single Flow Test Facility and ITS data to explain the discrepancy. Section 7.4 summaries this limited review, as well as other speculated reasons for the unexpected performance. However, because the decision was made to end the project after the Interteck testing, investigators are unable to provide a definitive explanation for the discrepancy between the Intertek results and expectations based on prior INL and other work.

The recorded data at ITS do not have any parameters that can help to determine the cause of the observed unexpected heat transfer performance of the toe-in (Concept 2) configuration design. Such information could be flow behavior between the fins, heat transfer thermal image, or any other such obvious data. However, no such data are available, and given the decision to end the project, additional research could not be done to provide such data. Therefore, merely speculative reasons coupled with engineering logic can be given here.

\subsection{Review of the INL Single Blow Test Facility Data}

During the tests performed at the INL Single Blow Test Facility (SBTF), the tubes in the bundles tested had differences in the number of fins per unit length (fin pitch). The baseline and toe-out (Concept 1) finned tubes had 11.2 fins per inch, whereas the toe-in (Concept 2) finned tube had 9 fins per inch. The following analysis makes an attempt to account for this discrepancy.

Sectin 3.3 definies Colburn j-factor, friction factor, Reynolds number, Re, and other related data reduction analysis. The hydraulic diameter, $\mathrm{D}_{\mathrm{h}}$, is $=4 \mathrm{LA}_{\min } / \mathrm{A}$, where $\mathrm{A}_{\min }$ is the minimum flow crosssectional area (accounting for tube diameter obstruction), and $\mathrm{A}$ is the total heat transfer surface area (top and bottom fin surfaces, fin edges, and bare tube surface). Values for $L, A_{\min }, A$, and $D_{h}$, defined on a unit-cell basis, are listed in Table 7-3 (used in calculations in Section 3.3) for the three fin-tube configurations considered by INL. Note that the values listed in Table 7-3 and the analysis presented here are for the tube and a pair of fins (not the full-size tube bundle). Also, note that the $\mathrm{D}_{\mathrm{h}}$ ratio is about equivalent to the fin pitch ratio (11.2/9).

Table 7-3. Unit-cell dimensions and hydraulic diameter for the three INL test bundles in SBTF.

\begin{tabular}{lcccc}
\hline & $\mathrm{L}(\mathrm{cm})$ & $\mathrm{A}_{\min }\left(\mathrm{cm}^{2}\right)$ & $\mathrm{A}\left(\mathrm{cm}^{2}\right)$ & $\mathrm{D}_{\mathrm{h}}(\mathrm{cm})$ \\
\hline Baseline - 0 & 5.5 & 0.8089 & 43.20 & 0.4119 \\
Toe-out (Concept 1) & 5.5 & 0.8089 & 43.20 & 0.4119 \\
Toe-in (Concept 2) & 5.5 & 0.9942 & 43.59 & 0.5017 \\
\hline
\end{tabular}

The initial emphasis in reviewing the data from the SBTF testing was to try to account for unexpected pressure drop results from the Intertek testing. During the SBTF testing, at equivalent face velocities, the pressure drop for the toe-in (Concept 2 ) configuration was slightly less $(\sim 10-20 \%)$ than that for the baseline configuration. Investigators compensated for the effect of the different fin pitch using an 
approach that is summarized in the following discussion of the pressure drop in the flow channel formed by two adjacent fins.

The friction factor can be specified as follows:

$$
f=\frac{\Delta P \rho}{\frac{\left(G D_{h}\right)^{2}}{2}\left(\frac{4 L}{D_{h}^{3}}\right)}
$$

The friction factor for Concept $2, f_{2}$, can be shown to be related to the friction factor for the baseline case, $f_{o}$, by:

$$
\left[\frac{f_{2}}{f_{o}}\right]=\left[\frac{\Delta P_{2}}{\Delta P_{o}}\right]\left(\frac{\left(G D_{h}\right)_{o}}{\left(G D_{h}\right)_{2}}\right)^{2}\left(\frac{D_{h 2}}{D_{h o}}\right)^{3}=\left[\frac{\Delta P_{2}}{\Delta P_{o}}\right]\left(\frac{\left(G D_{h}\right)_{o}}{\left(G D_{h}\right)_{2}}\right)^{2}\left(\frac{n_{o}}{n_{2}}\right)^{3}
$$

where $\mathrm{n}_{\mathrm{o}}$ and $\mathrm{n}_{2}$ are the fin pitch (number of fins/in.) values for the baseline and toe-in (Concept 2) cases, respectively. It can be shown that the hydraulic diameter ratio is about equivalent to the fin pitch ratio if the distance between fins is small relative to the distance between tubes, as well as large relative to the fin thickness. It is also assumed that the heat transfer surface areas $\left(\mathrm{A}_{\mathrm{o}}\right.$ and $\left.\mathrm{A}_{2}\right)$ for the baseline and toe-in (Concept 2) cases are nearly equal. If the fin thickness is fairly large and fin pitch values for two cases are also different, then a ratio $\left(A_{0} / A_{2}\right)$ has to be included on the right side of Equation (7-2). Equation (7-2) is applicable to any two situations with different fin pitch, mass flow rate, or hydraulic diameter values. If the pressure drop/friction factor data are compared at the same Reynolds number (based on hydraulic diameter), Equation (7-2) will reduce to Equation (7-3), in which the subscript $\operatorname{Re}_{D h}$ indicates values at specified Reynolds numbers.

$$
\left[\frac{f_{2}}{f_{o}}\right]_{\operatorname{Re}_{D h}}=\left[\frac{\Delta P_{2}}{\Delta P_{o}}\right]_{\operatorname{Re}_{D h}}\left(\frac{D_{h 2}}{D_{h o}}\right)^{3}=\left[\frac{\Delta P_{2}}{\Delta P_{o}}\right]_{\operatorname{Re}_{D h}}\left(\frac{n_{o}}{n_{2}}\right)^{3} .
$$

Values for the $\Delta \mathrm{P}$ ratio at fixed- $\mathrm{Re}_{D h}$ can be obtained from the raw data acquired from the INL Single Blow Test Facility. A plot of this raw pressure-drop data for the baseline and toe-in (Concept 2) cases (obtained with two different fin pitch values) is presented in Figure 7-5. By multiplying the baseline pressure drop data by an arbitrary ratio factor, $r$, the pressure drop ratio at a fixed $R_{D h}$ can be obtained. The best overall fit for the data is obtained for a value of $\mathrm{r}=0.69$, i.e., for a given $\mathrm{Re}_{D h}$; the raw pressure drop for toe-in case was $\sim 31 \%$ less than that for the baseline case, $\left(\Delta \mathrm{P}_{2} / \Delta \mathrm{P}_{\mathrm{o}}\right)_{\mathrm{ReDh}}=0.69$. Friction factors for the baseline and toe-in (Concept 2) cases obtained from the pressure drop values obtained in the INL Single Blow Test Facility are presented in Figure 7-6. The "estimated" friction factor curve is obtained from the baseline friction factors, using Equation (7-3), with a pressure drop ratio at fixed $\operatorname{Re}_{D h}$, of 0.69 and the values of hydraulic diameter listed in Table 7-3. The good agreement between the "estimated" values and the actual toe-in (Concept 2) friction factors indicate the validity of the relationship given in Equation (7-3). 


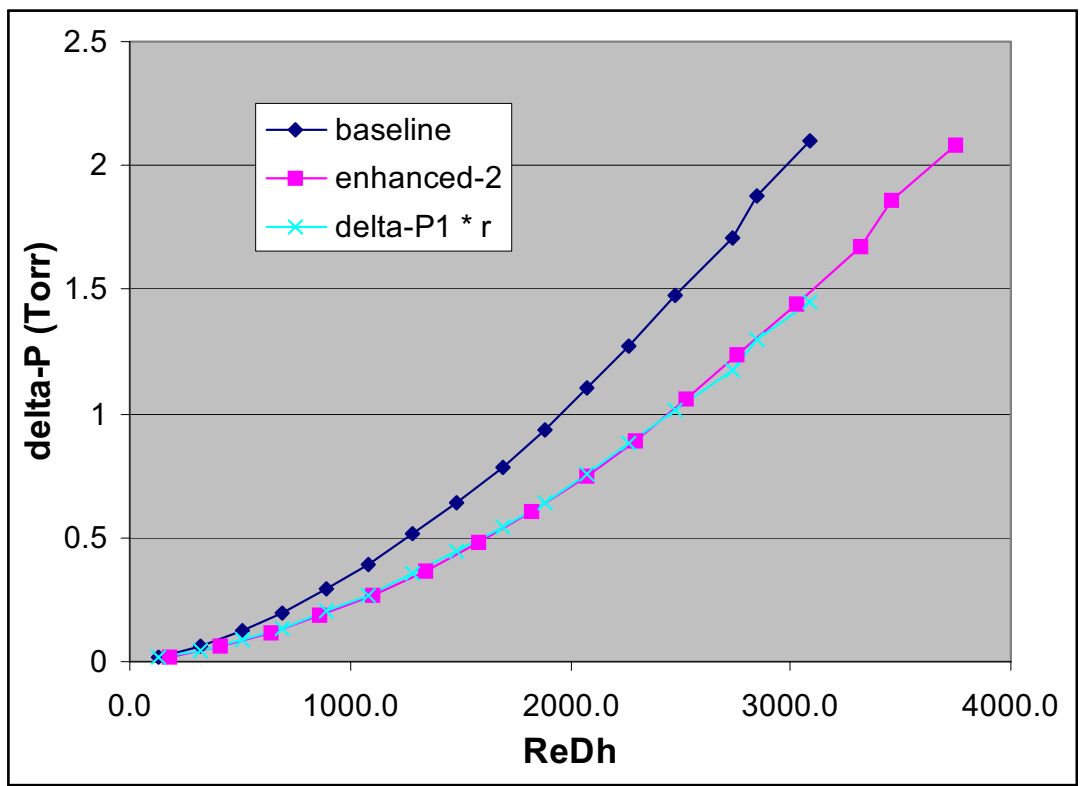

Figure 7-5. Raw pressure drop data versus Reynolds number from the Single Blow Facility.

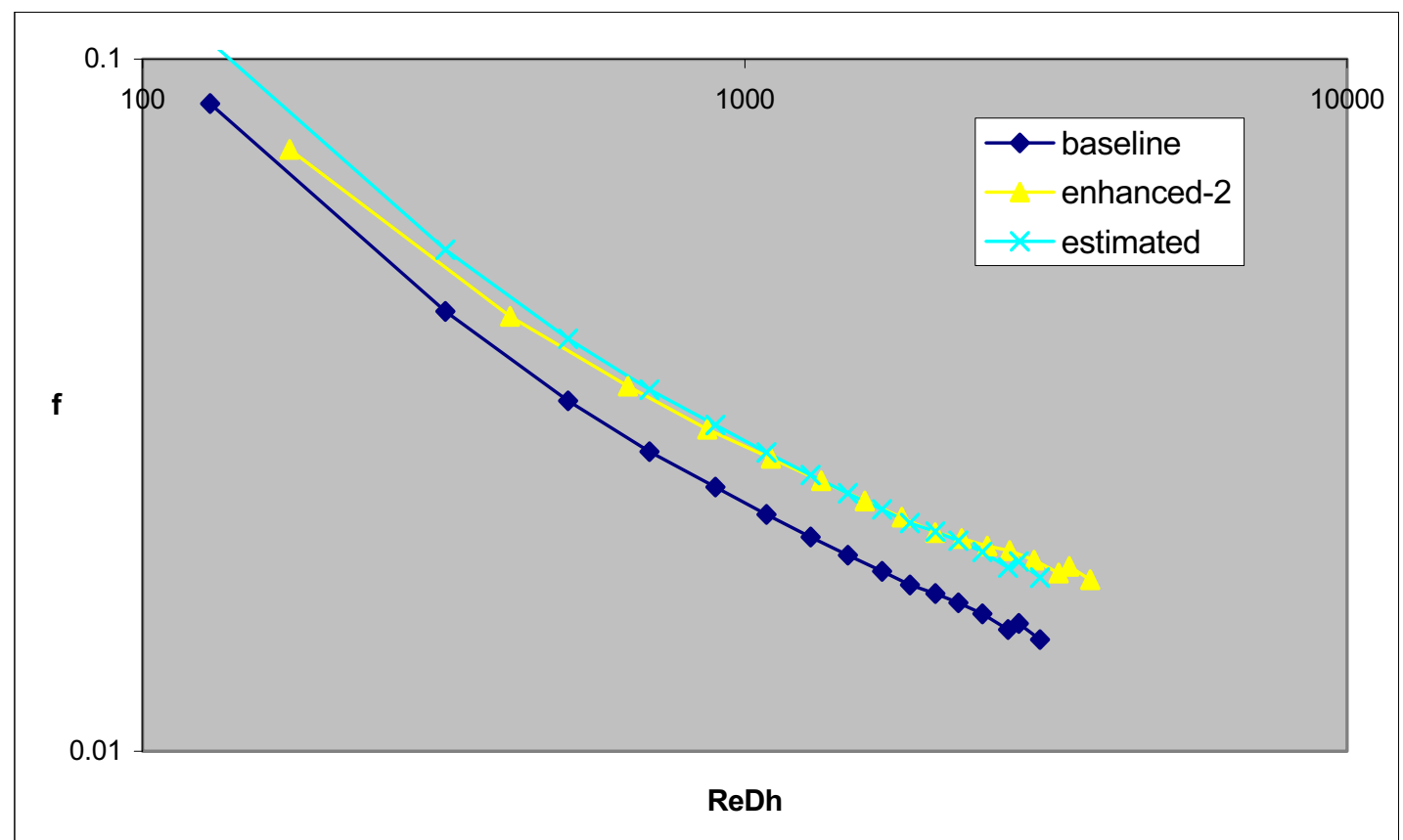

Figure 7-6. Friction factors for the baseline and toe-in (Concept 2) cases.

Equation (7.2) can also be expressed as:

$$
\left[\frac{f_{2}}{f_{o}}\right]=\left[\frac{\Delta P_{2}}{\Delta P_{o}}\right]\left(\frac{\left(G D_{h}\right)_{o}}{\left(G D_{h}\right)_{2}}\right)^{2}\left(\frac{D_{h 2}}{D_{h o}}\right)^{3}=\left[\frac{\Delta P_{2}}{\Delta P_{o}}\right]\left(\frac{(G)_{o}}{(G)_{2}}\right)^{2}\left(\frac{D_{h 2}}{D_{h o}}\right) .
$$


This equation can be used to account for the impact of the different fin pitch values at the same face velocity (mass flux). As indicated, at equivalent flow rates the pressure drop for the toe-in (Concept 2) case was $\sim 10-20 \%$ lower than for the baseline tube bundle. Using the same premise that the ratio of the hydraulic diameters is equivalent to the inverse ratio of the fin pitch values, one can estimate that at the same fluid velocity, the friction factor for the toe-in (Concept 2) case would be $\sim 1$ to 1.12 times that of the baseline configuration.

An analysis was also made using an approach described by Heat Transfer Research, Inc. It calculates the pressure drop based on a maximum mass flux flowing through the minimum duct area (the tube bundle frontal area minus the flow area occupied by the tubes and fins). In this approach, the pressure drop per row of tubes is proportional to the product of the friction factor and the square of the maximum mass flux, with no direct diameter term in the relationship. Using this analysis, the ratio of friction factors is inversely proportional to the square of the minimum flow areas. Because the fins occupy only a small portion of the total flow area, by increasing the fin pitch by $20 \%$, the minimum flow area for the toe-in (Concept 2) case increases by $\sim 1.75 \%$. Using the corresponding change in the mass flux, it was estimated that the friction factor for the toe-in (Concept 2 ) case is $\sim 93 \%$ of the friction factor for the base case corresponding to an equal face velocity of $2 \mathrm{~m} / \mathrm{s}$.

The above calculations for the pressure drop are not necessarily exact and conclusive. However, it does suggest that at equivalent flow rates one might expect the pressure drop for the toe-in (Concept 2) tube bundle to be about similar to that of the plain finned tube bundle.

A similar relation can be developed for Colburn $\mathrm{j}$-factors by rewriting the definition of the $\mathrm{j}$-factor as:

$$
j=\frac{h D_{h}}{G D_{h} c_{p}} \operatorname{Pr}^{2 / 3}
$$

Therefore, for a fixed value of $G D_{h}$,

$$
\left[\frac{j_{2}}{j_{o}}\right]_{\operatorname{Re}_{D h}}=\left(\frac{h_{2}}{h_{o}}\right)_{\operatorname{Re}_{D h}} \frac{D_{h 2}}{D_{h o}}=\left(\frac{h_{2}}{h_{0}}\right)_{\operatorname{Re}_{D h}} \frac{n_{o}}{n_{2}}
$$

A plot of Colburn j-factors obtained for the baseline and toe-in (Concept 2) cases obtained from the INL Single Blow Test Facility is presented in Figure 7-7. The "estimated" curve in this figure is based on Equation. (7-6), with a best-fit $\left(\mathrm{h}_{2} / \mathrm{h}_{\mathrm{o}}\right)_{\text {ReDh }}$ ratio of 1.13 . So, while the $\mathrm{j}$-factor values for the toein (Concept 2) case are about 33\% higher than those for the baseline case, the heat transfer coefficients associated with the toe-in (Concept 2) case are only $\sim 13 \%$ higher than those for the baseline case when compared at the same Reynolds number. Note that the results obtained from the INL Single Blow Test Facility only relate to the air-side heat transfer coefficients, with no fin efficiency or contact resistance effects. If it is assumed that a similar level of air-side heat transfer enhancement would be obtained with a fixed fin spacing, and examine the overall heat transfer situation corresponding to the ITS tests, accounting for inside film coefficient, tube-wall heat conduction, fin contact resistance, and fin efficiency effects, the expected overall heat transfer enhancement associated with an air-side enhancement of $13 \%$ is only $\sim 1.3 \%$. This result appears to be consistent with the experimental results obtained from the ITS tests, which also showed about $\pm 1 \%$ enhancement for a pressure drop increase of $\sim 3-6 \%$ for the toe-in (Concept 2) configuration. 


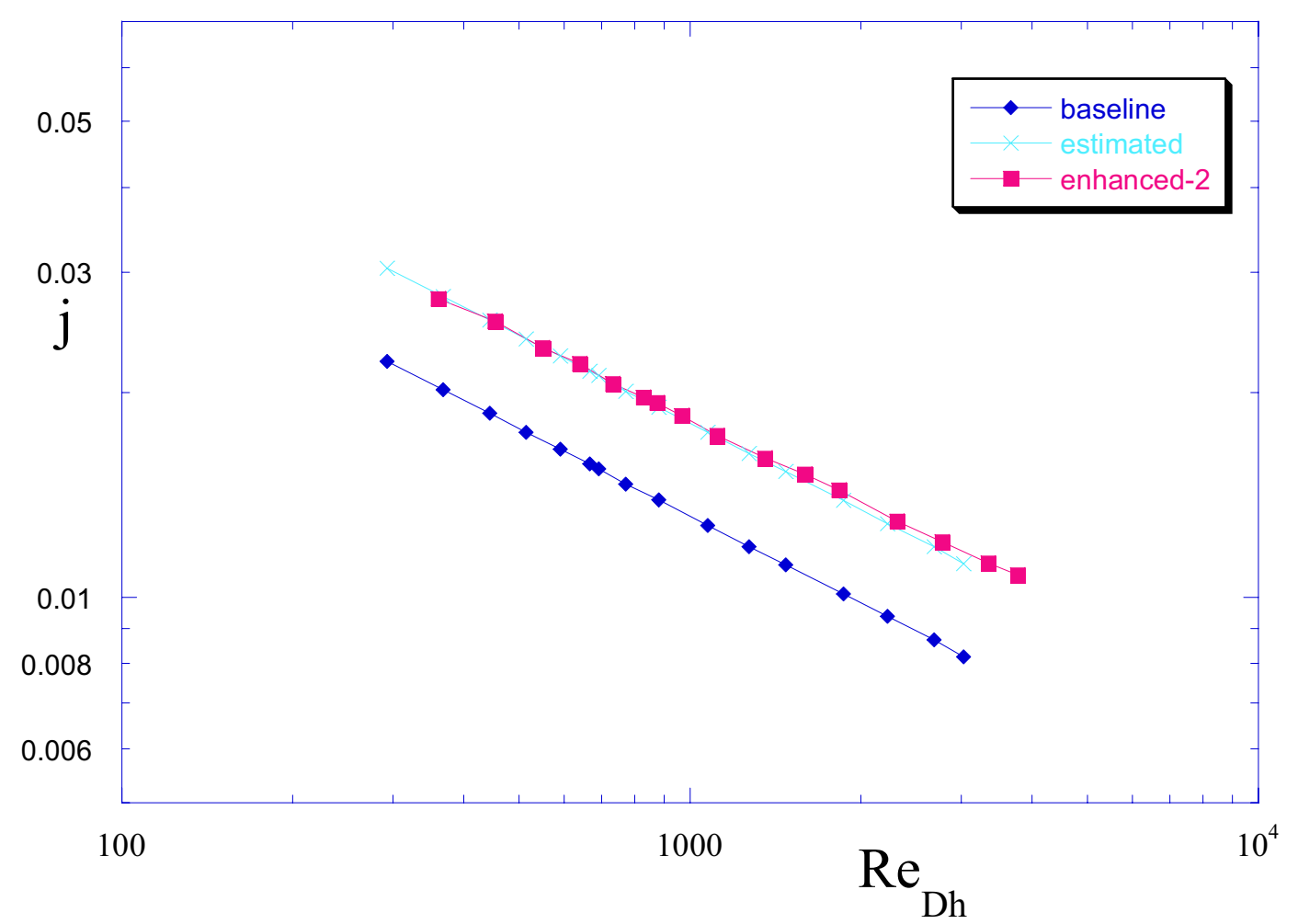

Figure 7.7. Colburn j-factors for the baseline and toe-in (Concept 2) cases from the Single Blow Test Facility.

Finally, it should be noted that the Colburn $\mathrm{j}$-factor and friction factors results are not directly representing heat transfer and pressure drop values. The same thought was also expressed earlier in an INL paper, ${ }^{70}$ which is reproduced here:

"Note that since the fin pitch and resultant unit-cell dimensions for the Concept 2 ("toe-in") case were different than for the baseline case, the values of friction factor and Colburn-j factor do not directly reflect increased pressure-drop and heat transfer coefficient values. In fact, pressure drop values at a specified Reynolds number were significantly lower for the Concept 2 case than for the baseline case, due to the larger fin spacing."

The Intertek data indicate that the Single Blow Test data for the toe-in (Concept 2) performance may have been interpreted in an overly favorable manner when data were compared on the basis of an equivalent Reynolds number. Although resources were not available to repeat the tests with toe-in (Concept 2) tubes having the same fin pitch or perform an extensive analysis of the Single Blow Test data, the brief analysis presented here suggests that earlier conclusions of toe-in (Concept 2) giving better performance than that by toe-out (Concept 1) may not have been based on solid reasoning.

\subsection{Possible Reasons for the Unexpected Performance of the Enhanced Tube Bundle}

Based on the subsequent analysis of the Single Blow Test data, it is possible that the performance of toe-in (Concept 2) winglet configuration was considered to be more optimistic than it might have been in a full-size heat exchanger system. There are also other factors that skewed the actual performance of toein (Concept 2) case. These factors are summarized below. It must be kept in mind that these are merely 
speculative reasons that point out the incomplete and imperfect nature of this research. They also serve to identify potential issues that will require resolution before any final verdict can be issued regarding the value of vortex generators as an enhancement device, especially the toe-in winglet design.

- The flow behavior past the winglets on the fin surface might have changed as a result of expanding the tubes by Super Radiator Coils to obtain good contact between the tube and the fins. It is speculated that the outer radius of the tubes might have increased by about $\sim 5-6 \%$. If indeed there was such an increase in the tube diameter, the winglets would move a bit toward the back/wake region of the tube bundle. This is likely to reduce the heat transfer enhancement.

- Flow behavior between the fins might have been somewhat nonideal, as the fins were not perfectly flat. It can be clearly seen in Figure 7-4 that during mounting and shipping, some of the fins did not remain perfectly flat. This may not be a significant factor, but the exact effect is not known

- Thermal contact resistance between the tube and the fins may be different in the enhanced fin case compared to that in the baseline case. Even an equal but high thermal contact resistance value can mask heat transfer enhancement for the toe-in (Concept 2) case. A few fins (5-6 on one tube) were observed to be loose on the tube. However, the integrity of the middle row of tubes could not be checked. After INL sent the fins to SRC, INL did not perform any quality control tests regarding the uniformity of thermal contact resistance of the finned tubes in the baseline and enhanced finned tubes. As a rough estimate, it is difficult to expect that nonuniform thermal contact resistance could negate the anticipated heat transfer enhancement.

- $\quad$ As noted in all Intertek (ITS) data, there was always an energy balance error within $\pm 3.5 \%$ between the heat absorbed by the cooling air and heat lost by the hot water. It was noted that, generally, heat lost by hot water was higher than the heat absorbed by air. However, for another set of tube bundle tests at ITS (not presented in this report) for a couple of cases, heat lost by water was lower than the heat absorbed by the air. It shows that the relative values of various measuring instruments can change trends from one set of tests to another. Experimental errors of $\pm 2-3 \%$ are possible. If so, an enhancement in heat transfer coefficient of $\sim 1-2 \%$ can get masked by such experimental errors.

- $\quad$ The shape, size, and location of the punched winglets were also checked by punching out a few fins with winglets after the ITS tests. There was no fabrication error in the size, shape, or location of the winglets. However, no test of a full-scale heat exchanger system with enhanced finned tubes was performed in the laboratory at INL before the ITS tests. The Single Blow Test tested only the fins without the participation of the tubes. Without any experimental visualization method, it is difficult to speculate whether the winglets are generating longitudinal vortices, as shown in several numerical results in Sections 4 and 6. If the flow near a winglet is parallel/tangential to the direction of the winglets, there is very little possibility of generating the longitudinal vortices. As mentioned, this is also just speculation.

If one or more of the above possible reasons indeed were encountered, a heat transfer enhancement in overall heat transfer coefficient of $\sim 2-5 \%$ could be difficult to measure. Again, this is speculation; none of the reasons given above could be proven with certainty.

\subsection{Conclusions}

An enhanced finned tube bundle was fabricated by Super Radiator Coils with winglets punched on fins by INL and independently tested by Intertek Testing Services. The performance of the enhanced bundle during this testing did not meet expectations that were based upon the Single Blow Test Facility investigations. It is believed that the test data taken at either facility were not in error; the data likely accurately reflect the performance of the configurations tested at each location. Based upon the available data and related information, it is believed that the methods used to account for the different fin pitch in 
the bundles tested at the Single Blow Test Facility did not adequately reflect the effect of this difference, and overestimated the impact that toe-in (Concept 2) winglet configuration would have on both thermal and hydraulic performance.

The Intertek testing represents a setback in the development of the concept of using winglets to enhance heat transfer performance, and has overshadowed extensive work at INL, as well as the work performed by the various NEDO investigators. As shown in the previous discussions, depending upon how one evaluates the Single Blow Test data to account for the differences in fin pitch, one could show that the Intertek data should have been expected. This would validate the data from the Single Blow Test Facility and indicate that if toe-in (Concept 1) winglets had been chosen for the Intertek tests, the results would have been similar to those obtained at the Single Blow Test Facility. This conclusion is consistent with the results of the investigations conducted by the other NEDO investigators that have been completed. These results suggest that the optimal configuration will likely not be similar to either of the two concepts considered by INL. With an optimized winglet design and precise fabrication, it is possible to have an enhanced tube bundle with a winglet design that provides a small increase in pressure drop with a moderate increase in the overall heat transfer. 


\section{SUMMARY AND CONCLUSIONS}

This report summarizes work by the Idaho National Laboratory (INL) to develop strategies to enhance air-side heat transfer in geothermal air-cooled condensers such that they should neither significantly increase pressure drop nor parasitic fan pumping power. The enhancement was to be accomplished without increasing the capital or operating cost of the total heat rejection system. An effective strategy can result in a reduction in condenser size and/or power consumption. The work was sponsored by NEDO (New Energy and Industrial Technology Development Organization)of Japan, Yokohama National University, and the Indian Institute of Technology, Kanpur, India.

INL performed an experimental study to examine the local and mean heat transfer performance of circular and oval tubes with vortex-generator winglets. Heat transfer measurements were obtained using a transient technique. Corresponding local fin-surface heat transfer coefficients were then calculated. Pressure drop measurements have also been obtained for similar circular and oval tubes with and without winglet geometries using a separate single-channel, multiple-tube-row pressure-drop apparatus. The data were obtained over a Reynolds number range based on a duct height of about 600-6500. Local heat transfer results clearly indicate areas of high fin-surface heat transfer in the stagnation region of the tube, along the side of the tube, and downstream of the winglets. Highest heat transfer coefficients are in the tube stagnation region. The local heat transfer enhancement associated with both the primary vortex and the corner, horseshoe-type vortices produced by each winglet are visible in the heat transfer images. Highest mean heat transfer coefficients were observed for the case of a circular tube plus winglets, with the winglets located on the downstream side of the cylinder in a toe-out (common flow down) or "toe-in" (common flow up) configuration. Evaluation of mean fin-surface heat transfer coefficients indicated that the addition of the single winglet pair to the oval-tube geometry yielded significant heat transfer enhancement, with Colburn $\mathrm{j}$-factor averaging 38\% higher than the oval-tube without any winglets. The corresponding increase in friction factor for an oval tube was very modest, less than $10 \%$ at a Reynolds number of 500 and less than $5 \%$ at a Reynolds number of 5000.

A large flow loop for the purpose of testing a tube bundle designated as the INL Single Blow Test Facility was developed. This facility can accommodate a prototypic tube bundle, with $25.4-\mathrm{cm}$ tube lengths in a $4 \times 4$ bundle. With the heater and test section in place, the flow rate through the loop can yield a Reynolds number range based on fin spacing from 0 to 1500 . Results of the experimental studies show that the heat transfer enhancement levels of 20-30\% were obtained over the tested Reynolds number range of 100-3000, with an increase in pressure drop of only $4-12 \%$ over the same range. Toe-in (common flow up or Concept 2) configuration appeared to be the preferred choice, considering performance.

A computational effort was also completed at INL in coordination with the INL experimental effort. A commercially available computational fluid dynamics code, FLUENT, was used to model heat transfer in various tube-fin configurations. Oval tubes with a 3:1 axis ratio were analyzed because they have a significantly smaller frontal area and form drag than circular tubes for the same cross-sectional area. Numerical simulations for heat transfer performance were compared to experimental results for the Reynolds number (based on fin spacing) $=670$ and 1224, corresponding to air frontal velocities of $1.0 \mathrm{~m} / \mathrm{s}$ and $2.5 \mathrm{~m} / \mathrm{s}$. The optimal configuration is a triangular winglet generator and an oval tube, which produced the best configuration of maximum heat transfer from the fin surface at a minimal pressure drop. The results of this analysis show a favorable performance ratio (Colburn $\mathrm{j}$-factor increase ratio/friction factor increase ratio) of $\sim 2.22$ over the range of the Reynolds number analyzed. Four oval tube-winglet configurations were analyzed. These results show that a toe-in configuration (Configuration 3) suggested by the Yokohama National University team for circular in-line and staggered tube bundles modified for oval tubes produced the best results. The performance ratio averaged $\sim 1.13$ over the range of ReH tested. Detailed interrelated fluid flow and heat transfer pattern analysis show that the configurations that produced only secondary effects produced considerably better performance results. 
The selected configuration was tested for both in-line and staggered oval tube bundle arrangements. This configuration produced good results for the in-line tube bundle, with the best results being produced for one row of winglets in the first tube row. Favorable performance ratio results were achieved across the Reynolds number range of 660-1630. Additional rows of winglets were not justified in this case, since less favorable performance results were obtained with additional rows of winglets. Significantly better results were obtained for the staggered tube bank arrangement with the analyzed winglet configurations. For the staggered tube bank arrangement, the best performance was obtained with three rows of winglets, giving performance ratios of 1.10-1.16 for ReH values of 660-1630, respectively. This analysis has shown that winglets orientated as shown in Configuration 3 have a very favorable performance ratio over the range of operation for both in-line and staggered tube bank arrangements.

The Yokohama National University team measured heat transfer enhancement and also visualized the flow behavior in a tube bundle with vortex generators. After initial tests, the university team proposed a novel strategy that could augment heat transfer and also reduce pressure loss in a finned circular tube heat exchanger in a relatively low Reynolds number flow, by deploying delta winglet-type vortex generators. The winglets are placed in a toe-in (common flow up) configuration. For the case of threerow staggered tube banks, the heat transfer (Colburn j-factor) was successfully augmented by 10 to $30 \%$, and the corresponding friction factor was reduced by 55 to 34\% for the Reynolds number (based on two times channel height) ranging from 350 to 2100. The winglets were employed only in the first (transverse) row of tubes. For the case of the in-line tube bundle, the winglets increase the heat transfer (j-factor) by 10 to $20 \%$ and decrease the pressure drop by 8 to $15 \%$ for the Reynolds numbers ranging from 350 to 2100 . The nozzle-like flow passages created by the delta winglet pair and the aft region of the circular tube promote acceleration to bring about a separation delay and form drag reduction of the tube, and remove the zone of poor heat transfer from the wake.

Local Nusselt number distributions on the bottom surface of the channel with built-in tube-bundles with winglet-pairs simulating a single passage of a fin-tube heat exchanger were obtained using an infrared camera by a transient heat transfer measurement technique. For the case of the channel with a built-in single row of tube bundles with winglet pairs, the experimental results show good agreement with the numerical results obtained by G. Biswas of the Indian Institute of Technology. For three rows of tube bundles with a single front row of winglet pair, the details of the heat transfer enhancement distribution were obtained. The heat transfer enhancement of span-averaged Nusselt number reaches the maximum value near the middle between the first and second rows of the tube, and persists far downstream up to the third row. Even behind the third tube-row, the heat transfer of the so-called dead zone was enhanced well by the winglet pairs.

A particle-image-velocimetry (PIV) technique was successfully developed to visualize qualitatively the complex flow structure of the channel with built-in tube bundles with winglets, and elucidated well the mechanism of the heat transfer enhancement. For the case of the channel with a built-in single row of tube bundles with winglet pairs, the experimental results show good agreement with the numerical results by G. Biswas (IITK). For the three rows of tube bundles with a single front row of winglet pair, details were obtained of the heat transfer enhancement distribution. The heat transfer enhancement of spanaveraged Nusselt number reaches the maximum value near the middle between the first and second rows of the tube and persists far downstream up to the third row. Even behind the third tube-row, the heat transfer of the so-called dead zone was enhanced. The configurations of tubes and winglets proposed by the YNU team have the favorable effect of introducing high-momentum fluid into the stagnant region behind the tubes. This effect of winglets, coupled with their capability of creating longitudinal vortical motions, explains the remarkable heat-transfer enhancement. The wall shear stress distributions evaluated from the whole-field velocity data are consistent, at least qualitatively, with the observed heat-transfer enhancement. The present flow fields exhibit significant unsteadiness in the regions where vortical motions exist. The presence of unsteadiness implies that any interpretation and prediction based on the 
assumption of steadiness will fail, in quantitative manner, to capture the true mechanisms happening in the flow fields.

The Indian Institute of Technology, Kanpur, India team developed a three-dimensional numerical study on the flow and heat transfer characteristics in a narrow rectangular duct with a built-in circular and oval tube in a cross-flow configuration. The duct was designed to simulate a passage formed by any two neighboring fins in a fin-tube heat exchanger. The horseshoe vortices are generated at the junction of the forward stagnation line and the bottom plate. The horseshoe vortices travel downstream, inducing a flow structure that is primarily a longitudinal vortex system. However, this natural horseshoe vortex system does not persist downstream. At the rear of a circular, it leads to a screw-like motion of a helical vortex tube. Limiting streamlines on the tube surface showed typical separation lines. Limiting streamlines on the bottom wall clearly established the presence of a saddle point of separation, a nodal point of attachment in front of the tube and bottom wall junction, and a horseshoe vortex system that wraps around the tube and extends to the rear of the tube. The span-averaged Nusselt number distribution and the isoNusselt number distribution clearly establish the high heat transfer near the leading edge of the fins and in the region influenced by the horseshoe vortex system. Poor heat transfer in the dead wake region is observed. The zone of poor heat transfer can possibly be removed by inducing a swirling motion in the wake region.

Longitudinal vortices can be created by winglet-type vortex generators. The present analysis reveals that the combination of circular tube and delta winglet pair with toe-in (common-flow-up) configuration improves the heat transfer performance even more. The nozzle-like flow passages created by the delta winglet pair and the aft region of the circular tube promote acceleration and thereby remove the zone of poor heat transfer from the near wake. At the end of a channel, the span-averaged Nusselt number (based on channel height) near the exit of a plane channel is 3.98 for a Reynolds number of 1000 . The spanaveraged value of Nusselt number (based on channel height) near the channel exit is 5.7 for the same Reynolds number. The average Nusselt number of the entire bottom plate for the case of a channel with a built-in circular tube is 5.37 for a Reynolds number of 1000 . The average Nusselt number of the plate improves to a value of 7.27 for the same Reynolds number when the winglet pair is added following the toe-in (common-flow-up) configuration.

A study was completed to determine the flow structure and heat transfer in a rectangular channel with a built-in oval tube and delta-winglet type vortex generators in various configurations. The performance of a heat exchanger module with the oval tube and the delta winglet pair appears to be the best of the three configurations examined in this investigation. The present study reveals that combinations of oval tube and the winglet pairs improve the heat transfer significantly, especially in the dead wake region. The mean span-averaged Nusselt number for the case of four winglet pairs, each two in sequence having a staggered configuration (inner pair in toe-out and the outer pair in toe-in arrangement) is about $100 \%$ higher compared to the no-winglet case at a Reynolds number of 1000 . The enhancement in heat transfer compared to a finned oval tube as the base line case, is $43.86 \%$ for the case of two winglet pairs in staggered mode. A comparison of heat transfer for the cases of one, two, and three winglet pairs (all in toe-out configuration) confirms that the addition of each extra winglet pair causes further enhancement of heat transfer.

The longitudinal vortices generated by the winglets located away from the tube wall are stronger and last longer. Vortical motion produced by the staggered winglet arrangements are stronger than that produced by the in-line arrangements. The winglets that are farther away from the tube bring about more heat transfer enhancement than the nearer winglets. The winglets located far downstream produce higher local heat transfer but, due to the domain size, their effect is not fully utilized. From the span-averaged Nusselt number distribution, Conf-9 emerges as the best configuration. Mean (overall) Nusselt number enhancement is $35.37 \%$ higher compared to the no-winglet case. 
An enhanced finned tube bundle was fabricated by Super Radiator Coils and INL. It was independently tested by Intertek Testing Services. The performance results were not as impressive as had been anticipated after the Single Blow Test data. A possible reason, or a combination of reasons, listed above for unexpected heat transfer performance can only be speculated. It is also highly likely that the ITS test results are correct, leading to the thought that the winglet design for circular tubes is yet to be optimized. The available ITS data are not sufficient to pass judgment on the applicability of an optimized winglet design for air-cooled condensers. With an optimized winglet design and precise manufacturing technique, it may be possible to have an enhanced bundle that gives low pressure drop increase and moderate increase in overall heat transfer.

Experimental and numerical modeling work by three independent research institutions present a consistent picture of the impact of winglet type vortex generators. It is obvious that the winglets yield performance enhancement, but their design needs further optimization. These conclusions can be summarized as follows.

1. Triangular (delta) type vortex generators can enhance air-side heat transfer (generally Colburn jfactor) by about $10-30 \%$, depending on several physical parameters, i.e., winglet size, winglet location, and flow parameters. As a general rule, the toe-in (common flow up) type winglets give a better performance ratio (enhancement increase ratio/friction factor increase ratio) compared to the toe-out (common flow down) type winglets.

2. A toe-in winglet design based on Yokohama National University findings (located a bit on the upstream side and with a smaller angle of attack) should be further examined for practical applications. An optimum design of this winglet can have a very small pressure drop increase with moderate heat transfer enhancement.

3. The heat transfer rate and pressure drop are not identical to the Colburn j-factor and friction factor, respectively. Therefore, the performance of a heat exchanger bundle may be different than the Colburn $\mathrm{j}$-factor and friction factor obtained in laboratory-scale experiments.

4. If manufacturing costs can be kept under control, oval tubes with a special toe-in winglet design should be considered for further investigation, as the oval tubes inherently reduce the pressure drop compared to circular tubes. Numerical modeling has shown their improved performance.

5. Even though the performance test results (heat transfer rate and pressure drop) of INL's finned tube bundle with enhanced winglets appear to be reasonable, the test raised some questions relating to the winglet design, quality control of the bundle fabrication, and testing procedure.

6. Based on the numerical results, it can be seen that in a full-scale tube bundle heat exchanger, the flow distribution after the first row of tubes, can be somewhat different than that corresponding to the first row of tubes. Hence, the angle between the flow streamlines and winglet angle of attack can be different than corresponding to the first row. Therefore, for a full-scale tube bundle design, it has to be decided whether it is beneficial to have enhancement beyond the first or second tube rows. If there is a need for such enhancement, the design of the enhancement vortex generator may have to be fine tuned for optimum performance. 


\section{REFERENCES}

1. C. E. Holt, "Next generation Geothermal Power Plants," EPRI RP 3657-01, September 1995.

2. M. Fiebig, "Vortices Generators and Heat Transfer," Chemical Engineering Research and Design (Trans. IChemE), Vol., 76, Part A2, 1998, pp. 108-123.

3. A. M. Jacobi, and R. K. Shah, "Heat Transfer Surface Enhancement through the Use of Longitudinal Vortices: A Review of Recent Progress," Experimental Thermal and Fluid Science, Vol. 11, 1995, pp. 295-309.

4. R. L. Webb and A. Iyengar, "Oval Finned Tube Condenser and Design Pressure Limits," presented at the International Conference and Exhibit on Heat Exchangers for Sustainable Development, Lisbon, Portugal, June 15-18, 1998.

5. S. Tiggelbeck, N. K.Mitra, and M. Fiebig, "Experimental Investigations of Heat Transfer Enhancement and Flow Losses in a Channel with Double Rows of Longitudinal Vortex Generators," International Journal of Heat and Mass Transfer, Vol. 36, No. 9, 1993, pp. 2327-2337.

6. K. Torii, K., Nishino, and K. Nakayama, "Mechanism of Heat Transfer Augmentation by Longitudinal Vortices in a Flat Plate Boundary Layer," Proceedings of Tenth International Heat Transfer Conference, Brighton, England, Vol. 6, 1994, pp. 123-128, Hemisphere Publishing Co., New York.

7. M. Fiebig and N. K. Mitra, "Experimental and Numerical Investigation of Heat Transfer Enhancement with Wing-Type Vortex Generators," Chapter 8 in Computer Simulations in Compact Heat Exchangers, B. Sünden and M. Faghri (eds.), Computational Mechanics Publications, 1997.

8. Y. Chen, M. Fiebig, and N. K. Mitra, "Conjugate Heat Transfer of a Finned Oval Tube Part B: Heat Transfer Behaviors," Numerical Heat Transfer, Part A, Vol. 33, 1998, pp. 387-401.

9. Y. Chen, M. Fiebig, and N. K. Mitra, "Heat Transfer Enhancement of a Finned Oval Tube with Punched Longitudinal Vortex Generators In-line," International Journal of Heat and Mass Transfer, Vol. 41, 1998, pp. 4151-4166.

10. Y. Chen, M. Fiebig, and N. K. Mitra, "Heat Transfer Enhancement of Finned Oval Tubes with Staggered Punched Longitudinal Vortex Generators," International Journal of Heat and Mass Transfer, Vol. 43, 2000, pp. 417-435.

11. J. E. O’Brien and M. S. Sohal, "Local Heat Transfer for Finned-Tube Heat Exchangers using Oval Tubes," Proceedings of the 2000 ASME National Heat Transfer Conference, Pittsburgh, Pennsylvania, August 2000, paper NHTC2000-12093.

12. S. Carlsen, "TIFF revision 6.0," Aldus Developers Desk (http://icib.igd.fhg.de/icib/it/defacto/ company/aldus/read.html\#SEC_2), Aldus Corporation, 1992.

13. F. P. Incropera and D. P. DeWitt, Fundamentals of Heat and Mass Transfer, $3^{\text {rd }}$ edition, New York: John Wiley and Sons, 1990.

14. R. J. Moffatt, "Contributions to the Theory of Single-Sample Uncertainty Analysis," Journal of Fluids Engineering, Vol. 104, 1982, pp. 250-260.

15. W. Krückels, and V. Kottke, "Untersuchung über die Verteilung des Wärmeübergangs an Rippen und Rippenrohr-Modellen," Chemie Ingenieur Technik, Vol. 42, No. 6, 1970, pp. 355-362.

16. R. J. Goldstein and J. Karni, "The Effect of a Wall Boundary Layer on Local Mass Transfer from a Cylinder in Crossflow," Journal of Heat Transfer, Vol. 106, 1984, pp. 260-267 
17. P. T. Ireland, and T. V. Jones, "Detailed Measurements of Heat Transfer on and around a Pedestal in Fully Developed Passage Flow," Proceedings of $8^{\text {th }}$ International Heat Transfer Conference, San Francisco, California, Vol. 3, 1986, pp. 975-980.

18. C. J. Baker, "The Laminar Horseshoe Vortex,” Journal of Fluid Mechanics, Vol. 95, part 2, 1979, pp. 347-367.

19. M. Fiebig, A. Valencia, and N. K. Mitra, "Wing-Type Vortex Generators for Fin-and-Tube Heat Exchangers," Experimental Thermal Fluid Science, Vol. 7, 1993, pp. 287-296.

20. B. S. Petukhov, "Heat Transfer in Turbulent Pipe Flow with Variable Physical Properties," Advances in Heat Transfer, Vol. 6, T. F. Irvine and J. P. Hartnett, eds., New York: Academic Press, 1970, pp. 504-564.

21. S. Mochizuki, Y. Yagi, and W. J. Yang, "Advances in Single-Blow Method for Performance Evaluation of Heat Transfer Surfaces," Proceedings, $2^{\text {nd }}$ International Symposium on Heat Transfer, Beijing, China, Vol. 1, 1988, pp. 284-291.

22. J. E. O'Brien and M. S. Sohal, "Heat Transfer Enhancement for Finned-Tube Heat Exchangers with Winglets," Proceedings, 2000 ASME International Congress and Exposition, Orlando, Florida, HTD-Vol. 365, November 2000, pp. 137-146.

23. W. Roetzel, "Transient Analysis in Heat Exchangers/Single-Blow Testing Technique, Off-line Data Processing System," version 2.0, April, 2001.

24. X. Luo, and W. Roetzel, "The Single-Blow Transient Testing Technique for Plate-Fin Heat Exchangers," International Journal of Heat and Mass Transfer, Vol. 44, 2001, pp. 3745-3753.

25. R. J. Moffatt, "Describing the Uncertainties in Experimental Results," Experimental Thermal and Fluid Science, Vol. 1, 1988, pp. 3-17.

26. R. L. Webb, Principles of Enhanced Heat Transfer, New York: Wiley, 1994.

27. T. D. Foust, Heat Transfer Enhancement at Minimal Pressure Drop Through the Combined Use of Oval Tubes and Winglet Vortex Generators, Ph. D. Thesis, University of Idaho, Moscow, ID 2004.

28. K. N. Atkinson, R. Drakulic, M. R. Heikal and T. A. Cowell, "Two and Three-Dimensional Numerical Models of Flow and Heat Transfer over Louvered Fin Arrays in Compact Heat Exchangers," International Journal of Heat and Mass Transfer, Vol. 41, 1998, pp. 4063-4089.

29. Y. Chen, M. Fiebig, and N. K. Mitra, "Conjugate Heat Transfer of a Finned Oval Tube with a Punched Longitudinal Vortex Generator in Form of a Delta Winglet Parametric Investigations of the Winglet," International Journal of Heat and Mass Transfer, Vol. 41, 1998, pp. 3961-3978.

30. L. W. Zhang, D.K. Tafli, F.M. Najjar, and S. Balachandar, "Computations of Flow and Heat Transfer in Parallel-Plate Fin Heat Exchangers on the CM-5: Effects of Flow Unsteadiness and Three-Dimensionality," International Journal of Heat and Mass Transfer, Vol. 40, 1997, pp. 13251341.

31. T. Cebeci and P. Bradshaw, Physical and Computational Aspects of Convective Heat Transfer, New York: Springer-Verlag Inc., 1984.

32. A. V. Luikov, "Conjugate Convective Heat Transfer Problem," International Journal of Heat and Mass Transfer, Vol. 17, 1974, pp. 257-265.

33. E. M. Sparrow and S. Acharya, "A Natural Convection Fin with a Solution-Determined Nonmonotonically Varying Heat Transfer Coefficient," Journal of Heat Transfer, Vol. 103, 1981, pp. 218-225. 
34. E. M. Sparrow and M. K. Chyu, "Conjugate Forced Convection-Conduction Analysis of Heat Transfer in a Plate Fin,” Journal of Heat Transfer, Vol. 104, 1982, pp. 204-206.

35. Gambit 1.0 Modeling Guide, Fluent Incorporated, 1998.

36. S. A. Canann, Y. C. Liu and A. V. Mobley, "Automatic 3D Surface Meshing to Address today's Industrial Needs," Finite Elements in Analysis and Design, Vol. 25, 1997, pp. 185-198.

37. J. Liang and J. Yang, "Experimental and 3-D Numerical Analysis of the Thermal-Hydraulic Characteristics of Elliptic Finned-Tube Heat Exchangers," Heat Transfer Engineering, Vol. 19, No. 6, 1998, pp. 55-67.

38. J. S. Leu, M. S. Liu, J. S. Liaw, and C. C. Wang, "A Numerical Investigation of Louvered Fin-andTube Heat Exchangers having Circular and Oval Tube Configurations," International Journal of Heat and Mass Transfer, Vol. 44, 2001, pp. 4235-4243.

39. V. Prabhakar, G. Biswas and V. Eswaran, "Numerical Prediction of Heat Transfer in a Channel with a Built-in Oval Tube and Two Different Shaped Vortex Generators," Numerical Heat Transfer, Part A, Vol. 41, 2002, pp. 307-329.

40. C. M. B Russell, T. V. Jones, and G. H. Lee, "Heat Transfer Enhancement using Vortex Generators," Proceedings of $7^{\text {th }}$ International Heat Transfer Conference, Vol. 3, pp. 283-288, Munich, Germany, 1982, Hemisphere Publishing Co, Washington, DC.

41. K. Torii and J. I. Yanagihara, "The Effects Of Longitudinal Vortices On Heat Transfer Of Laminar Boundary Layer,” JSME International Journal, Series II, Vol. 32, 1989, pp. 395-402.

42. K. Torii, J. I. Yanagihara, and Y. Nagai, "Heat Transfer Enhancement by Vortex Generators," Proceedings of 3rd ASME/JSME Thermal Engineering Joint Conference, J. R. Lloyd and Y. Kurosaka (eds.), 1991, ASME Book I0309C, pp. 77-83.

43. J. I. Yanagihara and K. Torii, "Enhancement of Laminar Boundary Layer Heat Transfer by a Vortex Generator," JSME International Journal, Series II, Vol. 35, 1992, pp. 400-405.

44. J. I. Yanagihara and K. Torii, "Heat Transfer Characteristics of Laminar Boundary Layers in the Presence of Vortex Generators," Proceedings of $9^{\text {th }}$ International Heat Transfer Conference, Jerusalem, Israel, G. Hetsroni, ed., Vol. 6, 1990, pp. 323-328, Hemisphere Publishing Co, Washington, DC.

45. J. I. Yanagihara and K. Torii, "Enhancement of laminar boundary layer heat transfer by longitudinal vortices," Proceedings of 4th International Symposium on Transport Phenomena in Heat and Mass Transfer, G. V. Davis, ed., Washington, DC: Hemisphere Publishing Co., 1991, pp. 942-953.

46. J. I. Yanagihara and K. Torii, "Heat Transfer Augmentation by Longitudinal Vortices Rows," Experimental Heat Transfer, Fluid Mechanics and Thermodynamics, 1993, pp. 560-567.

47. M. Fiebig, P. Kallweit, N. K. Mitra, and S. Tiggelbeck, "Heat Transfer Enhancement and Drag by Longitudinal Vortex Generators in Channel Flow, Experimental Thermal and Fluid Science, Vol. 4, 1991, pp. 103-114.

48. S. Tiggelbeck, N. K. Mitra, and M. Fiebig, "Experimental Investigations of Heat Transfer Enhancement and Flow Losses in a Channel with Double Rows of Longitudinal Vortex Generators," International Journal of Heat and Mass Transfer, Vol. 36, 1993, pp. 2327-2337.

49. St. Tiggelbeck, Experimentalle Untersuchungen an Kanalströmungen mit Einzel- und Doppelwirbelerzeuger-Reihen für den Einsatz in kompakten Wärmetauschern, Ph. D. Dissertation, Institut für Thermo- und Fluiddynamik, Ruhr-Universität Bochum, Germany, 1990. 
50. Th. Gäüntermann, Dreidimensionale stationäre und selbsterregt-schwingende Strömungs- und Temperaturfelder in Hochleistungs-Wärmeübertragern mit Wirbelerzeugern, Ph. D. Dissertation, Institut für Thermo- und Fluiddynamik, Ruhr-Universität Bochum, Germany, 1992.

51. A. Valencia, Wärmeübergang und Druckverlust in Lamellen-Rohr-Wärmeübertragern mit Längswirbelerzeugern, Ph. D. Dissertation, Institut für Thermo- und Fluiddynamik, Ruhr-Universität Bochum, Germany, 1993. Also, A. Valencia, M. Fiebig, and N. K. Mitra, "Heat Transfer Enhancement by Longitudinal Vortices in Fin-Tube Heat Exchanger Element with Flat Tubes," Journal of Heat Transfer, Vol. 118, 1996, pp. 209-211.

52. G. Biswas, and H. Chattopadhyay, "Heat Transfer in a Channel with Built-in Wing-Type Vortex Generators," International Journal of Heat and Mass Transfer, Vol. 35, 1992, pp. 803-814.

53. V. Prabhakar, G. Biswas, and V. Eswaran, "Numerical Prediction of Heat Transfer in a Channel with a Built-in Oval Tube and Two Different Shaped Vortex Generators," Numerical Heat Transfer, Part A, Vol. 41, 2002, pp. 307-329.

54. K. Torii, K. M. Kwak, and K. Nishino, "Heat Transfer Enhancement Accompanying Pressure-Loss Reduction with Winglet-type Vortex Generators for Fin-Tube Heat Exchangers," International Journal of Heat and Mass Transfer, Vol. 45, 2002, pp. 3795-3801.

55. A. Jain, G. Biswas, and D Maurya, "Winglet-Type Vortex Generators with Common-Flow-up Configuration for Fin-Tube Heat Exchangers," Numerical Heat Transfer, Part A, Vol. 42, 2003, pp. 201-219.

56. R. Kawai, K. Nishino and K. Torii, "PIV Measurement of 3-D Velocity Distribution Around Finned Tubes with Vortex Generators," 4th International Symposium on Particle Image Velocimetry, Göttingen, Germany, September 17-19, 2001, p. 1100.

57. J. Westerweel, Digital Image Velocimetry - Theory and Application, Ph. D. Thesis, Delft University of Technology, Delft, The Netherlands, 1993.

58. J. Westerweel, "Fundamentals of Digital Particle Image Velocimetry," Measurement Science and Technology, Vol. 8, 1997, pp. 1379-1392.

59. C. D. Meinhart, A. K. Prasad, and R. J. Adrian, "A parallel Digital Processor System for Particle Image Velocimetry," Measurement Science and Technology, Vol. 4, 1993, pp. 619-626.

60. I. Orlanski, “A Simple Boundary Condition for Unbounded flows,” J. Computational Physics, Vol. 21, 1976, pp. 251-269.

61. V. Eswaran and S. Prakash, "A Finite Volume Method for Navier-Stokes Equations," Proceedings of the Third Asian CFD Conference, Bangalore, India, Vol. 1, 1998, pp. 127-133.

62. F. H. Harlow and W. E. Welch, "Numerical Calculation of Time-Dependent Viscous Incompressible Flow of Fluid with Free Surface," Physics of Fluids, Vol. 8, 1965, pp. 2182-2188.

63. W. Kordulla and M. Vinokur, "Effcient Computation of Volume in Flow Predictions," AIAA Journal, Vol. 21, 1983, pp. 917-918.

64. M. Peric, A Finite Volume Method for the Prediction of Three-Dimensional Fluid Flow in Complex Ducts, Ph.D. Thesis, University of London, 1985.

65. J. H. Ferziger and M. Peric, Computational Methods for Fluid Dynamics, Springer-Verlag, Berlin, New York, NY, 1999.

66. T. D. Foust, J. E. O'Brien, and M. S. Sohal, "Numerical and Experimental Methods for Heat Transfer Enhancement for Finned Tube Heat Exchangers with Oval Tubes," Proceeding of the NHTC'01 35th National Heat Transfer Conference, Anaheim, CA, 2001. 
67. M. Hagedorn, Untersuchung des Wä rmeüberganges und des Strömungsverlustes an Lamellen Rohr Wärmeübertragermodulen unter Berücksichtigung der Wärmeleitung in den Lamellen, Diplomarbeit No. 9507, Institut für Thermo- und Fluiddynamik, RuhrUniversität Bochum, Germany, 1995.

68. G. Biswas, K. Torii, D. Fujii, and K. Nishino, "Numerical and Experimental Determination of Flow Structure and Heat Transfer Effects of Longitudinal Vortices in a Channel Flow," International Journal of Heat and Mass Transfer, Vol. 39, 1996, pp. 3441-3451.

69. G. Biswas and N. K. Mitra, Longitudinal Vortex Generators for Enhancement of Heat Transfer in Heat Exchanger Applications, Proceedings of the $11^{\text {th }}$ International Heat Transfer Conference, Kyongju, Korea, Vol. 5, 1998, pp. 339-344.

70. J. E. O'Brien, M. S. Sohal, and P. C. Wallstedt, "Heat Transfer Testing of Enhanced Finned-Tube Bundles using the Single-Blow Technique," Proceedings of the 2003 ASME Summer Heat Transfer Conference, Las Vegas, NV, July 21-23, 2003. 